\title{
Approaches for Transition Metal-Free Photocatalytic Oxidation
}

Reactions

Dissertation

for the award of the degree

"Doctor rerum naturalium"

of the Georg August University Göttingen

GEORG-AUGUST-UNIVERSITÄT

Within the doctoral program of chemistry of the

Georg August University School of Science (GAUSS)

Submitted by

Waldemar Schilling

From Duschanbe

Göttingen, 2021 



\section{Approaches for Transition Metal-Free Photocatalytic Oxidation}

Reactions

Dissertation

for the award of the degree

"Doctor rerum naturalium"

of the Georg August University Göttingen

GEORG-AUGUST-UNIVERSITÄT

Within the doctoral program of chemistry of the

Georg August University School of Science (GAUSS)

Submitted by

Waldemar Schilling

From Duschanbe

Göttingen, 2021 



\section{Thesis committee}

Prof. Dr. Shoubhik Das, Institute of Organic and Biomolecular Chemistry, Georg August University Göttingen / Department of Chemistry, University of Antwerp (Belgium)

Prof. Dr. Manuel Alcarazo, Institute of Organic and Biomolecular Chemistry, Georg August University Göttingen

\section{Examination Board}

Reviewer: Prof. Dr. Shoubhik Das, Institute of Organic and Biomolecular Chemistry, Georg August University Göttingen / Department of Chemistry, University of Antwerp (Belgium) Second Reviewer: Prof. Dr. Manuel Alcarazo, Institute of Organic and Biomolecular Chemistry, Georg August University Göttingen

\section{Further Members of the Examination Board}

Prof. Dr. Dietmar Stalke, Institute of Inorganic Chemistry, GeorgAugust University Göttingen

Prof. Dr. Konrad Koszinowski, Institute of Organic and Biomolecular Chemistry, Georg August University Göttingen

Prof. Dr. Lutz Tietze, Institute of Organic and Biomolecular Chemistry, Georg August University Göttingen Jun.-Prof. Dr. Johannes Walker, Institute of Organic and Biomolecular Chemistry, Georg August University Göttingen 



\section{Acknowledgment}

First and foremost, I would like to thank my dedicated supervisor and I would like to express my sincerest gratitude to Prof. Dr. Shoubhik Das as my doctoral thesis supervisor. He provided me the opportunity to perform research in an interesting field of chemistry and the possibilities to investigate the physical and technical aspects of our research.

Secondly, I would like to thank my second supervisor Prof. Dr. Manuel Alcarazo for his kind support and crucial scientific and administrative feedback which guided me through my time of research for this thesis.

Furthermore, I would like to thank the members of the examination board Prof. Dr. Konrad Koszinowski, Prof. Dr. Lutz Tietze, Jun.-Prof. Dr. Johannes Walker and Prof. Dr. Dietmar Stalke.

Additionally, I am grateful to several members of the faculty, namely Dr. Andrea Dietrich, Ralf Gerke, Hans-Peter Kroll for their kind support in the administrative and technical aspects of the work. Also, special thanks to Dr. Holm Frauendorf, Frank Hambloch and Hans-Peter Kroll for the direct access to their analytical equipment which enabled a fast and efficient work progress. From the faculty of physics, I would like to thank Karin Ahlborn, Kerstin Born and Prof. Dr. Volkert for the access to their high temperature oven equipment, which was used for the synthesis of carbon nitrides.

Thirdly, I would also like to thank all the active and former members of the research group for their comradeship and assistance, which urged and motivated all of us to never give up, overcome any obstacles and to share afterwards the joyful moments in the final successes.

Last but not least, I thank my parents for raising me and providing me the constant support to become a scientist and special thanks to my wife Christina Koutra Ketsetzi, who supported me in all possible ways during this time. 



\section{Contents}

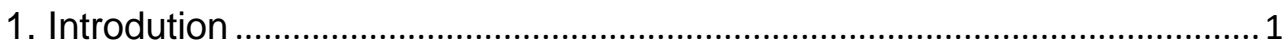

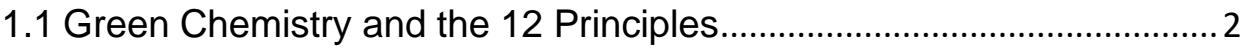

1.2 Photochemistry and Photocatalysis..................................................... 6

1.2.1 The Impact of Experimental Design ............................................. 8

1.2.2 Photocatalysts and the Design of Photocatalysts .........................12

1.2.3 Design of Heterogeneous Photocatalysts and Supporting Structures, Graphitic Carbon Nitrides ................................................... 16

1.3 Oxygen and its Activation to Reactive Oxygen Species...................... 20

1.4 Mechanisms of Organic Photocatalysts .............................................. 22

1.5 The Oxidation of Small Molecules and their Applications ...................2 24

1.5.1 Alcohol Oxidation Methods ............................................................ 24

1.5.2 Heterocyclic Compounds, Strategic Methodologies in the Synthesis of Complex Structure including the Indole, Pyrrole, Isatin and Maleimide Structural Motives............................................................ 30

1.5.3 Reactions of Singlet Oxygen and their interactions with

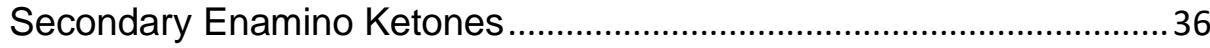

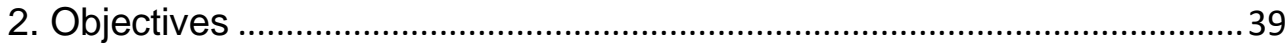

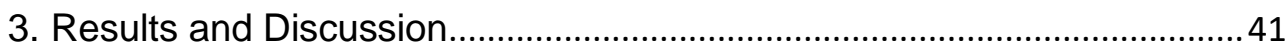

3.1 Oxidation of Primary and Secondary Alcohols...................................... 45

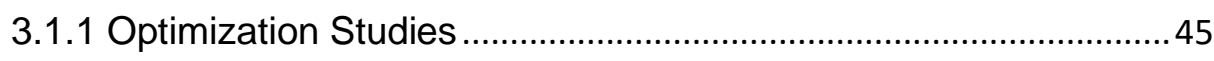

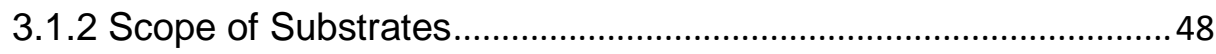

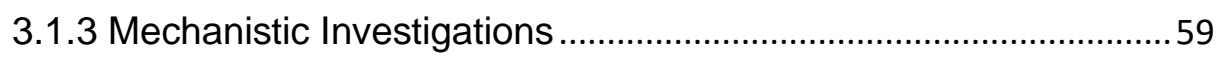

3.1.4 Transfer of Results to Flow Chemistry............................................69

3.1.5 Summary and Outlook of the Project ........................................... 72

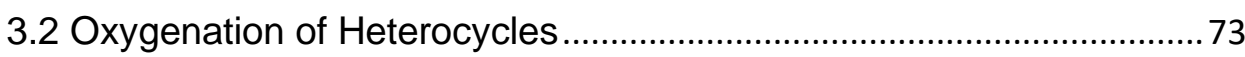

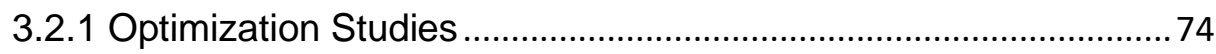

3.2.2 Scope of Substrates and Applications ……................................... 76

3.2.3 Application of the Photocatalytic Oxygenation Reaction...............83

3.2.4 Mechanistic Investigations and Proposed Mechanism..................86

3.2.4 Concluding Remarks and Outlook of the Chapter..........................94

3.3 Oxidation Combined with 1,2-Acyl Migration of Secondary Enamino

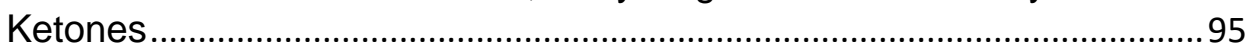

3.3.1 Synthesis of Starting Materials and Optimization Studies ........... 95

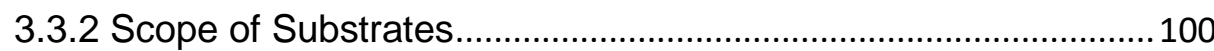

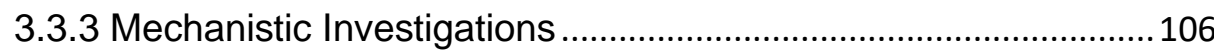


3.4 Design and Development of Graphitic Carbon Nitride as Photocatalysts

3.4.1 Design of Reaction Setup

3.4.2 Synthesis of Modified Carbon Nitrides and Their Activity

3.4.3 Summary and Outlook of the Application of $g-\mathrm{C}_{3} \mathrm{~N}_{4}$ 129

4. Summary and Outlook of the Entire Work 130

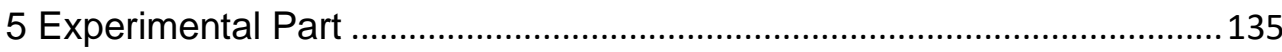

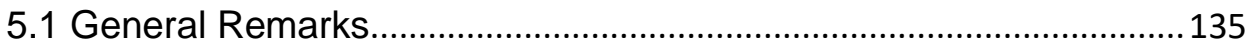

5.2 Experimental Procedure and Details for Alcohol Oxidation ............... 136

5.3 Experimental Procedure and Details for The Oxygenation of Heterocycles

5.4 Experimental Procedure and Details for Oxidation Combined with 1,2-Acyl Migration of Secondary Enamino Ketones ................................. 158

5.5 Synthesis of Graphitic Carbon Nitrides ........................................... 163

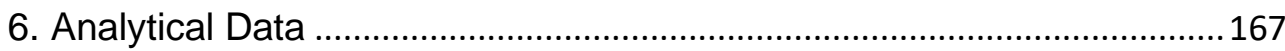

6.1 Analytical Data of Synthesized Compounds ...................................... 167

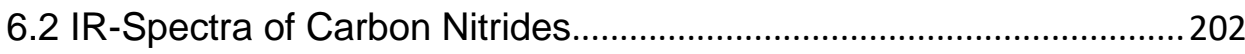

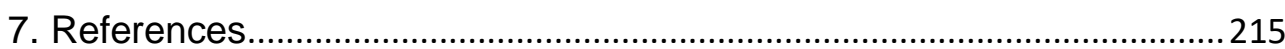

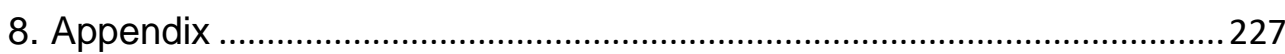

8.1 Additional Data from Coauthors of Publications ...................................227

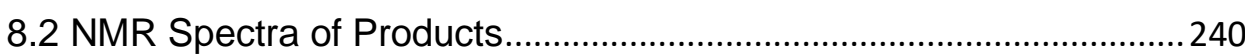




\section{List of Abbreviations}

ACN Acetonitrile

aq aqueous

Ar Aryl

atm atmospheric pressure (101325 Pa)

BC before christ

BHT 3,5-di-tert-butyl-4-hydroxytoluene

$\mathrm{Bn} \quad$ benzyl

Boc tert-butyloxycarbonyl

$\mathrm{Bu} \quad$ butyl

calc. calculated

cat. catalytic

CD conduction band

$\delta \quad$ chemical shift

d doublet

DBU 1,5-diazabicyclo[5.4.0]undec-7-ene

DFT density functional theory

DMA $\quad \mathrm{N}, \mathrm{N}$-dimethylacetamide

DMF $\quad N, N$-dimethylformamide

DMSO dimethyl sulfoxide

EA ethyl acetate

ed. Editor, edition

E-Factor environmental factor

EPR electron paramagnetic resonance

eq. equivalents

ESI electron spray ionization

ET energy transfer

et. al. and others (et alii)

Et ethyl

$\mathrm{EtOH} \quad$ Ethanol

g gram

GC(-FID) gas chromatography (flame ionization detector)

GC-MS gas chromatography-coupled mass spectrometry

$g-\mathrm{C}_{3} \mathrm{~N}_{4} \quad$ graphitic carbon nitride 


$\begin{array}{ll}\mathrm{h} & \text { hour } \\ \text { HOMO } & \text { highest occupied molecular orbital } \\ \mathrm{HRMS} & \text { high resolution mass spectrometry } \\ \mathrm{Hz} & \text { Hertz } \\ i & \text { iso } \\ \mathrm{KIE} & \text { kinetic isotope effect } \\ \text { LED } & \text { Light-emitting diode } \\ \text { LUMO } & \text { lowest unoccupied molecular orbital } \\ \mathrm{m} & \text { multiplet } \\ \mathrm{M} & \text { molar } \\ \text { [M+] } & \text { molecular ion peak } \\ \mathrm{Me} & \text { Methyl } \\ \mathrm{MeOH} & \text { Methanol } \\ \mathrm{MFC} & \text { mass flow controller } \\ \mathrm{mg} & \text { milligram } \\ \mathrm{mpg} & \text { meso porous graphitic } \\ \mathrm{MHz} & \text { megahertz } \\ \mathrm{min} & \text { minute(s) } \\ \mathrm{mL} & \text { milliliter } \\ \mathrm{mmol} & \text { millimole } \\ \mathrm{MS} & \text { mass spectrometry } \\ \mathrm{m} / z & \text { mass to charge ratio } \\ \mathrm{n} . \mathrm{r} . & \text { no reaction } \\ \mathrm{NMR} & \text { nuclear magnetic resonance } \\ o & \text { ortho } \\ \mathrm{OLED} & \text { organic light-emitting diode } \\ \mathrm{p} & \text { para } \\ \mathrm{PC} & \text { photocatalyst } \\ \mathrm{Ph} & \text { phenyl } \\ \mathrm{ppm} & \text { parts per million } \\ \mathrm{q} & \text { quartet } \\ \mathrm{rt} & \text { room temperature } \\ \mathrm{SET} & \text { single electron transfer } \\ t & \text { tert } \\ & \end{array}$




$\begin{array}{ll}\mathrm{t} & \text { triplet } \\ \mathrm{T} & \text { temperature } \\ \Delta \mathrm{T} & \text { increased temperature } \\ \text { TEMPO } & \text { 2,2,6,6-tetramethylpiperidine- } N \text {-oxide } \\ \text { TMP } & \text { 2,2,6,6-tetramethylpiperidine } \\ \text { THF } & \text { Teramethylfuran } \\ \text { TS } & \text { transition state } \\ \text { UV } & \text { ultraviolet } \\ \text { UV-Vis } & \text { ultraviolet - visible light } \\ \text { VB } & \text { valence band }\end{array}$




\section{INTRODUTION}

The oxidation of chemical compounds to form new compounds with the desired attributes, is a process which is as ancient as the founding of civilization. The oxidation of alcohol to vinegar and its use for conservation and medicinal application is reported to date back to $6000 \mathrm{BC} .^{[1]}$ With the development of civilization and the further advances in science and technology, new processes and fields were added to the collective capabilities, to improve and accelerate the production and yields. With the discovery of the concept of catalysis by Berzelius in the early $19^{\text {th }}$ century, chemical transformations have been accelerated and the reaction conditions improved. ${ }^{[2]}$ With the advances in chemistry, the golden age of chemistry and chemical industry surfacing in the $19^{\text {th }}$ and $20^{\text {th }}$ century, resulting in the production of bulk chemicals, pharamceuticals, functional materials and many more. ${ }^{[3]}$ With advances in technology and production, environmental impact became more and more visible and with the rise of global warming and environmental damages in our ecosystems, a new awareness for environmental aspects and human impact on the ecosystem and climate are becoming common in our society. ${ }^{[4]}$

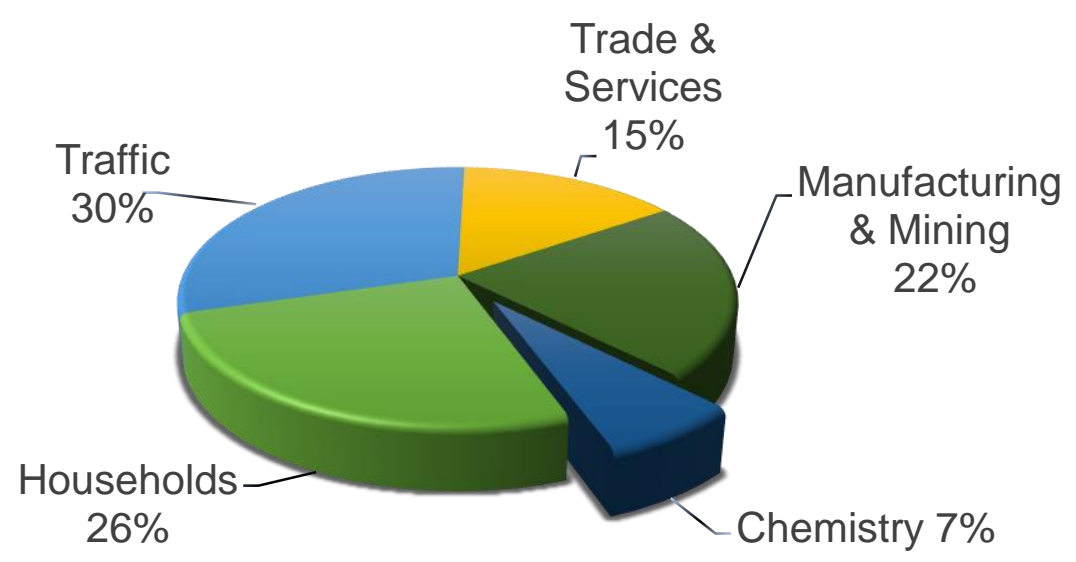

Figure 1: Energy consumption of different sectors in Germany. ${ }^{[5]}$

With the chemical industry as one of the dominant and most important contributors of modern society, a considerable part with around $10 \%$ (7\% in case of Germany $\left.{ }^{[5]}\right)$ of the world energy demand is utilized for this sector alone. ${ }^{[6]}$ As a major contribution sector of the global energy consumption, the chemical industry and chemical 
academic society are aware of their responsibilities and the necessity to address and deal with these new and therefore problematic issus. The priniciples of green chemistry are one of those concepts[7]. Initiatives and journals for the conversion of energy are being founded and receiving growing attention, e.g. Green Chemistry with an impact factor of currently $9.4,{ }^{[8]}$ or Energy and Environmental Science with an impact factor of 30.3. ${ }^{[8]}$ With the growing awareness of enviromental and energy impact, as also new developments in technology, the field of photochemistry and photocatalysis are gaining again growing interest. ${ }^{[9]}$

\subsection{GREEN CHEMISTRY AND THE 12 PRINCIPLES ${ }^{[7]}$}

Green Chemistry is a field of Chemistry focusing on the energy aspects and impact of chemistry on the environment and society. With the twelve principles of green chemistry the different aspects for the evaluation of chemical reactions are defined. The twelve principles are: 1) the prevention of waste, 2) atom economy, 3) less hazardous synthesis, 4) safer chemicals, 5) safer solvents and auxiliaries, 6) energy efficiency, 7) renewable feedstocks, 8) reduced derivatives, 9) application of catalysis, 10) design of degradation of waste products, 11) real-time analysis of the pollution and 12) accident prevention (Figure 2). The twelve principles are thereby interconnected and create several networks interlinking them.

Three examples are provided here to illustrate this. A) Interaction of principles $3^{\text {rd }}, 4^{\text {th }}$, $5^{\text {th }}$ and $12^{\text {th: }}$ Designing a less hazardous synthesis includes safer solvents, which are less toxic, flammable and easy to store, utilizing additionally safer chemicals, like more harmless bases and acids, applies in general for this, too. The application of these two concepts for the realization of $3^{\text {rd }}$ concept fulfills the $12^{\text {th }}$ principle additionally, due to the safe reaction conditions, the risk of accidents is minimized. B) The prevention of waste $\left(1^{\text {st }}\right)$ is closely related to the atom efficiency $\left(2^{\text {nd }}\right)$, the degradation of waste $\left(3^{\text {rd }}\right)$ and reduction of derivates $\left(8^{\text {th }}\right)$, by choosing synthetic routes that minimize the resulting byproducts and maximizing the target product, less waste is produced and therefore the atom efficiency is maximized. Additionally, if the forming byproducts are 
not merely considered contamination, but can be further utilized, this results in valuable byproducts, which can fulfill the $10^{\text {th }}$ principle.

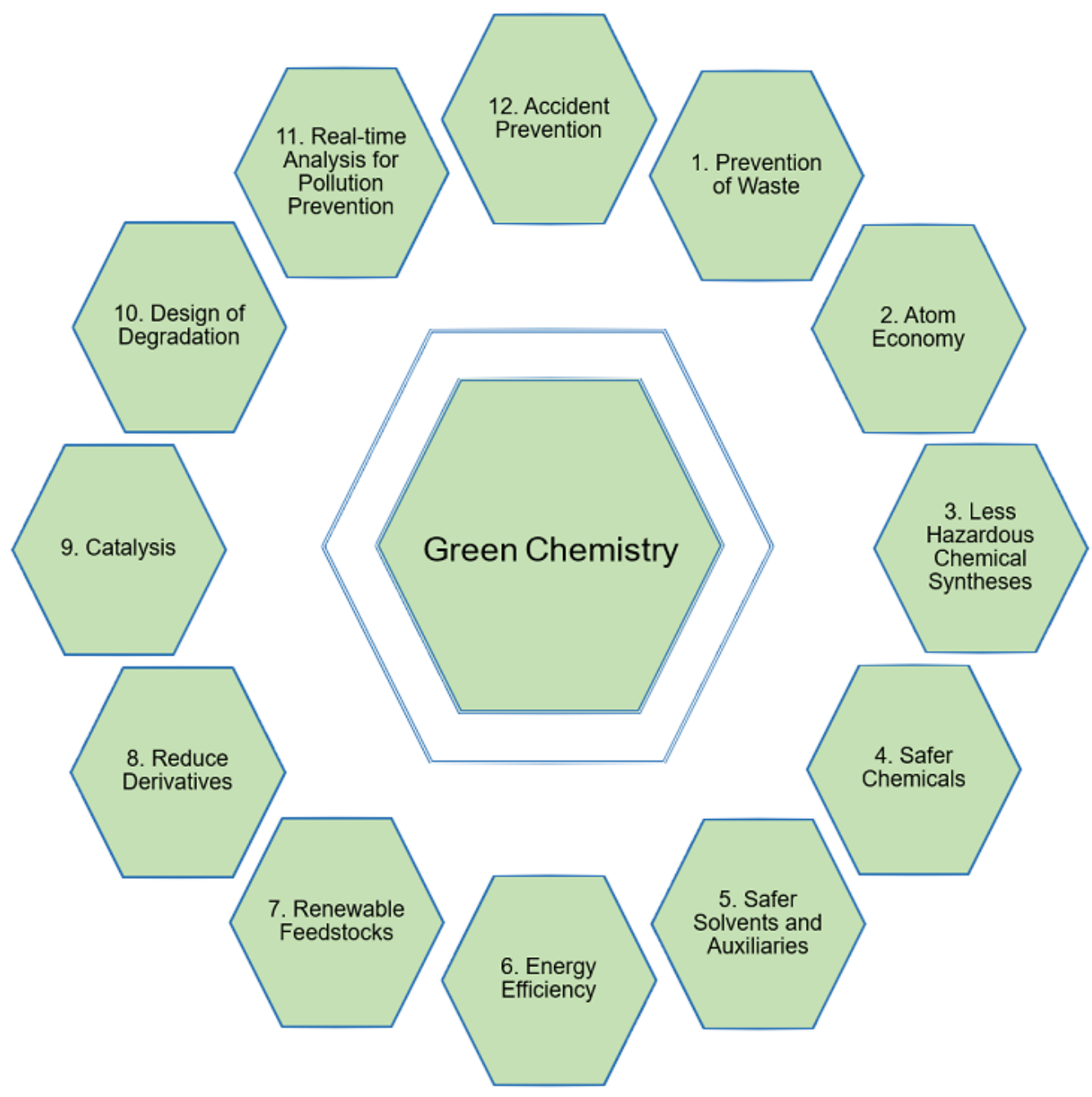

Figure 2: The 12 principles of Green Chemistry, based on Warner and Anastas. ${ }^{[7]}$

C) The oxidation of alcohols can be achieved through application of (over-) stoichiometric amounts of oxidants, applying the principles of catalysis for this process, reaction temperature and use of additives can be minimized. Therefore, the principle of catalysis $\left(9^{\text {th }}\right)$ is closely related to the $1^{\text {st }}, 2^{\text {nd }}$, and $6^{\text {th }}$ principle.

Based on the close interplay of the different principles, different interpretations of the sustainability of green chemistry reactions are possible. Several studies and meta studies were performed for the evaluation of solvents and the consideration of what can be defined as a green solvent. ${ }^{[10]}$ Based on these works different solvents can by classified as green solvents, possible green solvents and non-green solvents. Thereby 
three different criteria are usually compared, the safety of the solvent, the hazardousness and the energy consumption in its generation and disposal. Making easy distinctions between them, like the use of $i$-amyl alcohol is highly recommendable as a green solvent, due to high safety, low risk and low energy consumption. [11] Compared to this, the use of dimethyl sulfoxide (DMSO), which has high safety attributes and low hazardousness, but high energy demands in its production. Consequently, its use in synthesis of bulk chemicals can be classified as non-green. But the use for the production of specialized fine chemicals and pharmaceuticals, especially when DMSO is also the solvent of application, can be classified as a highly green solvent. The use of dimethylformamide (DMF) is under all consideration a nongreen solvent and is not part of any considerations. But this is a perspective solely based on consideration and evaluation of the solvent for general reactions; optimization of DMF specific reaction with benefits in other areas can still achieve improvements in the concept of Green Chemistry. Application of the remaining principles can improve on reactions to compensate for this. In the case of DMF as a non-green solvent, combined with the introduction of catalysis, reduction of additives and energy requirement of the reaction, can still justify the attribution of a green chemistry approach.

For example, B) the prevention of waste and the atom efficiency of a chemical reaction, a special concept was derived, the E-factor, as a valid description of the ratio of produced waste and product to compare synthetic concepts more objectively. The mathematical definition is thereby simple, due to the fact that it is the quotient of the mass of waste divided by the mass of the product. ${ }^{[121]}$ Based on the complexity of different target structures the E-factor can grow rapidly. This results in the fact that different industries have a different typical E-factor (Table 1). ${ }^{[13]}$ 
Table 1: Different production tonnages and E-factors of different chemical industries.

\begin{tabular}{ccc}
\hline Industry & Product tonnage & E-factor \\
\hline Oil refining & $10^{6}-10^{8}$ & $<0.1$ \\
Bulk chemicals & $10^{4}-10^{6}$ & $<1-5$ \\
Fine chemicals & $10^{2}-10^{4}$ & $5-50$ \\
Pharmaceuticals & $10-10^{3}$ & $25-100$ \\
\hline
\end{tabular}

Hence, it has to be considered that the E-factor only considers impact of the physical and chemical transformation of a substrate to the product. Oil refining mostly consists of purifications of crude oil, therefore, has high efficiency, but fossil fuel has high energy amounts stored in it due to natural processes. The overall trend is that from bulk chemicals, over fine chemicals to pharmaceuticals, the structural complexity is increasing and thereby the necessary chemical steps to form the desired product increases drastically. Furthermore, a growing number of different concepts exist and are being developed to evaluate different aspects. This all results into the possibility of defining the perfect synthesis in green chemistry (Figure 3). ${ }^{[7]}$

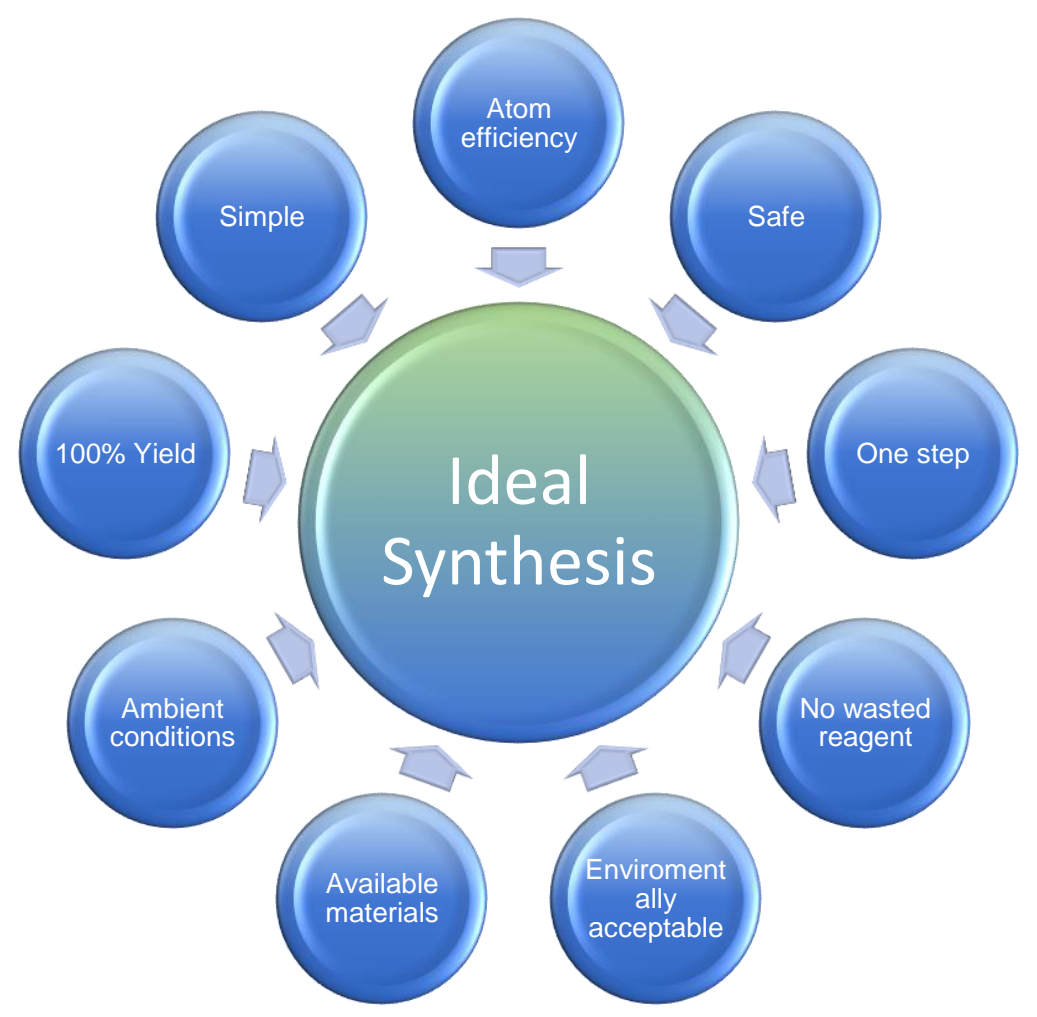

Figure 3: The ideal synthesis based on green chemistry principles..$^{[7]}$ 
Another point of focus of green chemistry is the use of safe and abundant reagents, as the use of new methodologies to reduce the energy consumption of reactions. Therefore the use of carbon dioxide from flue gas as a renewable feedstock as $\mathrm{C} 1$ building block ${ }^{[14]}$ and the use of atmospheric oxygen as an abundant oxidizing source are ideal candidates for green chemistry. ${ }^{[15]} \mathrm{A}$ recently growing field in green chemistry is thereby the concept of photocatalysis. ${ }^{[16]}$ Based on this concept, the utilization of irradiation as the primary source of energy is investigated, to achieve new reactivities and low energy demands of chemical transformations. Recent reports show that via photocatalysis the utilization of $\mathrm{CO}_{2}$ and $\mathrm{O}_{2}$ become easily available. ${ }^{[17]}$ The combined trend between those two concepts is shown in Figure $\mathbf{4}$ which depicts the number of cited publications including the terms green chemistry and photocatalysis. ${ }^{[18]}$

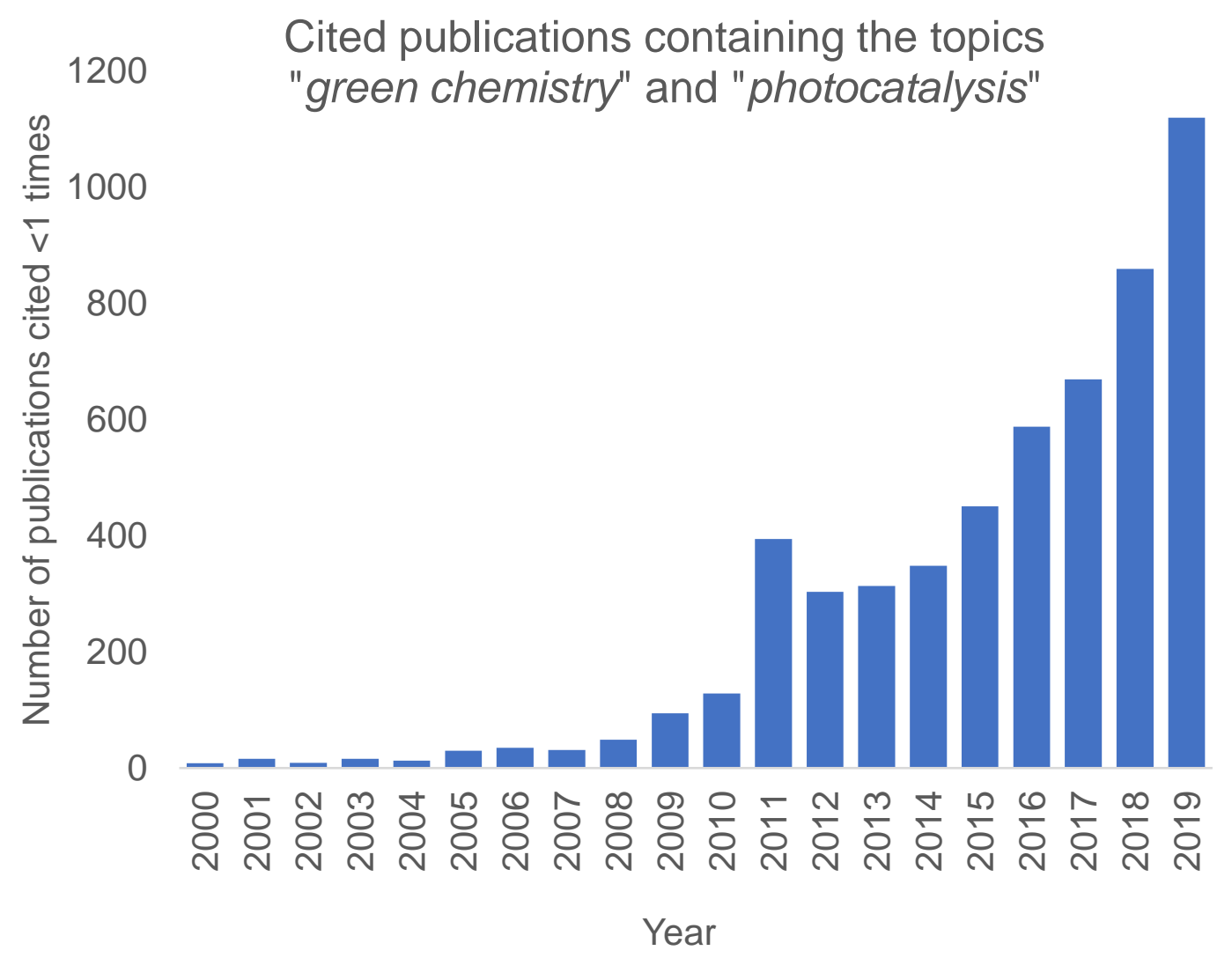

Figure 4: Exported statistical report from Dimensions, for the number of cited research publications containing the topics "green chemistry" and "photocatalysis". ${ }^{[18]}$

\subsection{PHOTOCHEMISTRY AND PHOTOCATALYSIS}

The term photocatalysis was first coined by Eibner in 1911. ${ }^{[19]}$ Thereafter, the concept of photochemistry, the utilization of light as an energy source for chemical 
transformation was known for nearly 100 years. As the first work in this field, the investigations of Trommsdorff in the year 1834 laid the groundwork for photochemistry. After initial success and advances in the field of photochemistry and forming photocatalysis, the interest declined with the limited success of upscaling the reactions. ${ }^{[20]}$ In the middle of the $20^{\text {th }}$ century photocatalysis regained a new interest for nearly 30 years. This was based on new methodologies for the investigation of the reaction and therefore enabling the possibility of intense mechanistic studies and theory derived reaction planning. ${ }^{[21]}$ But also, here the limitation in scalability forced a decline in the interest.

Since the beginning of the $21^{\text {th }}$ century, a new attempt has been made in photochemistry. ${ }^{[22]}$ The driving force is the growing interest in green chemistry and thereby new concepts to lower energy demands of chemical transformations. ${ }^{[23]}$ Again, in this case, the advancement of mechanistic studies enables new synthetic possibilities. The downfall of the first two periods of photochemistry was the limitation of the possible scale up and the limited possibilities to utilize light. Both limitations have currently been overcome and this is largely due to new developments which can achieve industrial scalable solar reaction, with enormous efficacy and the use of solar light. Several different prototypes reactors were examined, with PROPHIS as an prominent example (Figure 5). ${ }^{[24]}$ The difference between failed attempts in the $20^{\text {th }}$ century and the new attempts, are not based on the advances in photochemistry alone, but more on the effect of advances on the conception of individualized reaction setup. Through the innovation of 3D-printable designs to be utilized in chemistry, new reaction concepts can be realized and adapted for the requirements of photochemistry. The second and more significant innovation was the advance in flow chemistry and the combination of flow chemistry and its utilization for multi-phase photochemistry. ${ }^{[25]}$ 


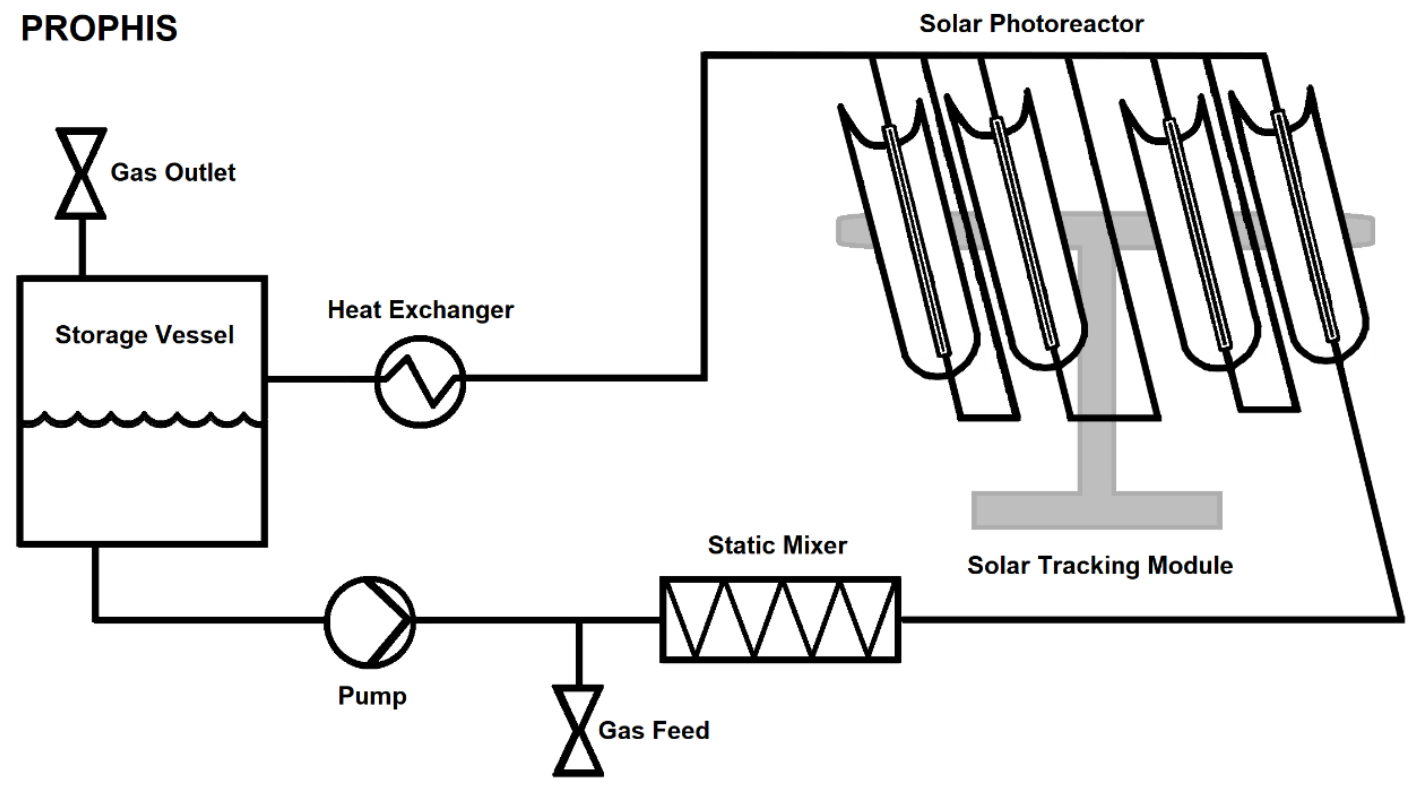

Figure 5: Schematics of the PROPHIS large scale photochemical project for utilizing solar energy for chemical transformations in multi kilogram scale. [figure based on 25]

\subsubsection{THE IMPACT OF EXPERIMENTAL DESIGN}

With the advances in 3D-print technology, new possibilities were found for the application of photochemical reaction design. ${ }^{[26]}$ Along with these advances, the design of the reaction setup was investigated and clear differences between all of the utilized components were defined.[27] Compared to classical thermal control, the use of light broadens the selectivity and possible ways to apply irradiation. Different sources of light produce different wavelength distributions and therefore have to be investigated, categorized and validated for the use in photochemistry. ${ }^{[27,28]}$ A red emitting LED cannot be utilized for a photocatalytic system based on blue light absorption. ${ }^{[29]}$ Based on this the characterization of the light source is an essential part of photochemical investigations. Blue light emitting LED might be classified as visible light, but possible UV-irradiation, of the light source can perform the reaction instead of the visible light. The concept of LED and OLED are powerful light emitting concepts, ${ }^{[30]}$ but in consideration of green chemistry, can be misused. The application of LED with $200 \mathrm{~W}$ power, is thereby not a green concept anymore, which requires 
intense cooling to reduce the thermal effect on the solvent and the reaction. The use of light concentrators, which collect wavelengths and concentrate, or even change to specific wavelengths contribute strongly to this. ${ }^{[31]}$ Concluding on these aspects, the declaration and classification of the irradiation source is a crucial aspect of photochemistry. Some of the organic molecules are well known for their photocatalytic characteristics, but investigating a developing field of chemistry with new methodologies require complete analysis of the involved mechanism. ${ }^{[27]}$ The impact of irradiation has thereby a further consideration, the geometry of the reaction setup and the distances between the single components further impacts the reaction. ${ }^{[32]}$ The classical round neck flask of the conventional chemical reaction is being replaced to new designs for the utilization of irradiation. The focus thereby is on the maximization of the surface area without the increase of volume. ${ }^{[33]}$ This process is due to the limitation of irradiation-based chemistry, the light interacts with the reaction mixture, or more precisely with the chromophores in the reaction mixtures. The target wavelengths are fast and cannot penetrate far into the reaction mixture to activate further chromophores. Furthermore, other wavelengths, with no valid absorption potential, bypass the reaction mixture without being utilized for the reaction. ${ }^{[33]}$ This problem was the limiting factor for the efficiency and scalability of the photochemical reactions in the $20^{\text {th }}$ century. The limitation is based on the Lambert-Beer law and nowadays several concepts have been designed to maximize the efficacy of the application of this law on the reaction design. ${ }^{[33]}$ The simplest is the change of geometry to improve the relation of surface to volume. The second concept is the transformation of unsuitable wavelengths into the target wavelength. The application of absorption-emission 3D-printable OLED materials (concentrators) provides a feasible approach to reduce the number of unsuitable wavelengths and thereby also increase the target wavelengths in power. ${ }^{[30]}$

The application of green chemistry principles on the photocatalysis offered new possibilities. The use of gas from our atmosphere and other viable sources like flue gases. ${ }^{[25]}$ Photochemistry has an efficient approach to utilize such gases due to its unique reaction mechanisms. Carbon dioxide $\left(\mathrm{CO}_{2}\right)$ from flue gas thereby is a renewable feedstock ${ }^{[34]}$ and atmospheric oxygen is a highly abundant resource, but hence, also creating new challenges to incorporate the gas phase into the reaction design. ${ }^{[25]}$ The major new concept is the incorporation of photocatalysis in flow 
chemistry as a valid approach for the use of gas phases and solutions and for up scaling. The combination of gas and liquid phases will be discussed first. Regular batch principles in flasks offer dead volume to be filled with gas phase and therefore provide an excess amount of possible gas phase reactant with a defined border between liquid and gas phase including well studied phase transfer kinetics. In a continuous flow an alternative design has to be realized to provide continuous flows to mix in a singular reaction area during the reaction segment. The realization of this was achieved in quite a simple way (Figure 6). ${ }^{[25,31]}$

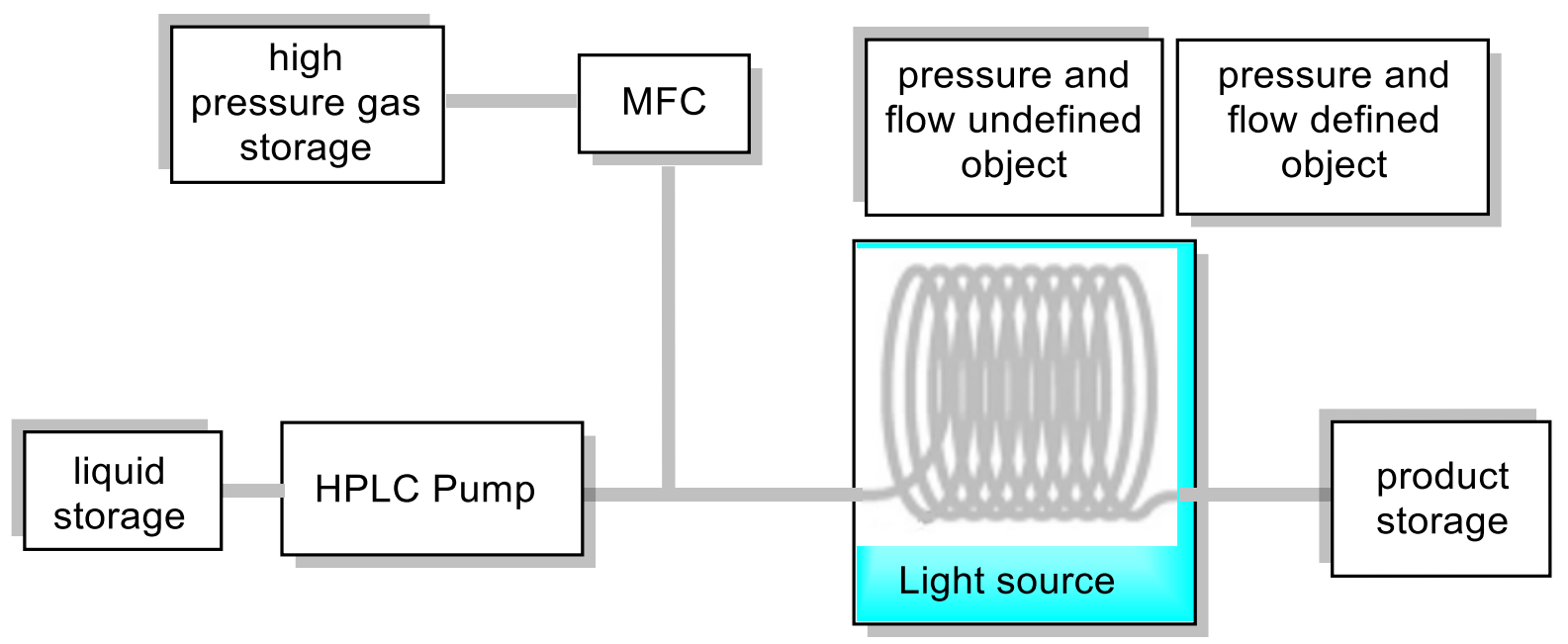

Figure 6: Concept of a continuous flow photochemical setup. [based on 25,31]

A gas storage of the required atmosphere, here oxygen, has a mass-flow-controller (MFC), which regulates the flow of utilized gas and its applied system pressure. An additional HPLC pump provides the flow of the liquid phase and regulates the applied system pressure. Both supply lines need to be adjusted to operate at the same pressure, otherwise a mixture of both cannot be achieved. Combining both lines into a singular line achieves an alternating system of bubbles and liquid drops. The two alternating phases have thereby a defined border between the two phases and so, the gas phase is available for the reaction medium. In this way, an ideal mixing between the both is realized due to the Taylor-regime and improved mixing, due to the Taylor recirculation (Figure 7). ${ }^{[25,31]}$ 


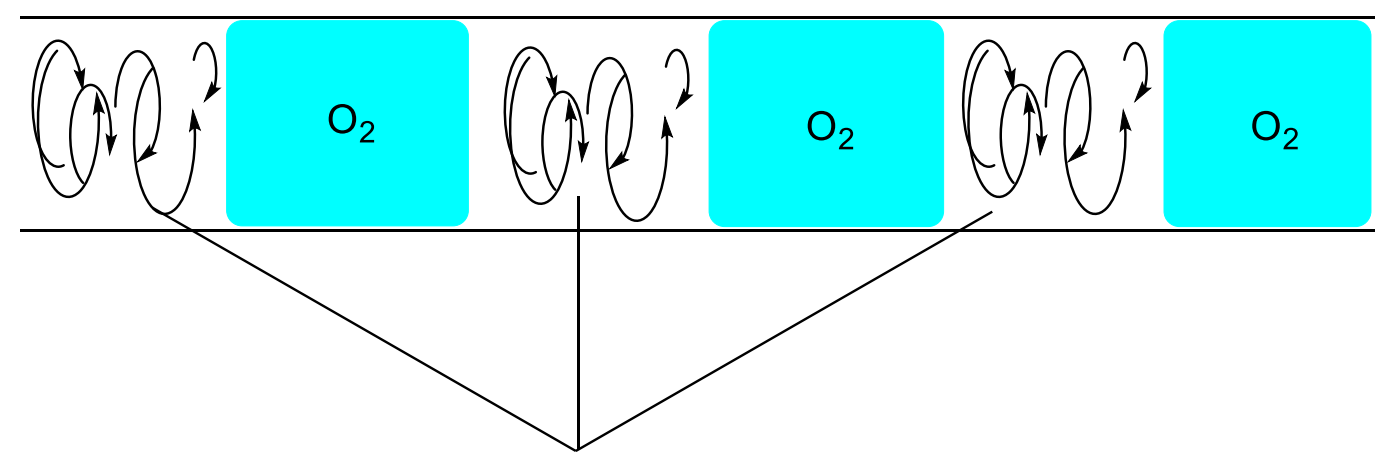

Taylor recirculation

Figure 7: Taylor-regime controlled flow after mixing of both lines, alternating droplets and gas bubbles. The droplets mixed due to Taylor recirculation. [based on 25,31]

Flow chemistry provides an essential befit of scalability, due to the concept of "numbering up" (Figure 8). The concept of "numbering up" consists of the idea that several irradiated tubes can be bundled with the light sources and therefore achieve the highest surface to volume ratio. ${ }^{[35]}$ Hence, several aspects can be combined for the single lines, minimizing technical requirements. In a numbering up concept, pumps and MFC can be utilized to provide the necessary flow for a multitude of different reaction lines. An additional benefit in case of failure of a single line, is that the remaining lines still operate independently and the resulting overall yield is reduced only by the number of failed lines. By these means industrial scale reactions in continuous flow can be achieved.

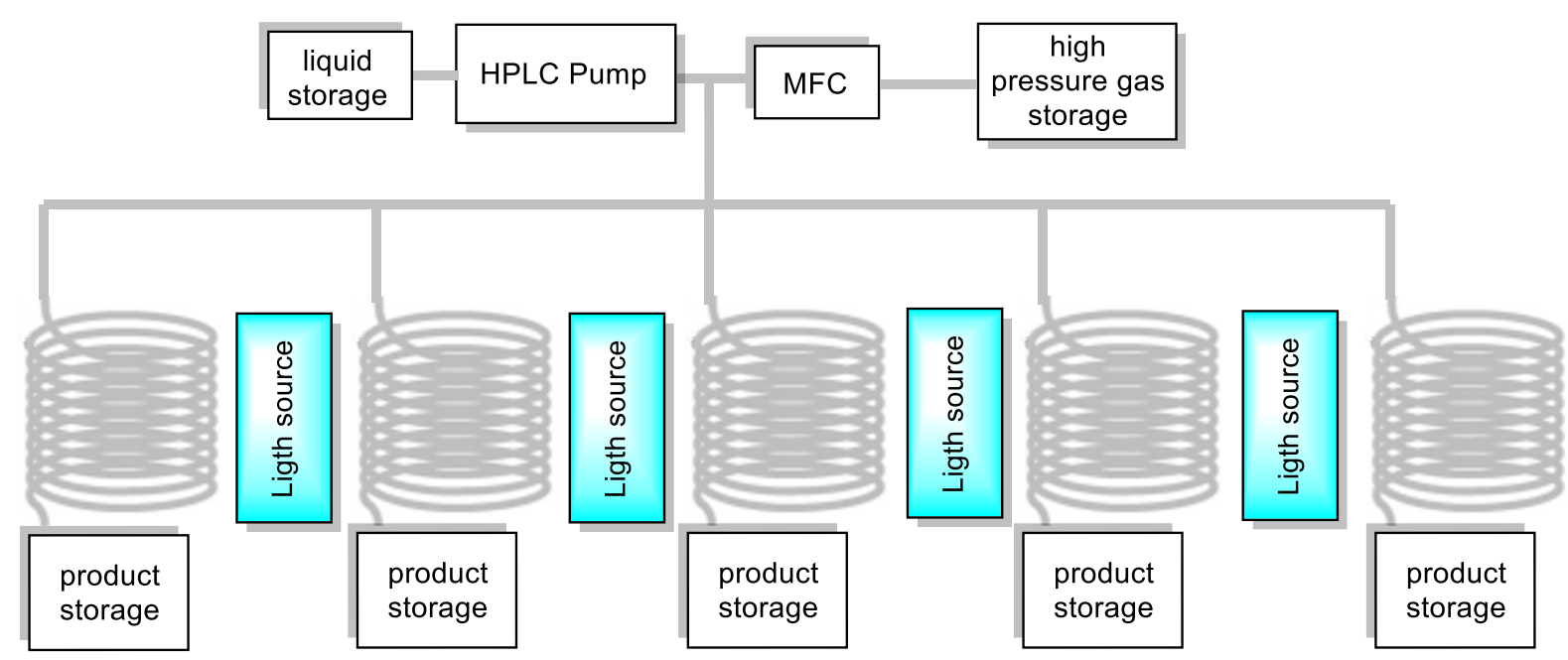

Figure 8: Schematic representation of a numbering up approach. [based on 32] 
A second continuous flow concept is called the falling film reactor concept. ${ }^{[30]}$ This provides high surface to volume ratio, but lacks the numbering aspect, or upscale aspect, of the alternative designs. Utilization of a double walled glass container to transport gravity-based liquid in thin films is used to guide the reaction mixture by the light source. An applied gas supply working in the opposite direction can provide substantial gas phase access.

\subsubsection{PhOtOcatalysts and the Design Of PhOtOcatalysts}

The technical aspects are exceeded by the possibilities of the design of photocatalysts. A variety of different precious metal based photocatalysts are known, like $\mathrm{Ru}^{\left[{ }^{[36]},\right.}$, Ir ${ }^{[37]}$ (Figure 9), $\mathrm{Pt}^{[38]}, \mathrm{Ti}^{[39]}$ etc. ${ }^{[40]}$ Each of the respective central metals can be modified to improve reactivity and optical properties based on the utilized ligands. This offers a substantial advantage to metal-based photocatalysts.

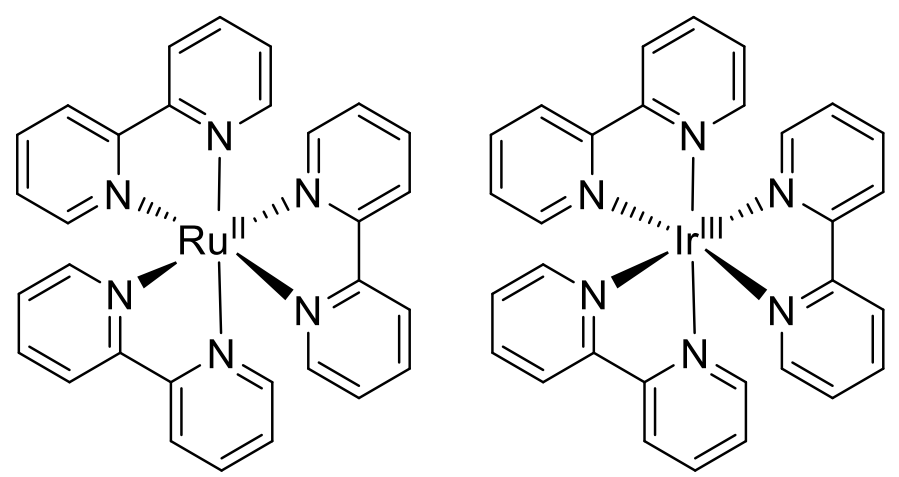

Figure 9: Examples for typical Ru- and Ir-based photocatalysts. ${ }^{[33]}$

On the other hand, organic molecule based photocatalysts are a wide group of over 10000 different chromophores with different redox potentials and chemical structural motives and functional groups. A part of these chromophores is reported and characterized, due to their reported applications as photocatalysts and photosensitizers, but the substantial part of them remains uninvestigated. The most prominent organic based metal free photocatalysts are shown in Figure $\mathbf{1 0}$ and Figure 11. ${ }^{[41]}$ 
What is displayed in Figure $\mathbf{1 0}$ is a selection of photocatalysts with simple organic structural motives of cyanoarenes ${ }^{[42]}$, benzophenones ${ }^{[43]}$ and quinones ${ }^{[44]}$. Based on their structural motif, the structures show absorption bands at UV-light and/or visible light. Additionally, the selected motives exhibit a broad spectrum of varied characteristics, raging in half-life time of the excited state, over different redox potentials and absorption band maxima. The aim of the selection of the different entries of the selected structural motives, is to illustrate the broad differences of closely related structural groups.
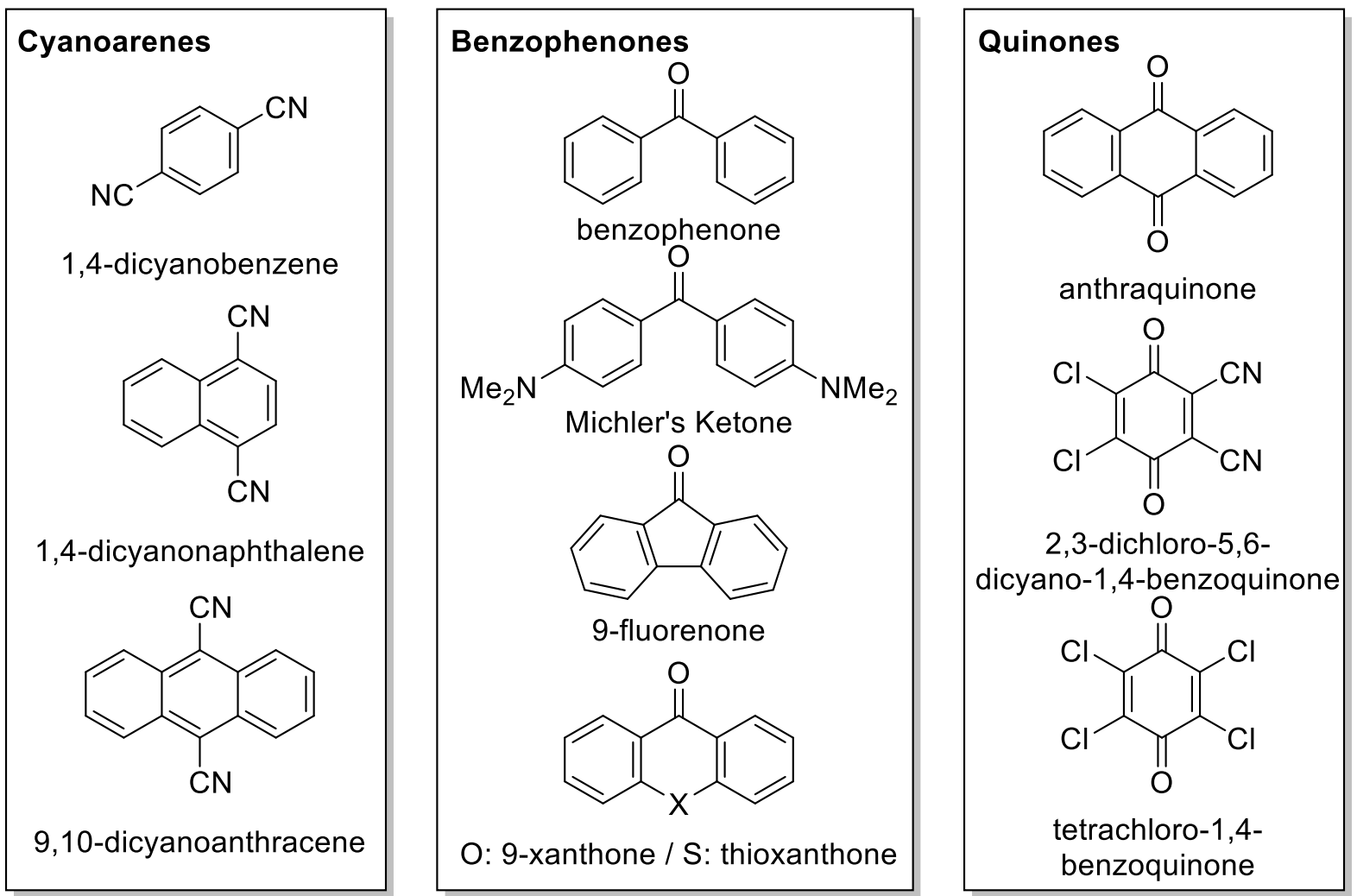

Figure 10: Examples of commonly used transition metal-free photocatalysts with uncharged ground states of the photocatalysts. ${ }^{[38]}$

Figure 11 displays a selection of different photocatalysts which are shown with solutions-based protonation behavior and the possibility to be present as ionic forms in solution. These photocatalysts exist in different forms and are commercially available in the different forms, as salts like thiazines (methylene blue) ${ }^{[45]}$, acridinium ${ }^{[42,46]}$ and xanthenes ${ }^{[47]}$, or the unprotonated forms as their natural acid forms, like eosin $\mathrm{Y}$ and rose bengal. Based on the reaction conditions in the solution different protonated forms of the photocatalyst can exist; based on the respective state the redox potentials and absorption maxima can shift due to the structural changes in the respective photocatalyst. The specific effects of the conditions and the 
performances of the photocatalyst were studied by A. Jakobi von Wangelin et al. to illustrate the effects of the protonation behavior and their photocatalytic performance. ${ }^{[48]}$ Some of the catalysts are further known based on this behavior as indicators for quantitative and qualitative analysis, like methylene blue, which exhibits intensive color changes based on the precise $\mathrm{pH}$-value. ${ }^{[45]}$

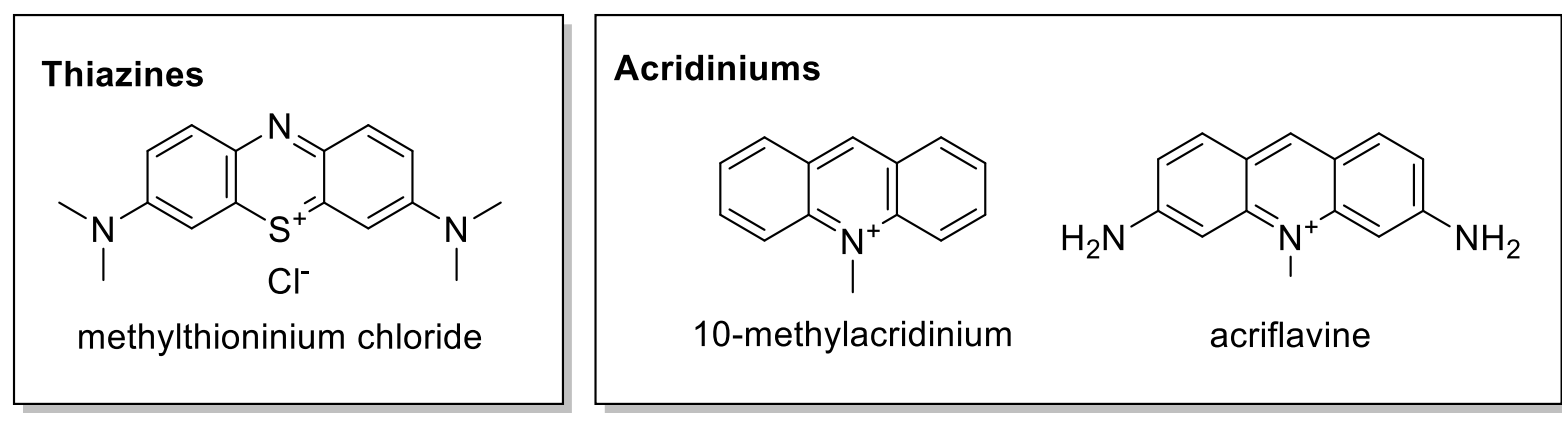<smiles></smiles>

eosin $\mathrm{Y}$ deprotonated<smiles>O=C1OC2(c3cc(I)c([O-])c(I)c3Oc3c2cc(I)c([O-])c3I)c2c(Cl)c(Cl)c(Cl)c(Cl)c21</smiles>

rose bengal deprotonated<smiles>CCN(CC)c1ccc2c(-c3ccccc3C(=O)O)c3ccc(=[N+](CC)CC)cc-3oc2c1</smiles>

rhodamine B<smiles>O=C1OC2(c3ccc(O)cc3Oc3cc(O)ccc32)c2ccccc21</smiles>

fluorescein<smiles>CCN=c1cc2oc3cc(NCC)c(C)cc3c(-c3ccccc3C(=O)OCC)c-2cc1C</smiles>

rhodamine $6 \mathrm{G}$

Figure 11: Examples of commonly used transition metal-free photocatalysts with possible charges for the salts and protonated forms of the photocatalysts. ${ }^{[41]}$

The $\mathrm{pH}$-dependent possibility to adjust the behavior of the photocatalyst and therefore change the possible application of the photocatalyst extended the application of transition metal-free organic photocatalyst. For the neutral equivalents like cyanoarenes, benzophenones and quinones, the work of G. M. Miyake et al. ${ }^{[49]}$ and Zeitler et al. ${ }^{[50]}$ provided a guideline on how to modify the structural motif and the photochemical properties of the photocatalyst. They applied synthetic protocols to 
modify phenoxazines (Miyake) and cyanoarenes (Zeitler) to introduce $\mathrm{N}$-substitution and to build extended frameworks at the aromatic rings to create symmetrical and nonsymmetrical derivatives of their base structure. In the case of Miyake et al., the resulting 19 different derivates were analyzed and characterized. The absorption and emission behavior and also the reduction and oxidation potentials were examined for their ground and excited state. This provided access to a "roadmap" to tailored redox potential of small organic neutral photocatalysts bridging the gap between organic based photocatalysts and former superior metal based photocatalysts. A selection of different entries of their "roadmap" with their respective redox potentials is given in Figure 12.

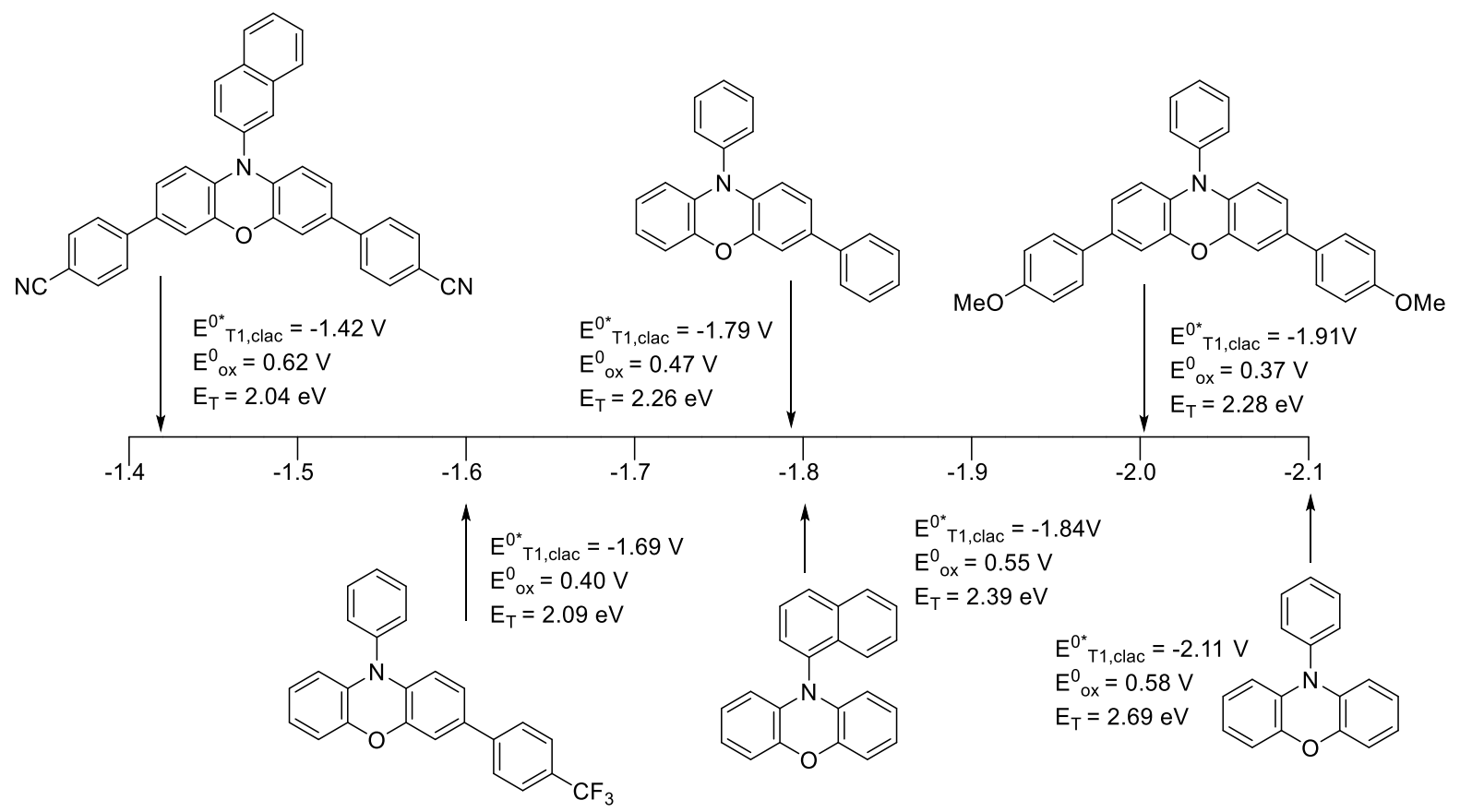

Figure 12: Selection of entries of Miyake et al., roadmap to tailored redox potentials of organic photocatalysts. ${ }^{46]}$ 


\subsubsection{Design Of Heterogeneous PhOtocatalystS AND SupPorting Structures, Graphitic Carbon Nitrides}

Based on the already mentioned advances in homogenous photocatalysts, several of those photocatalysts were combined with solid support structures, like titanium dioxide $\left(\mathrm{TiO}_{2}\right),{ }^{[39]}$ graphitic carbon nitrides ${ }^{[51]}\left(g-\mathrm{C}_{3} \mathrm{~N}_{4}\right)$, graphene ${ }^{[52]}$ and more. ${ }^{[53]}$ Based on initial studies several different beneficial results were found for the use of solid support concepts for the application of photocatalysts. The concept for the application of solid support materials was the creation of solid phase photocatalysts. Based on the fact that the photocatalyst is in a different phase, separation of the catalyst and recycling of this becomes possible. This reduces the costs and the environmental impact of the application, due to the possibility of recycling the catalyst. Additionally, the effort of product purification decreases due to the simple removal of the catalyst. ${ }^{[54]}$

However, the utilization of heterogenous photocatalysts has also drawbacks. In most cases, the charge separation is reduced and the recovery rate to the ground state is increased, which lowers the achieved quantum yield of the reaction drastically. The heterogeneity of the reaction mixture, with local high concentration of the heterogenous catalyst results in inhomogeneous mixtures, which decrease the reaction speed, due to required mixing. These drawbacks result in higher catalyst loading to achieve similar reaction speed compared to homogenous systems.

For different semiconducting support materials like the mentioned $\mathrm{TiO}_{2}, g-\mathrm{C}_{3} \mathrm{~N}_{4}$ and xenolithic structures, additional beneficial effects were found due to the semiconducting characteristics. These can interact with the photocatalyst and stabilize the charge separation by transferring the charge into the conducting band of the semiconductor and therefore increase the lifetime of the excited charge separated state. Additionally, transport of the charge separation is possible over the semiconducting support to additional reactive centers (Figure 13). Further studies of the support materials revealed that $\mathrm{TiO}_{2}$ and $g-\mathrm{C}_{3} \mathrm{~N}_{4}$ have photocatalytic properties of their own. Based on a variety of different support materials were investigated for their individual and combined photocatalytic capabilities. Through the focus of graphitic carbon nitride in this work, a detailed presentation of these effects and characteristics is shown only for carbon nitride semiconductors. 


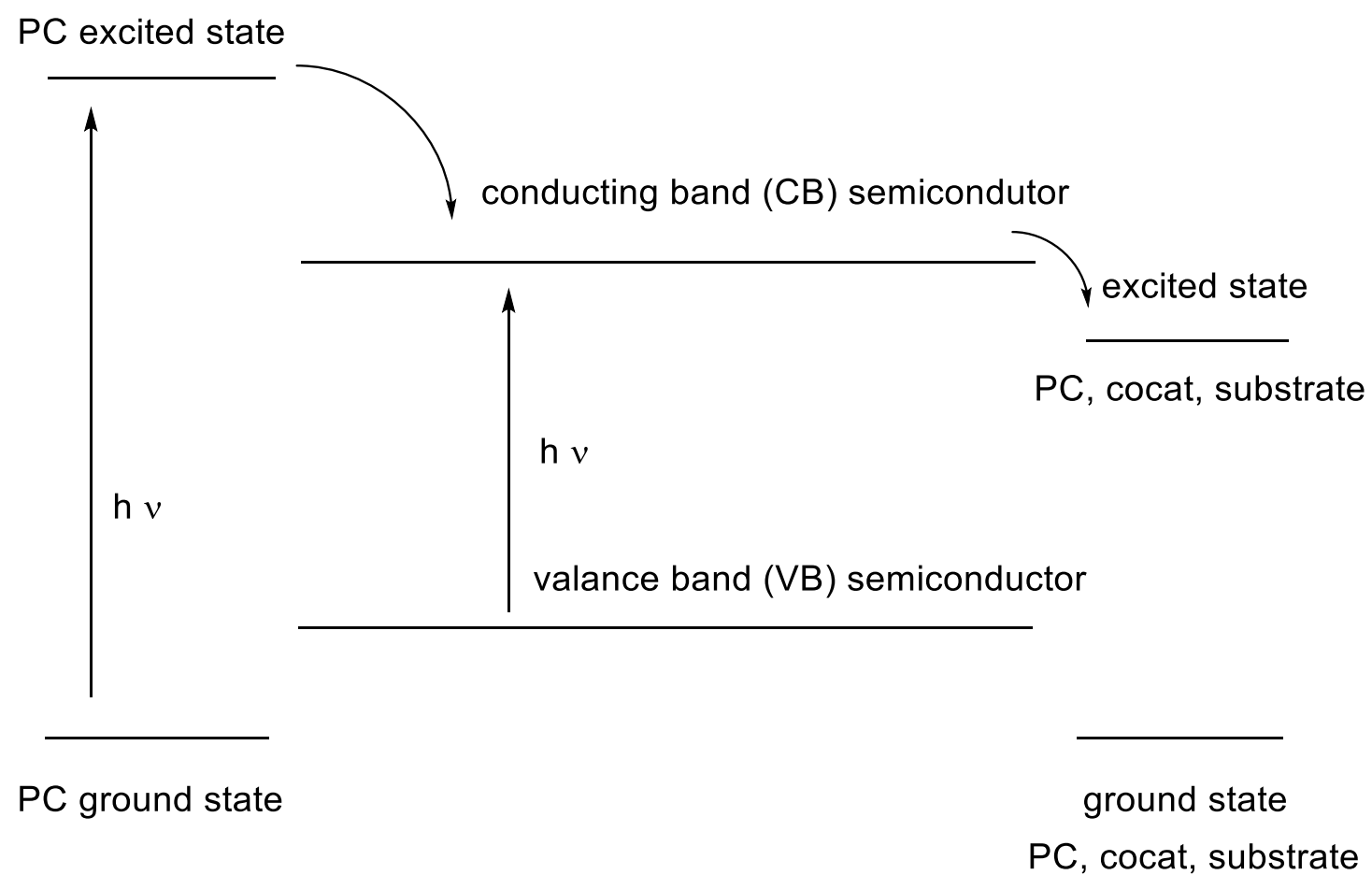

Figure 13: Schematic presentation of the interaction of photocatalytic materials with photocatalytic support materials and their ability to transfer activation on further objects. ${ }^{[39,51]}$

Synthesis of $g-\mathrm{C}_{3} \mathrm{~N}_{4}$ is based on urea and its higher condensed forms by thermal based deamination, the process is called pyrolysis. Based on different temperatures, various condensed materials can be obtained. Urea first condenses to 2cyanoguanidine, then further to melamine, to melon, to polymeric carbon nitride and then the formation of graphitic carbon nitride. The process is illustrated in the Scheme 1. Based on the utilized precursors, the photocatalytic behavior of the resulting carbon nitride changes. ${ }^{[55]}$

The thermal synthesis of $g-\mathrm{C}_{3} \mathrm{~N}_{4}$ offers a simple and highly modular system for the generation of transition metal containing, Additionally, a broad variety of purely organic semiconducting materials is accessible. Due to the distinctively separated deamination steps, the modification can be introduced at the different stages. If a heterostructure like other organic molecules or transition metals are mixed with the selected precures the incorporation of this additional modification occurs at specific positions. Based on the utilized modification, $\mathrm{n}$ - and $\mathrm{p}$-doping can be achieved for the formed semiconducting material. Mixtures with graphene can create nano sized conducting domains inside the semiconductor. A variety of studies exists for the 
incorporation of different transition metals and their enhanced reactivity are reported. ${ }^{[56]}$ Various modifications of the surface structure, as well incorporation of different organic molecules to the graphitic structure are reported. ${ }^{[57]}$ Several examples of different structures are given in Figure 14.

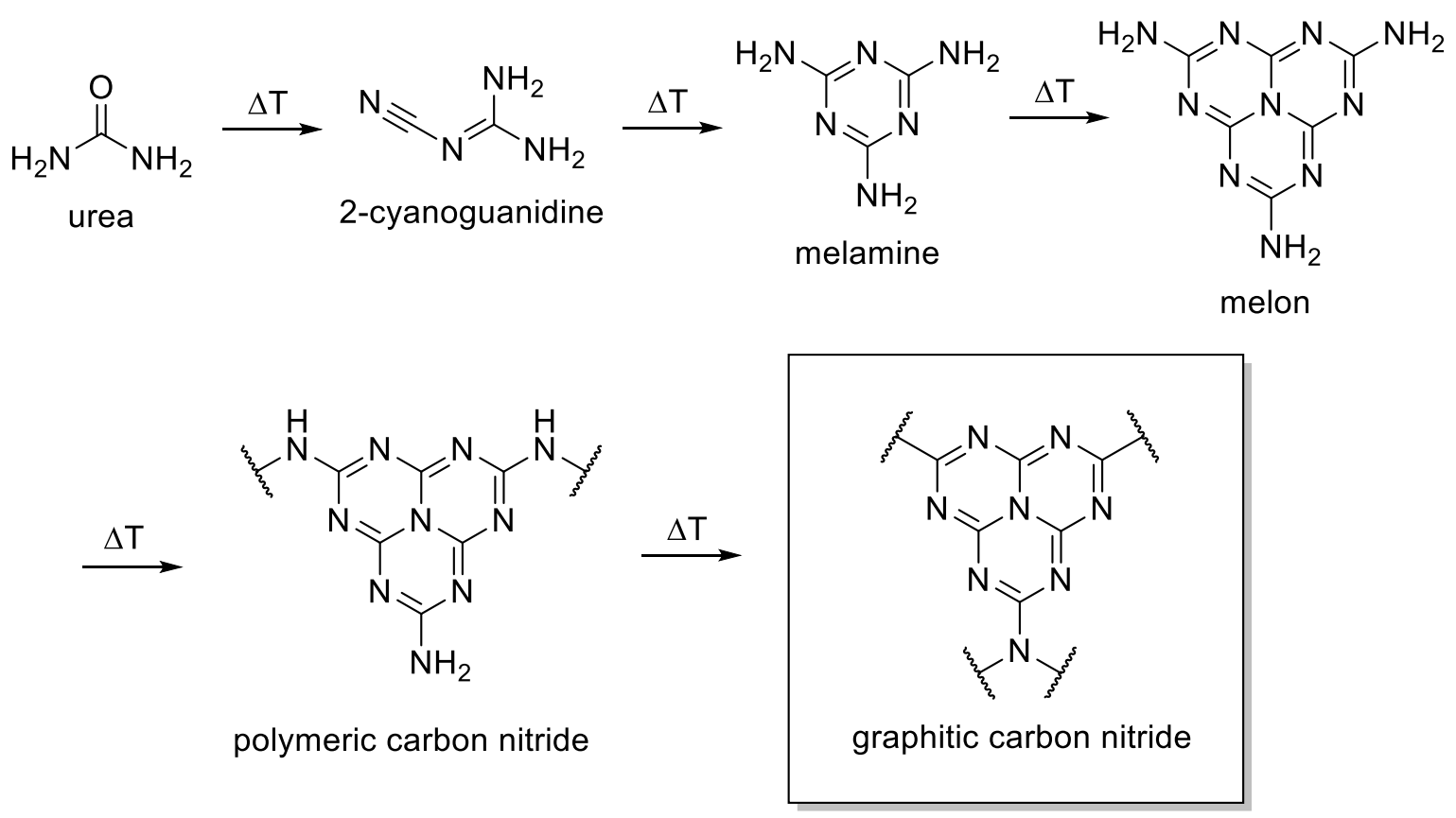

Scheme 1: Thermal reaction cascade for the synthesis of graphitic carbon nitride, between each step ammonia is released, as the byproduct.

The possible modification of the $g-\mathrm{C}_{3} \mathrm{~N}_{4}$-structure can be divided into two main categories. The first is the post synthesis modification, creating different modified structures by reactions of primary amines at the surface of the carbon nitride grid. In this case, different means exist for a metal ligand system to covalent bond with the semiconductor. For organic molecules as modifications, typical reactions of primary amines with the corresponding functions of the modification molecule can be performed. A typical reaction for this synthetic strategy is the formation of Schiff's bases, or the amidation with anhydride (Figure 14, first entry from the left).

The second possibility is the addition of additives to the reaction mixture during the pyrolysis. Utilizing urea with formamide creates nitrogen deficiencies, which create gaps, or holes, in the grid (Figure 14, second entry form the left). These gaps can be replaced by different other structural motives, as an example by combining melamine 
with aniline results in a phenyl ring instead of the gap (Figure 14, third entry). Instead of transition metals, organic molecules can be used to bond with the surface of the $g$ $\mathrm{C}_{3} \mathrm{~N}_{4}$, if these molecules have their own photochemical properties these can act efficiently as organic photocatalysts replacing transition metals.
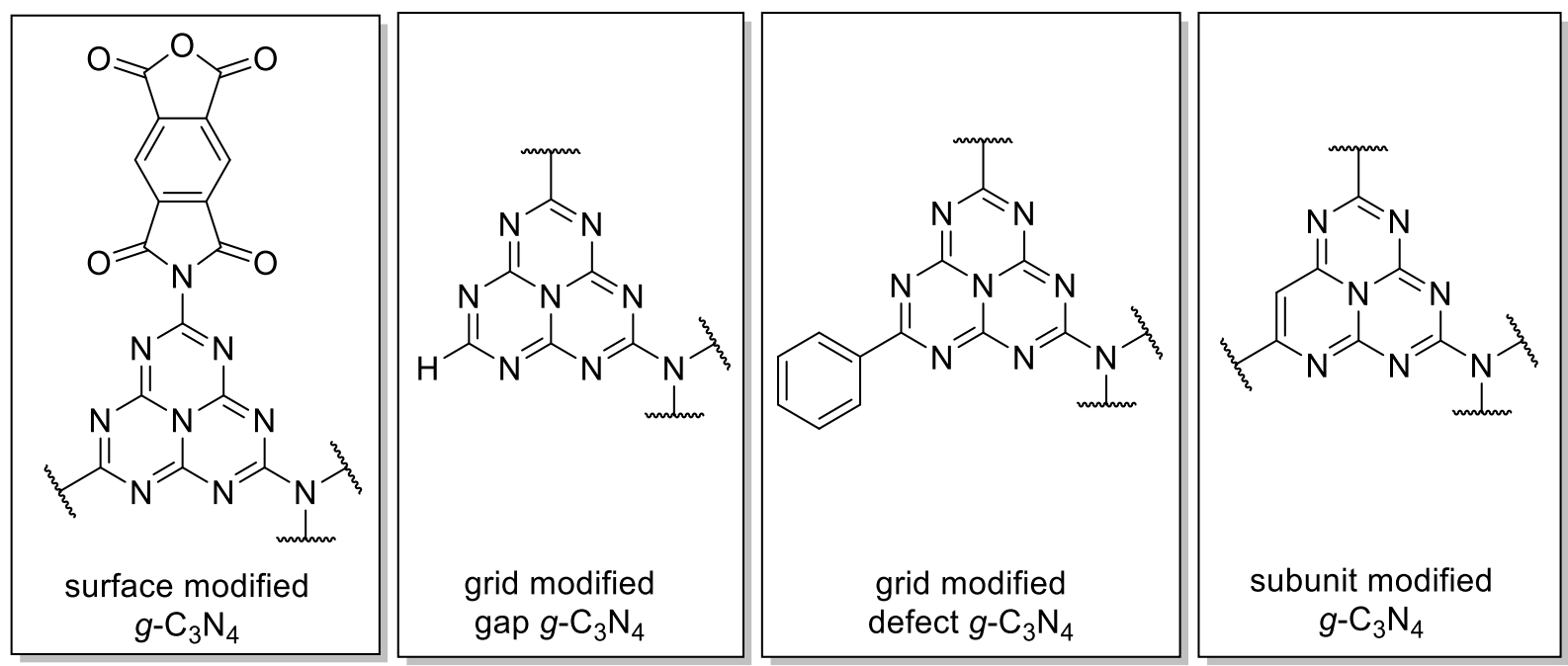

Figure 14: Different examples of modified graphitic carbon nitride structures, structures are the assumed synthesized modifications of this work.

The primary focus of heterogeneous photocatalysts and the utilization of graphitic carbon nitrides is based on their ability for the degradation of water pollutants and for the production of hydrogen from water. There are also reports on the direct use of these materials for the direct application for chemical transformation as a catalyst. ${ }^{[52,54,55-57]}$ 


\subsection{OXYGEN AND ITS ACTIVATION TO REACTIVE OXYGEN SPECIES}

The focus of this thesis is the utilization of oxygen for the chemical transformation of small molecules. Oxygen is one of the most abundant gases in our atmosphere with $20.95 \mathrm{Vol} \%$. The total percentage of the oxygen element content in our lithosphere is $49.4 \%{ }^{[58]}$ and thus, the most abundant element on the surface of the earth. ${ }^{[59]}$ The ground state of molecular oxygen $\left(\mathrm{O}_{2}\right)$ is the triplet state, which is relatively inert. Only at higher concentration the gas becomes reactive. ${ }^{[60]}$

The term reactive oxygen species (ROS) summarizes all the reactive forms of oxygen containing active forms and intermediates of oxygen. ${ }^{[61]}$ Namely these consist of ozone $\left(\mathrm{O}_{3}\right)$, singlet oxygen $\left({ }^{1} \mathrm{O}_{2}\right)$, hypochlorite anions $\left(\mathrm{OCl}^{-}\right)$, hydroperoxides $(\mathrm{ROOH})$, hydrogen peroxide $(\mathrm{HOOH})$, alkoxy radicals (RO-), peroxyl radicals (ROO-), hydroperoxide radicals (HOO.), hydroxy radicals ( $\mathrm{HO} \cdot)$, and the superoxide radical anion $\left(\mathrm{O}_{2} \cdot{ }^{-}\right)$. The four major ROS are recognized as superoxide radical anion, hydrogen peroxide, singlet oxygen and hydroxy radicals. In consequence, it should be mentioned that singlet oxygen exists in two different electronic configurations, but usually changes fast into the spin coupled state, which is longer living and less reactive. The different ROS are discussed based on their relations (Figure 15). Visible light at the earth's surface is not capable of activating atmospheric oxygen without catalysts, but at high altitudes harsher ionizing irradiation is responsible for the formation of ozone, which absorbs most of the ionizing irradiation coming from the space. Therefore, at standard conditions, only four major principles are applied to increase the reactivity of oxygen, 1) biological, 2) chemical, 3) use of high energy irradiation, 4) application of photocatalysis. Some biological means are well known like the chlorophyll of plants and the application of the photosystem 1 and 2 during the photosynthesis. Other biological enzymes also transform water and solvated oxygen to produce reactive oxygen species, for metabolic transformations and for signal transmission in organic systems. ${ }^{[62]}$ 


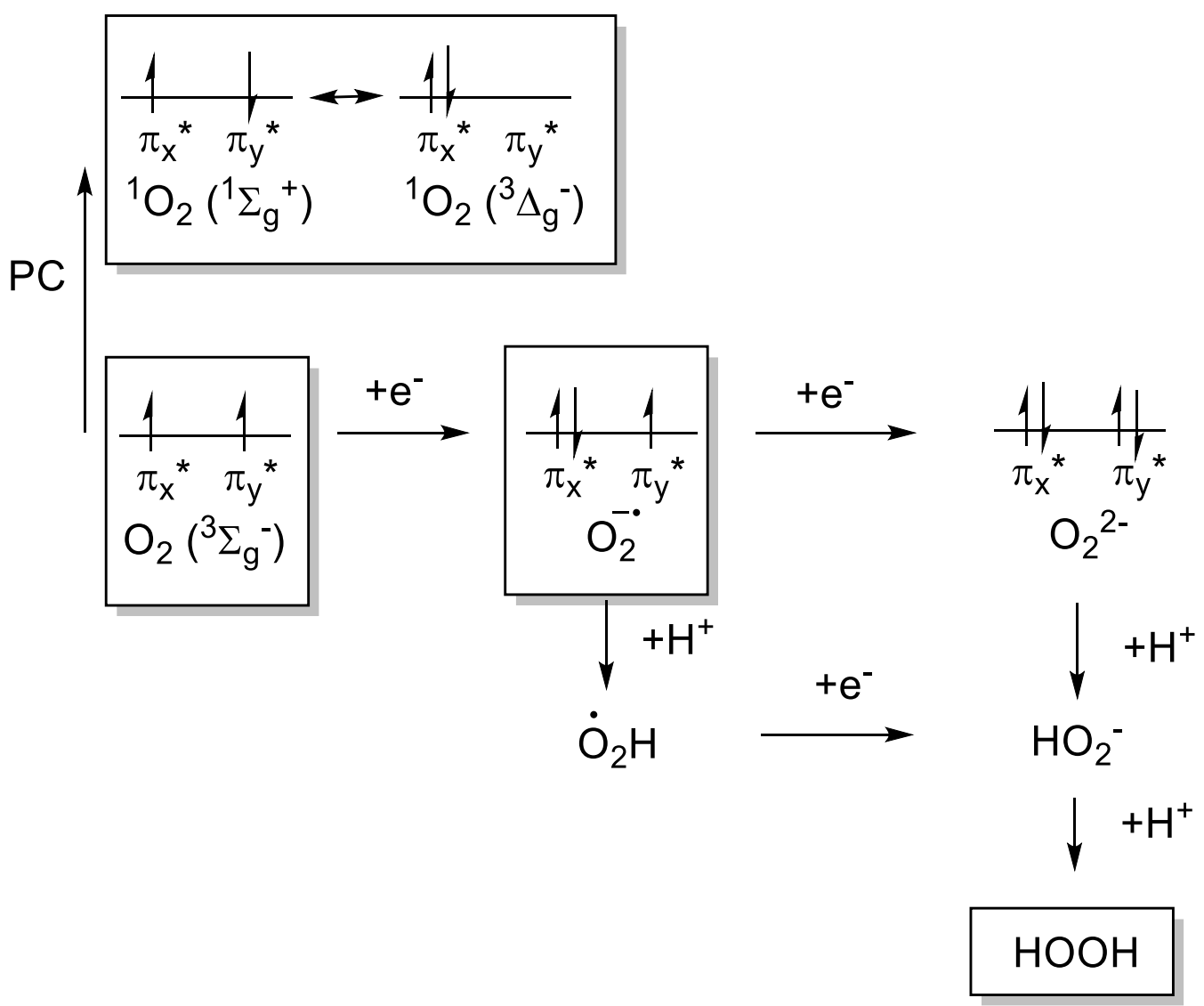

Figure 15: Energy independent representation of different ROS species. Marked states are: ground state oxygen $\left(\mathrm{O}_{2}\right)$, singlet oxygen $\left({ }^{1} \mathrm{O}_{2}\right)$, superoxide radical anions $\left(\mathrm{O}_{2 \cdot-}\right)$ and hydrogen peroxide $(\mathrm{HOOH}) \cdot{ }^{[61]}$

The chemical-based generation of ROS is based on high energetic molecules which release ROS during chemical reactions, like hypochlorite anions $\left(\mathrm{OCl}^{-}\right)$, radical activation of the peroxides to their higher reactive radical forms. Most concepts are not applicable for atmospheric oxygen, therefore not discussed in further detail. Utilizing high energy irradiation is not a valid concept for the transformation of oxygen to singlet oxygen, due to a spin forbidden transition. As mentioned earlier, the product would be ozone. ${ }^{[63]} A$ way to bypass this is the utilization of photocatalysts which can interact with the oxygen via direct energy transfer from the excited photocatalyst (PC) to form singlet oxygen, or via single electron transfer, forming a radical cation anion pair, which provides direct access to superoxide oxygen. ${ }^{[61]}$ The utilization of PC thereby provides access to the spin uncoupled singlet oxygen with has a shorter lifetime higher reactivity. 


\subsection{MECHANISMS OF ORGANIC PHOTOCATALYSTS}

Organic photocatalysts follow three different basic reaction pathways. The first one is the energy transfer (ET) and is considered as the Type 1 photocatalyst. ${ }^{[41]}$ Thereby, the photocatalyst absorbs a photon and is elevated to a higher state. This higher energy state can collide with an oxygen molecule transferring the energy directly to the oxygen molecule and forming the highly reactive singlet oxygen. Alternatively, other molecules can interact by collision with the photocatalyst and obtain the energy via energy transfer from the excited photocatalyst (Scheme 2)

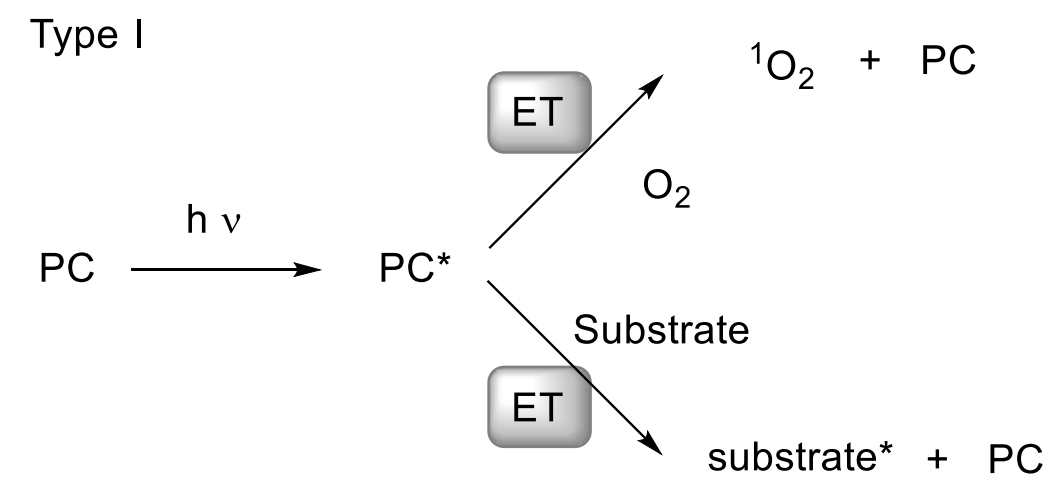

Scheme 2: Type 1 photocatalytic pathway via energy transfer (ET). ${ }^{[38]}$

The Type II photocatalytic pathway is divided into two different possible ways based on the redox potentials of the photocatalyst and the molecule interacting with the photocatalyst. So, an oxidative and reductive pathway become possible (Scheme 3). In the case of Type II (Scheme 3), the photocatalyst is excited by the irradiation. Based on the redox potential of the photocatalyst it can undergo oxidative, or reductive quenching. A) oxidative quenching pathway: the substrate or oxidant interacts with the activated photocatalyst to form a radical cation anion pair via singlet electron transfer (SET), with the photocatalyst as a radical cation and a corresponding radical anion. This radical cation photocatalyst can now interact with a second partner (substrate or reductant) and transfer the radical cation charge via a secondary SET. The photocatalyst is returned to its original state. The formed radical cation anion pairs are highly reactive and can perform a chemical reaction. 


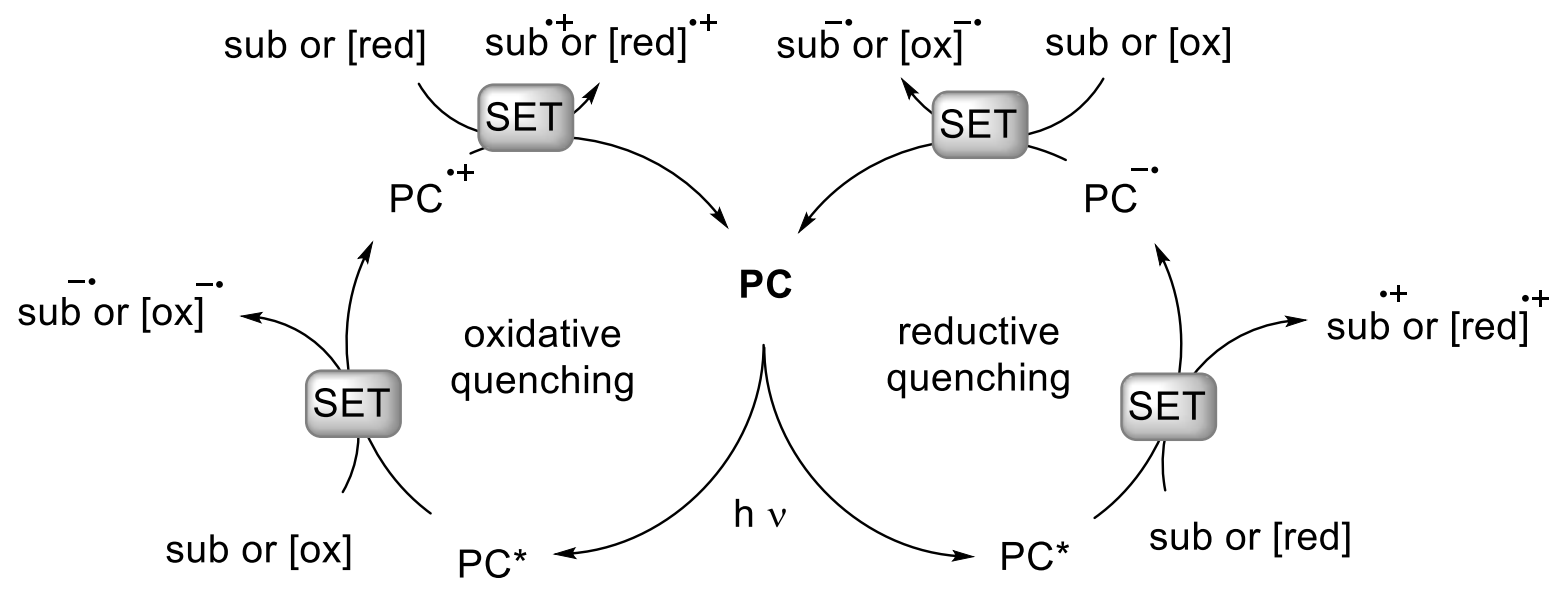

Scheme 3: Type 2 photocatalytic pathway via single electron transfer (SET) for the oxidative and reductive quenching pathways. Sub = substrate, [red] reductive species, [ox] oxidative species. ${ }^{[41]}$

B) Reductive quenching pathway: the substrate of reductant interacts with the photocatalyst to form a radical cation anion pair, via a singlet electron transfer (SET), with the photocatalyst as a radical anion and a corresponding cation radical. This radical anion photocatalyst can now interact with a second partner (substrate or oxidant) and transfer the radical anion charge via a secondary SET. The photocatalyst is returned to its original state. The remaining radical cation anion pairs are activated and can perform chemical reaction. Based on the redox potentials a substrate might not react in a desired way for optimal conditions, therefore the use of oxidants and reductants can offer viable bypass strategies. A third non-discussed pathway is the photocatalyzed atom transfer, which is based on the activation, which usually enables the transfer of a hydrogen atom between photocatalyst and the reagent. 


\subsection{THE OXIDATION OF SMALL MOLECULES AND THEIR APPLICATIONS}

Oxidation of small molecules is an omnipresent topic in organic chemistry. A broad variety of different methodologies exist for the transformation of innumerable substrates. The scope of possible organic substrates for the investigations is limitless and only the substrate groups and their products as also their possible uses, are discussed. The chosen four substrate groups are alcohols, indoles, pyrrole, secondary enamino ketones and their products. The use of stochiometric and over stochiometric amounts of oxidizing agents is one of the simplest approaches. ${ }^{[7]}$ This approach includes high amounts of produced waste products. The general use of catalysts is advisable to achieve the aims of green chemistry. Transition metal-based catalysts show good reactivity but have disadvantages. Many transition metal-based catalysts are difficult to handle, not stable to air, or water, need non-commercial ligands, or exhibit relatively high toxicity. By utilization of organocatalysts some, or even all, draw backs can be avoided. This positive influence of transition metal-free catalysts have thereby high interest for the pharmaceutical industry, due to possible lower toxicity and lower cost. ${ }^{[7,65]}$

\subsubsection{Alcohol Oxidation Methods}

The production of alcohol and its further reactions are ancient processes, which go along with the beginning of civilization. ${ }^{[64]}$ Oxidation products of common alcohols were already used for medical uses during the time of Hippocrates in ancient Greece, as western medicine was formed. ${ }^{[1]}$ The generation of carboxylic acids thereby appears under aerobic conditions gradually, but naturally. The application of oxidants and catalyst can accelerate this process. For primary alcohols the oxidation to the carboxylic acid is easily achieved. In contrast, the selective oxidation of primary alcohols to their corresponding aldehydes faces many challenges. ${ }^{[6]}$ The selective oxidation of primary alcohols has no generally applicable method and is a researched topic of modern organic chemistry. Selective oxidation protocols were discovered and 
became well known for their contributions like the Jones-, ${ }^{[67]}$ Swern-, ${ }^{[68]}$ DessMartin-, ${ }^{[69]}$ Collins-, ${ }^{[70]}$ Corey-Suggss ${ }^{[71]}$ and Corey-Schmidt-oxidations ${ }^{[72]}$ (Scheme 4).

Corey-Schmidt

Scheme 4: Selective oxidation protocols for the oxidation of primary alcohols. ${ }^{[67-73]}$

Each of these name reactions for the oxidation of alcohols have limitations and are not a generalized ideal oxidation. Jones-oxidation is not selective and results in overoxidation to the carboxylic acid. The Swern oxidation is a selective and efficient oxidation. The toxic intermediates are generated in situ and only partially toxic byproducts exist after the reaction. The efficiency of the reaction is thereby also a nonoptimal characteristic of the reaction, so that the reaction has to be performed at lower temperatures than $-40^{\circ} \mathrm{C}$ due to the high reactivity of the in situ formed reactive intermediates. Even with the reduced toxicity of the reaction, substantial amounts of 
toxic reagents are required for the reaction. The Collins-, Corey-Suggs- and CoreySchmidt-oxidations all utilize toxic chromium catalysts and environmentally unfriendly reaction conditions. In industry, the Pfitzner-Moffatt oxidation ${ }^{[73]}$ is one of the main oxidation protocols for the selective oxidation of primary alcohols, also in this case the use of stoichiometric amounts of toxic $N, N^{\prime}$-dicyclohexylcarbodiimide has to be utilized (Scheme 4, last entry)
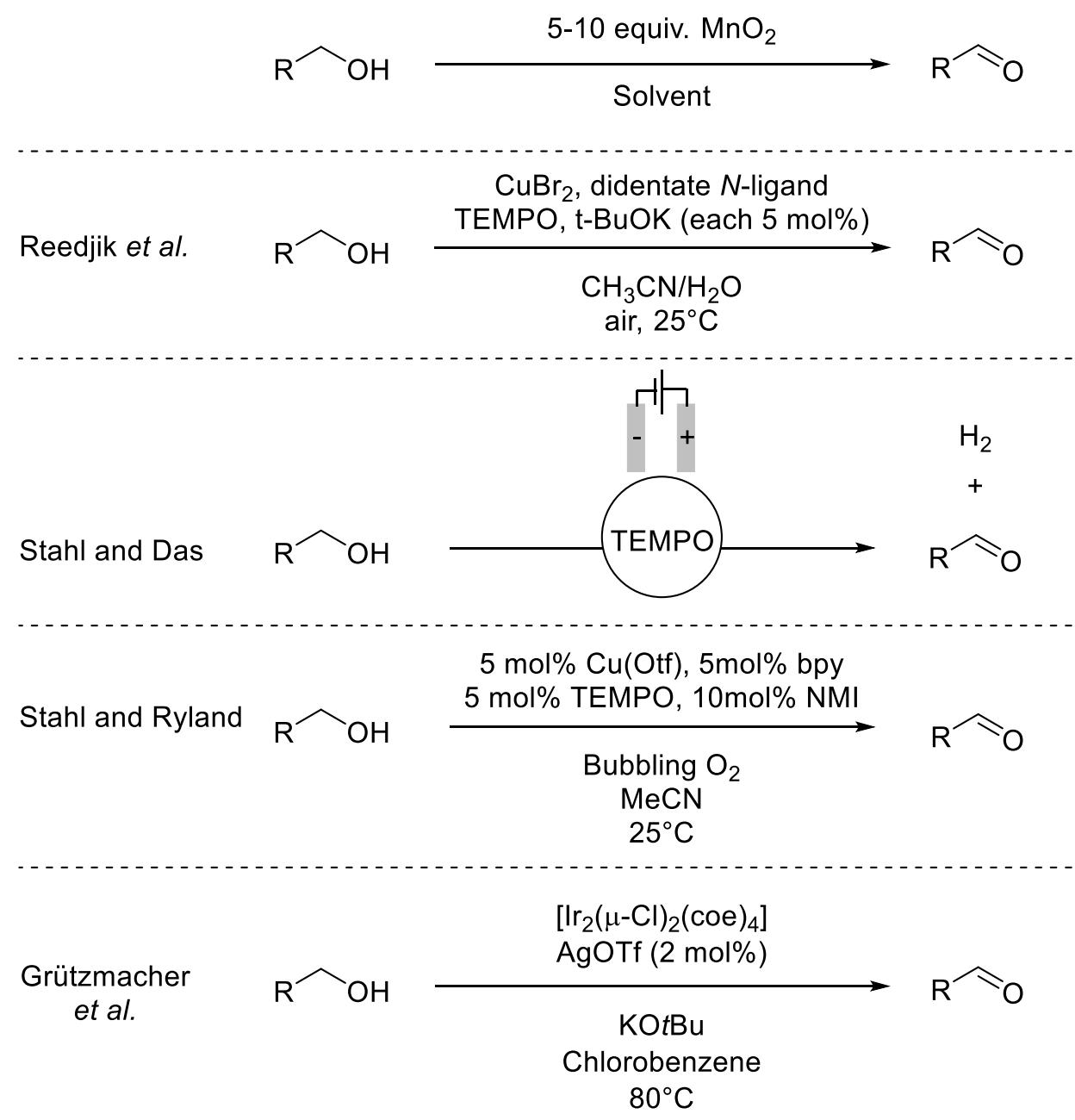

Scheme 5: Different catalytic approaches for the selective oxidation of primary alcohols. ${ }^{\text {[74-78] }}$ 
The limitation of the name reaction for the selective oxidation of alcohols leads to numerous further investigated ways for selective oxidation. The use of manganese dioxide $\left(\mathrm{MnO}_{2}\right)$ is stoichiometric approach which is over 55 years old based on Blackadder et al. and Wildman et al.; the conditions are still subject to research for further improvement. ${ }^{[74]}$

Many different attempts were performed to find mild and environmental friendly conditions for the selective oxidation of primary alcohols (Scheme 5 and $\mathbf{6}$ ), like the $\mathrm{CuBr}_{2} / \mathrm{TEMPO}$ catalyzed oxidation by Reddijk et al., but with a limited substrate scope (Scheme 5). ${ }^{[75]}$ An electrochemical approach was performed by Das and Stahl $(\text { Scheme } 5)^{[76]}$, by the use of electrodes and catalytic amounts of TEMPO. An non electrochemical oxidation protocol by Hoover, Ryland and Stahl (Scheme 5) utilizing $\mathrm{Cu}(\mathrm{OTf})_{2}$ in combination of bpy, TEMPO, NMI and DBU as additives for the oxidation was reported at the same time. ${ }^{[77]}$ Both approaches were versatile and efficient under mild conditions, but the experimental preparation for the electrochemical approach was quite extensive and for the wet chemical approach a five-component system had to be utilized for the catalytic reaction. Grützemacher et al. (Scheme 5) showed the effect of an iridium-aminyl-radical-complex capable of oxidizing benzylic and unactivated alcohols but had to utilize iridium as a precious metal. ${ }^{[78]}$ In $2004 \mathrm{Hu}$ et al. (Scheme 6) showed a metal-free approach for the selective oxidation of primary benzylic and secondary benzylic alcohols. ${ }^{[79]}$ Applying thermal conditions, TEMPO, bromide, $\mathrm{NaNO}_{2}$ at $80^{\circ} \mathrm{C}$, the reaction was efficient under mild conditions, but suffered by a limited substrate scope of benzylic substrates. In 2017, Das et al. (Scheme 6) showed a $\mathrm{CO}_{2}$-catalysed metal-free environmental-friendly selective oxidation protocol, but also in this case the reactivity was limited to benzylic substrates. ${ }^{[80]}$ 
Hu et al. $\quad \mathrm{R}_{\mathrm{OH}}$
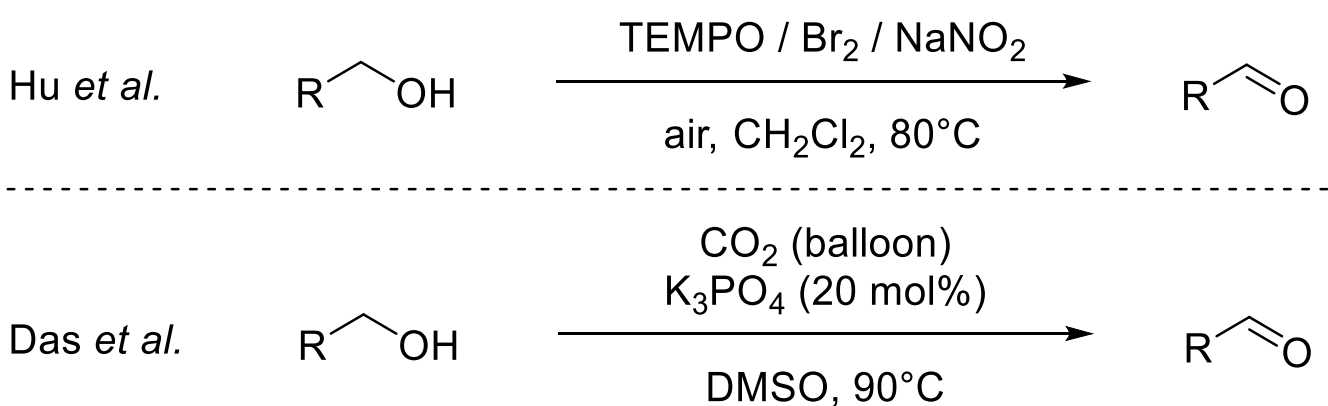

DMSO, $90^{\circ} \mathrm{C}$

Scheme 6: Transition metal-free approaches for the selective oxidation of primary and secondary benzylic alcohols. ${ }^{[79-80]}$

Based on the limitations discussed, an interest has arisen in photocatalytic approaches for the selective oxidation of primary alcohols. Antonietti et al. reported the utilization of mesoporous graphitic carbon nitride for the thermal photochemical secondary alcohols (Scheme 7). ${ }^{[81]}$ Utilizing 8 bar of oxygen pressure at $100^{\circ} \mathrm{C}$ reaction temperature and the application of photoactivation resulted in medium to good conversions for benzylic and unactivated secondary alcohols. Primary alcohols were not reported and the fact that several reports showed catalytic activity of graphitic carbon nitrides at elevated temperatures over $80^{\circ} \mathrm{C}$ without the use of visible light irradiation, ${ }^{[82]}$. This showed possible doubts about the reported mechanism of the study of Antonietti et al. Two major reports occurred in 2017. Jiang et al. reported that preparation of different $\mathrm{Pt} / \mathrm{CN}-224(\mathrm{M})$ catalysts using several different transition metals (Scheme 7). ${ }^{[83]}$ The catalysts were able to induce photothermal effects in combination with singlet oxygen production, which resulted in selective oxidation of several activated benzylic alcohols. The results were based only on GC-quantification and were never isolated. In the same year, Zhang et al. showed the utilization of covalent triazine frameworks as an efficient photocatalytic material for the selective oxidation of primary and secondary benzylic alcohols via the Type II photocatalytic pathway (Scheme 7). ${ }^{[84]}$ The photocatalytic performance for benzylic alcohols was excellent, but the substrate scope was limited.

Secondary alcohols have no possibility of overoxidations, due to the fact that it is in the highest oxidized form of the functional group. But the oxidation of secondary nonbenzylic alcohols has to be discussed thoroughly. A general summary of the reported methodologies exposed, that methodologies for the oxidation of primary, secondary 
alcohols without limitations, include toxic and partially harsh reaction conditions. Different approaches for milder, environmentally friendlier methodologies exist for selective oxidation under different aspects which in most of the cases, limit or deny the possibility to oxidize secondary non-benzylic alcohols.
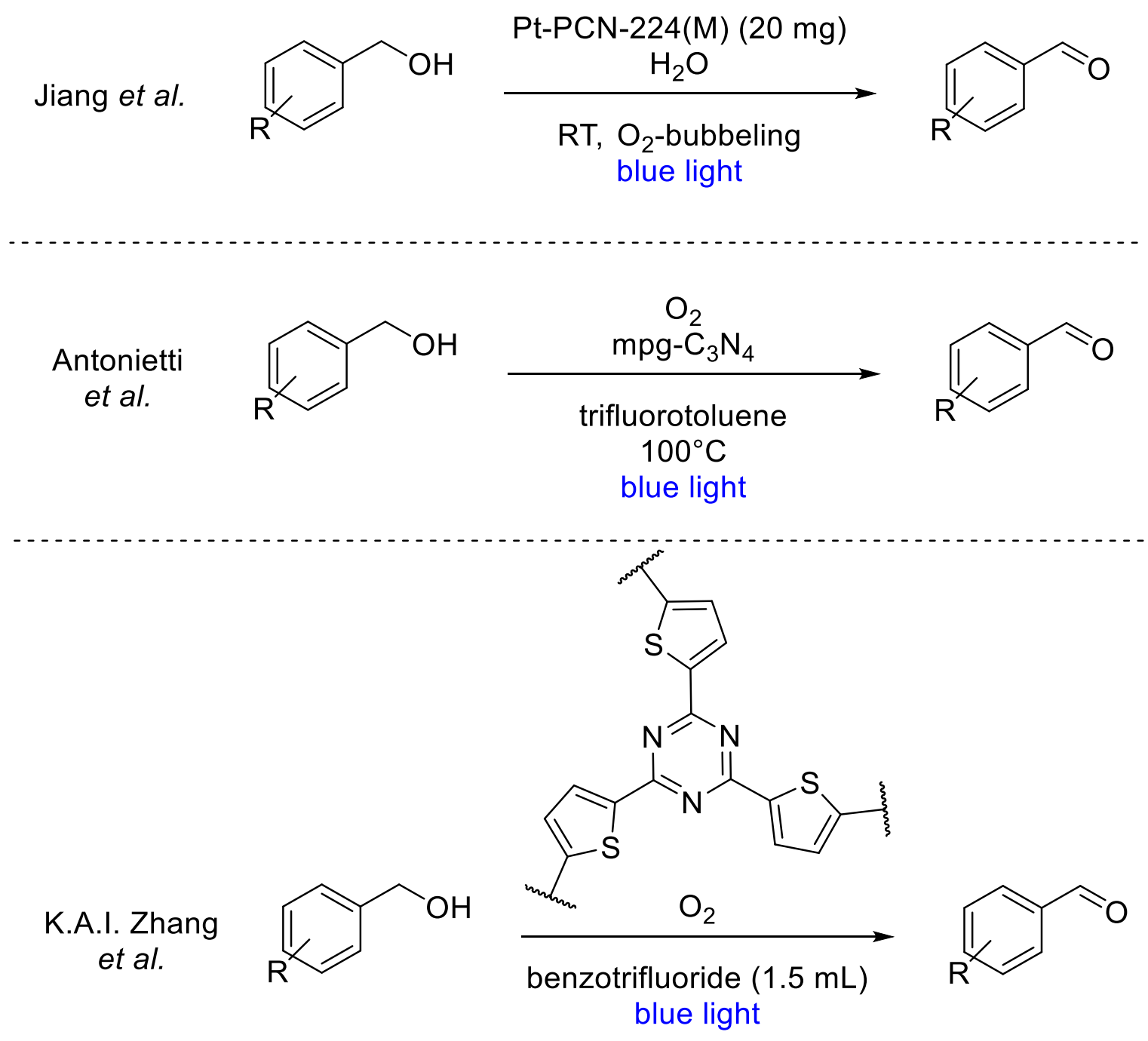

Scheme 7: Photocatalytic approaches for the selective oxidation of primary and secondary alcohols. ${ }^{[81,83-84]}$ 


\subsubsection{Heterocyclic Compounds, Strategic Methodologies IN the SYNTHESIS OF COMPLEX StRUCTURE INCLUDING THE INDOLE, PYRROLE, ISATIN ANd Maleimide StRuctural Motives}

Heterocycles are one of the most prominent structural groups in organic chemistry. Among these, indole, pyrrole, isatin and maleimides are prominent structural motives

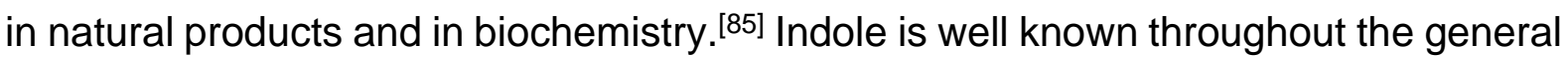
populace due to its presence in several flowers like jasmine and clove; in higher concentrations it changes the odor characteristics and is responsible for an essential part of the smell of human and animal feces. ${ }^{[86]}$ The beginning of indole-based chemistry in the $19^{\text {th }}$ century was related to the synthesis of the indigo dye which is based on the indole framework. In the 1930's indole research intensified with the discovery of the indole framework inside alkaloids and other natural products. Therefore, it was shown that the indole framework is an essential part of the animal and plant metabolism, especially being the foundation of one of the amino acids (tryptophan). ${ }^{[85]}$
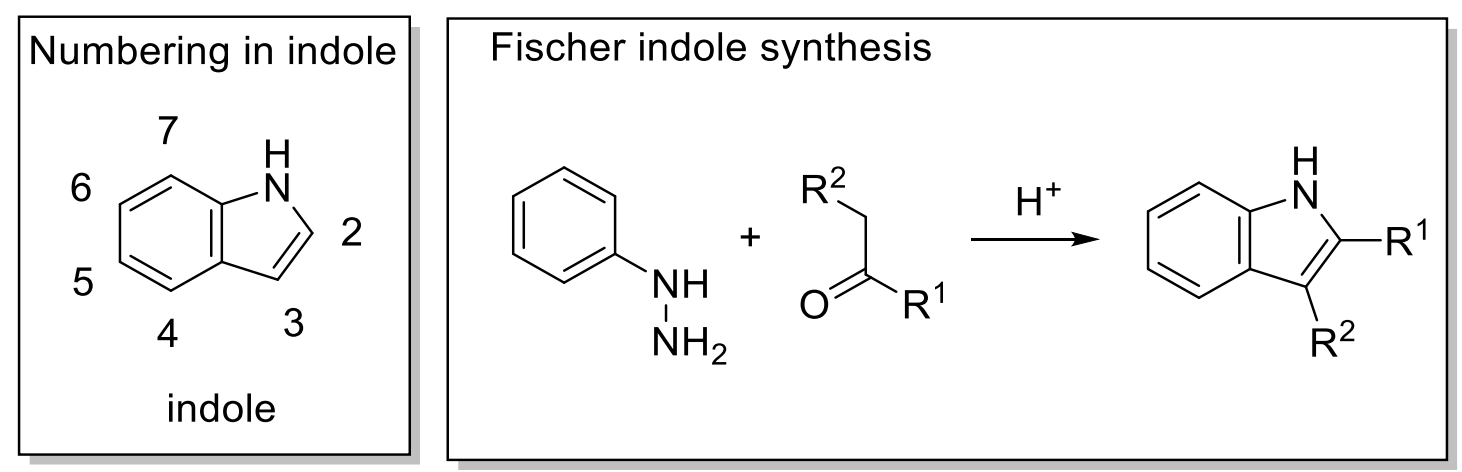

Scheme 8: Numbering in the indole structure and the Fischer indole synthesis. ${ }^{[85]}$

The synthesis of complex natural products and biochemical compounds containing these frameworks are limited and result in a variety of different strategies to form the target structure. The reactivity of indole is limited and involves in many cases the oxygenation of the indole scaffold in positions 2 and 3 (Scheme 8). ${ }^{[85]}$ The oxygenation, or dearomatization of indole to isatin usually includes harsh reaction conditions, which limit functional group tolerance and make this a complex step in the total synthesis of these compounds. Taking into consideration that a long inability of these dearomatizations, most total syntheses rely on the formation of the scaffold from 
different precursors. New approaches have been investigated for over 100 years of research in this field. After the formation of the indole-based scaffold, the reactivity is limited and results in direct approaches of final substituted scaffolds. The field would benefit from synthetic tools to afford the dearomatization of indoles under mild conditions with high functional group tolerance, to provide new concepts in the total synthesis of different compounds. Besides the biosynthesis of indoles three dominant technical routes exist. First of all is the Fischer indole synthesis from the $19^{\text {th }}$ century. ${ }^{[87]}$ The drawback of the Fischer indole synthesis is that in its original form usually only substituted indoles with substitution in position 2 and 3 could be obtained. Several other reactions exist similarly to the Fischer indole synthesis, like 1) the Bartoli indole synthesis, via grinard reagents, ${ }^{[88]}$ 2) Bischler-Möhlau, ${ }^{[89]} 3$ ) Fukuyama, which utilizes AIBN and Bu3 $\mathrm{SnH}$ for cyclisation, ${ }^{[90]}$ 4) Gassman,, ${ }^{[91]}$ 5) Hemetsberger, ${ }^{[92]} 6$ ) Larock, ${ }^{[93]}$ 7) Madelung, ${ }^{[94]}$ 8) Nenitzescu, ${ }^{[95]} 9$ 9) Reissert, ${ }^{[96]}$ 10) Baeyer-Emmerling indole synthesis. ${ }^{[97]}$ In the first eight reactions of indole syntheses, at least one of the two positions (2/3) will be substituted. Reissert and Baeyer-Emmerling are capable for the 2,3-unsubstituted indole synthesis. In the beginning of the $21^{\text {st }}$ century. Microwave assisted methodologies were developed for the indole synthesis to improve on that limitation. ${ }^{[98]}$ The resulting amount and wide variations of the reactions are due to the targeted synthetic approach to form the desired complex structure.

The second major synthetic route is the Leimgruber-Batcho indole synthesis utilizing Raney-Nickel. ${ }^{[99]}$ With this strategy, a wide range of different indole derivatives can be synthesized, which is mostly used in pharmaceutical industries due to the complex indole-based structures that compose an integral part of many pharmaceuticals (Scheme 9).[100] A technical large-scale synthesis of substituted indoles is based on high temperature catalyzed reaction of aniline derivatives with ethylene glycol (Scheme 9). ${ }^{[101]}$ 
LeimgruberBatcho indole synthesis<smiles>Cc1ccccc1[N+](=O)[O-]</smiles>

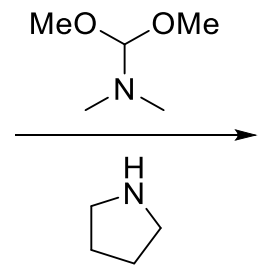<smiles>O=[N+]([O-])c1ccccc1/C=C/N1CCCC1</smiles><smiles>c1ccc2[nH]ccc2c1</smiles>

\footnotetext{
Technical aproach gas phase reaction Ube industries
}<smiles>[R]c1ccc(N)c([R])c1[R]</smiles><smiles>[R]c1cc2cc[nH]c2c([R])c1[R]</smiles>

Scheme 9: Leimgruber-Batcho indoles synthesis and industrial technical synthesis. ${ }^{[99,101]}$

The substitution of indoles in position 2 and 3 is thereby on the essential positions. The 2-oxoindole and 3-oxoindole, as also 2,3-dioxoindole (isatin) are reactive derivatives for the further modification of the indole framework. The number of methodologies to obtain this is very limited and recent. In 2019 Chandra et al. showed the oxygenation utilizing NIS and IBX in DMSO.[102] Muneer et al. showed photocatalyzed oxygenation in aqueous solution with titanium dioxide (Scheme 10). ${ }^{[103]}$ In 2016 Jiang et al. showed a versatile chemo selective methodology utilizing dicyanopyrazine-dervied chromophores for the formation of different isatins from indoles. Based on the chosen conditions several other reaction types could occur. ${ }^{[104]}$ The of limitations in synthetic strategies and the novelty of direct oxygenation of indoles gave rise to new possibilities in the synthetic pathways to form complex indole-containing structures. 
Muneer et al.<smiles>c1ccc2[nH]ccc2c1</smiles>

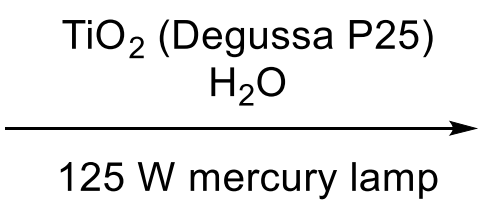<smiles>O=C1Nc2ccccc2C1=O</smiles>

Chandra et al.<smiles>[R]c1cc2ccn([R4])c2c([R])c1[R]</smiles>

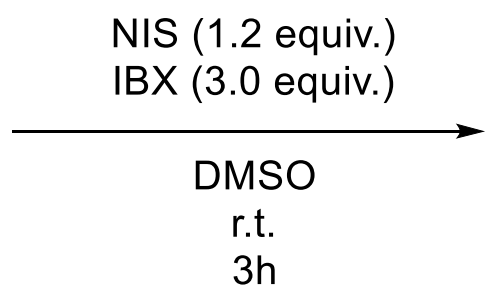<smiles>[R]c1cc2c(c([R])c1[R])N([R])C(=O)C2=O</smiles>

Jiang et al.<smiles>[R][X]c1ccc2ccn([R])c2c1</smiles><smiles></smiles>
Blue LED<smiles>[R][R]1ccc2c(c1)N([R])C(=O)C2=O</smiles>
r.t.

Scheme 10: Oxidations of indole to isatins and further functionalization. ${ }^{[102-104]}$

Pyrroles and maleimides are also essential building blocks in nature and form an integral part of the synthesis of porphyrinogens like heme and chlorophyll.[105] In this case the oxygenation of pyrrole to maleimide is easy to achieve but includes other obstacles. Based on the importance of pyrrole in synthetic chemistry and nature also here many reactions carry important names (Scheme 11), like Hantzsch pyrrole synthesis, ${ }^{[106]}$ Knorr pyrrole synthesis, ${ }^{[107]}$ Pall-Knorr pyrrole synthesis, ${ }^{[108]}$ Van Leusen reaction, ${ }^{[109]}$ Barton-Zard synthesis, ${ }^{[110]}$ Piloty-Robinson pyrrole synthesis ${ }^{[111]}$ and more. ${ }^{[112]}$ In this case the reactivity of the positions 2 and 4, which are adjunctive to the nitrogen are essential and need oxygenation to be modified if the ring formation is already concluded. Besides the natural occurrence of pyrrole in heterocycles, the more reactive oxygenated form of maleimide possesses great importance in the creation of biopolymers and bioconjugates. ${ }^{[95]}$ 
Hantzsch<smiles>[R]OC(=O)CC([R])=O</smiles><smiles>[R]C(=O)C([R1])Cl</smiles><smiles>[R]OC(=O)c1c([R])[nH]c([R3])c1[R]</smiles>

\section{Knorr}

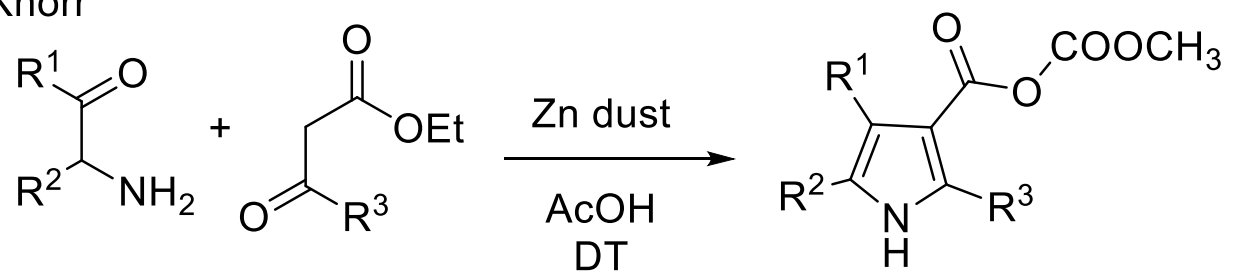

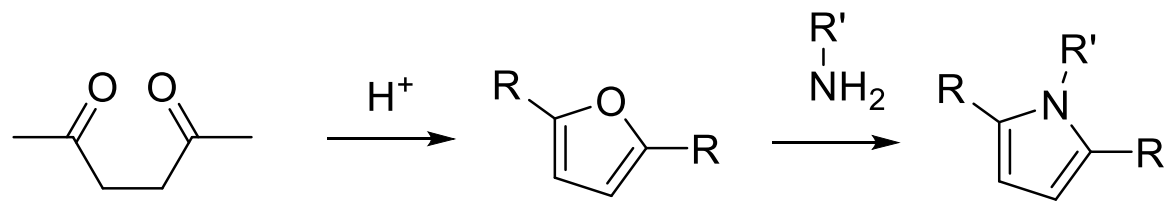

\section{Barton-Zard}<smiles>[R]/C=C(/[R])[N+](=O)[O-]</smiles><smiles>[R]C(=O)c1[nH]cc([R])c1[R]</smiles>

Scheme 11: Selected named reactions for the synthesis of pyrroles. ${ }^{[98-103]}$

The oxygenated form of pyrrole, the maleimide, is thereby a highly reactive reagent which has a multitude of different applications (Scheme 12). ${ }^{[113]}$ It is used for thermoset polymer synthesis, ${ }^{[114]}$ as preparation of SMCC reagents which is one of the most widely used bioconjugations in biotechnology. ${ }^{[115]}$ It is as a result, one of the important conjugates for the synthesis of antibody-drug conjugates. The central problem is the instability considered to basicity and acidity, thermal conditions and further influences. ${ }^{[12]}$ This makes mild conditions and direct oxygenation approaches feasible for this structure group. 


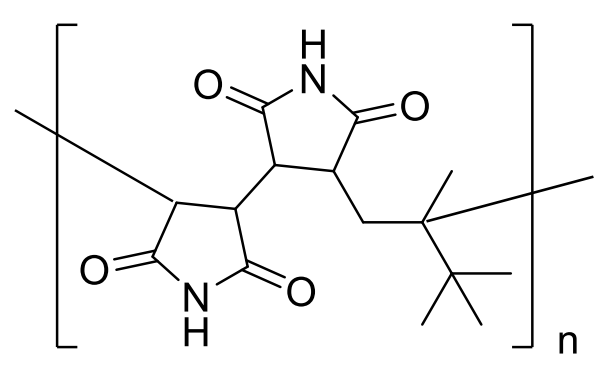

Polymers

(Also as dimeric polymerisation starters)

(Crosslinker)<smiles>[R]C(=O)ON1C(=O)CCC1C</smiles><smiles>[R]NC(=O)NCCCCC(NC(C)C)C(=O)NC(C)C</smiles>

Bioconjugation between antibody and possible drug as $\mathrm{R}$

Scheme 12: Utilizations of maleimides in biotechnology, pharmaceuticals and polymer science. ${ }^{[105,113-115]}$ 


\subsubsection{REACTIONS OF SINGLET OXYGEN AND THEIR INTERACTIONS WITH SECONDARY ENAMINO KETONES}

The use of singlet oxygen in photochemistry is a long-used concept, which is far longer than the physical and mechanistic understanding of the state of singlet oxygen. In the $19^{\text {th }}$ century different chromophores were used to facilitate different chemical reactions, several of which were based on singlet oxygen, without the proper means to investigate the underlying mechanism. In the 1940's, these reports led Günther Otto Schenck to demonstrate an industrial scale photocatalytic plant in his garden. The plant was used for the oxidation of terpinene by sunlight and the reaction was catalyzed by chlorophyll from spinach. With his success in upscaling the reactions, he additionally postulated the concept of photosensitized oxygenation and mechanistic theory of sens ${ }^{\text {rad }} \mathrm{O}_{2}$. With his theories he laid the foundations for the development of Schenck-ene reactions and the classification of singlet oxygen by advanced spectroscopic means, which did not exist at his time. ${ }^{[116]}$

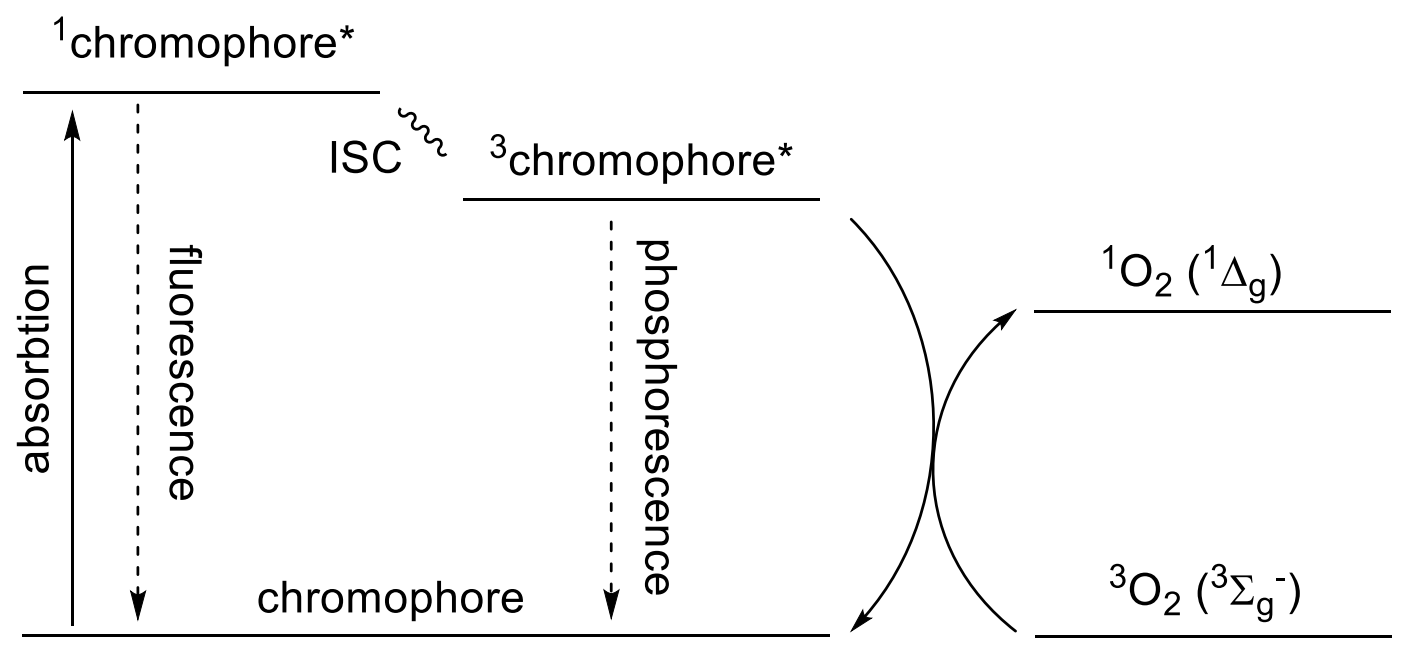

Figure 16: Jablonski diagram of the photosensitized activation of oxygen to singlet oxygen. ${ }^{[177]}$

With the advances in spectroscopic methodology, the precise photocatalytic excitation of molecular oxygen by visible light could be described by a Jablonski diagram (Figure 16). ${ }^{[117]}$ A chromophore absorbs the visible light, which excites the chromophore into the singlet excited state. The short-lived excited state undergoes an intersystem crossing to form the long-lived triplet state of the excited chromophore. This can perform an energy transfer (ET) to the inactive triplet oxygen from the atmosphere via 
collision and excite this to single oxygen. The chromophore can additionally deactivate itself by fluorescence quenching from the singlet excited state, or by phosphorescence from the triplet excited state.

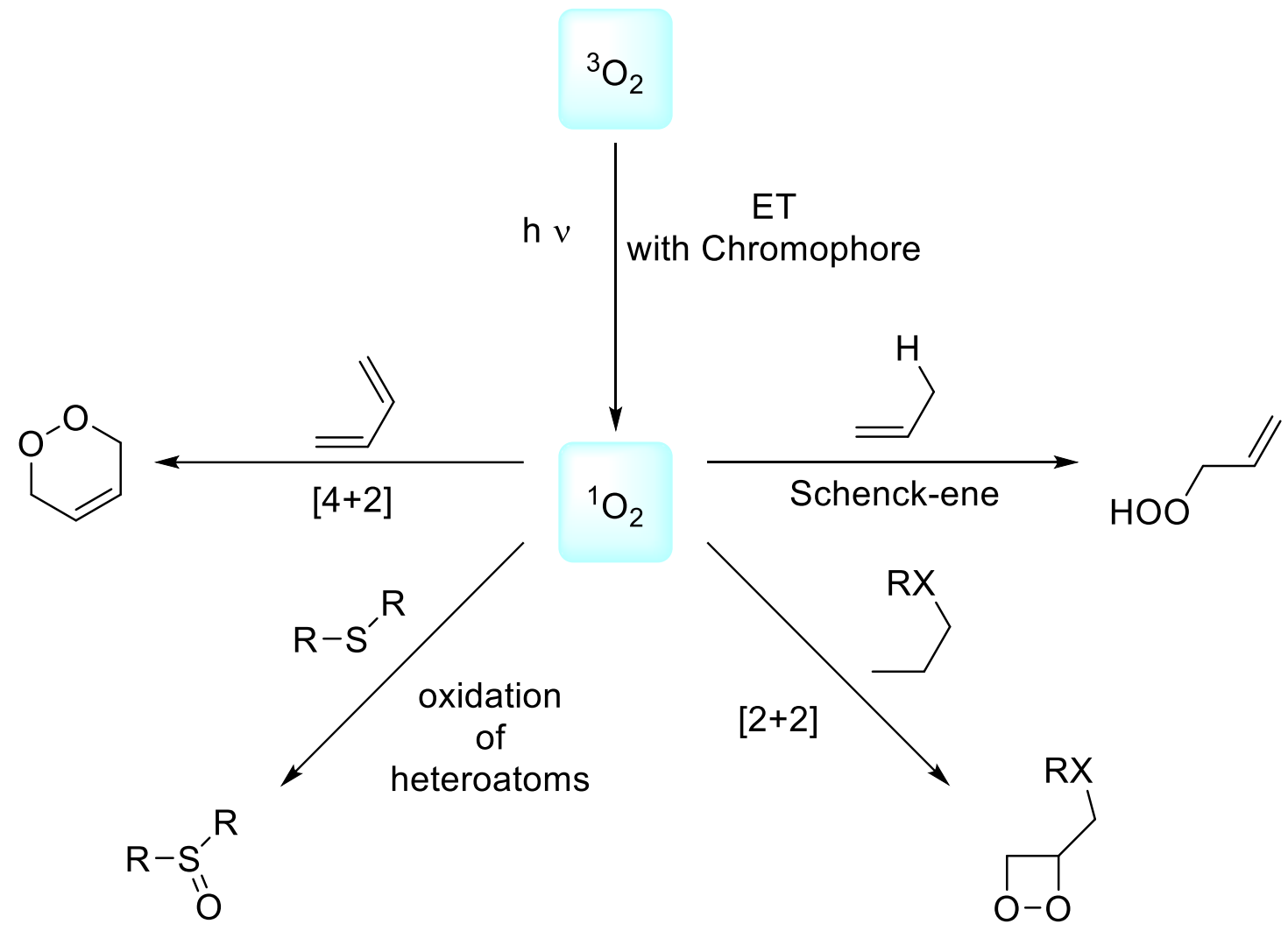

Scheme 13: The four most common reaction types of singlet oxygen by photochemical activation. ${ }^{[118]}$

With the advances in mechanistic investigations and experience in photochemistry, four different main classes of photochemical singlet oxygen could be classified (Scheme 13). ${ }^{[118]}$ The oxidation of 1,3-dienes to form endoperoxides via a [4+2] cycloaddition. The oxidation of heteroatoms, for example sulfides to sulfoxides. By reaction of electron rich alkenes with singlet oxygen a [2+2] cycloaddition occurs to form 1,2-dioxetanes. The fourth class is the Schenck-ene type reaction. Considering the long time Schenck-ene reactions are known, a full mechanistic investigation was only done recently by W. Adam et al. and respectively by Axel Jacobi von Wangelin et al. in which they explained several possible transition states and postulated rules for possible reactions. ${ }^{[119]}$

Classical cleavage reactions of secondary enamino ketones were already reported in the 1970 by Ando et al. ${ }^{[120]}$ and Wasserman et al. ${ }^{[121]}$ Additionally, many further 
alternative oxidations were performed on the substrate class and resulted in cleavage reaction yielding two carbonyl compounds. In 2014, Fan and $\mathrm{Li}^{\left[{ }^{[122]}\right.}$ as also Wu et al., ${ }^{[123]}$ reported independently that they observed an alternative reaction applying photochemical approaches (Scheme 14). Both groups investigated the results and found that they obtained 3-Oxo- $N$-phenyl-isovaline methyl ester from (E)-4(phenylamino)pent-3-en-2-one via photocatalytic reaction with their respective chromophore. Mechanistic investigations of both groups revealed that the use of singlet oxygen resulted in an ene-type reaction, followed by an esterification and an 1,2 -acylshift reaction. These results allowed access to a new structural class of compounds with so far unknown potential.

Ando

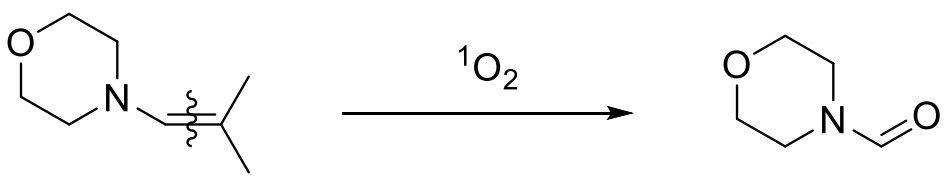<smiles>CC(C)=O</smiles><smiles>CC(=O)C(C)=O</smiles>

Fan and $\mathrm{Li}$

Wu et al.
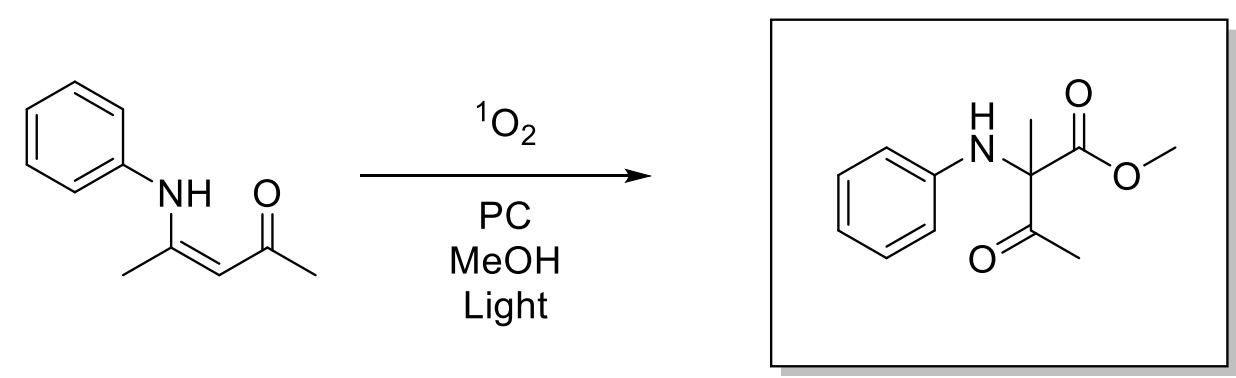

Scheme 14: Summary of reported reactions between singlet oxygen and enamines. ${ }^{[120-123]}$ 


\section{OBJECTIVES}

The resurface of photocatalysis was accompanied by the technological and mechanistic improvements, which enable new possibilities for chemical reactions to fulfill the target of green chemistry. The utilization of organic compounds, as photocatalysts, brings the befit to achieve well known reactions in greener and safer ways, with the addition of less toxic and complex technical aspects. The primary objective of this thesis was the investigation of organic molecules for photocatalytic oxidation of several substrate groups and the optimization of the oxidation to achieve high yield, minimized use of additives and low catalyst loadings. In general, the aims are greener, safer and milder reaction conditions, ideally under low energy LED-light, or utilizing direct solar radiation.

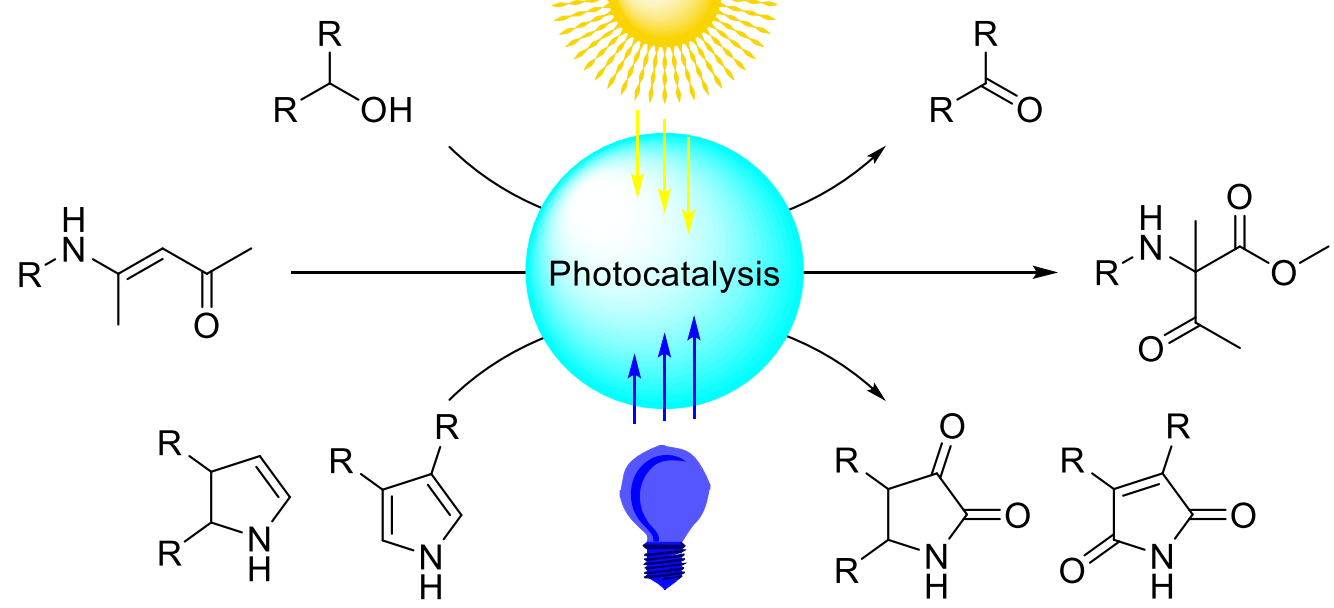

Figure 17: Concept of utilizing photocatalysis for the oxidation of small molecules by utilizing solar light, or alternatively LED light. The shown transformation are the chosen model substrates for the investigated oxidation reactions.

The targeted substrate groups consisted of three different substance classes with increasing complexity in their reactivity towards oxidation reactions. The first class was the oxidation of alcohols. The oxidation of alcohols is one of the oldest known chemical transformations in the history of mankind and has been achieved in many possible ways. Still green and environmentally friendly selective oxidation methodologies are missing. Therefore, new concepts should be developed. 
Based on the results of the selective oxidation of alcohols, the efficient oxygenation/dearomatization of heterocycles for the use in further syntheses was investigated. Heterocycles offer vast possibilities in the synthesis of natural compounds and pharmaceuticals but are limited to specific reactivities and synthetic protocols. New pathways in the synthetic strategies have been investigated and optimized for the step and atom efficiency.

Secondary enamino ketones were chosen as the third target group and their efficient oxidation via singlet oxygen was achieved. This field has been recently advancing with reports of new reactivities based on the concepts of photocatalysis. An alternative transition metal-free concept has been developed and examined for its possible application.

Additionally, the mechanisms of these reactions have been studied in detail to gain a deeper understanding of the mechanistic pathways, so that the results could lead to the design of new reaction concepts.

The secondary objective was the investigation of the physical properties of the LEDsetup and possible further developments to optimize the efficiency, applications and possibilities of the photochemical setups. Further alternative reaction setups shall be investigated and designed to affiliate new possibilities.

An additional objective was the design of new means for the synthesis of heterogenous graphitic carbon nitride photocatalysts, which can utilize the prior results. Therefore, the design of new setups for the synthesis of carbon nitrides was developed, examined and utilized for the synthesis of heterogenous photocatalysts based on graphitic carbon nitrides $\left(g-\mathrm{C}_{3} \mathrm{~N}_{4}\right)$ with different redox potentials. 


\section{RESULTS AND DISCUSSION}

To achieve the objectives, a variety of different approaches were chosen. The first part consists of different substrate groups for oxidation reactions utilizing different photocatalysts in different solvents to achieve maximum efficiency. An additional criterion for these investigations were safety and environmental concerns, therefore less-toxic reagents, byproducts and additives were preferred. Furthermore, the mechanism of the investigated transformation should be studied and the involvement of all parts of the reaction should be discussed for their influence in the reaction. Each chapter will include insights of the prior mechanistic conclusions, which will be summarized to form a detailed conclusion based on all performed investigations.

Prior to the specific results of the concluded project, the evaluation and design of the photocatalytic setup will be discussed. Based on the theoretical background discussed in Chapter 1.2, the reaction is severely influenced by geometry and characteristics of the chosen setup. For this thesis a standardized setup was chosen and used, if not stated differently. During the thesis mostly the original design of the Das group was used (Figure 18).

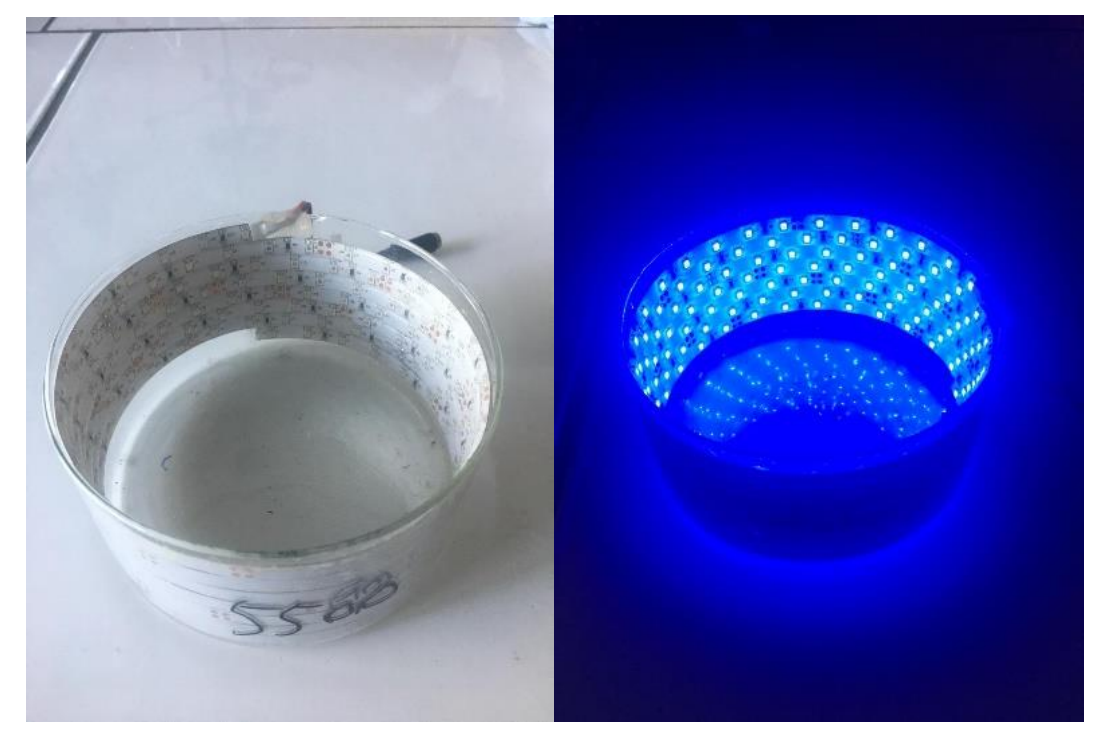

Figure 18: Led-glass setup of the Das group. 
The glass setup showed good possibilities but had also several disadvantages. The thermal conductivity of glass is limited and therefore the lifetime of the LED-strip is reduced. Additionally, the setup cannot divert heat by itself and has to be cooled by a computer fan, which has to be adjusted additionally. The position of the fan can have an impact on the resulting temperature of the reaction setup. To improve on these disadvantages, a metal-based concept was derived. By gluing the LED-stripes into the inner surface of a commercial paint can, high thermal conductivity was ensured. The bottom layer was removed from the paint can and thereby replaced by a fixed computer fan. This modification enabled a stable temperature of the reaction setup of $19-21^{\circ} \mathrm{C}$, even after long periods of irradiation. The improved thermal conductivity of the metal provided the possibility to increase the number of utilized LED-diodes, which made it possible to install enough diodes to yield up $36 \mathrm{~W}$ performance, without the risk of overheating. After initial examinations the setup was refined by additional safety grids, to separate the fan from the glassware of the reaction vessel. The metal concept provided a further safety benefit, the metal limited the angles in which the LED light could exit the reaction area, which was providing a safer workspace environment, due to the indirect light source. Additional variants with red LED and RGB-LED were realized afterwards.

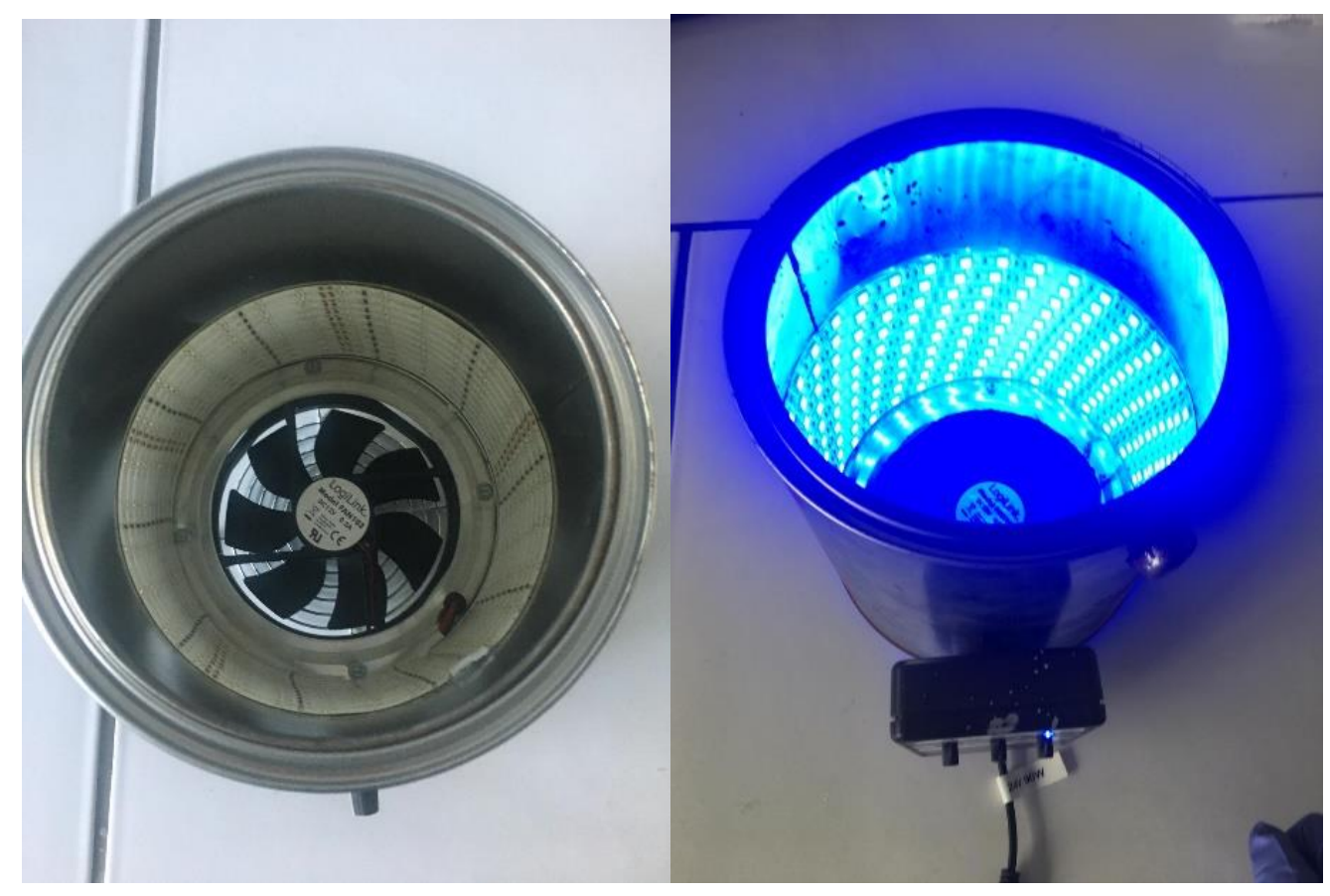

Figure 19: Metal-framed-LED design by Waldemar Schilling and Daniel Riemer and realized by the Das group. 
Emission spectra for both concepts were recorded (Figure 20). For both concepts no UV-irradiation was detected. For the two different blue LED-setups, maxima of emission were recorded at $456 \mathrm{~nm}$ for each. Lowest emission wavelength was at $404 \mathrm{~nm}$ and therefore excluding UV-emissions. The maximal detected wavelengths were recorded at 552 and $553 \mathrm{~nm}$. The investigations of the glass-based red-light emitting LED setup resulted in the following measured parameters: $\lambda_{\max }=631 \mathrm{~nm}$; $\lambda_{\text {low, end }}=575 \mathrm{~nm} ; \lambda_{\text {high,end }}=677 \mathrm{~nm}$.
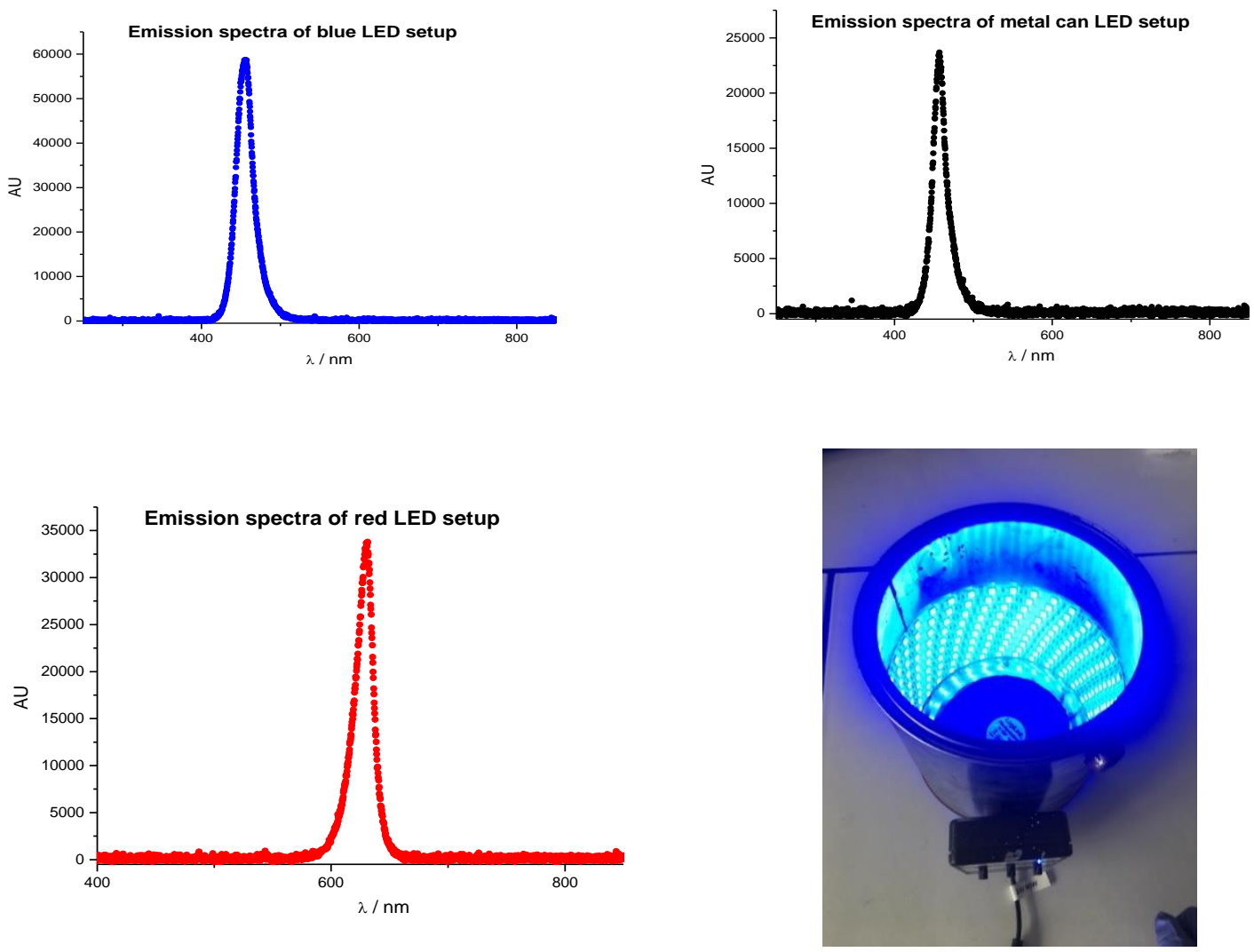

Figure 20: Emission spectra of different LED-setups. (Blue: blue-LED on glass, black: blue-LED on metal, red: red-LED on glass and a photo of the blue metal-LED setup) 
For the aspects of glassware and geometry, $10 \mathrm{~mL}$ round neck flasks were chosen, which could be placed at equidistant position inside the LED-setup. Their position could be adjusted and controlled by specifically designed distillation collectors to confirm the position and allow the possibility to attach connectors to balloons and Schlenk Line for changes of utilized atmospheres. Initial experiments of the positioning of the flask revealed that at the center and at a $66 \%$ radius, the highest results could be achieved. At $33 \%$ radius and direct contact with the LED stripe had slightly reduced yields (Figure 21). The position at $66 \%$ radius was chosen as the optimal position, as it allowed the reaction of four different reaction vessels in one setup without significant loss of yield. The results of the position dependent yields of this reaction show, the importance of specific geometries in the field of photocatalysis.

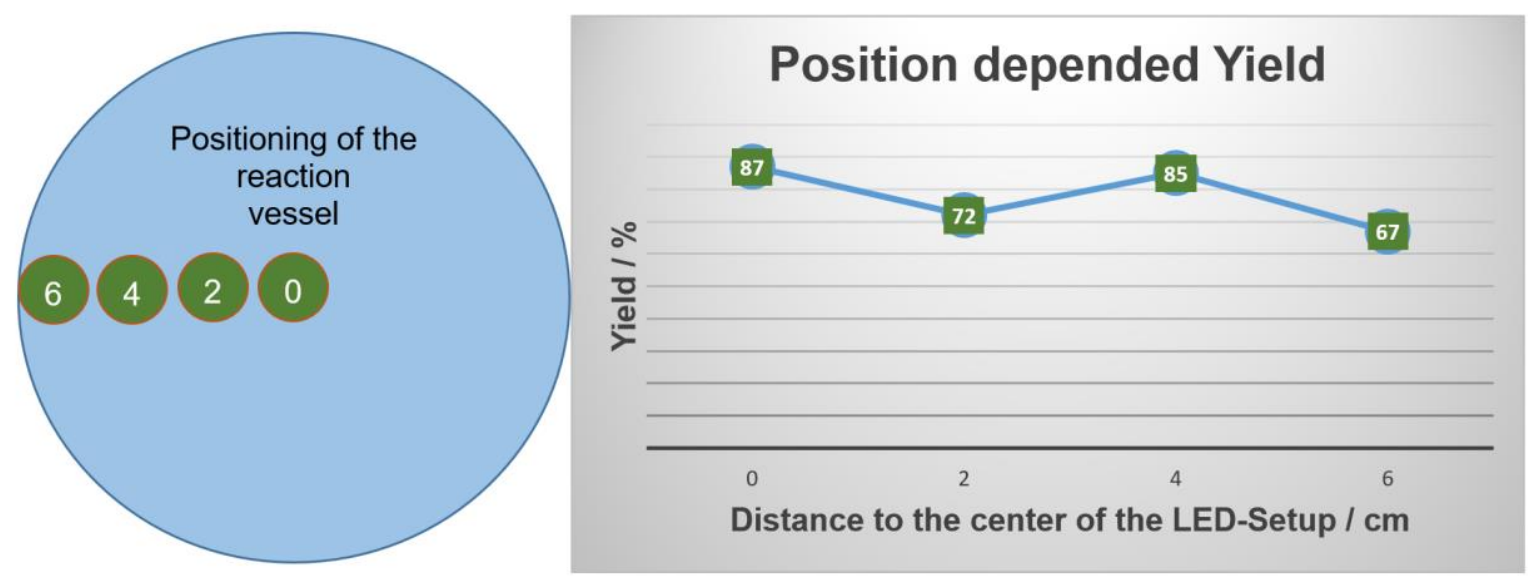

Figure 21: Position dependent yield for the oxidation of benzylic alcohol. Round neck flask center was placed at specific distances from the LED-Setup. Reaction conditions $2.9 \mathrm{mmol}$ of benzylic alcohol, $0.0079 \mathrm{mmol}(3 \mathrm{~mol} \%)$ photocatalyst, $16 \mathrm{~h}$ reaction time, blue LED irradiation at $12 \mathrm{~W} 420 \mathrm{~nm}$. Yield was determined by GC using $n$-dodecane as an internal standard. 


\subsection{OXIDATION OF PRIMARY AND SECONDARY ALCOHOLS}

The first substrate group of primary and secondary alcohols was chosen based on prior research on this topic. The oxidation potentials of most alcohol substrates are well known and many approaches have been established for the selective oxidation of alcohols to form efficiently targeted aldehydes and ketones. Nevertheless, most known approaches for the selective oxidation of alcohols are limited based on several aspects. Systems which are capable of oxidizing a wide variety of alcohol substrates, are based on complex and expensive transition-metal complexes. Alternative concepts like the shown oxidation by $\mathrm{MnO}_{2}$ utilizes between 5-20 equivalents of oxidation reagent. Furthermore, these 5-20 equivalents must be activated at high temperatures prior to its use. Other concepts have also high E-factors. Photocatalytic transition metal-free approaches were already shown, but had disadvantages considering the substrate scope and yield. Based on information in the field and initial reactivity of rose bengal in the oxidation of benzylic alcohol, optimization studies were conducted. ${ }^{[80]}$

\subsubsection{Optimization Studies}

Based on the carbon dioxide assisted oxidation of primary alcohols from our group, ${ }^{\text {[80] }}$ an alternative oxidation protocol for aerobic conditions was investigated. As a model substrate, benzylic alcohol was chosen. The first idea was to transfer the protocol by adding a photocatalyst to provide the energy for the chemical transformation. Initial investigations of 9-fluorenone and different bases revealed that the bases were inhibiting the reaction (Table 2, entries 1-4). Based on the clear inhibiting effect of different bases, the optimization was continued with screening of different photocatalysts and solvents. The results of the screening were that eosin $Y$ and 9fluorenone were the best photocatalysts under oxygen-balloon-atmosphere (entries 511). A change of the utilized solvent led to drastically decreased yields (entries 14-17). To achieve better reaction conditions in the aspect of simplicity and safety, the best 
results were repeated under ambient air conditions. 9-flourenone revealed a slight decrease in reactivity compared to almost full inhibition of eosin $\mathrm{Y}$ in photocatalytic performance (entries 12-13). Upon literature investigation two possible reasons for the better performance of 9 -fluorenone, compared to eosin $\mathrm{Y}$, could be found. The first reason is the longer half-life time of the 9-fluorenone, which was around six times greater than eosin $Y_{.}^{[41]}$ The second effect is a stabilizing effect of DMSO, DMF, DMA and $\mathrm{ACN}$ for excited states of photocatalysts. Both effects should contribute to a long lifetime of 9-fluorenone, which gave 9-fluorenone the possibility to react efficiently even at lower oxygen concentrations. In the case of eosin $Y$, the lifetime of the excited state should be limited to a shorter timeframe in which it had low chances of interaction with oxygen, at low oxygen concentrations. This gave high interest to optimize further with 9-flourenone under ambient air, based on the consideration that ambient air provides severe benefits compared to reaction under pure oxygen. Pure oxygen provided additional safety concerns especially in radical involving reaction, resulting in a low risk of explosion. Therefore, different concentrations of the reaction mixture were investigated (entries 18-21). A doubled catalyst loading revealed increased yield in the same time frame. Alternatively, different amounts of DMSO as a solvent were examined with intensively improved yields. An optimum could be observed at $1.0 \mathrm{~mL}$ DMSO (entry 20). This observation can be explained by the ratio of irradiated area to volume which is higher at low solvent amounts and higher concentrations. Additionally, a sufficient amount of solvent was present to solve sufficient amounts of oxygen from the air, to achieve optimal performance. The achieved results for the optimization of benzylic alcohol were repeated for eosin $\mathrm{Y}$. The inefficiency of other solvents were comparable and higher yields could be achieved by reducing the amount of solvent, but the inefficiency under ambient air conditions continued (entry 22). A special feature about the optimization was that in none of the cases, an overoxidation has been observed, with the only exclusion of entry $\mathbf{2 3}$, which revealed around $13 \%$ of carboxylic acid formation. 
Table 2: Optimization studies for the photocatalytic oxidation of benzylic alcohol. (a)

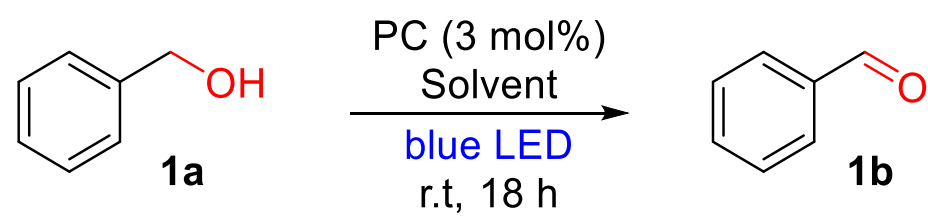

\begin{tabular}{|c|c|c|c|c|c|c|}
\hline Entry & Catalyst & Base & Solvent & $\begin{array}{l}\text { Volume } \\
/ \mathrm{mL}\end{array}$ & Atmosphere & $\begin{array}{c}\text { Yield } / \\
\%\end{array}$ \\
\hline 1 & 9-Fluorenone & DBU & DMSO & 2.5 & oxygen & 3 \\
\hline 2 & 9-Fluorenone & $\mathrm{K}_{3} \mathrm{PO}_{4}$ & DMSO & 2.5 & oxygen & 2 \\
\hline 3 & 9-Fluorenone & $\mathrm{LiOH}$ & DMSO & 2.5 & oxygen & 0 \\
\hline 4 & 9-Fluorenone & - & DMSO & 2.5 & oxygen & 31 \\
\hline 5 & 9,10-Dicyanoanthracene & - & DMSO & 2.5 & oxygen & 0 \\
\hline 6 & Rose bengal & - & DMSO & 2.5 & oxygen & 2 \\
\hline 7 & Riboflavine & - & DMSO & 2.5 & oxygen & 10 \\
\hline 8 & Fluorescein & - & DMSO & 2.5 & oxygen & 10 \\
\hline 9 & Rhodamine 6G & - & DMSO & 2.5 & oxygen & 19 \\
\hline 10 & Eosin $Y$ & - & DMSO & 2.5 & oxygen & 64 \\
\hline 11 & 9-Fluorenone & - & DMSO & 2.5 & oxygen & 34 \\
\hline 12 & Eosin $Y$ & - & DMSO & 2.5 & air & 2 \\
\hline 13 & 9-Fluorenone & - & DMSO & 2.5 & air & 26 \\
\hline 14 & 9-Fluorenone & - & DMF & 2.5 & oxygen & 1 \\
\hline 15 & 9-Fluorenone & - & DMA & 2.5 & oxygen & 2 \\
\hline 16 & 9-Fluorenone & - & THF & 2.5 & oxygen & 3 \\
\hline 17 & 9-Fluorenone & - & Toluene & 2.5 & oxygen & 0 \\
\hline 18 & 2x 9-Fluorenone & - & DMSO & 2.5 & air & 52 \\
\hline 19 & 9-Fluorenone & - & DMSO & 1.5 & air & 73 \\
\hline 20 & 9-Fluorenone & - & DMSO & 1.0 & air & 98 \\
\hline 21 & 9-Fluorenone & - & DMSO & 0.5 & air & 86 \\
\hline 22 & Eosin $Y$ & - & DMSO & 1.0 & air & 6 \\
\hline 23 & Eosin $Y$ & - & DMSO & 1.0 & oxygen & 83 \\
\hline
\end{tabular}




\subsubsection{SCOPE OF SUBSTRATES}

After optimizing the reaction protocol, the substrate scope was explored. The beginning was made by activated benzylic primary alcohol substrates. The reactions were monitored by GC-MS and TLC and substrates were performed for up to $24 \mathrm{~h}$. If a substrate provided more than $60 \%$ yield based on the GC-MS results, the product was attempted to be isolated. The isolated yields for successful substrates are reported in Scheme 15. It could be shown that methoxy (2a, 4a, 6a), aldehyde (3a) and aromatic extensions $(\mathbf{5} \mathbf{a}, \mathbf{7} \mathbf{a})$ performed excellently under the chosen conditions. In the case of $3 a$, no overoxidation of the present and forming aldehyde functionality could be overserved. Substrates $1 \mathrm{a}$ and $\mathbf{4 a}$ could be upscaled to $5 \mathrm{mmol}$ scale without significant impact, but the reaction time had to be increase.

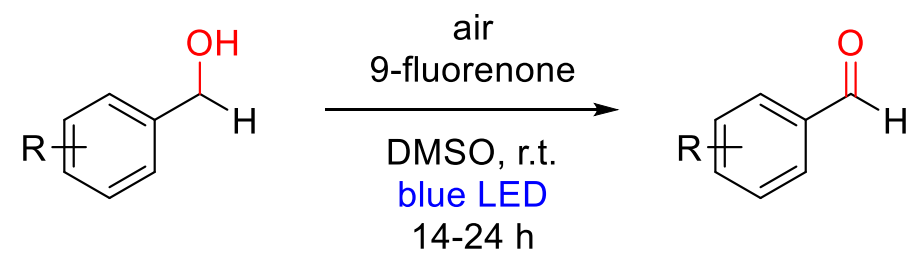<smiles>OCc1ccccc1</smiles>

1a: $98 \%$<smiles>OCc1ccc(-c2ccccc2)cc1</smiles>

5a: $77 \%$<smiles>COc1ccc(CO)cc1</smiles>

2a: $98 \%$<smiles>O=Cc1ccc(CO)cc1</smiles>

3a: $90 \%$<smiles>COc1ccc(CO)cc1OC</smiles>

4a: $64 \%$<smiles>COc1cc(CO)cc(OC)c1OC</smiles>

6a: $76 \%$<smiles>COC(=O)c1ccc(CO)cc1</smiles>

7a: $61 \%$

8a: $63 \%$

Scheme 15: Isolated yields of substrates using the 9-fluorenone. Reaction conditions: substrate $(0.25 \mathrm{mmol})$, 9-fluorenone (3 mol\%), DMSO $(1 \mathrm{~mL}), 14-24 \mathrm{~h}$. All are isolated yield.

Several further substrates showed low reactivity and were not isolated (Scheme 16). Striking is the observation that electron withdrawing groups in meta- and ortho-position seemed to have a decreased effect on the efficiency of the oxidation. 


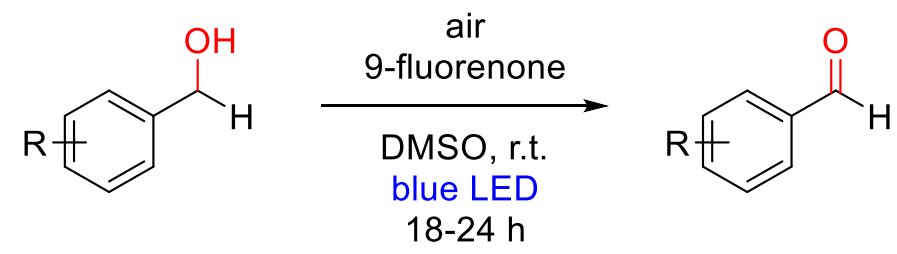<smiles>OCc1c(Cl)cccc1Cl</smiles>

$\mathrm{MeO}$<smiles>OCc1ccccc1</smiles><smiles>OCc1ccc2ccccc2c1</smiles>

9a: $\sim 5$

10a: $5 \%$

11a: $\sim 48 \%$<smiles>OCc1ccc(Br)cc1</smiles><smiles>CN(C)c1cccc(CO)c1</smiles><smiles>N#Cc1ccc(CO)cc1</smiles>

12a: $5 \%$

13a: $5 \%$

14a: $\sim 40 \%$<smiles>OCc1cccc(F)c1</smiles>

15a: 10\%<smiles>O=[N+]([O-])c1cccc(CO)c1</smiles>

16a: 25\%<smiles>O=[N+]([O-])c1ccc(CO)cc1</smiles>

17a: 50\%

Scheme 16: Substrates with insufficient conversion and yield based on GC-MS evaluation for the fluorenone based oxidation protocol. Reaction conditions: Substrates $(0.25 \mathrm{mmol}), 9$-fluorenone $(3 \mathrm{~mol} \%)$, DMSO $(1 \mathrm{~mL}), 18-24 \mathrm{~h}$. Part of the experiments were performed with the assistance of Nareh Hatami.

To further investigate the possible substrate scope of primary alcohols, further substrates including heterocyclic and non-activated alcohols were investigated. Primary heterocyclic activated alcohols also performed well but revealed lower reactivity in the same timeframe (Scheme 17, 18a-20a). During the investigations several substrates revealed insufficient yields and as a compensation catalyst loading was doubled and atmosphere was changed to pure oxygen to achieve fast conversions in the reaction. In the case of extended $\pi$-electron systems of 2Phenylethan-1-ol (21a) and 2-phenylethynol (22a) showed no selectivity for the oxidation to aldehyde but converted mostly to the corresponding carboxylic acid. Investigating different non-activated primary alcohols showed no trace amount of activity, with $\beta$-phenylbenzene ethanol (23a) as barrier molecule in terms of activation. With these results the substrate scope continued with the investigation of secondary alcohols as possible substrates. 

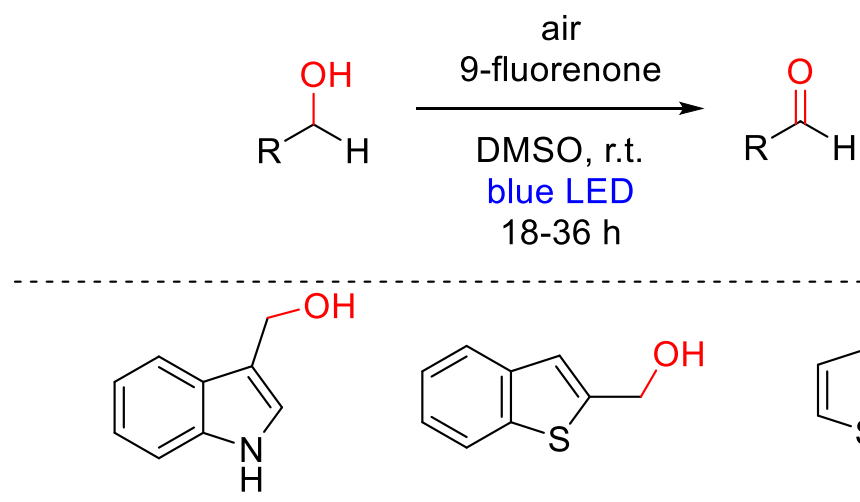<smiles>OCc1cc2ccccc2s1</smiles><smiles>OCc1cccs1</smiles>

18a: $52 \%$ (72\%) 19a: $51 \%$ (84\%) 20a: $48 \%$<smiles>OC/C=C/c1ccccc1</smiles><smiles>OCC#Cc1ccccc1</smiles>

21a: $24 \%$<smiles>OCC(c1ccccc1)c1ccccc1</smiles><smiles>OCC1CCCCC1</smiles>
(68\% acid)

23a: $\sim 5 \%$ 24a: n.r.<smiles>OCC1CCCO1</smiles>

25a: n.r.

Scheme 17: Substrates with insufficient conversion and yield based on GC-MS. Reaction conditions: Substrates $(0.25 \mathrm{mmol}), 9$-fluorenone $(3 \mathrm{~mol} \%)$, DMSO $(1 \mathrm{~mL})$, 1-24 h. (in parenthesis the yields of double catalyst loading and pure oxygen atmosphere for the heterocyclic compounds and detected amount of overoxidized products for substrates 20a and 21a). Part of the experiments were performed with the assistance of Nareh Hatami. 


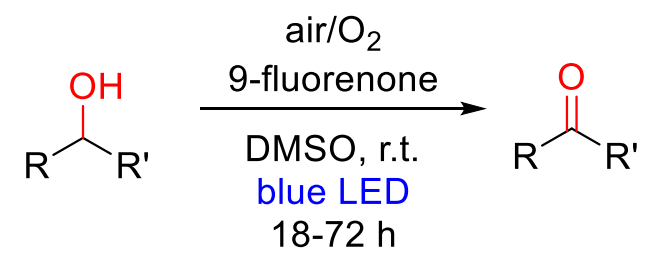

Aromatic secondary substrates<smiles>OC(c1ccccc1)c1ccc(Br)cc1</smiles>

26a: $99 \%$ 27a: $81 \%$<smiles>OC(c1ccc(Cl)cc1)c1ccc(Cl)cc1</smiles>

28a: $86 \%$<smiles>OC(c1ccccc1)C(O)c1ccccc1</smiles>

29a: $92 \%$<smiles>CC(O)c1ccccc1</smiles>

30a: $71 \%$<smiles>OC1CCCc2ccccc21</smiles>

31a: $95 \%$<smiles>OC1CCc2ccccc21</smiles>

32a: $91 \%$<smiles>CC(C)(O)C=Cc1ccccc1</smiles>

33a: $61 \%$<smiles>OC1Cc2cccc3cccc1c23</smiles>

34a: $85 \%$

Aromatic secondary substrates with heteroatoms<smiles>OC(c1ccccc1)c1ccco1</smiles>

35a: $88 \%$<smiles>OC1c2ccccc2Oc2ccccc21</smiles>

36a: $89 \%$<smiles>OC1CCOc2ccccc21</smiles>

37a: $84 \%$<smiles>CC(O)c1ccccn1</smiles>

38a: $86 \%$

Scheme 18: Isolated yields of substrates based on the fluorenone based oxidation protocol. Reaction conditions: substrates $(0.25 \mathrm{mmol})$, 9-fluorenone (3-6 mol\%), DMSO (1 mL), 18-72 h. All are isolated yield. Part of the experiments were performed with the assistance of Nareh Hatami.

In the case of aromatic activated secondary alcohols, all the investigated substrates performed with good to excellent yields. In the case of double activation, the reaction performed smoothly without any complications. In addition, halogenated substrates have shown no high activity, in the case of single halogenation (27a) as well as in the double halogenated case (28a). In the case of $18 \mathbf{a}$, complications occurred, the presence of the allylic bond in the substrate led to a secondary reaction in which a cleavage reaction occurred. Cyclic and non-cyclic aliphatic-groups were tolerated and revealed no direct influence. The incorporation of heteroatoms (35a-38a) in the aromatic structure also yielded the respective ketone in excellent yields. 


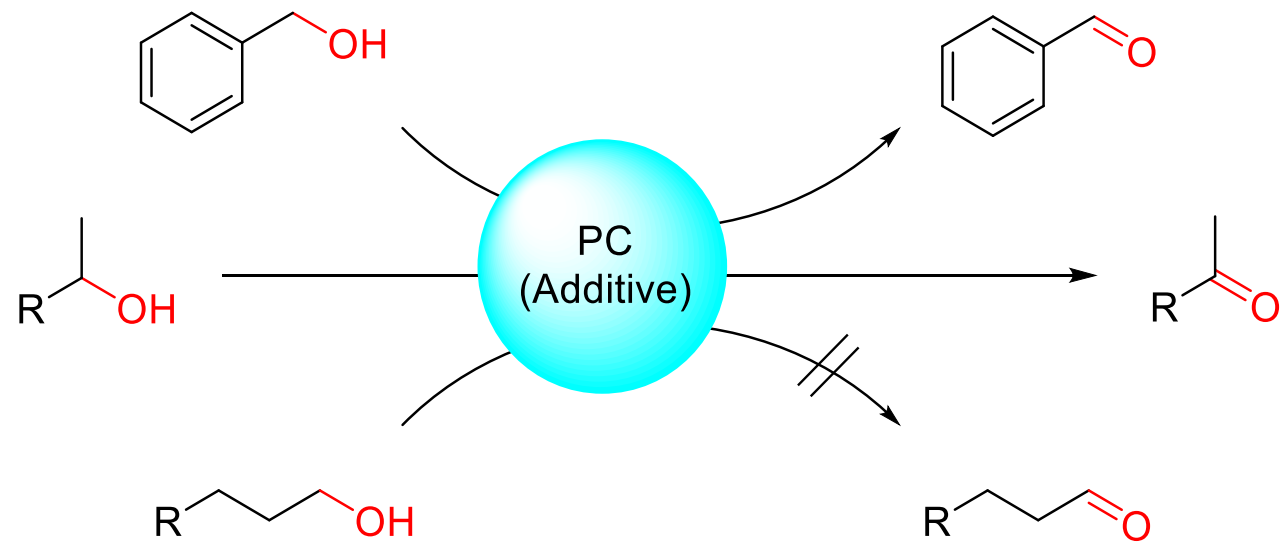

Figure 22: Summary of the three model substrates for general studies to oxidize different alcohols.

After investigating the reactivity of benzylic alcohols, the substrate scope was extended towards more challenging substrate groups. Several substrates were chosen to see possible alternatives for the extension of the substrate scope. As new substrates 2-octanol, 1-octanol, cyclohexanemethanol and furfural (Figure 22) were selected. 2-Octanol showed $32 \%$ yield within $18 \mathrm{~h}$. 1-Octanol showed trace amounts of 1-octanal, which were not present before the reaction start. The cyclohexanemethanol and furfural showed no reactivity. Based on these results, a second optimization was concluded to modify the reaction protocol to achieve the oxidation of activated benzylic alcohol and additionally, the oxidation of primary aliphatic alcohols. The primary consideration was that non-activated alcohols have higher redox potentials, this should be lowered by utilization of additives. Additionally, the reactions were repeated with eosin $Y$ under oxygen atmosphere for double confirmation of the results. For this purpose, different bases and acids were investigated and especially strong Lewis acids with known interaction between alcohols (Table 3), like $\mathrm{AlCl}_{3}$, but with no visible effect on the oxidation under the chosen photocatalytic conditions. A change of solvent showed that the prior inhibiting effect of other solvents was still present. The oxidation of aliphatic primary alcohols unsuccessful and the substrate scope was extended towards secondary aliphatic alcohols. 
Table 3: Optimization of the oxidation of 1-octanol.

\begin{tabular}{|c|c|c|c|c|c|}
\hline & $\mathrm{OH}$ & $\begin{array}{c}\text { 9-fluoreno } \\
\text { DMSO (1 } \\
\text { blue LEL } \\
\text { r.t, } 18 \mathrm{~h} \\
\text { Additive }\end{array}$ & & & \\
\hline Entry & Catalyst & Additive & Solvent & Atmosphere & $\begin{array}{c}\text { Yield / } \\
\%\end{array}$ \\
\hline 1 & 9-Fluorenone & --- & DMSO & air & $<2$ \\
\hline 2 & Rose Bengal & --- & DMSO & oxygen & $<2$ \\
\hline 3 & Riboflavine & --- & DMSO & oxygen & $<2$ \\
\hline 4 & 9-10-Dicyanoantracene & --- & DMSO & oxygen & $<2$ \\
\hline 5 & Eosin $Y$ & --- & DMSO & oxygen & \\
\hline 6 & 9-Fluorenone & $\mathrm{LiOH}$ & DMSO & air & $<2$ \\
\hline 7 & 9-Fluorenone & $\mathrm{LiCl}$ & DMSO & air & $<2$ \\
\hline 8 & 9-Fluorenone & $\mathrm{LiBr}$ & DMSO & air & $<2$ \\
\hline 9 & 9-Fluorenone & $\mathrm{AlCl}_{3}$ & DMSO & air & $<2$ \\
\hline 10 & 9-Fluorenone & $\mathrm{B}\left(\mathrm{C}_{6} \mathrm{~F}_{5}\right)_{3}$ & DMSO & air & $<2$ \\
\hline 11 & 9-Fluorenone & $\mathrm{B}(\mathrm{OH})_{3}$ & DMSO & air & $<2$ \\
\hline 12 & 9-Fluorenone & $\mathrm{B}(\mathrm{Ph})_{3}$ & DMSO & air & $<2$ \\
\hline 13 & 9-Fluorenone & $\mathrm{AlCl}_{3}$ & DMSO & air & $<2$ \\
\hline 14 & $3 \times 9$-Fluorenone & $\mathrm{AlCl}_{3}$ & DMSO & oxygen & $<2$ \\
\hline 15 & $3 \times 9$-Fluorenone & --- & DMSO & oxygen & $<2$ \\
\hline 16 & $3 \times \operatorname{Eosin} Y$ & --- & DMSO & oxygen & $<2$ \\
\hline 17 & $3 \times$ Eosin $Y$ & $\mathrm{AlCl}_{3}$ & DMSO & air & $<2$ \\
\hline
\end{tabular}

(a) Reaction conditions: $3.1 \mathrm{mmol}$ of 1 -octanol, $0.0083 \mathrm{mmol} \quad(3 \mathrm{~mol} \%)$ photocatalyst, $18 \mathrm{~h}$ reaction time, blue LED irradiation at $12 \mathrm{~W} 420 \mathrm{~nm}$. Yield determined by GC using $n$-dodecane as an internal standard.

After the excellent yield of secondary alcohols and the shown reactivity of 2-octanol, the substrate scope was extended towards aliphatic and alicyclic substrates to examine the efficiency of the system. After the additional screening most of the 
compounds showed reactivity, but with lower yields. In consequence the protocol was modified by increasing the reaction time, utilizing an oxygen balloon to provide atmosphere and in some cases with double catalyst loading to achieve higher conversions. It is assumed that the substrates with less activation have a low overlap of redox potential and therefore only reacted on fewer occasions with the catalyst. In the case of $41 \mathrm{a}$ both stereo isomers were investigated in pure form and as mixtures. This showed no difference in their reactivity.

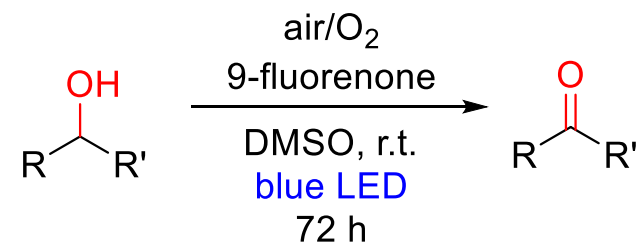

aliphatic and allylic secondary alcohols

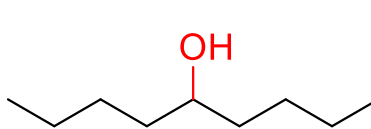

39a: $68 \%$<smiles>CCCC(C)(C)O</smiles>

40a: $71 \%$<smiles>CCCC(C)(C)O</smiles>

$41 a^{*}: 78 \%$<smiles>CCCC(C)(C)O</smiles>

42a: $64 \%$<smiles>CCCC(C)(C)O</smiles>

43a: $67 \%$

Alyciclic secondary alcohol<smiles>OC1C=CCCC1</smiles>

$44 a^{*}: 87 \%$

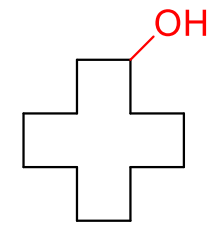

45a: $83 \%$<smiles>OC1CCCC1</smiles>

46a: $63 \%$<smiles>OC1CCCCC1</smiles>

47a: $59 \%$

Scheme 19: Isolated yields of substrates based on the fluorenone based oxidation protocol. Reaction conditions: substrates $(0.25 \mathrm{mmol})$, 9-fluorenone $(3-6 \mathrm{~mol} \%)$, DMSO (1 mL), 18-72 h. 41 and $\mathbf{4 4}$ yield were determined by GC using $n$-dodecane as the internal standard. Entry 44a was performed by of Nareh Hatami.

With the successful oxidation of unactivated substrates an interest in steroid oxidation arose. A variety of different steroid scaffolds were investigated for their activity. Testosterone (48a), stanelone (49a), androsterone (50a) performed as expected, based on the oxidation of aliphatic secondary alcohols. All of these three substrates showed slow but steady conversion into their corresponding ketones. Triamcinolone acetonide (51a) performed well but could not be isolated successfully in a pure form. A similar result was achieved for methyl cholate (52a) and HR-MS confirmed that the 
isolated product was a mixture of the seven possible oxidation products. 53a reacted with $62 \%$ yield of similar product, in this case an oxygenation in position 7, B-ring, occurred. Both compounds result in the same amount of proton signals but only by 2D-NMR the difference was fully distinguishable.

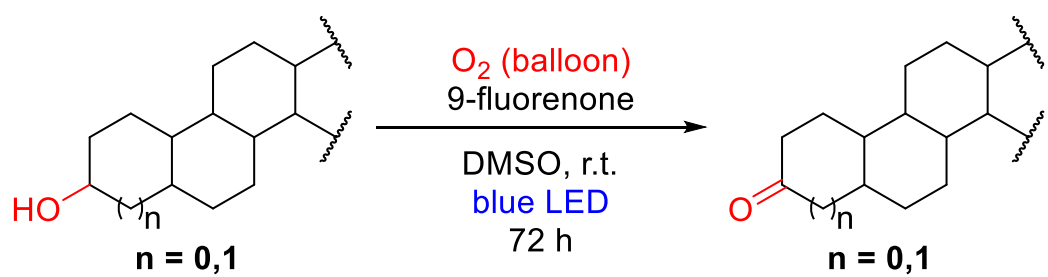

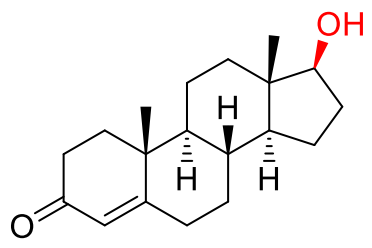

48a: $72 \%$ Testosterone<smiles>C[C@]12CC[C@H]3C(CC[C@@H]4CC(=O)CC[C@]34C)[C@@H]1CC[C@@H]2O</smiles>

49a: $62 \%$ Stanelone<smiles>C[C@]12CC[C@H]3[C@@H](CC[C@@H]4C[C@@H](O)CC[C@]34C)[C@@H]1CCC2=O</smiles>

50a: $72 \%$ Androsterone

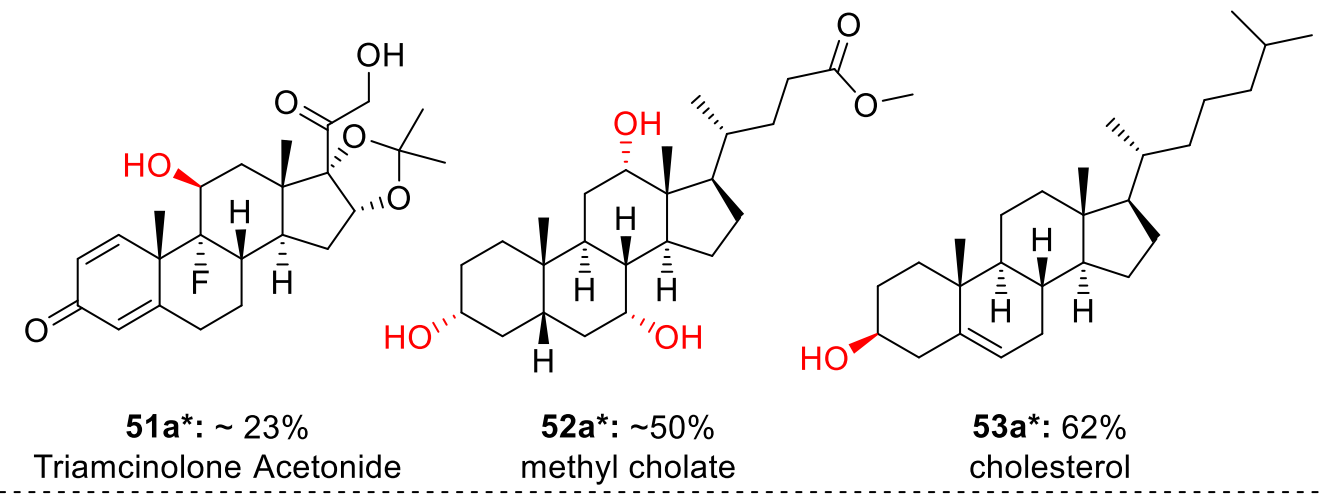

Scheme 20: Yields of steroid based substrates. Reaction conditions: substrates $(0.25$ $\mathrm{mmol})$, 9-fluorenone (6 mol\%), DMSO (1 mL), $72 \mathrm{~h}$. Yields of $\mathbf{5 1 - 5 3}$ by GC-MS. *) Annotation of complication: 51a: failed to be isolated. 52a: was collected as a mixture of 7 different oxidation products, 53a: reacted to a different product.

Extension of the reaction time for the case 52a to 7 days was still not sufficient to completely convert the entire sample. Cholesterol (53a) was oxidized and isolated without any problems. Upon intensive validation of the product's identity, several discrepancies were detected. HR-MS mass was incorrect, and the shifts of one signal was misplaced compared to published values in the ${ }^{1} \mathrm{H}$ - and ${ }^{13} \mathrm{C}$ NMR-spectra. After conclusion of the investigation the product was identified as a different product, namely 7-Ketocholesterol, with an oxygenation at the position 7 in the steroid $B$ ring. Comparison with literature was a perfect match and increment calculation. ${ }^{[124]}$ Based 
on intensive literature search several publications were found not differentiating between those two compounds correctly. In cases, in which ESI and 2D-NMR experiments are not available the two shifted signals in ${ }^{1} \mathrm{H}$ - and ${ }^{13} \mathrm{C}$-NMR are easily confused. A recent example of this is the work of V. Bhalla et al. ${ }^{[25]}$ about UV light promoted oxidation of alcohols. Based on only simple ${ }^{1} \mathrm{H}$-NMR results for their validation, they are claimed to be the first group performing the desired alcohol oxidation of the substrate 53a, without the use of transition metal catalysts and additives. The analytical evidence showed consistency for the oxidation product of the position 7 in the B-ring, but a full confirmation was not possible, based on the limited amount of analytics provided. [126]

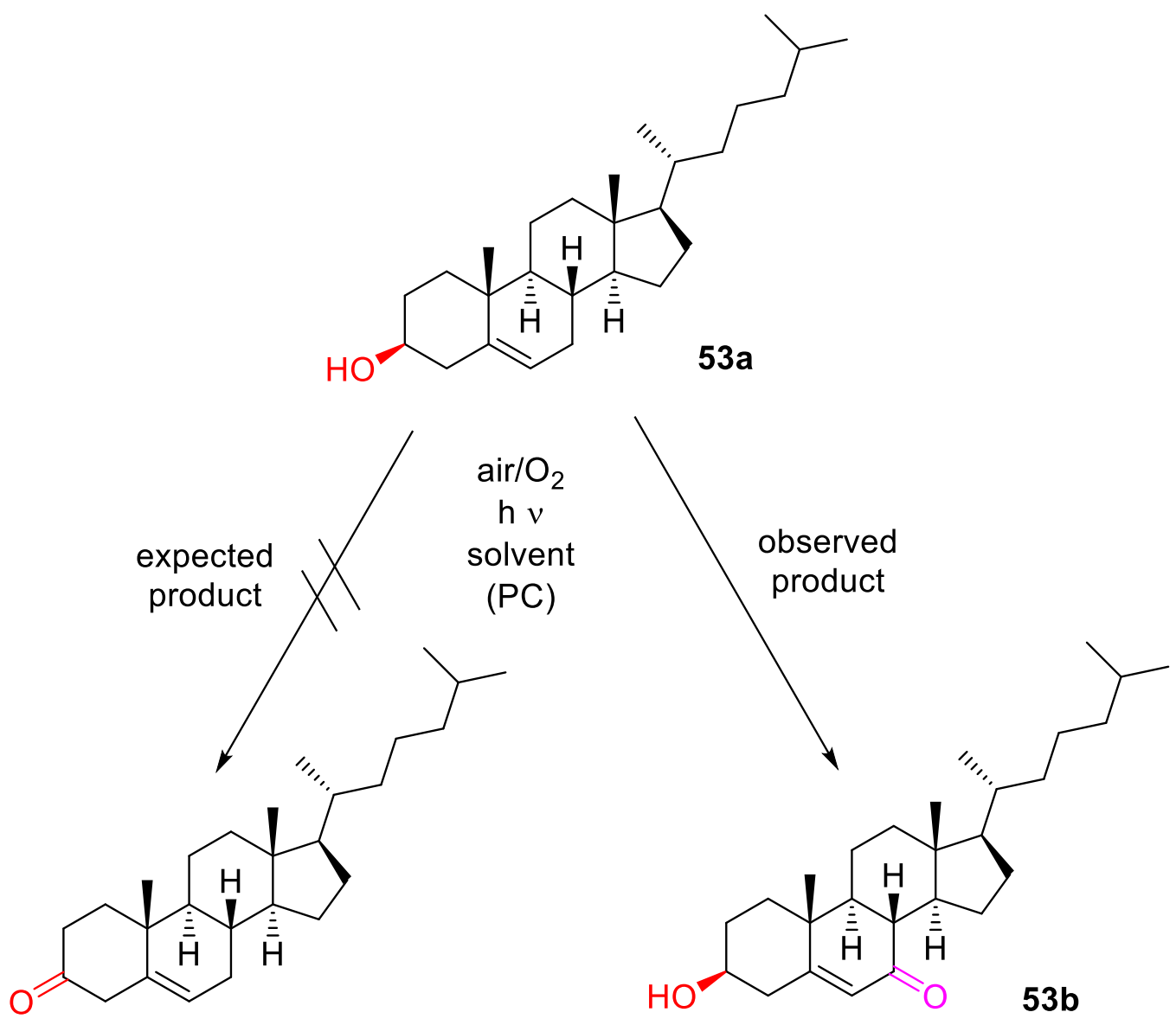

Scheme 21: Expected and observed/isolated product of the oxidation of cholesterol.

The broad substrate scope and the reactivity of steroids under this photocatalytic protocol created an interest to extend the substrate scope towards natural base compounds and biomolecules like, lignins, sugars and further structures (Figure 23). None of the other investigated biomolecules showed the desired alcohol oxidation 
product as their main product of the reaction. However, the resulted alternative products served as an inspiration for further studies of their own. Allylic compounds continued with the two observed characteristics and performed either overoxidation to the corresponding carboxylic acid or the cleavage product.

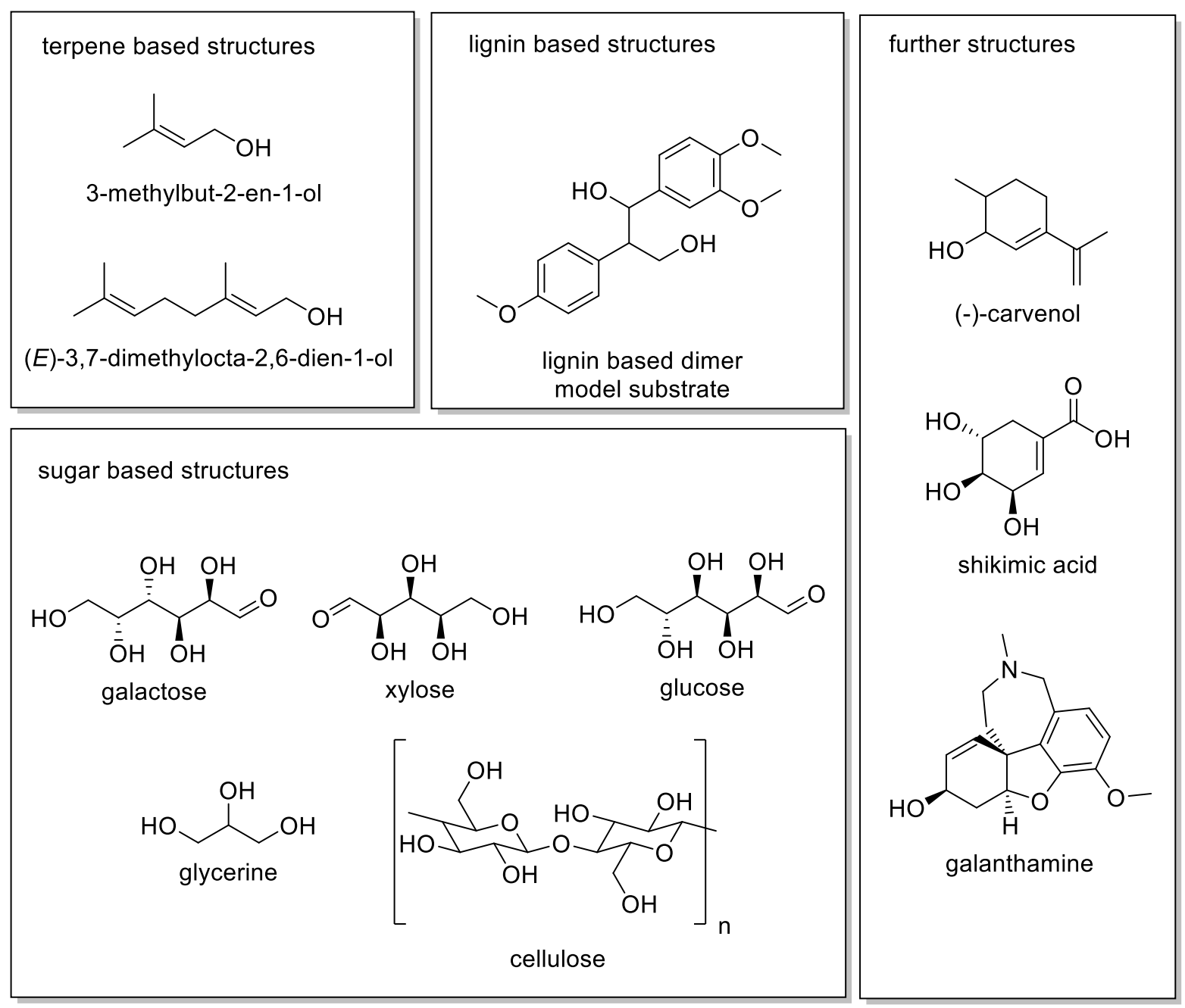

Figure 23: Further biomolecule-based substrates that were tried with the 9-fluorenone based oxidation protocol.

Terpenes showed full conversion, but no product could be isolated. GC-MS analysis found no product in the mass area of 50-500 $\mathrm{m} / \mathrm{z}$. Generation of a high mass product of the reaction could not be confirmed, nor excluded.

In the case of the lignin model compound the oxidation of the alcohol in the benzylic position could be observed, but in minor concentrations; a following reaction to cleave the dimer into its monomer parts seemed to be the dominating reaction. 
Carvenol showed similar behavior to the substrate cholesterol (53a) and performed mostly the oxygenation in the proximity of the double bond instead of the oxidation of the alcohol functionality.

For different sugar-based molecules, full conversion could be observed, but no oxidation product could be found at the beginning. Further repetition and investigation revealed the formation of formic acid as the main product of the reaction. Several reactions were performed in this regard and in the case of glucose $0.104 \mathrm{mmol}$ of formic acid was determined. For the other compounds $0.022 \mathrm{mmol}$ (cellulose), $0.078 \mathrm{mmol}$ (xylose), 0.036 (galactose), 0.022 (glycerin) were observed. Changing to eosin $\mathrm{Y}$ as the photocatalyst under oxygen atmosphere could increase the yield of the formic acid to $0.239 \mathrm{mmol}$. In the attempt to reproduce the results, the amount of formic acid was varying uncontrollably and showed yields of 0.003 up to $0.239 \mathrm{mmol}$ under all conditions. Applying different photocatalyst and solvents had no improving effect on the reliability of the formation of formic acid.

In the case of shikimic acid and galanthamine conversion of the starting material was observed, but no product was identified for these reactions.

Summarizing the substrate scope investigation showed that nearly all secondary alcohols can be oxidized with this protocol. In the case of primary alcohols an activation through a $\pi$-electron system seemed to be of the essence to achieve oxidation. All the substrates showed reactivity and in the case of slow process of the oxidation the reaction speed could be increased, by several adjustments. Firstly, the increase of oxygen in the used atmosphere improved the yields considerably. Secondly, the increase in catalyst loading to the double amount increased the yield, thirdly, was the increase of the reaction time, which showed that the reaction speed was applied constantly on the conversion. Alkyne and allylic functionalities near the targeted alcohol functionality showed indication to perform side reactions. In the case of specific structural motives alternative reaction pathways were found and investigated by other researchers of the group. 


\subsubsection{MECHANISTIC INVESTIGATIONS}

After the substrate scope, the mechanism of the reaction was investigated. Initially control experiments were performed to present first insights towards the possible mechanism (Table 4).

Table 4: Optimization studies for the photocatalytic oxidation of benzylic alcohol.(a)

\begin{tabular}{ccc}
\hline Entry & Controlled parameter & $\begin{array}{c}\text { 9-fluorenone } \\
\text { DMSO } \\
\text { blue LED } 18 \mathrm{~h}\end{array}$ \\
\hline $\mathbf{1}$ & Standard conditions $/ \%$ \\
$\mathbf{2}$ & Nitrogen atmosphere & $\mathbf{9 8}$ \\
$\mathbf{3}$ & No catalyst & $\mathbf{0}$ \\
$\mathbf{4}$ & No light (covered by aluminum foil) & $\mathbf{1}$
\end{tabular}

(a) Reaction conditions $2.9 \mathrm{mmol}$ of benzylic alcohol, $0.0079 \mathrm{mmol}$ (3 mol\%) 9fluorenone, $18 \mathrm{~h}$ reaction time, $12 \mathrm{~W}$ LED irradiation at $420 \mathrm{~nm}$. Yield was determined by GC-FID using $n$-dodecane as an internal standard.

The control experiments showed that the change to inert atmosphere by applying nitrogen atmosphere inhibited the reaction completely (entry 2 ). Therefore, it can be concluded that the oxygen in the ambient air was essential for the reaction. Irradiation by $420 \mathrm{~nm}$ wavelength light without the catalyst (entry 3) had no effect on the conversion of the model substrate into the aldehyde. Additional experiments about the LED-setup showed that the wavelength of the LED-setup was narrow and exhibited no signs that partial high energy UV-irradiation was present. For the final experiment (entry 4), the regular reaction mixture was covered completely by aluminum foil and checked that no area was left for partial exposure to the light. Afterwards, the reaction mixture was stirred together with other samples to include a possible thermal effect on the sample. A yield of around $1 \%$ was observed for this reaction. A possible explanation for this observation is that during the preparation of the experiment, daylight was present and already initiated oxidation and after the experiment was concluded the sample was prepared again under daylight conditions. Both exposures 
to daylight seemed to be sufficient to form a measurable amount of product in the reaction mixture.

The second point of interest was identification of byproducts of the reaction. During optimization studies, the conversion seemed to decrease with the increase of the product formation. With over $33 \%$ product formation, an increase of the starting material in the GC-FID chromatogram was overserved and reached up to $-400 \%$. The formed byproduct was evaluated as a substance with similar retention time to the substrate 1a. The temperature program of the GC-FID was as a result modified to separate the two compounds additionally. After isolation of the byproduct, it was characterized by GC-MS and revealed a molar mass of $94.2 \mathrm{~g} / \mathrm{mol}$, with a fitting fragmentation to methylsulfonylmethane. ${ }^{[127]}$ NMR measurement of the reaction mixture in DMSO- $D_{6}$ confirmed further the characterization with a fitting methyl-group signal at reported values for methylsulfonylmethane. ${ }^{[128]}$ Methylsulfonylmethane in analytical grade was compared to the isolated byproduct in retention times at the GCMS and GC-FID showed identical retention times for both the samples in both temperature programs. As a second byproduct hydrogen peroxide was assumed. The Luminol test was performed with the reaction mixture after irradiation and showed a positive result (chemiluminescence). ${ }^{[129]}$ Additionally, the hydrogen peroxide content was analyzed by Yu Zhang via UV/Vis spectroscopic measurement. The reaction mixture was treated with $\mathrm{TiCl}_{4}$ and acidified, as reported in literature. The resulting mixture was measured at $410 \mathrm{~nm}$ (Figure 24). The absorption spectrum was compared with artificial reaction mixtures of all components, and their counterpart with the addition of hydrogen peroxide. 


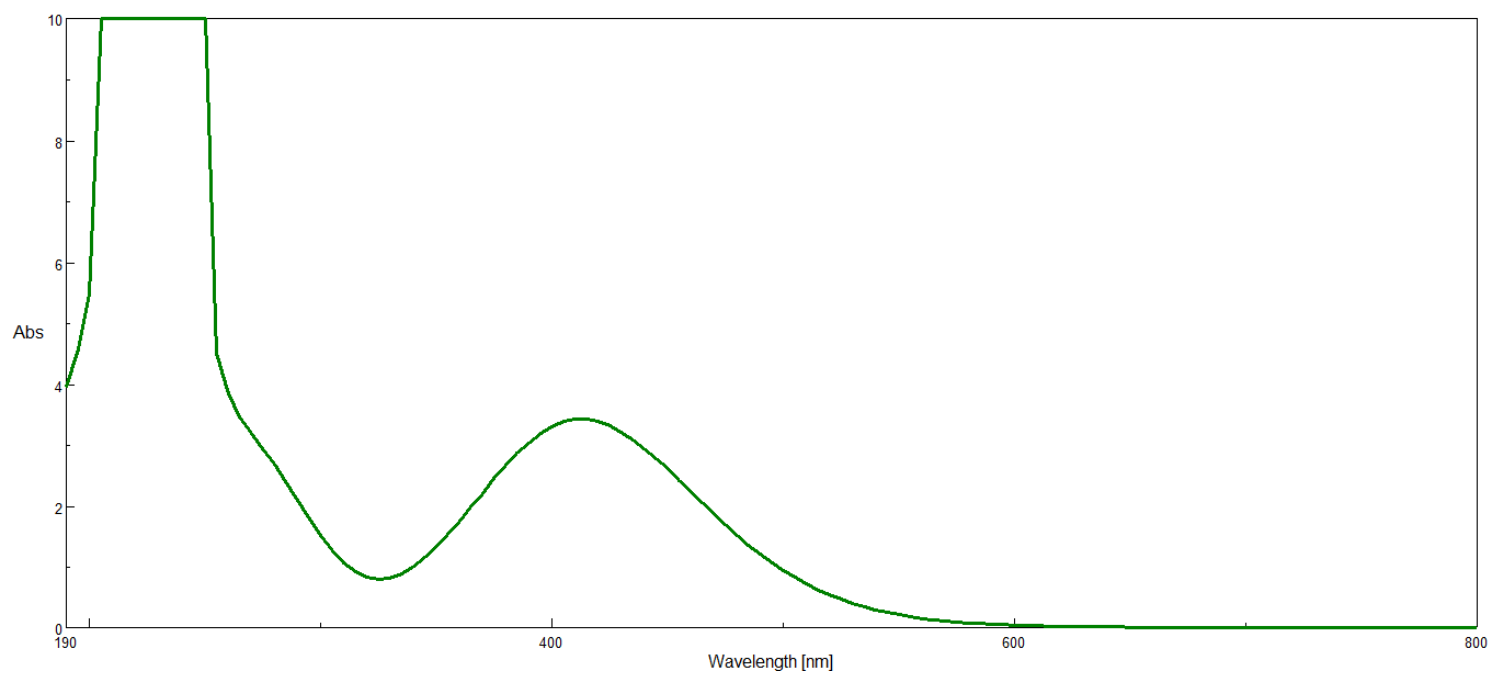

Figure 24: Absorption spectrum of the reaction mixture (irradiated) after treatment with $\mathrm{TiCl}_{4}$ and conc. $\mathrm{H}_{2} \mathrm{SO}_{4}$ at high dilution. The experiment and analysis was performed by Yu Zhang.

After confirmation of the byproducts, control experiments and investigations of the light dependence of the reaction were performed. The first reactions were performed with different light intensities to see how the yield changed within 18-hour time period (Figure 25). It was visible that the yield decreased nearly linear with the decrease in light intensity with an interception around 4-5 W LED-power. This was assumed to be the minimum needed light intensity to initiate the photocatalytic reaction. Both the samples (below $6 \mathrm{~W}$ ) showed minimal yields, which could be primarily achieved by daylight background irradiation, or a combination of daylight and LED-light. The results revealed that the chosen light intensity was ideal for the chosen time frame. Examination of shorter reaction times with higher light intensities were concluded, showing that the reaction speed could be further increased by increasing the light intensity further, indicating that the efficacy maximum was not reached, this corresponded to the observation of the substrate scope, that by doubling the catalyst concentration the yield could be increased in the same timeframe. 

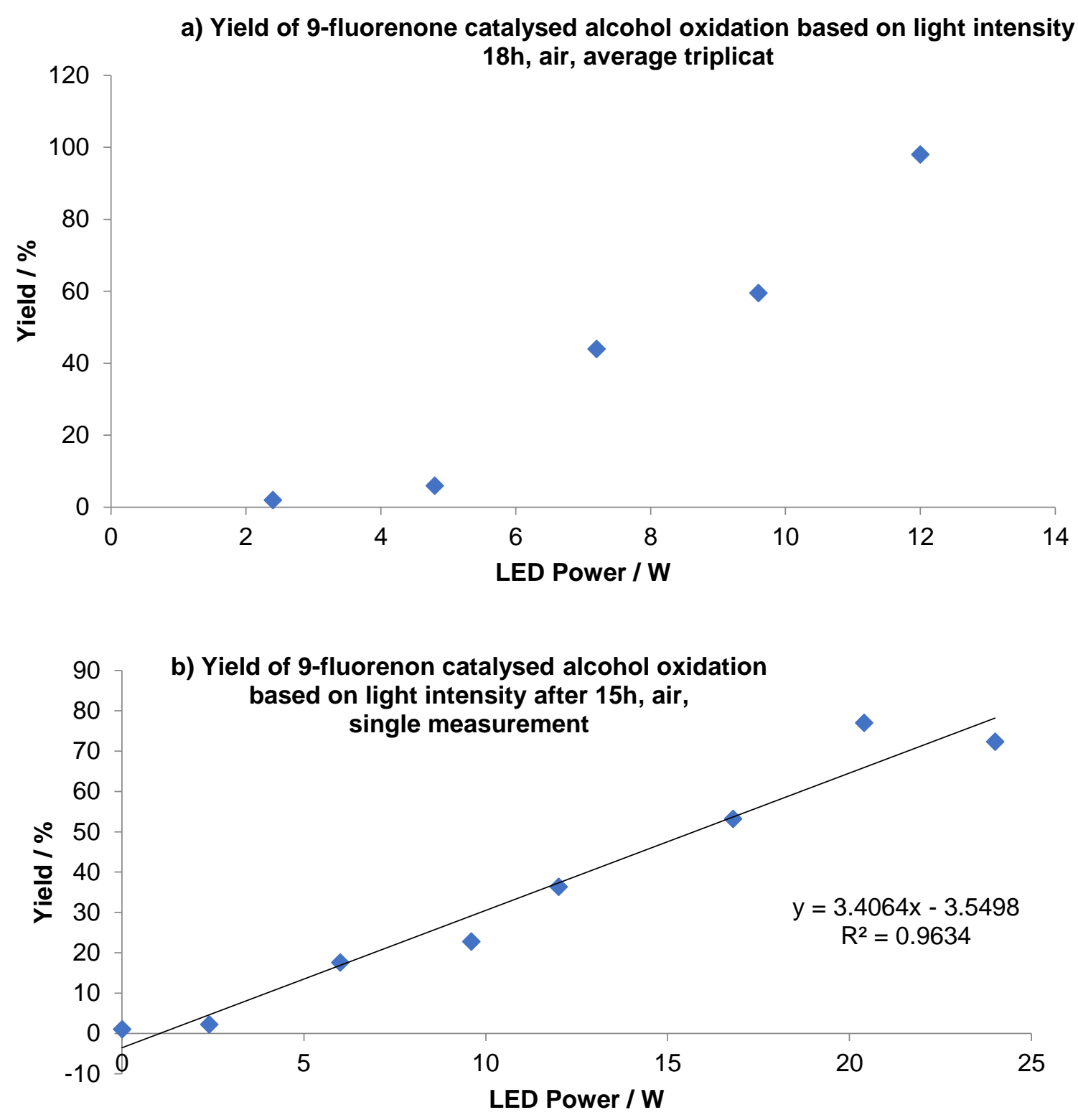

Figure 25: a) light intensity dependent yields (18 h) for the photocatalytic oxidation of benzyl alcohol utilizing 9-fluorenone. b) light intensity dependent yields including higher intensities $(15 \mathrm{~h}$ ) for the photocatalytic oxidation of benzyl alcohol utilizing 9fluorenone.

Additional experiments were performed to investigate if the reaction was performed in a linear fashion and the modified GC-FID temperature was used to have detailed information about the conversion during this period (Figure 26). Yield and conversion behaved in a direct relation with each other and showed that the reaction performed in three separable domains. The initial domain (starting period, shorter than $2 \mathrm{~h}$ reaction time) that a lower reaction constant would apply. After this initial domain the reaction constant nearly increased by factor 3 , till it reached partial saturation and exhibited a typical lower reaction constant due to the decreased amount of starting 
material. The initial domain indicated a further aspect concerning the minimal light intensity requirement of this reaction. Thus, a minimum activation seemed to be achieved to initialize the photocatalytic reaction mechanism.

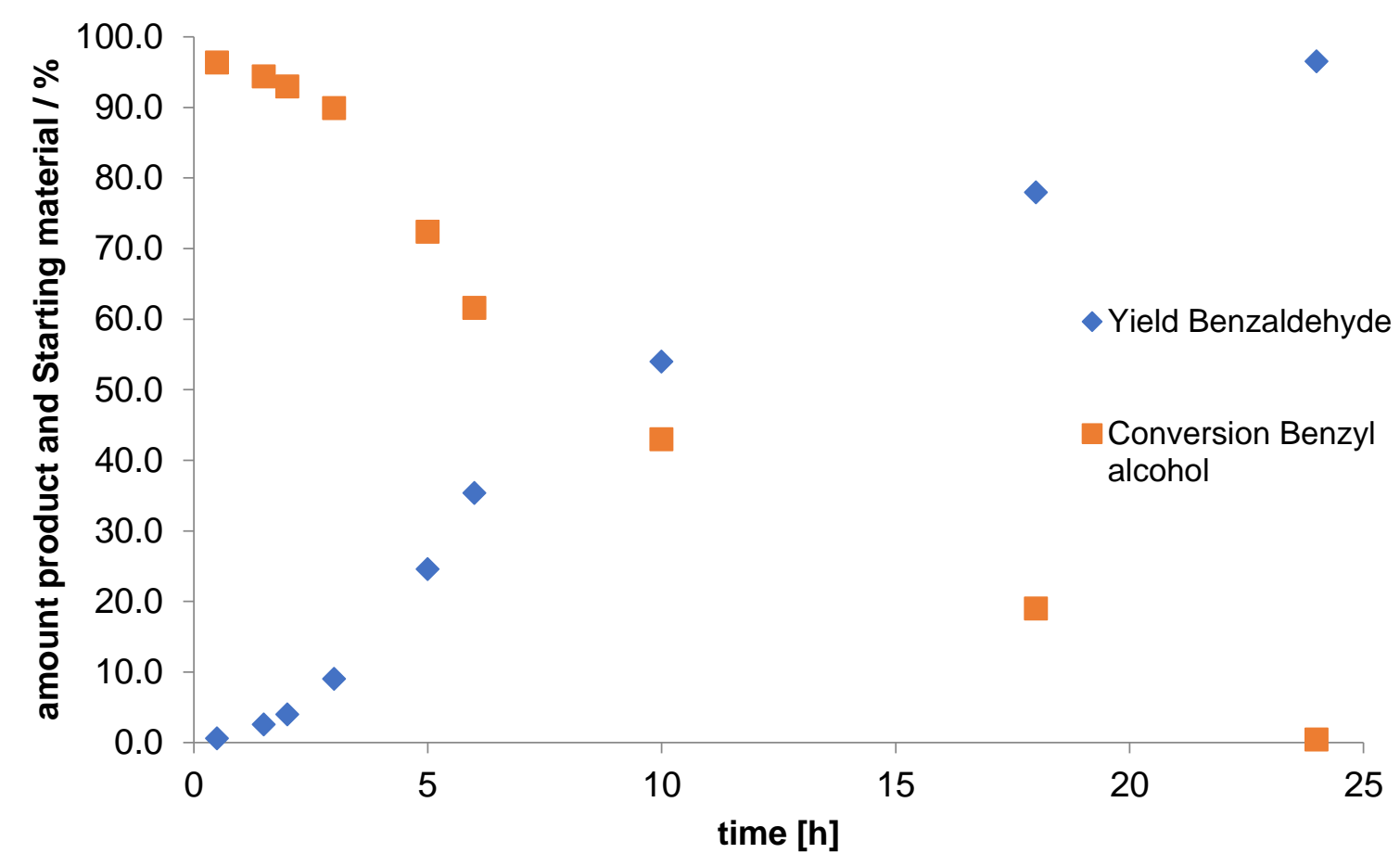

Figure 26: Yield and conversion based on passed reaction time, each point represents an independent experiment.

To investigate this aspect further and to identify the first step in the reaction SternVolmer experiments were performed. As, a full 3D-absorbtion-emission spectrum of 9-fluorenone in DMSO was recorded (Figure 27). The major absorption was at UVwavelengths, but a considerable absorption was present at visible light wavelength, primarily at the wavelength corresponding to blue light. A small absorption was present at green light corresponding wavelengths. The difference between emission wavelength $(515 \mathrm{~nm})$ and absorption wavelength $(290 \mathrm{~nm})$ was determined to perform Stern-Volmer Plots. Based on the simplicity of the reaction mixture, only dependence of substrate concentration (benzyl alcohol) and saturation of oxygen content were viable parameters (Figure 28). 

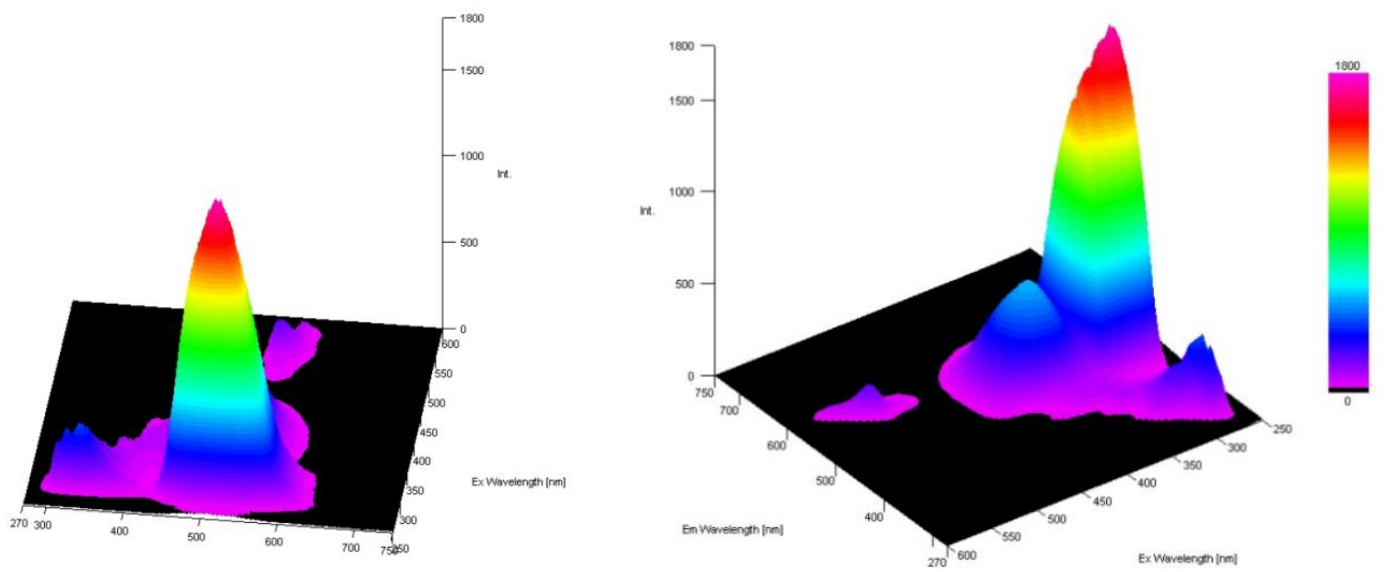

Figure 27: 3D-absorption-emission spectrum of 9-fluorenone in DMSO. Same spectrum at different angles. The spectrum was recorded in cooperation with Daniel Riemer.
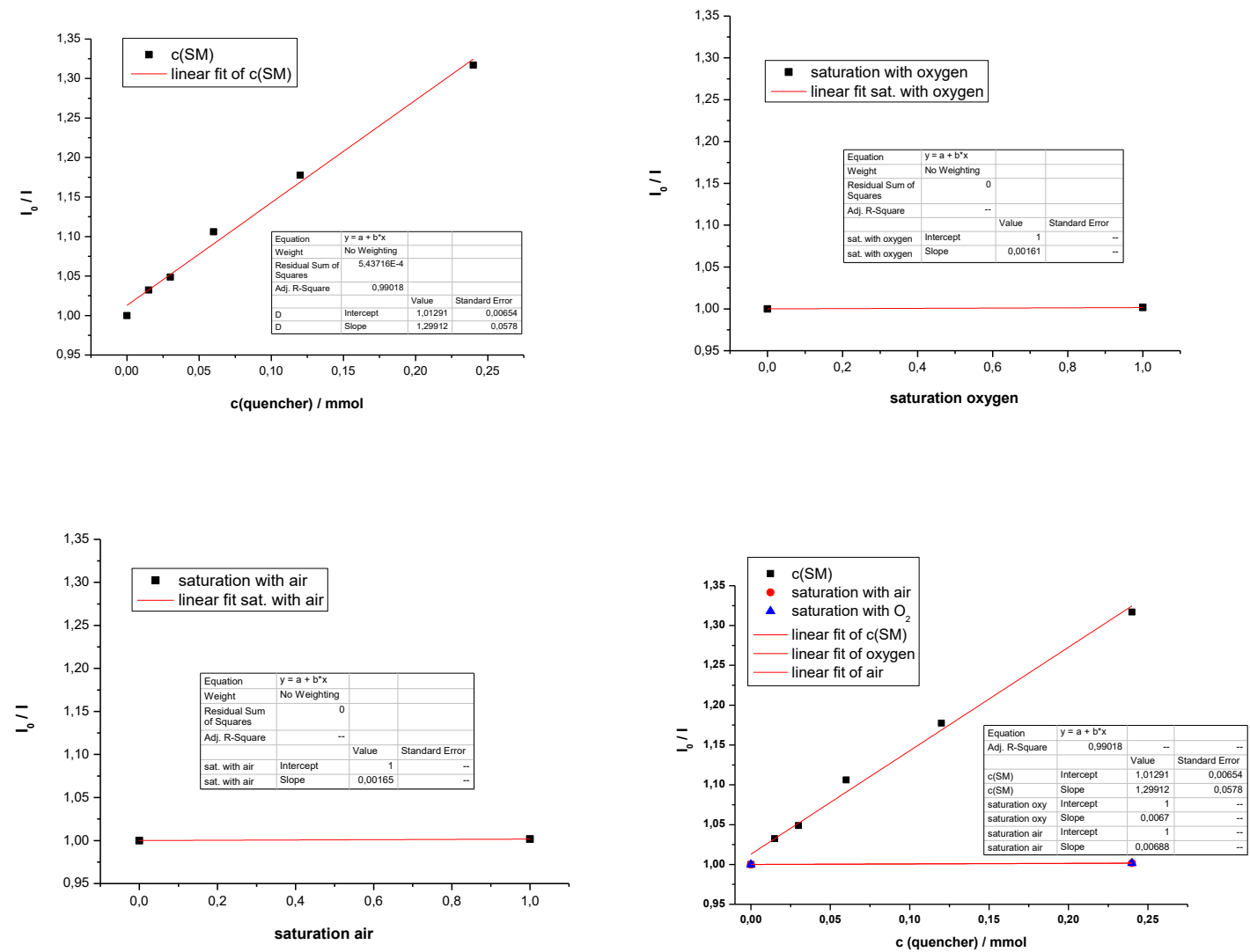

Figure 28: Stern-Volmer Plots of the investigated parameters; left upper corner: concentration of SM; right upper corner saturation with pure oxygen; left lower corner saturation with ambient air; right lower corner combined plot. 
The saturation of the sample with air/oxygen showed no visible effect on the catalyst. Increasing the concentration of the substrate had a corresponding quenching effect at the 9-fluorenone in the excited state interacting with the photocatalyst and diminishing the emission of photons. The substrate dependence was investigated under nitrogen and ambient air conditions with the same sample; the samples of different concentrations were prepared under inert conditions applying Schlenk-techniques. After the first measurement the sample was saturated for 10 minutes with ambient air utilizing a balloon syringe construction.

In addition to the performed reactions, labeling experiments (Scheme 22) were performed and combined with the kinetic isotope effect (KIE) experiments. Performing the reaction under isotope 18 oxygen atmosphere $\left({ }^{18} \mathrm{O}_{2}\right)$ resulted in non-labeled product formation with the formation of the labelled byproduct methylsulfonylmethane $\left(\mathrm{DMSO}^{18} \mathrm{O}\right.$ ), which was confirmed by GC-MS and HR-MS. This was a further confirmation for the product of hydrogen peroxide and a following further reaction between hydrogen peroxide with the solvent DMSO to form the oxidized form of the solvent. ${ }^{[130]}$ For the kinetic isotope effect (KIE), deuterium-labeled benzyl alcohol was synthesized and utilized for the model reaction. The model reaction was performed once again. The same procedure was applied for both the reactions at the same LEDsetup and the yield was compared by GC-FID using n-dodecane as the internal standard. The labeled product was additionally confirmed by GC-MS and HR-MS to be the mono-deuterated product compound. The compared yields showed a KIE with the value of 3.0 indicating that the rate determining step was the cleavage of the benzylic $\mathrm{C}-\mathrm{H}$ bond. 

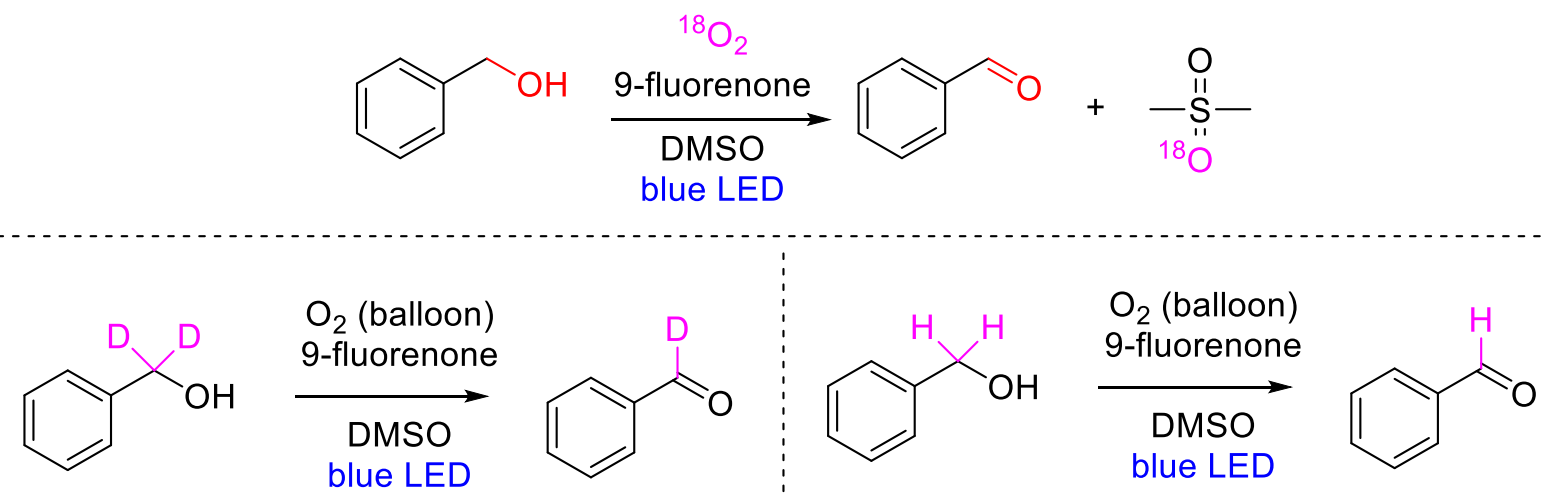

$$
0.0145 \mathrm{~mol}
$$

$0.0434 \mathrm{~mol}$

$$
\frac{\mathbf{k}_{H}}{\mathbf{k}_{\mathrm{D}}} \sim \frac{\mathbf{n}\left(\mathbf{P}_{\mathrm{H}}\right)}{\mathbf{n}\left(\mathbf{P}_{\mathrm{D}}\right)}=\frac{\mathbf{0 . 0 4 3 4}}{\mathbf{0 . 0 1 4 5}}=3.0
$$

Scheme 22: Performed labeling and KIE experiments. Phenylmethan- $d_{2}-\mathrm{ol}$ was synthetized by Daniel Riemer.

Table 5: Quenching experiments for the photocatalytic oxidation of benzylic alcohol. ${ }^{(a)}$<smiles>OCc1ccccc1</smiles>

1a

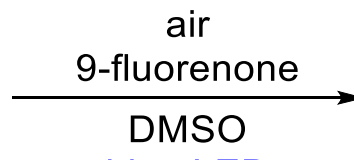

blue LED

Quencher

18 h, r.t.<smiles>O=Cc1ccccc1</smiles>

$1 b$

\begin{tabular}{cccc}
\hline Quencher & equivalents & Yield $/ \%$ & Notes \\
\hline BHT & 0.5 & 37 & Radical scavenger \\
BHT & 1.0 & 9 & Radical scavenger \\
TEMPO & 0.5 & 31 & Radical scavenger \\
TEMPO & 1.0 & 11 & Radical scavenger \\
tert-butanol & 1.0 & 81 & Hydroxide radical scavenger \\
CuCl 2 & 1.0 & 4 & Electron scavenger \\
Catalase & $100 \mathrm{mg}$ & 0 & Peroxide radical scavenger \\
Benzoquinone & 1.0 & 5 & Superoxide radical scavenger \\
Sodium azide & 1.0 & 22 & Singlet oxygen scavenger \\
\hline
\end{tabular}

(a) Reaction conditions: substrates (0.29 mmol), 9-fluorenone (3 mol\%), DMSO $(1 \mathrm{~mL})$, quenchers, $18 \mathrm{~h}$. [b] Yield determined by GC using $n$-dodecane as an internal standard. [Based on 84] 
An essential part of the mechanistic studies was the quenching experiments (Table 5). Several different compounds were utilized which were well known for their ability to capture different reactive intermediates. Each of the respective intermediates were investigated with its corresponding quencher. The addition of the radical scavengers, butylated hydroxytoluene (BHT) and 2,2,6,6-tetramethlpiperidinyloxyl (TEMPO), showed an intense decrease in yield when used in equimolar ratio and a considerable decrease at half concentration. This was a strong indication that the mechanism followed a radical pathway. The addition of tert-butanol showed no significant change in yield; therefore, it can be assumed that no hydroxy radicals were formed during the reaction. The addition of copper chloride $\left(\mathrm{CuCl}_{2}\right)$ almost completely inhibited the reaction and is a known quencher for single electron transformation. The addition of catalase, as very active enzyme, which is efficient for the transformation of reactive oxygen species and especially for peroxide radicals inhibited the reaction completely. The addition of benzoquinone had an intense decrease in yield as an effect showing the presence of superoxide radicals. The addition of sodium azide, to exclude the presence of singlet oxygen, had also a strong impact but lower than other quenchers. In total the quenching experiments had high accordance to a Type II single electron transfer (SET) pathway. The only inconsistency was the decreased yield through sodium azide, which could indicate a secondary pathway by Type I energy transfer (ET), which would be typically between the photocatalyst and the atmospheric oxygen. This assumption could be excluded from consideration according to the results of the Stern-Volmer plot, which excluded a direct energy transfer between photocatalyst and oxygen. This still left a possible complex interaction by generating singlet oxygen from reactive intermediates, which is rarely reported, due to the complexity of the mixture of the intermediates. EPR experiments were performed, which had short lived radicals and could not be measured directly. TEMPO was utilized as a source for stable radicals for the examination, which resulted in a complex mixture of many detectable radicals, which in addition showed dependence on the reaction time, with a corresponding change in ratio between them. During all the changes a highly complex mixture of different radicals could be observed. The primary signal had high similarity to the expected, superoxide radical intermediate, but could not be proven with certainty. The question remained, if the additional forming radicals in the EPR spectra were based on substrate-based radicals, or possible singlet oxygen radicals, or maybe even further forming radicals. 
All this information resulted towards a plausible mechanism for this reaction (Scheme 23). The photocatalyst 9 -fluorenone was excited by the irradiation of visible light. The excited state of the photocatalyst performed a singlet electron transfer with the substrate, forming a radical anion form of the catalyst and a radical cation of the substrate. The formed radical anion of the photocatalyst reacted further with oxygen from the atmosphere to form the starting state of the photocatalyst concluding a full cycle of the photocatalyst and superoxide radical anion as a reactive intermediate. The benzyl alcohol radical cation was attacked by the superoxide radical anion resulting in a proton abstraction to form an aldehyde radical and a hydrogen peroxide radical, followed by a second proton abstraction resulting in the main product and hydrogen peroxide. Based on literature research, several reports about the interaction of hydrogen peroxide with DMSO could be found. This is verifying a likely follow up reaction between those two reactants to form $\mathrm{DMSO}_{2}$. Further literature research yielded that the reduction potential of 9 -fluorenone resides at $-0.61 \mathrm{~V}$ vs SCE ${ }^{[43]}$ and the value for the formation of superoxide radical anions at $-0.56 \mathrm{~V}$ vs SCE. ${ }^{[84]}$ This is a sufficient value for the proposed SET reaction. The possibility of a secondary pathway was not excluded, several observations could not deny the possibility of the generation of singlet oxygen and its involvement in the mechanism. A direct generation via the photocatalytic pathway was not involved, but a possible complex combination of several reactive intermediates could still provide a viable source for the generation of singlet oxygen. This could also be an explanation of the slower reactivity in the beginning of the reaction, the requirement of higher light intensity above $4 \mathrm{~W}$ and the very broad substrate scope compared to similar reports in photocatalytic oxidation of alcohols. The high efficiency of the catalyst and long-time stability can be explained with the mechanism indirectly. 


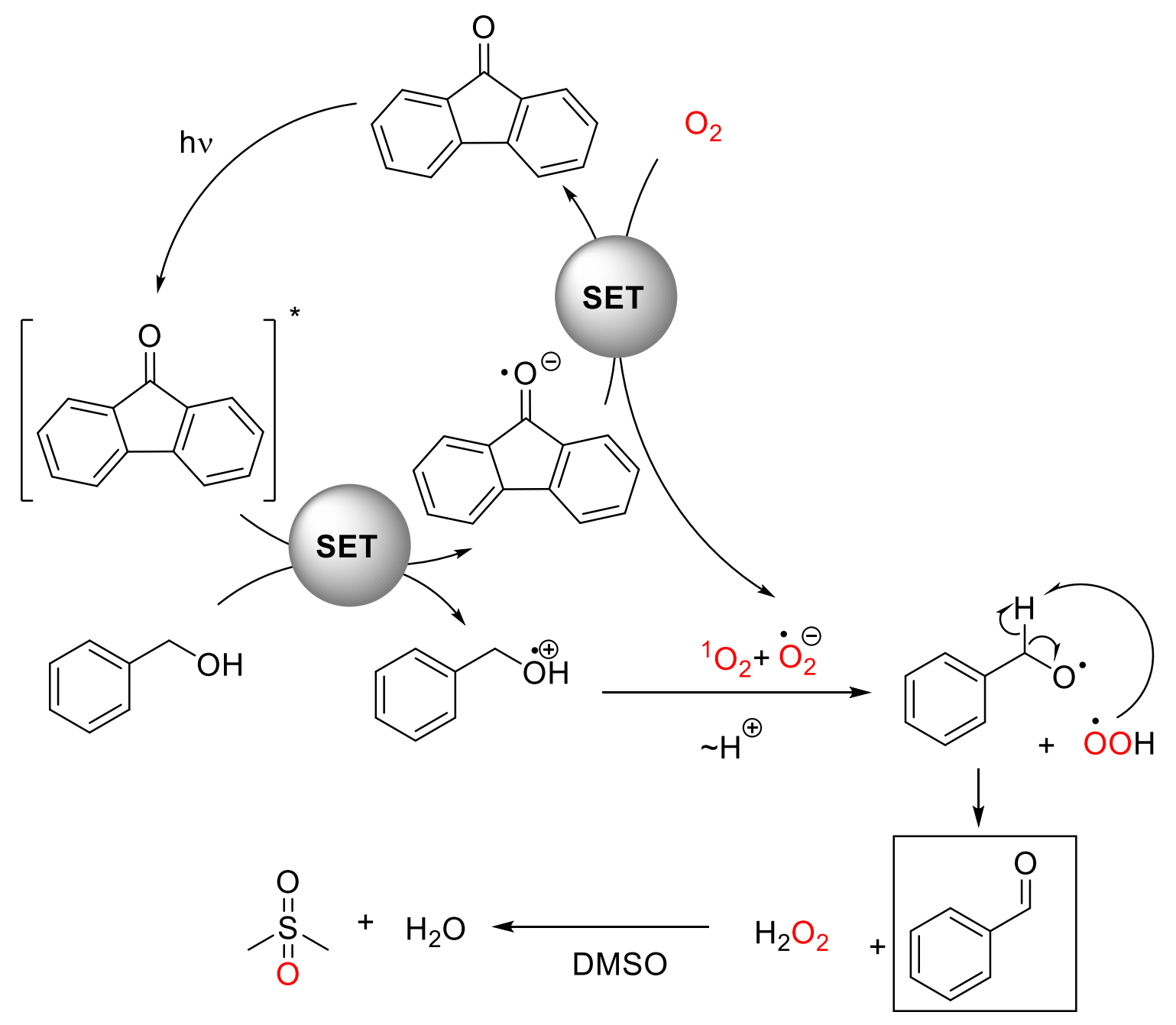

Scheme 23: Proposed mechanism for the 9-fluorenone based photocatalytic oxidation of alcohols.

\subsubsection{TRANSFER OF RESULTS TO FLOW CHEMISTRY}

The dependence of photochemistry on geometry and reaction setup construction was explained in the introduction. The benefit of lab scale reaction utilizing regular flasks provides benefits to the number of possible microscale reactions, but their up-scale potential is highly limited. The recent advances in the construction and utilization of flow photochemical setups enable the possibility to construct flow setups which can provide faster reaction times and the benefit of the number up concept to increase the scale of the reaction. Additionally, through continuous flow, no down times in the 
reaction process are given. The reported concepts are still quite expensive with around 10000 dollars $^{[131]}$ and commercial versions reaching easily up to 80000 dollars. The idea was to build a simpler and affordable alternative to directly utilize this concept. Therefore, an internal limit of around 1000 euro was set. Different ideas on how to achieve a mass flow control of the gas phase were investigated. Utilizing several pressure regulators to minimize the gas flow for lab scale achieved low gas flows. This provided the possibility to build a low cost photochemical continuous flow LED-setup (Figure 29).

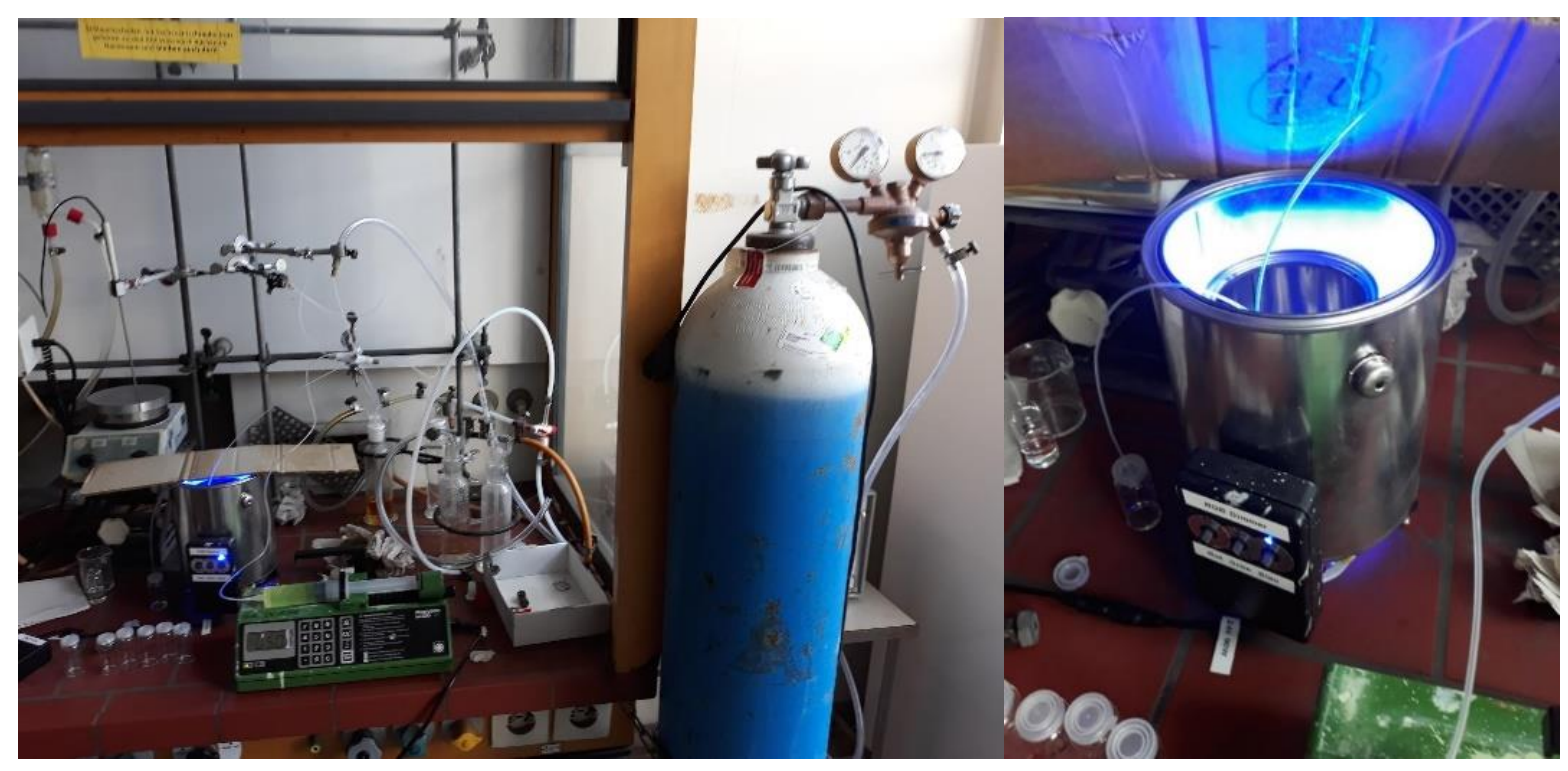

Figure 29: Experimental setup for the continuous flow photochemical reaction.

The setup was capable of utilizing gas from a standard gas bottle with a pressure regulator and also by typical pressurized air. Gas flow was regulated by an additional fine value. The liquid mass flow was regulated by a Perfusor pump. Mixing of both phases was performed by simple a PFT-T-piece. Tubing ( $5 \mathrm{~mL}$ total volume) was glued around a smaller metal can, which was small enough to be inserted into the already discussed metal setup. The concept was used to perform the alcohol oxidation and to compare the gained results with the flask-based batch concept. The concept was only able to achieve combined flow rates (gas phase and liquid phase) of lower than $10 \mathrm{~mL}$ per hour resulting in reaction times of 30 minutes. Reaction was performed for 3 full voluminal till a sample was collected to exclude initial flow disturbances in the setup. Based on the slow flow of the reaction mixture and the resulting effect of the Reynolds and Peclet numbers, a total of three setup voluminal was passed before 
measurement to counteract possible back mixing. After the 3 full voluminal, $1 \mathrm{~mL}$ sample was collected and was analyzed by GC-FID using $n$-dodecane as the internal standard. The comparison batch sample was prepared in the same fashion and compared (Table 6).

Table 6: Yields of photochemical continuous flow setup and batch comparison. ${ }^{\text {(a) }}$

\begin{tabular}{ccc}
\hline Reaction time / $\mathbf{m i n}$ & Yield flow setup / \% & Yield batch setup / \% \\
\hline 15 & 3.1 & 1.2 \\
30 & 9.5 & 3.2 \\
180 & --- & 9.1 \\
\hline
\end{tabular}

The effect of utilizing a flow setup resulted in an increase of reaction speed in the area of factor 2-4. Longer reaction times could not be achieved. A follow up concept for the continuous flow was designed utilizing also for the gas phase a second Perfusor pump. This modification provided a controlled mass flows of both phases with up to $10 \mathrm{~h}$ reaction time as its physical limit. 


\subsubsection{SUMmaRY AND OUTLOOK OF THE PROJECT}

The performed project was able to demonstrate a metal-free and additive-free selective oxidation of primary and secondary alcohols to their corresponding carbonyl compounds. The developed methodology was working under ambient mild conditions, precisely under ambient air and room temperature, utilizing commercially available low power LED and technical equipment. The methodology was intensively examined and could be increased further in efficiency, by utilizing pure oxygen atmosphere and increasing the catalyst loading, to achieve higher yields. The substrate scope showed a broad reactivity for different benzylic primary and secondary alcohols with a variety of different functional groups being tolerated. Among those substrates were also heteroaromatic compounds. In total an amount of 22 isolated products with good to excellent yield. 15 further substrates showed reactivity which the basic protocol. Reexamination of these substrates with extended reaction times, under the advanced conditions of pure oxygen atmosphere and increased catalyst loading should yield good results. The resulting reactivity led to the investigation of aliphatic secondary alcohols of which 14 further substrates could be isolated with good to excellent yields.

Additionally, it was the first metal-free photocatalytic approach for the oxidation of steroid substrates. Mechanistic investigations could show a plausible mechanism. This revealed that a simple organic compound could act as an efficient photocatalyst with state-of-the-art results and environmentally friendly conditions. The forming byproduct of the reaction, hydrogen peroxide, was interacting with the solvent DMSO and forming $\mathrm{DMSO}_{2}$ and water as the result, inactivating all reactive byproducts. Furthermore, these results can be transferred to continuous flow concepts and thus offering the possibility for up scaling into industrial scales. A new cost-efficient system could be developed based on this work, lowering the costs of continuous flow setups to under 1000 Euro. Based on the results of the investigations parts of this work were already published in ACS Catalysis ${ }^{[132]}$ and Nature Protocols. ${ }^{[133]}$ 


\subsection{OXYGENATION OF HETEROCYCLES}

After establishing the oxidation protocol for alcohols, new alternative substrate groups were investigated for the application of oxidation reaction. Several different model substrates were investigated for different possible oxidation products (Figure 30). Transformation of cyclic ethers to their corresponding esters were found, as also transformation of indoles to isatins. After initial consideration, the choice was made to focus on the transformation of indoles. To summarize the results of the ether to ester work, 46 optimization experiments were performed. At total of 8 photocatalysts, with 6 solvents and different bases were investigated, however, only trace amounts of desired product were found. Introduction of NHPI combined with utilization of base (DBN) and mixture of different solvents (DMF/ACN) resulted in drastically higher yields of up to $65 \%$. However, changes of concentrations, photocatalysts, solvents, did not further improve the result.
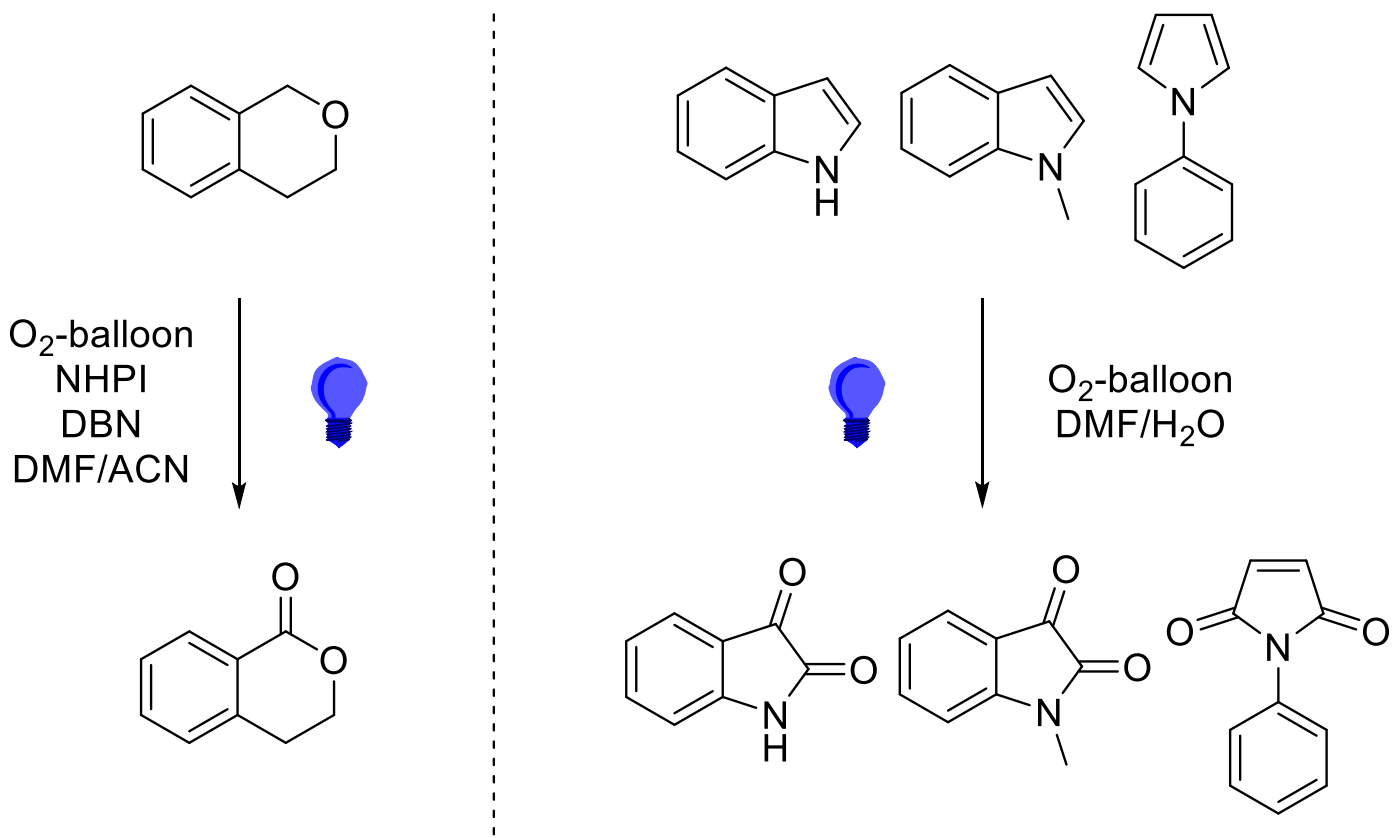

Figure 30: Overview of oxygenation reactions concepts investigated.

Protected indole, unprotected indole and pyrrole were chosen as the target substrates. Initial experiments showed that the previous protocol with 9-fluorenone showed low reactivity for 2 of the 3 chosen substrates and forming the corresponding isatins, but not the desired maleimide. Around $7 \%$ yield for the protected and $3 \%$ for the unprotected isatin were formed. In the case of maleimide, no detectable signal was 
found for the starting material, nor the product. During optimization, the third model substrate pyrrole was changed to phenylpyrrole.

\subsubsection{OptIMIZATION StUdies}

As the central model substrate unprotected indole was chosen, a broad screening was initiated with different solvents (DMSO, DMF, DMA, ACN, EtOH, water, toluene, $\mathrm{MeNO}_{2}$ ), catalysts (9-fluorenone, rose bengal, eosin $\mathrm{Y}$, acriflavine, xanthone, riboflavine, rhodamine 6G), additives (NHPI, KOH, DBU, DBN) and over 118 different combinations were attempted. Among them, only rose bengal in DMSO, DMF, DMA achieved yield of $\sim 10, \sim 12$ and $\sim 9 \%$ yield respectively. All the other combinations revealed lower reactivity. Utilization of $\mathrm{NHPI}$ as an activator did not improve the yield. Utilization of different solvent mixtures combined with water were investigated. Rose bengal with DMSO/water and DMF/water mixtures showed the highest reactivity with a 23 and $30 \%$ yield. Different ratios of both mixtures were investigated and resulted in DMSO/water 50:50 ratio and DMF/water 90:10 ratio achieving yields of 51 and $52 \%$. Cross referencing the results with protected indole and phenylpyrrole showed that protected indole was reacting in DMSO/water mixture with a $98 \%$ yield did not show any reactivity for the generation of phenylmalimide. In the case of the DMF/water mixture a high activity for all the substrates was observed, with $62 \%$ for the protected indole and $64 \%$ the phenylpyrrole. Addition of $10 \mathrm{~mol} \%$ of $\mathrm{LiCl}$ to the solution increased the yields by only $4 \%$. An increase of the reaction time resulted in a $93 \%$ yield for the unprotected indole as the substrate. The increase in yield by the increase of reaction time, showed a disproportional increase and was repeated 4 times with consistent results. The same experiment was performed for the 18-hour sample, with the same consistent results. The disproportional increase in yield was confirmed with this and marked for further studies during the mechanistic investigations. 
Table 7: Selection of the optimization experiments of the oxygenation of $1 \mathrm{H}$ indole to isatin. (a)

\begin{tabular}{cccc} 
Catalyst & Solvent & Water content / Vol\% & Yield / \% \\
\hline Rose bengal & DMSO & 0 & 12 \\
Fluorenone & DMSO & 0 & 3 \\
Eosin Y & DMSO & 0 & 8 \\
Xanthone & DMSO & 0 & 4 \\
Rhodamine 6G & DMSO & 0 & 2 \\
Rose bengal & DMSO & 20 & 23 \\
Rose bengal & DMSO & 33 & 28 \\
Rose bengal & DMSO & 50 & 51 \\
Rose bengal & DMSO & 66 & 15 \\
Rose bengal & DMA & 20 & 7 \\
Rose bengal & THF & 20 & 9 \\
Rose bengal & ACN & 20 & 30 \\
Rose bengal & DMF & 20 & 12 \\
Rose bengal & DMF & 50 & 40 \\
Rose bengal & DMF & 15 & 52 \\
Rose bengal & DMF & 10 & 93 \\
Rose bengal[b] & DMF & 10 & 18 \\
\hline
\end{tabular}

(a) Reaction conditions $0.25 \mathrm{mmol}$ of $1 \mathrm{H}$ indole, photocatalyst ( 3 mol\%), $18 \mathrm{~h}$ reaction time, blue LED irradiation at $12 \mathrm{~W} 420 \mathrm{~nm}$. Yield was determined by GC using biphenyl as an internal standard. [b] reaction time $24 \mathrm{~h}$. [c] full conversion, product was mostly an insoluble precipitate. 


\subsubsection{SCOPE OF SUBSTRATES AND APPLICATIONS}

For the substrate scope of the oxygenation reaction, a systematic approach was chosen. Different substitutions at different positions were investigated to estimate the impact of substitutions and its impact at different positions (Scheme 24). The first point of the investigation was to examine the reactivity towards indoline, to observe if aromatization followed by oxygenation could be achieved. Aromatization product with $\sim 50 \%$ yield was observed along with trace amounts of isatin. The combination of aromatization and a follow up oxygenation was determined as possible, but insufficient since the increased reaction time of $72 \mathrm{~h}$ could not further increase the yields.

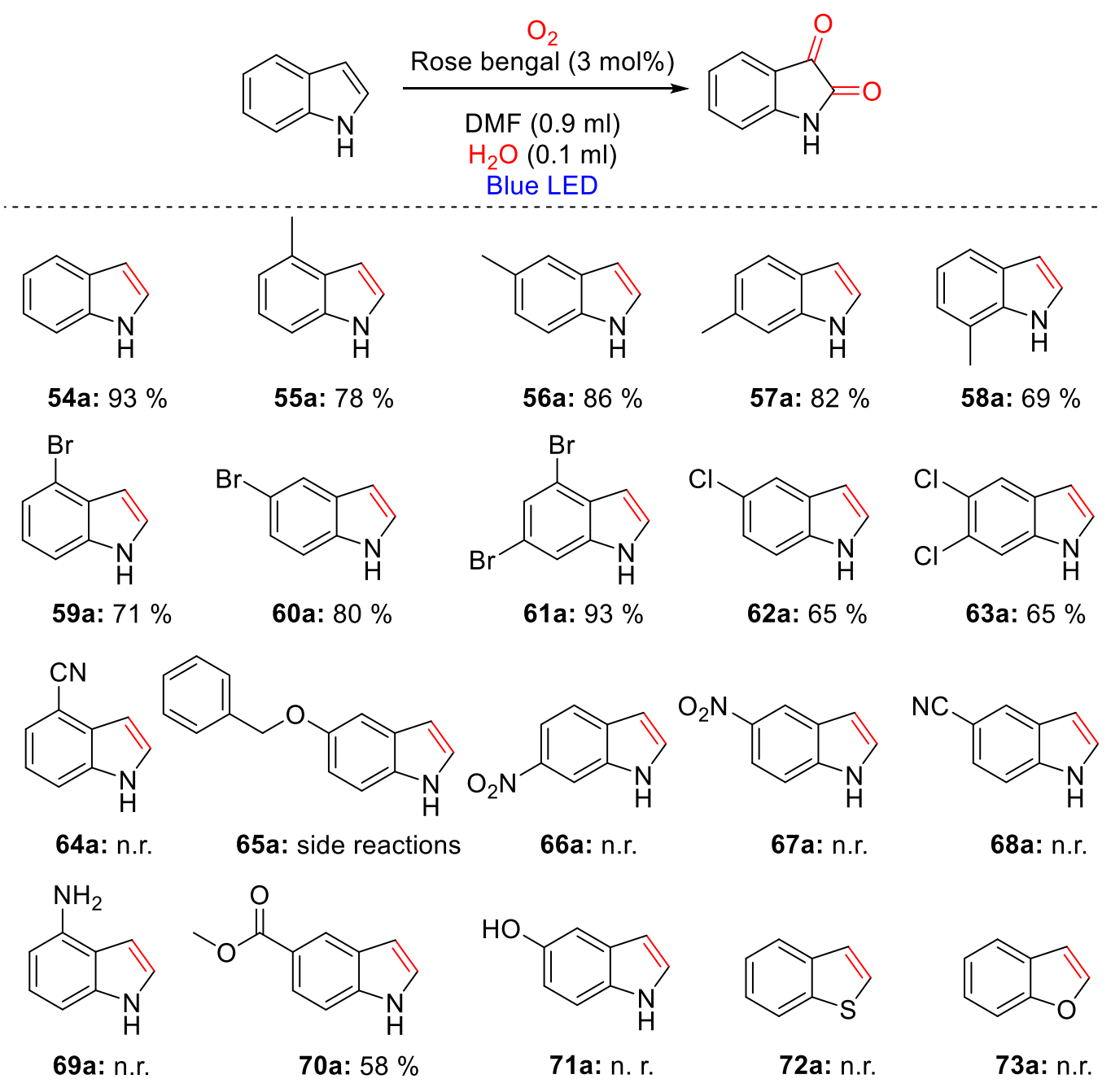

Scheme 24: Substrate scope of different indole-based derivatives. Percentages are the isolated yields. reaction conditions: substrate $(0.25 \mathrm{mmol})$, rose bengal $(3 \mathrm{~mol} \%)$, DMF (0.9 mL), H2O (0.1 mL), reaction time 16-48 h, blue LED light $420 \mathrm{~nm}$. 
The investigation of the substrate scope (Scheme 24) was initiated with methyl derivatives in the position of 4, 5, 6 and 7 (55-58a) of the indole system. The presence of the methyl groups in the positions 4-7 had no significant impact on the photocatalytic. In all four cases, a slight reduction in yield was observed and the positions 5 and 6 seem to be preferable compared to the positions 4 and 7. For $56 a$ with a methyl group in position 5 , the reaction performed in a faster manner and was concluded within $18 \mathrm{~h}$. Replacement with an electron withdrawing group in form of halogens showed that bromide performed also with good yield (59-60a). In the case of double substitution with bromide in position 4 and 6 (61a), a higher reactivity was be observed. Changing the bromide to chloride (62a) resulted in slightly lower yields. Double chloride substituted indole with the substitution in positions 5 and 6 (63a) was examined which should have a higher reactivity, but only performed comparably to the mono substituted indole (62a). Introducing a cyano group in position 4 (64a) showed no reactivity, change to position 5 (65a) also resulted in no reactivity. Examining the effect of nitro groups in position 5 (67a) and 6 (66a) also showed no reactivity. An amine containing derivative was investigated $(69 a)$ and had no reactivity as well. It was concluded that nitrogen containing functional group could interfere with the photocatalytic system and may inhibit the reaction. To investigate the effect of other functional groups such as methyl ester (70a) and hydroxy (71b), each in positions 5 were examined. Hydroxide also showed an inhibiting effect on the oxygenation reaction. The methyl ester was able to be oxygenated in good yield. The reaction was attempted with 5-(benzyloxy)indole (65a), but included several side reactions. The benzylic position was also oxygenated, cleavage occurred additionally for this product yielding the benzoic acid, the target isatin formed in a lower yield, as also a combination of the main and side reactions. It was not possible to separate the complex mixtures of the different products. The investigation of different performance of the indole framework continued. The next examined aspect included the change of the nitrogen to other heteroatoms like sulfur (benzothiophene, 72a) and oxygen (benzofuran, 73a). In both cases no reactivity could be observed. The next investigated changes were different substitution/protection groups at the indole nitrogen (Scheme 25). Methyl (74a) ethyl (75a), octyl (76a), benzyl (77a) and lilolidine (78a) as an alicyclic compound were examined and revealed good to excellent yields. $79 a$ was to investigate the tolerance for the boc-protecting group. The result was that 
the reaction occurred, but the forming isatin was directly deprotected in the photocatalytic process.

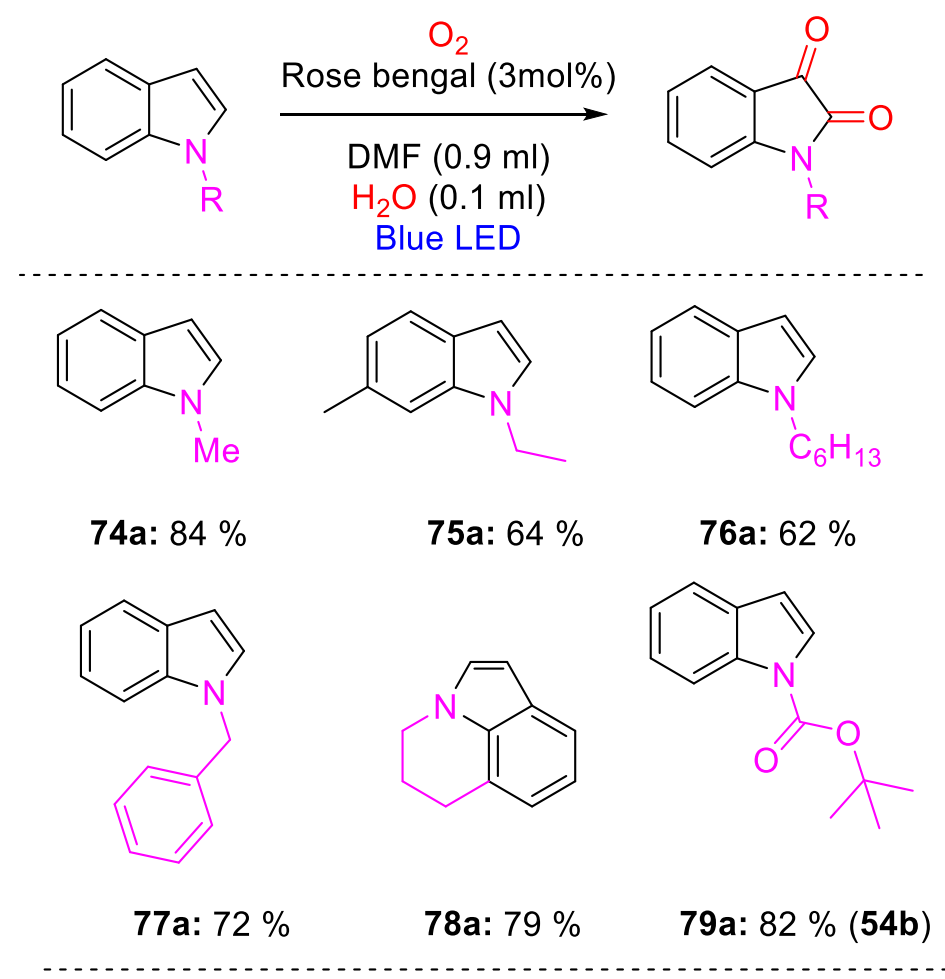

Scheme 25: Substrate scope of different indole-based derivatives with changes at the nitrogen. percentages are the isolated yields. Reaction conditions: Substrate (0.25 mmol), rose bengal (3 mol\%), DMF $(0.9 \mathrm{~mL}), \mathrm{H}_{2} \mathrm{O}(0.1 \mathrm{~mL})$, reaction time $48 \mathrm{~h}$, $12 \mathrm{~W}$ blue LED light $420 \mathrm{~nm}$.

The third group of possible derivatives was a change of the substitution in position 2 and 3 of the indole scaffold (Scheme 26). For the unprotected $1 \mathrm{H}$-indole carboxaldehyde (80a and 81a) and carboxylic acids (82a and 83a) each in position 2 (80a and 82a) and 3 (81a and 83a) were examined and for the protected $N$ methylindole with carboxylic acid in position 2 was examined (Scheme 26). In those cases, several observations could be made. The carboxaldehydes (80a/81a) were oxidized to the corresponding carboxylic acids (82a/83a). The carboxylic acids $(\mathbf{8 2} \mathbf{a} / \mathbf{8 3} \mathbf{a} / \mathbf{8 4 a})$ were decarboxylated forming the model substrate indoles $(\mathbf{5 4 a}$ and $\mathbf{7 4 a}$ ), which were then oxygenated to their corresponding isatin forms (54b and $\mathbf{7 4 b}$ ). The protected case (84a) also followed the same procedure. Based on the fact that for the carboxaldehydes a three-step reaction occurred, the yield in $48 \mathrm{~h}$ was considerably lower. Increasing the reaction time to $72 \mathrm{~h}$ showed further conversion to 
over $60 \%$ yield in both cases. It can be assumed that for all the cases full conversion to isatin can be achieved within sufficient reaction time.
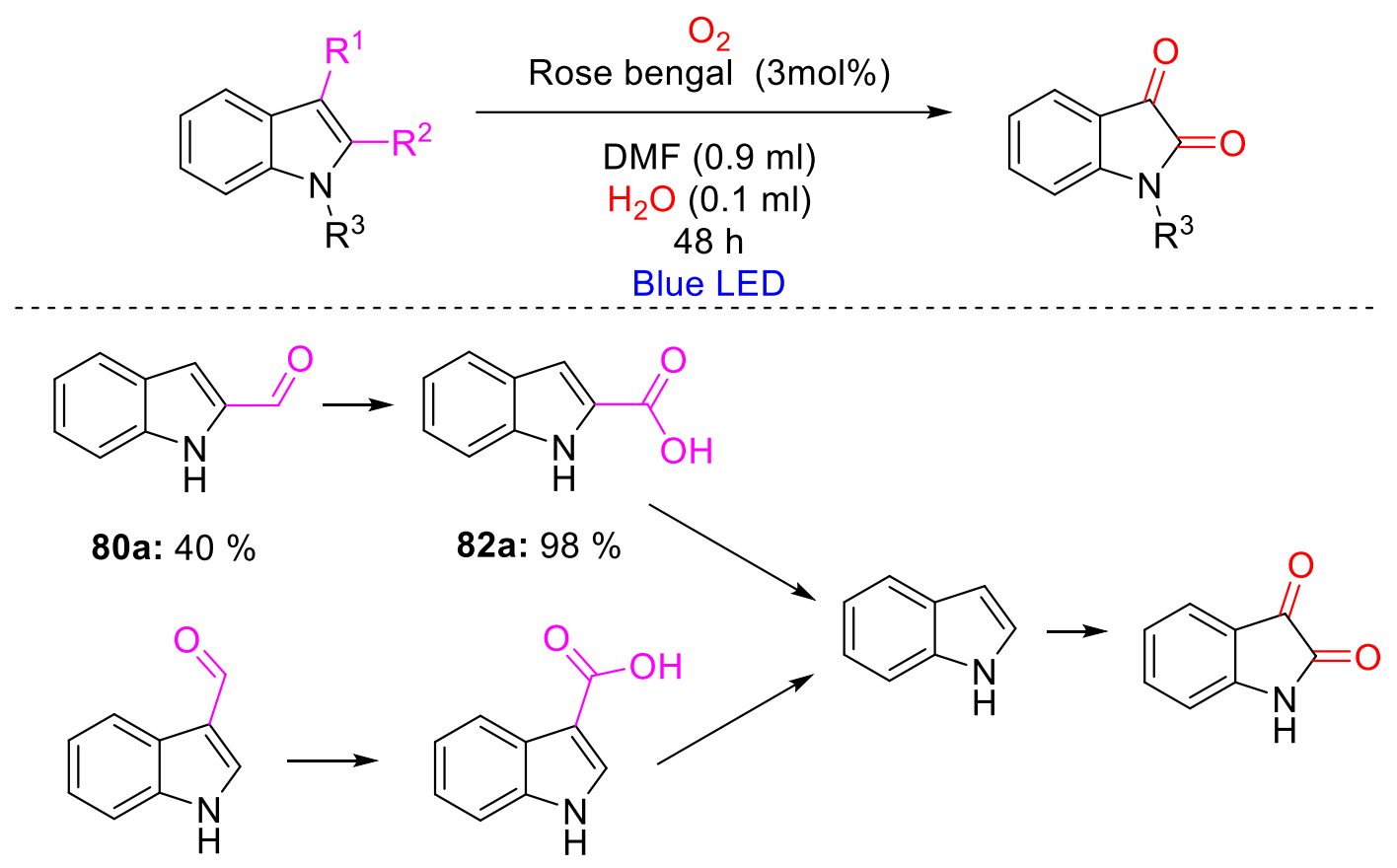

81a: $31 \%$

83a: $94 \%$

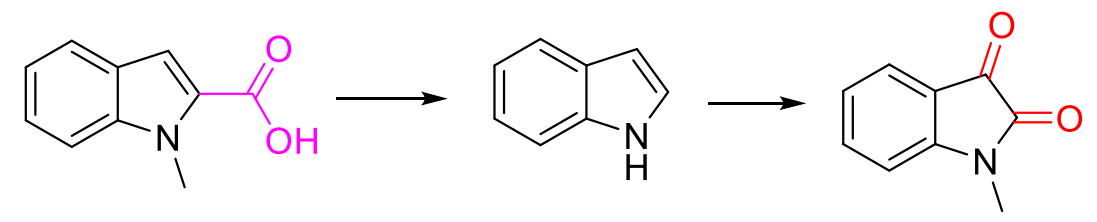

84a: $58 \%$

Scheme 26: Substrate scope of different indole-based derivatives with changes at the position 2 and 3 . Yields of the final product were determined by GC using biphenyl as an internal standard. Respective final products were isolated before. Reaction conditions: substrate $(0.25 \mathrm{mmol})$, rose bengal (3 mol\%), DMF $(0.9 \mathrm{~mL}), \mathrm{H}_{2} \mathrm{O}$ $(0.1 \mathrm{~mL})$, reaction time $48 \mathrm{~h}, 12 \mathrm{~W}$ blue LED light with a maximum at $420 \mathrm{~nm}$.

Changing the substitution in position 2 and 3 (Scheme 27) to other functional groups resulted in no formation of isatin. A cleavage reaction combined with the oxygenation reaction was observed. Yields of those reactions were unsatisfying between 22-38 \% yield for the investigated derivatives. Only the substrate (87a) reached the desired yield of $60 \%$ under the optimized conditions. Inspired by the work of Jiang et al., the addition of $50 \mathrm{~mol} \%$ of $\mathrm{K}_{3} \mathrm{PO}_{4}$ significantly increased the yield. The remaining substrates were reevaluated (Scheme 12). Four unprotected and three protected indoles with different substituents in position 2 and 3 were chosen from the present substrates and were investigated. A single methyl group in position 3 (86a) was 
sufficient for the change in the reactivity from the main product isatin to the cleavage product. The presence of methyl groups in each of the positions 2 and 3 (85a) resulted in an increase of the yield of the oxygenated cleavage product. Utilization of phenyl group (87b) in position 2 showed even higher reactivity and achieved sufficient yield without the additional use of $\mathrm{K}_{3} \mathrm{PO}_{4}$. An extension of the aromatic backbone (88b) was well tolerated with an excellent yield. The use of $\mathrm{N}$-methylindole with one methyl and two methyl groups could demonstrate similar results and observations. Also, in the $\mathrm{N}$ methylindole case, the use of a phenyl group showed higher yields. During the initial part of the substrate scope, an inhibiting effect of nitrogen containing functional groups was observed. To investigate this theory of an inhibiting effect, tryptamine (92a) as an amine containing derivative was examined. For this case, no reactivity could be observed either. It can be concluded that a possible effect on the entire catalytic system could be based on the presence of further nitrogen containing functional groups. 
<smiles>[R]c1c([R])n([R])c2ccccc12</smiles>

$\mathrm{O}_{2}$

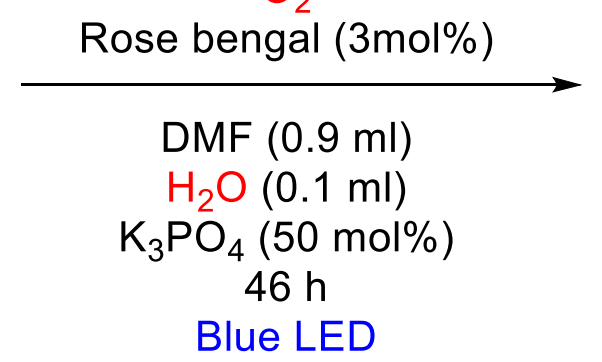<smiles>[R]C(=O)c1ccccc1N([R])C([R])=O</smiles><smiles>CC(=O)Nc1ccccc1C(C)=O</smiles>

85b: $82 \%$<smiles>CC(=O)c1ccccc1NC=O</smiles>

86b: $70 \%$<smiles>O=Cc1ccccc1NC(=O)c1ccccc1</smiles>

$87 b^{\text {a }}: 60 \%$<smiles>CC(=O)Nc1ccc2ccccc2c1C(C)=O</smiles>

88b: $85 \%$<smiles>CC(=O)N(C)c1ccccc1C=O</smiles>

89b: $79 \%$<smiles>CC(=O)c1c(N(C)C(C)=O)ccc2ccccc12</smiles>

90b: $84 \%$<smiles>CN(C(=O)c1ccccc1)c1ccccc1C=O</smiles>

91b: $92 \%$

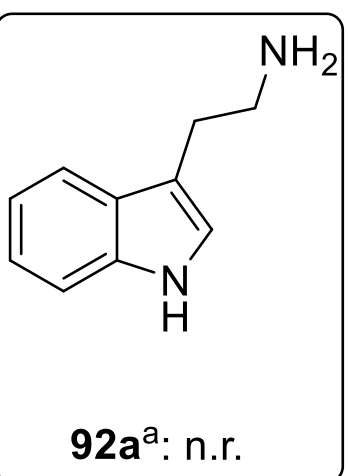

Scheme 27: Further substrate scope of different indole-based derivatives with changes at the position 2 and 3 . Isolated yields. Reaction conditions: substrate (0.25 mmol), rose bengal (3 mol\%), DMF $(0.9 \mathrm{~mL}), \mathrm{H}_{2} \mathrm{O}(0.1 \mathrm{~mL}), \mathrm{K}_{3} \mathrm{PO}_{4}$, reaction time $48 \mathrm{~h}, 12 \mathrm{~W}$ blue LED light $420 \mathrm{~nm}$. a) without the use of $\mathrm{K}_{3} \mathrm{PO}_{4}$. Entries 85b-91b were performed and isolated by Yu Zhang.

After showing a broad substrate scope for indole-based substrates, the substrate scope investigations continued with the oxygenation of pyrroles (Scheme 28). As stated in the beginning of the chapter, pyrrole was too volatile and the reaction scale was too small to be effectively investigated. Three different pyrrole derivatives were chosen with different substitutions in the backbone at position 3 and 4 . For the symmetrical case (93b) good yields for the formation of the products were observed. The compound showed high reactivity after the reaction was stopped and was not stable during purification. In the nonsymmetrical cases (94b and 95b) the stability of the maleimide product was even less and after fast work up and isolation yields of 50 
and $53 \%$ were obtained. The reactions were repeated, and the yield was verified by NMR using iodoform as an internal standard.<smiles>[R]c1cn([R1])cc1[R]</smiles>

$\mathrm{R}^{1}$

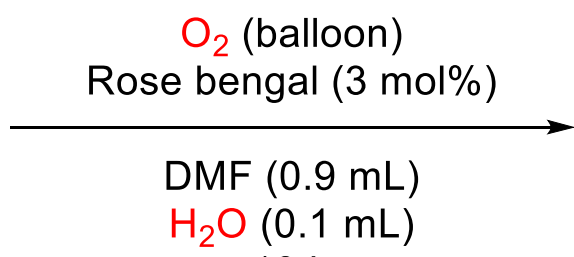

$18 \mathrm{~h}$<smiles>[R]C1=C([R])C(=O)N([R1])C1=O</smiles>

$\mathrm{R}^{1}$

Blue LED<smiles>CCC1=C(CC)C(=O)NC1=O</smiles>

93b: $75 \%$<smiles>CCOC(=O)C1=C(c2ccccc2)C(=O)NC1=O</smiles>

94b: $50 \%$<smiles>O=C1C=C(c2ccccc2)C(=O)N1</smiles>

95b: $53 \%$<smiles>O=C1C=CC(=O)N1c1ccccc1</smiles>

96b: $74 \%$<smiles>O=C1C=CC(=O)N1c1ccc(I)cc1</smiles>

97b: $69 \%$<smiles>COc1ccc(N2C(=O)C=CC2=O)cc1</smiles>

98b: $77 \%$<smiles>CCCCCCc1ccc(N2C(=O)C=CC2=O)cc1</smiles>

99b: active<smiles>NCCN1C(=O)C=CC1=O</smiles>

100b: n.r.

Scheme 28: Further substrate scope of different pyrrole-based derivatives with isolated yields. Reaction conditions: substrate $(0.25 \mathrm{mmol})$, rose bengal $(3 \mathrm{~mol} \%)$, DMF $(0.9 \mathrm{~mL}), \mathrm{H}_{2} \mathrm{O}(0.1 \mathrm{~mL})$, reaction time $18 \mathrm{~h}, 12 \mathrm{~W}$ blue LED light $420 \mathrm{~nm}$. Isolation of entries 93b-95b in cooperation with Yu Zhang and utilization of GPC.

It could be confirmed that after the reaction was concluded, yields of around $90 \%$ were present in all three cases (93b - 95b). The isolated yields were based on the speed and efficiency of the isolation and stabilization of the maleimides. Repetition of the experiment and determination of the product by crude NMR using iodoform as the internal standard confirmed this observation. The investigations continued with different substitutions at the pyrrole nitrogen. Electron withdrawing groups at the phenyl attachment (96b-98b) lowered the reactivity of the maleimide products and purification could be performed with more ease. For $99 \mathrm{~b}$, a high reactivity could be observed but all attempts to isolate it failed. The only confirmation was based on GC- 
MS, with an assumed yield of $89 \%$. To examine the hypothesis of nitrogen containing additional functional groups could inhibit the photocatalytic reaction, an amine containing pyrrole (100b) was examined and showed no reactivity.

\subsubsection{Application of the Photocatalytic Oxygenation Reaction}

After establishing a broad substrate scope for indoles and pyrroles, possible applications for the oxygenation protocol were investigated; different syntheses of bioactive compounds were selected. Beginning from indole derivatives, those compounds were synthesized. As a final examination of the nitrogen-inhibiting effect theory, 7-azaindole (101b) was used for the oxygenation protocol (Scheme 29). Azaisatins showed higher reactivity as anticancer drugs. ${ }^{[135]}$ The reaction was performed and could even be performed on a $5 \mathrm{mmol}$ scale with a longer reaction time.

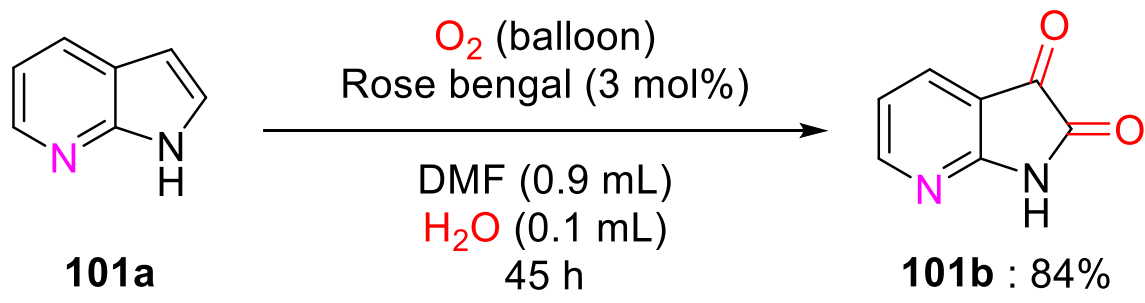

Scheme 29: Utilization of the oxygenation protocol for the generation of 7-azaisatin. Reaction conditions 7-azaindole $(0.25 \mathrm{mmol})$, rose bengal (3 mol\%), DMF $(0.9 \mathrm{~mL})$ $\mathrm{H}_{2} \mathrm{O}(0.1 \mathrm{~mL})$, reaction time $45 \mathrm{~h}$.

Rose bengal for photocatalytic oxidation reaction of different substrates and different conditions are well known, but rarely reported to their direct use in synthetic strategies. The second concept was the synthesis of 1,3-dihydro-3-(phenylimino)-2H-indol-2-one (102b) in a fast and efficient way beginning from indole (Scheme 30). The compound is known for its anti-convulsant and anti-inflammatory effects and currently, well established synthetic strategies are missing for this compound. ${ }^{[135]}$ Based on the reported reactivity of isatin with aniline in DMF, the hypothesis was that the reaction could be performed by oxygenating the indole and using the generated isatin directly in the reaction mixture to react with the aniline. Initial attempts to perform the reaction 
in a one pot manner failed. It was assumed that a similar inhibiting effect of amines on the photocatalytic system was occurring through the presence of aniline. After changing the reaction concept to a one pot multiple step approach, the target structure could be synthesized with a $92 \%$ yield. The photocatalytic reaction was first performed till sufficient isatin was formed and afterwards the aniline was added to the reaction mixture. After a further $24 \mathrm{~h}$ hours reaction time, the desired product could be obtained after purification. No purification in between was necessary and the rose bengal photocatalyst was proven not to be a hindrance for the further synthetic protocol.<smiles>c1ccc2[nH]ccc2c1</smiles><smiles>CCCCC</smiles>

$\mathrm{O}_{2}$-Balloon

Rose bengal DMF (0.9 mL) $\mathrm{H}_{2} \mathrm{O}(0.1 \mathrm{~mL})$ blue LED<smiles>Clc1ccc2[nH]ccc2c1</smiles><smiles>C1CCCCC1</smiles><smiles>O=C1Nc2ccccc2C1=O</smiles>
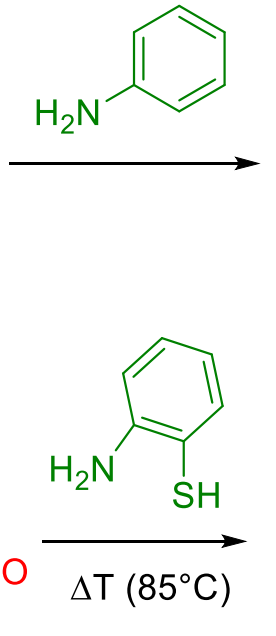<smiles>O=C1Nc2ccccc2/C1=N/c1ccccc1</smiles>

102b: $92 \%$ (anti-convulsant anti-inflammatory)<smiles>[R6][R6]#[R16]</smiles>

Scheme 30: Utilization of the oxygenation protocol for the generation of bio active compounds in a one-pot two step procedure. Reaction conditions 7-azaindole $(0.25 \mathrm{mmol})$, rose bengal $(3 \mathrm{~mol} \%)$, DMF $(0.9 \mathrm{~mL}) \mathrm{H} 2 \mathrm{O}(0.1 \mathrm{~mL})$, addition of corresponding benzyl amine $(0.25 \mathrm{mmol})$.

To prove the synthetic usability of the oxygenation protocol, the synthesis of spiro compounds like 5'-chloro-spiro[benzothiazole-2(3H),3'-[3H]indol]-2'(1'H)-one (103b) was attempted, beginning from 5-chloroindole (Scheme 30). This compound is known for anti-cancer activity. ${ }^{[136]}$ A one-pot two-step concept had to be applied to form the spiro compound. The additive was dissolved in the same solvent mixture and then was added via syringe. Increasing the time of the photocatalytic step and increasing the temperature of the second step to $85^{\circ} \mathrm{C}$, was sufficient to form the product with a $72 \%$ yield isolated. The obtained 102b was then used for the synthesis of trisindoline (104b), which is an anti-viral agent, with a $74 \%$ yield (Scheme 31). ${ }^{[137]}$ This result did not utilize directly the photocatalytic system but showed the possibilities of the 
oxygenation protocol in a multi-step synthetic protocol for the synthesis of drug molecules.

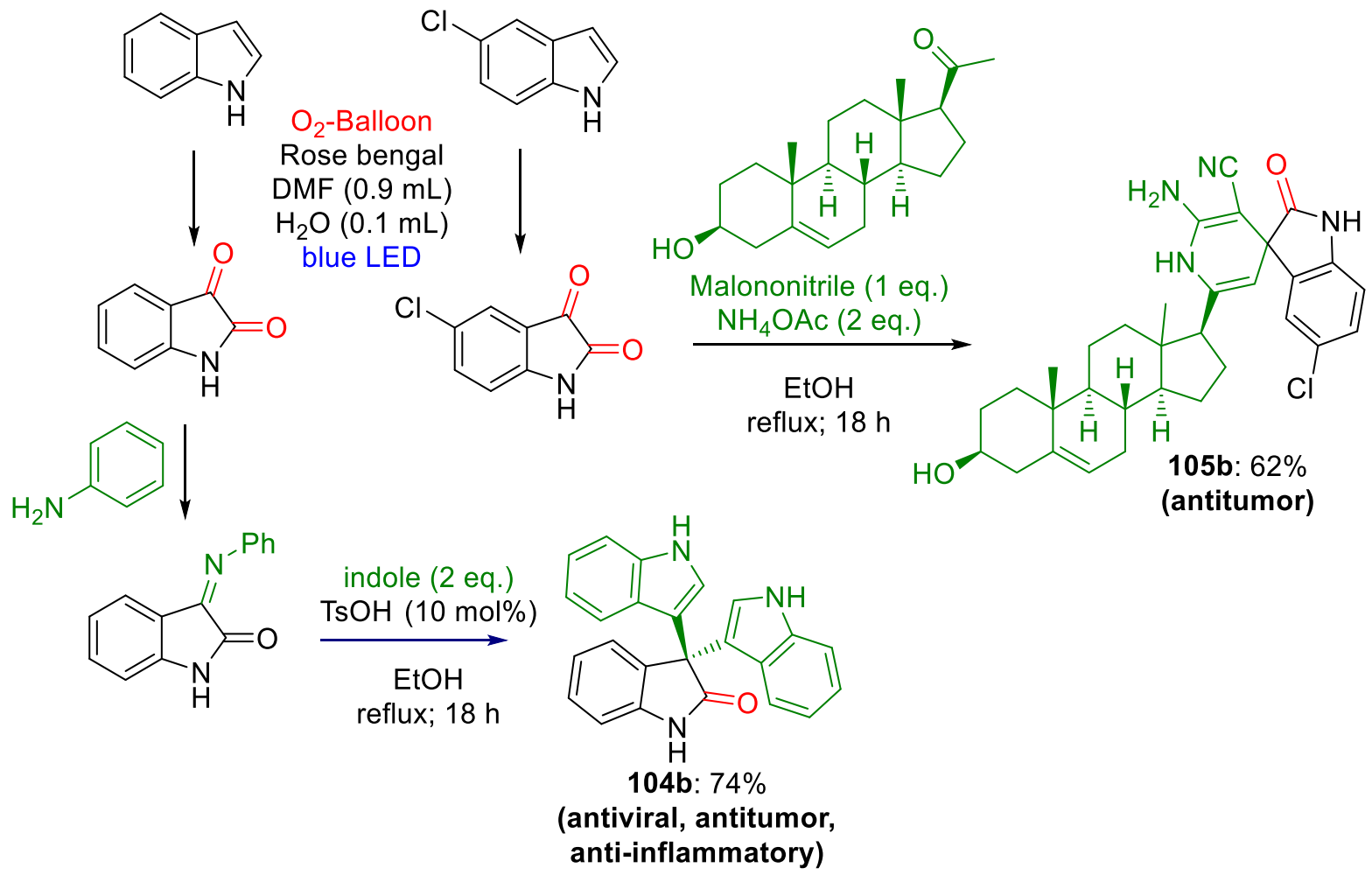

Scheme 31: Utilization of the oxygenation protocol for the total synthesis of complex drug molecules.

An alternative concept for the oxygenation and utilization of 5-chloroisatin (62a) from 5 -chloroindole (62b) was conceived. The oxygenation was repeated and afterwards the formed 5-chloroisatin (62b) was not isolated, but only an aqueous workup with lithium chloride followed by extraction with ethyl acetate was performed and the organic phase was removed under low pressure. The dried reaction mixture was directly used for a multicomponent one-pot reaction to synthesize 2'-Amino-5-chloro-

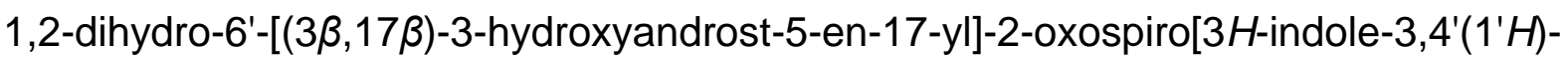
pyridine]-3'-carbonitrile (Scheme 16, 105b). This compound is a newly designed artificial steroid with high anti-cancer activity. ${ }^{[138]}$ The complexity to synthesize a highly steroidal drug in a complete transition metal-free approach was appealing. The high amount of side products from the reaction partners created a highly complex mixture for purification. Utilization of preparative HPLC was used to separate the compounds of the mixture and the desired $105 \mathrm{~b}$ was obtained with a $62 \%$ yield. 


\subsubsection{Mechanistic InVestigations and Proposed Mechanism}

Table 8: Control and quenching experiments for the oxygenation of $1 \mathrm{H}$ indole to isatin.

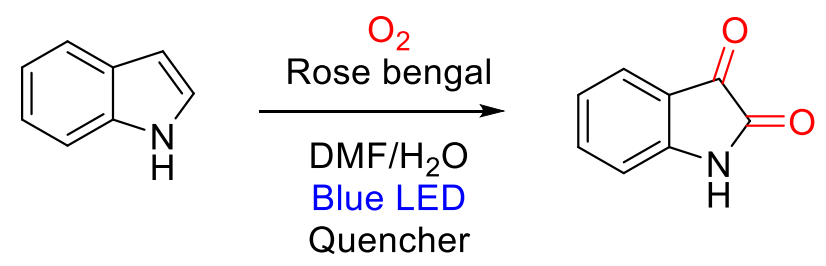

\begin{tabular}{cccc}
\hline Entry & Conditions / $^{\text {changes }}{ }^{[\mathrm{a}]}$ & Quencher $^{[\mathrm{b}]}$ & Yield $/ \%$ \\
\hline $\mathbf{1}$ & N2-atmosphere $_{2}$ & --- & 0 \\
$\mathbf{2}$ & No light & -- & 0 \\
$\mathbf{3}$ & No water & --- & 28 \\
$\mathbf{4}$ & No catalyst & -- & $32 / 33^{*}$ \\
$\mathbf{5}$ & STD & BHT & 35 \\
$\mathbf{6}$ & STD & TEMPO & 23 \\
$\mathbf{7}$ & STD & tert-butanol & 87 \\
$\mathbf{8}$ & STD & CuCl 2 & 29 \\
$\mathbf{9}$ & STD & Benzoquinone & 32 \\
$\mathbf{1 0}$ & STD & Sodium azide & 0 \\
$\mathbf{1 1}$ & STD & tert-butanol[c] & 21
\end{tabular}

[a] changes to standard conditions (STD); [b] 1.0 equivalent quencher; *) yield after $72 \mathrm{~h}$. [c] 7 equivalents used (mechanistic part). Yield was determined by GC with biphenyl as the internal standard.

After the investigation of the substrate scope and utilization of the oxygenation protocol, the mechanism was investigated. The first aspect of the mechanistic investigations was the control and quenching experiments (Table 8). Replacing the oxygen atmosphere with inert nitrogen atmosphere (entry 1 ) resulted in no conversion at all, indicating a reaction with oxygen as its primary source for the oxygenation. Covering the flask by aluminum foil to achieve complete darkness in the reaction vessel (entry 2) resulted in no conversion at all. Reaction without water (entry 3) was still possible, but with substantially lower yields, indicating that water played an important role in the reaction. The reaction yielded product even without the catalyst 
with around a 33\% yield at full conversion (entry 4). An increase of the reaction time resulted in nearly the same result (entry $4^{\star}$, change $1 \%$ ). Since indole exhibits a low absorption band at $435 \mathrm{~nm}$, it was assumed that it can act as its own photosensitizer. Based on the fact that only one third of the product was formed at full conversion, indicated that this process sacrificed two indole molecules in the process to photooxidize itself. The possibility of high yields utilizing rose bengal could explain that the absorption band of rose bengal exceeded that of indole efficiently, shielding it from photo excitation. Utilizing different quenchers showed conflicting results. The use of BHT (entry 5) and TEMPO (entry 6) as radical quenchers, resulted in very low yields of 35 and $23 \%$. The result indicated a possible radical interaction in the mechanism but was not sufficiently clear. The use of tert-butanol (entry 7) resulted in no significant change and showed no hydroxy radicals were essential for the mechanism. The effect of the electron quencher copper chloride (entry 8) was revealed, like the radical quencher, a partially quenching effect was observed. Those results were further confirmed by the use of benzoquinone (entry 9) as a superoxide quencher which resulted in similar low yields. Based on these observations, a radical pathway via SET seemed to be involved, but not the central part of the reaction. The use of sodium azide (entry 10) resulted in a full inhibition of the reaction indicated the presence of singlet oxygen and an ET as central step of the reaction. The results indicated that a SET and ET could be involved in the mechanism, giving cause of concern due to a possible complex mechanism. The use of seven equivalents of tert-butanol showed a decrease in reaction yield. 
To have further insight into a possible mechanism, possible byproducts of the reaction were investigated. GC-MS indicated no formation of higher mass byproducts and no solvent interaction adducts. Smaller molecule byproducts were assumed and the primary candidate was hydrogen peroxide. After the reaction, an aqueous workup was performed, and the separated phases were examined for hydrogen peroxide. Two qualitative tests were chosen for this. First was the "glowing splint tests". The addition of $\mathrm{MnO}_{2}$ catalyzed the degradation of hydrogen peroxide and thereby releasing oxygen gas which could be detected by a glowing wooden piece. The aqueous phase reacted positive for this test. As the second test, the "luminol test" was performed. High concentrations of peroxide in the aqueous phase were confirmed. For both cases the organic phases were examined with negative results. This was assumed to be sufficient proof for the formation of hydrogen peroxide as the byproduct.

The results were investigated further by Stern-Volmer absorption emission quenching experiments. Firstly, the 3D absorption-emission spectra for rose bengal (Figure 31) and indole (Figure 32) were recorded for detailed analysis of the usable parameters. For rose bengal, high absorption and emission values could be found at visible light conditions with an excitation maximum at $565 \mathrm{~nm}$ and emission maximum at $575 \mathrm{~nm}$.
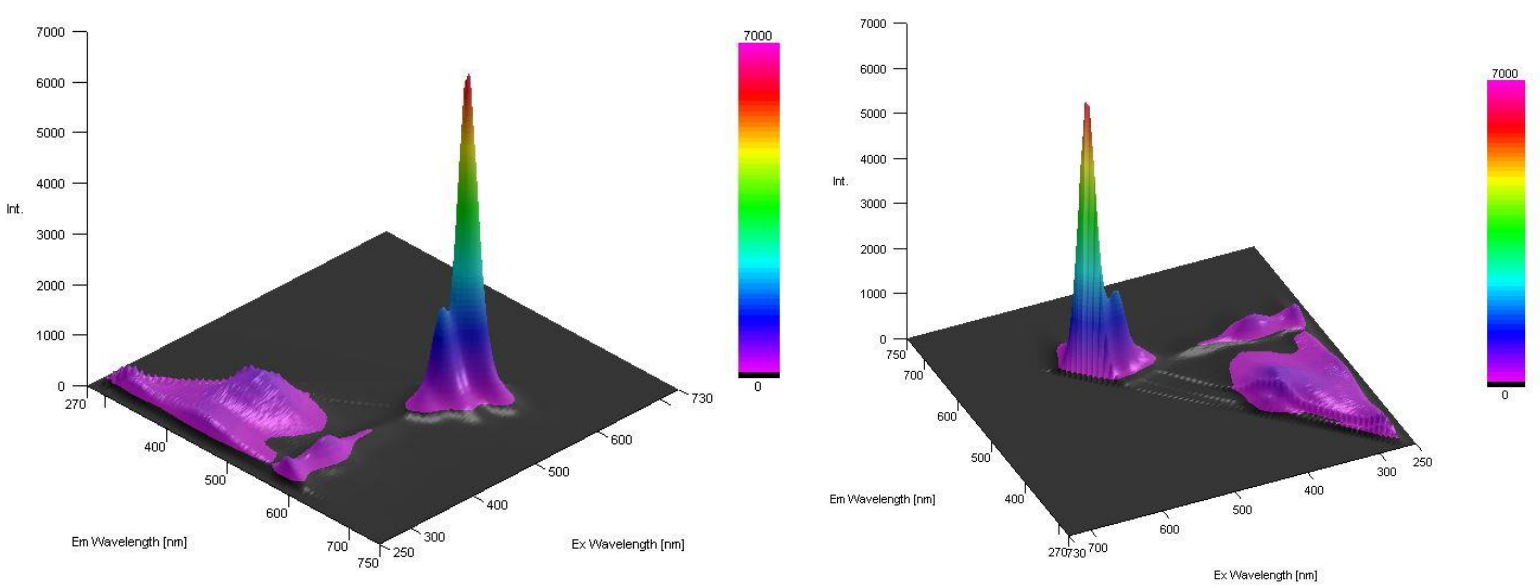

Figure 31: 3D-absorption-emission spectrum of rose bengal in DMF. 
As the initial control experiments indicated the absorption emission spectrum of $1 \mathrm{H}$ indole showed in DMF an absorption band between 400 and $500 \mathrm{~nm}$ (Figure 32). The absorption at this wavelength was not intense anymore, compared to the classically reported UV-absorption bands of indole. The comparison of absorptions of indole and rose bengal and $1 \mathrm{H}$-indole at the same concentrations, indicated a difference in absorption close to the factor of 1000 higher for rose bengal.

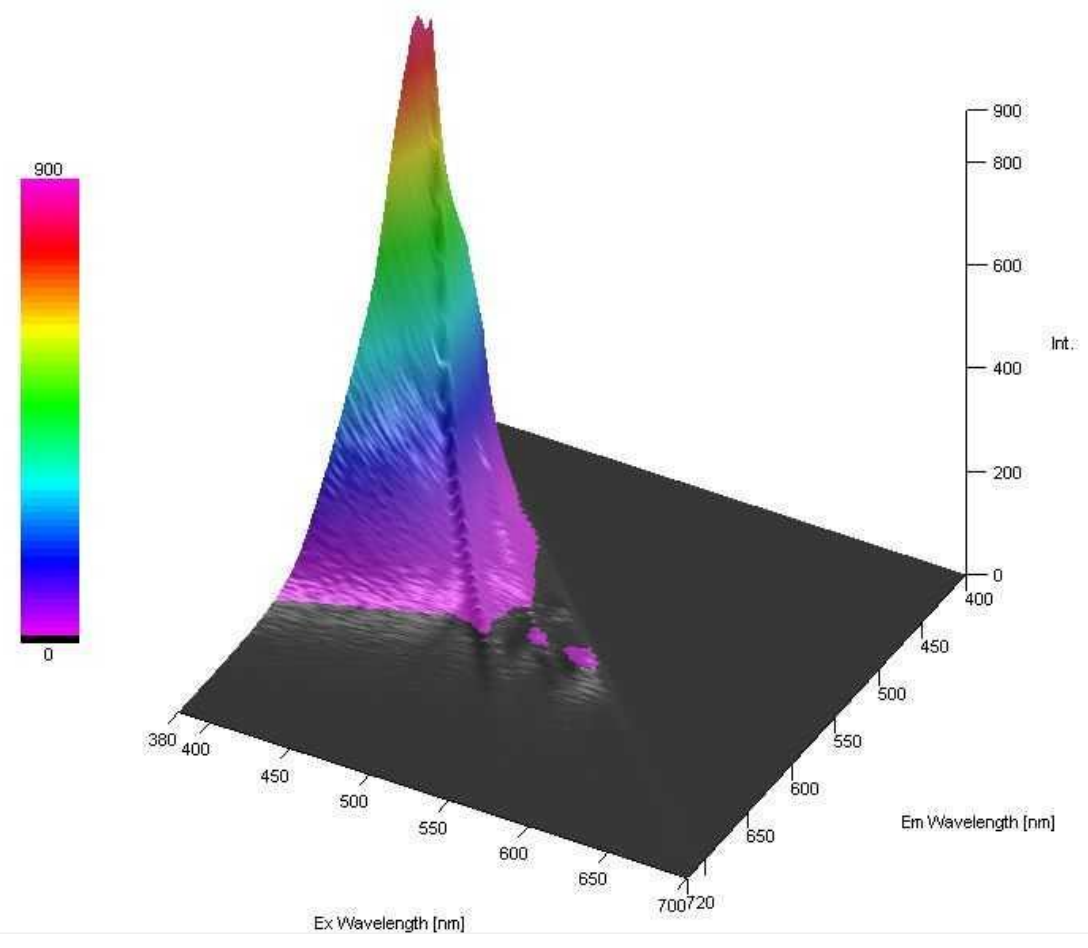

Figure 32: 3D-absorption-emission spectrum of $1 \mathrm{H}$-indole in DMF.

Performed Stern-Volmer-pots (Figure 33) were executed for indole, oxygen and water at different concentrations. In the case of indole, a quenching effect could be observed. The oxygen and water concentrations had quenching effects on the catalyst. The quenching effect of different water contents resulted in a nonlinear behavior. This observation was based on the large quantities of water added to the solvent, resulted in a solvent mixture and therefore induced a solvent based band shift of the excitation maximum. Moreover, it is assumed that high amounts of water changed the solubility of the reaction mixture and therefore also changed concentrations slightly. Overall, a quenching effect of water can be assumed by this result. 

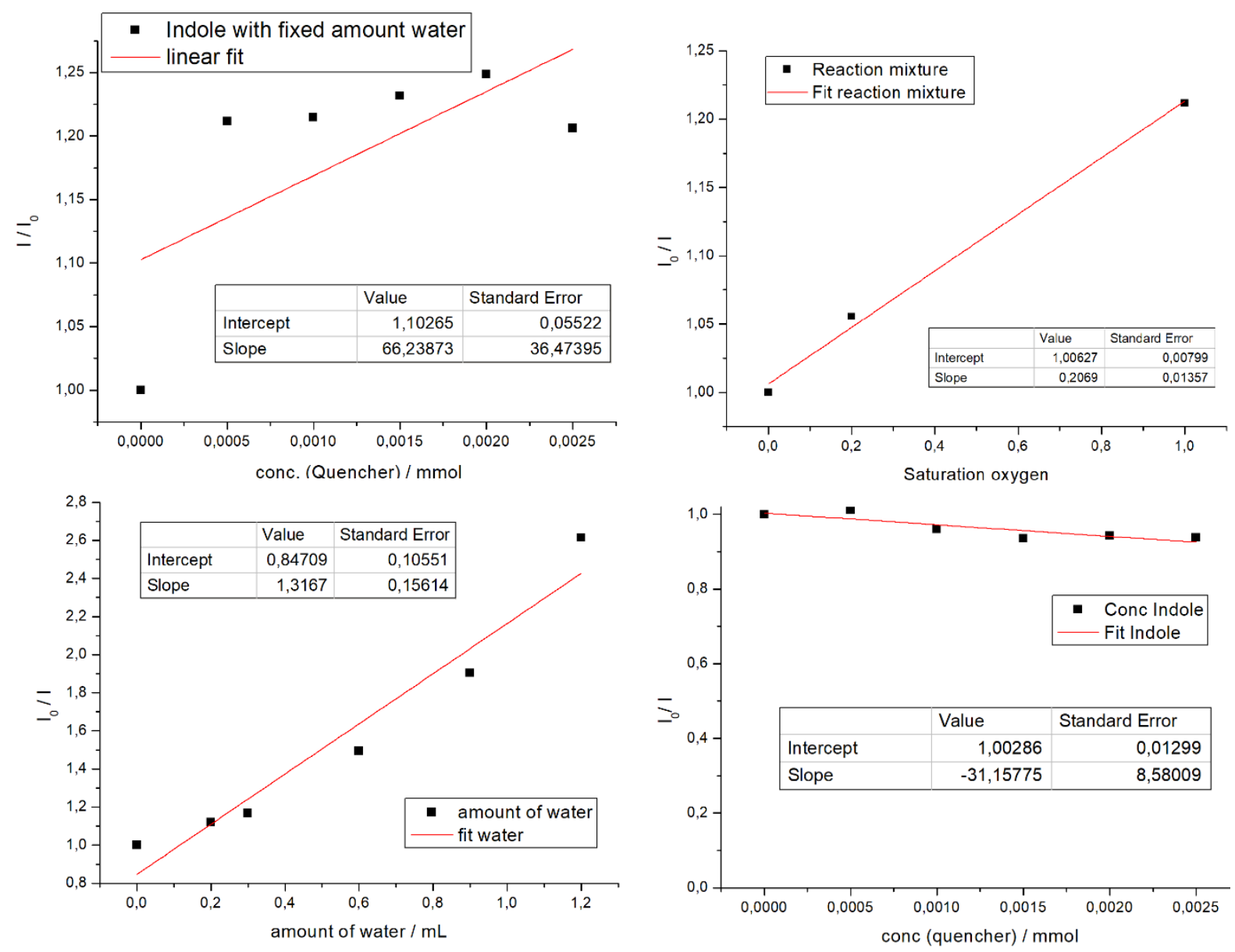

Figure 33: Stern-Volmer-plots for $1 \mathrm{H}$-indole, oxygen and water at different concentrations.

To gain more insight into the reaction mechanism, labeling experiments were performed to observe from where the oxygen for the reaction was originating (Scheme 32). Three different labeling experiments were performed Firstly utilizing ${ }^{18} \mathrm{O}_{2}$-atmosphere and $\mathrm{H}_{2} \mathrm{O}$, secondly regular $\mathrm{O}_{2}$-atmosphere and labeled $\mathrm{H}_{2}{ }^{18} \mathrm{O}$, thirdly combined labeling with ${ }^{18} \mathrm{O}_{2}$-atmosphere and $\mathrm{H}_{2}{ }^{18} \mathrm{O}$. The formed products with different isotope distribution were investigated by ESI-HR-MS. Due to the close structural similarity, their integrals could be compared. Using only one labeled possible oxygen source for the oxygenation resulted in different ratios of labeled and unlabeled products. The use of ${ }^{18} \mathrm{O}_{2}$-atmosphere had mono-labelled product as its main product, but also considerable amount of unlabeled product and low amounts of double labeled product. The use of $\mathrm{O}_{2}$-atmosphere and labeled $\mathrm{H}_{2}{ }^{18} \mathrm{O}$ resulted in a different distribution of the labelled products. The main product was the unlabeled isatin, with considerable amount of mono-labeled and low amounts of double labeled product. 
Utilization of both possible sources labeled resulted in solely double labeled isatin. The results indicated that oxygen and water were both sources of oxygen for the oxygenation of indole to isatin. The central source of the oxygen was the $\mathrm{O}_{2}$ atmosphere with an additional contribution of the oxygen from water.

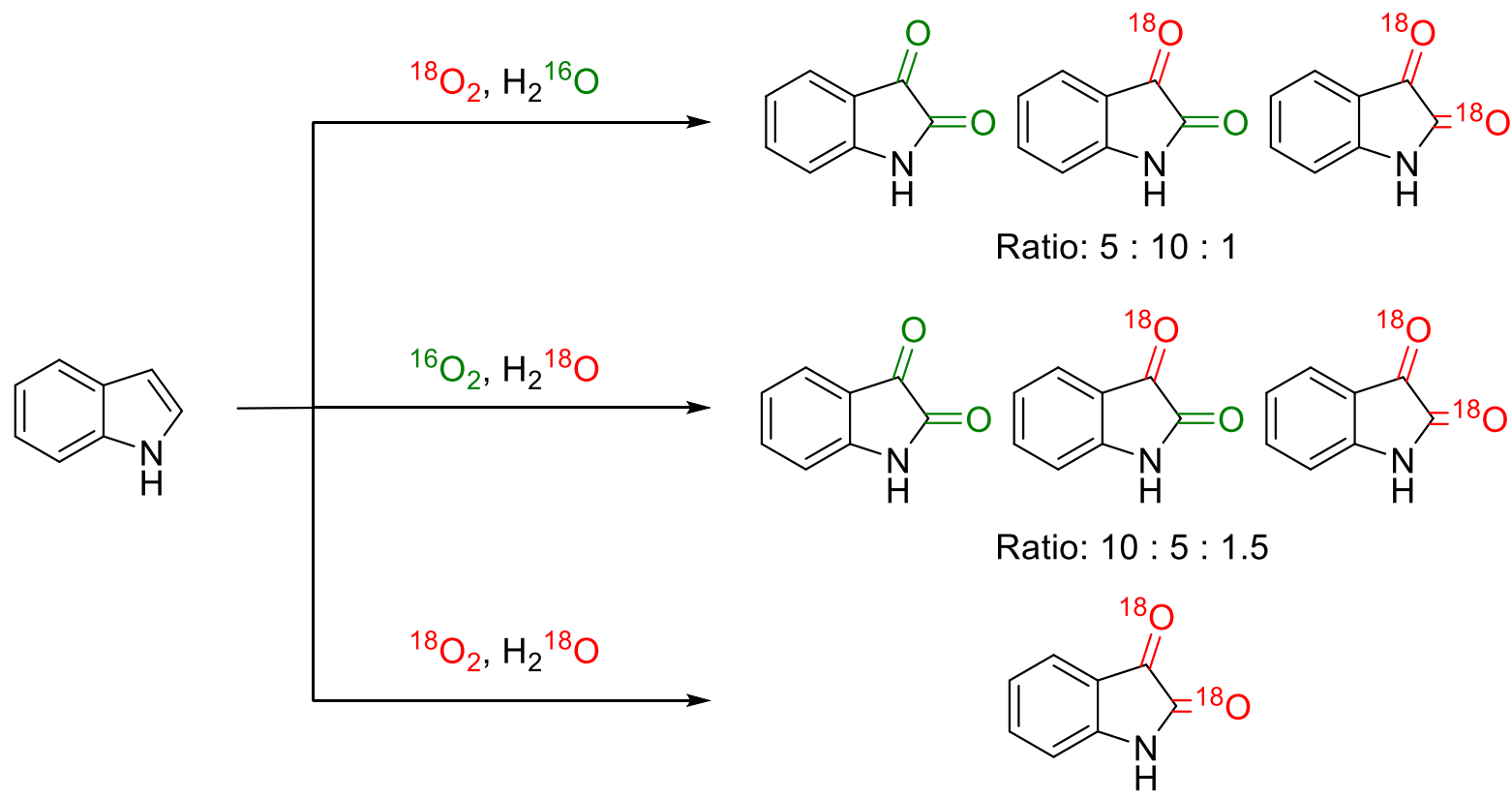

Scheme 32: Results of the labeling experiments with observed ratios in the ESI-HRMS.

The results of the mechanistic investigations were sufficient to postulate a plausible mechanism (Scheme 33). After due consideration, a two-step involving two different photochemical interactions were considered based on the observed quenching experiments and that showed that an ET and SET reactions were involved. The photochemical oxygenation reaction began with rose bengal being excited by a photon (Cycle A). The excited photosensitizers transferred its energy via an energy transfer (ET) to atmospheric oxygen forming highly reactive singlet oxygen. The singlet oxygen interacted with the indole and formed a peroxo species (II). The peroxo species can undergo a second reaction. An excited rose bengal photocatalyst, from a second photoactivation, interacted with the peroxo species (II) by single electron transfer to form a radical cation (III) and a rose bengal radical anion (Cycle $\mathbf{B}$ ). The reactive intermediate III reacted further by disaffiliating a hydroxy radical. The hydroxy radical interacted with the present water content and interchanged protons with the water. 


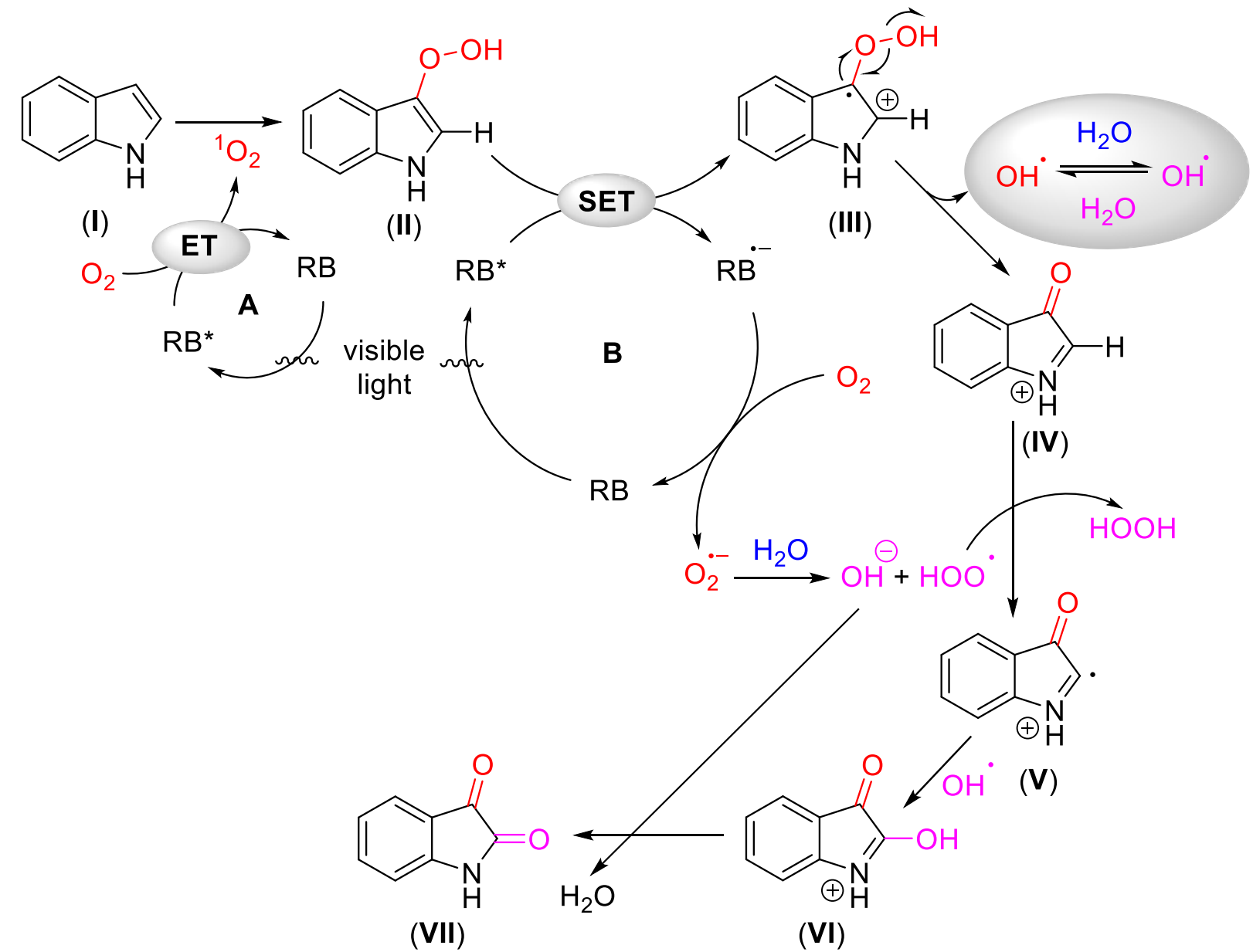

Scheme 33: Proposed mechanism for the oxygenation of indoles based on experimental and literature research.

The scrambling with the water was assumed to be the reason behind the complex ratios of the labeling experiments. The photocatalyst radical anion interacted in the meantime with atmospheric oxygen and formed the superoxide radical anion. This was known to interact with water to form hydrogen peroxide radicals and hydroxide anions. The hydrogen peroxide radical abstracted a proton from intermediate IV forming the monooxygenated radical intermediate $\mathbf{V}$. Recombination with the earlier formed hydroxide radical formed the cation intermediate VI. The remaining hydroxy anion postulated by the reaction between superoxide radical anions and water was responsible for a deprotonation of the intermediate cation $\mathbf{V I}$ forming the final product isatin (VII). In total, the mechanism resulted in the formation of one isatin molecule and one hydrogen peroxide molecule as the sole products. Interactions of the formed 
reactive intermediate species, formed from atmospheric oxygen with water, resulted in scrambling of the atoms and exchanged with water and hydroxy radicals explaining the ratios of the labeled products during labeling experiments. An additional contribution of the formed hydrogen peroxide to activate the starting material indole, initiating a non-photochemical activation, was possible. The hypothesis of the mechanism was examined by a further quenching experiment. The use of tert-butanol had no significant impact on the reaction yield utilizing one equivalent. Increasing the content of tert-butanol to 7 equivalents (Table 8, entry 11) showed significant impact on the formed reaction yield, which was consistent with the postulated reaction mechanism.

For the oxygenative cleavage reaction, which occurred for the derivatives with substitution in position 2 and 3 ; the sterically hinderance of the substitutions was responsible for a different coordination of the attacking reactive oxygen. A side on coordination would be in this case preferable, stabilizing a possible intermediate which would make a cleavage reaction possibly desirable.

The final consideration to the mechanism was a comparison to the work of Jiang et al. ${ }^{104]}$ Their reported catalytic system is quite similar and additionally the extent of this protocol showed for several different possible reactions pathway. Contrary to the present postulated mechanism, they postulated a mechanism including only a SET reaction with their photocatalyst and an uninvestigated influence of the present water in their system. The mechanistic investigations were for both cases the same, only this work and the published results extended the mechanistic investigations further and all experiments concluded a further complexity of the system which cannot be simply explained by a single photocatalytic step. 


\subsubsection{ConCluding REMARKS AND OUTLOOK OF THE CHAPTER}

Based on the results of the selective oxidation of alcohols, a new methodology for the oxygenation of indoles and pyrroles was developed. This methodology utilized rose bengal as the photocatalyst. Through fine tuning of the solvent conditions this methodology was used for a broad spectrum of different heterocycles, based on unprotected, protected indoles and additionally pyrroles. The substitution pattern of different positions resulted in the targeted synthesis of isatin, ortho-formyl/acyl anilide and maleimide derivatives. In total of 30 different structures were obtained in good to excellent yields. The methodology was examined, and a good functional group tolerance could be demonstrated and the limits of it could be defined. The examination showed additionally the influence of different substitution pattern and their influence on the reactivity of the structure. For carboxylic substitutions in position 2 and 3, effective decarboxylation reactions with followed oxygenation were investigated and described, which could extend the future applications of this methodology.

Derived from these results of this investigation, the methodology was used to synthesize four different bioactive structures and a complex steroid structure with a high step and atom efficiency. The investigation of the cleavage reaction of substitutions in position 2 and 3 led to successful synthesis of a prominent pesticide by the Das group to further expand the applications of this methodology.

A detailed mechanistic investigation showed the role of oxygen, water in this reaction and provided a plausible reaction mechanism. Notably the results of the mechanistic experiments led to a mechanism involving a reaction based on an energy transfer (ET) and a singlet electron transfer (SET) reaction step, which were both catalyzed by the same photocatalyst.

Part of the results were published in Chemistry a European Journal. ${ }^{[139]}$ 


\subsection{OXIDATION COMBINED WITH 1,2-ACYL MIGRATION OF SECONDARY ENAMINO KETONES}

After establishing two synthetic methods for photocatalytic oxidations interest arose in the transformation of secondary enamino ketones. The aim of the investigations was set to increase the performance of a photocatalytic system and to find an organophotocatalyst to replace the transition metal catalysts of prior works on this topic. ${ }^{122-}$ 123] It was reported that the use of singlet oxygen in photocatalytic systems could achieve new reactivities for secondary enamino ketones. The new reactivity could provide new synthetic methods. The reported reaction showed a three-step reaction based on the activation of atmospheric oxygen reacting with the substrate, initiating a 1,2-acyl shift followed by an esterification of the reactive intermediate by utilizing the solvent as a reaction partner for the esterification.

\subsubsection{Synthesis OF StARTING MATERIALS AND OptimizATION STUdiES}

Most of suitable secondary enamine ketones for this reaction were not commercially available. After different concepts for the synthesis of the required starting materials, several suitable ways were found for the synthesis of different secondary enamino ketones to be utilized for the targeted reaction. Initially amine precursor, a diketone precursor and cyanuric chloride were added to an algae mortar. ${ }^{[140]}$ Upon grinding the reaction was initiated. The concept was excellent for syntheses of model secondary enamino ketone. The reaction conditions were changed to achieve better result in the synthesis of further starting materials. Stirring at room temperature in ethanol achieved the same result. The use of cyanuric chloride seemed to be useful only in the case of grinding. The reactivity of amines with high basicity showed low performance. After acidifying the mixture and applying reflux conditions, the reaction performed fast and efficient, for all attempted substrate combinations. 
The initial concept was to find a transition metal-free photocatalyst for the transformation of $(E)$-4-(phenylamino)pent-3-en-2-one to 3-Oxo- $N$-phenyl-isovaline methyl ester in one step. First screening via GC-MS analysis indicated high conversion and resulting isovaline methyl ester for graphitic carbon nitride, acriflavine and eosin $Y$ (Table 9). Methylene blue and fluorenone showed no reactivity. Further attempts to isolate the product were not successful. A variety of 24 different conditions, different solvents, higher light intensity and reaction times reconfirmed the first observations. All the reactions were measured by GC-FID utilizing $n$-dodecane as an internal standard. A united combination of a large-scale reaction and twelve further reactions yielded $184.2 \mathrm{mg} 3-\mathrm{Oxo}-\mathrm{N}$-phenyl-isovaline methyl ester. The obtained product was used to perform a GC-FID calibration and compared former results with the thereby available calibration of the GC-FID. The results confirmed that almost full conversion was achieved, but mostly no product was formed. Several attempts were conducted to identify possible byproducts and could confirm that different cleavage products of the formed product could be observed, namely aniline, $N$-methylaniline and a third unidentified product. A too high oxidation potential of the catalyst was assumed to be the reason for the low product yield, which led to high amounts of cleavage and degradation products. An increase in irradiation power of the LED source was attempted and could increase the obtained yields, in the case of $g-\mathrm{C}_{3} \mathrm{~N}_{4}$ catalyst, but for the other photocatalysts only insignificant changes were observed. A variation of different other photocatalysts were investigated. Among those were Sudan Black, Fuchsine, carbon nano dots, tetra-p-tolyl-porphyrin (Table 9). Only porphyrin showed activity, which was also the highest activity among the examined photocatalysts. Based on the results, different porphyrin compounds and commercially available natural chlorophyll from TCI were examined. Under the chosen conditions the natural chlorophyll showed almost full conversion with selective formation of the desired product. Attempts to add the central metal ion as an addition to the artificial porphyrins had no effect on their catalytic performance. The present natural chlorophyll contained mostly remaining membrane residue of leaves, which remained from the purification process. Extraction of the chlorophyll by methanol and filtration of the mostly unsolvable remains, showed a minor reduction in catalytic performance (84\% yield). 
Table 9: Selected entries for the optimization table of the multi-step oxidation reaction of secondary enamino ketones.<smiles>CC(=O)/C=C(\C)Nc1ccccc1</smiles>

Air

Catalyst (5 mol\%)

Methanol

$18 \mathrm{~h}$

Blue LED<smiles>COC(=O)C(Nc1ccccc1)(C(C)=O)C(C)=O</smiles>

\begin{tabular}{cccc}
\hline Catalyst & Light intensity / \% & GC-MS notes & Yield / \% \\
\hline Commercial and & 25 & Full conversion & 4 \\
synthesized $g-\mathrm{C}_{3} \mathrm{~N}_{4}$ & & No reactivity & 0 \\
Fluorenone & 25 & No reactivity & 0 \\
Methylene blue & 25 & Partial conversion & $<2$ \\
Eosin Y & 25 & Partial conversion & $<2$ \\
Acriflavine & 25 & No reactivity & \\
Sudan black & 75 & No reactivity & \\
Fuchsine & 75 & No reactivity & 31 \\
Carbon nanodots & 75 & Partial conversion & \\
Tetra-p-tolyl- & 75 & & 32 \\
porphyrin & & Partial conversion & \\
Tetra-p-tolyl- & 75 & & \\
porphyrin + MgSO 4 & & Partial conversion & \\
Chlorophyll $6 \%$ & 75 & & \\
TCl[b] & &
\end{tabular}


Table 10: Optimization table for the chlorophyll catalyzed multi-step oxidation reaction of secondary enamino ketones.<smiles>CC(=O)/C=C(\C)Nc1ccccc1</smiles>
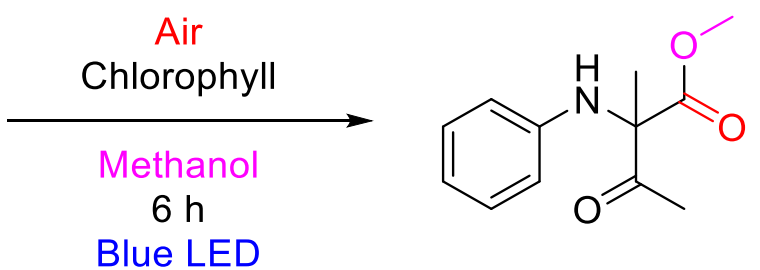

\begin{tabular}{ccccc}
\hline Entry & Catalyst loading / $\mathbf{~ o l} \%$ & Substrate / $\mathbf{~ m o l}$ & Solvent $/ \mathbf{~ m L}$ & Yield / \% \\
\hline $\mathbf{1}$ & 5 & 0.25 & 2.0 & 63 \\
$\mathbf{2}$ & 4 & 0.25 & 2.0 & 52 \\
$\mathbf{3}$ & 2 & 0.25 & 2.0 & 42 \\
$\mathbf{4}$ & 2.5 & 0.50 & 2.0 & 45 \\
$\mathbf{5}$ & 1.25 & 1.00 & 2.0 & 24 \\
$\mathbf{5}^{\star}$ & 1.25 & 1.00 & 2.0 & 64 \\
$\mathbf{6}$ & 5 & 0.25 & 1.5 & 59 \\
$\mathbf{7}$ & 5 & 0.25 & 2.5 & 56 \\
$\mathbf{8}$ & 5 & 0.25 & 3.0 & 68 \\
$\mathbf{9}$ & 5 & 0.25 & 3.5 & 92 \\
\hline
\end{tabular}

Different conditions were applied for the utilization of natural chlorophyll (Table 10) to attain faster reaction times and more favorable reaction conditions. A change in catalyst loading had only a non-linear effect on the catalytic performance and the substrate concentration also seemed to have no linear dependence. These results indicated that the presence of oxygen in the solvent was the primary limiting factor. Utilization of oxygen atmosphere was considered less favorable, therefore different amounts of solvent were applied, resulting in a $92 \%$ yield within 6 hours utilizing $3.5 \mathrm{~mL}$ of methanol. Based on the fact that the natural chlorophyll contains a mixture of chlorophyll $A$ and $B$ an absorption spectrum of the catalyst was recorded. Present LED-setups of diverse wave lengths were utilized to investigate the use of different wavelengths for the performance of the catalytic system (Figure 34). According to the absorption bands of the catalyst, the catalytic performance yielded similar values. The most prominent absorption band resided at $460 \mathrm{~nm}$ and a yield of $92 \%$ was obtained. The secondary absorption band resided at $675 \mathrm{~nm}$ and utilization of $680 \mathrm{~nm}$ red-LED resulted in a $68 \%$ yield, close to the relative height of the absorption band. The use 
of green-LED light at $560 \mathrm{~nm}$ resulted in $8 \%$ yield similar to the low absorption potential at this wavelength. The use of direct solar irradiation resulted in $98 \%$ yield, indicating a beneficial effect of using several different wavelengths. Upscaling the reaction could be achieved. For $5 \mathrm{mmol}$ scale of the reaction, $87 \%$ yield was obtained within $36 \mathrm{~h}$. $5 \mathrm{mmol}$ scale reaction exhibited full conversion with a $94 \%$ yield after $8 \mathrm{~h}$ of direct solar irradiation.

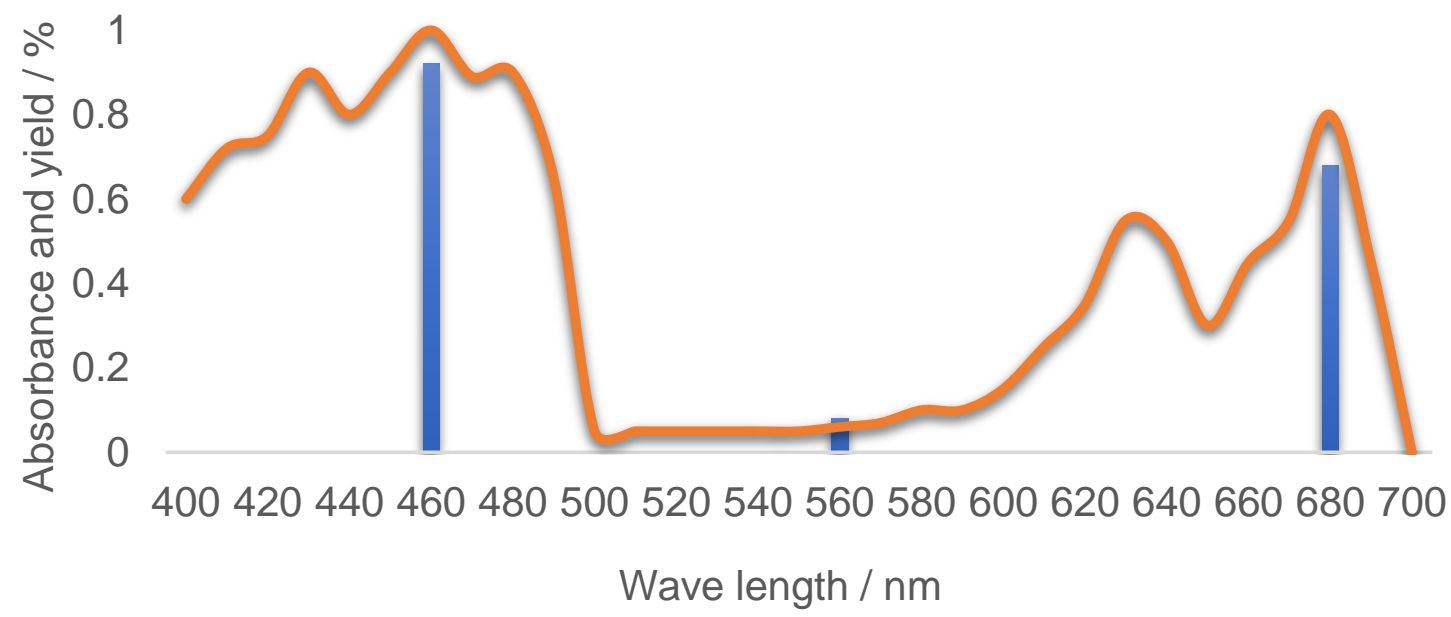

Figure 34: Wavelength dependent yields, overlaid with the absorption spectra of the chlorophyll catalyst in the reaction mixture.

The final part of the investigations was the possibility to combine the starting material synthesis with the actual reaction. Most of the substrates were synthesized in ethanol, by stirring respective aniline and diketone at room temperature, or under refluxing conditions. In some cases, the addition of a small amount of TFA increased the conversion rates. The simplicity of the starting material synthesis gave rise to the idea that both reactions could be combined. Initial experiments showed that the final product can be formed by combining the respective precursors to the photocatalytic reaction mixture. Reaction scale of $1.0 \mathrm{mmol}$ was chosen, due to the volatility of diketone and the possible risk of evaporation. It was possible to achieve $5 \%$ yield of the final product within 8 hours. An increase in reaction time to 48 hours increased the yield of the final product to $39 \%$ and mostly transformation of aniline to the secondary enamino ketone was observed. The photocatalytic system seemed to have a decreasing effect on the synthesis of the starting material. The lower yields, achieved by combination of both the reaction steps into one, seemed inefficient. A sequential 
approach was thereby the most efficient. The aniline and diketone were stirred in the alcohol and then the photocatalyst in alcohol solvent was added afterwards and was irradiated. After 2 hours of stirring and 8 hours of irradiation, the reaction resulted in a $72 \%$ yield. An addition of one up to two drops of TFA did not inhibit the second reaction step, nor accelerated the first.

\subsubsection{SCOPE OF SUBSTRATES}

After optimization a substrate scope was investigated. Considering that the utilized solvent was also a reaction partner, it was examined if the reaction would perform with stochiometric amount of methanol in a different solvent. Lower yields could be observed (yield $31 \%$ ). Changing the solvent to other alcohols resulted in the respective product ester of the utilized solvent (Scheme 34). Performing the reaction without alcohol to obtain a possible intermediate were not successful with DMSO as a solvent.

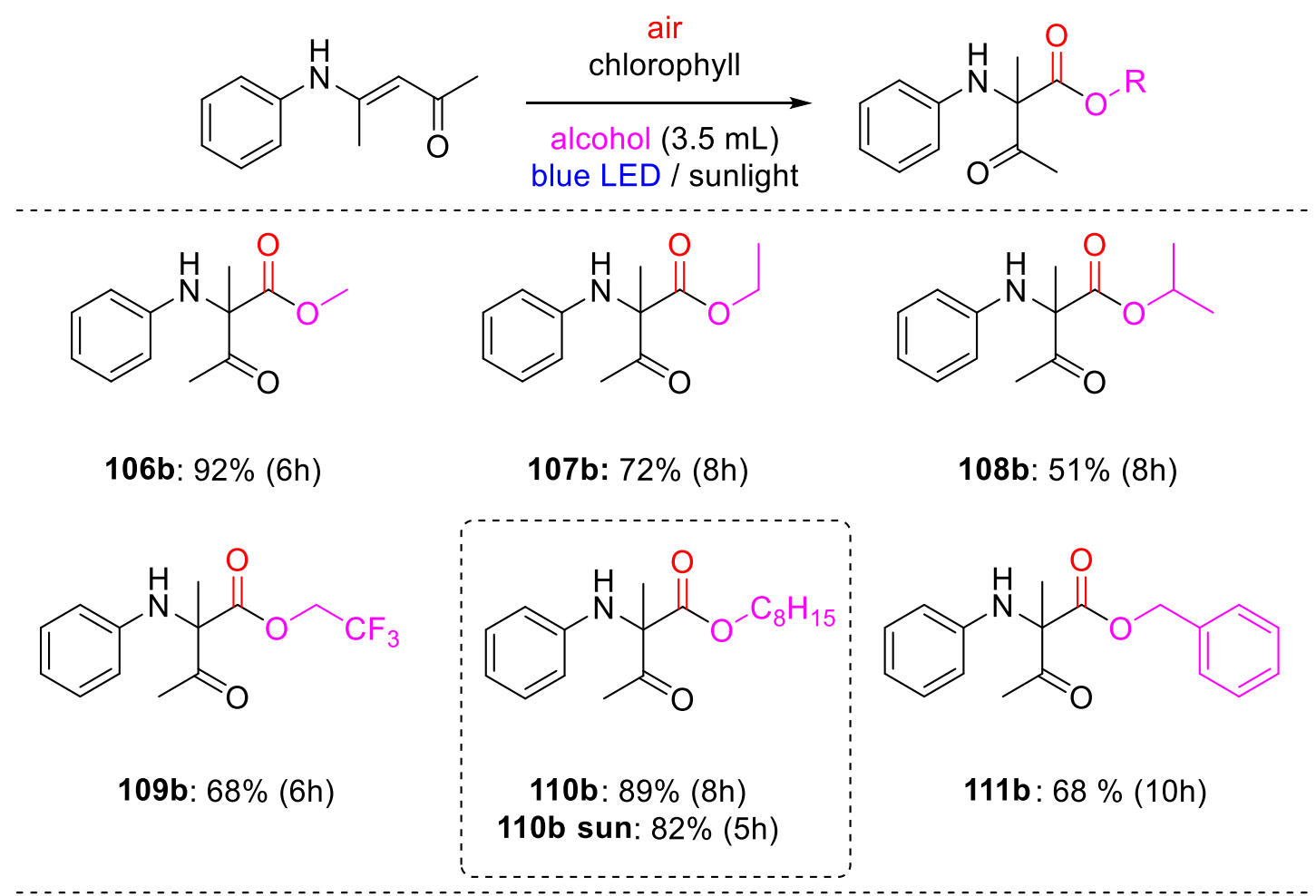

Scheme 34: Substrate scope based on different solvents. Reaction conditions: substrate $(0.25 \mathrm{mmol})$, catalyst $(0.3 \mathrm{~mol} \%)$, respective alcohol $(3.5 \mathrm{~mL})$, excitation at $460 \mathrm{~nm}$ wavelength (blue LED). 
Utilization of ethanol (107b) as the solvent resulted in a slower reaction performance. This effect was even more pronounced with the use of iso-propanol (108b). A possible influence of the viscosity and polarity of the solvent could be assumed. To obtain higher yields, the reaction time was increased. A less pronounced influence was observed for tri-fluoroethanol (109b), which could yield sufficient product within sixhour reaction time. In the case of octanol (110b), the highest viscosity was present but resulted in excellent yields with shortly increased reaction time. For the use of benzylic alcohol as the solvent/reaction partner, considerably lower yields were obtained. This was due to additional oxidation of the solvent forming benzaldehyde and carboxylic acid during the reaction. The use of different alcohols present was examined utilizing methanol, ethanol and benzyl alcohol. Analyzing the results via GC-MS showed that all three products were formed. A precise distribution was not possible.

After utilizing different solvent/esterification agents, the substitution pattern of the starting material was investigated. First different substitutions at the nitrogen function were examined. Scheme 35 shows the successful substrates for this reaction and the tolerated aliphatic compounds (112b-114b). In the case of 123b, having two ethyl in both ortho position, only degradation products were observed. Steric hindrance in the ortho position was assumed to be a limiting factor in the reaction scope. Substrate $114 \mathrm{~b}$ and 120b were repeated under solar conditions and resulted in higher yields at shorter times. 


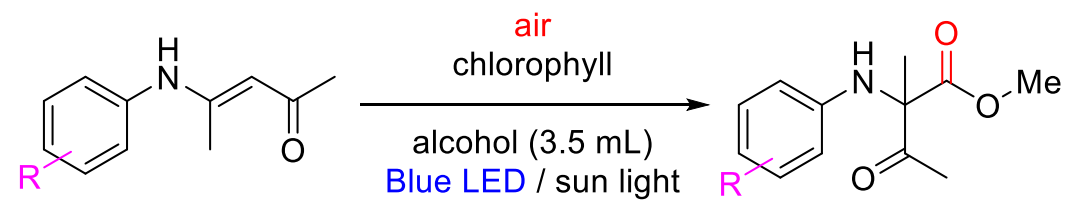<smiles>CCc1ccc(NC(C)(C(C)=O)C(=O)OC)cc1</smiles>

112b: $77 \%$ (8 h)<smiles>COC(=O)C(Nc1ccc(C(C)(C)C)cc1)(C(C)=O)C(C)=O</smiles>

113b: $69 \%(8 h)$<smiles>COC(=O)C(C)(Nc1c(C)cccc1C)C(C)=O</smiles>

114b: $65 \%(6 \mathrm{~h})$ $114 b$ sun: $82 \%(5 h)$<smiles>COC(=O)C(C)(Nc1ccccc1F)C(C)=O</smiles>

118b: $78 \%(8 h)$<smiles>COC(=O)C(C)(Nc1cc(OC)c(OC)c(OC)c1)C(C)=O</smiles>

115b: $82 \%(12 h)$<smiles>COC(=O)C(C)(Nc1ccc(Cl)c(C)c1)C(C)=O</smiles>

119b: $41 \%(7 \mathrm{~h})$

$116 b: 48 \%(12 \mathrm{~h})$

117b: $64 \%(6 h)$<smiles>COC(=O)C(C)(NCc1ccccc1)C(C)=O</smiles>

121b: $71 \%(7 \mathrm{~h})$<smiles>CCc1cccc(CC)c1NC(C)(C(C)=O)C(=O)OC</smiles>

122b: active mostly side products

Scheme 35: Successful substrate scope based on different substitutions. Reaction conditions: substrate $(0.25 \mathrm{mmol})$, catalyst $(0.3 \mathrm{~mol} \%)$, respective alcohol $(3.5 \mathrm{~mL})$, excitation at $460 \mathrm{~nm}$ wavelength (blue LED).

In the case of trimethoxy substitution (115b) at the aromatic ring a longer reaction time had to be utilized but the product was formed without much complication. Different halogens (116b+, 117b, 118b) alone and in combination with alkyl substituents (119b, 120b) performed with lower yields, but without any further observations. Changing the aryl group to a benzyl group (121b) performed with good yield and no trace amounts of oxygenated byproduct could be found; slight appearances of further side products could be detected in GC-MS. 


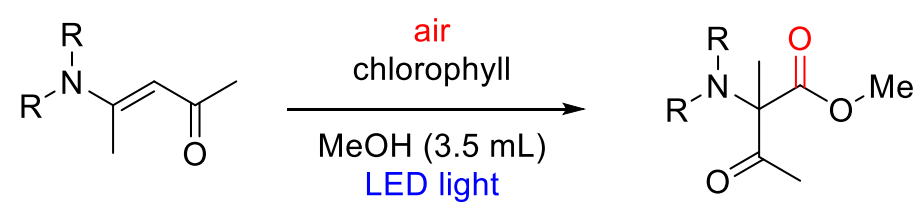<smiles>CC(=O)/C=C(\C)Nc1ccccc1I</smiles>

123a: degradation<smiles>CC(=O)/C=C(\C)Nc1ccc([N+](=O)[O-])cc1</smiles>

124a: n. r.<smiles>CCCN/C(C)=C/C(C)=O</smiles>

125a*: alternative<smiles>CCN(CC)/C(C)=C/C(C)=O</smiles>

126a*: alternative<smiles>CC(=O)/C=C(\C)NCC12CCCCC1(C)c1ccc(C(C)C)cc1CC2</smiles>

127a: alternative<smiles>CC(=O)/C=C(\C)NCCc1c[nH]c2ccccc12</smiles>

128a: alternative<smiles>CC(=O)C=C(C)N1CCC(O)CC1</smiles>

129a: alternative<smiles>CNC(=O)c1ccc(N/C(C)=C/C(C)=O)cc1</smiles>

130a: undefined activity failed to be isolated<smiles>CCOC(=O)/C=C/c1ccc(N/C(C)=C/C(C)=O)cc1</smiles>

131a: undefined activity failed to be isolated

Scheme 36: Unsuccessful substrates of this reaction. Reaction conditions: substrate $(0.25 \mathrm{mmol})$, catalyst $(0.3 \mathrm{~mol} \%)$, methanol $(3.5 \mathrm{~mL})$, excitation at $460 \mathrm{~nm}$ wavelength (blue LED).

To further confirm of possible degradation product, ortho-iodo-aryl compound (Scheme 36, 123a) was synthesized. The initial formation of the desired product could be observed but no higher concentration of it could be formed. Small substitutions like methyl and fluoro seemed to be the limits in the ortho position that can be performed for this transformation. A nitro functional group (124a) was examined and showed no reactivity for the reaction at all. The $n$-alkyl substrates were not isolated after synthesis. All attempts to isolate these compounds failed due to the low boiling point and vapor pressure. The starting material was synthesized in methanol. Synthesis reaction was performed until GC-MS and TLC showed quantitative transformation to the confirmed structure. Afterwards, chlorophyll was added to the mixture and the reaction performed. These reactions resulted in the formation of undefined reaction products, which were not isolated successfully. To examine these observations further several 
more complex and higher mass structures were synthesized (127a-130a). All reactions showed full conversion combined with the formation of an alternative product. All formed products showed a similar mass difference from the expected products. Entries 130a and 131a showed reactivity with no conclusive result of the GC-MS analysis. Both compounds could not be isolated successfully and remain open for further investigation. Several other compounds were attempted to be synthesized but did not formed the desired secondary enamino ketone (Scheme 37).

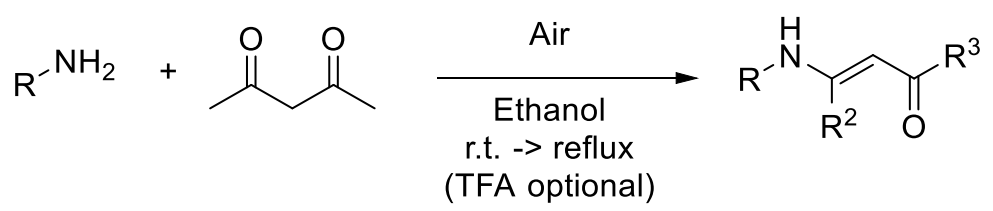<smiles>Nc1cccnc1</smiles><smiles>Nc1cc[nH]c(=O)n1</smiles><smiles>Nc1nc(N)nc(N)n1</smiles><smiles>COC(=O)C(=O)C1CNc2ccccc21</smiles><smiles>CCC(C)C(N)C(=O)O</smiles><smiles>CNC(CO)C(=O)OC</smiles>

Scheme 37: Unsuccessful utilized amines for the synthesis of secondary enamino ketones. 
In the final stage the ketone part was modified and investigated (Scheme 38). A symmetrical (131b) and a nonsymmetrical (132b) derivative were prepared. Both were able to facilitate the reaction and resulted in good yields. Replacing the allylic proton through a chloro substituent (133a) resulted in no activity to form the desired product.<smiles>CCCCC(=O)/C=C(\C)Nc1ccccc1</smiles><smiles>CCC(=O)/C=C(\CC)Nc1ccccc1</smiles>

$133 a$

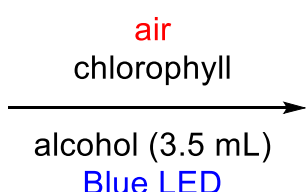

Blue LED

air

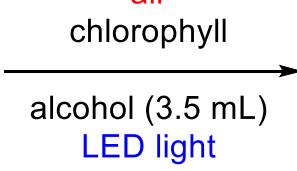

LED light<smiles>CC(=O)/C(Cl)=C(\C)Nc1ccccc1</smiles><smiles>C=C(C)C</smiles><smiles>CCCCCC(=O)C(C)(Nc1ccccc1)C(=O)OC</smiles>

132b: $71 \%$ (8h)<smiles>CCC(=O)C(Nc1ccccc1)(C(=O)CC)C(=O)OC</smiles>

133b: $81 \%(6 h)$

134a: $81 \%(6 h)$

Scheme 38: Substrate scope based on different substitutions at the ketone part. Reaction conditions: substrate $(0.25 \mathrm{mmol})$, catalyst $(0.3 \mathrm{~mol} \%)$, respective alcohol (3.5 $\mathrm{mL}$ ), excitation at $460 \mathrm{~nm}$ wavelength (blue LED). 


\subsubsection{MECHANISTIC INVESTIGATIONS}

The established substrate scope raised the quest for the underlying mechanism of the reaction. Control experiments were performed in combination of the quenching experiments (Table 11). Reaction under inert argon atmosphere (entry 1) inhibited all reactivity, indicating that the reacting oxygen is essential. The substrate without the presence of the photocatalyst (entry 2) stayed inert and had no reaction either. Therefore, a photochemical pathway should be shown by this. Performing the reaction without light (entry 3 ) decreased the yield to below $5 \%$, which was attributed to the high sensitivity and broad distribution of the photocatalysts to absorb visible light. This seemed to have a sufficient impact on the reaction mixture during preparation of the experiment and during sample preparation. Performing the reaction in DMF (entry 4) and DMSO (entry 5) without any presence of alcohol resulted in no reaction at all; both solvents are well known for their ability to stabilize reactive oxygen species like superoxide radical anions and singlet oxygen, which would have enabled an insolation of a possible intermediate. Minor concentrations of methanol (entry 6) resulted in the formation of the targeted product, but at lower yields. The quenching experiments showed nearly full inhibition with the use of sodium azide (entry 7 ), indicating that singlet oxygen could be the primary oxygen species for the reaction and the involvement of an ET pathway. The use of 1,4-benzoquinone (entry 8) and copper chloride (entry 9), which would be a quencher for a typical SET pathway resulted in full conversion of the starting material, but with nearly no formation of the desired product. A broad variety of different other oxidation products was also formed. This observation could indicate that the reaction was still initiated, but due to the presence of the quencher, different reaction pathways were enabled. The radical quencher BHT (entry 10) and TEMPO (entry 11) had no effect on the reaction. The use of tert-butanol (entry 11), as a typical hydroxy radical quencher, showed no effect on the reaction outcome either. 
Table 11: Control and quenching experiments for the chlorophyll photocatalyzed multi-step reaction of secondary enamino ketones. ${ }^{[a]}$
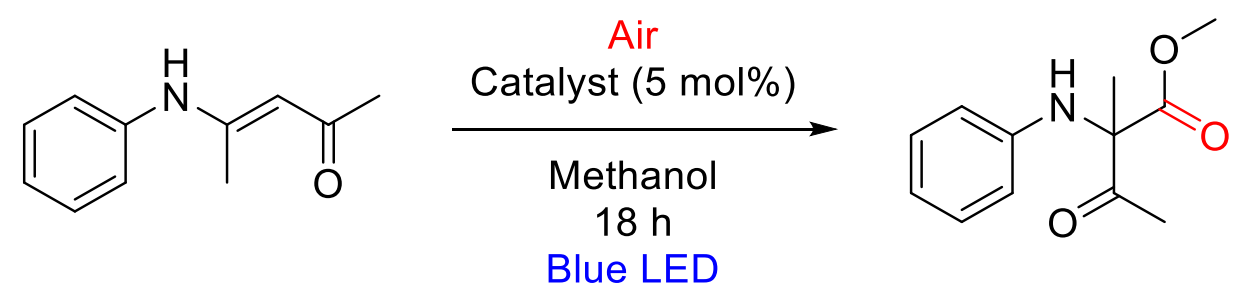

\begin{tabular}{|c|c|c|c|c|}
\hline Entry & Conditions / changes & Quencher & Quenching & $\begin{array}{c}\text { Yield / } \\
\%\end{array}$ \\
\hline 1 & Argon atmosphere & --- & --- & 0 \\
\hline 2 & No catalyst & --- & --- & 0 \\
\hline 3 & No light & --- & --- & $<2$ \\
\hline 4 & In DMF & --- & --- & 0 \\
\hline 5 & In DMSO & --- & --- & 0 \\
\hline \multirow[t]{2}{*}{6} & In DMSO 10 vol\% & --- & --- & 31 \\
\hline & $\mathrm{MeOH}$ & & & \\
\hline 7 & STD & $\mathrm{NaN}_{3}$ & Singlet oxygen & 0 \\
\hline \multirow[t]{2}{*}{8} & STD & $1,4-$ & Superoxide & $2^{[b]}$ \\
\hline & & Benzoquinone & & \\
\hline 9 & STD & $\mathrm{CuCl}_{2}$ & Electron & $0^{[b]}$ \\
\hline 10 & STD & BHT & Radical & 89 \\
\hline 11 & STD & TEMPO & Radical & 87 \\
\hline 12 & STD & tert-Butanol & $\begin{array}{l}\text { Hydroxide } \\
\text { radical }\end{array}$ & 86 \\
\hline
\end{tabular}

[A] Reaction conditions: substrate $(0.25 \mathrm{mmol})$, catalyst $(750 \mathrm{nmol})$, quencher $(0.25$ $\mathrm{mmol}) \mathrm{MeOH}(3.5 \mathrm{~mL})$, excitation at $460 \mathrm{~nm}$ wavelength (blue LED) irradiation for 12 h. [b] full conversion, without product formation.

Kinetic data of the reaction was also investigated to determine the Turn-Over-Number (TON) and Turn-Over-Frequency (TOF) of the reaction. The reaction was performed for different time lengths and the resulting yields were compared (Figure 35). The results were a nearly lineal increase of reaction yield with $15.7 \%$ product formation per hour. Based on the reaction scale this means $0.0393 \mathrm{mmol} / \mathrm{h}$; this would correlate with a TON of 307 and with a respective TOF of $1.7^{*} 10^{-2} \mathrm{~s}^{-1}$. 


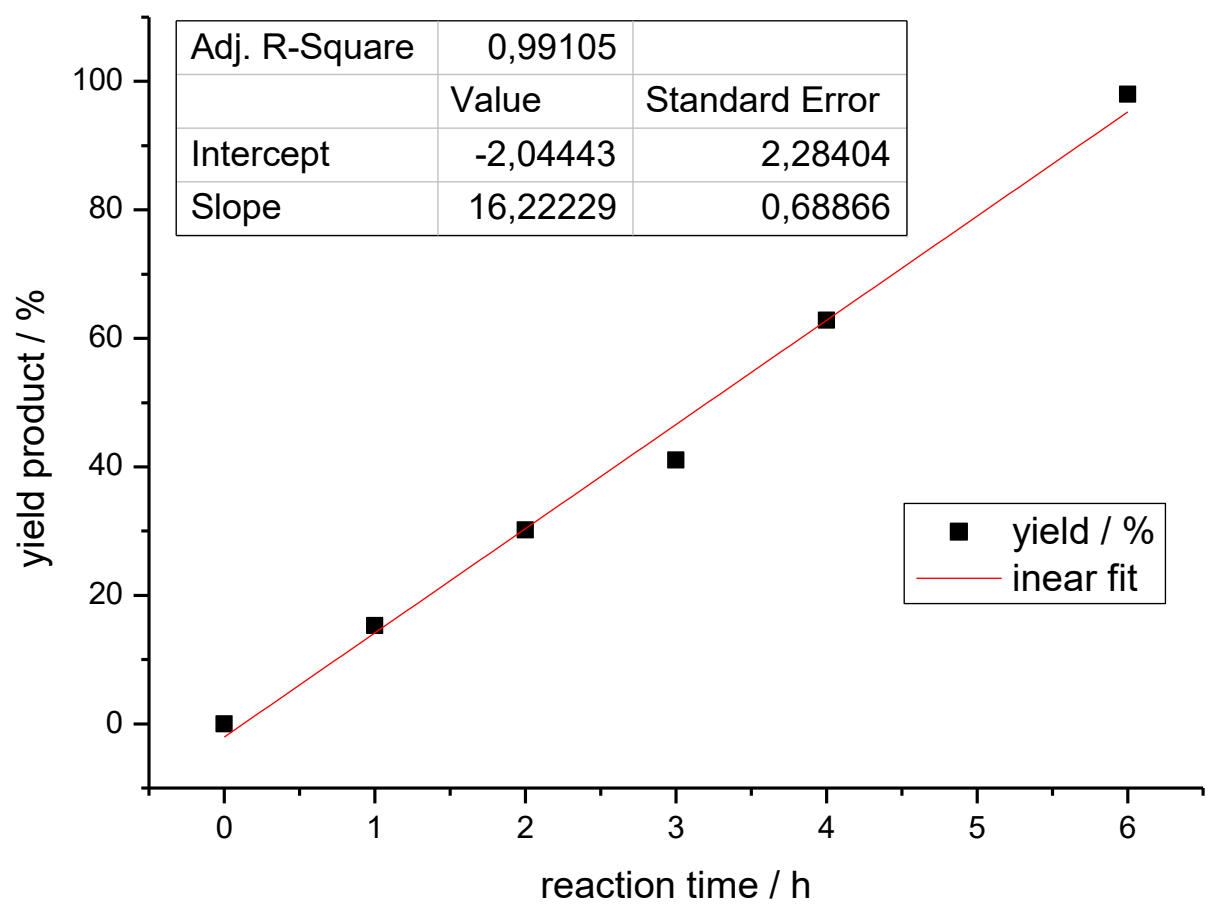

Figure 35: Time dependent yields for the photochemical reaction of secondary enamino ketones.

To further investigate the light dependence of the reaction, on and off experiments were performed (Figure 36 and 37). Alternating 30-minute periods of light and darkness were applied at the reaction mixtures and samples were taken to evaluate the yield by GC-FID with $n$-dodecane as an internal standard. After the irradiation period, a constant increase of around $8 \%$ yield could be observed. The concentration of the product increased in the darkness period by $4 \%$. This could imply that the initiation of the reaction required light but that essential part of the reaction could not be light dependent. To gain further insight into the reaction process during the darkness phase, a second experiment was designed. The sample was irradiated for 2 hours and afterwards the yields were determined in short periods of the darkness phase (Figure 37) 


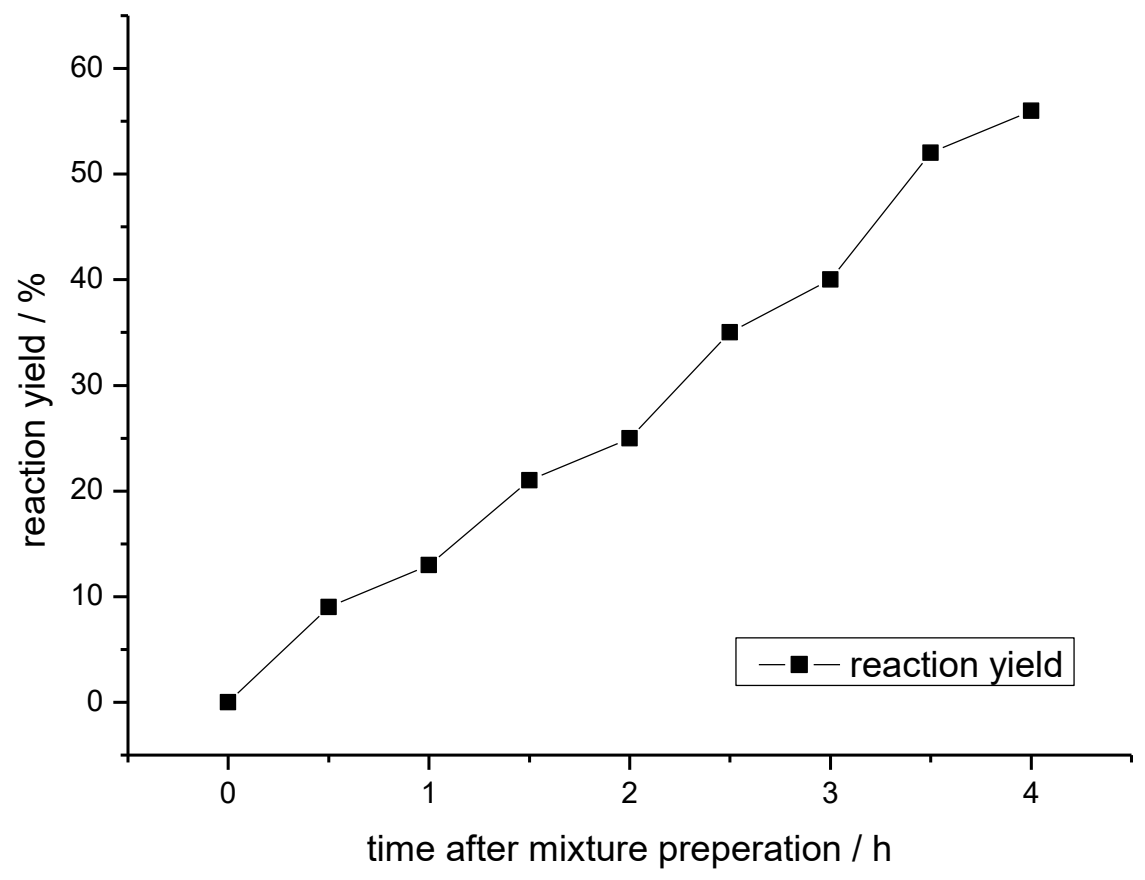

Figure 36: Resulting yield for on and off experiments for the photochemical reaction of secondary enamino ketones. periods from full hours to half hour irradiation and the altering 30 minutes darkness.

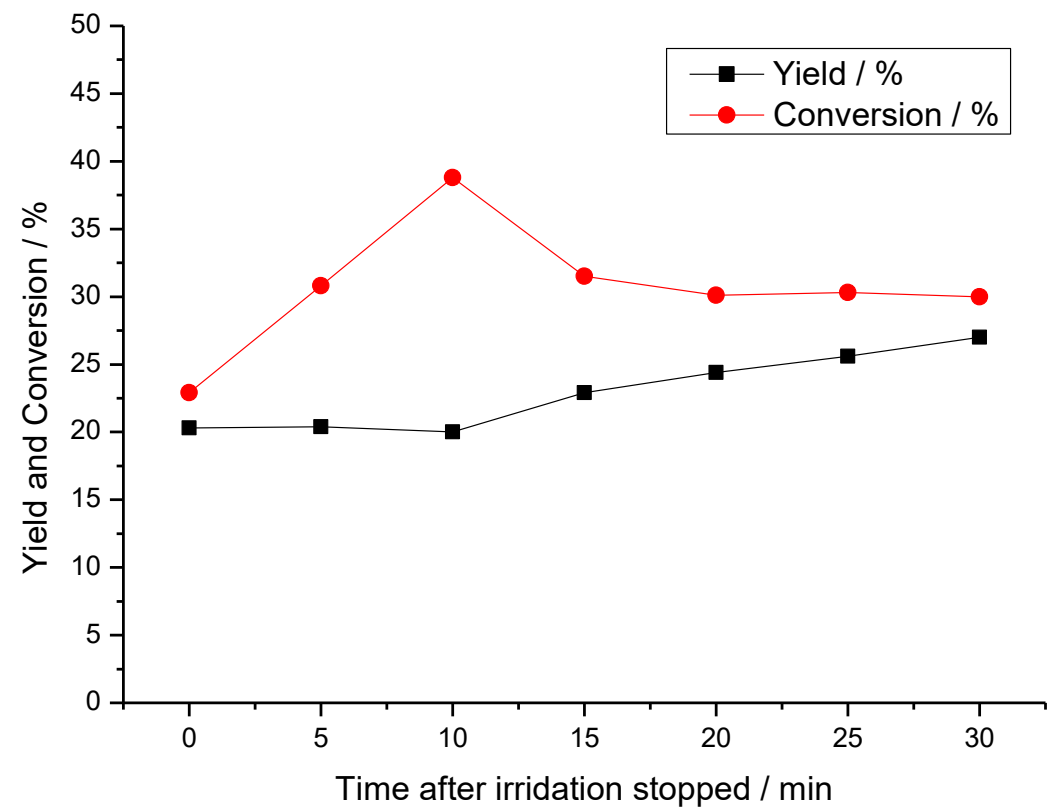

Figure 37: Resulting yields and conversion of the sample after 2 hours of irradiation for the photochemical reaction of secondary enamino ketones. 
The second concept to investigate the conversion and yield during the darkness phase showed that shortly after the irradiation stopped, the conversion of substrate continued for the following 10 minutes and afterwards started to decrease again, reforming starting material. After 10 minutes, the yield of the product started to increase forming yields similar to the conversion progress. These observations allowed the hypothesis, that during the irradiation the initial step was performed to start the reaction and the following steps would be performed without light. Formed intermediates seemed to progress through reversible reaction steps till the final product was formed. Therefore, it can be assumed that several steps of the reaction should be reversible in their nature.

To further resolve the underlying mechanism of the reaction labeling with ${ }^{18} \mathrm{O}_{2}$ atmosphere was conducted (see Experimental Part 5.4). The resulting product obtained an isotope labeled oxygen, indicating that one oxygen atom in the final product originated from the oxygen in the air. This result combined with the different utilized alcohols during the substrate scope investigation, would result in the following assumption that the three oxygen atoms in the final product originated from three different sources. One oxygen formed the substrate itself, one oxygen for the formation of the ester function from the oxygen containing atmosphere and one oxygen originated from the alcohol.

To gain further insights 3D-absorption-fluorescence spectra were recorded and the Stern-Volmer plots were conducted. The 3D-absorption-fluorescence behavior of the chlorophyll showed typical shape and values for the reported chlorophylls. If substrate was present in the measured reaction mixture, the absorption and emission behavior of the solution changed drastically, creating an additional band for the absorption and new emissions for the reaction solution (Figure 38). 


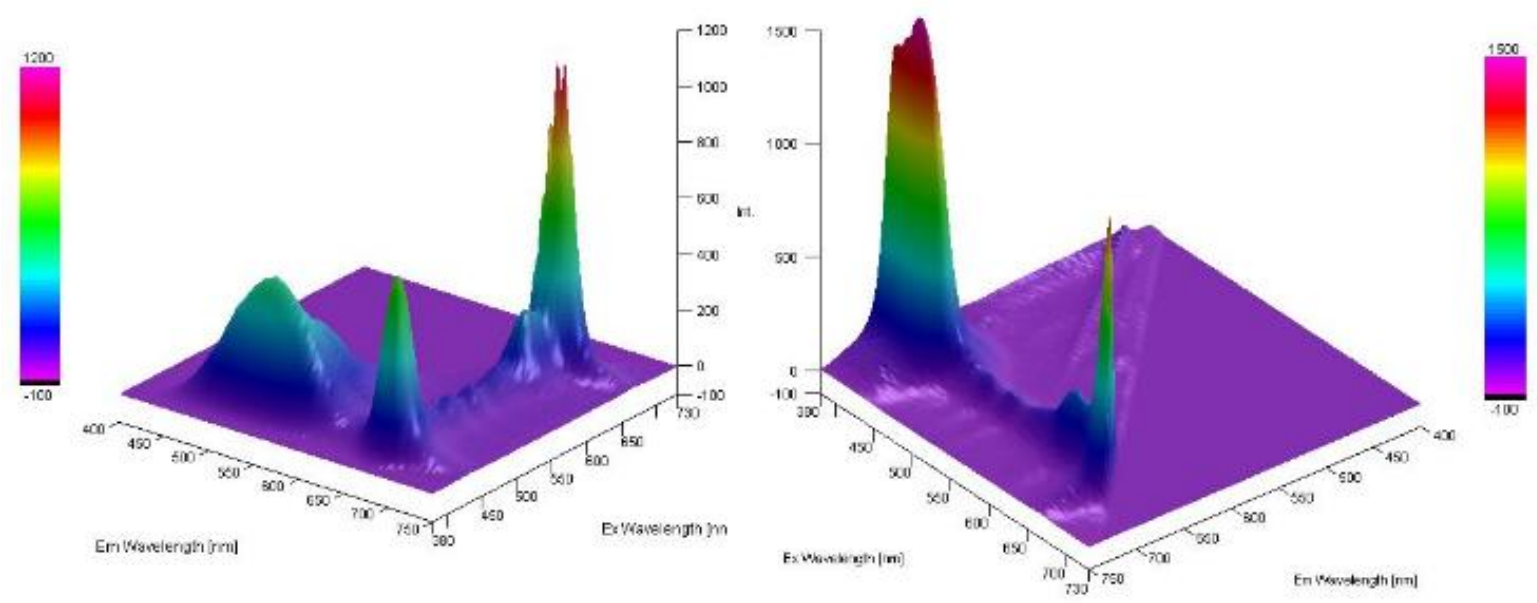

Figure 38: 3D-absorption-emission spectra for the reaction mixture (left) and the chlorophyll in methanol (right).

The Stern-Volmer plot for the chlorophyll in methanol, showed a quenching effect in dependence of the oxygen concentration in solution, further confirming a possible formation of singlet oxygen via energy transfer from the chlorophyll to oxygen (Figure 39, upper left). Different concentration of substrate in the reaction mixture showed that the catalyst was additionally changed by the presence of the substrate in solution (Figure 39, upper right). Investigating the influence of the oxygen concentration in solution of the reaction mixture also showed a quenching effect on the photocatalyst (Figure 39, bottom right). Based on these observations two conclusions could be made. Firstly, the substrate interacted with the chlorophyll. It could be assumed that substrate coordinated or associated with the catalyst. Secondly, an energy transfer from the chlorophyll to the oxygen was present, with and without the substrate's presence. Addition of sodium azide to the reaction mixture, did not alter the 3Dabsorption-fluorescence spectra. 

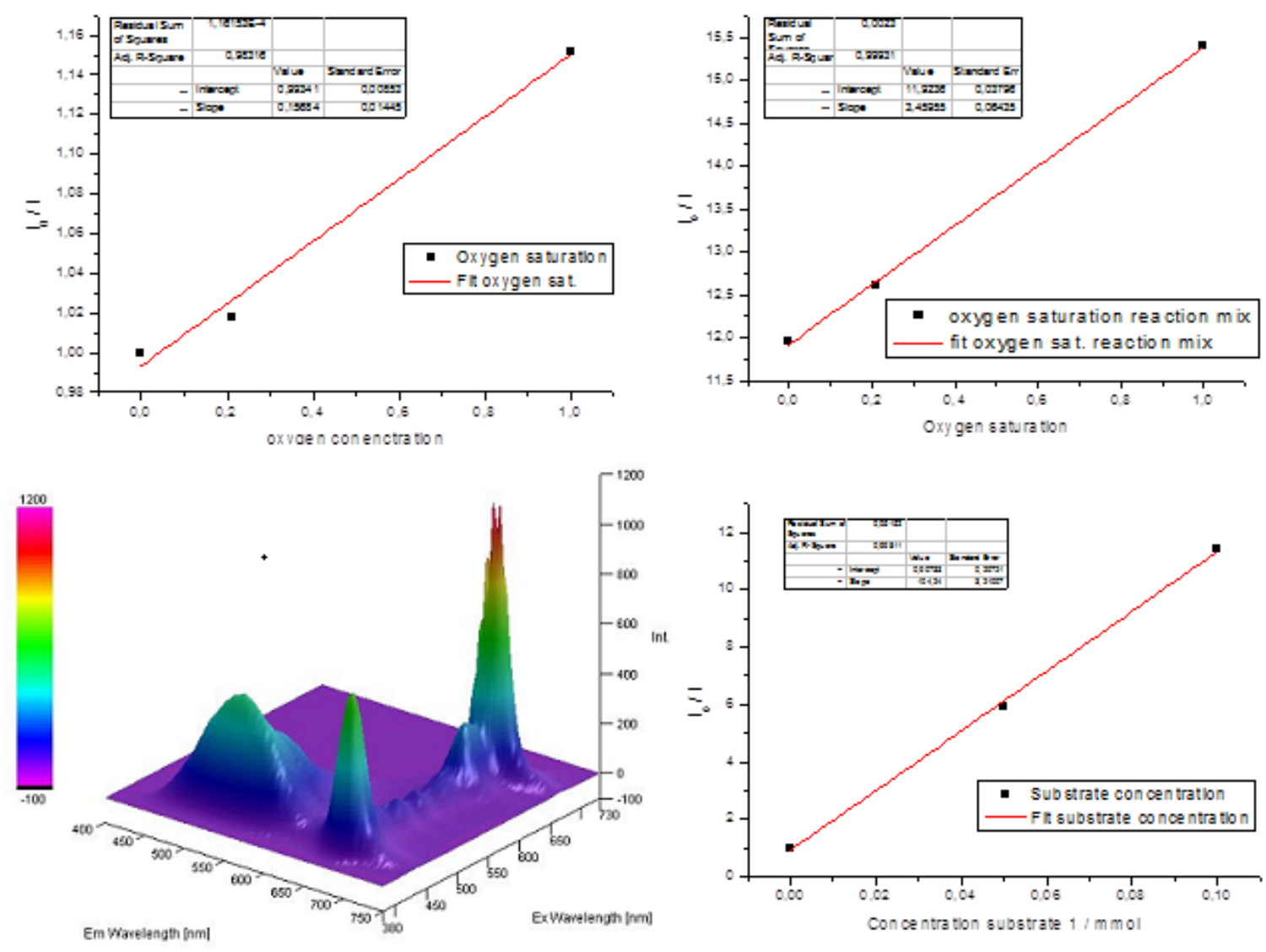

Figure 39: Stern-Volmer plots for the oxygen concentration in solution for chlorophyll in methanol (upper left), oxygen concentration in solution for the reaction mixture (upper right) and the substrate concentration (bottom right).

Additional EPR experiments were conducted (in collaboration with Samir Kumar Sarkar at the Institute for Inorganic Chemistry) to confirm the involvement of singlet oxygen in the reaction (Figure 40). Due to the short lifetime of singlet oxygen, a spin trapping agent was utilized. As a fitting agent TMP was reported and was utilized. Three samples were prepared. A) a sample of the reaction mixture under nitrogen atmosphere. B) the reaction mixture under aerobic conditions. C) Chlorophyll in methanol without the substrate. Each sample was irradiated for 10 minutes and was afterwards measured via EPR-spectrometry. All the samples, under aerobic conditions, showed positive results for single oxygen adducts. The control sample under nitrogen atmosphere showed only base line values. The observed EPR-spectra for the reaction mixture and catalyst solution showed a slight shift. Two possible explanations were derived from this. Option A: the presence of the substrate changed 
polarity of the solvent system and therefore changed the solvent effects which led to a minimal shift in the field. Option B: interaction of the substrate with the catalyst was present and that led to lower energy levels at the singlet oxygen.

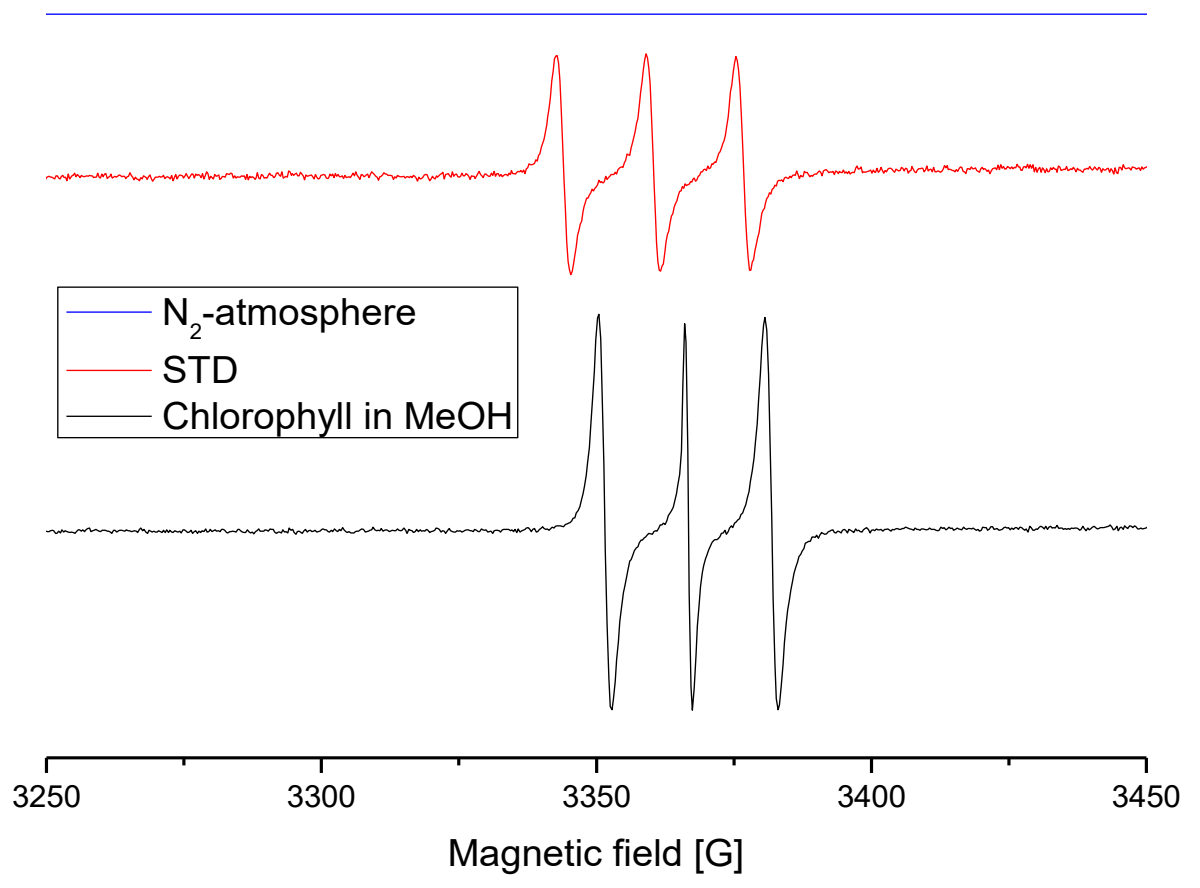

Figure 40: EPR spectra for the reaction mixture under nitrogen atmosphere, the reaction mixture under air and the catalyst in methanol under air, each after 10 minutes of irradiation at $460 \mathrm{~nm}$ wavelength. Experiments performed by Waldemar Schilling and Samir Kumar Sarkar.

The performed mechanistic experiments were deemed sufficient for the postulation of a plausible mechanism (Scheme 39). The chlorophyll was excited by irradiation of visible light, followed by two energy transfer (ET) based interaction. The first possible interaction was the transfer to triplet oxygen to form singlet oxygen. This was confirmed by the performed quenching experiments and through the EPR measurements. The second possible interaction was an energy transfer (ET) to the substrate resulting in a higher reactivity of the substrate. This was confirmed by the high interaction in the 3D-absorption-fluorescence spectra and the Stern-Volmer investigations. Further literature reports also indicated that the oxidation potential of 
the substrate and chlorophyll were incompatible. ${ }^{[122-123,141]}$ The substrate oxidation potential $\left(\mathrm{E}_{\mathrm{a} \cdot+/ \mathrm{a}}\right)$ was reported as $+1.24 \mathrm{~V}$ vs. SCE and therefore higher than the excited state potential of chlorophyll as $+0.94 \mathrm{~V}$ vs SCE. This further excluded a possible singlet electron transfer (SET), as assumed during the quenching experiments. Through the proximity of the activated substrate and singlet oxygen a dioxetane intermediate was reversibly formed in $\beta$-carbon position of the substrate. The intermediate was able to undergo dehydration to form the diketone intermediate. This was confirmed by the single labelled product during the labelling experiment (Experimental Part 5.4). The diketone intermediate was attacked by deprotonated alcohol initiating a 1,2-acyl shift reaction. After protonation of the intermediate the final product was obtained.

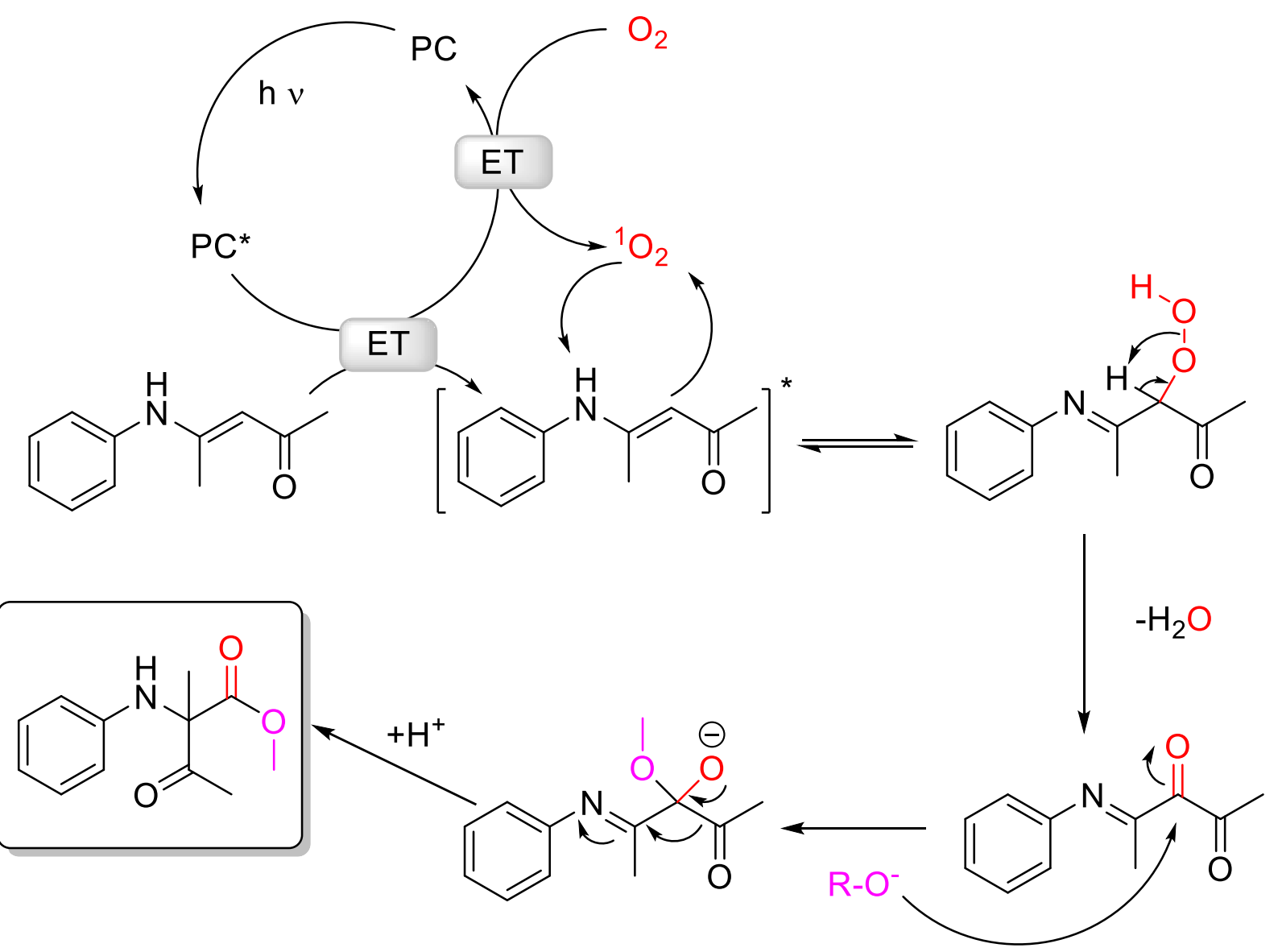

Scheme 39: Postulated mechanism for the chlorophyll catalyzed reaction of secondary enamino ketones.

Additional density functional theory (DFT) calculation at zb3lyp/6-311++g(d,p) level of theory in conjunction with the CPM solvent model for $\mathrm{MeOH}$ were performed by Samir 
Kumar Sarkar and provided a complete single-point energy profile for the reaction (see Appendix).[141] The provided profile showed fitting values for the postulated mechanism. Further DFT calculations by Gandhi Sivaraman showed fitting calculation for the singlet oxygen activation through the chlorophyll structure. ${ }^{[141]}$

\subsubsection{Summary and Outlook}

This project revealed that the utilization of commercially available natural chlorophyll can replace the use of known transition metal-catalyzed systems and could further improve the efficiency to industrial performance levels. The presented work showed the uses of polychromatic substances in photochemistry which can improve possible applications in photochemical synthesis and application of green chemistry principles. The developed methodology was able to perform the activation of oxygen to singlet oxygen, the oxygenation of the secondary enamine ketone, the dehydration, followed by an 1,2-acetylshift and protonation in a one-pot reaction. During the investigations the possibility of direct synthesis of the starting material in the final reaction mixture was possible. This showed decreased reactivity and slow accumulation of yield, but with additional investigations could be optimized to good efficiency. The methodology was able to perform at different wavelengths in the same pathway. Utilization of direct solar irradiation showed that utilization of several wavelengths can increase the performance of this reaction intensively. This enabled large scale reactions even at faster reaction times, than the blue-LED lab scale reactions. Therefore, the possibility of overcoming the well-known upscale problem of photochemical reactions could be bypassed.

Detailed mechanistic experiments showed the role of the chlorophyll, oxygen and solvent in the reaction and a plausible mechanism could be provided.

These results also provided new insights for studies which aim to identify the special contribution of membrane residues and interactions of chlorophyll with substrates to apply the effects targeted. 
Combining those results with flow concepts, with natural photocatalysts and solar light could show highly efficient photochemical systems, which could be capable of industrial application. Part of the results were published in Green Chemistry. ${ }^{[141]}$

\subsection{DESIGN AND DEVELOPMENT OF GRAPHITIC CARBON NITRIDE AS PHOTOCATALYSTS}

The last aspect of the work was the design of organic heterogenous materials with photocatalytic properties and the investigation of their modification; to enable tunable heterogenous photocatalysts without the utilization of transition-metals. Small scale attempts were performed to see if the synthesis was possible. The resulting activity was afterwards reproduced in a new concept for the large-scale synthesis of the $g$ $\mathrm{C}_{3} \mathrm{~N}_{4}$. The target of the subchapter was to establish new synthetic methods for the heterogenous photocatalysts based on organic materials. The gained insights in the synthesis should then pave the way for targeted synthesis of the heterogenous photocatalysts with a broad variety of different wavelengths and redox potentials, which enable tailored catalysts for future applications.

Initial attempts, utilizing a commercially available Gero ring oven to polymerize melamine revealed that photoactive graphitic carbon nitrides could be obtained in the area of $500-625^{\circ} \mathrm{C}$ reaction temperature. All results were characterized by FT-IR spectroscopy to confirm successful synthesis of the $g-\mathrm{C}_{3} \mathrm{~N}_{4}$ network. A full characterization should be applied during future application of $g-\mathrm{C}_{3} \mathrm{~N}_{4}$ materials for photocatalytic reactions.

The initially obtained $g-\mathrm{C}_{3} \mathrm{~N}_{4}$ based on melamine was examined for the reactivity to catalyze three separate reaction protocols and showed reactivity for all protocols of this work. With the initial results of this investigation, future efforts of tailored design could be achievable. 


\subsubsection{Design of REAction Setup}

Based on the target of synthesizing large quantities of $g-\mathrm{C}_{3} \mathrm{~N}_{4}$ the safety aspects should be considered. The synthesis of 20-30 g scale reactions means the use of up to $70 \mathrm{~g}$ of melamine as precursor. The directly visible mass difference was based on the separation of ammonia from the material. This was roughly $2.5 \mathrm{~mol}$ of ammonia. At room temperature this would mean $50 \mathrm{~L}$ of ammonia gas, but at temperatures of over $500^{\circ} \mathrm{C}$, a possible reaction setup must exhibit sufficient pressure, heat and ammonia resistance. The given safety restrictions led to the following concept (Figure 41). The main material for the reaction chamber and reaction vessel were used VA4 steel, for its reported inertness to ammonia conditions at normal and at high temperatures. For the production of heat a larger commercially Gero tube furnace was used and a second oven concept was rebuilt by the workshops.

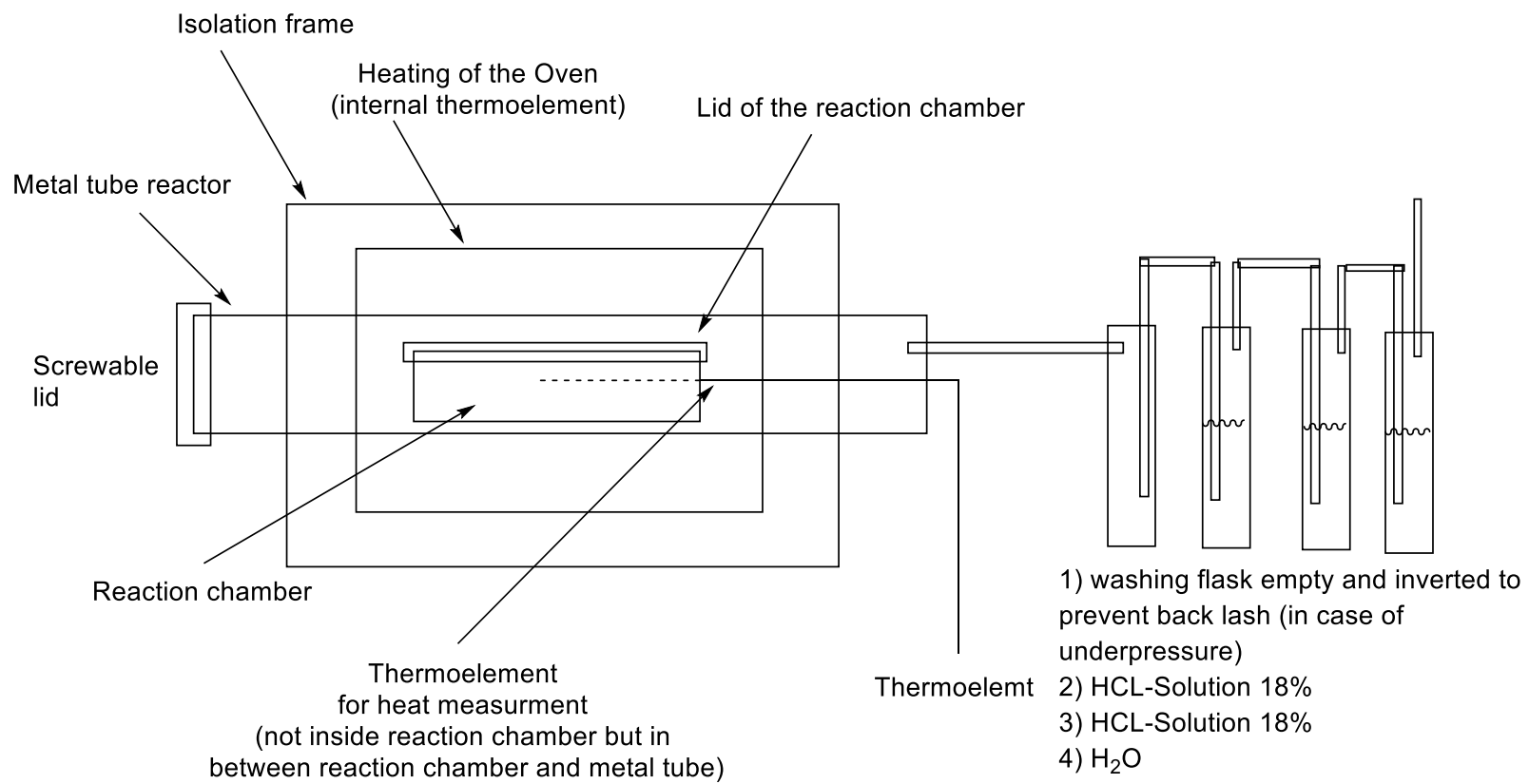

Figure 41: Schematic presentation of the designed large-scale reaction concept for the synthesis of graphitic carbon nitrides.

For the synthesis a VA4 steel chamber was constructed by the faculty workshops with a wall strength of $0.8 \mathrm{~mm}$, with sufficient material strength to withhold inner pressures of over $25 \mathrm{~atm}$. The selection of VA4 steel was based on its inertness towards ammonia gas and a general high corrosion and thermal persistence. The chamber 
was tube shaped and was sealed at the end with a small $5.0 \mathrm{~mm}$ diameter exit opening, connected to the gas neutralization system. This formed an open pressure system to remove excess forming gas from the reaction and preventing intake of ambient air. In two cases a blockage of the exit opening was formed by byproducts during the pyrolysis. The inner pressure was not recorded, but it can be assumed that several atm pressure was present at the highest temperature. After cooling to room temperature, only a slight over pressure was present in the chamber.

The opposite side was equipped with a screw able lid and a copper-based sealing ring. Based on the temperature inducing different expansion of the metals a sealing effect was achieved. A thermo conducting cooper paste was not deemed necessary in this case.

The commercial oven included a thermoelement for monitoring the temperature of the heating coil. A secondary thermoelement type $\mathrm{K}$ was used to monitor the temperature of the inside chamber. For the temperature area of $500-625^{\circ} \mathrm{C}$ (inside temperature) compared to the outside temperature of $35^{\circ} \mathrm{C}\left(585-655^{\circ} \mathrm{C}\right.$ outside set temperature $)$ was experimentally recorded. The temperature difference resulted from thermal transport of the heating chamber, to not heated areas of the setup, which stabilized after the heating ramp period with a $100^{\circ} \mathrm{C}$ lower temperature at the outside of the chamber wall. The achieved temperature difference of the inner and outer thermoelement was stable $\pm 1^{\circ} \mathrm{C}$ in the chosen temperature area.

The gas neutralization system consisted of four washing bottles in sequential connection. The first washing bottle acted as setback reserve in the case of unexpected under pressure events so that quenching solutions were not able to enter the metal tube reactor. The following washing bottles were filled with $18 \%-\mathrm{HCl}$ solutions to neutralize the formed ammonia. A last washing bottle with water acted as a closing against remaining gas contaminations. The designed concept showed essential benefits, based on chosen additives and lower mass precursors the reactions seemed to exhibit endothermic reaction steps resulting in a short period of cooling inside the reaction chamber, which led to short periods where the quenching solutions were transferred to the prior sequenced washing bottles. The event lasted shorter than one minute and pressure stabilized in all cases again. 
The reaction was performed inside a specially designed VA4-steel reaction vessel and a fitting loose lid. Possible contamination of the reaction vessel metal to the formed $g$ $\mathrm{C}_{3} \mathrm{~N}_{4}$ cannot be excluded but should be minimized by removal of the outer layer of the formed $g-\mathrm{C}_{3} \mathrm{~N}_{4}$. Four different synthetic protocols were developed for this concept to achieve different targeted modifications. Based on the utilized materials, several heating ramps had to be programmed, due to under pressure events, when urea and other low mass precursors were used, due to the endothermal reactions. The use of a fitting loose lid is a crucial concept of the design. The lid was loose to allow forming gas to exit the reaction vessel but was sufficiently tight to remain attached to the vessel constantly. Initial experiments resulted in the precursor material being lifted by the forming ammonia gas and distributed throughout the entire heating chamber. This resulted in high reduction of the obtained product, Additionally, the distributed precursor was still able to perform the pyrolysis reaction in the heating chamber, forming partially polymeric carbon nitrides and possible further polymeric ceramics with other impurities. 


\subsubsection{SyNTHESIS OF MODIFIED CARBON NITRIDES AND THEIR ACTIVITY}

The modified carbon nitrides were divided into several concepts. The first group was based on the utilized starting materials (urea thiourea, 2-cyanoguanidine, melamine). The second group utilized comonomers to introduce gaps, or impurities as structural deficits. The third group were post synthesis modifications of the surface. A schematic representation of the different classes of obtained $g-\mathrm{C}_{3} \mathrm{~N}_{4}$ is given in Figure 42.

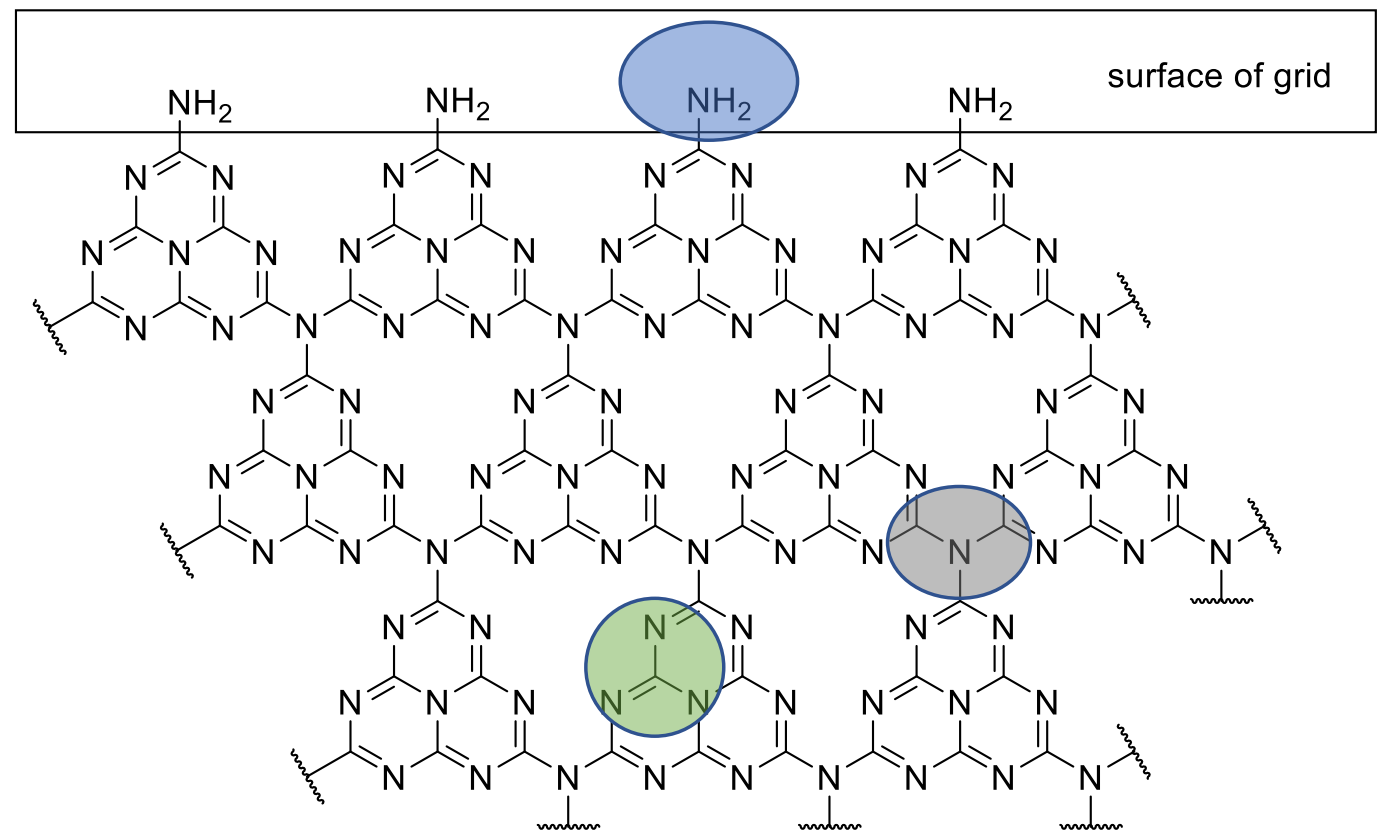

Figure 42: Schematic presentation of the obtained graphitic carbon nitrides. The marked areas showed the possible position of disconnection, impurity incorporation or surface modification into the grid of graphitic carbon nitrides.

All the obtained modifications of $g-\mathrm{C}_{3} \mathrm{~N}_{4}$ were compared for their ability and selectivity of photocatalytic oxidize benzyl alcohol in DMSO. The procedure was similar to the oxidation protocol described in Chapter 3.1; the catalyst was replaced by $10 \mathrm{mg}$ of $\mathrm{g}$ $\mathrm{C}_{3} \mathrm{~N}_{4}$. These results acted as a simplified evaluation of the benchmark test for the redox potentials of the obtained $g-\mathrm{C}_{3} \mathrm{~N}_{4}$ materials. The resulting overview is reported in Figure 43 and the details about the formed $g-\mathrm{C}_{3} \mathrm{~N}_{4}$ catalysts is discussed in the following part. Based on the consideration of possible better conversion and yields for the obtained $g-\mathrm{C}_{3} \mathrm{~N}_{4}$, the reaction time was set to 8 hours and a comparative reaction utilizing 9-fluorenone was performed (entry Flu). The different $g-\mathrm{C}_{3} \mathrm{~N}_{4}$ show a broad variety of different results in the benchmark reaction, ranging from high yields and 
fitting conversions (entries 1, 12, 17, 20), to no activity (entries 2, 19, 21, 22), or over proportional conversions (entries $2,6,15,18$ ), which indicated overoxidation to the benzoic acid. For future results this benchmark can be compared to the still missing characterizations of the band gap, redox potentials and energy values of the formed $g-\mathrm{C}_{3} \mathrm{~N}_{4}$, which would give access to simplify low effort pre-evaluation of the heterogenous photocatalysts. Distinctive differences are recognizable between the standard $g-\mathrm{C}_{3} \mathrm{~N}_{4}$ catalyst obtained from different precursors (Entries 1, 2, 3, 4).

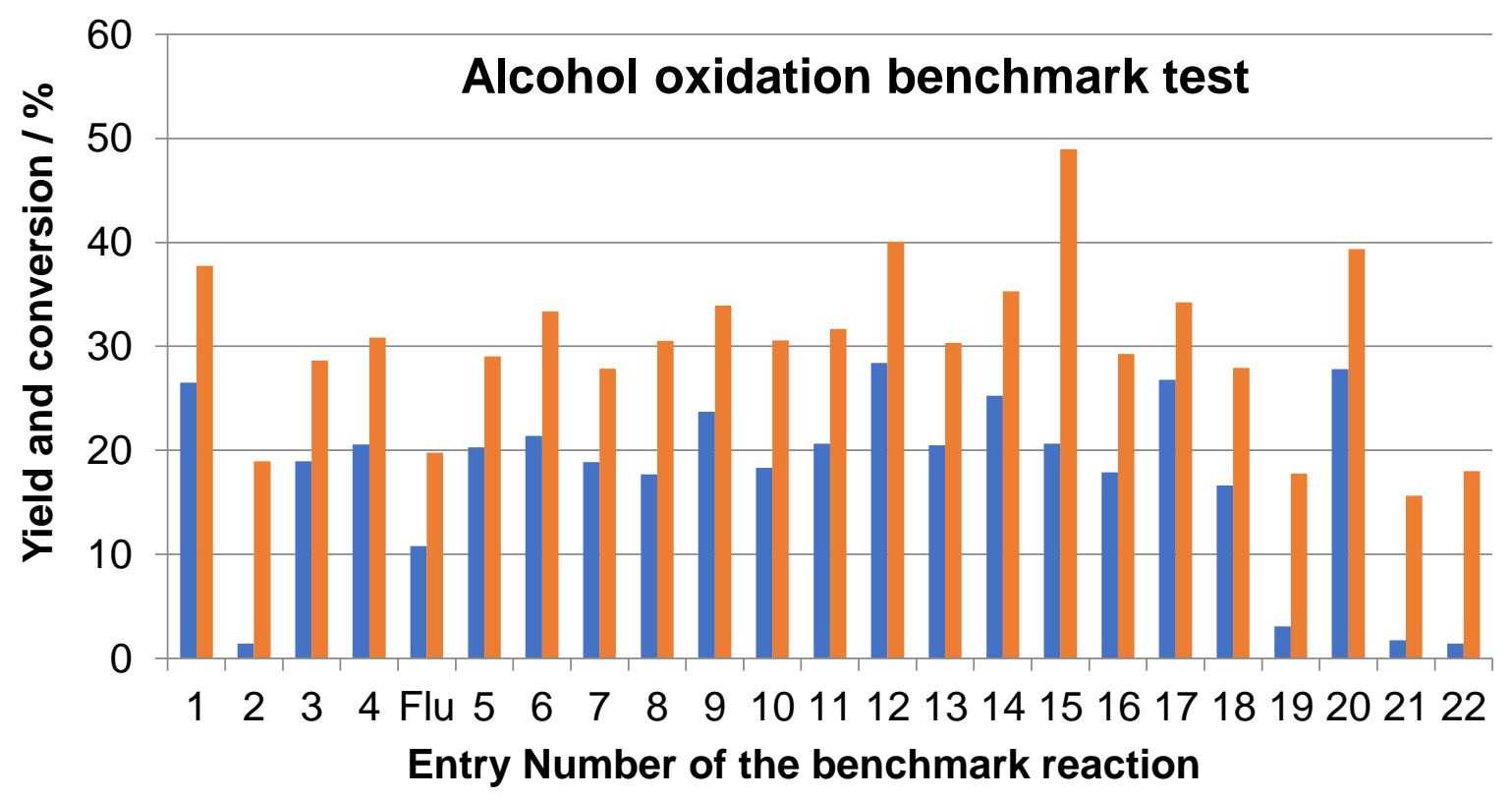

Figure 43: Yields (blue) and conversion (orange) for the benchmark reaction for the oxidation of benzyl alcohol. reaction time 8 hours.

\section{Utilization of different precursors}

Different precursors were utilized for the synthesis of $g-\mathrm{C}_{3} \mathrm{~N}_{4}$, each of the precursor materials should generate the same $g-\mathrm{C}_{3} \mathrm{~N}_{4}$-strcture as the final result. It was observed that the products exhibit different catalytic behavior and different macroscopic characteristics, like Bulk density, slight coloration differences and structural stability. Melamine and 2-cyanoguanidine based $g-\mathrm{C}_{3} \mathrm{~N}_{4}$ exhibited low coloration and high stoniness, the urea and thiourea based $g-\mathrm{C}_{3} \mathrm{~N}_{4}$ exhibited a loose flake stability. 


\section{Entry 1: Melamine}<smiles>Nc1nc(N)nc(N)n1</smiles>

Melamine

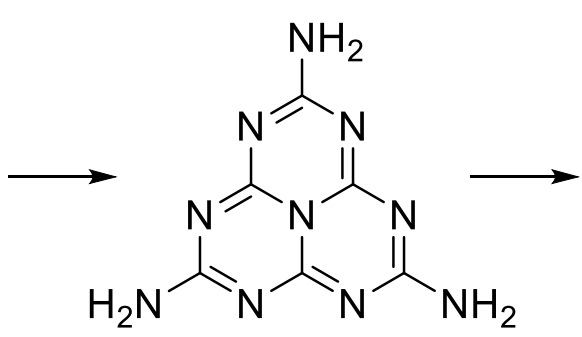

$\mathrm{H}_{2} \mathrm{~N}-\mathrm{NH}_{2}$

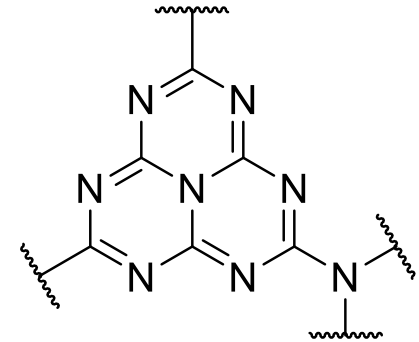

Scheme 40: Synthesis representation of the obtained carbon nitride.

Melamine based synthesis showed one of the best activities and conversions. The synthesis of higher mass precursor resulted in high mass conversion efficiencies of $44 \%$ and up to $33.4 \mathrm{~g}$ could be obtained with the designed reaction setup. Repeated batches were synthesized of this modification and performed similar to each other. An extended characterization of the obtained carbon nitride was performed during the published application to perform photocatalyzed aerobic C-C cleavage reactions, including reusability studies, scanning electron microscopy (SEM) and high resolution transmission electron microscopy (HRTEM) characterization of the particles, $2 \Theta X$-ray powder diffraction (XRD) UV/Vis, elemental analysis (see appendix, characterization was performed by the further coauthors of the publication). ${ }^{[139]}$ Elemental analysis showed typical values for carbon nitrides in the area of $35 \%$ carbon, $2 \%$ hydrogen and $62 \%$ nitrogen mass content. The further analysis showed similar results. SEM and HRTEM revealed that the catalyst is forming rounded plates with an average diameter of $30 \pm 10 \mathrm{~nm}$ and a thickness of the order of a few angstroms. The catalyst was analyzed before and after several cycles of use and separation and showed stability throughout the reaction cycles. FT-IR confirmed the typically IR-Bands. This catalyst performed well in the "benchmark test" and showed higher activity with similar selectivity compared to 9-fluorenone. The catalyst was examined as a replacement for the oxygenation of indoles, pyrroles. It showed around $34 \%$ yield which was not higher than the auto oxygenation of indole. For pyrrole no activity was observed. As a replacement for chlorophyll the catalyst showed activity, but degradation of the formed product was observed for longer time periods. The melamine based graphitic carbon nitride was used as a reference for the synthesis of further carbon nitrides. Based on a known reaction with defined redox potentials, an efficient benchmarking could be 
possible as an alternative characterization technique, if high-cost equipment is not available.

\section{Entry 2: Urea}<smiles>NC(=O)Nc1nc(N)nc2nc(N)nc3nc(N)nc(n1)-n-2-3</smiles><smiles>CC(C)C1=NC2=NC(C(C)(C)C)=NC3=NC(N(C(C)C)C(C)C)=NC(=N1)N32</smiles>

Scheme 41: Synthesis representation of the obtained carbon nitride.

Urea based synthesis showed almost no activity for the alcohol oxidation. Additionally, the low condensation state of the precursor had an effect that the mass conversion efficiency for the synthesis of carbon nitride could only reach around $4 \%$. This resulted that $70 \mathrm{~g}$ of Urea yielded only $2.1 \mathrm{~g}$ of carbon nitride. Comparation with literature showed that it was close to the best achieved results $(5.2 \%)$ for the synthesis. The utilization in other projects showed higher activities. It can be assumed that the precursor has a significant impact on the resulting morphology of the resulting carbon nitride. Further studies are necessary to find the precise influence. 


\section{Entry 3: Thiourea}<smiles>NC(=S)I</smiles>

thiourea<smiles>NC1=NC2=NC(N)=NC3=NC(N)=NC(=N1)N23</smiles><smiles>CC(C)C1=NC2=NC(C(C)(C)C)=NC3=NC(N(C(C)C)C(C)C)=NC(=N1)N32</smiles>

Scheme 42: Synthesis representation of the obtained carbon nitride.

Thiourea formally should yield no difference in the obtained grid, due to the fact that the sulfide was leaving during the polycondensation in the form of $\mathrm{H}_{2} \mathrm{~S}$ gas among the ammonia. Obtained grid showed a distinctive performance difference. Coloration and physical stability were drastically different. Based on literature research a possible explanation was found for the drastic differences between the obtained carbon nitrides. The forming $\mathrm{H}_{2} \mathrm{~S}$ can be placed between the forming grid levels and thereby effect a spatial separation between the graphitic layers. This was resulting in an intensively different catalytic and optical behavior. The obtained IR-spectrum showed no evidence of this. The mass efficiency for the synthesis was similar to urea as precursor. 


\section{Entry 4: Dicyandiamide}

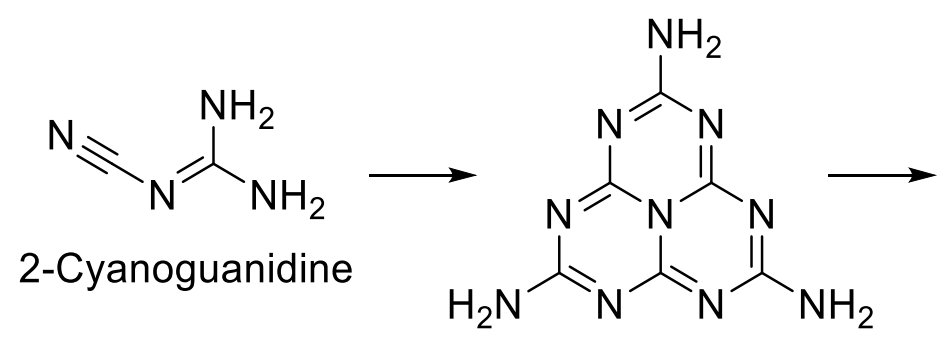<smiles>CCC1=NC2=NC(C(C)C)=NC3=NC(N(CC)C(C)C)=NC(=N1)N23</smiles>

Scheme 43: Synthesis representation of the obtained carbon nitride.

The utilization 2-cyanoguanidine, or dicyandiamide, resulted in high mass efficiency of $38 \%$ and high catalytic performance for the oxidation of benzylic alcohol. The obtained carbon nitrides have similar physical behavior to melamine-based carbon nitrides. Based on the low condensation state of the precursor the incorporation of modification can be applied to the precursor. The addition of additives should result in the incorporation of those additives into modified melamine and then result in structural defects in the final carbon nitride grid. This concept and the results will be discussed for the entries 7-17, where this strategy was investigated. 


\section{Post synthesis modification of the surface of carbon nitride}

\section{Entries 5 and 6 surface modifications}
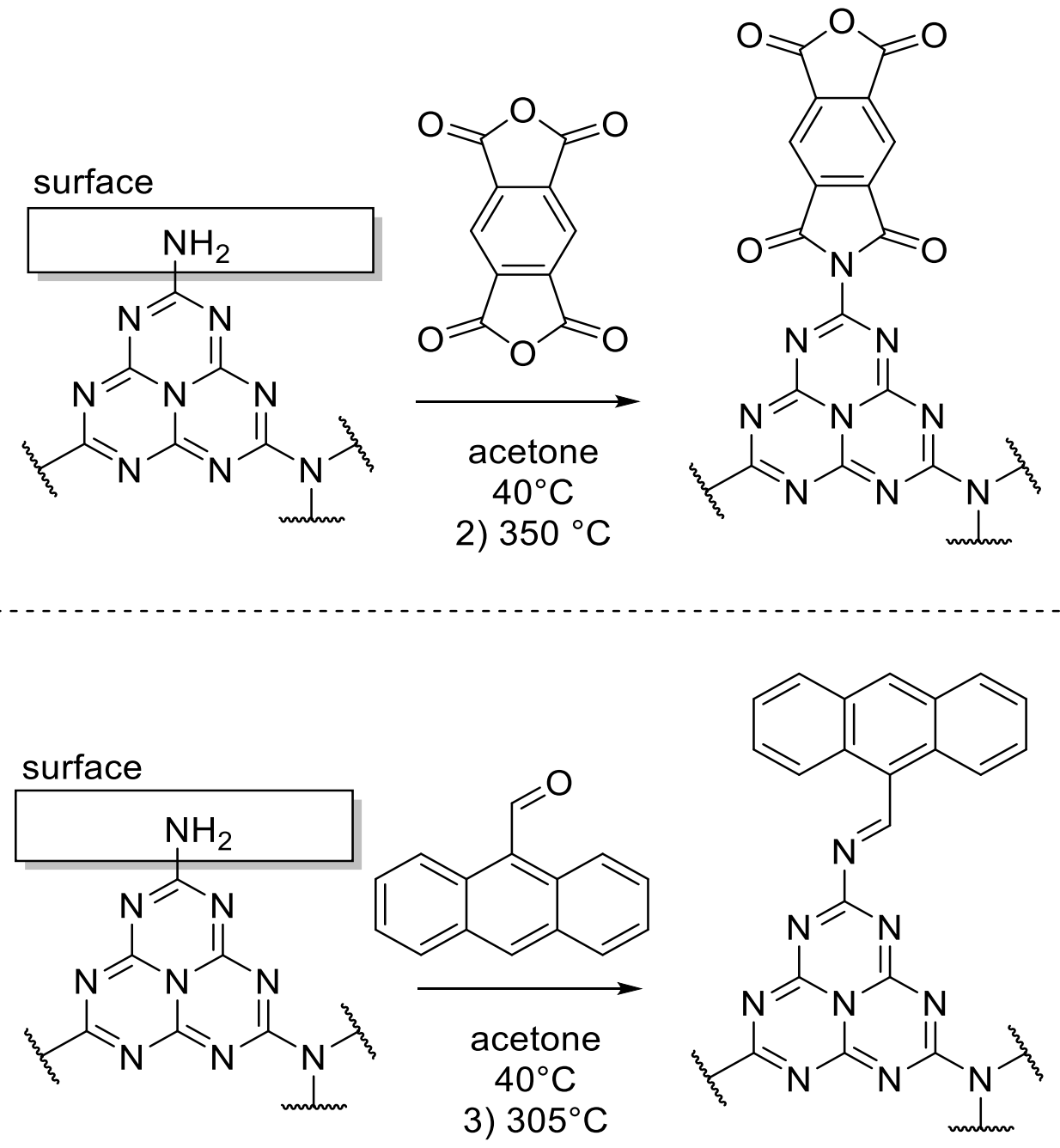

Scheme 44: Synthesis representation of the obtained carbon nitride.

Attempts to modify the surface of the obtained carbon nitride grid were investigated. The obtained carbon nitride was therefore mixed with modification agent and after evaporation of the solvent (acetone) a homogenous mixture was obtained. Heating the mixture at boiling temperature of the additive resulted in modification of the carbon nitride. The obtained materials showed a drastic change in color (entry 5 green and entry 6 brown). Further a change in catalytic behavior was observed and the IR additional IR-band could be observed. A thermal surface modification of the carbon nitride shows a possible synthetic route for the design setup. 


\section{Incorporation of grid defect in carbon nitride}

The utilization of dicyandiamide offers the possible creation of carbon nitride with statistical embedded defect in the grid structure. Several different additives were examined for the incorporation. The concept was based on the idea that cyano groups of additives can efficiently be incorporated into the formation of melamine derivates which further condense into the final carbon nitride (Scheme 44). A total of ten different additives were attempted with 5 mass $\%$ of the precursor, with distinctive changes in IR and catalytical behavior. The synthesis yields were comparable to the pure monomer.<smiles>N#CN=C(N)N</smiles><smiles>NC1=NC2=NC(N)=NC3=NC(N)=NC(=N1)N23</smiles><smiles>[R][X]</smiles><smiles>[R]C1=NC2=NC(N)=NC3=NC(N)=NC(=N1)N23</smiles><smiles>[R]C1=NC2=NC(N(CC)C(C)C)=NC3=NC(C)=NC(=N1)N32</smiles>

extended view of the graphitic grid of defect modified carbon nitride

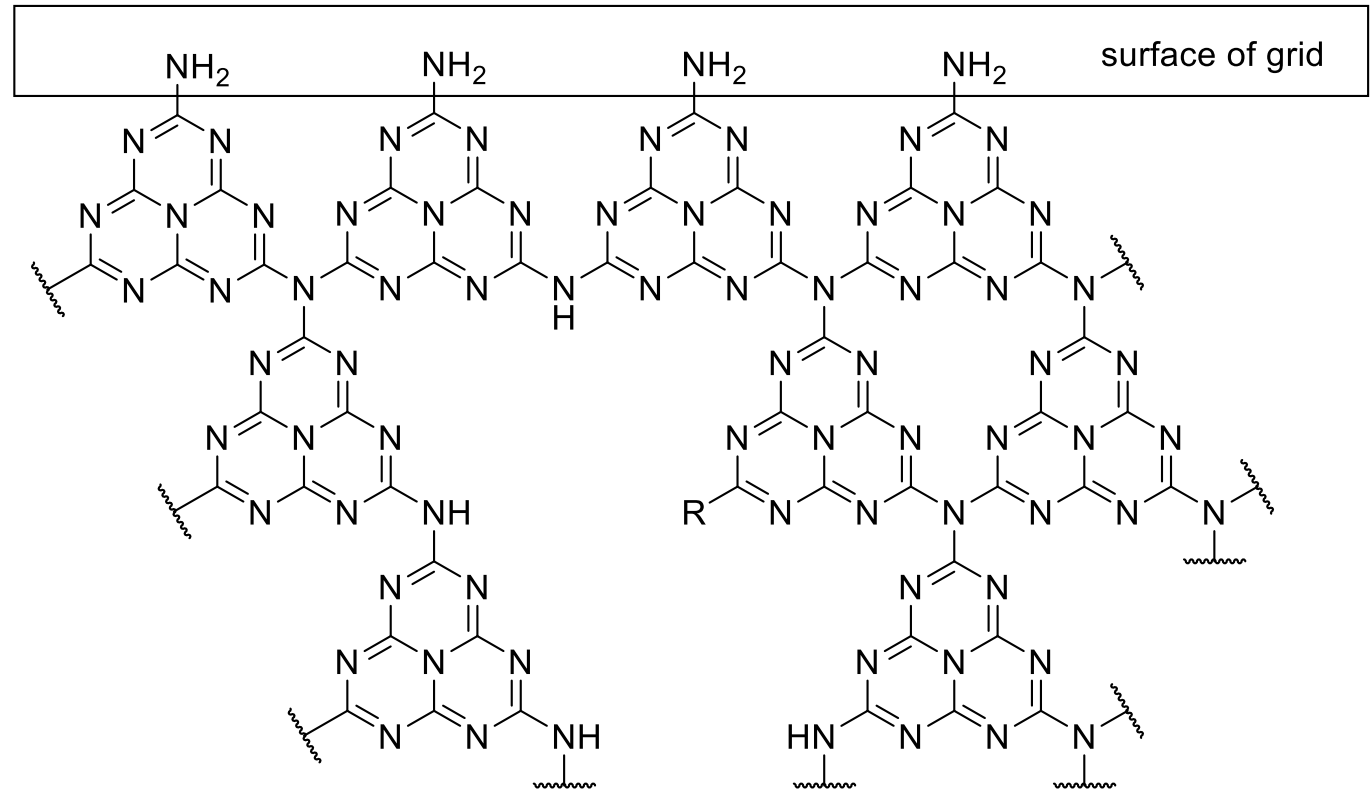

Scheme 45: Synthesis representation of the obtained carbon nitride. 


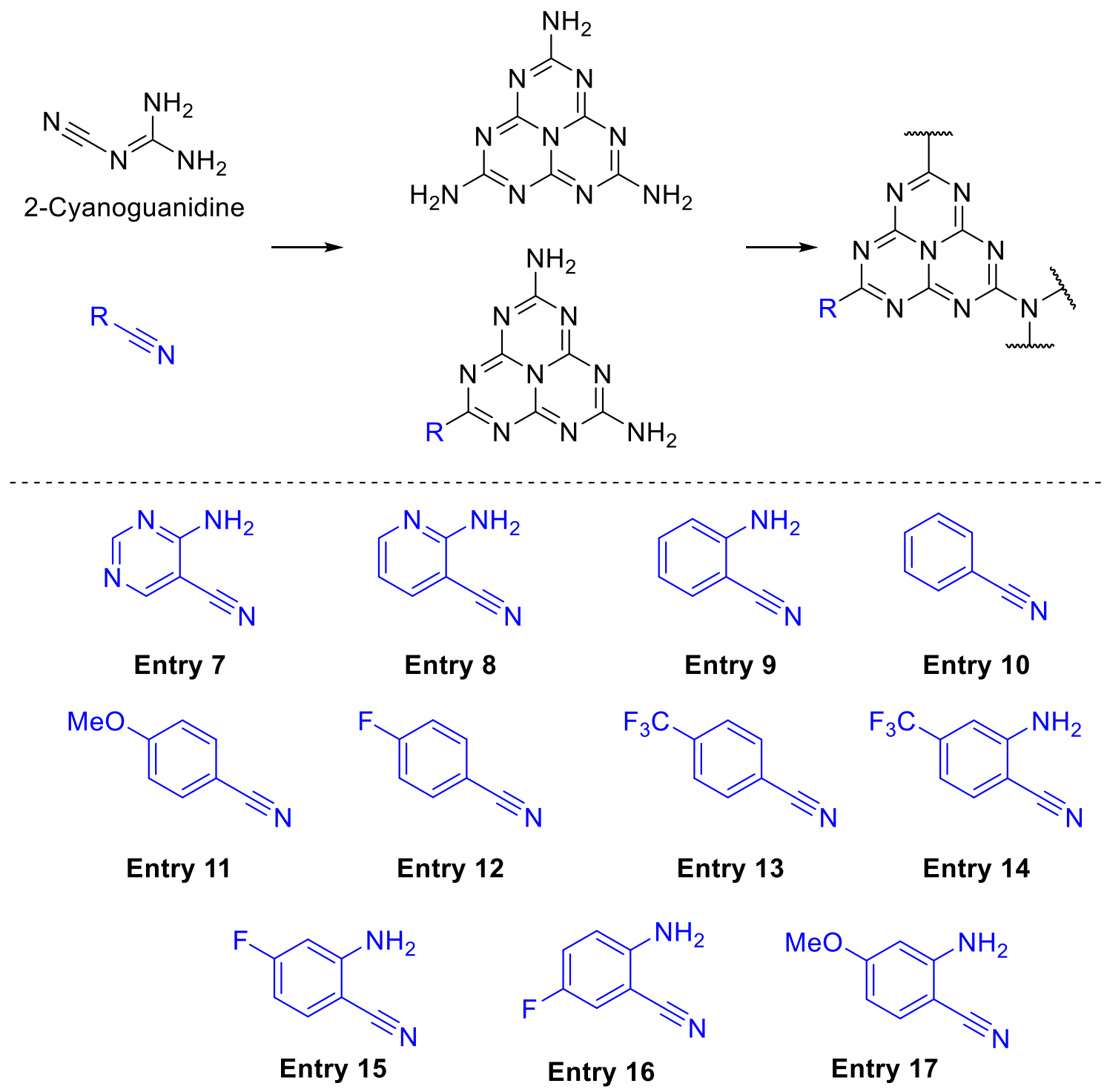

Scheme 46: Synthesis representation of the obtained carbon nitride.

Based on the obtained modified carbon nitride structures urea was modified with formamide resulting in a hydrogen atom as the formed defect, efficiently resulting in a simple gap (entry 18). The resulting structure showed improved efficacy compared to sole urea carbon nitrides for the oxidation of benzylic alcohol. Based on this result several attempts were performed to combine the incorporation of gaps and further additives and surface modifications. The resulting IR signals changed accordingly, and the catalytic performance changed to a better and worse catalytical performance. These results have to be studied further for a detailed explanation of the different combination effect. Entries 19 and 20 were the combination of gaps and surface modification with PMDA and Schiff's-base similar to entries $\mathbf{5}$ and $\mathbf{6}$. Entry 21 was the combination of gaps (Entry 18) and additive from entry 7 . This combination did not 
show any improvement on the catalytic behavior for benzylic alcohol. Entry 22 was an attempt to form gaps catalyst and functionalizing of oxamide functions, which is not further investigated so far. The utilization of oxalamide is reported to have a template effect on the forming carbon nitride. The necessary equipment and time were not present during this work. Modification of the inner grid (heptazine unit) were also prepared, but not fully characterized so far.

\subsubsection{Summary AND OUtLOOK OF THE APPLICATION OF $G-\mathrm{C}_{3} \mathrm{~N}_{4}$}

The investigations in the means of the synthesis of graphitic carbon nitride resulted in the successful development of a new design of high temperature furnaces. With the new synthesis concept, a broad variety of different carbon nitrides could be obtained. Modifications of the inner structure of the carbon nitride grid, as also at the surface of the grid could be achieved. First insights in the characterization and the synthetic protocols could be obtained by this work and could be utilized for further projects. A full characterization of the obtained carbon nitrides was done for the first obtained carbon nitride. It was not possible in the course of this work to fully characterize all the obtained modifications in detail and is given to lab members for further investigations/characterization. Through these results, graphitic carbon nitrides can be designed with modified surfaces for the absorption of different wave lengths and their utilization. Combining the initial results of this study a broad spectrum of further studies can be performed. Different reactivity of the heterogeneous photocatalyst graphitic carbon nitride could be seen, which gave the possibility to perform further studies of heterogeneous alternative methodologies. 


\section{SUMMARY AND OUTLOOK OF THE ENTIRE WORK}

The objective of this work was to investigate the new possibilities which accompanied the new advances in photochemistry of the 21st century. ${ }^{[22-23]}$ It focused on the new concepts of green chemistry to examine new methodologies to provide environment friendlier and simpler reaction concepts for the oxidation reactions in organic synthesis.

In summary, this work was able to find three new reaction methodologies, for the oxidation of alcohols, the oxygenation of heterocycles and the multicomponent reaction of secondary enamino ketones. All these three developed methodologies exhibited benefits concerning environmental friendliness, cost efficiency and safeness of the photocatalysts. All the developed methodologies were successfully applied for the synthesis of complex and bioactive compounds. Detailed mechanistic investigations provided mechanistic information of the reaction pathways. Considering the secondary objective, the physical properties of LED setups, a sufficient investigation was performed and resulted in the development of new advanced photochemical setups, which enabled higher performance and the functionalization of photochemistry in flow. The third objective was designing of graphitic carbon nitrides, this led to the successful development of large-scale synthesis of catalysts. Initial investigations of the graphitic carbon nitrides showed potential for future applications.

Firstly, the chosen oxidation of alcohols was successfully achieved by utilization of 9fluorenone in DMSO. This system was able to oxidize a broad spectrum of primary and secondary benzylic alcohols. Extended investigation showed that the oxidative protocol could be applied for the oxidation of heterocyclic and secondary aliphatic alcohols additionally. The concept was able to perform without the necessity of any additive to the reaction, which was beneficial considering the environmental friendliness and simplicity of the methodology. 


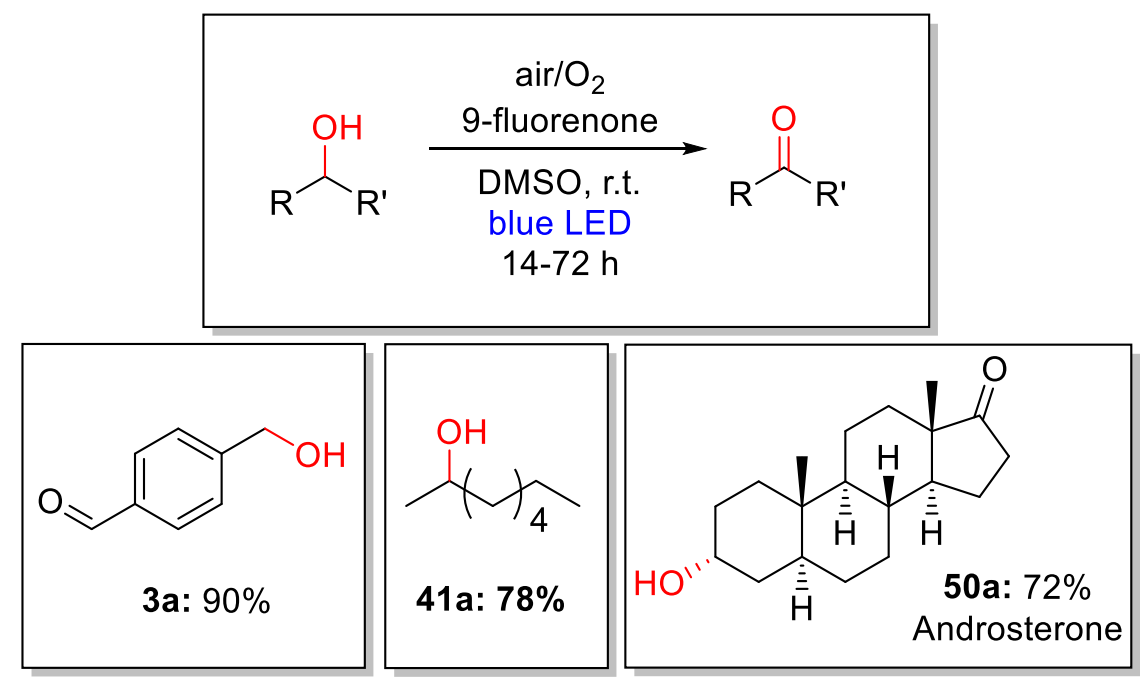

Scheme 47: Summary of the 9-fluorenone catalyzed alcohol oxidation and selected substrates.

Among the investigated substrate-product compounds was the product veratraldehyde which is a key intermediate in several synthetic procedures. ${ }^{[80]}$ The central achievement was the successful application of this methodology for the transition metal-free photocatalytic oxidation of steroid substrates, which was unpreceded at the begin of this work (e.g. Scheme 47, 50a). The examination of several different further natural substrate classes for this oxidation methodology showed a variety of further possible reactions for new research, like the generation of formic acid from sugars and cellulose. The degradation of alcohol containing structures like lignin and the oxygenation of alicyclic double bonds were found. Last of the mentioned reaction was shown by the substrate $\mathbf{5 3}$ b, utilizing cholesterol as a starting material. A later, performed investigation of another research groups showed a broad variety of transition metal-free oxidation protocols for this target compound. [126] The mechanistic investigations of this work provided a plausible mechanism for the reaction and lead to kinetic data which was useful for the examination of physical properties of the reaction setup and general mechanistic possibilities of photocatalysis. This led to the development and examination of an own concept for photocatalysis in flow. In summary, the oxidation of alcohol is an old topic of chemistry which is continuously receiving attention due to its importance to chemistry, especially with the renewed vigor of photochemical advances and green chemistry.

Secondly, the investigations of oxygenation of heterocycles were performed. Indoles and pyrroles offer viable starting material in the synthesis of complex natural 
compounds and bioactive compounds. The oxidation, or more precise oxygenation, at the positions 2 and 3 of the indole structure reversed the aromaticity of the system and increase the reactivity of the compound to form spiro-compounds and further other complex structures. By the utilization of rose bengal in a mixture of DMF and water the photocatalytic reactivity could be achieved with excellent yield. The developed methodology was able to oxygenate successfully protected and unprotected indoles equally. Additionally, it was able to oxygenate pyrroles to access highly reactive maleimides, which are valuable for the generation of bioconjugates and further applications. The protocol could decarboxylate aldehyde and carbonic acids at positions 2 and 3 , which were oxygenated afterwards in the same reaction. For other substitution patterns in positions 2 and 3 the methodology could be used to gain access to ortho-formyl/acyl anilidines. The yield of the anilidines could be increased by utilization of $\mathrm{K}_{2} \mathrm{CO}_{3}$ as an additive. After establishing and investigating a substrate scope of 30 different structures, the methodology was used for the synthesis of 5 different bioactive compounds, 7-azaisatin (Scheme 48, 101b), spiro-compounds (103b) and other more complex bioactive compounds via a multistep one-pot synthesis concept. In a final attempt, a complex steroid indole compound (105b) was created in a four component one-pot synthesis with good yield.
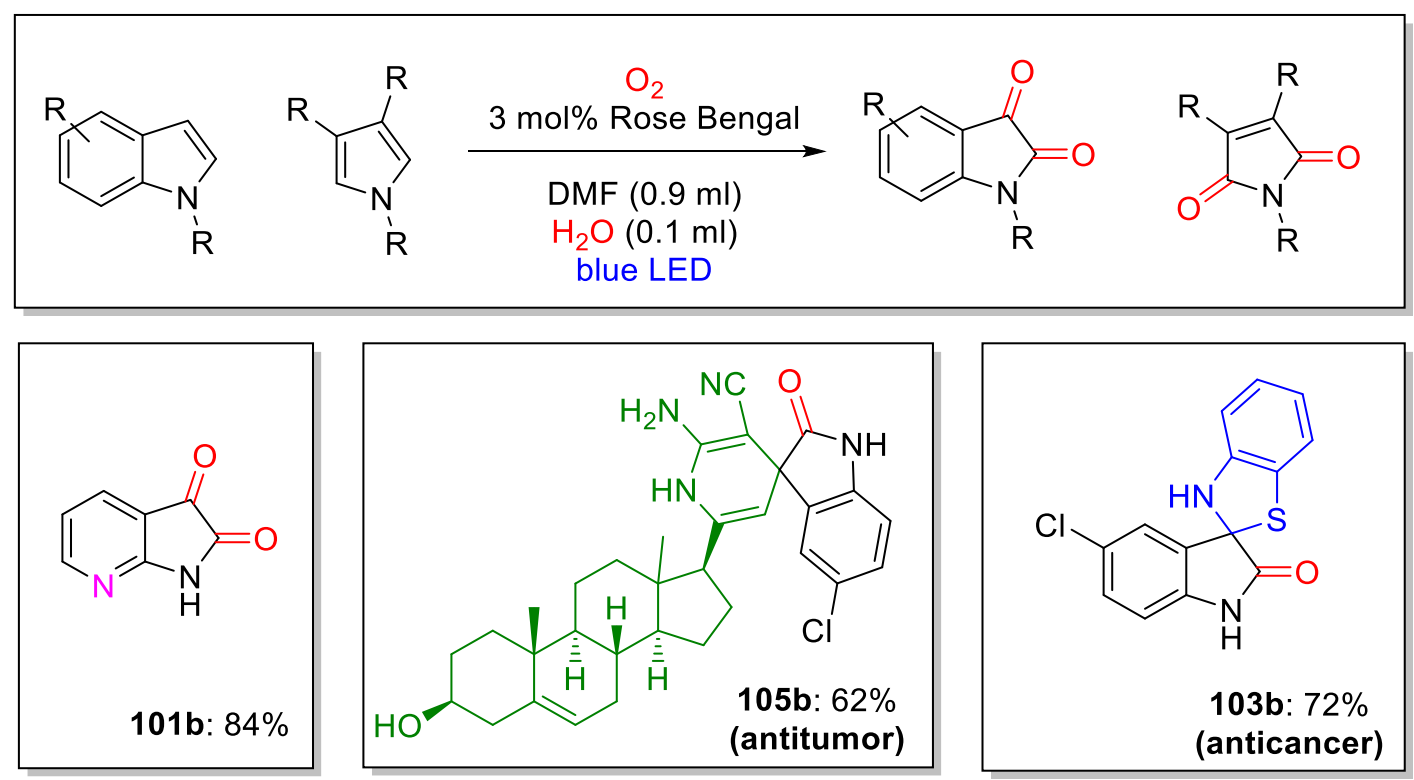

Scheme 48: Summary reaction of the rose bengal catalyzed oxygenation and three of the synthesized complex structures. 
The concluding mechanistic investigations showed new insights for the proposed mechanism which includes an energy transfer (ET) and a singlet electron transfer (SET). The individual reaction steps were performed via involvement of rose bengal, to provide the photochemical driving force of the reaction. In summary, a mild, efficient and broad protocol for the oxygenation of indole and pyrrole substrates for the use in complex synthetic protocols was achieved. For the perspective of green chemistry, the use of DMF as part of the solvent mixture has to be criticized to some extent, due to it toxicity and environmental impact. Compared to the multitude of different synthetic protocols involving these heterocycles two different solvents stand out. The first were alcohols and foremost ethanol. Many of the ethanol-based reaction included high temperatures and energy consuming pathways. The second solvent was DMF, which is used in many cases due to its excellent ability to solve the indole substrates. In this case, the use of DMF was not avoidable to achieve good reactivity. Based on many other protocols, the use of DMF was a viable green decision, due to the use of low energy consuming LED-light sources and the mild conditions, which provided energybased benefits to this new methodology.

Thirdly, the reactivity of secondary enamino ketones was investigated. The fourcomponent reaction was of interest due to its singlet oxygen initiated 1,2-acyl shift, which is unusual for singlet oxygen. Through the optimization process the methodology was able to achieve fast and efficient transformation within six hours. The solvent was also one of the reaction partners and was necessary for the formation of the final ester product. The only limitation of the methodology so far found is the necessity of an aromatic system attached to the amine. Different alcohols could be successfully utilized as reaction partners and solvents for the reaction. Based on a broad absorption spectrum of the chlorophyll, the reaction was able to perform at different wavelengths.

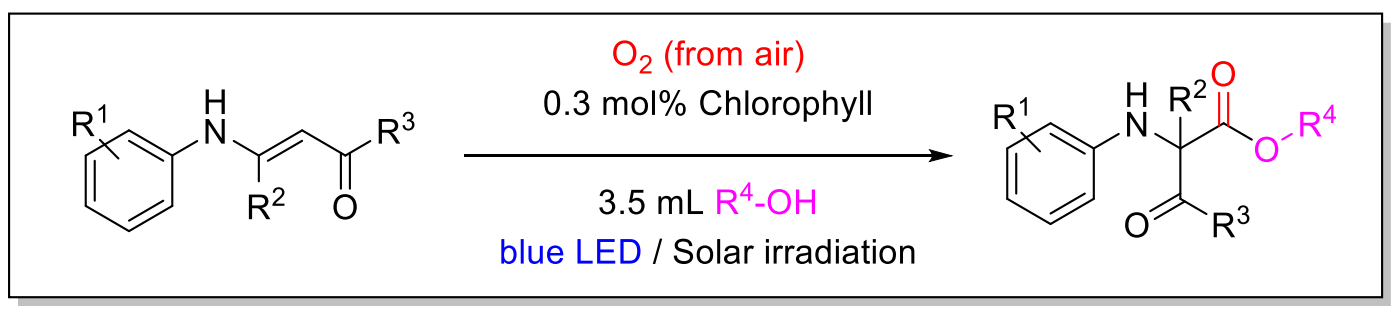

Scheme 49: Summary reaction of the rose bengal catalyzed oxygenation and three of the synthesized complex structures. 
Utilizing solar irradiation showed that the methodology can be used efficiently, even in the large scale. The investigations showed that the synthesis can be achieved starting from aniline and diketone directly, with the enamino ketones forming in situ. Through kinetic and mechanistic experiments, a plausible mechanism for the reaction was provided. The formed product showed a not naturally appearing structural stereo center, which could lead to the discovery of bioactivity in future research. In perspective of green chemistry this offers benefits. Bulk chemicals could be used as starting materials directly and the catalyst was plant based and easily available. Based on this work and similar works on this field suitable methodologies now exist for a possible library synthesis of this type of structure. A bioactivity screening should be investigated for this kind of structure class.

Fourthly, the synthesis of graphitic carbon nitride was performed in this work. Safe and inexpensive means were found to build multigram scale setups for the high temperature pyrolysis to form the photoactive semiconducting material. Initial smallscale experiments provided the necessary information needed to construct and define the required safety concerns and their solutions. Over 20 different modifications of graphitic carbon nitride were successfully synthesized in the course of this work. With further characterization of the different semiconductors and comparison with the developed benchmark test will provide valuable insights into this field of photocatalysis for researchers who are designing new forms of graphitic carbon nitrides. The field of carbon nitrides has been intensively investigated and expanded in the last 20 years and continues, due to its broad capabilities in wastewater purifications, hydrogen production and further reactivities. 


\section{EXPERIMENTAL PART}

\subsection{GENERAL REMARKS}

Commercial reagents were used without purification and reactions were run under $\mathrm{O}_{2}$ atmosphere with the exclusion of moisture from reagents using standard techniques for manipulating air-sensitive compounds.

${ }^{1} \mathrm{H}$ NMR spectra $(300,400$ and $500 \mathrm{MHz})$ and ${ }^{13} \mathrm{C}$ NMR spectra $(75.58,100.62$ and $125.71 \mathrm{MHz}$ ) were recorded using Bruker spectrometers AVANCE III 300, AVANCE III HD 400, AVANCE III 400, AVANCE III HD 500 and Varian spectrometers Mercury VX 300, VNMRS 300 and Inova 500 with $\mathrm{CDCl}_{3}$ and DMSO-D 6 as solvent. NMR spectra were calibrated using the solvent residual signals $\left(\mathrm{CDCl}_{3}: \delta^{1} \mathrm{H}=7.26, \delta^{13} \mathrm{C}\right.$ $=77.16)$.

ESI mass spectra were recorded on Bruker Daltonic spectrometers maXis (ESIQTOF-MS) and micrOTOF (ESI-TOF-MS). GC-MS mass spectra were recorded on Thermo Finnigan spectrometers TRACE (Varian GC Capillary Column; wcot fused silica coated CP-SIL 8CB for amines; $30 \mathrm{~m} \times 0.25 \mathrm{~mm} \times 0.25 \mu \mathrm{m}$ ) and DSQ (Varian FactorFour Capillary Column; VF- $5 \mathrm{~ms} \quad 30 \mathrm{~m} \times 0.25 \mathrm{~mm} \times 0.25 \mu \mathrm{m}$ ). Gas chromatography was performed on an Agilent Technologies chromatograph $7890 \mathrm{~A}$ GC System (Supelcowax 10 Fused Silica Capillary Column; $30 \mathrm{~m} \times 0.32 \mathrm{~mm} \times 0.25 \mu \mathrm{m})$. GC calibrations were carried out with authentic samples and $n$-dodecane, or biphenyl, as an internal standard.

In the section 6 mentioned citation refer to literature mention of analytic data (checked by SciFinder, or direct comparison with the literature source).

Absorption-emission spectra were recorded on a Jasco FP-8500 Spectrofluorometer and UV/Vis spectra were recorded on a Jasco V-770 Spectrophotometer. Reference for reported products was given after the name of the product. The emission spectrum of self-constructed LED reaction setups was recorded by an Ocean Optics Flame-T equipped with a P200-UV-Vis probe. 


\subsection{EXPERIMENTAL PROCEDURE AND DETAILS FOR ALCOHOL OXIDATION}

\section{Photochemical Setup}

The reaction setup is depicted in Figure S1. The reaction setup consisted of a selfconstructed light source configuration, made up of a crystallizing dish with a diameter of $140 \mathrm{~mm}$. Inside of the crystallizing dish, commercially available $5 \mathrm{~m}$ LED-Strip was glued with separable LED elements. In total, $3 \mathrm{~m}$ LED strip was used in a crystallizing dish, with a total power of $24 \mathrm{~W}$. Light intensity of the light source could be adjusted by a self-constructed dimmer. Construction of the reaction setup and the dimmer was performed by the electronic services of the faculty for chemistry of the Georg-AugustUniversität Göttingen. Cooling of the setup was performed by a commercially available $120 \mathrm{~mm}$ computer fan. To ensure constant room temperature the dimmer setting was used at $50 \%(12 \mathrm{~W})$. During the first experiment the temperature was monitored inside the crystallizing dish and did not exceed the room temperature $\left(25-30^{\circ} \mathrm{C}\right)$. Magnetic stirring was performed with $250 \mathrm{rpm}$.
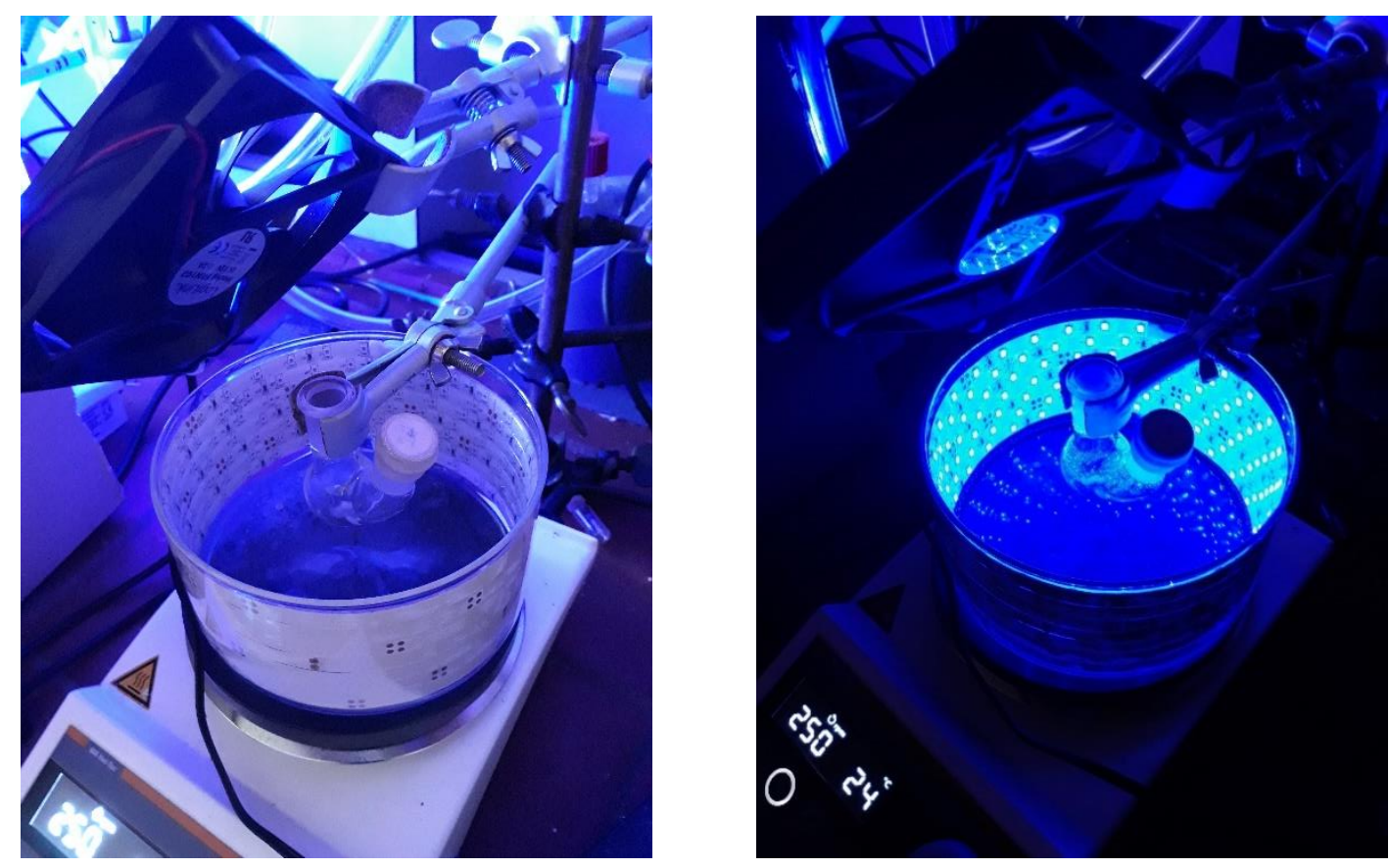

Figure 44: Photochemical setup for the alcohol oxidation under air conditions. 
<smiles>[R]C(O)c1ccccc1</smiles>

$$
\begin{gathered}
\text { PC }(3 \mathrm{~mol} \%) \\
\text { DMSO }(1.0 \mathrm{~mL}) \\
\underset{\text { blue LED }}{\text { r.t, } 14-72 \mathrm{~h}}
\end{gathered}
$$<smiles>[R]C(=O)c1ccccc1</smiles>

\section{Procedure 1A:}

$10 \mathrm{~mL}$ two-necked flask containing a stirring bar was charged with $0.25 \mathrm{mmol}$ substrate, $0.0075 \mathrm{mmol}$ of 9-fluorenone and $1.0 \mathrm{ml}$ DMSO. The resulting mixture was stirred for 14-72 $\mathrm{h}$ under blue LED irradiation (the progress can be monitored via GCMS or TLC). Then, the resulting mixture underwent an aqueous workup (using distilled water; or brine in case of slurry phase separation) and was extracted three times with ethyl acetate. The combined organic layers were dried over anhydrous $\mathrm{Na}_{2} \mathrm{SO}_{4}$, filtered and concentrated in vacuo. Products were purified using silica gel chromatography with ethyl acetate and $n$-hexane as solvents (typically 20:80 ethyl acetate: $n$-hexane). Compounds which were not visible under UV light were stained with $\mathrm{KMnO}_{4}$ solution.

\section{Procedure 1B:}

Modification to perform under pure oxygen atmosphere:

$10 \mathrm{~mL}$ two-necked flask containing a stirring bar was charged with $0.25 \mathrm{mmol}$ substrate, $0.0075 \mathrm{mmol}$ of 9 -fluorenone and $1.0 \mathrm{ml}$ DMSO. After purging the flask three times with vacuum and two times with nitrogen, $\mathrm{O}_{2}$ atmosphere was incorporated through an $\mathrm{O}_{2}$-filled balloon. Afterwards dry DMSO $(1.0 \mathrm{~mL})$ was added. The resulting mixture was stirred for 24-72 $\mathrm{h}$ under blue LED irradiation (the progress can be monitored via GC-MS or TLC). Then, the resulting mixture underwent an aqueous workup (using distilled water; or brine in case of slurry phase separation) and was extracted three times with ethyl acetate. The combined organic layers were dried over anhydrous $\mathrm{Na}_{2} \mathrm{SO}_{4}$, filtered and concentrated in vacuo. Products were purified using silica gel chromatography with ethyl acetate and $n$-hexane as solvents (typically 20:80 ethyl acetate:n-hexane). Compounds which were not visible under UV light were stained with $\mathrm{KMnO}_{4}$ solution. 


\section{KIE experiment}

Synthesis of deuterium-labeled benzyl alcohol<smiles>[2H]C([2H])(O)c1ccccc1</smiles>

Synthesis was performed according to the literature. ${ }^{[1]} \mathrm{LiAID}_{4}(63 \mathrm{mg}, 1.5 \mathrm{mmol}$, 0.6 eq) was suspended in THF $(5 \mathrm{~mL}, 2 \mathrm{~mL} / \mathrm{mmol}$ LiAID 4$)$ and cooled to $0{ }^{\circ} \mathrm{C}$. A solution of methyl benzoate $(0.31 \mathrm{~mL}, 2.5 \mathrm{mmol}, 1 \mathrm{eq})$ in THF $(5 \mathrm{~mL}, 0.15 \mathrm{~mL} / \mathrm{mmol}$ ester) was added dropwise via syringe. The reaction slurry was allowed to warm to room temperature over night. It was quenched by addition of $15 \mathrm{~mL}$ EtOAc. After additional $30 \mathrm{~min}, 3 \times 5 \mathrm{~mL} \mathrm{H}_{2} \mathrm{O}$ were added over $60 \mathrm{~min}$. The white suspension was filtered and the residue washed six times with $10 \mathrm{~mL}$ of $\mathrm{CH}_{2} \mathrm{Cl}_{2}$ each. The solution was dried over $\mathrm{Na}_{2} \mathrm{SO}_{4}$ and the solvent was removed under reduced pressure providing the product in >95 atom\% D and 99\% yield which was used without further purification.

MS (ESI): calcd. for $\mathrm{C}_{7} \mathrm{H}_{5} \mathrm{D}_{2} \mathrm{O}\left[\mathrm{M}+\mathrm{H}^{+}\right]: 111.0772$, found: 111.0771 .

\section{Oxidation of deuterium-labeled benzyl alcohol}

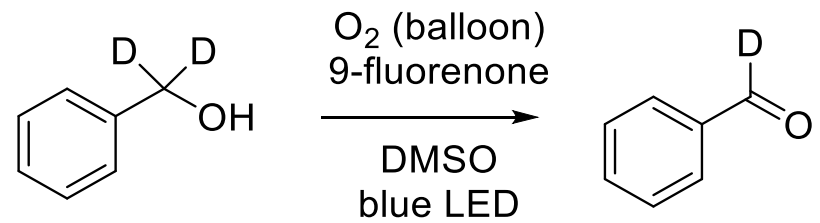

Deuterium-labelled benzyl alcohol was oxidized by the procedure described in section 5.2 Procedure B. under $\mathrm{O}_{2}$ atmosphere. After $4 \mathrm{~h}$, resulting products were characterized by GC using $n$-dodecane as internal standard and compared with a nondeuterated sample under same conditions. The calculated KIE is a result of the average of three independent runs. 


\section{Oxidation of benzyl alcohol under ${ }^{18} \mathrm{O}$-atmosphere}

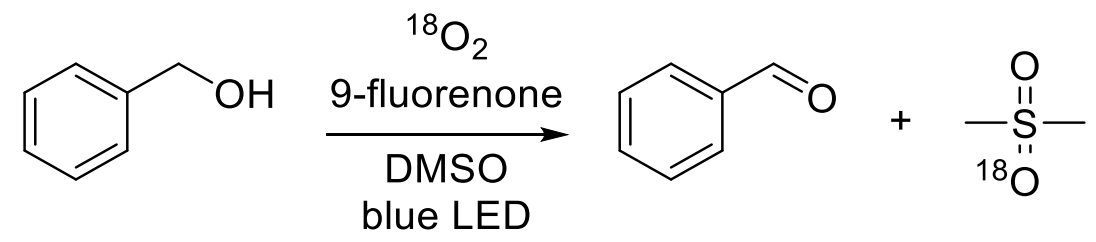

Benzyl alcohol was oxidized by the procedure described in section 4. with a 97 atom\% ${ }^{18} \mathrm{O}_{2}$ atmosphere. After $18 \mathrm{~h}$ the resulting products were characterized by GC-MS, HRMS and ESI.

MS (ESI): calcd. for $\mathrm{C}_{7} \mathrm{H}_{6} \mathrm{O}\left[\mathrm{M}+\mathrm{H}^{+}\right]:$107.0491, found: 107.0492.

MS (ESI): calcd. for $\mathrm{C}_{2} \mathrm{H}_{6} \mathrm{O}^{18} \mathrm{OS}\left[\mathrm{M}+\mathrm{H}^{+}\right]$: 97.0204 , found: 97.0203 .

MS (ESI): calcd. for $\mathrm{C}_{2} \mathrm{H}_{6} \mathrm{O}^{18} \mathrm{OS}\left[\mathrm{M}+\mathrm{Na}^{+}\right]: 119.0023$, found: 119.0023 . 


\section{Stern-Volmer Plot}

To determine the reactive species in the beginning of the photocatalytic reaction absorption-emission spectra for a Stern-Volmer plot were acquired. Firstly, a 3D spectrum for excitation and emission of 9-fluorenone was recorded in order to detect the maxima of absorption and emission. The resulting spectrum is depicted in Figure 45 with 3 absorption bands. The excitation maximum was measured at $290 \mathrm{~nm}$ and the emission maximum at $515 \mathrm{~nm}$. These wavelengths were used for further measurements.

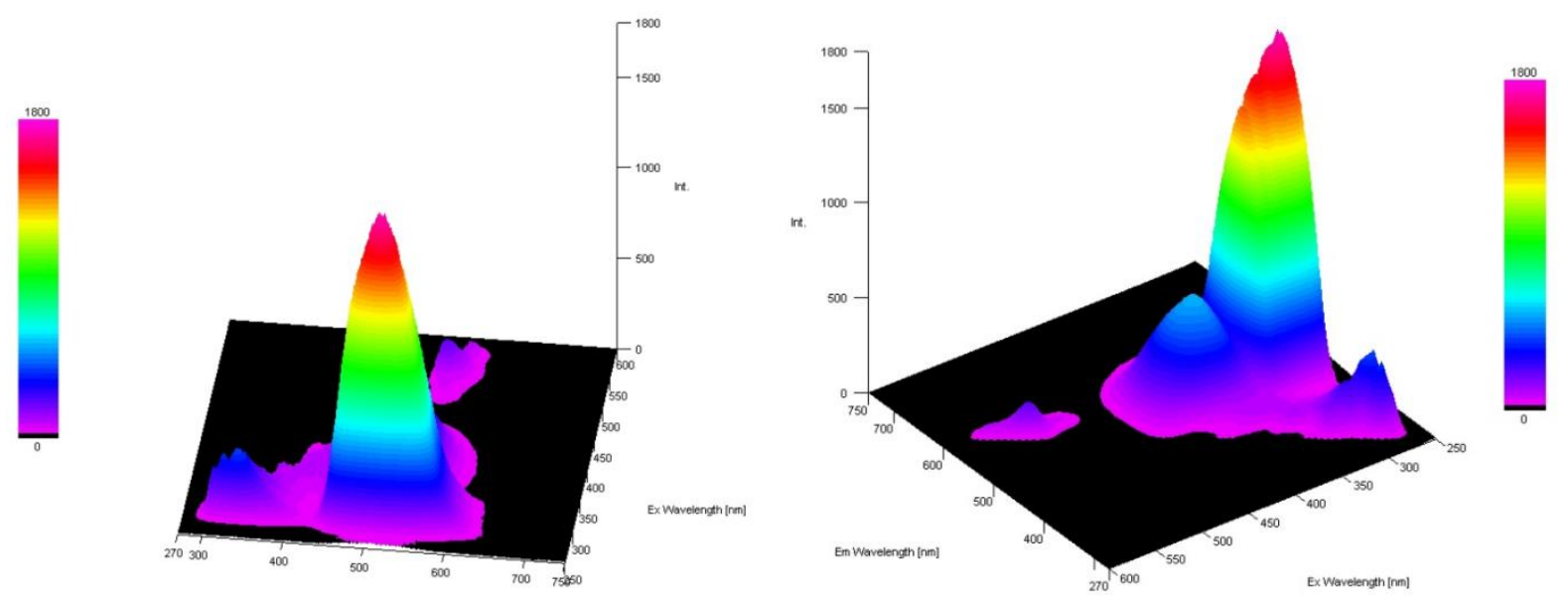

Figure 45: 3D absorbtion-emission spectrum of 9-fluorenone in DMSO.

A blank sample was recorded under $\mathrm{N}_{2}$ atmosphere without substrate and the received intensity was set as $I_{0}$. The effect of varied amounts of substrate was investigated, as also the saturation of the solution with air and oxygen. Figure $\mathbf{4 6}$ shows a summary of all three investigations. Figures 47-49 show the results for each parameter separately. Depending on the concentration of benzyl alcohol, the emission decreases significantly. Saturation with air and oxygen, respectively, shows only marginal effect in the magnitude of the uncertainty of the spectrometer. 


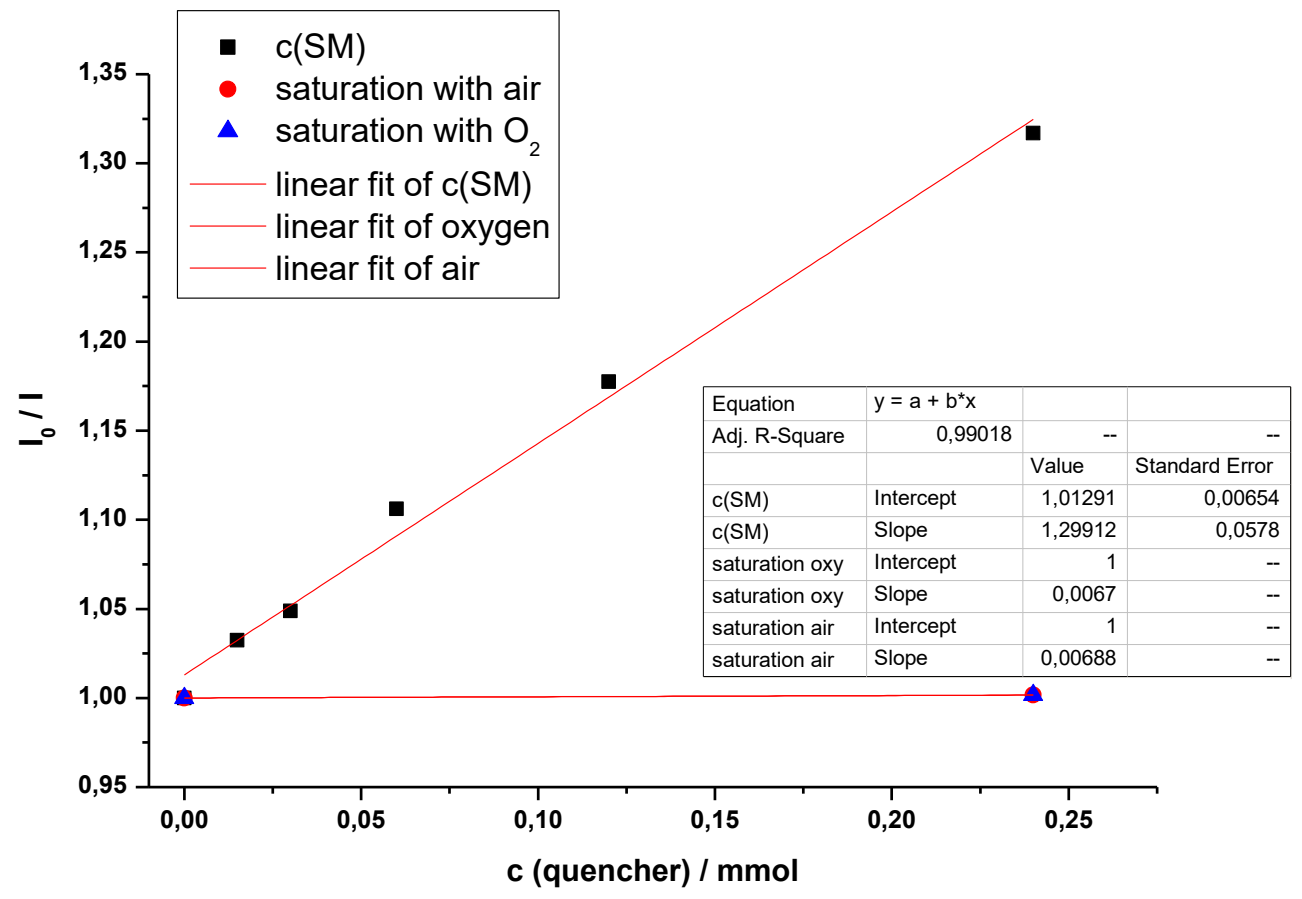

Figure 46: Stern-Volmer plot for all investigations (varied concentration of substrate and saturation with air and oxygen, respectively).

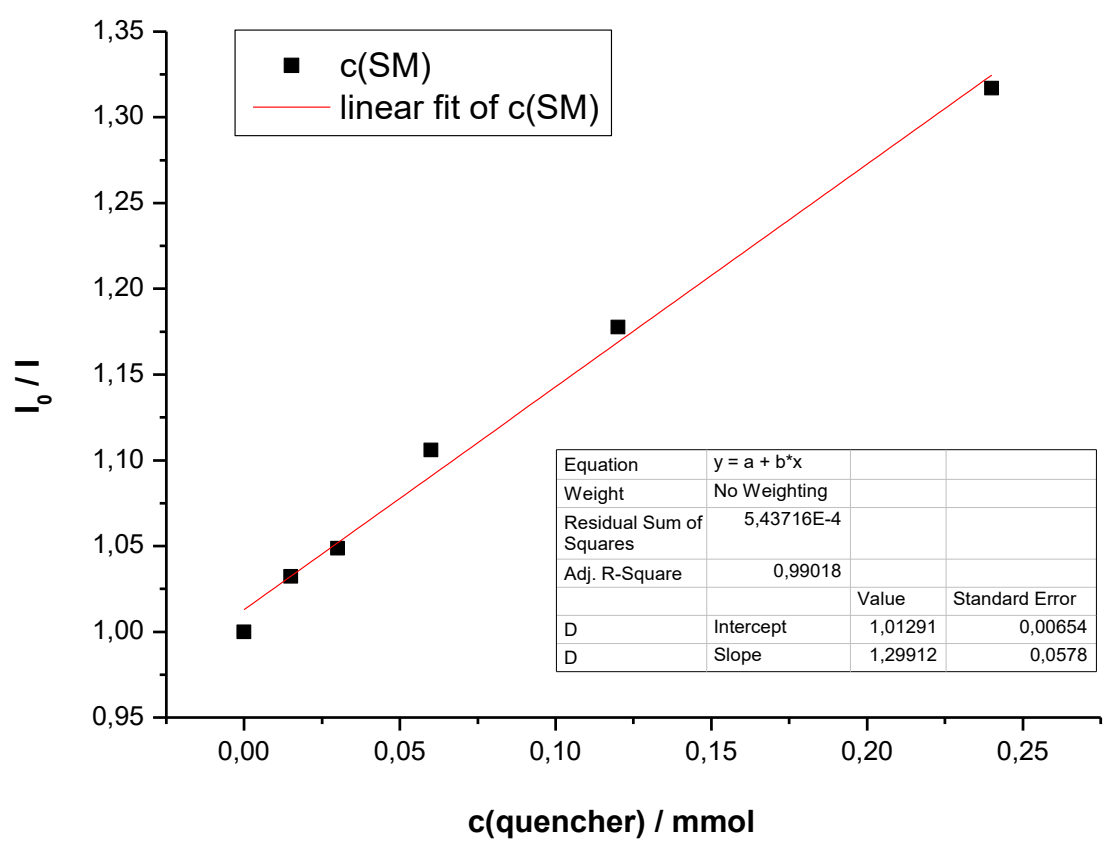

Figure 47: Quenching of the activated catalyst in dependence of the concentration of substrate benzyl alcohol. 


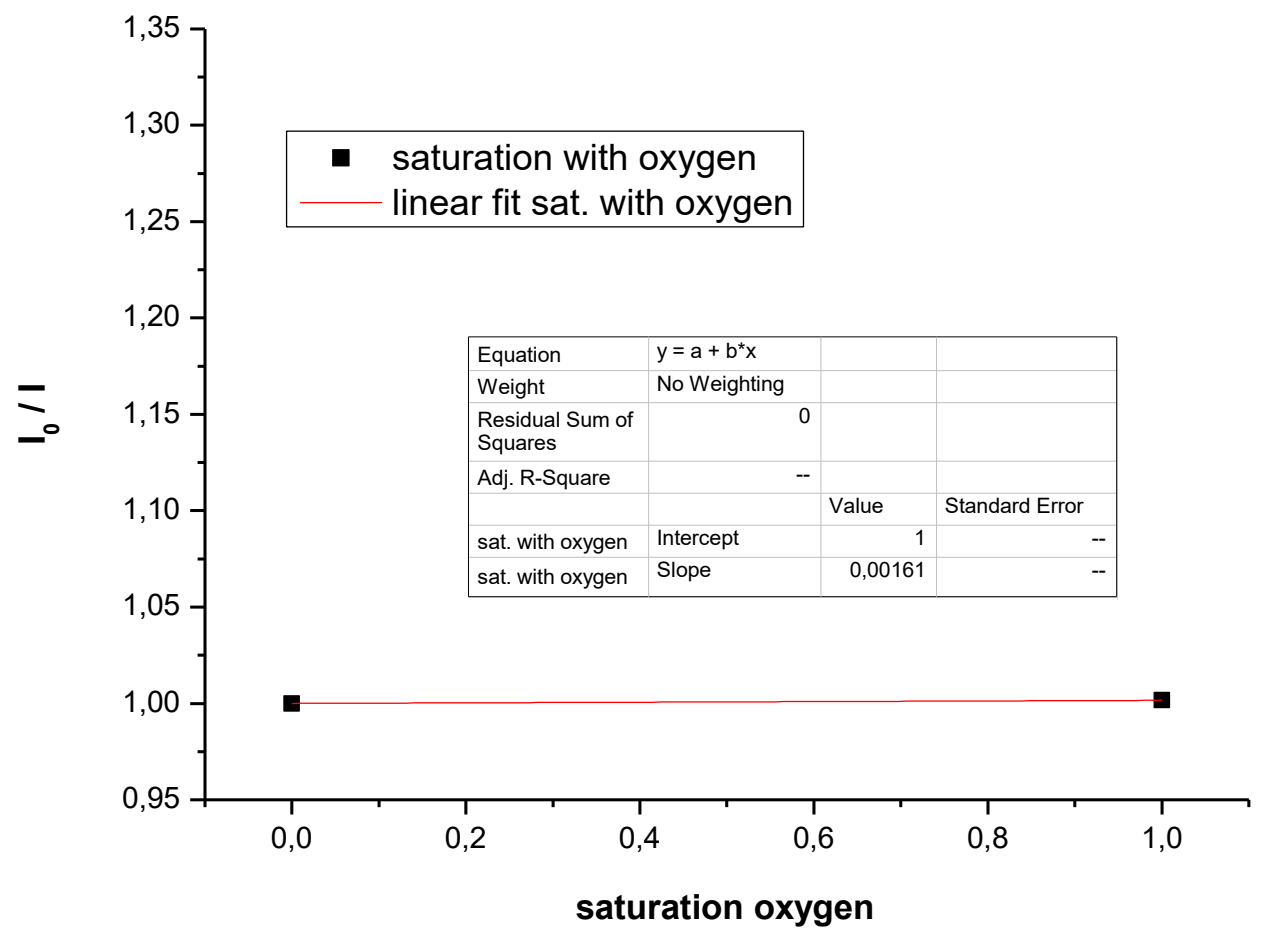

Figure 48: Influence of the saturation with oxygen.

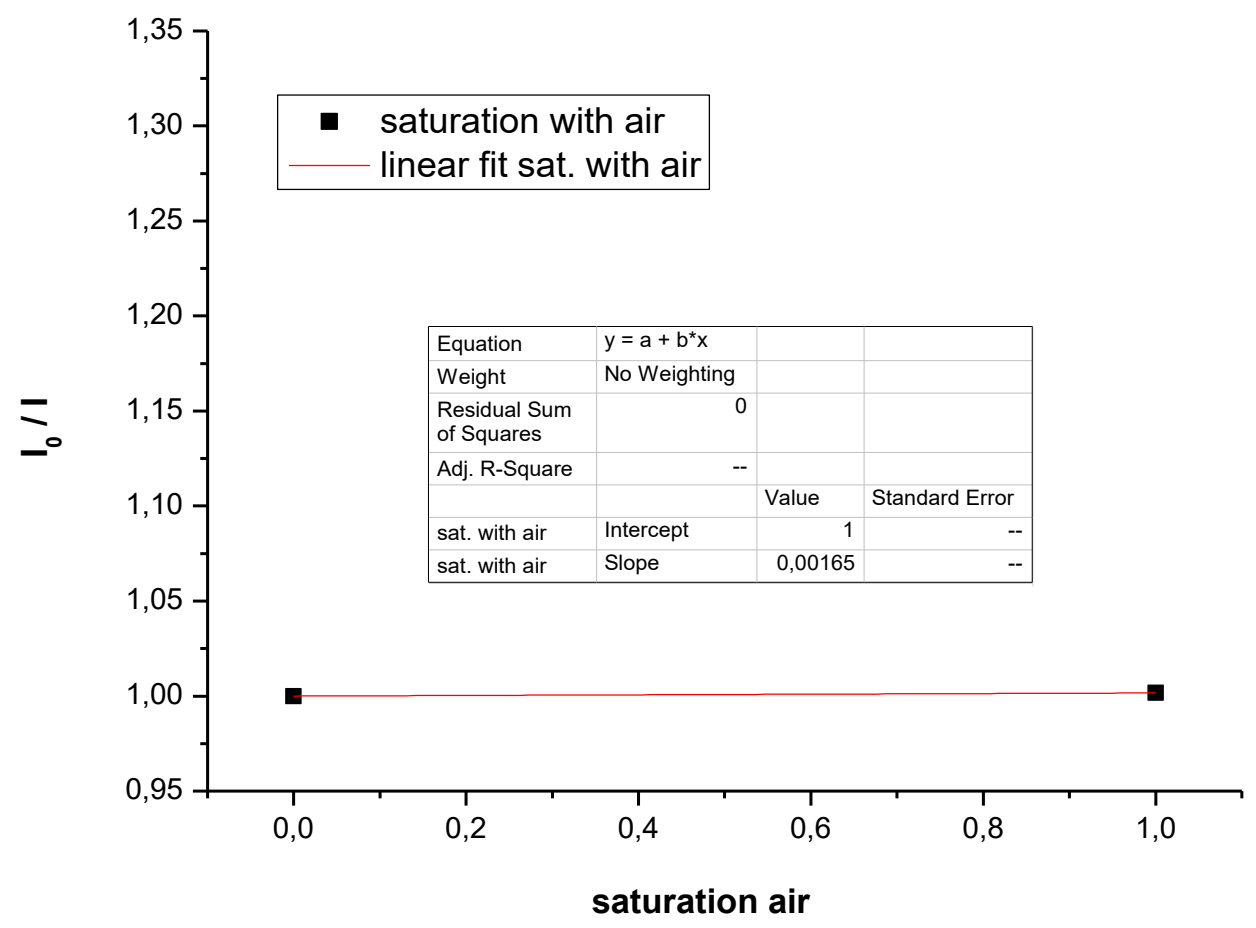

Figure 49: Influence of the saturation with air. 


\section{$\underline{\text { Test for hydrogen peroxide }}$}

To test the formation of hydrogen peroxide as side product, the reaction was performed according to the procedure described in section 5.2, Procedure 1a.

The resulting reaction mixture was extracted with ethyl acetate $(5 \mathrm{~mL})$, water $(5 \mathrm{~mL})$, aqueous and organic phase were tested for hydrogen peroxide with literature-known reactions.

$\mathrm{H}_{2} \mathrm{O}_{2}$ glowing splint test

$$
2 \mathrm{H}_{2} \mathrm{O}_{2} \stackrel{\mathrm{MnO}_{2}}{\longrightarrow} 2 \mathrm{H}_{2} \mathrm{O}+\mathrm{O}_{2}
$$

Procedure: $1 \mathrm{~mL}$ solution was taken, was transferred to a snap cap vial and $\mathrm{MnO}_{2}$ $(\sim 5 \mathrm{mg})$ was added, the forming gas was tested with a glowing splint:

Results:

Aqueous phase tested positive: production of flammable gas (oxygen) after addition of $\mathrm{MnO}_{2}$.

Organic phase tested negative.

Control aqueous phase tested negative.

Control organic phase tested negative. 
$\mathrm{H}_{2} \mathrm{O}_{2}$ luminol test

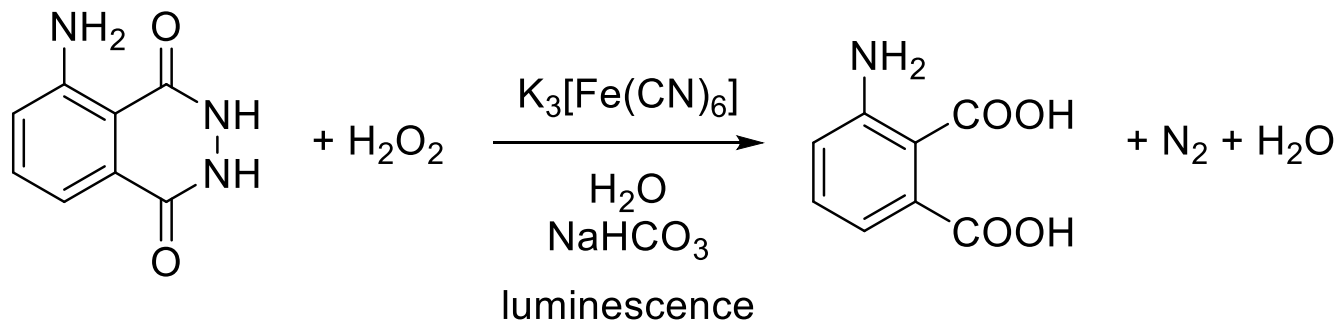

Procedure: Luminol (10 mg) was dissolved in $\mathrm{H}_{2} \mathrm{O}(5 \mathrm{~mL})$ and $\mathrm{NaHCO}_{3}(100 \mathrm{mg})$ was added to adjust the $\mathrm{pH}$ value. The resulting mixture was divided between 5 vials. For each vial, one drop of a $1 \mathrm{M} \mathrm{K}_{3}\left[\mathrm{Fe}(\mathrm{CN})_{6}\right]$ solution was added shortly before the test. $1 \mathrm{~mL}$ of the corresponding phases of the reaction mixture were added to the luminol solution. In case of peroxides, a luminescence was observed. Afterwards all samples were controlled by the addition of hydrogen peroxide solution $(30 \mathrm{~V} \%)$ for luminescence.

Results:

Aqueous phase tested positive with luminescence for over 2 minutes.

Organic phase tested negative.

Control aqueous phase tested negative.

Control organic phase tested negative. 


\subsection{EXPERIMENTAL PROCEDURE AND DETAILS FOR THE OXYGENATION OF HETEROCYCLES}

\section{Setup for photocatalytic reactions}

The reaction setup is depicted in Figure 50. The reaction setup consists of a self-constructed light source configuration, made up of a crystallizing dish with a diameter of $140 \mathrm{~mm}$. Inside of the crystallizing dish, commercially available $5 \mathrm{~m}$ LED-Strip is glued with separable LED elements. In total, $3 \mathrm{~m}$ LED strip is used in a crystallizing dish, with a total power of $24 \mathrm{~W}$. Light intensity of the light source can be adjusted by a self-constructed dimmer. Construction of the reaction setup and the dimmer was performed by the electronic services of the faculty for chemistry of the Georg-August-Universität Göttingen. Cooling of the setup is performed by a commercially available $120 \mathrm{~mm}$ computer fan. To ensure the constant room temperature, the dimmer setting was used at $50 \%(12 \mathrm{~W})$. During the first experiment the temperature was monitored inside the crystallizing dish and did not exceed room temperature $\left(25-30^{\circ} \mathrm{C}\right)$. Magnetic stirring was performed with $250 \mathrm{rpm}$. The wavelength of the LED setup is ranging from $404 \mathrm{~nm}$ to $553 \mathrm{~nm}$ with a maximum at $456 \mathrm{~nm}$.

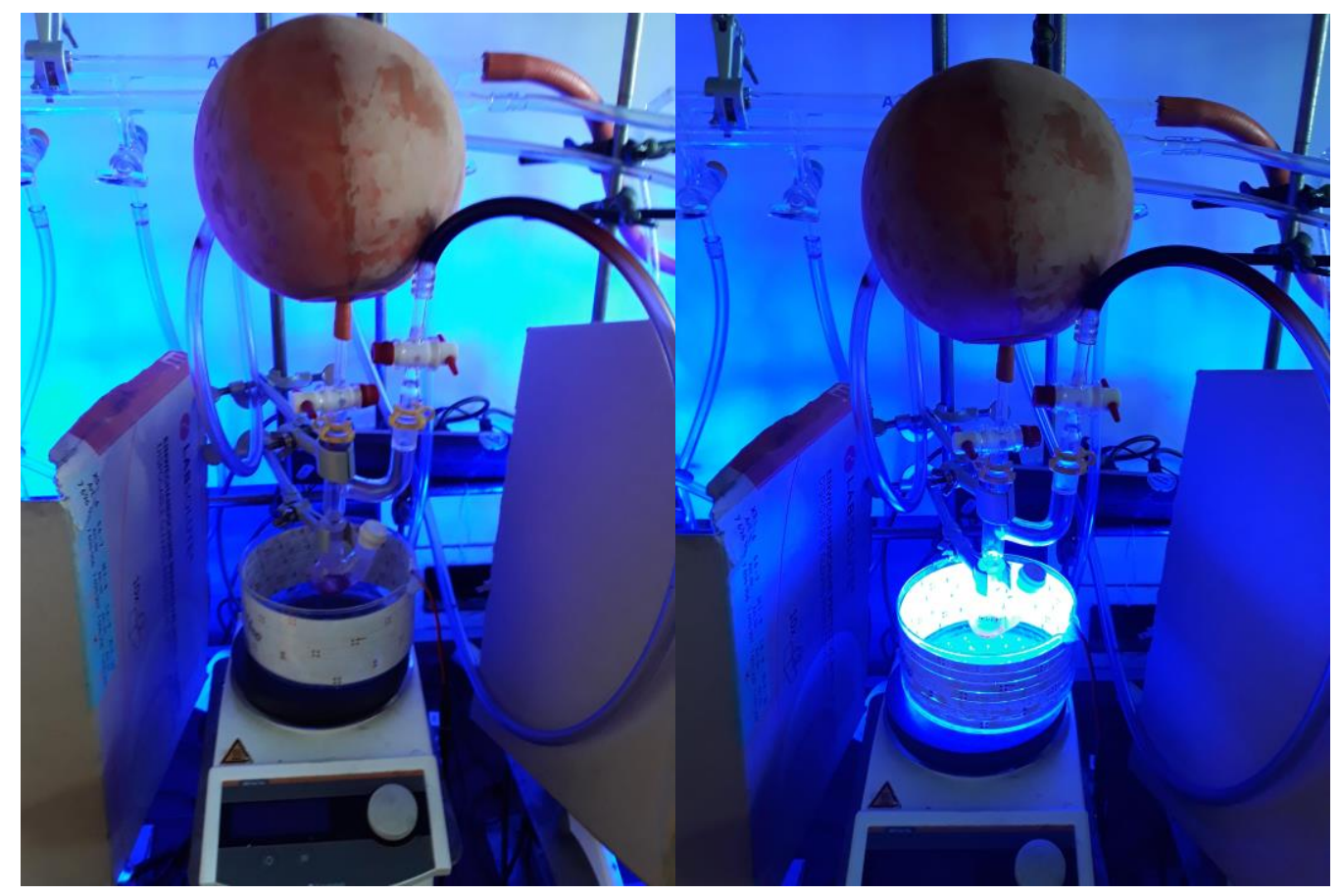

Figure 50: LED reaction setup for reactions under oxygen balloon atmosphere. 


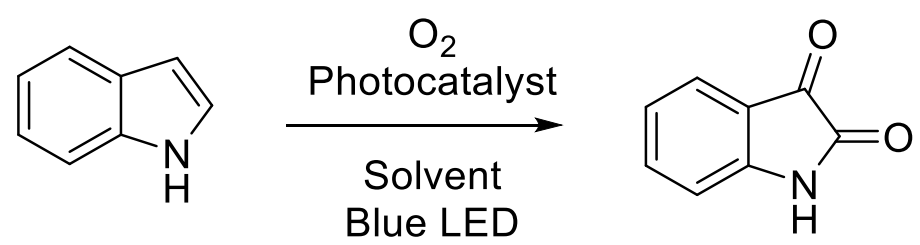

\section{Procedure 2A:}

$10 \mathrm{~mL}$ two-necked flask containing a stirring bar was charged with substrate (0.25 mmol), rose bengal $(0.0075 \mathrm{mmol})$, DMF $(0.9 \mathrm{~mL})$ and $\mathrm{H}_{2} \mathrm{O}(0.1 \mathrm{~mL})$. After purging the flask three times with vacuum and two times with nitrogen, $\mathrm{O}_{2}$ atmosphere was incorporated through an $\mathrm{O}_{2}$-filled balloon. Afterwards, dry DMF $(0.9 \mathrm{~mL})$ and degassed Milli- $Q$ water $(0.1 \mathrm{~mL})$ were added. The resulting mixture was stirred for $16-$ $48 \mathrm{~h}$ under blue LED irradiation (the progress can be monitored via TLC). Then, the resulting mixture underwent an aqueous workup (saturated $\mathrm{LiCl}$ solution) and was extracted three times with ethyl acetate (in several cases (unprotected isatins) extraction was performed with benzene). The combined organic layers were dried over anhydrous $\mathrm{Na}_{2} \mathrm{SO}_{4}$, filtered and concentrated in vacuo. Products were purified using silica gel chromatography with ethyl acetate and $n$-pentane as solvents (typically 50:50 ethyl acetate: $n$-pentane).

\section{Procedure 2B:}

$10 \mathrm{~mL}$ two-necked flask containing a stirring bar was charged with substrate (0.25 mmol), rose bengal $(0.0075 \mathrm{mmol}), \mathrm{K}_{3} \mathrm{PO}_{4}(0.125 \mathrm{mmol}) \mathrm{DMF}(0.9 \mathrm{~mL})$ and $\mathrm{H}_{2} \mathrm{O}$ $(0.1 \mathrm{~mL})$. After purging the flask three times with vacuum and two times with nitrogen, $\mathrm{O}_{2}$ atmosphere was incorporated through an $\mathrm{O}_{2}$-filled balloon. Afterwards, dry DMF $(0.9 \mathrm{~mL})$ and degassed Milli-Q water $(0.1 \mathrm{~mL})$ were added. The resulting mixture was stirred for 16-48 $\mathrm{h}$ under blue LED irradiation (the progress can be monitored via TLC). Then, the resulting mixture underwent an aqueous workup (saturated $\mathrm{LiCl}$ solution) and was extracted three times with ethyl acetate (in several cases (unprotected isatins) extraction was performed with benzene). The combined organic layers were dried over anhydrous $\mathrm{Na}_{2} \mathrm{SO}_{4}$, filtered and concentrated in vacuo. Products were purified using silica gel chromatography with ethyl acetate and $n$ pentane as solvents (typically 50:50 ethyl acetate: $n$-pentane). 


\section{Procedure 2C:}

Synthesis of 1,3-dihydro-3-(phenylimino)-2H-indol-2-one

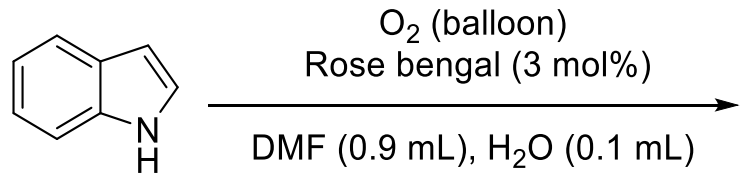<smiles>O=C1Nc2ccccc2C1=O</smiles>

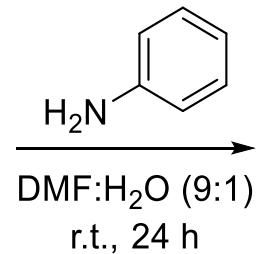

r.t., $24 \mathrm{~h}$<smiles>O=C1Nc2ccccc2/C1=N/c1ccccc1</smiles>

$1 \mathrm{H}$ Indole $(29.3 \mathrm{mg}, 0.25 \mathrm{mmol})$ and rose Bengal $(7.3 \mathrm{mg}, 0.0075 \mathrm{mmol})$ were prepared in a $10 \mathrm{~mL}$ two-necked flask. After purging the flask three times with vacuum and two times with nitrogen, $\mathrm{O}_{2}$ atmosphere was incorporated through an $\mathrm{O}_{2}$-filled balloon. Afterwards, dry DMF $(0.9 \mathrm{~mL})$ and degassed Milli-Q water $(0.1 \mathrm{~mL})$ was added. The resulting mixture was stirred for $24 \mathrm{~h}$ under blue LED irradiation (the progress can be monitored via TLC). After completion of the oxygenation reaction aniline $(27.9 \mathrm{mg}, 0.3 \mathrm{mmol})$ was the reaction was stirred for further $24 \mathrm{~h}$ at room temperature. Then, the resulting mixture underwent an aqueous workup and was extracted three times with ethyl acetate. The combined organic layers were dried over anhydrous $\mathrm{Na}_{2} \mathrm{SO}_{4}$, filtered and concentrated in vacuo. The product mixture was purified using silica gel chromatography with ethyl acetate and $n$-pentane as solvents (20:80 ethyl acetate: $n$-pentane). 


\section{Procedure 2D:}

Synthesis of Trisindoline<smiles>O=C1Nc2ccccc2/C1=N\c1ccccc1</smiles><smiles>Oc1cccc2cc[nH]c12</smiles><smiles>O=C1Nc2ccccc2C1(c1c[nH]c2ccccc12)c1c[nH]c2ccccc12</smiles>

1,3-Dihydro-3-(phenylimino)-2 $\mathrm{H}$-indol-2-one $(57.0 \mathrm{mg}, 0.25 \mathrm{mmol}), \mathrm{TsOH}(4.3 \mathrm{mg}$, $0.025 \mathrm{mmol})$ and $1 \mathrm{H}$ indole $(58.6 \mathrm{mg}, 0.5 \mathrm{mmol})$ were dissolved in ethanol $(5.0 \mathrm{~mL})$ and refluxed $18 \mathrm{~h}$ under stirring. Then, the resulting mixture underwent an aqueous workup and was extracted three times with ethyl acetate. The combined organic layers were dried over anhydrous $\mathrm{Na}_{2} \mathrm{SO}_{4}$, filtered and concentrated in vacuo. The product mixture was purified using silica gel chromatography with ethyl acetate and $n$-pentane as solvents (20:80 ethyl acetate: $n$-pentane). 


\section{Procedure 2E:}

Synthesis of 5'-chloro-spiro[benzothiazole-2(3H),3'- [3H] indol]-2'(1'H)-one:

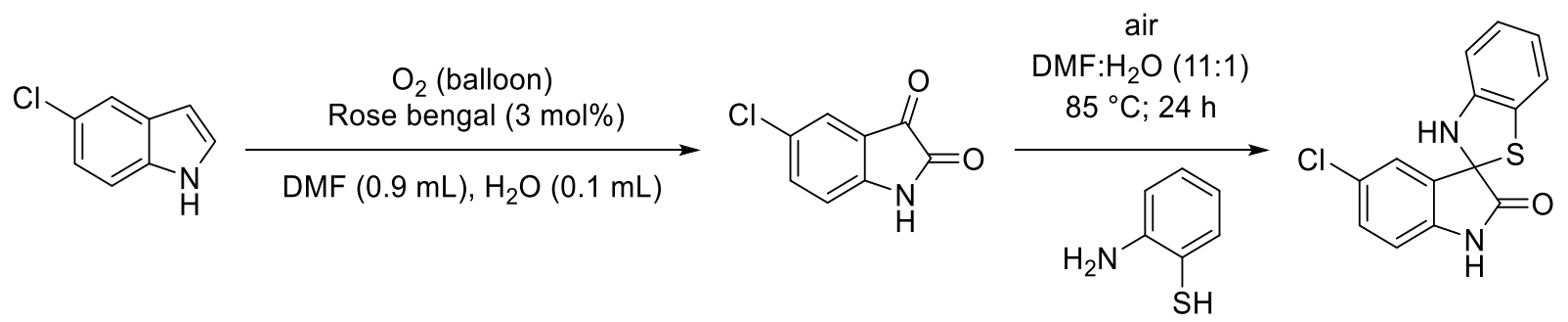

5-Chloroindole ( $37.8 \mathrm{mg}, 0.25 \mathrm{mmol})$ and rose bengal $(7.3 \mathrm{mg}, 0.0075 \mathrm{mmol})$ were prepared in a $10 \mathrm{~mL}$ two-necked flask. After purging the flask three times with vacuum and two times with nitrogen, $\mathrm{O}_{2}$ atmosphere was incorporated through an $\mathrm{O}_{2}$-filled balloon. Afterwards dry DMF $(0.9 \mathrm{~mL})$ and degassed Milli-Q water $(0.1 \mathrm{~mL})$ were added. The resulting mixture was stirred for $24 \mathrm{~h}$ under blue LED irradiation (the progress can be monitored via TLC). After completion of the oxygenation reaction 2amino-benzenethiol (37.5 mg, $0.3 \mathrm{mmol}$ ) in DMF $(0.2 \mathrm{~mL})$, was added and the LED setup replaced by an oil bath. The reaction was stirred for further $24 \mathrm{~h}$ at $85^{\circ} \mathrm{C}$. Then, the resulting mixture underwent an aqueous workup and was extracted three times with ethyl acetate. The combined organic layers were dried over anhydrous $\mathrm{Na}_{2} \mathrm{SO}_{4}$, filtered and concentrated in vacuo. The product mixture was purified using silica gel chromatography with ethyl acetate and $n$-pentane as solvents (50:50 ethyl acetate: $n$ pentane). 


\section{Procedure 2F:}

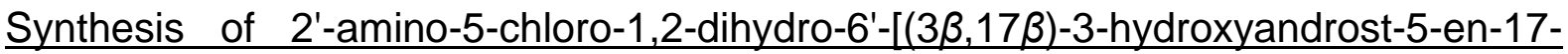
yl]-2-oxospiro[3H-indole-3,4' $\left(1^{\prime} H\right)$-pyridine]-3'-carbonitrile $(35 \mathrm{~b})$

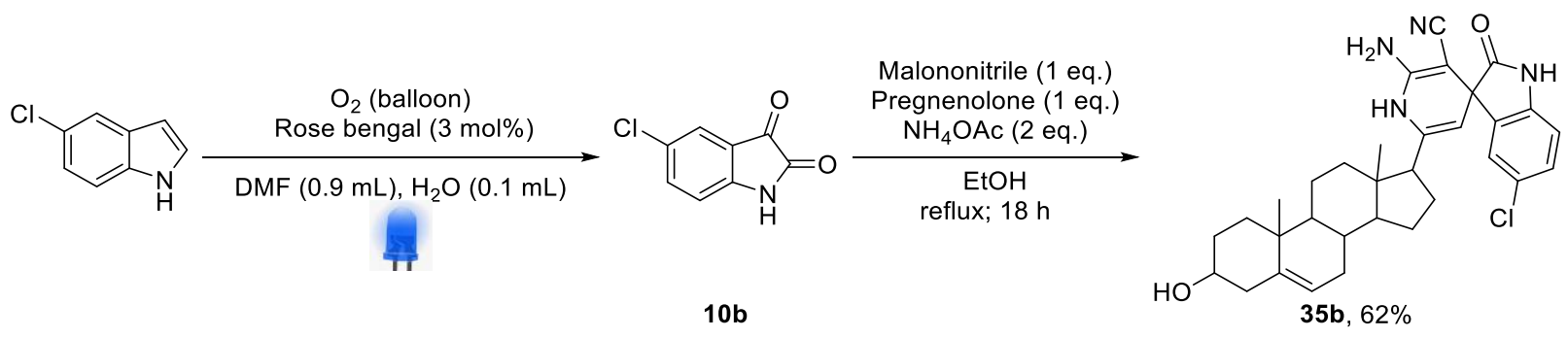

Step 1: 5-Chloroindole $(75.6 \mathrm{mg}, 0.5 \mathrm{mmol})$ and rose bengal $(14.6 \mathrm{mg}, 0.015 \mathrm{mmol})$ were prepared in a $10 \mathrm{~mL}$ two-necked flask. After purging the flask three times with vacuum and two times with nitrogen, $\mathrm{O}_{2}$ atmosphere was incorporated through an $\mathrm{O}_{2}$ filled balloon. Afterwards dry DMF (1.8 mL) and degassed Milli-Q water $(0.2 \mathrm{~mL})$ were added. The resulting mixture was stirred for $24 \mathrm{~h}$ under blue LED irradiation (the progress can be monitored via TLC). After completion of the oxygenation reaction the resulting mixture underwent an aqueous workup and was extracted three times with ethyl acetate. The combined organic layers were dried over anhydrous $\mathrm{Na}_{2} \mathrm{SO}_{4}$, filtered and concentrated in vacuo.

Additional purification of confirmation of the product after Step 1: The product mixture was purified using silica gel chromatography with ethyl acetate and $n$-pentane as solvents (ratio 50:50 ethyl acetate:n-pentane).

Step 2: 5-Chloroisatin ( $45.4 \mathrm{mg}, 0.25 \mathrm{mmol}$, purified/unpurified), Pregnenolone $(79.1 \mathrm{mg}, \quad 0.25 \mathrm{mmol})$, Malononitrile $(16.5 \mathrm{mg}, 0.25 \mathrm{mmol})$ and freshly dried ammonium acetate $(38.5 \mathrm{mg}, 0.5 \mathrm{mmol})$ were dissolved in ethanol $(5 \mathrm{~mL})$. The resulting mixture was refluxed for $18 \mathrm{~h}$. After cooling, the solvent was removed under reduced pressure. The product mixture was purified using HPLC with ACN and water as solvents (with a gradual shift in solvent ratio).

\section{Oxygenation under ${ }^{18} \mathrm{O}_{2}$ atmosphere}




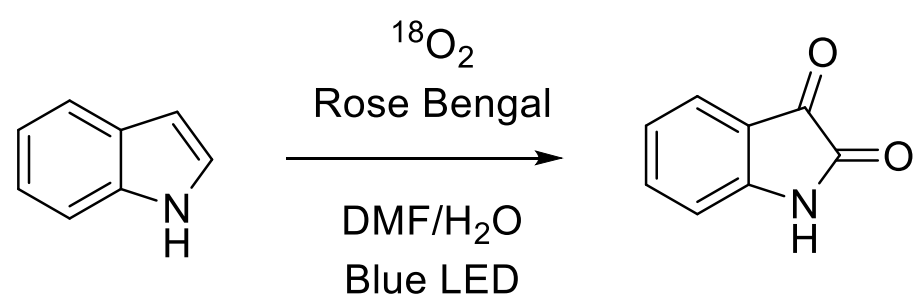

$1 H$ Indole was oxidized by the procedure described in section 5.3, Procedure 2A. with a 97 atom $\%{ }^{18} \mathrm{O}_{2}$ atmosphere. After $18 \mathrm{~h}$ the resulting products were characterized by GC-MS, HRMS and ESI.

MS (ESI): calcd. for $\mathrm{C}_{8} \mathrm{H}_{5} \mathrm{~N}^{16} \mathrm{O}_{2}\left[\mathrm{M}+\mathrm{H}^{+}\right]:$148.0393, found: 148.0401 .

MS (ESI): calcd. for $\mathrm{C}_{8} \mathrm{H}_{5} \mathrm{~N}^{18} \mathrm{O}^{16} \mathrm{O}\left[\mathrm{M}+\mathrm{H}^{+}\right]: 150.0441$, found: 150.0431 .

MS (ESI): calcd. for $\mathrm{C}_{8} \mathrm{H}_{5} \mathrm{~N}^{18} \mathrm{O}_{2}\left[\mathrm{M}_{+} \mathrm{H}^{+}\right]:$152.0478, found: 152.0471 .

Ratio: $5: 10: 1$

\section{Oxidation with $\mathrm{H}_{2}{ }^{18} \mathrm{O}$}

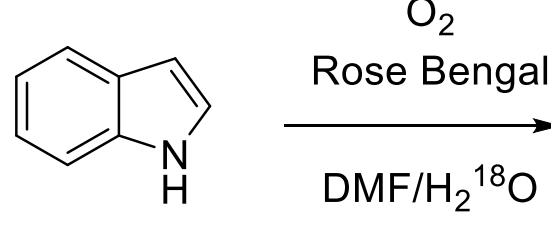

Blue LED<smiles>O=C1Nc2ccccc2C1=O</smiles>

$\mathrm{H}$

$1 H$ Indole was oxidized by the procedure described in section 5.3, Procedure 2A. with $\mathrm{H}_{2}{ }^{18} \mathrm{O}$, and an ${ }^{16} \mathrm{O}_{2}$-atmosphere. After $18 \mathrm{~h}$ the resulting products were characterized by GC-MS, HRMS and ESI.

MS (ESI): calcd. for $\mathrm{C}_{8} \mathrm{H}_{5} \mathrm{~N}^{16} \mathrm{O}_{2}\left[\mathrm{M}_{+} \mathrm{H}^{+}\right]:$148.0393, found: 148.0401 .

MS (ESI): calcd. for $\mathrm{C}_{8} \mathrm{H}_{5} \mathrm{~N}^{18} \mathrm{O}^{16} \mathrm{O}\left[\mathrm{M}+\mathrm{H}^{+}\right]: 150.0441$, found: 150.0431 .

MS (ESI): calcd. for $\mathrm{C}_{8} \mathrm{H}_{5} \mathrm{~N}^{18} \mathrm{O}_{2}\left[\mathrm{M}+\mathrm{H}^{+}\right]:$152.0478, found: 152.0471 .

Ratio: $10: 5: 1.5$ 
Oxidation with $\mathrm{H}_{2}{ }^{18} \mathrm{O}$ under ${ }^{18} \mathrm{O}_{2}$ atmosphere<smiles>c1ccc2[nH]ccc2c1</smiles><smiles></smiles>

Blue LED<smiles>[O+]=C1Nc2ccccc2C1=[OH+]</smiles>

$1 H$ Indole was oxidized by the procedure described in section 5.3, Procedure 2A. with $\mathrm{H}_{2}{ }^{18} \mathrm{O}$ and an 97 atom\% ${ }^{18} \mathrm{O}_{2}$ atmosphere. After $18 \mathrm{~h}$ the resulting products were characterized by GC-MS, HRMS and ESI.

MS (ESI): calcd. for $\mathrm{C}_{8} \mathrm{H}_{5} \mathrm{~N}^{18} \mathrm{O}_{2}\left[\mathrm{M}+\mathrm{H}^{+}\right]: 152.0478$, found: 152.0471 .

\section{Test for hydrogen peroxide}

To test the formation of hydrogen peroxide as side product, the reaction was performed according to the procedure described in section 5.3, Procedure 2A.

The resulting reaction mixture was extracted with ethyl acetate $(5 \mathrm{~mL})$, water $(5 \mathrm{~mL})$, aqueous and organic phase were tested for hydrogen peroxide with literature-known reactions.

\section{$\mathrm{H}_{2} \mathrm{O}_{2}$ glowing splint test}

$$
2 \mathrm{H}_{2} \mathrm{O}_{2} \stackrel{\mathrm{MnO}_{2}}{\longrightarrow} 2 \mathrm{H}_{2} \mathrm{O}+\mathrm{O}_{2}
$$

Procedure: $1 \mathrm{~mL}$ solution was taken, was transferred to a snap cap vial and $\mathrm{MnO}_{2}$ ( $5 \mathrm{mg}$ ) was added, the forming gas was tested with a glowing splint:

Results:

Aqueous phase tested positive: production of flammable gas (oxygen) after addition of $\mathrm{MnO}_{2}$.

Organic phase tested negative.

Control aqueous phase tested negative.

Control organic phase tested negative. 
$\mathrm{H}_{2} \mathrm{O}_{2}$ luminol test

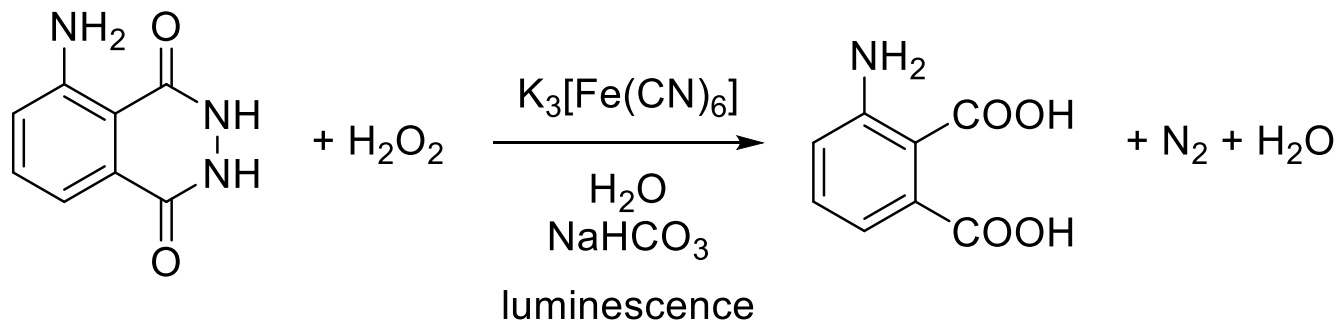

Procedure: Luminol (10 mg) was dissolved in $\mathrm{H}_{2} \mathrm{O}(5 \mathrm{~mL})$ and $\mathrm{NaHCO}_{3}(100 \mathrm{mg})$ was added to adjust the $\mathrm{pH}$ value. The resulting mixture was divided between 5 vials. For each vial, one drop of a $1 \mathrm{M} \mathrm{K}_{3}\left[\mathrm{Fe}(\mathrm{CN})_{6}\right]$ solution was added shortly before the test. $1 \mathrm{~mL}$ of the corresponding phases of the reaction mixture were added to the luminol solution. In case of peroxides, a luminescence was observed. Afterwards all samples were controlled by the addition of hydrogen peroxide solution $(30 \mathrm{~V} \%)$ for luminescence.

Results:

Aqueous phase tested positive with luminescence for over 2 minutes.

Organic phase tested negative.

Control aqueous phase tested negative.

Control organic phase tested negative. 


\section{Stern-Volmer plot}

To determine the reactive species, in the beginning of the photocatalytic reaction absorption-emission spectra for a Stern-Volmer plot were acquired. Firstly, a 3D spectrum for excitation and emission of rose bengal in DMF was recorded in order to detect the maxima of absorption and emission. The resulting spectrum is depicted in Figure 51 with 3 absorption bands. The excitation maximum was measured at $565 \mathrm{~nm}$ and the emission maximum at $575 \mathrm{~nm}$. These wavelengths were used for further measurements.
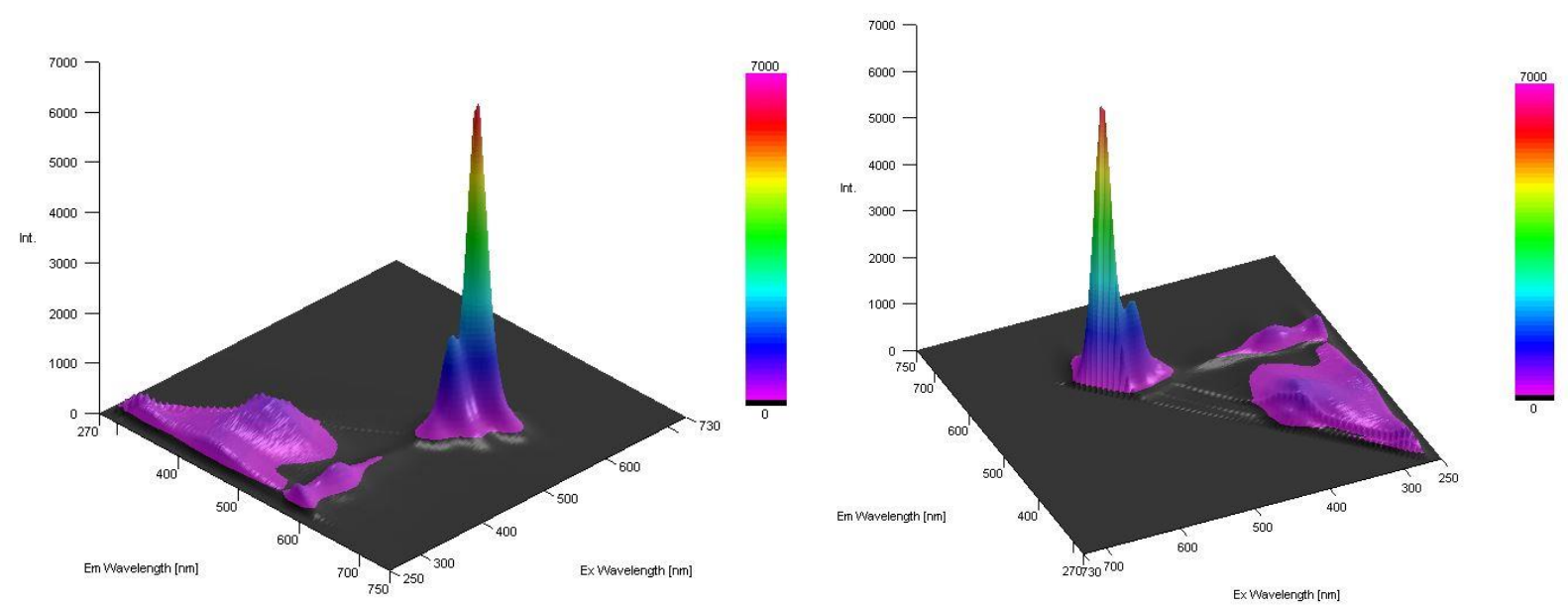

Figure 51: 3D absorbtion-emission spectrum of Rose Bengal in DMF.

A blank sample was recorded under $\mathrm{N}_{2}$ atmosphere without substrate, water, oxygen and the received intensity was set as 10 . The effect of varied amounts of substrate was investigated, as also the saturation of the solution with air and oxygen. The effect of varied amounts of water was investigated. Figures 52-55 show the results for each parameter separately. Depending on the concentration of oxygen and water, the emission decreases significantly. The amount of substrate had no effect on the emission of Rose Bengal.

Based in the mechanistic investigation the 3D spectrum for excitation and emission of $1 H$ indole was recorded additionally, which shows an absorption band at blue light. 


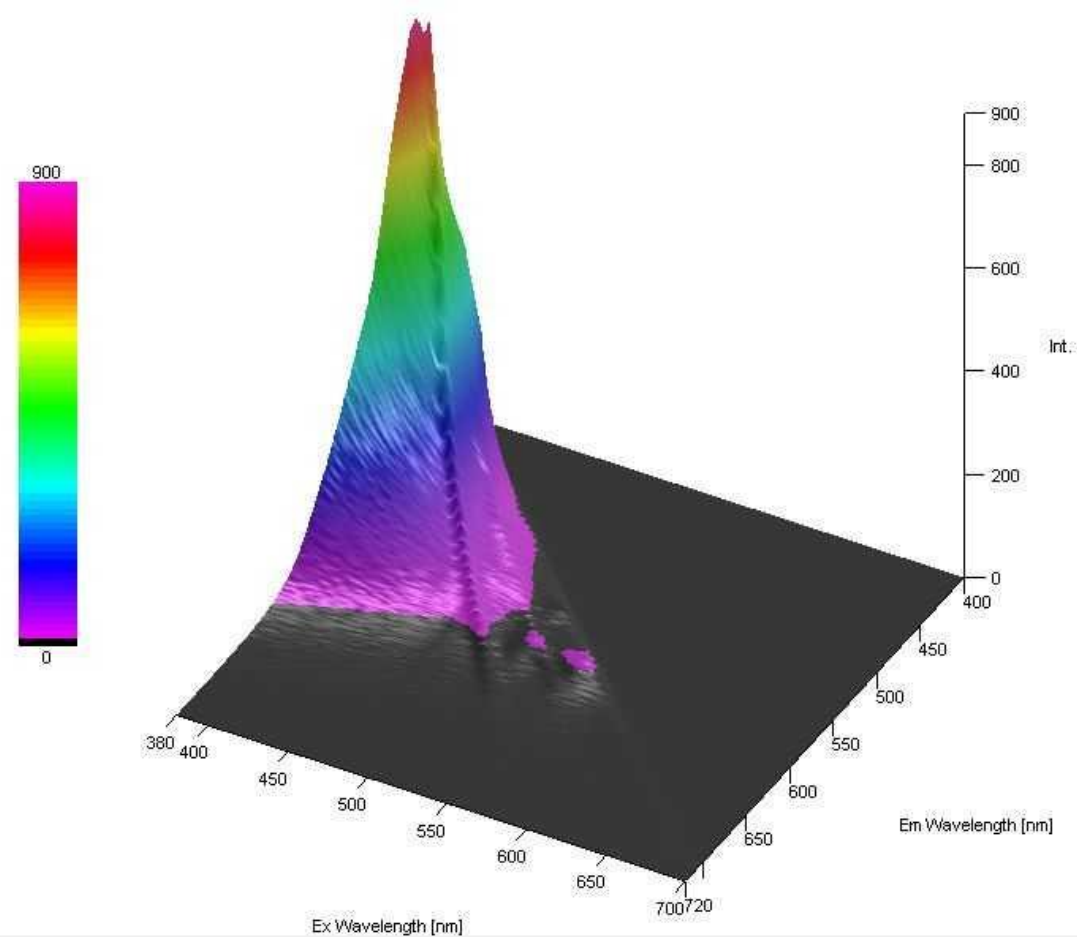

Figure 52: 3D absorbtion-emission spectrum of $1 \mathrm{H}$ indole in DMF.

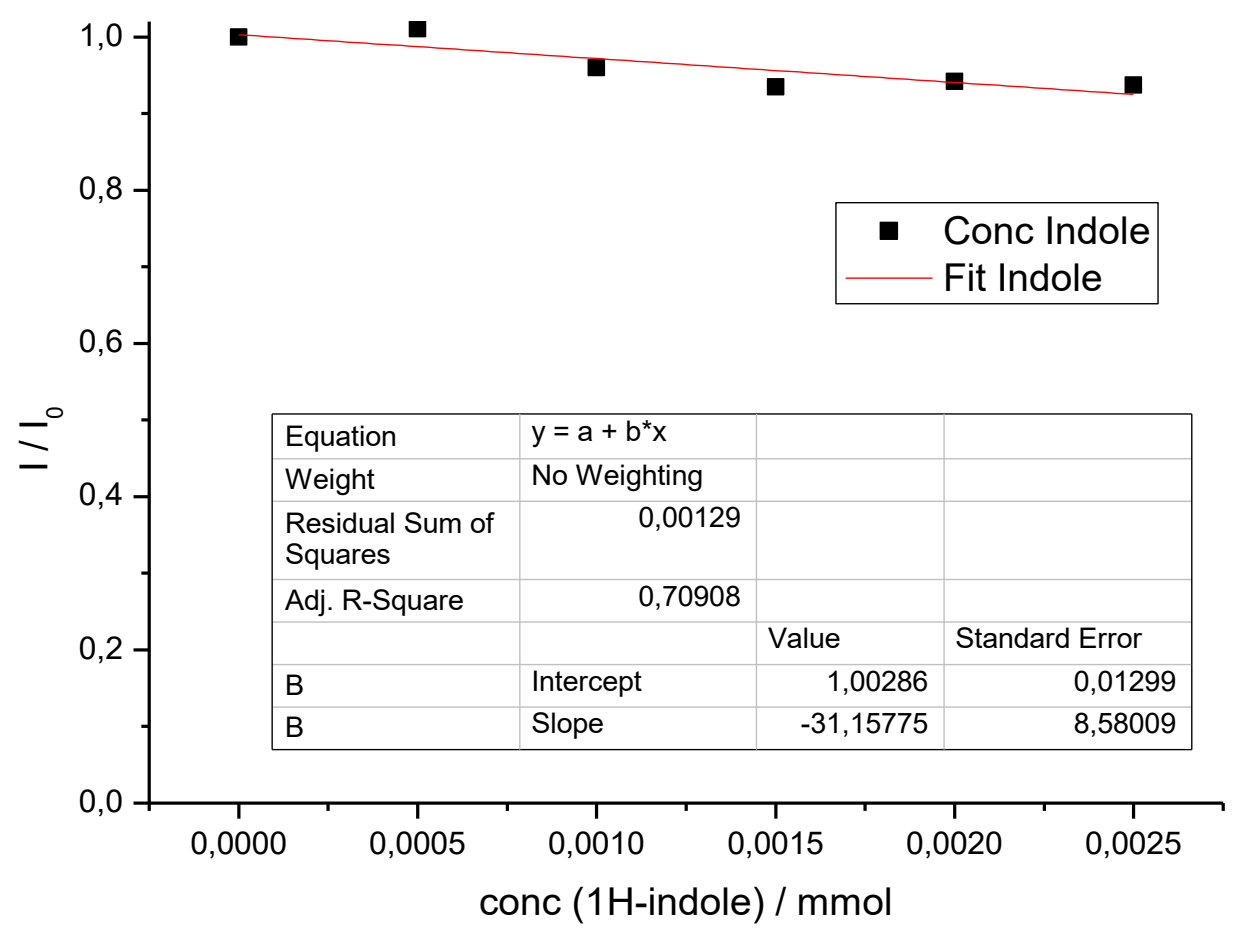

Figure 53: Stern-Volmer plot for different concentration of $1 \mathrm{H}$ indole. 


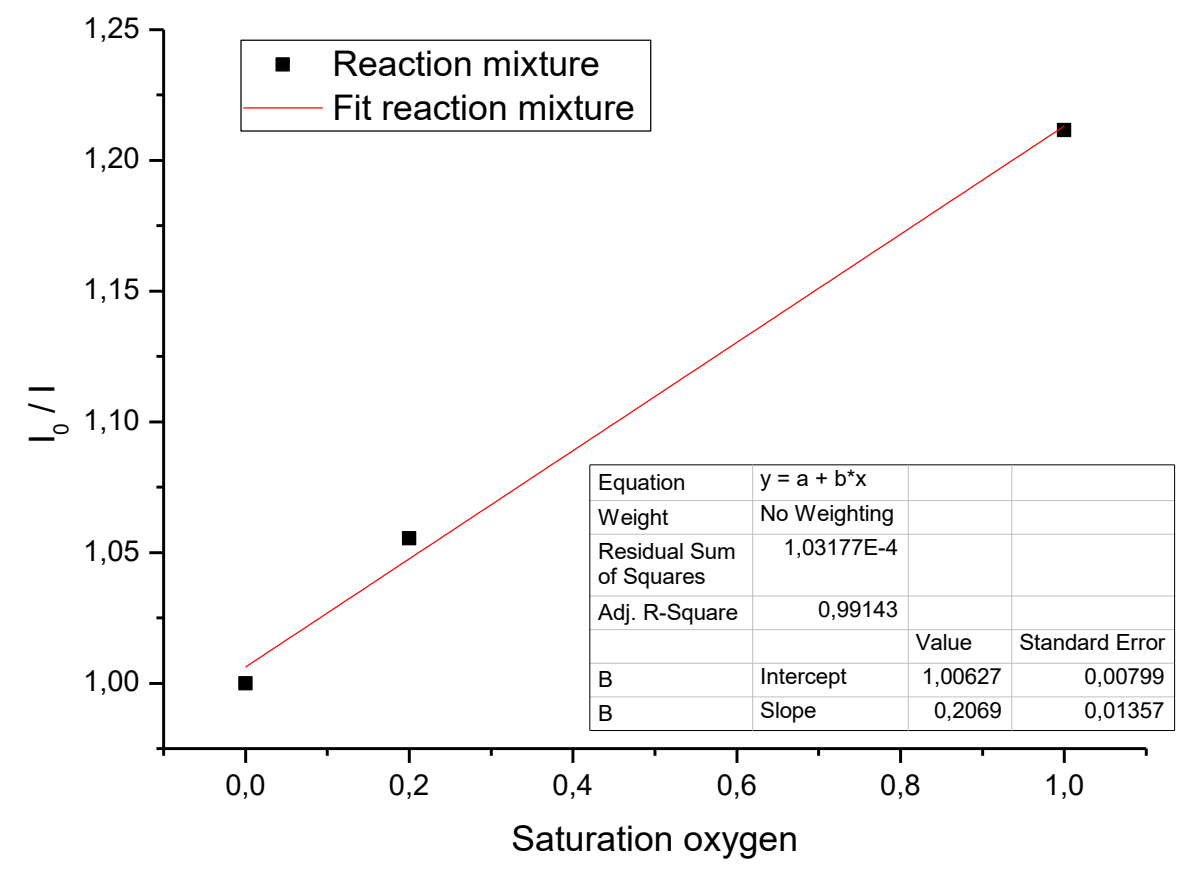

Figure 54: Stern-Volmer plot for different oxygen concentrations for the reaction mixture.

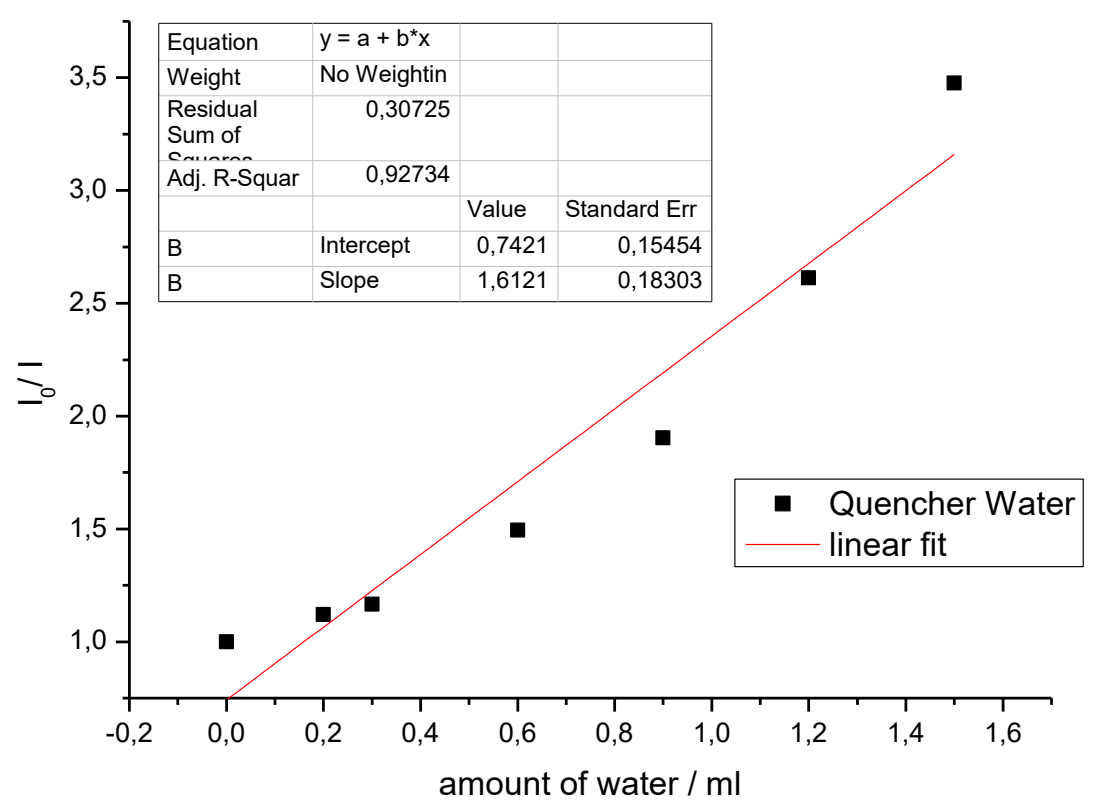

Figure 55: Stern-Volmer plot for different amount of water for mixtures of indole and rose bengal in DMF. Total volume constant $3.5 \mathrm{ml}$. Concentration of indole and rose bengal accordingly. 


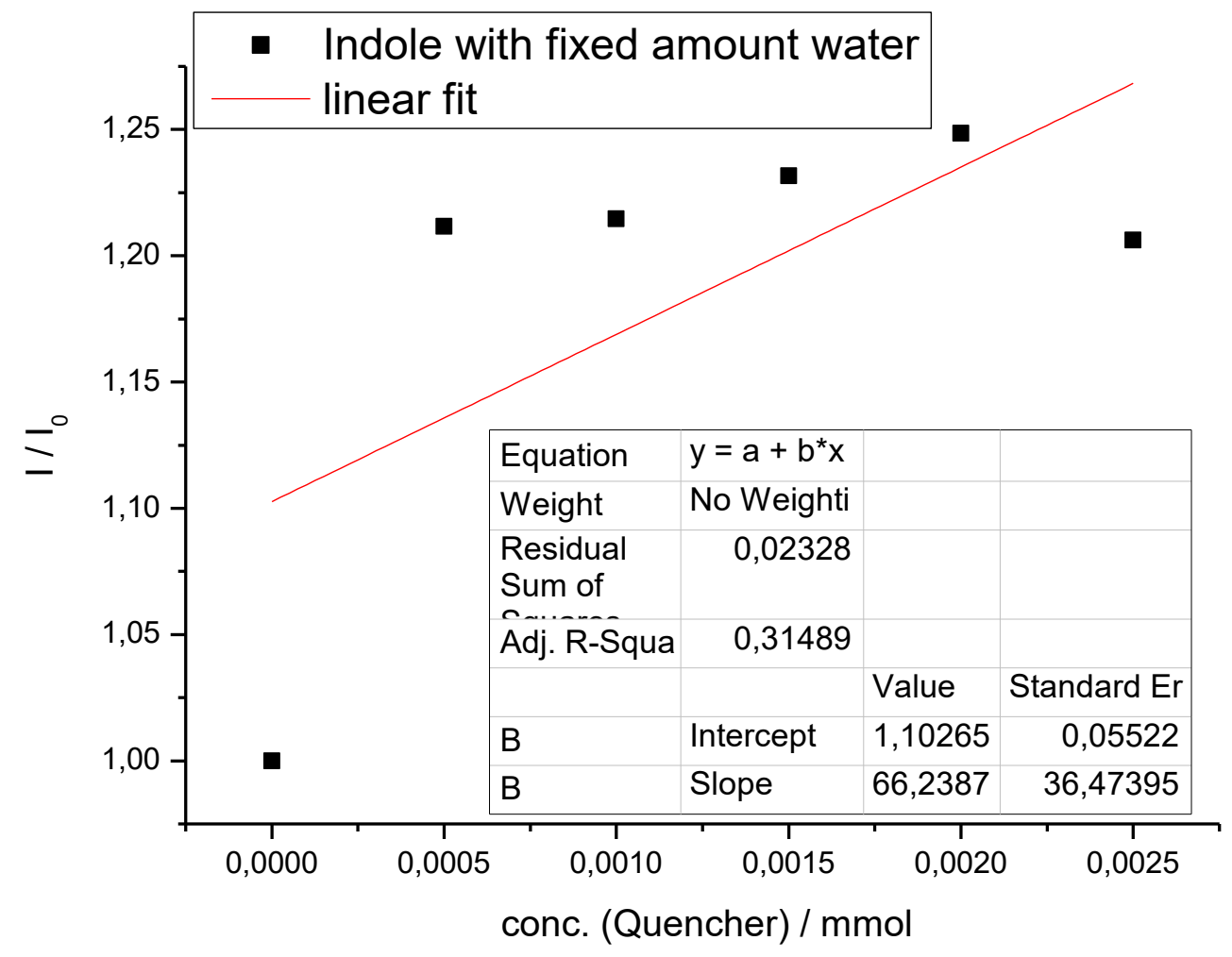

Figure 56: Stern-Volmer plot for the reaction mixture with different concentrations of indole. 


\subsection{EXPERIMENTAL PROCEDURE AND DETAILS FOR OXIDATION COMBINED WITH 1,2-ACYL MIGRATION OF SECONDARY ENAMINO KETONES}

The reaction setup is depicted in Figure 56-57. The reaction setup consists of a selfconstructed light source configuration, made up of a crystallizing dish with a diameter of $140 \mathrm{~mm}$. Inside of the crystallizing dish, commercially available $5 \mathrm{~m}$ LED-Strip is glued with separable LED elements. In total, $3 \mathrm{~m}$ LED strip is used in a crystallizing dish, with a total power of $24 \mathrm{~W}$. Light intensity of the light source can be adjusted by a self-constructed dimmer. Construction of the reaction setup and the dimmer was performed by the electronic services of the faculty for chemistry of the Georg-AugustUniversität Göttingen. Cooling of the setup is performed by a commercially available $120 \mathrm{~mm}$ fan. To ensure the constant room temperature, the dimmer setting was used at $80 \%(19.2 \mathrm{~W})$. During the first experiment, the temperature was monitored inside the crystallizing dish and did not exceed room temperature $\left(25-32{ }^{\circ} \mathrm{C}\right)$. Magnetic stirring was performed at $250 \mathrm{rpm}$. The wavelength of the blue LED setup is ranging from $404 \mathrm{~nm}$ to $553 \mathrm{~nm}$ with a maximum at $456 \mathrm{~nm}$.

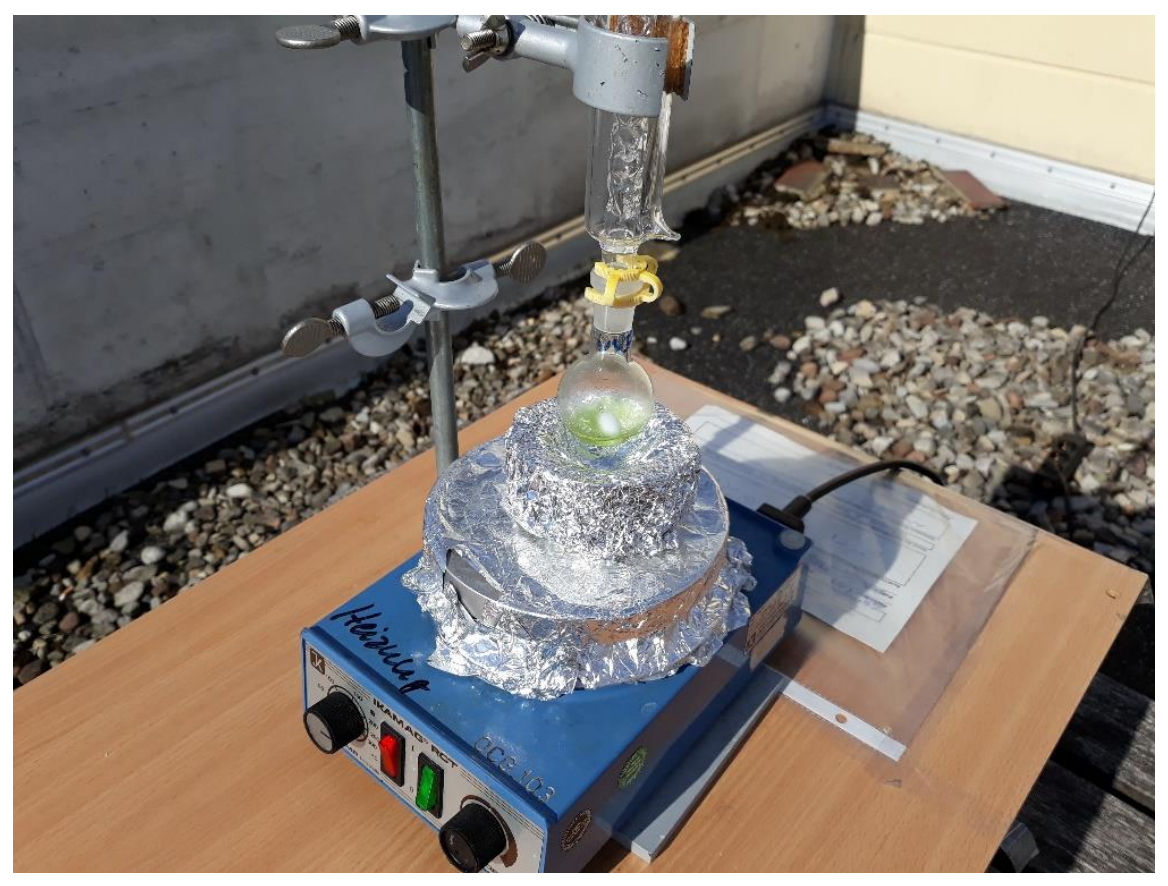

Figure 57: Reaction Setup for solar irradiation photochemical reactions. 


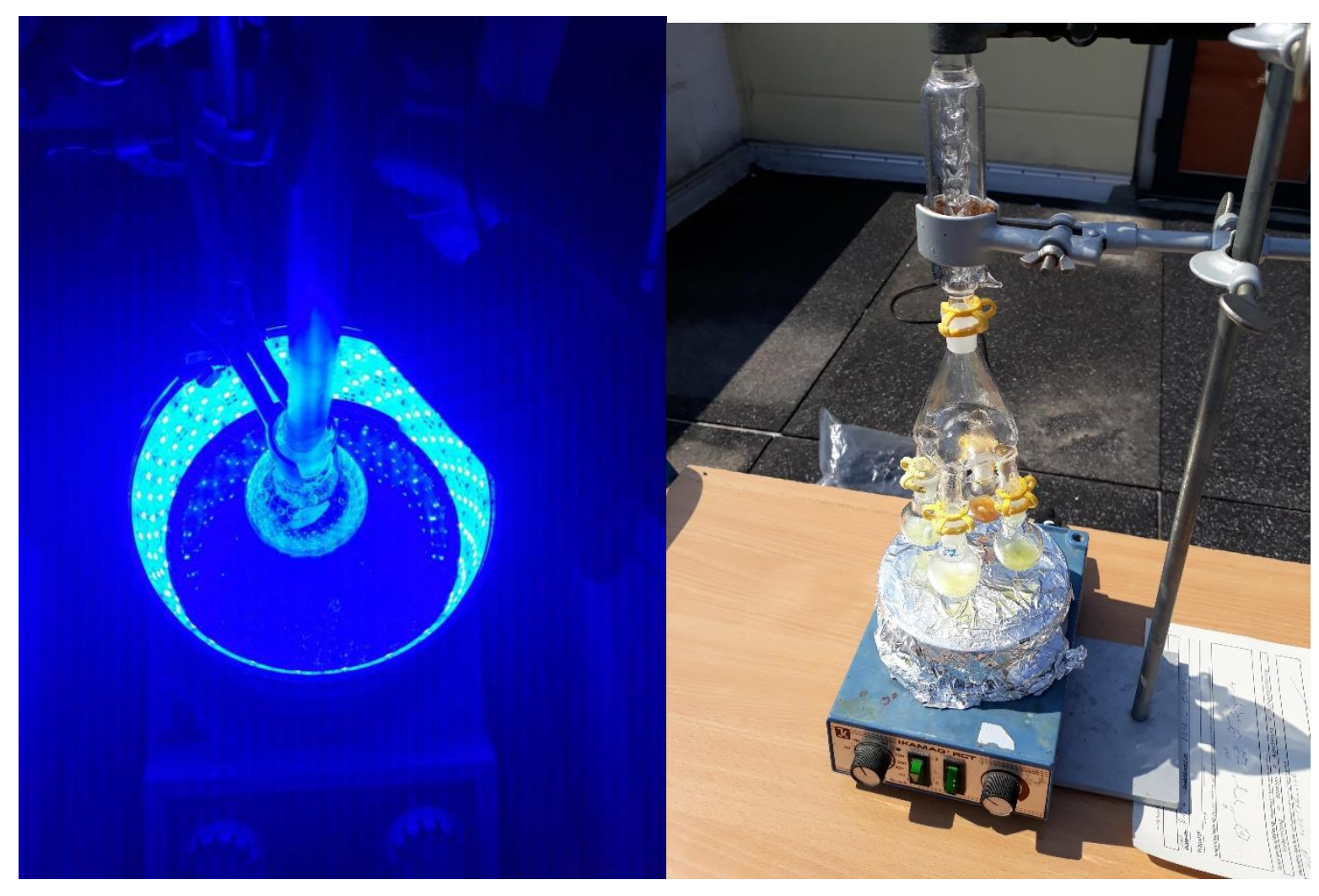

Figure 58: Led and Sunlight reaction setup. 


\section{Procedure 3A:}

Synthesis of secondary enamine ketones<smiles>[R]C(=O)CC([R])=O</smiles>

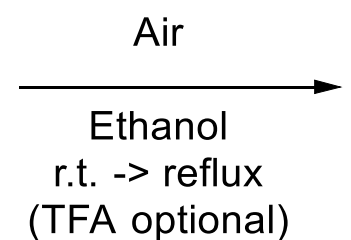<smiles>[R][R]C([R])=CC([R])=O</smiles>

(TFA optional)

Used reaction procedure is based on published works. A $25 \mathrm{ml}$ flask containing a stirring bar was charged with benzamine $(5 \mathrm{mmol})$ and corresponding diketone substrate $(7.5 \mathrm{mmol})$ in ethanol $(20 \mathrm{ml})$. Resulting mixture was stirred for $24-72 \mathrm{~h}$. In case of unsatisfying yields, the reaction was heated to reflux condition and stirred for further 24-72 h. Addition of triflouro acetic acid can also increase reaction speed and yield. 


\section{Procedure 3B}<smiles>[R]O[R]C(Nc1cc[R7](C)cc1)(C([R])=O)C(=O)O[R]</smiles>

$10 \mathrm{~mL}$ two-necked flask containing a stirring bar was charged with substrate $(0.25$ $\mathrm{mmol})$, chlorophyll (6\%-mass content, $750 \mathrm{nmol})$ and alcohol $(3.5 \mathrm{~mL})$. The resulting mixture was stirred for 4-24 $\mathrm{h}$ under blue LED irradiation (the progress can be monitored via TLC, or GC-MS). A Vigreux column was attached to minimize evaporation of solvent. Then, the resulting mixture underwent an aque-ous workup (using distilled water) and was extracted three times with ethyl acetate. The combined organic layers were dried over anhydrous $\mathrm{Na2SO} 4$, filtered and concentrated in vacuo. Products were purified using silica gel chromatography with ethyl acetate and n-pentane as solvents (typical-ly 10:90 ethyl acetate:n-pentane).

In case of sunlight experiments, the LED setup was removed and replaced by a cork ring wrapped in aluminum foil.

Additional information, in one case of a new batch chlorophyll the top layer without mixing showed a lower activity, after removing the top layer of the bottle, the activity was restored. In some cases, a base contamination was present, in those cases a lower activity was observed. Further repetition of the experiments (without base) always showed normal activity.

Chlorophyll information: Supplier: TCI, Product Number: C0780, this product is a natural pigment from a plant-derived chlorophyll. The natural pigment consists of chlorophyll contained substances (extracted from a plant), Gum Arabic and Lactose.

Handling of the catalyst was performed without any additional requirement. Filtration of the catalyst before the reaction resulted in minor reduction in the yield but afforded a clear solution. 


\section{Calculation of TON and TOF values}

Calculation of the product-based TON and TOF values was performed

$$
\frac{\mathrm{m}_{P} \cdot \mathrm{M}_{P}}{\mathrm{n}_{\text {catalyst }}}=\mathrm{TON}
$$

Alternatively:

$$
\frac{\text { yield }_{G C}}{\mathrm{n}_{\text {catalyst }}}=\mathrm{TON}
$$

$$
\frac{\mathrm{TON}}{\mathrm{t}}=\mathrm{TOF}
$$

$\mathrm{m}_{\mathrm{p}}$ : weight of the product after isolation in $\mathrm{g}$

$\mathrm{Mp}$ : molar mass of the product in $\mathrm{g}^{*} \mathrm{~mol}^{-1}$

$\mathrm{n}_{\text {catalyst: effective amount of catalyst in mol }}$

TON: Turn over number

yieldGc: Yield of the reaction determined by GC-FID calibration, $n$-dodecane as internal standard. In mol

$\mathrm{t}$ : reaction time in $\mathrm{h}$

TOF: turn over frequency in $\mathrm{h}^{-1}$

\section{Labeling experiment under ${ }^{18} \mathrm{O}_{2}$-Atmosphere}<smiles>CC(=O)/C=C(\C)Nc1ccccc1</smiles>

1a

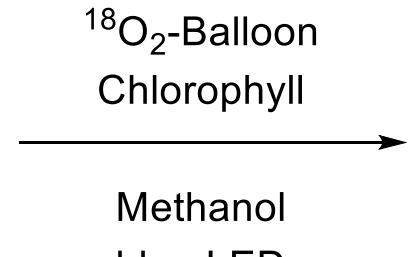

blue LED<smiles>COC(=O)C(Nc1ccccc1)(C(C)=O)C(=O)OC</smiles>

$1 \mathrm{~b}$

Secondary enaminone 106a was transformed by following the procedure described in section 5.4, Procedure 3B. under ${ }^{18} \mathrm{O}_{2}$ atmosphere. After $6 \mathrm{~h}$, the resulting products were characterized by GC-MS, HRMS and ESI. Yield was $86 \%$ for the labelled product.

MS (ESI): calcd. for $\mathrm{C}_{12} \mathrm{H}_{15} \mathrm{~N}^{16} \mathrm{O}_{2}{ }^{18} \mathrm{O}\left[\mathrm{M}+\mathrm{H}^{+}\right]$: 224.1165, found: 244.1167 .

MS (ESI): calcd. for $\mathrm{C}_{12} \mathrm{H}_{15} \mathrm{~N}^{16} \mathrm{O}_{2}{ }^{18} \mathrm{O}\left[\mathrm{M}+\mathrm{Na}^{+}\right]$: 246.0990, found: 246.0987 . 


\subsection{SYNTHESIS OF GRAPHITIC CARBON NITRIDES}
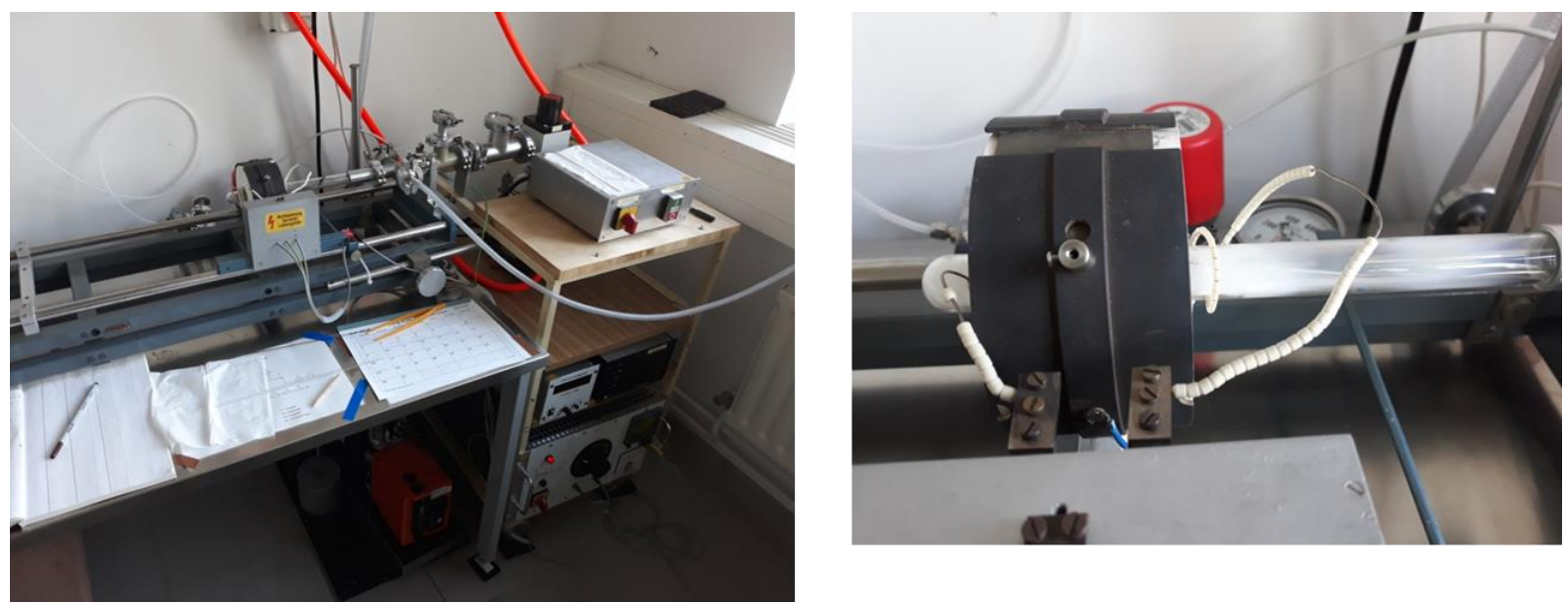

Figure 59: Electrical and heating control of the gero ring oven for small scale synthesis of graphitic carbon nitrides.
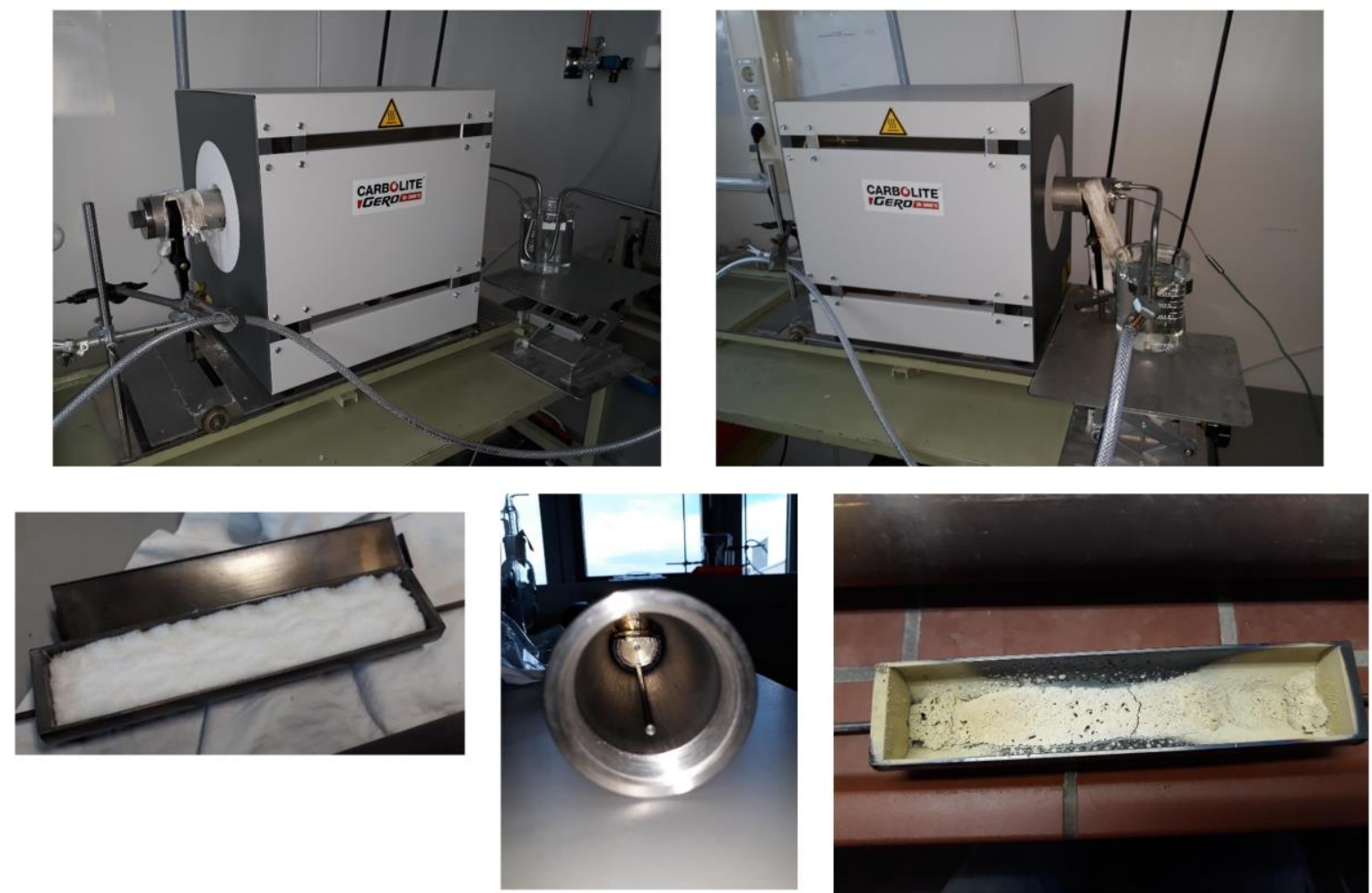

Figure 60: Large scale concept for the synthesis of graphitic carbon nitrides. Heating and electrical control not visible. 


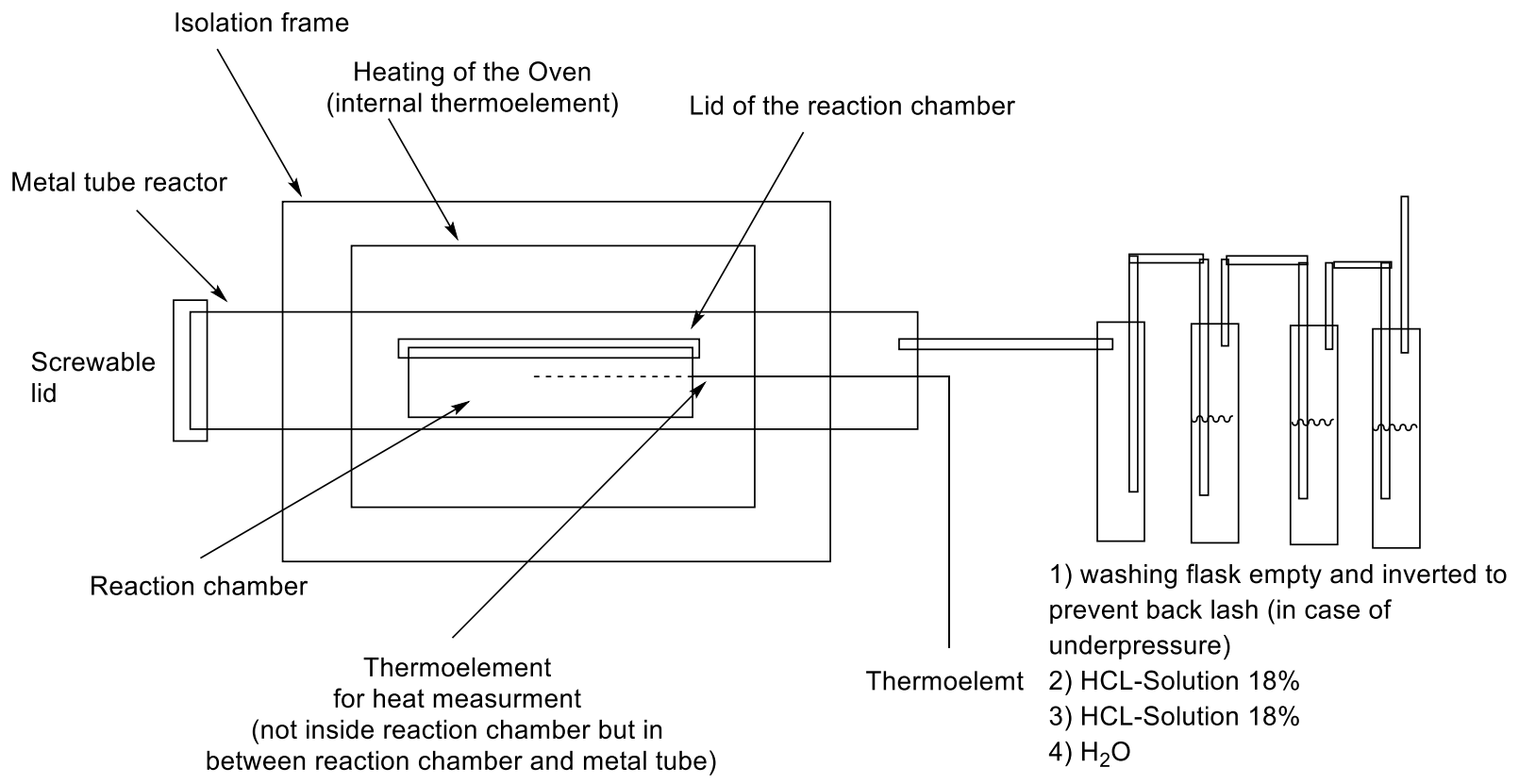

Figure 61: Schematic representation of the designed large-scale reaction concept for the synthesis of graphitic carbon nitrides.

For the synthesis a VA4 steel chamber was constructed by the faculty workshops with a wall strength of $0.8 \mathrm{~mm}$, with sufficient material strength to withhold inner pressures of over $25 \mathrm{~atm}$. The chamber was tube shaped and sealed at the end with a small $5.0 \mathrm{~mm}$ diameter exit opening, connected to the gas neutralization system. The opposite side was equipped with a screwable lid and a copper-based sealing ring. Based on the temperature inducing different expansion of the metals a sealing effect was achieved. The commercial oven included a thermoelement for monitoring the temperature of the heating coil. A secondary thermoelement type $K$ was used to monitor the temperature of the inside chamber. For the temperature area of 500$625^{\circ} \mathrm{C}$ (inside temperature) compared to the outside temperature of $35^{\circ} \mathrm{C}\left(585-655^{\circ} \mathrm{C}\right.$ outside set temperature) was experimentally recorded. The temperature difference results from thermal transport of the heating chamber, to not heated areas of the setup, which stabilized after the heating ramp period with a $100{ }^{\circ} \mathrm{C}$ lower temperature at the outside of the chamber wall. The gas neutralization system consisted of four washing bottles in sequential connection. The first washing bottle acted as setback reserve in the case of unexpected under pressure events so that quenching solutions were not able to enter the metal tube reactor. The following washing bottles were filled with $18 \%-\mathrm{HCl}$ solutions to neutralize the formed ammonium. A last washing bottle with 
water acted as a closing against remaining gas contaminations. The reaction was performed inside a specially designed VA4-steel reaction vessel and a fitting loose lid. Possible contamination of the reaction vessel metal to the formed $g-\mathrm{C}_{3} \mathrm{~N}_{4}$ cannot be excluded but should be minimized by removal of the outer layer of the formed $g-\mathrm{C}_{3} \mathrm{~N}_{4}$. Four different synthetic protocols were developed for this concept to achieve different targeted modifications. Based on the utilized materials, several heating ramps had to be programmed, due to under pressure events, when urea and other low mass precursors were used, due to the endothermal reactions.

\section{Procedure 4A:}

Melamine (15 g), was loaded in a stainless-steel chamber with a semi closed lid. Prepared chamber inserted into a stainless-steel heating chamber, which was heated to $600^{\circ} \mathrm{C}$ (inside temperature) by setting the temperature of the GERO carbolite oven to $635^{\circ} \mathrm{C}$ (outside temperature at the heating coil) (type F70-200, power: $1.5 \mathrm{~kW}$ ). The heating ramp was set to achieve target temperature in 30 minutes. The temperature was maintained for about two hours, followed by cooling the chamber to room temperature in 6 hours. 


\section{Procedure 4B:}

Urea/Dicyan (15 g), was loaded in a stainless-steel chamber with a semi closed lid. Prepared chamber inserted into a stainless-steel heating chamber, which was heated to $600^{\circ} \mathrm{C}$ (inside temperature) by setting the temperature of the GERO carbolite oven to $635^{\circ} \mathrm{C}$ (outside temperature at the heating coil) (type F70-200, power: $1.5 \mathrm{~kW}$ ). The heating ramp was set to achieve target temperature in 244 minutes. The temperature was maintained for about two hours, followed by cooling the chamber to room temperature in 6 hours.

\section{Procedure 4C:}

Urea/Dicyan (15 g) stated mass\% additive, were loaded in a stainless-steel chamber with a semi closed lid. Prepared chamber inserted into a stainless-steel heating chamber, which was heated to $550^{\circ} \mathrm{C}$ (inside temperature) by setting the temperature of the GERO carbolite oven to $585^{\circ} \mathrm{C}$ (outside temperature at the heating coil) (type F70-200, power: $1.5 \mathrm{~kW}$ ). The heating ramp was set to achieve target temperature in 244 minutes. The temperature was maintained for about four hours, followed by cooling the chamber to room temperature in 6 hours.

\section{Procedure 4D:}

Graphitic carbon nitride and surface modification were loaded stainless-steel chamber with a semi closed lid. Prepared chamber inserted into a stainless-steel heating chamber, which was heated to the boiling temperature of the surface modification (inside temperature) by setting the temperature of the GERO carbolite oven to $35^{\circ} \mathrm{C}$ above the boiling temperature (outside temperature at the heating coil) (type F70-200, power: $1.5 \mathrm{~kW}$ ). The heating ramp was set to achieve target temperature in 244 minutes. The temperature was maintained for about four hours, followed by cooling the chamber to room temperature in 6 hours. 


\section{ANALYTICAL DATA}

\subsection{ANALYTICAL DATA OF SYNTHESIZED COMPOUNDS}<smiles>O=Cc1ccccc1</smiles>

3 mol\% 9-fluorenone, air, $18 \mathrm{~h}$, benzaldehyde (1b) ${ }^{[142]}:{ }^{1} \mathbf{H}$ NMR $\left(300 \mathrm{MHz}, \mathrm{CDCl}_{3}\right): \delta$ $10.01(\mathrm{t}, J=0.5 \mathrm{~Hz}, 1 \mathrm{H}), 7.91-7.83(\mathrm{~m}, 2 \mathrm{H}), 7.67-7.58(\mathrm{~m}, 1 \mathrm{H}), 7.56-7.45(\mathrm{~m}$, 2H); ${ }^{13}$ C NMR (75 MHz, CDCl 3$): \delta 192.47,134.53,129.80,129.06 ;$ MS (GC-MS): m/z $106\left(\mathrm{M}^{+}\right) ; 98 \%$ yield.<smiles>COc1ccc(C=O)cc1</smiles>

3 mol\% 9-fluorenone, air, $18 \mathrm{~h}$, 4-methoxybenzaldehyde (2b) ${ }^{[143]}$ : ${ }^{1} \mathbf{H}$ NMR (300 $\mathrm{MHz}_{\mathrm{CDCl}}$ ): $\delta 9.87(\mathrm{~s}, 1 \mathrm{H}), 7.82(\mathrm{~d}, J=8.7 \mathrm{~Hz}, 2 \mathrm{H}), 6.99(\mathrm{~d}, J=8.7 \mathrm{~Hz}, 2 \mathrm{H}), 3.87$ (s, 3H); ${ }^{13} \mathrm{C}$ NMR (75 MHz, CDCl $): \delta 190.87,164.71,132.06,130.07,114.41,55.67$; MS (GC-MS): $m / z 136\left(\mathrm{M}^{+}\right) ; 98 \%$ yield.<smiles>O=Cc1ccc(C=O)cc1</smiles>

3 mol\% 9-fluorenone, air, $14 \mathrm{~h}$, terephthalaldehyde (3b) ${ }^{[144]}$ : ${ }^{1} \mathbf{H}$ NMR (300 MHz, $\left.\mathrm{CDCl}_{3}\right): \delta 10.14(\mathrm{~s}, 2 \mathrm{H}), 8.05(\mathrm{~s}, 4 \mathrm{H}) ;{ }^{13} \mathrm{C} \mathrm{NMR}$ (75 MHz, $\left.\mathrm{CDCl}_{3}\right): \delta 191.58,140.18$, 130.27; MS (GC-MS): m/z $134\left(\mathrm{M}^{+}\right) ; 90 \%$ yield.<smiles>COc1ccc(C=O)cc1OC</smiles>

3 mol\% 9-fluorenone, air, 24 h, 3,4-dimethoxybenzaldehyde (4b) [145]: ${ }^{1} \mathbf{H}$ NMR (300 $\mathrm{MHz}_{\mathrm{CDCl}}$ ): $\delta 9.81(\mathrm{~s}, 1 \mathrm{H}), 7.41$ (dd, $\left.J=1.9 \mathrm{~Hz}, 2 \mathrm{H}\right), 7.36(\mathrm{~d}, J=1.9 \mathrm{~Hz}, 1 \mathrm{H}), 6.94$ (d, $J=8.2 \mathrm{~Hz}, 1 \mathrm{H}), 3.92$ (s, 3H), 3.90 (s, 3H); ${ }^{13} \mathrm{C}$ NMR (75 MHz, CDCl $)$ : $\delta$ 190.86, 154.50, 149.64, 130.16, 126.83, 110.44, 108.99, 56.18, 56.01; MS (GC-MS): m/z 166 
$\left(\mathrm{M}^{+}\right) ; 64 \%$ yield; $10 \mathrm{mmol}$ scale $\left(4 \mathrm{~b}^{\star}\right)$ : 3 mol\% 9-fluorenone, air, $120 \mathrm{~h}, 6 \mathrm{~W}$ LED, $10 \mathrm{ml} \mathrm{DMSO}, 79 \%$ yield.<smiles>O=Cc1ccc(-c2ccccc2)cc1</smiles>

3 mol\% 9-fluorenone, air, $24 \mathrm{~h}$, benzophenone[1,1'-biphenyl]-4-carbaldehyde (5b) [146]: ${ }^{1} \mathrm{H}$ NMR (300 MHz, $\left.\mathrm{CDCl}_{3}\right): \delta 10.06(\mathrm{~s}, 1 \mathrm{H}), 8.06-7.89(\mathrm{~m}, 2 \mathrm{H}), 7.82-7.68$ $(\mathrm{m}, \mathrm{Hz}, 2 \mathrm{H}), 7.68-7.58(\mathrm{~m}, 2 \mathrm{H}), 7.54-7.38(\mathrm{~m}, 3 \mathrm{H}) ;{ }^{13} \mathrm{C} \mathrm{NMR}\left(75 \mathrm{MHz}, \mathrm{CDCl}_{3}\right): \delta$ 191.88, 147.19, 139.72, 135.22, 130.26, 129.01, 128.47, 127.68, 127.36; MS (GCMS): $m / z 182\left(\mathrm{M}^{+}\right) ; 77 \%$ yield.<smiles>COc1cc(C=O)cc(OC)c1OC</smiles>

3 mol\% 9-fluorenone, air, 24 h, 3,4,5-trimethoxybenzaldehyde (6b) ${ }^{[147]}$ : ${ }^{1} \mathbf{H}$ NMR $\left(300 \mathrm{MHz}, \mathrm{CDCl}_{3}\right): \delta 9.85(\mathrm{~s}, 1 \mathrm{H}), 7.11(\mathrm{~s}, 2 \mathrm{H}), 3.92(\mathrm{~s}, 3 \mathrm{H}), 3.91(\mathrm{~s}, 6 \mathrm{H}) ;{ }^{13} \mathrm{C}$ NMR (75 $\mathrm{MHz}_{\mathrm{CDCl}}$ ): $\delta 191.12,153.74,143.72,131.81,106.83,61.08,56.37$; MS (GCMS): $m / z 196\left(\mathrm{M}^{+}\right) ; 76 \%$ yield.<smiles>O=Cc1ccc2ccc3cccc4ccc1c2c34</smiles>

3 mol\% 9-fluorenone, air, $18 \mathrm{~h}$, pyrene-1-carbaldehyde (7b) ${ }^{[148]}$ : ${ }^{1} \mathbf{H}$ NMR $(300 \mathrm{MHz}$, $\left.\mathrm{CDCl}_{3}\right): \delta 10.73(\mathrm{~s}, 1 \mathrm{H}), 9.34(\mathrm{dd}, J=9.3,6.6 \mathrm{~Hz}, 1 \mathrm{H}), 8.43-7.98(\mathrm{~m}, 8 \mathrm{H}) ;{ }^{13} \mathrm{C} \mathrm{NMR}$ $\left(75 \mathrm{MHz}_{\mathrm{CDCl}}\right): \delta$ 192.96, 135.42, 134.61, 131.24, 130.97, 130.87, 130.71, 130.34, 129.02, 127.12, 126.98, 126.77, 126.50, 124.54, 124.45, 123.96, 122.91; MS (GCMS): $m / z 230\left(\mathrm{M}^{+}\right) ; 61 \%$ yield. 
<smiles>COC(=O)c1ccc(C=O)cc1</smiles>

3 mol\% 9-fluorenone, air, $18 \mathrm{~h}$, methyl 4-formylbenzoate (8b) ${ }^{[149]}:{ }^{1} \mathbf{H}$ NMR (300 $\left.\mathrm{MHz}, \mathrm{CDCl}_{3}\right) \delta 10.12(\mathrm{~s}, 1 \mathrm{H}), 8.36-8.12(\mathrm{~m}, 2 \mathrm{H}), 8.09-7.84(\mathrm{~m}, 2 \mathrm{H}), 3.98(\mathrm{~s}, 3 \mathrm{H})$; ${ }^{13} \mathrm{C}$ NMR $\left(75 \mathrm{MHz}, \mathrm{CDCl}_{3}\right): \delta 191.61,166.05,139.16,135.10,130.19,129.51,52.57$; MS (GC-MS): $m / z 164\left(\mathrm{M}^{+}\right) ; 63 \%$ yield.<smiles>O=Cc1c[nH]c2ccccc12</smiles>

3 mol\% 9-fluorenone, air, 18h, 1H-indole-3-carbaldehyde (18b) ${ }^{[150]}$ : ${ }^{1} \mathrm{H}$ NMR (300 MHz, DMSO- $\left.d_{6}\right): \delta 12.03(\mathrm{~s}, 1 \mathrm{H}), 9.95(\mathrm{~s}, 1 \mathrm{H}), 8.28(\mathrm{~s}, 1 \mathrm{H}), 8.19-8.05(\mathrm{~m}, 1 \mathrm{H}), 7.65$ - $7.46(\mathrm{~m}, 1 \mathrm{H}), 7.24(\mathrm{~m}, 2 \mathrm{H}) ;{ }^{13} \mathrm{C}$ NMR (75 MHz, DMSO-d6): $\delta 184.93,138.40,137.07$, 124.14, 123.44, 122.11, 120.83, 118.18, 112.41; MS (GC-MS): m/z $145\left(\mathrm{M}^{+}\right) ; 52 \%$ yield, b) $\mathrm{O}_{2}, 24 \mathrm{~h} 72 \%$.<smiles>O=Cc1cc2ccccc2s1</smiles>

3 mol\% 9-fluorenone, air, 18h, benzo[b]thiophene-2-carbaldehyde (19b) ${ }^{[151]:}{ }^{1} \mathbf{H}$ NMR (300 MHz, CDCl 3 ): $\delta 10.11(\mathrm{~s}, 1 \mathrm{H}), 8.03(\mathrm{~d}, J=0.8 \mathrm{~Hz}, 1 \mathrm{H}), 7.97-7.87(\mathrm{~m}, 2 \mathrm{H})$, $7.47(\mathrm{~m}, 2 \mathrm{H}) ;{ }^{13} \mathrm{C}$ NMR $\left(75 \mathrm{MHz}, \mathrm{CDCl}_{3}\right): \delta 184.76,143.50,142.81,138.67,134.55$, 128.29, 126.39, 125.38, 123.43; MS (GC-MS): $m / z 162\left(\mathrm{M}^{+}\right) ; 51 \%$ yield. b) 6 mol\% 9fluorenone, $\mathrm{O}_{2}, 24 \mathrm{~h} 84 \%$.<smiles>O=Cc1cccs1</smiles>

3 mol\% 9-fluorenone, air, 18h, thiophene-2-carbaldehyde (20b) ${ }^{[152]}$ : ${ }^{1} \mathrm{H}$ NMR (300 $\mathrm{MHz}_{\mathrm{CDCl}}$ ): $\delta 9.92(\mathrm{~d}, J=1.3 \mathrm{~Hz}, 1 \mathrm{H}), 7.86-7.65(\mathrm{~m}, 2 \mathrm{H}), 7.20$ (dd, J = 4.9, 3.8 Hz, 
1H); $\left.{ }^{13} \mathbf{C ~ N M R ~ ( 7 5 ~ M H z , ~} \mathrm{CDCl}_{3}\right): \delta 183.04,144.10,136.39,135.18,128.40$; MS (GCMS): $m / z 112\left(\mathrm{M}^{+}\right) ; 76 \%$ yield. b) 6 mol\% 9-fluorenone, $\mathrm{O}_{2}, 24 \mathrm{~h} 84 \%$.<smiles>O=C(c1ccccc1)c1ccccc1</smiles>

3 mol\% 9-fluorenone, air, $38 \mathrm{~h}$, benzophenone (26b) ${ }^{[153]:}{ }^{1} \mathrm{H}$ NMR $\left(300 \mathrm{MHz}, \mathrm{CDCl}_{3}\right)$ : $\delta 7.85-7.78(\mathrm{~m}, 4 \mathrm{H}), 7.63-7.55(\mathrm{~m}, 2 \mathrm{H}), 7.52-7.45(\mathrm{~m}, 4 \mathrm{H}) ;{ }^{13} \mathrm{C}$ NMR $(75 \mathrm{MHz}$, $\left.\mathrm{CDCl}_{3}\right): \delta 196.87,137.75,132.53,130.18,128.40 ;$ MS (GC-MS): $\mathrm{m} / \mathrm{z} 182\left(\mathrm{M}^{+}\right) ; 99 \%$ yield.<smiles>O=C(c1ccccc1)c1ccc(Br)cc1</smiles>

3 mol\% 9-fluorenone, $\mathrm{O}_{2}, 48 \mathrm{~h}$, (4-bromophenyl)phenylmethanone $(27 \mathrm{~b})^{[154]}{ }^{1}{ }^{1} \mathrm{H}$ NMR (300 MHz, $\left.\mathrm{CDCl}_{3}\right): \delta 7.81-7.75(\mathrm{~m}, 2 \mathrm{H}), 7.71-7.56(\mathrm{~m}, 5 \mathrm{H}), 7.54-7.45(\mathrm{~m}$, $2 \mathrm{H}) ;{ }^{13} \mathrm{C}$ NMR $\left(75 \mathrm{MHz}, \mathrm{CDCl}_{3}\right): \delta 195.76,137.31,136.45,132.81,131.75,131.70$, 130.07, 128.55, 127.65; MS (GC-MS): 260/262 (M+/M+); 81\% yield.<smiles>O=C(c1ccc(Cl)cc1)c1ccc(Cl)cc1</smiles>

3 mol\% 9-fluorenone, $\mathrm{O}_{2}, 48 \mathrm{~h}$, bis(4-chlorophenyl)methanone (28b) ${ }^{[155]}:{ }^{1} \mathbf{H}$ NMR $\left(300 \mathrm{MHz}, \mathrm{CDCl}_{3}\right): \delta 7.76-7.67(\mathrm{~m}, 4 \mathrm{H}), 7.50-7.42(\mathrm{~m}, 4 \mathrm{H}) ;{ }^{13} \mathrm{C} \mathbf{N M R}(75 \mathrm{MHz}$, $\left.\mathrm{CDCl}_{3}\right): \delta$; MS (GC-MS): $250\left(\mathrm{M}^{+}\right) ; 86 \%$ yield. 
<smiles>O=C(C(=O)c1ccccc1)c1ccccc1</smiles>

3 mol\% 9-fluorenone, air, $70 \mathrm{~h}$, benzil (29b) ${ }^{[156]: ~}{ }^{1} \mathrm{H}$ NMR $\left(300 \mathrm{MHz}, \mathrm{CDCl}_{3}\right): \delta 8.01-$ $7.94(\mathrm{~m}, 4 \mathrm{H}), 7.72-7.62(\mathrm{~m}, 2 \mathrm{H}), 7.56-7.47(\mathrm{~m}, 4 \mathrm{H}) ;{ }^{13} \mathrm{C}$ NMR $\left(75 \mathrm{MHz}, \mathrm{CDCl}_{3}\right): \delta$ 194.68, 135.00, 133.16, 130.04, 129.15; MS (GC-MS): m/z $210\left(\mathrm{M}^{+}\right) ; 92 \%$ yield.<smiles>CC(=O)c1ccccc1</smiles>

3 mol\% 9-fluorenone, air, $24 \mathrm{~h}$, acetophenone (30b) [157]: ${ }^{1} \mathbf{H}$ NMR (300 MHz, CDCl 3 ): $\delta 8.01-7.85(\mathrm{~m}, 2 \mathrm{H}), 7.56-7.49(\mathrm{~m}, 1 \mathrm{H}), 7.46-7.38(\mathrm{~m}, 2 \mathrm{H}), 2.56(\mathrm{~s}, 3 \mathrm{H}) ;{ }^{13} \mathrm{C}$ NMR (75 $\mathrm{MHz}_{\mathrm{CDCl}}$ ): 198.12, 137.10, 133.08, 128.55, 128.28, 26.56; MS (GC-MS): $\mathrm{m} / \mathrm{z}$ $120\left(\mathrm{M}^{+}\right) ; 75 \%$ yield.<smiles>O=C1CCCc2ccccc21</smiles>

3 mol\% 9-fluorenone, air, 64 h, 3,4-dihydronaphthalen-1(2H)-one (31b) ${ }^{[158]}$ : ${ }^{1}$ H NMR $\left(300 \mathrm{MHz}, \mathrm{CDCl}_{3}\right): \delta 8.05-8.00(\mathrm{~m}, 1 \mathrm{H}), 7.49-7.42(\mathrm{~m}, 1 \mathrm{H}), 7.37-7.15(\mathrm{~m}, 2 \mathrm{H})$, $2.96(\mathrm{t}, J=6.1 \mathrm{~Hz}, 2 \mathrm{H}), 2.74-2.56(\mathrm{~m}, 2 \mathrm{H}), 2.24-2.00(\mathrm{~m}, 2 \mathrm{H}) ;{ }^{13} \mathrm{C}$ NMR $(75 \mathrm{MHz}$, $\left.\mathrm{CDCl}_{3}\right): \delta 198.40,144.57,133.46,132.74,128.86,127.26,126.71,39.27,29.81$, 23.40; MS (GC-MS): m/z $146\left(\mathrm{M}^{+}\right)$; 95\% yield.<smiles>O=C1CCc2ccccc21</smiles>

3 mol\% 9-fluorenone, air, 64 h 2,3-dihydro-1H-inden-1-one (32b) ${ }^{[158]}$ : ${ }^{1} \mathrm{H}$ NMR (300 $\left.\mathrm{MHz}, \mathrm{CDCl}_{3}\right): \delta 7.79-7.72(\mathrm{~m}, 1 \mathrm{H}), 7.62-7.54(\mathrm{~m}, 1 \mathrm{H}), 7.51-7.44(\mathrm{~m}, 1 \mathrm{H}), 7.42-$ $7.32(\mathrm{~m}, 1 \mathrm{H}), 3.18-3.11(\mathrm{~m}, 2 \mathrm{H}), 2.74-2.64(\mathrm{~m}, 2 \mathrm{H}) ;{ }^{13} \mathrm{C} \mathrm{NMR}\left(75 \mathrm{MHz}^{\mathrm{C}} \mathrm{CDCl}_{3}\right): \delta$ 207.16, 155.26, 137.21, 134.70, 127.39, 126.81, 123.83, 36.34, 25.93; MS (GC-MS): $\mathrm{m} / \mathrm{z} 132\left(\mathrm{M}^{+}\right) ; 91 \%$ yield. 
<smiles>CCCCCCCC(=O)/C=C/c1ccccc1</smiles>

3 mol\% 9-fluorenone, air, 70 h, (E)-1-phenyldec-1-en-3-one (33b) ${ }^{[159]:}{ }^{1} \mathrm{H}$ NMR (600 $\left.\mathrm{MHz}_{\mathrm{CDCl}}\right): \delta 7.60-7.51(\mathrm{~m}, 3 \mathrm{H}), 7.42-7.38(\mathrm{~m}, 3 \mathrm{H}), 6.74(\mathrm{~d}, J=16.2 \mathrm{~Hz}, 1 \mathrm{H})$, 2.66 (t, $J=7.5 \mathrm{~Hz}, 2 \mathrm{H}), 1.68(\mathrm{p}, J=7.4 \mathrm{~Hz}, 2 \mathrm{H}), 1.37-1.13(\mathrm{~m}, 8 \mathrm{H}), 0.89$ (t, $J=$ $7.2 \mathrm{~Hz}, 3 \mathrm{H}) ;{ }^{13} \mathrm{C}$ NMR $\left(126 \mathrm{MHz}, \mathrm{CDCl}_{3}\right): \delta$ 200.63, 142.32, 134.73, 130.42, 129.00, 128.31, 126.40, 41.21, 31.93, 29.54, 29.34, 24.66, 22.86, 14.32; MS (GC-MS): m/z $230\left(\mathrm{M}^{+}\right) ; 63 \%$ yield.<smiles>O=C1Cc2cccc3cccc1c23</smiles>

3 mol\% 9-fluorenone, air, $24 \mathrm{~h}$, acenaphthylen-1(2H)-one (34b) ${ }^{[160]}$ : ${ }^{1} \mathrm{H}$ NMR (300 $\mathrm{MHz}_{\mathrm{CDCl}}$ ): $\delta 8.10$ (dd, $\left.J=8.1,0.8 \mathrm{~Hz}, 1 \mathrm{H}\right), 7.97$ (dd, $J=7.0,0.7 \mathrm{~Hz}, 1 \mathrm{H}$ ), 7.83 (dd, $J=8.4,0.8 \mathrm{~Hz}, 1 \mathrm{H}), 7.72(\mathrm{dd}, J=8.1,7.0 \mathrm{~Hz}, 1 \mathrm{H}$ ), 7.61 (dd, $J=8.4,6.9 \mathrm{~Hz}, 1 \mathrm{H}), 7.52$ - $7.44(\mathrm{~m}, 1 \mathrm{H}), 3.83(\mathrm{t}, J=0.9 \mathrm{~Hz}, 2 \mathrm{H}) ;{ }^{13} \mathrm{C}$ NMR $\left(75 \mathrm{MHz} \mathrm{CDCl}_{3}\right): \delta 203.08,141.59$, 135.17, 134.86, 131.62, 131.11, 128.52, 128.15, 124.10, 121.59, 121.18, 42.16; MS (GC-MS): $m / z 168\left(\mathrm{M}^{+}\right) ; 85 \%$ yield.<smiles>O=C(c1ccccc1)c1ccco1</smiles>

3 mol\% 9-fluorenone, air, $70 \mathrm{~h}$, furan-2-yl(phenyl)methanone (35b) ${ }^{[161]}$ : ${ }^{1} \mathbf{H}$ NMR $\left(300 \mathrm{MHz}, \mathrm{CDCl}_{3}\right): \delta 7.93-7.82(\mathrm{~m}, 2 \mathrm{H}), 7.74-7.63(\mathrm{~m}, 2 \mathrm{H}), 7.62-7.55(\mathrm{~m}, 1 \mathrm{H})$, $7.54-7.45(\mathrm{~m}, 2 \mathrm{H}), 7.16(\mathrm{dd}, J=5.0,3.8 \mathrm{~Hz}, 1 \mathrm{H}) ;{ }^{13} \mathrm{C} \mathbf{N M R}\left(75 \mathrm{MHz}, \mathrm{CDCl}_{3}\right): \delta$ $188.29,143.73,138.25,134.93,134.29,132.36,129.25,128.51,128.06$; MS (GCMS): $m / z 172\left(\mathrm{M}^{+}\right) ; 88 \%$ yield. 
<smiles>O=c1c2ccccc2oc2ccccc12</smiles>

3 mol\% 9-fluorenone, air, 72 h, 9H-xanthen-9-one (36b) ${ }^{[162]: ~}{ }^{1} \mathbf{H}$ NMR (300 MHz, $\left.\mathrm{CDCl}_{3}\right): \delta 8.39-8.26(\mathrm{~m}, 2 \mathrm{H}), 7.77-7.67(\mathrm{~m}, 2 \mathrm{H}), 7.52-7.43(\mathrm{~m}, 2 \mathrm{H}), 7.40-7.32$ $(\mathrm{m}, 2 \mathrm{H}) ;{ }^{13} \mathrm{C}$ NMR $\left(75 \mathrm{MHz}, \mathrm{CDCl}_{3}\right): \delta 177.31,156.29,134.92,126.85,124.02,121.98$, 118.09; MS (GC-MS): m/z $196\left(\mathrm{M}^{+}\right) ; 89 \%$ yield.<smiles>O=C1CCOc2ccccc21</smiles>

3 mol\% 9-fluorenone, air, $18 \mathrm{~h}$, chroman-4-one (37b) ${ }^{[163]}{ }^{1}{ }^{1} \mathbf{H}$ NMR (300 MHz, CDCl 3$)$ : $\delta 8.00-7.78(\mathrm{~m}, 1 \mathrm{H}), 7.50-7.41(\mathrm{~m}, 1 \mathrm{H}), 7.08-6.90(\mathrm{~m}, 2 \mathrm{H}), 4.64-4.41(\mathrm{~m}, 2 \mathrm{H})$, $2.90-2.71(\mathrm{~m}, 2 \mathrm{H}) ;{ }^{13} \mathrm{C}$ NMR $\left(75 \mathrm{MHz}, \mathrm{CDCl}_{3}\right): \delta 191.83,161.94,136.03,127.21$, 121.44, 117.96, 67.10, 37.88; MS (GC-MS): $m / z 148\left(\mathrm{M}^{+}\right) ; 78 \%$ yield.<smiles>CC(=O)c1ccccn1</smiles>

3 mol\% 9-fluorenone, air, 72 h, 1-(pyridine-2-yl)ethan-1-one (38b) ${ }^{[164]}$ : ${ }^{1} \mathrm{H}$ NMR (300 $\left.\mathrm{MHz}_{\mathrm{CDCl}}\right): \delta 8.52(\mathrm{~m}, 1 \mathrm{H}), 7.86(\mathrm{dt}, J=7.9,1.1 \mathrm{~Hz}, 1 \mathrm{H}), 7.67(\mathrm{~m}, 1 \mathrm{H}), 7.31(\mathrm{~m}$, $1 \mathrm{H}), 2.55(\mathrm{~s}, 3 \mathrm{H}) ;{ }^{13} \mathrm{C}$ NMR $\left(75 \mathrm{MHz}, \mathrm{CDCl}_{3}\right): \delta$ 199.75, 153.42, 148.83, 136.64, 126.92, 121.39, 25.56; MS (GC-MS): $m / z 121\left(\mathrm{M}^{+}\right) ; 86 \%$ yield.<smiles>CCCCC(=O)CCCC</smiles>

6 mol\% 9-fluorenone, $\mathrm{O}_{2}, 72 \mathrm{~h}$, nonan-5-one (39b) ${ }^{[165]}:{ }^{1} \mathrm{H}$ NMR $\left(300 \mathrm{MHz}, \mathrm{CDCl}_{3}\right): \delta$ $2.47-2.28(\mathrm{~m}, 4 \mathrm{H}), 1.62-1.43(\mathrm{~m}, 4 \mathrm{H}), 1.40-1.13(\mathrm{~m}, 4 \mathrm{H}), 0.86(\mathrm{t}, J=7.3 \mathrm{~Hz}, 6 \mathrm{H})$; ${ }^{13} \mathrm{C}$ NMR $\left(75 \mathrm{MHz} \mathrm{CDCl}_{3}\right): \delta 211.48,42.44,25.94,22.32,13.78 ;$ MS (GC-MS): $\mathrm{m} / \mathrm{z}$ $142\left(\mathrm{M}^{+}\right) ; 68 \%$ yield. 
<smiles>CCCCCC(C)=O</smiles>

6 mol\% 9-fluorenone, $\mathrm{O}_{2}, 72 \mathrm{~h}$, heptan-2-one (40b) ${ }^{[166]}:{ }^{1} \mathrm{H}$ NMR (300 MHz, CDCl 3$)$ : $\delta 2.38(\mathrm{t}, J=7.5 \mathrm{~Hz}, 2 \mathrm{H}), 2.09(\mathrm{~s}, 3 \mathrm{H}), 1.60-1.46(\mathrm{~m}, 2 \mathrm{H}), 1.26(\mathrm{~m}, 4 \mathrm{H}), 0.94-0.76$ $(\mathrm{m}, 3 \mathrm{H}) ;{ }^{13} \mathrm{C}$ NMR $\left(75 \mathrm{MHz}, \mathrm{CDCl}_{3}\right): \delta 209.24,43.71,31.31,29.75,23.51,22.39$, 13.84; MS (GC-MS): m/z $114\left(\mathrm{M}^{+}\right) ; 71 \%$ yield.<smiles>CCCCCCC(C)=O</smiles>

6 mol\% 9-fluorenone, $\mathrm{O}_{2}, 72$ h, 2-octanone (41b) ${ }^{[167]}$ : yield by GC with calibration of the pure product and substrate with $n$-dodecane as internal standard. ${ }^{1} \mathbf{H}$ NMR (300 MHz, Chloroform- $d$ ): $\delta 2.38$ (t, $J=7.4 \mathrm{~Hz}, 2 \mathrm{H}$ ), 2.09 (s, 3H), $1.68-1.44(\mathrm{~m}, 2 \mathrm{H}$ ), 1.24 (qd, $J$ $=3.7,2.5 \mathrm{~Hz}, 6 \mathrm{H}), 0.96-0.68(\mathrm{~m}, 3 \mathrm{H}) ;{ }^{13} \mathrm{C} \mathrm{NMR}\left(75 \mathrm{MHz}, \mathrm{CDCl}_{3}\right) \delta 209.13,43.71,31.52$, 29.71, 28.78, 23.76, 22.41, 13.92; MS (GC-MS): $m / z 128\left(\mathrm{M}^{+}\right) ; 78 \%$ yield.<smiles>CCCCCCCCC(C)=O</smiles>

6 mol\% 9-fluorenone, $\mathrm{O}_{2}, 72 \mathrm{~h}$, decan-2-one (42b) ${ }^{[168]}$ : ${ }^{1} \mathrm{H} \mathrm{NMR}\left(300 \mathrm{MHz}, \mathrm{CDCl}_{3}\right): \delta$ 2.40 (t, J=7.4 Hz, 2H), $2.11(\mathrm{~s}, 3 \mathrm{H}), 1.65-1.50(\mathrm{~m}, 2 \mathrm{H}), 1.26(\mathrm{~m}, 10 \mathrm{H}), 0.96-0.76$ $(\mathrm{m}, 3 \mathrm{H}) ;{ }^{13} \mathrm{C}$ NMR $\left(75 \mathrm{MHz}, \mathrm{CDCl}_{3}\right): \delta 209.41,43.94,31.94,29.94,29.48,29.31$, 29.25, 24.01, 22.76, 14.19; MS (GC-MS): $m / z 156\left(\mathrm{M}^{+}\right) ; 64 \%$ yield.

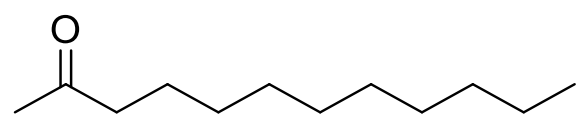

6 mol\% 9-fluorenone, $\mathrm{O}_{2}, 72 \mathrm{~h}$, dodecan-2-one (43b) ${ }^{[169]}$ : ${ }^{1} \mathrm{H}$ NMR (300 MHz, CDCl 3$)$ : $\delta 2.38$ (t, J = $7.4 \mathrm{~Hz}, 2 \mathrm{H}$ ), 2.09 (s, 3H), 1.53 (t, J = 7.2 Hz, 2H), 1.23 (t, J = 3.0 Hz, 14H), $0.95-0.75(\mathrm{~m}, 3 \mathrm{H}) ;{ }^{13} \mathrm{C}$ NMR (75 MHz, $\left.\mathrm{CDCl}_{3}\right)$ : $\delta$ 209.29, 43.88, 31.98, 29.87, 29.65, 29.56, 29.49, 29.39, 29.28, 23.97, 22.76, 14.16; MS (GC-MS): m/z $184\left(\mathrm{M}^{+}\right)$; $67 \%$ yield. 
<smiles>O=C1C=CCCC1</smiles>

3 mol\% 9-fluorenone, air, 72 h, cyclohex-2-en-1-one (44b) ${ }^{[169]}$ : analyzed by GC with calibration of product and substrate with $n$-dodecane as internal standard; MS (GCMS): $m / z 96\left(\mathrm{M}^{+}\right) ; 87 \%$ yield.

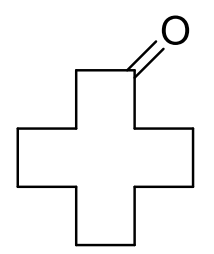

3 mol\% 9-fluorenone, air, $72 \mathrm{~h}$, cyclododecanone (45b) ${ }^{[170]}$ : ${ }^{1} \mathbf{H}$ NMR (300 MHz, $\left.\mathrm{CDCl}_{3}\right): \delta 2.53-2.39(\mathrm{~m}, 4 \mathrm{H}), 1.80-1.64(\mathrm{~m}, 4 \mathrm{H}), 1.39-1.18(\mathrm{~m}, 14 \mathrm{H}) ;{ }^{13} \mathrm{C} \mathrm{NMR}$ (75 $\mathrm{MHz}_{\mathrm{CDCl}}$ ): $\delta$ 212.85, 40.38, 24.77, 24.62, 24.25, 22.57, 22.37; MS (GC-MS): $\mathrm{m} / \mathrm{z} 182\left(\mathrm{M}^{+}\right) ; 83 \%$ yield.

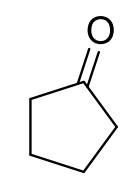

6 mol\% 9-fluorenone, $\mathrm{O}_{2}, 72 \mathrm{~h}$, cyclopenatnone (46b) ${ }^{[171]}$ : ${ }^{1} \mathbf{H}$ NMR $(300 \mathrm{MHz}$, $\left.\mathrm{CDCl}_{3}\right): \delta 2.2$ - $2.05(\mathrm{~m}, 4 \mathrm{H}), 2.05-1.83(\mathrm{~m}, 4 \mathrm{H}) ;{ }^{13} \mathrm{C} \mathrm{NMR}\left(75 \mathrm{MHz}, \mathrm{CDCl}_{3}\right): \delta 220.67$, 38.41, 23.31; MS (GC-MS): $m / z 84\left(\mathrm{M}^{+}\right) ; 63 \%$ yield.<smiles>O=C1CCCCC1</smiles>

6 mol\% 9-fluorenone, $\mathrm{O}_{2}, 72 \mathrm{~h}$, cyclohexanone (47b) ${ }^{[170]:}{ }^{1} \mathbf{H} \mathrm{NMR}\left(300 \mathrm{MHz}, \mathrm{CDCl}_{3}\right)$ : $\delta 2.27-2.20(\mathrm{~m}, 4 \mathrm{H}), 1.83-1.77(\mathrm{~m}, 4 \mathrm{H}), 1.70-1.58(\mathrm{~m}, 2 \mathrm{H}) ;{ }^{13} \mathrm{C}$ NMR $(75 \mathrm{MHz}$, $\left.\mathrm{CDCl}_{3}\right): \delta 211.85,41.84,26.92,24.88 ;$ MS (GC-MS): $\mathrm{m} / \mathrm{z} 98\left(\mathrm{M}^{+}\right) ; 59 \%$ yield. 


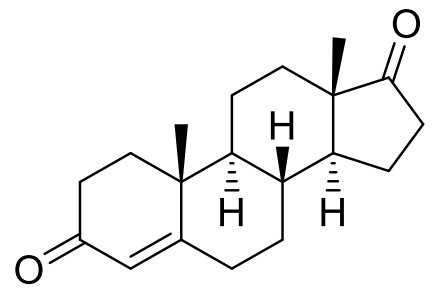

6 mol\% 9-fluorenone, $\mathrm{O}_{2}, 72 \mathrm{~h}$, androst-4-ene-3,17-dione (48b) ${ }^{[172]}$ : ${ }^{1} \mathrm{H}$ NMR (300 $\left.\mathrm{MHz}, \mathrm{CDCl}_{3}\right): \delta 5.75(\mathrm{~s}, 1 \mathrm{H}), 2.57-1.03(\mathrm{~m}, 22 \mathrm{H}), 0.91(\mathrm{~s}, 3 \mathrm{H}) ;{ }^{13} \mathrm{C} \mathrm{NMR}(75 \mathrm{MHz}$, $\left.\mathrm{CDCl}_{3}\right): \delta 220.45,199.45,170.46,124.28,53.98,51.01,47.65,38.79,35.88,35.32$, $34.04,32.71,31.44,30.91,21.89,20.47,17.53,13.85$; MS (ESI): $\mathrm{m} / \mathrm{z}$ calcd. for $\mathrm{C}_{19} \mathrm{H}_{26} \mathrm{O}_{2}\left[\mathrm{M}+\mathrm{H}^{+}\right]$: 287.2006 , found $287.1997 ; 72 \%$ yield.<smiles>C[C@]12CC[C@H]3[C@@H](CC[C@@H]4CC(=O)CC[C@]34C)[C@@H]1CCC2=O</smiles>

6 mol\% 9-fluorenone, $\mathrm{O}_{2}, 72 \mathrm{~h},(5 \alpha)$-androstane-3,17-dione (49b/50b) ${ }^{[173]}$ : ${ }^{1} \mathrm{H}$ NMR $\left(300 \mathrm{MHz}, \mathrm{CDCl}_{3}\right): \delta 2.51-1.20(\mathrm{~m}, 22 \mathrm{H}), 1.03(\mathrm{~s}, 3 \mathrm{H}), 0.88(\mathrm{~s}, 3 \mathrm{H}) ;{ }^{13} \mathrm{C}$ NMR $(75$ $\left.\mathrm{MHz}, \mathrm{CDCl}_{3}\right): \delta 220.84,211.53,53.90,51.24,47.72,46.60,44.57,38.44,38.06$, $35.81,34.96,31.49,30.53,28.62,21.78,20.71,13.80,11.45$; MS (GC-MS): $\mathrm{m} / \mathrm{z} 288$ $\left(\mathrm{M}^{+}\right)$; 33b: $62 \%$ yield; 34b: $72 \%$ yield.

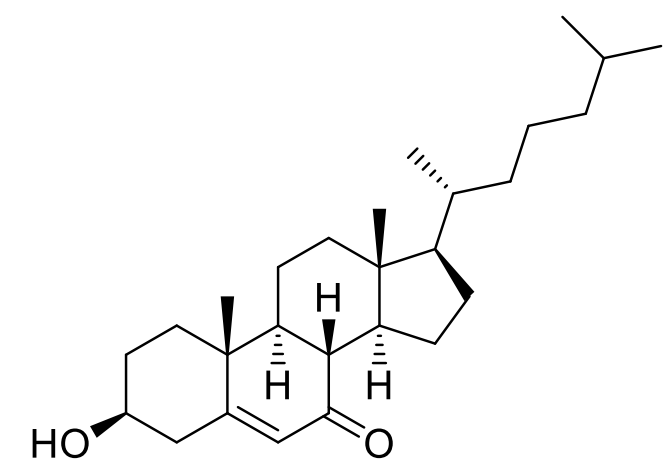

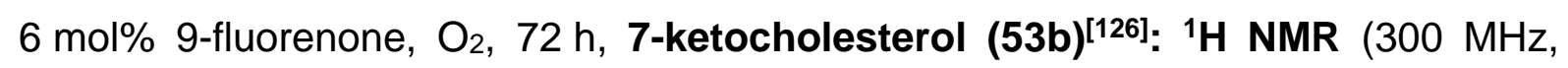
$\mathrm{CDCl}_{3}$ ): $\delta 5.69$ (d, $J=1.8 \mathrm{~Hz}, 1 \mathrm{H}$ ), 3.67 (ddd, $J=11.2,6.7,4.5 \mathrm{~Hz}, 1 \mathrm{H}$ ), $2.55-1.03$ (m, 29H), 0.92 (d, $J=6.5 \mathrm{~Hz}, 3 \mathrm{H}$ ), 0.86 (dd, $J=6.6,1.4 \mathrm{~Hz}, 6 \mathrm{H}$ ), 0.68 (s, 3H). ; ${ }^{13} \mathrm{C}$ NMR $\left(75 \mathrm{MHz}, \mathrm{CDCl}_{3}\right): 202.33,165.07,126.13,70.53,54.79,49.92,45.41,43.10$, 
$41.81,39.48,38.70,38.27,36.34,36.18,35.72,31.19,28.55,28.00,26.32,23.83$, 22.82, 21.22, 18.87, 17.32, 11.97 ; MS (GC-MS): m/z $400\left(\mathrm{M}^{+}\right) ; 62 \%$ yield.<smiles>O=C1Nc2ccccc2C1=O</smiles>

3 mol\% rose bengal, $\mathrm{O}_{2}, 24 \mathrm{~h}$, isatin (54b) ${ }^{[174]}:{ }^{1} \mathrm{H}$ NMR (300 MHz, DMSO-d6): $\delta 11.03$ (s, $1 \mathrm{H}), 7.73-7.30(\mathrm{~m}, 2 \mathrm{H}), 7.22-6.72(\mathrm{~m}, 2 \mathrm{H}) ;{ }^{13} \mathrm{C}$ NMR $(75 \mathrm{MHz}$, DMSO-d6): $\delta$ 184.4, 159.4, 150.8, 138.4, 124.7, 122.8, 117.8, 112.3; MS (GC-MS): m/z $147\left(\mathrm{M}^{+}\right)$; $93 \%$ yield.<smiles>Cc1cccc2c1C(=O)C(=O)N2</smiles>

3 mol\% rose bengal, $\mathrm{O}_{2}, 36 \mathrm{~h}$, 4-methylisatin (55b) ${ }^{[175]}$ : ${ }^{1} \mathbf{H}$ NMR $(300 \mathrm{MHz}, \mathrm{DMSO}-$ d6): $\delta 11.00(\mathrm{~s}, 1 \mathrm{H}), 7.44(\mathrm{t}, J=8 \mathrm{~Hz}, 1 \mathrm{H}), 6.86(\mathrm{dt}, J=8 \mathrm{~Hz}, 1 \mathrm{H}), 6.71$ (dt, $J=7.8 \mathrm{~Hz}$, $1 \mathrm{H}), 2.44(\mathrm{~s}, 3 \mathrm{H}) ;{ }^{13} \mathrm{C}$ NMR (75 MHz, DMSO-d6): $\delta 184.4,159.4,150.8,139.4,137.6$, 124.5, 115.6, 109.2, 17.2; MS (GC-MS): m/z $161\left(\mathrm{M}^{+}\right) ; 78 \%$ yield.<smiles>Cc1ccc2c(c1)C(=O)C(=O)N2</smiles>

3 mol\% rose bengal, $\mathrm{O}_{2}, 18 \mathrm{~h}$, 5-methylisatin (56b) ${ }^{[175]}$ : ${ }^{1} \mathrm{H}$ NMR (300 MHz, DMSOd6): $\delta 10.94(\mathrm{~s}, 1 \mathrm{H}), 7.46-7.37(\mathrm{~m}, 1 \mathrm{H}), 7.34-7.30(\mathrm{~m}, 1 \mathrm{H}), 6.81(\mathrm{~d}, \mathrm{~J}=8.0 \mathrm{~Hz}, 1 \mathrm{H})$, 2.26 (s, 3H); ${ }^{13} \mathrm{C}$ NMR (75 MHz, DMSO-d6): $\delta 185.0,159.9,149.0,139.2,132.4,125.2$, 118.2, 112,5, 20,5; MS (GC-MS): $m / z 161\left(\mathrm{M}^{+}\right) ; 86 \%$ yield. 
<smiles>Cc1ccc2c(c1)NC(=O)C2=O</smiles>

3 mol\% rose bengal, $\mathrm{O}_{2}, 48 \mathrm{~h}, 6$-methylisatin (57b) ${ }^{[176]}$ : ${ }^{1} \mathrm{H}$ NMR (300 MHz, DMSOd6): $\delta 11.00(\mathrm{~s}, 1 \mathrm{H}), 7.40(\mathrm{~d}, J=7.7 \mathrm{~Hz}, 1 \mathrm{H}), 6.97-6.84(\mathrm{~m}, 1 \mathrm{H}), 6.78-6.69(\mathrm{~m}, 1 \mathrm{H})$, $2.35(\mathrm{~s}, 3 \mathrm{H}) ;{ }^{13} \mathrm{C}$ NMR (75 MHz, DMSO-d6): $\delta 184.1,160.4,151.6,150.6,125.1,124.0$, 116.0, 113.1, 22.7; MS (GC-MS): $m / z 161\left(\mathrm{M}^{+}\right) ; 82 \%$ yield.<smiles>Cc1cccc2c1NC(=O)C2=O</smiles>

3 mol\% rose bengal, $\mathrm{O}_{2}, 18 \mathrm{~h}, \mathbf{7}-$ methylisatin (58b) ${ }^{[177]}:{ }^{1} \mathbf{H}$ NMR (300 MHz, DMSOd6): $\delta 11.08(\mathrm{~s}, 1 \mathrm{H}), 7.50-7.39(\mathrm{~m}, 1 \mathrm{H}), 7.38-7.27(\mathrm{~m}, 1 \mathrm{H}), 6.98(\mathrm{t}, J=7.5 \mathrm{~Hz}, 1 \mathrm{H})$, $2.18(\mathrm{~s}, 3 \mathrm{H}) ;{ }^{13} \mathrm{C}$ NMR $(75 \mathrm{MHz}$, DMSO-d6): $\delta 184.8,160.0,149.3,139.5,122.6,122.0$, 121.6, 117.6, 15.4; MS (GC-MS): $m / 2161\left(\mathrm{M}^{+}\right) ; 69 \%$ yield.<smiles>O=C1Nc2cccc(Br)c2C1=O</smiles>

3 mol\% rose bengal, $\mathrm{O}_{2}, 48 \mathrm{~h}$, 4-bromoisatin (59b) ${ }^{[178]}$ : ${ }^{1} \mathrm{H}$ NMR (300 MHz, DMSOd6): $\delta 11.18(\mathrm{~s}, 1 \mathrm{H}), 7.45(\mathrm{t}, J=8.0 \mathrm{~Hz}, 1 \mathrm{H}), 7.21(\mathrm{dd}, J=8.1,0.7 \mathrm{~Hz}, 1 \mathrm{H}), 6.88$ (dd, J $=7.8,0.8 \mathrm{~Hz}, 1 \mathrm{H}) ;{ }^{13} \mathrm{C}$ NMR $\left(75 \mathrm{MHz}\right.$, DMSO- $\left.d_{6}\right): \delta 181.7,158.6,152.5,138.9,126.7$, 119.4, 116.5, 111.4; MS (GC-MS): m/z $225\left(\mathrm{M}^{+}\right)$; 71\% yield.<smiles>O=C1Nc2ccc(Br)cc2C1=O</smiles>

3 mol\% rose bengal, $\mathrm{O}_{2}, 48 \mathrm{~h}$, 5-bromoisatin (60b) ${ }^{[179]:}{ }^{1} \mathrm{H}$ NMR (300 MHz, DMSOd6): $\delta 11.13(\mathrm{~s}, 1 \mathrm{H}), 7.73(\mathrm{dd}, J=8.3,2.1 \mathrm{~Hz}, 1 \mathrm{H}), 7.65(\mathrm{~d}, J=2.0 \mathrm{~Hz}, 1 \mathrm{H}), 6.87$ (dd, 
$J=8.3,0.5 \mathrm{~Hz}, 1 \mathrm{H}) ;{ }^{13} \mathrm{C}$ NMR $\left(75 \mathrm{MHz}, \mathrm{DMSO}-d_{6}\right): \delta 183.6,159.4,150.0,140.5$, 131.1, 127.4, 120.4, 114.7; MS (GC-MS): $m / 2225\left(\mathrm{M}^{+}\right) ; 80 \%$ yield.<smiles>O=C1Nc2cc(Br)cc(Br)c2C1=O</smiles>

3 mol\% rose bengal, $\mathrm{O}_{2}, 48 \mathrm{~h}, 0,1 \mathrm{mmol}$ scale, 4,6-dibromoisatin (61b) ${ }^{[180]}$ : ${ }^{1} \mathrm{H}$ NMR (300 MHz, DMSO-d6): $\delta 11.27(\mathrm{~s}, 1 \mathrm{H}), 7.51$ (d, $J=1.5 \mathrm{~Hz}, 1 \mathrm{H}), 7.05(\mathrm{~d}, J=1.5 \mathrm{~Hz}$, $1 \mathrm{H}) ;{ }^{13} \mathrm{C}$ NMR (75 MHz, DMSO-d6): $\delta 180.7,158.7,153.0,131.4,128.6,120.2,116.0$, 114.1; MS (GC-MS): m/z $303\left(\mathrm{M}^{+}\right) ; 93 \%$ yield.<smiles>O=C1Nc2ccc(Cl)cc2C1=O</smiles>

3 mol\% rose bengal, $\mathrm{O}_{2}, 48 \mathrm{~h}, 5$-chloroisatin (62b) ${ }^{[176]}$ : ${ }^{1} \mathrm{H}$ NMR $(300 \mathrm{MHz}, \mathrm{DMSO}-$ d6): $\delta 11.13(\mathrm{~s}, 1 \mathrm{H}), 7.60$ (dd, $J=8.4,2.3 \mathrm{~Hz}, 1 \mathrm{H}), 7.53(\mathrm{~d}, J=2.2 \mathrm{~Hz}, 1 \mathrm{H}), 6.91$ (d, J $=8.3 \mathrm{~Hz}, 1 \mathrm{H}) ;{ }^{13} \mathrm{C}$ NMR $\left(75 \mathrm{MHz}\right.$, DMSO- $\left.d_{6}\right): \delta 182.3,159.2,149.2,137.3,126.8$, 124.1, 119.1, 113.8; MS (GC-MS): $m / z 181\left(\mathrm{M}^{+}\right)$; 73\% yield.<smiles>O=C1Nc2cc(Cl)c(Cl)cc2C1=O</smiles>

$3 \mathrm{~mol} \%$ rose bengal, $\mathrm{O}_{2}, 48 \mathrm{~h}$, 5,6-dichloroisatin $(63 \mathrm{~b})^{[181]}$ : ${ }^{1} \mathbf{H}$ NMR $(300 \mathrm{MHz}$, DMSO-d $): \delta 11.25(\mathrm{~s}, 1 \mathrm{H}), 7.78(\mathrm{~s}, 1 \mathrm{H}), 7.13(\mathrm{~s}, 1 \mathrm{H}) ;{ }^{13} \mathrm{C}$ NMR $\left(75 \mathrm{MHz}\right.$, DMSO- $\left.d_{6}\right)$ : $\delta 182.7,159.6,150.1,140.1,126.5,125.5,118.6,114.4$; MS (GC-MS): m/z $215\left(\mathrm{M}^{+}\right)$; $65 \%$ yield. 
<smiles>COC(=O)c1ccc2c(c1)C(=O)C(=O)N2</smiles>

3 mol\% rose bengal, $\mathrm{O}_{2}, 48 \mathrm{~h}$, methyl-5-carboxylateisatin (70b) ${ }^{[182]}$ : ${ }^{1} \mathrm{H}$ NMR (300 MHz, DMSO-d6): $\delta 11.40$ (s, $1 \mathrm{H}), 8.16$ (dd, $J=8.3,1.8 \mathrm{~Hz}, 1 \mathrm{H}), 7.93$ (dd, $J=1.9,0.6$ $\mathrm{Hz}, 1 \mathrm{H}), 7.02$ (dd, J = 8.3, $0.6 \mathrm{~Hz}, 1 \mathrm{H}), 3.85$ (s, 3H); ${ }^{13} \mathrm{C} \mathrm{NMR}(75 \mathrm{MHz}$, DMSO-d6): $\delta$ 183.3, .165.2, 159.7, 154.1, 138.9, 124.9, 123.8, 118.0, 112.3, 52.2; MS (GC-MS): $m / z$ $205\left(\mathrm{M}^{+}\right) ; 58 \%$ yield.<smiles>CN1C(=O)C(=O)c2ccccc21</smiles>

3 mol\% rose bengal, $\mathrm{O}_{2}, 48 \mathrm{~h}, \mathbf{1}$-methylisatin (74b) ${ }^{[183]}$ : ${ }^{1} \mathrm{H}$ NMR (300 MHz, DMSOd6): $\delta 7.68$ (dd, $J=7.8,1.4 \mathrm{~Hz}, 1 \mathrm{H}), 7.59-7.46(\mathrm{~m}, 1 \mathrm{H}), 7.17-7.05(\mathrm{~m}, 2 \mathrm{H}), 3.13(\mathrm{~s}$, $3 \mathrm{H}) ;{ }^{13} \mathrm{C}$ NMR (75 MHz, DMSO- $\left.d_{6}\right): \delta 183.4,158.1,151.3,138.2,124.2,123.2,117.2$, 110.5, 26.0; MS (GC-MS): m/z $161\left(\mathrm{M}^{+}\right) ; 84 \%$ yield.<smiles>CCN1C(=O)C(=O)c2ccc(C)cc21</smiles>

3 mol\% rose bengal, $\mathrm{O}_{2}, 48$ h, 6-methyl-1-ethylisatin (75b) ${ }^{[184]}$ : ${ }^{1} \mathbf{H}$ NMR $(300 \mathrm{MHz}$, $\left.\mathrm{CDCl}_{3}\right): \delta 7.42(\mathrm{t}, J=7.8 \mathrm{~Hz}, 1 \mathrm{H}), 6.87(\mathrm{dd}, J=7.8,1.0 \mathrm{~Hz}, 1 \mathrm{H}), 6.70(\mathrm{~d}, J=7.8 \mathrm{~Hz}$, $1 \mathrm{H}), 3.77$ (q, $J=7.2 \mathrm{~Hz}, 2 \mathrm{H}), 2.56(\mathrm{~s}, 3 \mathrm{H}), 1.29$ (t, $J=7.2 \mathrm{~Hz}, 3 \mathrm{H}) ;{ }^{13} \mathrm{C} \mathrm{NMR}(75 \mathrm{MHz}$, $\left.\mathrm{CDCl}_{3}\right): \delta 184.3,157.9,150.8,141.5,137.6,126.1,115.9,107.4,35.0,18.2,12.7 ; \mathrm{MS}$ (GC-MS): $m / z 189\left(\mathrm{M}^{+}\right) ; 64 \%$ 
<smiles>CCCCCCN1C(=O)C(=O)c2ccccc21</smiles>

3 mol\% rose bengal, $\mathrm{O}_{2}, 48 \mathrm{~h}, \mathbf{1}$-hexylisatin (76b) ${ }^{[185]}$ : ${ }^{1} \mathbf{H}$ NMR $\left(300 \mathrm{MHz}, \mathrm{CDCl}_{3}\right): \delta$ $7.64-7.52(\mathrm{~m}, 2 \mathrm{H}), 7.10(\mathrm{~m}, 1 \mathrm{H}), 6.89(\mathrm{dd}, J=7.9,0.8 \mathrm{~Hz}, 1 \mathrm{H}), 3.76-3.65(\mathrm{~m}, 2 \mathrm{H})$, $1.75-1.61(\mathrm{~m}, 2 \mathrm{H}), 1.40-1.22(\mathrm{~m}, 6 \mathrm{H}), 0.95-0.79(\mathrm{~m}, 3 \mathrm{H}) ;{ }^{13} \mathrm{C} \mathrm{NMR}(75 \mathrm{MHz}$, $\left.\mathrm{CDCl}_{3}\right): \delta 183.7,151.1,138.3,125.4,123.6,121.2,117.6,110.1,40.3,31.4,27.2$, 26.6, 22.5, 14.0; MS (GC-MS): $m / z 231\left(\mathrm{M}^{+}\right) ; 62 \%$ yield.<smiles>O=C1C(=O)N(Cc2ccccc2)c2ccccc21</smiles>

3 mol\% rose bengal, $\mathrm{O}_{2}, 48 \mathrm{~h}, 1$-benzylisatin (77b) ${ }^{[186]}:{ }^{1} \mathbf{H}$ NMR $\left(300 \mathrm{MHz}, \mathrm{CDCl}_{3}\right)$ : $\delta 7.67-7.61(\mathrm{~m}, 1 \mathrm{H}), 7.55-7.48(\mathrm{~m}, 1 \mathrm{H}), 7.43-7.34(\mathrm{~m}, 5 \mathrm{H}), 7.12(\mathrm{t}, J=7.6 \mathrm{~Hz}$, $1 \mathrm{H}), 6.80(\mathrm{~d}, J=8.0 \mathrm{~Hz}, 1 \mathrm{H}), 4.96(\mathrm{~s}, 2 \mathrm{H}) ;{ }^{13} \mathrm{C} \mathrm{NMR}\left(75 \mathrm{MHz}, \mathrm{CDCl}_{3}\right): \delta 189.94$, $168.88,154.62,129.05,128.17,127.42,125.43,123.86,110.98,44.07$; MS (GC-MS): $\mathrm{m} / \mathrm{z} 237\left(\mathrm{M}^{+}\right) ; 72 \%$ yield.<smiles>O=C1C(=O)N2CCCc3cccc1c32</smiles>

3 mol\% rose bengal, $\mathrm{O}_{2}, 48 \mathrm{~h}, \quad 5,6$-dihydro-4H-pyrrolo[3,2,1-ij]quinoline-1,2dione(78b) [187]: ${ }^{1} \mathrm{H}$ NMR $\left(300 \mathrm{MHz}\right.$, DMSO- $\left.d_{6}\right): \delta 7.43(\mathrm{dd}, J=7.6,1.0 \mathrm{~Hz}, 1 \mathrm{H}), 7.35$ $-7.31(\mathrm{~m}, 1 \mathrm{H}), 7.00(\mathrm{t}, J=7.6 \mathrm{~Hz}, 1 \mathrm{H}), 3.70-3.55(\mathrm{~m}, 2 \mathrm{H}), 2.72(\mathrm{t}, J=6.1 \mathrm{~Hz}, 2 \mathrm{H})$, 
1.98 - $1.85(\mathrm{~m}, 2 \mathrm{H}) ;{ }^{13} \mathrm{C}$ NMR (75 MHz, DMSO- $\left.d 6\right): \delta 184.0,156.6,147.5,136.9$, 122.6, 122.1, 122.0, 115.5, 37.9, 23.2, 19.7; MS (GC-MS): m/z 187 (M+); 79\% yield.<smiles>CC(=O)Nc1ccccc1C(C)=O</smiles>

$3 \mathrm{~mol} \%$ rose bengal, $\mathrm{O}_{2}, 46 \mathrm{~h}, \mathbf{N}$-(2-acetylphenyl)acetamide (85b) ${ }^{[188]}$ : ${ }^{1} \mathrm{H}$ NMR (300 $\left.\mathrm{MHz}, \mathrm{CDCl}_{3}\right): \delta 11.72(\mathrm{~s}, 1 \mathrm{H}), 8.76(\mathrm{~d}, \mathrm{~J}=8.4 \mathrm{~Hz}, 1 \mathrm{H}), 7.93-7.89(\mathrm{~m}, 1 \mathrm{H}), 7.60-$ $7.54(\mathrm{~m}, 1 \mathrm{H}), 7.13(\mathrm{t}, J=7.5 \mathrm{~Hz}, 1 \mathrm{H}), 2.69(\mathrm{~s}, 3 \mathrm{H}), 2.25(\mathrm{~s}, 3 \mathrm{H}) ;{ }^{13} \mathrm{C} \mathrm{NMR}(\mathrm{CDCl}$, $75 \mathrm{MHz}): \delta 202.8,169.4,141.0,135.2,131.6,122.3,121.7,120.7,28.6,25.6$; MS (GC-MS): m/z $177\left(\mathrm{M}^{+}\right) ; 76 \%$ yield.<smiles>CC(=O)c1ccccc1NC=O</smiles>

3 mol\% rose bengal, $\mathrm{O}_{2}, 46 \mathrm{~h}, \mathbf{N}$-(2-acetylphenyl)formamide (86b) [189]: ${ }^{1} \mathrm{H}$ NMR (300 $\mathrm{MHz}_{\mathrm{CDCl}}$ ): $\delta 11.62(\mathrm{br}-\mathrm{s}, 1 \mathrm{H}), 8.79-8.76(\mathrm{~d}, \mathrm{~J}=8.81 \mathrm{H}), 8.52(\mathrm{br}-\mathrm{s}, 1 \mathrm{H}), 7.95-7.92$ (dd, J=8.0, 1.6 Hz, 1H), $7.61-7.56$ (t, J = 8.8, 1H), $7.21-7.17(\mathrm{~m}, 1 \mathrm{H}), 2.70(\mathrm{~s}, 3 \mathrm{H})$; ${ }^{13} \mathrm{C}$ NMR $\left(\mathrm{CDCl}_{3}, 75 \mathrm{MHz}\right): \delta$ 202.8, 159.9, 139.9, 135.2, 131.7, 123.1, 122.0, 121.6, 28.6; MS (GC-MS): m/z $163\left(\mathrm{M}^{+}\right) ; 70 \%$ yield.<smiles>O=Cc1ccccc1NC(=O)c1ccccc1</smiles>

3 mol\% rose bengal, $\mathrm{O}_{2}, 46 \mathrm{~h}, \mathbf{N}$-(2-formylphenyl)benzamide (87b) ${ }^{[190]}$ : ${ }^{1} \mathbf{H}$ NMR (300 MHz, $\left.\mathrm{CDCl}_{3}\right): \delta 12.12(\mathrm{~s}, 1 \mathrm{H}), 10.03(\mathrm{~s}, 1 \mathrm{H}), 8.99(\mathrm{~d}, J=8.4 \mathrm{~Hz}, 1 \mathrm{H}), 8.11-8.09$ $(\mathrm{m}, 2 \mathrm{H}), 7.79$ - $7.68(\mathrm{~m}, 2 \mathrm{H}), 7.63$ - $7.53(\mathrm{~m}, 3 \mathrm{H}), 7.39$ - $7.34(\mathrm{~m}, 1 \mathrm{H}) ;{ }^{13} \mathbf{C ~ N M R}\left(\mathrm{CDCl}_{3}\right.$, 
$75 \mathrm{MHz}): \delta$ 195.9, 166.2, 141.3, 136.4, 136.2, 134.3, 132.2, 128.9, 127.5, 123.1 , 122.0, 120.0; MS (GC-MS): $m / z 225\left(M^{+}\right) ; 60 \%$ yield.<smiles>CC(=O)Nc1ccc2ccccc2c1C(C)=O</smiles>

$3 \mathrm{~mol} \%$ rose bengal, $\mathrm{O}_{2}, 46 \mathrm{~h}, \mathbf{N}$-(1-formylnaphthalen-2-yl)acetamide (88b): ${ }^{1} \mathbf{H}$ NMR $\left(300 \mathrm{MHz}, \mathrm{CDCl}_{3}\right): \delta=9.11(\mathrm{~s}, 1 \mathrm{H}), 8.21-8.17(\mathrm{~d}, J=9.0 \mathrm{~Hz}, 1 \mathrm{H}), 7.88-7.83$ (m, 2H), $7.79-7.76(\mathrm{dd}, J=8.4,1.2 \mathrm{~Hz}, 1 \mathrm{H}), 7.56-7.45(\mathrm{~m}, 2 \mathrm{H}), 2.72(\mathrm{~s}, 3 \mathrm{H}), 2.22$ (s, 3H); ${ }^{13} \mathrm{C}$ NMR $\left(\mathrm{CDCl}_{3}, 75 \mathrm{MHz}\right): \delta 206.2,169.2,133.3,131.8,130.7,129.8,128.7$, 127.4, 126.9, 125.5, 124.7, 122.0, 32.8, 24.7; ESI-HRMS: $\mathrm{m} / z$ calcd. for $\mathrm{C}_{14} \mathrm{H}_{13} \mathrm{NO}_{2}$ $[\mathrm{M}+\mathrm{H}]^{+}: 228.1025 \mathrm{~m} / \mathrm{z}$, found $228.1019 \mathrm{~m} / \mathrm{z} ; 73 \%$ yield.<smiles>CC(=O)N(C)c1ccccc1C=O</smiles>

3 mol\% rose bengal, $\mathrm{O}_{2}, 46 \mathrm{~h}, \mathbf{N}$-(2-formylphenyl)- $\mathbf{N}$-methylacetamide (89b) ${ }^{[191]}{ }^{1}{ }^{1} \mathbf{H}$ NMR $\left(300 \mathrm{MHz}, \mathrm{CDCl}_{3}\right): \delta 10.16(\mathrm{~s}, 1 \mathrm{H}), 8.01-7.99(\mathrm{dd}, J=7.6,1.6 \mathrm{~Hz}, 1 \mathrm{H}), 7.75-7.69$ (dd, $J=7.6,1.6 \mathrm{~Hz}, 1 \mathrm{H}), 7.58-7.53(\mathrm{~m}, 1 \mathrm{H}), 7.32-7.28(\mathrm{~m}, 1 \mathrm{H}), 3.31(\mathrm{~s}, 3 \mathrm{H}), 1.82(\mathrm{~s}$, $3 \mathrm{H}) ;{ }^{13} \mathrm{C}$ NMR $\left(\mathrm{CDCl}_{3}, 75 \mathrm{MHz}\right): \delta 189.3,170.4,146.2,135.8,132.5,130.3,129.2$, 129.0, 37.9, 22.4; MS (GC-MS): $m / z 177\left(\mathrm{M}^{+}\right) ; 67 \%$ yield.<smiles>CC(=O)c1c(N(C)C(C)=O)ccc2ccccc12</smiles>

3 mol\% rose bengal, $\mathrm{O}_{2}, 46 \mathrm{~h}, \mathrm{~N}$-(1-acetylnaphthalen-2-yl)- $\mathrm{N}$-methylacetamide (90b): ${ }^{1} \mathrm{H}$ NMR $\left(300 \mathrm{MHz}, \mathrm{CDCl}_{3}\right): \delta=7.98-7.88(\mathrm{~m}, 2 \mathrm{H}), 7.75-7.71(\mathrm{~m} 1 \mathrm{H}), 7.60-$ $7.55(\mathrm{~m}, 2 \mathrm{H}), 7.27-7.22(\mathrm{~m}, 1 \mathrm{H}), 3.24(\mathrm{~s}, 3.0 \mathrm{H}), 2.57(\mathrm{~s}, 3.0 \mathrm{H}), 1.86(\mathrm{~s}, 3.0 \mathrm{H}) ;{ }^{13} \mathrm{C}$ 
NMR $\left(\mathrm{CDCl}_{3}, 75 \mathrm{MHz}\right): \delta 204.2,171.1,137.5,137.1,132.7,131.4,129.4,128.4$, 128.0, 127.2, 125.1, 124.7, 37.3, 32.2, 22.4; ESI-HRMS: $\mathrm{m} / z$ calcd. for $\mathrm{C}_{15} \mathrm{H}_{13} \mathrm{NO}_{2}$ $[\mathrm{M}+\mathrm{H}]^{+}: 242.1181 \mathrm{~m} / \mathrm{z}$, found $242.1176 \mathrm{~m} / \mathrm{z} ; 84 \%$ yield.<smiles>CN(C(=O)c1ccccc1)c1ccccc1C=O</smiles>

3 mol\% rose bengal, $\mathrm{O}_{2}, 46 \mathrm{~h}, \mathbf{N}$-(2-formylphenyl)- $\mathrm{N}$-methylbenzamide (91b) ${ }^{[192]}$ : ${ }^{1} \mathrm{H}$ NMR $\left(300 \mathrm{MHz}, \mathrm{CDCl}_{3}\right): \delta=10.12(\mathrm{~s}, 1 \mathrm{H}), 7.77(\mathrm{~m} 1 \mathrm{H}), 7.52(\mathrm{~m}, 1 \mathrm{H}), 7.36(\mathrm{~m}$, $1 \mathrm{H}), 7.20-7.10(\mathrm{~m}, 6 \mathrm{H}), 3.48(\mathrm{~s}, 3 \mathrm{H}) ;{ }^{13} \mathrm{C}$ NMR $\left(\mathrm{CDCl}_{3}, 75 \mathrm{MHz}\right): \delta 189.2,171.0,146.4$, 135.2, 135.0, 131.9, 130.8, 130.0, 129.4, 128.4, 128.0, 127.9, 39.2; MS (GC-MS): $\mathrm{m} / \mathrm{z}$ $239\left(\mathrm{M}^{+}\right) ; 92 \%$ yield.<smiles>CCC1=C(CC)C(=O)NC1=O</smiles>

3 mol\% rose bengal, $\mathrm{O}_{2}, 18 \mathrm{~h}$, 3,4-diethylmaleimide (93b) ${ }^{[192]:}{ }^{1} \mathbf{H}$ NMR $(300 \mathrm{MHz}$, $\left.\mathrm{CDCl}_{3}\right): \delta 7.62$ (bs, $\left.1 \mathrm{H}\right) 2.40$ (q, $\left.J=7.6 \mathrm{~Hz}, 4 \mathrm{H}\right), 1.14(\mathrm{t}, J=7.6 \mathrm{~Hz}, 6 \mathrm{H}) ;{ }^{13} \mathrm{C}$ NMR $(75$ $\left.\mathrm{MHz}, \mathrm{CDCl}_{3}\right): \delta$ 172.0, 143.0, 17.0, 13.4; MS (GC-MS): $\mathrm{m} / \mathrm{z} 153\left(\mathrm{M}^{+}\right) ; 75 \%$ yield.<smiles>CCOC(=O)C1=C(c2ccccc2)C(=O)NC1=O</smiles>

3 mol\% rose bengal, $\mathrm{O}_{2}, 18 \mathrm{~h}, 3$-[ethyl ester]-4-phenyl-1 $\mathrm{H}$-pyrrole-2,5-dione (94b): ${ }^{1} \mathrm{H}$ NMR $\left(300 \mathrm{MHz}, \mathrm{CDCl}_{3}\right): \delta 7.69$ (dd, $\left.J=7.8,1.7 \mathrm{~Hz}, 2 \mathrm{H}\right), 7.55-7.41(\mathrm{~m}, 3 \mathrm{H}), 4.37$ (q, $J=7.1 \mathrm{~Hz}, 2 \mathrm{H}), 1.30(\mathrm{t}, J=7.1 \mathrm{~Hz}, 3 \mathrm{H}) ;{ }^{13} \mathrm{C}$ NMR $\left(75 \mathrm{MHz}, \mathrm{CDCl}_{3}\right): \delta 168.8,166.4$, 161.3, 143.0, 131.6, 130.1, 128.6, 127.0, 62.4, 13.9; ESI-HRMS: $\mathrm{m} / \mathrm{z}$ calcd. for $\mathrm{C}_{13} \mathrm{H}_{11} \mathrm{NO}_{4}[\mathrm{M}+\mathrm{H}]^{+}: 245.0688 \mathrm{~m} / \mathrm{z}$, found $245.0697 \mathrm{~m} / \mathrm{z} ; 50 \%$ yield. 


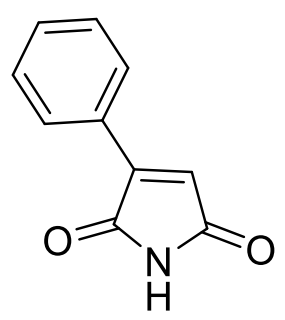

3 mol\% rose bengal, $\mathrm{O}_{2}, 18 \mathrm{~h}, \mathbf{2}-$ phenylmaleinimide (95b) ${ }^{[193]:}{ }^{1} \mathbf{H}$ NMR $(300 \mathrm{MHz}$, $\left.\mathrm{CDCl}_{3}\right): \delta 7.94(\mathrm{dd}, J=7.5,2.3 \mathrm{~Hz}, 2 \mathrm{H}), 7.60-7.42(\mathrm{~m}, 3 \mathrm{H}), 6.76(\mathrm{~s}, 1 \mathrm{H}) ;{ }^{13} \mathrm{C} \mathrm{NMR}$ $\left(\mathrm{CDCl}_{3}, 75 \mathrm{MHz}\right): \delta{ }^{13} \mathrm{C} \mathrm{NMR}\left(126 \mathrm{MHz}, \mathrm{CDCl}_{3}\right) \delta$ 170.4, 169.7, 144.7, 131.3, 129.0, 128.7, 128.5, 124.7; MS (GC-MS): m/z $173\left(\mathrm{M}^{+}\right)$; yield 53\%.<smiles>O=C1C=CC(=O)N1c1ccccc1</smiles>

3 mol\% rose bengal, $\mathrm{O}_{2}, 18 \mathrm{~h}, \mathbf{N}$-phenylmaleimide (96b) ${ }^{[194]}:{ }^{1} \mathbf{H}$ NMR $(300 \mathrm{MHz}$, DMSO-d6): $\delta 7.51(\mathrm{~d}, J=7.0 \mathrm{~Hz}, 2 \mathrm{H}), 7.49-7.34(\mathrm{~m}, 3 \mathrm{H}), 7.16(\mathrm{~d}, J=0.8 \mathrm{~Hz}, 2 \mathrm{H}) ;{ }^{13} \mathrm{C}$ NMR (75 MHz, DMSO-d6): $\delta$ 169.9, 134.6, 131.6, 128.9, 127.7, 126.7; MS (GC-MS): $\mathrm{m} / \mathrm{z} 173\left(\mathrm{M}^{+}\right) ; 74 \%$ yield.<smiles>O=C1C=CC(=O)N1c1ccc(I)cc1</smiles>

$3 \mathrm{~mol} \%$ rose bengal, $\mathrm{O}_{2}, 18 \mathrm{~h}, \mathbf{N}$-para-iodophenylmaleimide (97b) ${ }^{[195]}{ }^{1}{ }^{1} \mathrm{H}$ NMR (300 MHz, DMSO-d6): $\delta 7.86(\mathrm{~d}, \mathrm{~J}=8.5 \mathrm{~Hz}, 2 \mathrm{H}), 7.20(\mathrm{~s}, 2 \mathrm{H}), 7.17(\mathrm{~d}, \mathrm{~J}=8.5 \mathrm{~Hz}, 2 \mathrm{H}){ }^{13} \mathrm{C}$ NMR $\left(75 \mathrm{MHz}, \mathrm{CDCl}_{3}\right): \delta 161.78,158.74,138.76,138.07,121.70,120.55,88.11$; MS (GC-MS): $m / z 299\left(\mathrm{M}^{+}\right) ; 69 \%$ yield. 
<smiles>COc1ccc(N2C(=O)C=CC2=O)cc1</smiles>

3 mol\% rose bengal, $\mathrm{O}_{2}, 18 \mathrm{~h}, \mathbf{N}$-para-methoxyphenylmaleimide (98b) ${ }^{[196]}$ : ${ }^{1} \mathbf{H}$ NMR $\left(300 \mathrm{MHz}, \mathrm{CDCl}_{3}\right): \delta 7.54(\mathrm{~d}, J=9.0 \mathrm{~Hz}, 2 \mathrm{H}), 7.10(\mathrm{~d}, J=8.8 \mathrm{~Hz}, 2 \mathrm{H}), 6.81$ (dd, $J=$ 9.1, 7.1 Hz, 2H), 3.80 (d, J=3.2 Hz, 3H); ${ }^{13} \mathrm{C}$ NMR (75 MHz, CDCl 3 ): $\delta 162.8,158.7$, 121.8, 121.7, 114.9, 114.3, 55.5; MS (GC-MS): 203 (M+); 77\% yield.<smiles>O=C1Nc2ncccc2C1=O</smiles>

3 mol\% rose bengal, $\mathrm{O}_{2}$, $48 \mathrm{~h}, 7$-azaisatin (101b) ${ }^{[197]}$ : ${ }^{1} \mathrm{H}$ NMR (300 MHz, DMSO-d6): $\delta 11.61$ (s, $1 \mathrm{H}$ ), 8.40 (dd, J=5.2, 1.7 Hz, 1H), 7.88 (dd, J=7.4, 1.7 Hz, 1H), 7.11 (dd, $J=7.4,5.2 \mathrm{~Hz}, 1 \mathrm{H}) ;{ }^{13} \mathrm{C}$ NMR (75 MHz, DMSO- $\left.d 6\right): \delta 183.4,164.5,160.4,155.7$, 133.1, 119.4, 113.3; MS (GC-MS): m/z $148\left(\mathrm{M}^{+}\right)$; yield: 84\%; 3 mol\% Rose Bengal, $72 \mathrm{~h}, \mathrm{O}_{2}, 5 \mathrm{mmol}$ scale: $74 \%$ yield.<smiles>O=C1Nc2ccccc2/C1=N/c1ccccc1</smiles>

$3 \mathrm{~mol} \%$ rose bengal, $\mathrm{O}_{2}, 24 \mathrm{~h}+24 \mathrm{~h}, 1,3-$ dihydro-3-(phenylimino)-2H-indol-2-one (102b) ${ }^{[198]}:{ }^{1} \mathrm{H}$ NMR $\left(300 \mathrm{MHz}_{\mathrm{CDCl}}\right.$ ): $\delta 9.59$ (s, 1H), $7.44(\mathrm{t}, J=7.8 \mathrm{~Hz}, 2 \mathrm{H}), 7.37-$ $7.22(\mathrm{~m}, 2 \mathrm{H}), 7.04$ (dd, J=7.7, 1.2 Hz, 2H), $6.94(\mathrm{~d}, J=7.9 \mathrm{~Hz}, 1 \mathrm{H}), 6.74$ (td, J = 7.6 $\mathrm{Hz}, 1 \mathrm{H}) 6.66(\mathrm{dd}, J=7.8 \mathrm{~Hz}, 1.2 \mathrm{~Hz}, 1 \mathrm{H}) ;{ }^{13} \mathrm{C} \mathrm{NMR}\left(\mathrm{CDCl}_{3}, 75 \mathrm{MHz}\right): \delta 165.6,154.8$, $150.2,145.8,134.5,129.6,126.5,125.6,122.9,118.0,116.3,112.0$; MS (GC-MS): $\mathrm{m} / \mathrm{z} 222\left(\mathrm{M}^{+}\right) ; 92 \%$ yield. 
<smiles>O=C1Nc2ccc(Cl)cc2C12Nc1ccccc1S2</smiles>

$3 \mathrm{~mol} \%$ rose bengal, $\mathrm{O}_{2}, 24 \mathrm{~h}+24 \mathrm{~h}, 5^{\prime}$-chloro-spiro[benzothiazole-2(3H),3'-[3H] indol]-2'(1'H)-one (103b) ${ }^{[199]: ~}{ }^{1} \mathrm{H}$ NMR (300 MHz, CDCl 3 ): $\delta=10.53$ (s, 1H), 7.55 (d, $J=2.2 \mathrm{~Hz}, 1 \mathrm{H}), 7.41-7.28(\mathrm{~m}, 2 \mathrm{H}), 7.06(\mathrm{dd}, J=7.6,1.2 \mathrm{~Hz}, 1 \mathrm{H}), 6.95-6.85(\mathrm{~m}$, $2 \mathrm{H}), 6.63$ (ddd, $J=27.2,7.6,1.1 \mathrm{~Hz}, 2 \mathrm{H}) ;{ }^{13} \mathrm{C} \mathrm{NMR}\left(\mathrm{CDCl}_{3}, 75 \mathrm{MHz}\right): \delta 176.4,147.4$, 140.6, 132.5, 130.8, 126.8, 126.3, 125.9, 124.3, .121.6, 109.4, 112.2, 109.1, 74.7; MS (GC-MS): $m / z 288\left(\mathrm{M}^{+}\right) ; 72 \%$ yield.<smiles>O=C1Nc2ccccc2C1(c1c[nH]c2ccccc12)c1c[nH]c2ccccc12</smiles>

trisindoline (104b) ${ }^{\text {[200]: }}{ }^{1} \mathrm{H}$ NMR (300 MHz, DMSO-d6): $\delta=11.00(\mathrm{~d}, J=2.6 \mathrm{~Hz}, 2 \mathrm{H})$, $10.66(\mathrm{~s}, 1 \mathrm{H}), 7.41(\mathrm{~d}, J=8.2 \mathrm{~Hz}, 2 \mathrm{H}), 7.36-7.21(\mathrm{~m}, 4 \mathrm{H}), 7.11-7.02(\mathrm{~m}, 3 \mathrm{H}), 7.00$ - $6.92(\mathrm{~m}, 3 \mathrm{H}), 6.85$ (ddd, $J=8.0,7.0,1.0 \mathrm{~Hz}, 2 \mathrm{H}$ ); ${ }^{13} \mathrm{C}$ NMR (DMSO-d6, $75 \mathrm{MHz}$ ): $\delta$ 179.3, 170.8, 141.8, 137.5, 135.1, 128.3, 126.2, 125.4, 124.8, 121.9, 121.4, 121.3, $118.7,114.9,112.1,110.1,60.2,53.1,21.2,14.5$; MS (GC-MS): $m / z 363\left(\mathrm{M}^{+}\right) ; 74 \%$ yield. 
<smiles>CC12CCC(O)CC1=CCC1C2CCC2(C)C(C3=CC4(C(=O)Nc5ccc(Cl)cc54)C(C#N)=C(N)N3)CCC12</smiles>

2'-amino-5-chloro-1,2-dihydro-6'-[(3 $\beta, 17 \beta)$-3-hydroxyandrost-5-en-17-yl]-2-

oxospiro[3H-indole-3,4'(1'H)-pyridine]-3'-carbonitrile (105b) ${ }^{\text {[201]: }}{ }^{1} \mathrm{H}$ NMR (300 $\left.\mathrm{MHz}_{\mathrm{CDCl}}\right): \delta=10.25(\mathrm{~s}, 1 \mathrm{H}), 7.47-7.39(\mathrm{~m}, 1 \mathrm{H}), 7.27-7.14(\mathrm{~m}, 1 \mathrm{H}), 7.02(\mathrm{~d}, \mathrm{~J}=$ $2.2 \mathrm{~Hz}, 1 \mathrm{H}), 6.79(\mathrm{~d}, \mathrm{~J}=8.2 \mathrm{~Hz}, 1 \mathrm{H}), 5.78(\mathrm{~s}, 2 \mathrm{H}), 5.25(\mathrm{~d}, \mathrm{~J}=4.7 \mathrm{~Hz}, 1 \mathrm{H}), 4.58(\mathrm{~d}, \mathrm{~J}$ $=4.4 \mathrm{~Hz}, 1 \mathrm{H}), 4.07(\mathrm{~s}, 1 \mathrm{H}), 3.24(\mathrm{~s}, 1 \mathrm{H}), 2.20-0.85(\mathrm{~m}, 23 \mathrm{H}) 0.56(\mathrm{~s}, 3 \mathrm{H}) ;{ }^{13} \mathrm{C}$ NMR $\left(\mathrm{CDCl}_{3}, 75 \mathrm{MHz}\right): \delta 180.6,153.7,141.8,139.9,136.7,128.5,126.4,125.2,121.3$, 120.7, 111.3, 96.6, 96.5, 70.5, 56.3, 55.8, 53.7, 52.5, 51.9, 50.3, 43.9, 43.7, 42.7, 37.4, 36.6, 31.9, 31.1, 30.1, 24.3, 23.6, 19.7, 13.4; ESI-HRMS: $m / z$ calcd. for $\mathrm{C}_{32} \mathrm{H}_{37} \mathrm{CIN}_{4} \mathrm{O}_{2}$ $545.2678(\mathrm{M}+\mathrm{H})^{+}$found: $545.2689(\mathrm{M}+\mathrm{H})^{+} ; 62 \%$ yield.<smiles>CC(=O)C=C(C)Nc1ccccc1</smiles>

(E)-4-(phenylamino)pent-3-en-2-one (106a) [202]: ${ }^{1} \mathrm{H}$ NMR $\left(300 \mathrm{MHz}, \mathrm{CDCl}_{3}\right) \delta 12.47$ (s, 1H), 7.32 (dd, J = 8.5, 7.1 Hz, 2H), $7.21-7.14(\mathrm{~m}, 1 \mathrm{H}), 7.09$ (dd, J = 7.3, $1.5 \mathrm{~Hz}$, 2H), $5.18(\mathrm{~s}, 1 \mathrm{H}), 2.09$ (s, 3H), $1.98(\mathrm{~s}, 3 \mathrm{H}) ;{ }^{13} \mathrm{C} \mathrm{NMR}\left(75 \mathrm{MHz}, \mathrm{CDCl}_{3}\right) \delta$ 196.2, 160.3, 138.8, 129.2, 125.6, 124.8, 97.7, 29.2, 19.9; MS (GC-MS): m/z $175\left(\mathrm{M}^{+}\right)$; $99 \%$ yield.<smiles>COC(=O)C(C)(Nc1ccccc1)C(C)=O</smiles>

3-oxo- $\mathrm{N}$-phenyl-isovaline methyl ester (106b) ${ }^{[122]}$ : ${ }^{1} \mathrm{H}$ NMR (400 $\left.\mathrm{MHz}, \mathrm{CDCl}_{3}\right): \delta$ $7.17(\mathrm{dd}, J=8.6,7.3 \mathrm{~Hz}, 2 \mathrm{H}), 6.78-6.73(\mathrm{~m}, 1 \mathrm{H}), 6.55(\mathrm{dt}, J=7.7,1.0 \mathrm{~Hz}, 2 \mathrm{H}), 5.17$ (bs, $1 \mathrm{H}) 3.81(\mathrm{~s}, 3 \mathrm{H}), 2.22(\mathrm{~s}, 3 \mathrm{H}), 1.71(\mathrm{~s}, 3 \mathrm{H}) ;{ }^{13} \mathrm{C}$ NMR $\left(100 \mathrm{MHz}, \mathrm{CDCl}_{3}\right): \delta 203.5$, 171.7, 143.9, 129.4, 118.4, 114.1, 69.6, 53.6, 24.7, 18.4; MS (GC-MS): m/z $221\left(\mathrm{M}^{+}\right)$; $92 \%$ yield. 
<smiles>CCOC(=O)C(C)(Nc1ccccc1)C(C)=O</smiles>

Ethyl 3-oxo-2-(phenylamino)butanoate (107b) ${ }^{[123]}:{ }^{1} \mathrm{H}$ NMR $\left(400 \mathrm{MHz}^{\mathrm{CDCl}} 3\right): \delta$ $7.14(\mathrm{dd}, J=8.6,7.3 \mathrm{~Hz}, 2 \mathrm{H}), 6.73(\mathrm{tt}, J=7.4,1.1 \mathrm{~Hz}, 1 \mathrm{H}), 6.57-6.47(\mathrm{~m}, 2 \mathrm{H}), 5.28$ (s, 1H), 4.25 (q, J=7.1 Hz, 2H), $2.20(\mathrm{~s}, 3 \mathrm{H}), 1.68(\mathrm{~s}, 3 \mathrm{H}), 1.23(\mathrm{t}, J=7.1 \mathrm{~Hz}, 3 \mathrm{H}) ;{ }^{13} \mathrm{C}$ NMR $\left(100 \mathrm{MHz}_{\mathrm{CDCl}}\right): \delta 203.9,171.3,144.1,129.5,118.5,114.3,69.8,62.9,24.8$, 18.7, 14.1 ; MS (GC-MS): m/z $235\left(\mathrm{M}^{+}\right) ; 72 \%$ yield.<smiles>CC(=O)C(C)(Nc1ccccc1)C(=O)OC(C)C</smiles>

Isopropyl 3-oxo-2-(phenylamino)butanoate (108b) ${ }^{[122]: ~}{ }^{1} \mathbf{H}$ NMR (400 MHz, CDCl $)$ : $\delta 7.16(\mathrm{dd}, J=8.6,7.3 \mathrm{~Hz}, 2 \mathrm{H}), 6.75$ (tt, $J=7.4,1.0 \mathrm{~Hz}, 1 \mathrm{H}), 6.63-6.49(\mathrm{~m}, 2 \mathrm{H}), 5.28$ (bs, 1H), 5.12 (p, J=6.2 Hz, 1H), $2.20(\mathrm{~s}, 3 \mathrm{H}), 1.68$ (s, 3H), 1.27 (d, J=6.2 Hz, 3H), 1.19 (d, $J=6.3 \mathrm{~Hz}, 3 \mathrm{H}) ;{ }^{13} \mathrm{C}$ NMR $\left(100 \mathrm{MHz}, \mathrm{CDCl}_{3}\right): \delta$ 203.8, 170.6, 144.2, 129.4, 118.3, 114.2, 70.6, 24.6, 21.4, 21.3, 18.5; MS (GC-MS): m/z 249 (M+); ESI-HRMS: $m / z$ calcd. For $\mathrm{C}_{14} \mathrm{H}_{19} \mathrm{NO}_{3}[\mathrm{M}+\mathrm{H}]^{+}: 250.1438$, found: 250.1441 ; reaction time: $8 \mathrm{~h} ; 51 \%$ yield,<smiles>CC(=O)C(C)(Nc1ccccc1)C(=O)OCC(F)(F)F</smiles>

2,2,2-Trifluoroethyl 3-oxo-2-(phenylamino)butanoate (109b): ${ }^{1} \mathrm{H}$ NMR (400 MHz, $\left.\mathrm{CDCl}_{3}\right): \delta 7.19-7.13(\mathrm{~m}, 2 \mathrm{H}), 6.80-6.74(\mathrm{~m}, 1 \mathrm{H}), 6.58-6.51(\mathrm{~m}, 2 \mathrm{H}), 5.24(\mathrm{~s}, 1 \mathrm{H})$, 4.65 (dq, $J=12.6,8.2$ Hz, 1H), 4.47 (dq, $J=12.6,8.2$ Hz, 1H), 2.24 (s, 3H) 1.75 (s, $3 \mathrm{H}) ;{ }^{13} \mathrm{C}$ NMR $\left(100 \mathrm{MHz}, \mathrm{CDCl}_{3}\right): \delta 201.85,170.17,143.68,129.55,122.50$ (d, J = 277.7 Hz), 118.94, 114.48, 69.86, 61.43 (q, $J=37.1 \mathrm{~Hz}$ ), 24.50, 19.07; MS (GC-MS): m/z $289\left(\mathrm{M}^{+}\right)$; ESI-HRMS: $\mathrm{m} / z$ calcd. For $\mathrm{C}_{13} \mathrm{H}_{14} \mathrm{~F}_{3} \mathrm{NO}_{3}[\mathrm{M}+\mathrm{H}]^{+}: 312.0816$, found: 312.0820 ; reaction time: $6 \mathrm{~h} ; 68 \%$ yield. 
<smiles>CCCCCCCCOC(=O)C(C)(Nc1ccccc1)C(C)=O</smiles>

Octyl 3-oxo-2-(phenylamino)butanoate (110b): ${ }^{1} \mathrm{H}$ NMR $\left(400 \mathrm{MHz}, \mathrm{CDCl}_{3}\right): \delta^{1} \mathrm{H}$ NMR (400 MHz, $\left.\mathrm{CDCl}_{3}\right) \delta 7.21-7.11(\mathrm{~m}, 2 \mathrm{H}), 6.75(\mathrm{tt}, J=7.3,1.1 \mathrm{~Hz}, 1 \mathrm{H}), 6.59-$ $6.51(\mathrm{~m}, 2 \mathrm{H}), 5.33(\mathrm{~s}, 1 \mathrm{H}), 4.20$ (td, $J=6.6,1.7 \mathrm{~Hz}, 2 \mathrm{H}), 2.22(\mathrm{~s}, 3 \mathrm{H}), 1.71(\mathrm{~s}, 3 \mathrm{H})$, 1.61 (hept, $J=6.7,6.0 \mathrm{~Hz}, 2 \mathrm{H}), 1.38-1.22(\mathrm{~m}, 10 \mathrm{H}), 0.90$ (t, $J=6.9 \mathrm{~Hz}, 3 \mathrm{H}) ;{ }^{13} \mathrm{C}$ NMR (100 MHz, $\left.\mathrm{CDCl}_{3}\right): \delta$ 203.6, 171.2, 144.0, 129.4, 118.3, 114.1, 69.7, 66.9, 31.7, 29.1, 28.4, 25.6, 24.7, 22.6, 18.6, 14.1; MS (GC-MS): m/z $319\left(\mathrm{M}^{+}\right)$; ESI-HRMS: $\mathrm{m} / \mathrm{z}$ calcd. For $\mathrm{C}_{19} \mathrm{H}_{29} \mathrm{NO}_{3}[\mathrm{M}+\mathrm{H}]^{+}: 320.1326$, found: 320.1332 ; reaction time: $8 \mathrm{~h} ; 89 \%$ yield; 110b-s: 5 mmol scale, sunlight, $5 \mathrm{~h}, 82 \%$.<smiles>CC(=O)C(C)(Nc1ccccc1)C(=O)OCc1ccccc1</smiles>

Benzyl 3-oxo-2-(phenylamino)butanoate (111b) ${ }^{\text {[203]: }}{ }^{1} \mathrm{H}$ NMR $\left(400 \mathrm{MHz}, \mathrm{CDCl}_{3}\right): \delta$ $7.41-7.31(\mathrm{~m}, 3 \mathrm{H}), 7.31-7.24(\mathrm{~m}, 2 \mathrm{H}), 7.16(\mathrm{dd}, J=8.6,7.3 \mathrm{~Hz}, 2 \mathrm{H}), 6.76$ (tt, $J=$ 7.3, 1.1 Hz, 1H), $6.54(\mathrm{dt}, J=7.7,1.1 \mathrm{~Hz}, 2 \mathrm{H}), 5.33(\mathrm{~s}, 1 \mathrm{H}), 5.23(\mathrm{~s}, 2 \mathrm{H}), 2.11(\mathrm{~s}, 3 \mathrm{H})$, 1.72 (s, 3H); ${ }^{13} \mathrm{C}$ NMR (100 MHz, $\left.\mathrm{CDCl}_{3}\right): \delta 203.2,171.0,143.9,134.7,129.4,128.7$, 128.4, 118.4, 114.2, 69.8, 68.2, 24.6, 18.7; MS (GC-MS): m/z $297\left(\mathrm{M}^{+}\right)$; reaction time: $10 \mathrm{~h} ; 68 \%$ yield.<smiles>CCc1ccc(N/C(C)=C/C(C)=O)cc1</smiles>

(E)-4-((4-ethylphenyl)amino)pent-3-en-2-one (112a) [202]: ${ }^{1} \mathrm{H} \quad \mathrm{NMR}(400 \mathrm{MHz}$, $\left.\mathrm{CDCl}_{3}\right): \delta 12.43(\mathrm{~s}, 1 \mathrm{H}), 7.17(\mathrm{~d}, J=8.3 \mathrm{~Hz}, 2 \mathrm{H}), 7.03(\mathrm{~d}, J=8.4 \mathrm{~Hz}, 2 \mathrm{H}), 5.18(\mathrm{~s}, 1 \mathrm{H})$, $2.65(\mathrm{~d}, J=7.6 \mathrm{~Hz}, 2 \mathrm{H}), 2.10(\mathrm{~s}, 3 \mathrm{H}), 1.98(\mathrm{~s}, 3 \mathrm{H}), 1.24(\mathrm{t}, J=7.6 \mathrm{~Hz}, 3 \mathrm{H}) ;{ }^{13} \mathrm{C} \mathrm{NMR}$ $\left(100 \mathrm{MHz}, \mathrm{CDCl}_{3}\right): \delta$ 195.8, 160.9, 141.9, 136.2, 128.5, 124.9, 97.2, 29.0, 28.3, 19.8, 15.5; MS (GC-MS): $m / z 203\left(\mathrm{M}^{+}\right) ; 87 \%$ yield. 
<smiles>CCc1ccc(NC(C)(C(C)=O)C(=O)OC)cc1</smiles>

Methyl 2-((4-ethylphenyl)amino)-2-methyl-3-oxobutanoate (112b) ${ }^{[123]}$ : ${ }^{1} \mathrm{H}$ NMR ((400 MHz, $\left.\mathrm{CDCl}_{3}\right): \delta 7.07$ - $6.97(\mathrm{~m}, 2 \mathrm{H}), 6.53-6.45(\mathrm{~m}, 2 \mathrm{H}), 5.17(\mathrm{~s}, 1 \mathrm{H}), 3.81$ (s, 3H), 2.55 (q, J = 7.6 Hz, 2H), $2.22(\mathrm{~s}, 3 \mathrm{H}), 1.69$ (s, 3H), 1.20 (t, J = 7.6 Hz, 3H); ${ }^{13} \mathrm{C}$ NMR (100 MHz, $\left.\mathrm{CDCl}_{3}\right): \delta$ 204.0, 172.0, 141.8, 134.4, 128.8, 114.5, 69.8, 53.6, 28.0, 24.9, 18.8, 15.9; MS (GC-MS): $m / z 249\left(\mathrm{M}^{+}\right)$; reaction time: $8 \mathrm{~h} ; 69 \%$ yield<smiles>CC(=O)/C=C(\C)Nc1ccc(C(C)(C)C)cc1</smiles>

(E)-4-((4-(tert-butyl)phenyl)amino)pent-3-en-2-one (113a)[202]: ${ }^{1} \mathrm{H}$ NMR (400 MHz, $\left.\mathrm{CDCl}_{3}\right): \delta 12.43(\mathrm{~s}, 1 \mathrm{H}), 7.34(\mathrm{~d}, J=8.6 \mathrm{~Hz}, 2 \mathrm{H}), 7.03(\mathrm{~d}, J=8.6 \mathrm{~Hz}, 2 \mathrm{H}), 5.16(\mathrm{~s}, 1 \mathrm{H})$, 2.09 (s, 3H), $1.98(\mathrm{~s}, 3 \mathrm{H}), 1.31$ (s, 9H); ${ }^{13} \mathrm{C} \mathrm{NMR}\left(100 \mathrm{MHz}, \mathrm{CDCl}_{3}\right): \delta$ 195.9, 160.9, 148.8, 136.1, 126.0, 124.5, 97.4, 34.6, 31.4, 29.2, 20.0; MS (GC-MS): m/z $231\left(\mathrm{M}^{+}\right)$; $96 \%$ yield.<smiles>COC(=O)C(C)(Nc1ccc(C(C)(C)C)cc1)C(C)=O</smiles>

Methyl 2-((4-(tert-butyl)phenyl)amino)-3-oxobutanoate (113b) ${ }^{[122]:}{ }^{1} \mathrm{H}$ NMR ((400 $\left.\mathrm{MHz}_{\mathrm{CDCl}}\right)$ : $\delta 7.07$ - $6.97(\mathrm{~m}, 2 \mathrm{H}), 6.53-6.45(\mathrm{~m}, 2 \mathrm{H}), 5.17(\mathrm{~s}, 1 \mathrm{H}), 3.81(\mathrm{~s}, 3 \mathrm{H})$, $2.55(\mathrm{q}, J=7.6 \mathrm{~Hz}, 2 \mathrm{H}), 2.22(\mathrm{~s}, 3 \mathrm{H}), 1.69(\mathrm{~s}, 3 \mathrm{H}), 1.20(\mathrm{t}, J=7.6 \mathrm{~Hz}, 3 \mathrm{H}) ;{ }^{13} \mathbf{C ~ N M R}$ $\left(100 \mathrm{MHz}, \mathrm{CDCl}_{3}\right): \delta 204.0,172.0,141.8,134.4,128.8,114.5,69.8,53.6,28.0,24.9$, 18.8, 15.9; MS (GC-MS): $m / z 249\left(\mathrm{M}^{+}\right)$; reaction time: $8 \mathrm{~h} ; 69 \%$ yield.<smiles>CC(=O)/C=C(\C)Nc1c(C)cccc1C</smiles> 
(E)-4-((2,6-dimethylphenyl)amino)pent-3-en-2-one (114a) ${ }^{[204]}$ : ${ }^{1} \mathrm{H}$ NMR $(400 \mathrm{MHz}$, $\mathrm{CDCl}_{3}$ ): $\delta 11.96$ (s, 1H), 7.11 (q, J = $5.4 \mathrm{~Hz}, 3 \mathrm{H}$ ), 5.23 (s, 1H), 2.22 (s, 6H), 2.13 (s, $3 \mathrm{H}), 1.64(\mathrm{~s}, 3 \mathrm{H}) ;{ }^{13} \mathrm{C}$ NMR $\left(100 \mathrm{MHz}, \mathrm{CDCl}_{3}\right): \delta 196.1,162.9,136.6,136.2,128.3$, 127.5, 95.9, 29.1, 18.9, 18.3; MS (GC-MS): m/z $203\left(\mathrm{M}^{+}\right) ; 78 \%$ yield.<smiles>COC(=O)C(Nc1c(C)cccc1C)C(C)=O</smiles>

Methyl 2-((2,6-dimethylphenyl)amino)-2-methyl-3-oxobutanoate (114b) ${ }^{[122]}:{ }^{1} \mathrm{H}$ NMR $\left(400 \mathrm{MHz}, \mathrm{CDCl}_{3}\right): \delta 6.97$ (d, $\left.J=7.4 \mathrm{~Hz}, 2 \mathrm{H}\right), 6.89(\mathrm{dd}, J=8.2,6.6 \mathrm{~Hz}, 1 \mathrm{H}), 4.93$ (s, $1 \mathrm{H}), 3.83(\mathrm{~s}, 3 \mathrm{H}), 2.30(\mathrm{~d}, J=1.2 \mathrm{~Hz}, 3 \mathrm{H}), 2.17(\mathrm{~s}, 6 \mathrm{H}), 1.17(\mathrm{~d}, J=1.2 \mathrm{~Hz}, 3 \mathrm{H})$; ${ }^{13} \mathrm{C} \mathrm{NMR}\left(100 \mathrm{MHz}, \mathrm{CDCl}_{3}\right): \delta 203.6,171.9,154.1,140.6,91.9,61.2,56.1,53.7,34.3$, 24.9, 22.5, 18.9, 14.2; MS (GC-MS): $\mathrm{m} / \mathrm{z} 249\left(\mathrm{M}^{+}\right)$; ESI-HRMS: $\mathrm{m} / \mathrm{z}$ calcd. For $\mathrm{C}_{14} \mathrm{H}_{19} \mathrm{NO}_{3}[\mathrm{M}+\mathrm{H}]^{+}: 250.1438$, found: 250.1441 ; reaction time: $6 \mathrm{~h} ; 65 \%$ yield; $114 \mathrm{~b}-$ s: 5 h, $82 \%$.<smiles>COc1cc(N/C(C)=C/C(C)=O)cc(OC)c1OC</smiles>

(E)-4-((4-fluoro-2-methylphenyl)amino)pent-3-en-2-one (115a) [202]: ${ }^{1} \mathrm{H}$ NMR (400 $\left.\mathrm{MHz}, \mathrm{CDCl}_{3}\right): \delta 12.12(\mathrm{~s}, 1 \mathrm{H}), 7.00(\mathrm{dd}, J=8.6,5.3 \mathrm{~Hz}, 1 \mathrm{H}), 6.96-6.89(\mathrm{~m}, 1 \mathrm{H}), 6.88$ - $6.79(\mathrm{~m}, 1 \mathrm{H}), 5.17(\mathrm{~s}, 1 \mathrm{H}), 2.21(\mathrm{~s}, 3 \mathrm{H}), 2.06(\mathrm{~s}, 3 \mathrm{H}), 1.77(\mathrm{~s}, 3 \mathrm{H}) ;{ }^{13} \mathrm{C}$ NMR (100 MHz, $\mathrm{CDCl}_{3}$ ): $\delta$ 196.2, 161.7, 161.0 (d, $J=245.6 \mathrm{~Hz}$ ), 135.1 (d, $J=325.9 \mathrm{~Hz}$ ), 128.2, 117.3 (d, $J=22.2 \mathrm{~Hz}$ ), 113.2 (d, $J=22.5 \mathrm{~Hz}$ ), 97.0, 29.0, 19.5, 18.2; MS (GCMS): $m / z 265\left(\mathrm{M}^{+}\right) ; 90 \%$ yield.<smiles>COC(=O)C(C)(Nc1cc(OC)c(OC)c(OC)c1)C(C)=O</smiles> 
Methyl 2-methyl-3-oxo-2-((3,4,5-trimethoxyphenyl)amino)butanoate (115b): ${ }^{\mathbf{1}} \mathbf{H}$ NMR (400 MHz, CDCl $): \delta 5.76(\mathrm{~s}, 2 \mathrm{H})$ 3.80-3.75 (m, 12H), $2.20(\mathrm{~s}, 3 \mathrm{H}) 0.88(\mathrm{~s}, 3 \mathrm{H})$; ${ }^{13} \mathrm{C}$ NMR $\left(100 \mathrm{MHz} \mathrm{CDCl}_{3}\right): \delta 203.6,171.9,154.1,140.6,91.9,61.2,56.1,53.7,34.3$, 24.9, 22.5, 18.9, 14.2; MS (GC-MS): $\mathrm{m} / \mathrm{z} 311\left(\mathrm{M}^{+}\right)$; ESI-HRMS: $\mathrm{m} / \mathrm{z}$ calcd. For $\mathrm{C}_{15} \mathrm{H}_{21} \mathrm{NO}_{6}[\mathrm{M}+\mathrm{H}]^{+}: 312.1442$, found: 312.1431 ; reaction time: $12 \mathrm{~h} ; 82 \%$ yield.<smiles>CC(=O)/C=C(\C)Nc1ccc(Br)cc1</smiles>

(E)-4-((4-(tert-bromo)phenyl)amino)pent-3-en-2-one (116a) ${ }^{[205]}$ : ${ }^{1} \mathrm{H} \quad$ NMR (400 $\mathrm{MHz}_{\mathrm{CDCl}}$ ): $\delta 12.39(\mathrm{~s}, 1 \mathrm{H}), 7.41(\mathrm{~d}, J=8.7 \mathrm{~Hz}, 2 \mathrm{H}), 6.94(\mathrm{~d}, J=8.7 \mathrm{~Hz}, 2 \mathrm{H}), 5.17$ (s, 1H), 2.06 (s, 3H), 1.95 (s, 3H); ${ }^{13} \mathrm{C}$ NMR (100 MHz, CDCl $)$ : $\delta$ 196.6, 159.8, 137.9, 132.2, 126.1, 118.8, 98.3, 29.2, 19.8; MS (GC-MS): $m / z 253\left(\mathrm{M}^{+}\right) ; 91 \%$ yield.<smiles>COC(=O)C(C)(Nc1ccc(Br)cc1)C(C)=O</smiles>

Methyl 2-((4-iodophenyl)amino)-3-oxobutanoate (116b) ${ }^{[123]}$ : ${ }^{1} \mathrm{H}$ NMR (400 MHz, $\left.\mathrm{CDCl}_{3}\right): \delta 7.51-7.32(\mathrm{~m}, 2 \mathrm{H}), 6.42-6.21(\mathrm{~m}, 2 \mathrm{H}), 5.36(\mathrm{~s}, 1 \mathrm{H}), 3.78(\mathrm{~d}, J=1.4 \mathrm{~Hz}$, $3 \mathrm{H}), 2.18(\mathrm{~d}, J=1.3 \mathrm{~Hz}, 3 \mathrm{H}), 1.66(\mathrm{~d}, J=1.4 \mathrm{~Hz}, 3 \mathrm{H}) ;{ }^{13} \mathrm{C} \mathrm{NMR}\left(100 \mathrm{MHz}, \mathrm{CDCl}_{3}\right): \delta$ 202.8, 171.5, 143.6, 138.1, 116.3, 79.4, 69.5, 53.8, 24.7, 18.6; MS (GC-MS): m/z 347 $\left(\mathrm{M}^{+}\right)$; ESI-HRMS: $\mathrm{m} / \mathrm{z}$ calcd. For $\mathrm{C}_{12} \mathrm{H}_{14} \mathrm{INO}_{3}[\mathrm{M}+\mathrm{H}]^{+}$: 348.0091, found: 348.0093; reaction time: $6 \mathrm{~h}, 64 \%$ yield. 
<smiles>CC(=O)/C=C(\C)Nc1ccc(I)cc1</smiles>

(E)-4-((4-(tert-iodo)phenyl)amino)pent-3-en-2-one (117a) ${ }^{\text {[202]: }}{ }^{1} \mathbf{H}$ NMR $(400 \mathrm{MHz}$, $\mathrm{CDCl}_{3}$ ): $\delta 12.57$ - 12.23 (bs, $\left.1 \mathrm{H}\right), 7.65$ (d, $\left.J=8.6 \mathrm{~Hz}, 2 \mathrm{H}\right), 6.87$ (d, J = 8.6 Hz, 2H), $5.22(\mathrm{~s}, 1 \mathrm{H}), 2.11(\mathrm{~s}, 3 \mathrm{H}), 2.01$ (s, 3H).; ${ }^{13} \mathrm{C}$ NMR (100 MHz, $\left.\mathrm{CDCl}_{3}\right): \delta$ 196.7, 159.5, 138.3, 126.4, 117.4, 98.5, 89.5, 29.4, 20; MS (GC-MS): m/z $301\left(\mathrm{M}^{+}\right) ; 92 \%$ yield.<smiles>COC(=O)C(C)(Nc1ccc(I)cc1)C(C)=O</smiles>

Methyl 2-((4-iodophenyl)amino)-3-oxobutanoate (117b): ${ }^{1} \mathrm{H}$ NMR (400 MHz, $\left.\mathrm{CDCl}_{3}\right): \delta 7.51-7.32(\mathrm{~m}, 2 \mathrm{H}), 6.42-6.21(\mathrm{~m}, 2 \mathrm{H}), 5.36(\mathrm{~s}, 1 \mathrm{H}), 3.78(\mathrm{~d}, J=1.4 \mathrm{~Hz}$, $3 \mathrm{H}), 2.18(\mathrm{~d}, J=1.3 \mathrm{~Hz}, 3 \mathrm{H}), 1.66(\mathrm{~d}, J=1.4 \mathrm{~Hz}, 3 \mathrm{H}) ;{ }^{13} \mathrm{C}$ NMR (100 MHz, CDCl $): \delta$ 202.8, 171.5, 143.6, 138.1, 116.3, 79.4, 69.5, 53.8, 24.7, 18.6; MS (GC-MS): m/z 347 $\left(\mathrm{M}^{+}\right) ; 64 \%$ yield.<smiles>CC(=O)/C=C(\C)Nc1ccccc1F</smiles>

(E)-4-((2-fluorophenyl)amino)pent-3-en-2-one (118a) ${ }^{\text {[202]: }}{ }^{1} \mathrm{H}$ NMR ((300 MHz, $\left.\mathrm{CDCl}_{3}\right) \delta 12.25(\mathrm{~s}, 1 \mathrm{H}), 7.32-6.94(\mathrm{~m}, 4 \mathrm{H}), 5.27(\mathrm{~s}, 1 \mathrm{H}), 2.12(\mathrm{~s}, 3 \mathrm{H}), 1.95(\mathrm{~s}, 3 \mathrm{H})$; ${ }^{13} \mathrm{C}$ NMR $\left(75 \mathrm{MHz}, \mathrm{CDCl}_{3}\right) \delta$ 196.8, 160.6, 156.8 (d, J=248.0 Hz), 127.3 (d, J=11.0 Hz), 124.4 (d, $J=4.0 \mathrm{~Hz}$ ), 116.4 (d, $J=20.2 \mathrm{~Hz}$ ), 98.3, 29.3, 19.6; MS (GC-MS): $\mathrm{m} / \mathrm{z}$ $193\left(\mathrm{M}^{+}\right) ; 92 \%$ yield. 
<smiles>COC(=O)C(C)(Nc1ccccc1F)C(C)=O</smiles>

Methyl 2-((2-fluorophenyl)amino)-2-methyl-3-oxobutanoate (118b) ${ }^{[123]}$ : ${ }^{1} \mathrm{H}$ NMR (400 MHz, $\left.\mathrm{CDCl}_{3}\right): \delta$ 7.03-6.99 (m, 1H), 6.92.6.87 (m, 1H), 6.70-6.62 (m, 1H), 6.48 $6.35(\mathrm{~m}, 1 \mathrm{H}), 3.80(\mathrm{~s}, 3 \mathrm{H}), 2.21(\mathrm{~s}, 3 \mathrm{H}), 1.68(\mathrm{~s}, 3 \mathrm{H}) ;{ }^{13} \mathrm{C} \mathrm{NMR}\left(100 \mathrm{MHz}, \mathrm{CDCl}_{3}\right): \delta$ 202.93, 171.34, 152.06 (d, $J=239.8 \mathrm{~Hz}), 132.40,124.43,118.06$ (d, $J=6.9 \mathrm{~Hz})$, 115.06 (d, $J=18.9 \mathrm{~Hz}), 113.35,69.29,53.61,24.61,18.66$; MS (GC-MS): $m / z 239$ $\left(\mathrm{M}^{+}\right) ; 78 \%$ yield.<smiles>CC(=O)/C=C(\C)Nc1ccc(Cl)c(C)c1</smiles>

(E)-4-((4-chloro-3-methylphenyl)amino)pent-3-en-2-one (119a): ${ }^{1} \mathrm{H}$ NMR (400 $\mathrm{MHz}_{\mathrm{CDCl}}$ ): $\delta 12.40$ (s, 1H), 7.27 (d, J = 8.4 Hz, 1H), $6.96(\mathrm{~d}, J=2.6 \mathrm{~Hz}, 1 \mathrm{H}), 6.87$ (dd, $J=8.5,2.6 \mathrm{~Hz}, 1 \mathrm{H}), 5.19(\mathrm{~s}, 1 \mathrm{H}), 2.34(\mathrm{~s}, 3 \mathrm{H}), 2.09(\mathrm{~s}, 3 \mathrm{H}), 1.97(\mathrm{~s}, 3 \mathrm{H}) ;{ }^{13} \mathrm{C} \mathrm{NMR}$ $\left(100 \mathrm{MHz}_{\mathrm{CDCl}}\right): \delta$ 196.3, 160.1, 137.2, 137.0, 131.3, 129.5, 127.0, 123.3, 97.9, 29.5, 20.1, 19.8; MS (GC-MS): $m / z 223\left(\mathrm{M}^{+}\right) ; 54 \%$ yield.<smiles>COC(=O)C(C)(Nc1ccc(Cl)c(C)c1)C(C)=O</smiles>

Methyl 2-((4-chloro-3-methylphenyl)amino)-2-methyl-3-oxobutanoate (119b): ${ }^{\mathbf{1}} \mathbf{H}$ NMR (400 MHz, CDCl $)$ ): $\delta 7.08(\mathrm{~d}, J=8.6 \mathrm{~Hz}, 1 \mathrm{H}), 6.48-6.39(\mathrm{~m}, 1 \mathrm{H}), 6.27$ (dd, $J=$ 8.6, $2.9 \mathrm{~Hz}, 1 \mathrm{H}), 3.78(\mathrm{~s}, 3 \mathrm{H}), 2.26(\mathrm{~s}, 3 \mathrm{H}), 2.19$ (s, 3H), 1.66 (s, 3H); ${ }^{13} \mathrm{C}$ NMR $\left(100 \mathrm{MHz}, \mathrm{CDCl}_{3}\right): \delta 203.1,171.5,142.5,136.9,129.6,123.6,116.7,112.6,69.5$, 53.6, 24.6, 20.3, 18.6; MS (GC-MS): $\mathrm{m} / \mathrm{z} 269\left(\mathrm{M}^{+}\right)$; ESI-HRMS: $\mathrm{m} / \mathrm{z}$ calcd. For $\mathrm{C}_{13} \mathrm{H}_{16} \mathrm{CINO}_{3}[\mathrm{M}+\mathrm{H}]^{+}:$270.0891, found: $270.0894 ; 41 \%$ yield. 
<smiles>CC(=O)/C=C(\C)Nc1ccc(F)cc1C</smiles>

(E)-4-((4-fluoro-2-methylphenyl)amino)pent-3-en-2-one (120a): ${ }^{1} \mathrm{H}$ NMR (400 $\mathrm{MHz}_{\mathrm{CDCl}}$ ): $\delta 12.12(\mathrm{~s}, 1 \mathrm{H}), 7.00(\mathrm{dd}, J=8.7,5.4 \mathrm{~Hz}, 1 \mathrm{H}), 6.93-6.88(\mathrm{~m}, 1 \mathrm{H}), 6.87$ - $6.79(\mathrm{~m}, 1 \mathrm{H}), 5.17(\mathrm{~s}, 1 \mathrm{H}), 2.21(\mathrm{~s}, 3 \mathrm{H}), 2.06(\mathrm{~s}, 3 \mathrm{H}), 1.77(\mathrm{~s}, 3 \mathrm{H}) ;{ }^{13} \mathrm{C}$ NMR (100 MHz, $\left.\mathrm{CDCl}_{3}\right): \delta$ 196.2, 161.7, 161.0 (d, $\left.J=245.6 \mathrm{~Hz}\right), 135.1$ (d, $\left.J=325.9 \mathrm{~Hz}\right)$, 128.2, 117.3 (d, $J=22.2 \mathrm{~Hz}$ ), 113.2 (d, $J=22.5 \mathrm{~Hz}$ ), 97.0, 29.0, 19.5, 18.2; MS (GCMS): $m / z 207\left(\mathrm{M}^{+}\right)$; ESI-HRMS: $m / z$ calcd. For $\mathrm{C}_{12} \mathrm{H}_{14} \mathrm{FNO}[\mathrm{M}+\mathrm{H}]^{+}:$208.1132, found: 208.1132; $90 \%$ yield.<smiles>COC(=O)C(C)(Nc1ccc(F)cc1C)C(C)=O</smiles>

Methyl 2-((4-fluoro-2-methylphenyl)amino)-3-oxobutanoate (120b): ${ }^{1} \mathrm{H}$ NMR (400 $\mathrm{MHz}_{\mathrm{CDCl}}$ ): $\delta 6.83(\mathrm{dd}, J=9.2,3.1 \mathrm{~Hz}, 1 \mathrm{H}), 6.71(\mathrm{td}, J=8.5,3.1 \mathrm{~Hz}, 1 \mathrm{H}), 6.22$ (dd, $J=8.8,4.7 \mathrm{~Hz}, 1 \mathrm{H}), 5.15(\mathrm{~s}, 1 \mathrm{H}), 3.78(\mathrm{~d}, J=0.9 \mathrm{~Hz}, 3 \mathrm{H}), 2.24(\mathrm{~s}, 3 \mathrm{H}), 2.19(\mathrm{~s}, 3 \mathrm{H})$, $1.66(\mathrm{~s}, 3 \mathrm{H}) ;{ }^{13} \mathrm{C}$ NMR (100 MHz, $\left.\mathrm{CDCl}_{3}\right): \delta 203.38,171.92,155.71(\mathrm{~d}, J=236.1 \mathrm{~Hz})$, 138.16, 125.44 (d, J=7.1 Hz), 117.51 (d, $J=22.4 \mathrm{~Hz}), 112.74$ (d, J=21.7 Hz), 111.98 (d, $J=7.8 \mathrm{~Hz}), 69.78,53.54,24.54,18.74,17.78$; MS (GC-MS): $m / z 253\left(\mathrm{M}^{+}\right) ; 62 \%$ yield. ESI-HRMS: $\mathrm{m} / \mathrm{z}$ calcd. For $\mathrm{C}_{13} \mathrm{H}_{16} \mathrm{FNO}_{3}[\mathrm{M}+\mathrm{H}]^{+}:$254.1187, found: 254.1188; 120b-s: sunlight; reaction time: $5 \mathrm{~h}, 72 \%$ yield.<smiles>CC(=O)/C=C(\C)NCc1ccccc1</smiles>

(E)-4-(benzylamino)pent-3-en-2-one (121a): ${ }^{1} \mathrm{H}$ NMR (400 MHz, $\mathrm{CDCl}_{3}$ ): $\delta 11.15$ (s, $1 \mathrm{H}), 7.41-7.14(\mathrm{~m}, 5 \mathrm{H}), 5.05(\mathrm{~s}, 1 \mathrm{H}), 4.45(\mathrm{~d}, J=6.3 \mathrm{~Hz}, 2 \mathrm{H}), 2.02(\mathrm{~s}, 3 \mathrm{H}), 1.92$ (s, $3 \mathrm{H}) ;{ }^{13} \mathrm{C}$ NMR $\left(100 \mathrm{MHz}, \mathrm{CDCl}_{3}\right): \delta 195.3,163.4,138.0,128.8,127.4,126.7,95.9$, 46.7, 28.8, 18.9; MS (GC-MS): $m / z \quad 169\left(\mathrm{M}^{+}\right)$; ESI-HRMS: $m / z$ calcd. for $\mathrm{C}_{12} \mathrm{H}_{15} \mathrm{NO}[\mathrm{M}+\mathrm{H}]^{+}:$190.1226, found: $190.1228 ; 61 \%$ yield. 
<smiles>COC(=O)C(C)(NCc1ccccc1)C(C)=O</smiles>

Methyl 2-(benzylamino)-2-methyl-3-oxobutanoate (121b): ${ }^{1} \mathrm{H}$ NMR (400 MHz, $\left.\mathrm{CDCl}_{3}\right)$ : $\delta 7.38-7.30(\mathrm{~m}, 5 \mathrm{H}), 3.76(\mathrm{~s}, 3 \mathrm{H}), 3.64(\mathrm{~s}, 2 \mathrm{H}), 2.24(\mathrm{~s}, 3 \mathrm{H}), 1.57(\mathrm{~s}, 3 \mathrm{H})$; ${ }^{13} \mathrm{C}$ NMR $\left(100 \mathrm{MHz}, \mathrm{CDCl}_{3}\right): \delta 204.9,172.4,139.8,128.6,128.4,127.4,72.1,52.9$, 47.9, 25.2, 19.0; MS (GC-MS): $m / z 235\left(\mathrm{M}^{+}\right)$; ESI-HRMS: $\mathrm{m} / z$ calcd. For $\mathrm{C}_{13} \mathrm{H}_{17} \mathrm{NO}_{3}[\mathrm{M}+\mathrm{H}]^{+}:$: 236.1281, found: $236.1280 ; 71 \%$ yield.<smiles>CCc1cccc(CC)c1N/C(C)=C/C(C)=O</smiles>

(E)-4-((2,6-diethylphenyl)amino)pent-3-en-2-one (122a) ${ }^{[207]}:{ }^{1} \mathbf{H}$ NMR (400 MHz, Chloroform- $d$ ): $\delta 12.08$ (s, 1H), 7.24 (dd, $J=8.3,6.8 \mathrm{~Hz}, 1 \mathrm{H}), 7.14(\mathrm{~d}, J=7.6 \mathrm{~Hz}, 2 \mathrm{H})$, 5.23 (s, 1H), 2.57 (dq, J=22.8, 7.3 Hz, 4H), 2.14 (s, 3H), 1.65 (s, 3H), 1.20 (t, J= 7.6 $\mathrm{Hz}, 6 \mathrm{H}) ;{ }^{13} \mathrm{C}$ NMR (101 MHz, $\left.\mathrm{CDCl}_{3}\right): \delta$ 195.94, 163.02, 141.84, 135.17, 127.88, 126.44, 95.69, 29.05, 24.78, 19.00, 14.59; MS (GC-MS): m/z 231 (M+); 84\% yield.<smiles>CC(=O)/C=C(\C)Nc1ccccc1I</smiles>

(E)-4-((2-iodophenyl)amino)pent-3-en-2-one (123a) ${ }^{\text {[208]: }}{ }^{1} \mathrm{H} \quad \mathrm{NMR} \quad(400 \mathrm{MHz}$, Chloroform- $d$ ): $\delta 12.29$ (s, 1H), 7.88 (dd, $J=7.9,2.1 \mathrm{~Hz}, 1 \mathrm{H}), 7.36-7.30(\mathrm{~m}, 1 \mathrm{H})$, 7.17 (dd, $J=8.0,2.0 \mathrm{~Hz}, 1 \mathrm{H}), 6.95$ (td, $J=7.7,1.9 \mathrm{~Hz}, 1 \mathrm{H}), 5.25(\mathrm{~d}, J=2.3 \mathrm{~Hz}, 1 \mathrm{H})$, $2.13(\mathrm{t}, J=1.9 \mathrm{~Hz}, 3 \mathrm{H}), 1.85$ (t, $J=1.8 \mathrm{~Hz}, 3 \mathrm{H}) ;{ }^{13} \mathrm{C}$ NMR (101 MHz, CDCl 3$): \delta$ 196.74, 159.75, 141.09, 139.54, 128.78, 127.86, 127.19, 97.80, 29.31, 19.72; MS (GC-MS): $\mathrm{m} / \mathrm{z} 301\left(\mathrm{M}^{+}\right) ; 61 \%$ yield. 
<smiles>CC(=O)C=C(C)Nc1ccc([N+](=O)[O-])cc1</smiles>

(E)-4-((4-nitrophenyl)amino)pent-3-en-2-one (124a): ${ }^{1} \mathrm{H} \quad \mathrm{NMR} \quad(400 \mathrm{MHz}$, Chloroform-d) $\delta 12.75$ (s, 1H), 8.18 (d, J = 9.1 Hz, 2H), $7.16(\mathrm{~d}, J=9.0 \mathrm{~Hz}, 2 \mathrm{H}), 5.32$ (s, 1H), $2.51-1.59(\mathrm{~m}, 6 \mathrm{H})$, MS (GC-MS): $\mathrm{m} / \mathrm{z} 220\left(\mathrm{M}^{+}\right) ; 57 \%$ yield.<smiles>CCCN/C(C)=C/C(C)=O</smiles>

(E)-4-(propylamino)pent-3-en-2-one (125a) ${ }^{\text {209]: }}$ MS (GC-MS): m/z 141( $\left.\mathrm{M}^{+}\right)$.<smiles>CCN(CC)/C(C)=C/C(C)=O</smiles>

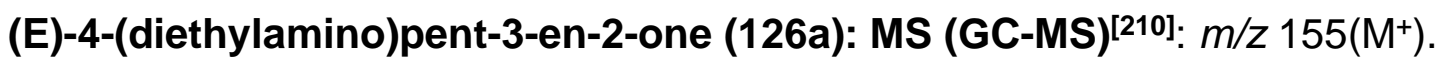<smiles>CC(=O)/C=C(\C)NCC1(C)CCCC2(C)c3ccc(C(C)C)cc3CCC12</smiles>

(E)-4-(((7-isopropyl-1,4a-dimethyl-1,2,3,4,4a,9,10,10a-octahydrophenanthren-1yl)methyl)amino)pent-3-en-2-one (127a): ${ }^{1} \mathrm{H}$ NMR (400 MHz, Chloroform- $d$ ): $\delta 7.18$ (d, $J=8.2 \mathrm{~Hz}, 1 \mathrm{H}), 6.98$ (dd, $J=8.1,2.0 \mathrm{~Hz}, 1 \mathrm{H}), 6.89$ (t, $J=2.3 \mathrm{~Hz}, 1 \mathrm{H}), 3.71$ (d, $J=$ $1.8 \mathrm{~Hz}, 3 \mathrm{H}$ ), 3.49 (d, $J=1.7 \mathrm{~Hz}, 1 \mathrm{H}), 3.24$ (dd, $J=16.4,7.0 \mathrm{~Hz}, 1 \mathrm{H}$ ), 2.86 (ddd, $J=$ 23.1, 12.7, $6.9 \mathrm{~Hz}, 3 \mathrm{H}), 2.32(\mathrm{~d}, J=9.4 \mathrm{~Hz}, 1 \mathrm{H}), 2.05(\mathrm{~d}, J=11.7 \mathrm{~Hz}, 1 \mathrm{H}), 1.66-1.46$ $(\mathrm{m}, 4 \mathrm{H}), 1.39-1.13(\mathrm{~m}, 15 \mathrm{H}), 0.89(\mathrm{~d}, J=8.2 \mathrm{~Hz}, 3 \mathrm{H}) \cdot{ }^{13} \mathrm{C}$ NMR $(101 \mathrm{MHz}, \mathrm{CDCl}): \delta$ 192.23 , 176.97, 147.71, 145.60, 135.13, 126.93, 124.47, 123.93, 108.57, 59.78, 59.07, 57.96, 51.78, 45.19, 44.72, 38.62, 37.58, 36.20, 33.59, 30.41, 25.40, 24.14, 19.50, 19.00; MS (GC-MS): $m / z$ 368( $\left.\mathbf{M}^{+}\right) ; 73 \%$ yield. 
<smiles>CC(=O)/C=C(\C)NCCc1c[nH]c2ccccc12</smiles>

(E)-4-((2-(1H-indol-3-yl)ethyl)amino)pent-3-en-2-one (128a) [211]: ${ }^{1} \mathrm{H} \quad$ NMR (300 MHz, Chloroform-d) $\delta 10.93(\mathrm{~s}, 1 \mathrm{H}), 8.86(\mathrm{~s}, 1 \mathrm{H}), 7.58$ (d, J = 7.7 Hz, 1H), 7.35 (dd, J $=7.9,1.2 \mathrm{~Hz}, 1 \mathrm{H}), 7.23-6.94(\mathrm{~m}, 3 \mathrm{H}), 4.95(\mathrm{~s}, 1 \mathrm{H}), 3.57(\mathrm{q}, J=6.5 \mathrm{~Hz}, 2 \mathrm{H}), 3.05(\mathrm{t}$, $J=6.8 \mathrm{~Hz}, 2 \mathrm{H}), 2.01(\mathrm{~s}, 3 \mathrm{H}), 1.86(\mathrm{~s}, 3 \mathrm{H}) ;{ }^{13} \mathrm{C}$ NMR $\left(75 \mathrm{MHz}, \mathrm{CDCl}_{3}\right) \delta 194.69,163.28$, 136.56, 127.00, 123.07, 121.87, 119.19, 118.27, 111.73, 111.54, 95.24, 43.51, 28.70, 26.05, 18.99. MS (GC-MS): $m / z 242\left(\mathrm{M}^{+}\right) ; 52 \%$ yield.<smiles>CC(=O)/C=C(\C)N1CCC(O)CC1</smiles>

(E)-4-(4-hydroxypiperidin-1-yl)pent-3-en-2-one (129a): MS (GC-MS): $1 \mathrm{H} \mathrm{NMR}$ (400 MHz, Chloroform-d): $\delta 11.10-10.81(\mathrm{~m}, 1 \mathrm{H}), 4.88(\mathrm{~s}, 1 \mathrm{H}), 3.34(\mathrm{tt}, \mathrm{J}=9.1,4.7$ $\mathrm{Hz}, 1 \mathrm{H}), 1.95(\mathrm{~s}, 3 \mathrm{H}), 1.91(\mathrm{~s}, 3 \mathrm{H}), 1.85-1.69(\mathrm{~m}, 4 \mathrm{H}), 1.60-1.49(\mathrm{~m}, 1 \mathrm{H}), 1.31(\mathrm{t}$, $\mathrm{J}=9.8 \mathrm{~Hz}, 4 \mathrm{H}) ;{ }^{13} \mathrm{C}$ NMR $\left(101 \mathrm{MHz}, \mathrm{CDCl}_{3}\right): \delta 194.27,161.78,94.86,51.45,33.76$, 28.67, 25.28, 24.37, 18.56. $\mathrm{m} / \mathrm{z} 183\left(\mathrm{M}^{+}\right) ; 41 \%$ yield.<smiles>CNC(=O)c1ccc(N/C(C)=C/C(C)=O)cc1</smiles>

(E)-N-methyl-4-((4-oxopent-2-en-2-yl)amino)benzamide (130a): ${ }^{1} \mathrm{H}$ NMR (400 $\mathrm{MHz}$, Chloroform-d) $\delta 12.56(\mathrm{~s}, 1 \mathrm{H}), 7.75(\mathrm{~d}, J=8.6 \mathrm{~Hz}, 2 \mathrm{H}), 7.09$ (d, J = 8.7 Hz, 2H), $5.23(\mathrm{~s}, 1 \mathrm{H}), 2.99(\mathrm{~d}, J=4.8 \mathrm{~Hz}, 3 \mathrm{H}), 2.10(\mathrm{~d}, J=1.1 \mathrm{~Hz}, 3 \mathrm{H}), 2.05(\mathrm{~s}, 3 \mathrm{H}) ;{ }^{13} \mathrm{C}$ NMR $\left(101 \mathrm{MHz}, \mathrm{CDCl}_{3}\right) \delta 196.94,167.55,159.17,141.78,131.13,128.67,128.14$, 123.50, 114.24, 99.10, 29.48, 26.98, 20.27; MS (GC-MS): $m / z$ 232(M+); 64\% yield. 
<smiles>CCOC(=O)/C=C/c1ccc(N/C(C)=C/C(C)=O)cc1</smiles>

ethyl (E)-3-(4-(((E)-4-oxopent-2-en-2-yl)amino)phenyl)acrylate (131a): ${ }^{1} \mathrm{H}$ NMR (400 MHz, Chloroform-d): $\delta 12.59$ (s, 1H), 7.63 (d, J=15.9 Hz, 1H), $7.54-7.44$ (m, $2 \mathrm{H}), 7.16-7.03(\mathrm{~m}, 2 \mathrm{H}), 6.37(\mathrm{~d}, J=16.0 \mathrm{~Hz}, 1 \mathrm{H}), 5.22(\mathrm{~s}, 1 \mathrm{H}), 4.54-4.01(\mathrm{~m}, 2 \mathrm{H})$, $2.08(\mathrm{dd}, J=12.3,1.2 \mathrm{~Hz}, 6 \mathrm{H}), 1.32$ (td, $J=7.1,1.2 \mathrm{~Hz}, 3 \mathrm{H}) ;{ }^{13} \mathrm{C}$ NMR $(101 \mathrm{MHz}$, $\left.\mathrm{CDCl}_{3}\right): \delta 196.80,167.06,158.96,143.60,140.82,131.12,129.05,123.90,117.82$, 99.02, 60.61, 29.44, 20.29, 14.44; MS (GC-MS): m/z 273(M+); 59\% yield.<smiles>CCCCC(=O)/C=C(\C)Nc1ccccc1</smiles>

(E)-2-(phenylamino)non-2-en-4-one (132a): ${ }^{1} \mathrm{H}$ NMR (300 MHz, $\left.\mathrm{CDCl}_{3}\right) \delta 12.56$ (s, $1 \mathrm{H}), 7.33$ (dd, $J=8.3,7.2 \mathrm{~Hz}, 2 \mathrm{H}), 7.24-7.15(\mathrm{~m}, 1 \mathrm{H}), 7.15-7.06(\mathrm{~m}, 2 \mathrm{H}), 5.19(\mathrm{~s}$, $1 \mathrm{H}), 2.41-2.28(\mathrm{~m}, 2 \mathrm{H}), 2.01(\mathrm{~s}, 3 \mathrm{H}), 1.76-1.57(\mathrm{~m}, 2 \mathrm{H}), 1.44-1.28(\mathrm{~m}, 4 \mathrm{H}), 1.02$ - 0.85 (m, 3H); ${ }^{13} \mathrm{C}$ NMR $\left(75 \mathrm{MHz}, \mathrm{CDCl}_{3}\right) \delta 199.4,160.1,138.9,129.1,125.4,124.5$, 97.3, 42.3, 31.8, 25.7, 22.6, 20.0, 14.0; MS (GC-MS): m/z $231\left(\mathrm{M}^{+}\right)$; ESI-HRMS: $\mathrm{m} / \mathrm{z}$ calcd. For $\mathrm{C}_{15} \mathrm{H}_{21} \mathrm{NO}[\mathrm{M}+\mathrm{H}]^{+}:$232.1696, found: $232.1700 ; 87 \%$ yield.<smiles>CCCCCC(=O)C(C)(Nc1ccccc1)C(=O)OC</smiles>

Methyl 2-methyl-3-oxo-2-(phenylamino)octanoate (132b): ${ }^{1} \mathrm{H}$ NMR (400 MHz, $\left.\mathrm{CDCl}_{3}\right)$ : $\delta 7.20-7.07(\mathrm{~m}, 2 \mathrm{H}), 6.81-6.66(\mathrm{~m}, 1 \mathrm{H}), 6.54-6.46(\mathrm{~m}, 2 \mathrm{H}), 5.33(\mathrm{~s}, 1 \mathrm{H})$, $3.76(\mathrm{~s}, 3 \mathrm{H}), 2.55-2.41(\mathrm{~m}, 2 \mathrm{H}), 1.68(\mathrm{~s}, 3 \mathrm{H}), 1.57$ (h, J = $7.0 \mathrm{~Hz}, 2 \mathrm{H}), 1.27-1.18$ $(\mathrm{m}, 6 \mathrm{H}), 0.85(\mathrm{t}, J=7.1 \mathrm{~Hz}, 3 \mathrm{H}) ;{ }^{13} \mathrm{C}$ NMR (100 MHz, $\left.\mathrm{CDCl}_{3}\right): \delta 205.77,171.88$, 143.93, 129.35, 118.24, 114.10, 69.45, 53.38, 36.85, 31.09, 23.52, 22.35, 18.66, 13.86; MS (GC-MS): m/z $277\left(\mathrm{M}^{+}\right)$; ESI-HRMS: $m / z$ calcd. For $\mathrm{C}_{16} \mathrm{H}_{23} \mathrm{NO}_{3}[\mathrm{M}+\mathrm{H}]^{+}$: 278.1751 , found: $278.1748 ; 71 \%$ yield. 
<smiles>CCC(=O)/C=C(\CC)Nc1ccccc1</smiles>

(E)-5-(phenylamino)hept-4-en-3-one (133a) ${ }^{\text {[212]: }}{ }^{1} \mathrm{H}$ NMR $\left(400 \mathrm{MHz}, \mathrm{CDCl}_{3}\right): \delta 12.52$ $(\mathrm{s}, 1 \mathrm{H}), 7.35(\mathrm{t}, J=7.8 \mathrm{~Hz}, 2 \mathrm{H}), 7.25-7.18(\mathrm{~m}, 1 \mathrm{H}), 7.17-7.09(\mathrm{~m}, 2 \mathrm{H}), 5.24(\mathrm{~s}, 1 \mathrm{H})$, $2.48-2.25(\mathrm{~m}, 4 \mathrm{H}), 1.13(\mathrm{~m}, 6 \mathrm{H}){ }^{13} \mathrm{C}$ NMR $\left(100 \mathrm{MHz}, \mathrm{CDCl}_{3}\right): \delta 200.2,165.9,138.7$, 129.1, 125.7, 125.2, 94.4, 35.3, 25.2, 12.5, 9.9; MS (GC-MS): m/z 203 (M+); $91 \%$ yield.<smiles>CCC(=O)C(Nc1ccccc1)(C(=O)CC)C(=O)OC</smiles>

Methyl 2-ethyl-3-oxo-2-(phenylamino)pentanoate (133b) ${ }^{[122]}$ : ${ }^{1} \mathrm{H}$ NMR $(400 \mathrm{MHz}$, CDCl3) $\delta 7.20-7.08(\mathrm{~m}, 2 \mathrm{H}), 6.79-6.65(\mathrm{~m}, 1 \mathrm{H}), 6.54-6.46(\mathrm{~m}, 2 \mathrm{H}), 3.77(\mathrm{~s}, 3 \mathrm{H})$, $2.58-2.43(\mathrm{~m}, 2 \mathrm{H}), 2.36-2.29(\mathrm{~m}, 2 \mathrm{H}), 1.04(\mathrm{t}, J=7.3 \mathrm{~Hz}, 3 \mathrm{H}), 0.66(\mathrm{t}, J=7.5 \mathrm{~Hz}$, $3 \mathrm{H}) ;{ }^{13} \mathrm{C}$ NMR $\left(101 \mathrm{MHz}, \mathrm{CDCl}_{3}\right) \delta 206.74,171.13,143.94,129.41,125.12,118.20$, 114.13, 94.41, 53.34, 30.25, 22.64, 8.26, 7.13. MS (GC-MS): $m / z 249\left(\mathrm{M}^{+}\right) ; 81 \%$ yield<smiles>CC(=O)/C(Cl)=C(\C)Nc1ccccc1</smiles>

(Z)-3-chloro-4-(phenylamino)pent-3-en-2-one (134a): ${ }^{1} \mathrm{H} \quad \mathrm{NMR} \quad(400 \mathrm{MHz}$, Chloroform- $d$ ): $\delta 12.68(\mathrm{~s}, 1 \mathrm{H}), 7.30-7.22(\mathrm{~m}, 2 \mathrm{H}), 7.13(\mathrm{td}, J=7.3,1.3 \mathrm{~Hz}, 1 \mathrm{H}), 7.03$ - $6.95(\mathrm{~m}, 2 \mathrm{H}), 2.27(\mathrm{~d}, J=1.0 \mathrm{~Hz}, 3 \mathrm{H}), 2.08(\mathrm{~d}, J=0.9 \mathrm{~Hz}, 3 \mathrm{H}) ;{ }^{13} \mathrm{C}$ NMR $(101 \mathrm{MHz}$, $\left.\mathrm{CDCl}_{3}\right): \delta 194.55,158.42,138.47,129.29,126.23,125.42,104.52,28.73,18.33$; MS (GC-MS): $m / z 209\left(\mathrm{M}^{+}\right) ; 46 \%$ yield. 


\subsection{IR-SPECTRA OF CARBON NITRIDES}

Summary table of the FTIR signals

\begin{tabular}{|c|c|c|c|c|c|c|c|c|c|c|c|c|}
\hline Name & Entry & & & & & IR & & & & & & \\
\hline Com. & 0 & & 806 & & & 1230 & 1314 & 1396 & & 1540 & & 1635 \\
\hline WW4 & 0 & & 805 & & & 1232 & 1315 & 1396 & & 1540 & & 1633 \\
\hline WW7 & 1 & & 808 & & 1207 & 1234 & 1316 & 1397 & 1456 & 1540 & & 1635 \\
\hline WW11 & 2 & & 806 & 1132 & & 1233 & 1315 & 1401 & 1453 & & & 1630 \\
\hline WW12 & 3 & & 812 & & 1206 & 1233 & 1314 & 1404 & 1456 & 1537 & & 1633 \\
\hline WW10 & 4 & & 804 & 1130 & 1204 & 1230 & 1314 & 1397 & 1453 & & & 1524 \\
\hline FWW2 & 5 & 772 & 806 & 1132 & 1204 & 1231 & 1314 & 1395 & 1455 & 1537 & & 1632 \\
\hline FWW6 & 6 & & 804 & 1130 & 1202 & 1232 & 1314 & 1396 & 1456 & 1540 & & 1632 \\
\hline FWW13 & 7 & & 809 & & 1207 & 1235 & 1315 & 1404 & 1457 & 1540 & 1558 & 1634 \\
\hline FWW3 & 8 & & 804 & & 1204 & 1232 & 1314 & 1396 & 1456 & 1540 & 1559 & 1624 \\
\hline FWW4 & 9 & & 804 & 1135 & 1205 & 1233 & 1314 & 1396 & 1456 & & & 1619 \\
\hline FWW5 & 10 & & 804 & & 1202 & 1232 & 1314 & 1398 & 1456 & 1539 & & 1625 \\
\hline FWW15 & 11 & & 804 & & 1204 & 1232 & 1314 & 1396 & 1456 & & 1558 & 1620 \\
\hline FWW16 & 12 & & 804 & & 1204 & 1232 & 1314 & 1396 & 1456 & & 1576 & 1617 \\
\hline FWW17 & 13 & 733 & 803 & 1132 & 1205 & 1232 & 1315 & 1396 & 1456 & & & 1622 \\
\hline FWW20 & 14 & & 804 & & 1204 & 1232 & 1314 & 1396 & 1456 & & 1558 & 1620 \\
\hline FWW21 & 15 & & 801 & & 1204 & 1232 & 1314 & 1396 & 1456 & & & 1617 \\
\hline FWW22 & 16 & & 804 & 1075 & 1204 & 1230 & 1314 & 1393 & 1456 & 1540 & 1576 & 1622 \\
\hline FWW23 & 17 & & 804 & & 1204 & 1232 & 1316 & 1396 & 1456 & & & 1620 \\
\hline FWW24 & 18 & & 804 & & 1207 & 1232 & 1316 & 1396 & 1456 & & 1558 & 1635 \\
\hline FWW8 & 19 & & 809 & 1207 & 1236 & 1278 & 1330 & 1404 & 1457 & 1539 & 1568 & 1633 \\
\hline FWW9 & 20 & & 809 & & 1207 & 1235 & 1316 & 1405 & 1457 & 1542 & 1568 & 1632 \\
\hline FWW10 & 21 & & 809 & & & 1239 & 1319 & 1409 & & 1431 & 1555 & 1632 \\
\hline FWW11 & 22 & & 809 & & & 1240 & 1318 & 1407 & 1456 & 1540 & 1557 & 1634 \\
\hline
\end{tabular}




\section{Commercial g- $\mathrm{C}_{3} \mathrm{~N}_{4}$}

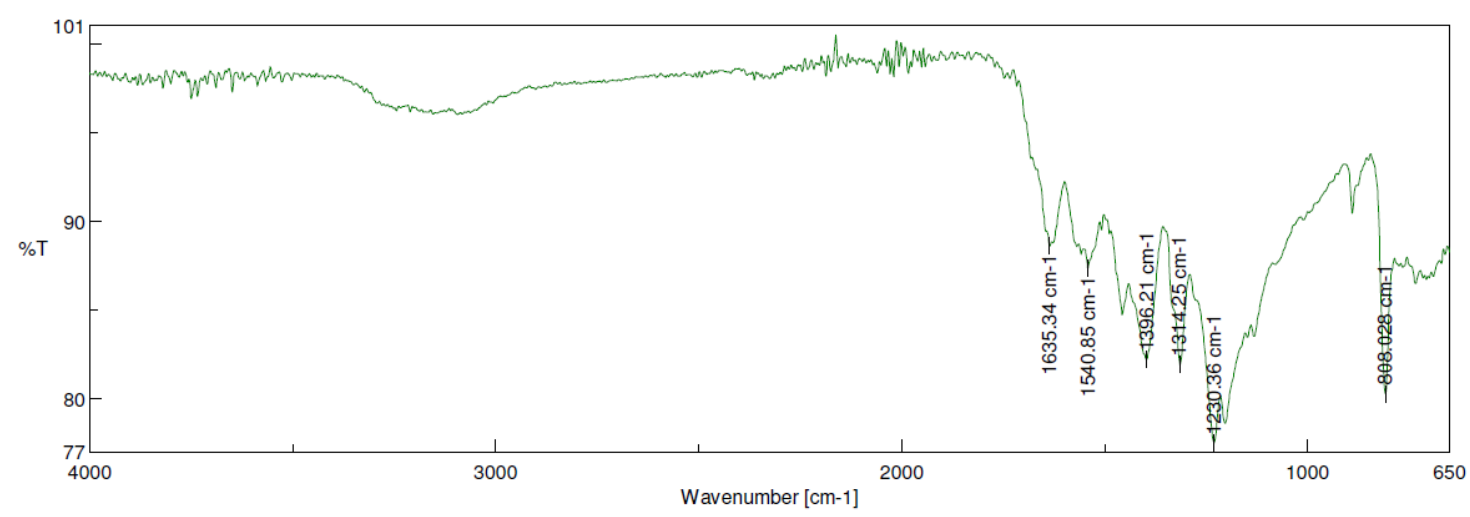

\begin{tabular}{llll} 
[Comments] & & \multicolumn{2}{l}{ [Measurement Information] } \\
$\begin{array}{llll}\text { Sample name } \\
\text { Comment }\end{array}$ & g-C3N4_commercial & Model Name & FT//R-4100typeA \\
User & & Serial Number & C188561016 \\
Division & AK Das & Measurement Date & $11.10 .201815: 00$ \\
Company & Univ. Göttingen Org. + Biomol. Chemie & & \\
& & Light Source & Standard \\
& & Detector & TGS \\
[Detailed Information] & Accumulation & 32 \\
Creation date & $11.10 .201815: 02$ & Resolution & $4 \mathrm{~cm}-1$ \\
& Zero Filling & On \\
Data array type & Linear data array & Apodization & Cosine \\
Horizontal axis & Wavenumber [cm-1] & Gain & Auto $(8)$ \\
Vertical axis & $\%$ T & Aperture & Auto $(7.1 \mathrm{~mm})$ \\
Start & $649.893 \mathrm{~cm}-1$ & Scanning Speed & Auto $(2 \mathrm{~mm} / \mathrm{sec})$ \\
End & $4000.6 \mathrm{~cm}-1$ & Filter & Auto $(30000 \mathrm{~Hz})$ \\
Data interval & $0.964233 \mathrm{~cm}-1$ & & \\
Data points & 3476 & & \\
& & &
\end{tabular}

WW4 Melamine no Entry (small scale attempt)

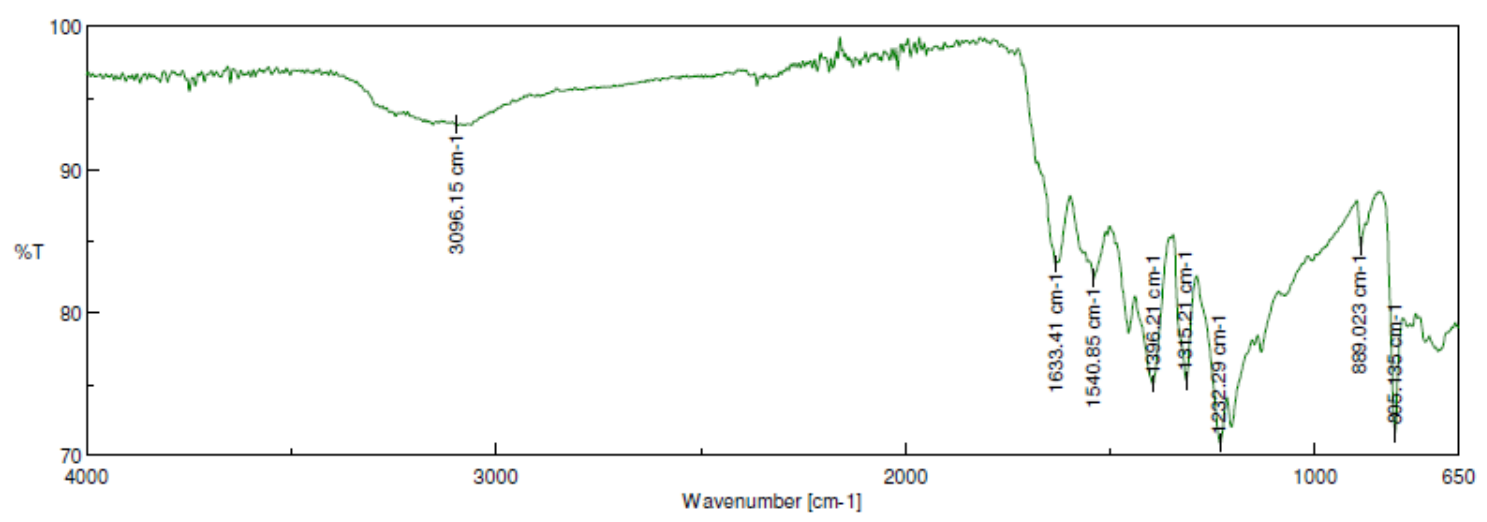

\begin{tabular}{|c|c|c|c|}
\hline \multicolumn{2}{|l|}{ Comments] } & \multicolumn{2}{|c|}{ [Measurement Information] } \\
\hline Sample name & g-C3N4 WW4 & Model Name & $F T / I R$ \\
\hline $\begin{array}{l}\text { Comment } \\
\text { User }\end{array}$ & & Serial Number & C188561 \\
\hline Division & AK Das & Measurement Date & $11.10 .201814: 56$ \\
\hline Comp & Univ. Gottingen Org. + Biomol. Chemie & Light & \\
\hline etall & ation. & Detec & \\
\hline Creatio & $11.10 .201814: 58$ & $\begin{array}{l}\text { Accumulation } \\
\text { Resolution }\end{array}$ & $\begin{array}{l}32 \\
4 \mathrm{~cm}-1\end{array}$ \\
\hline ta array & Une & $\mathrm{Ze}$ & \\
\hline rizo & Wave & Apodization & Cosine \\
\hline itice & & Gain & Auto (8) \\
\hline art & 649.893 & Ape & Auto \\
\hline terval & 4000.6 & Scanning Speed & $\mathrm{nm} / \mathrm{sec}$ ) \\
\hline ata interval & $0.964233 \mathrm{~cm}-1$ & Filter & Auto $(30000 \mathrm{~Hz})$ \\
\hline
\end{tabular}


WW7 Melamine Entry 1 (large scale)

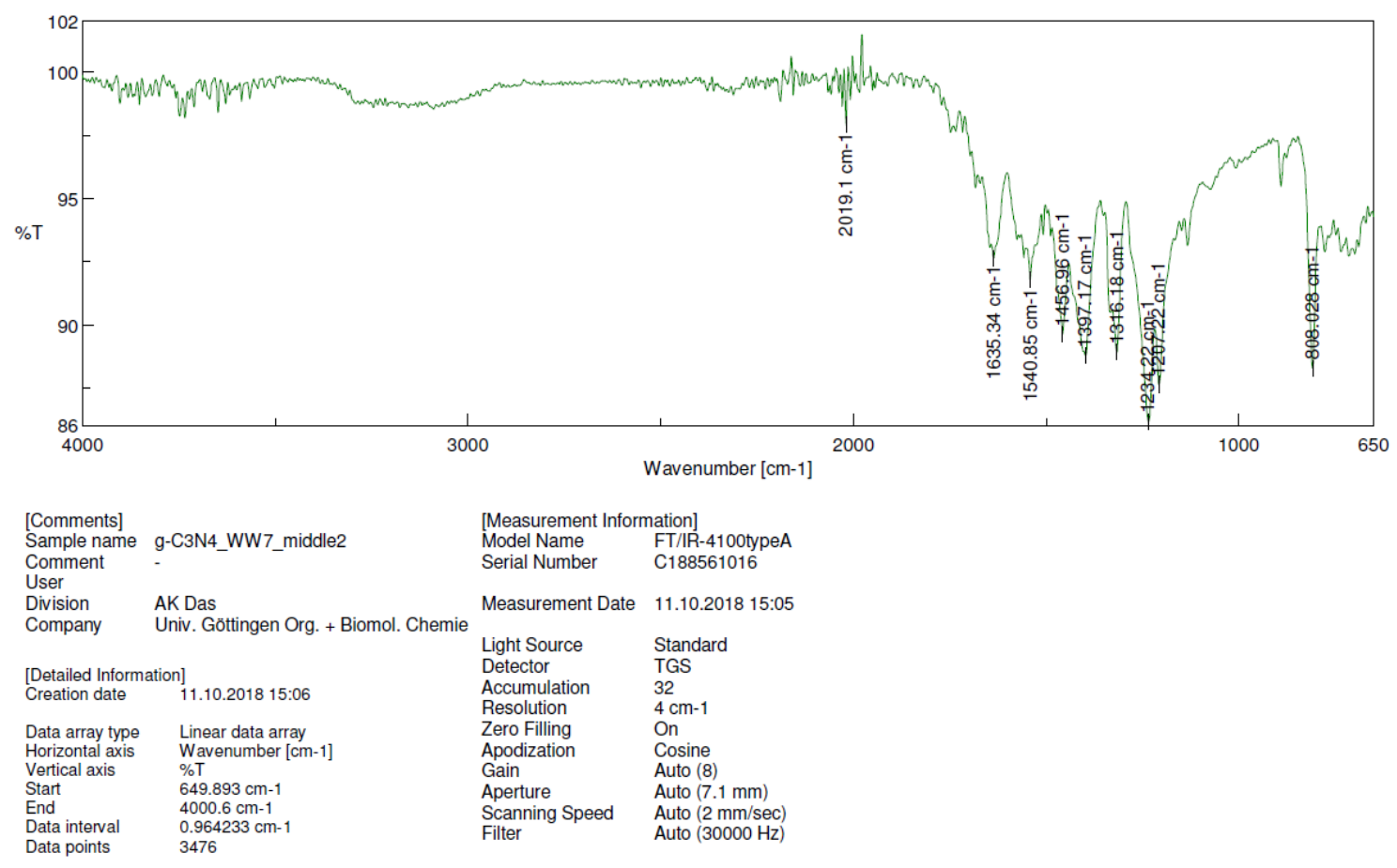

\section{WW12 Urea Entry 2}

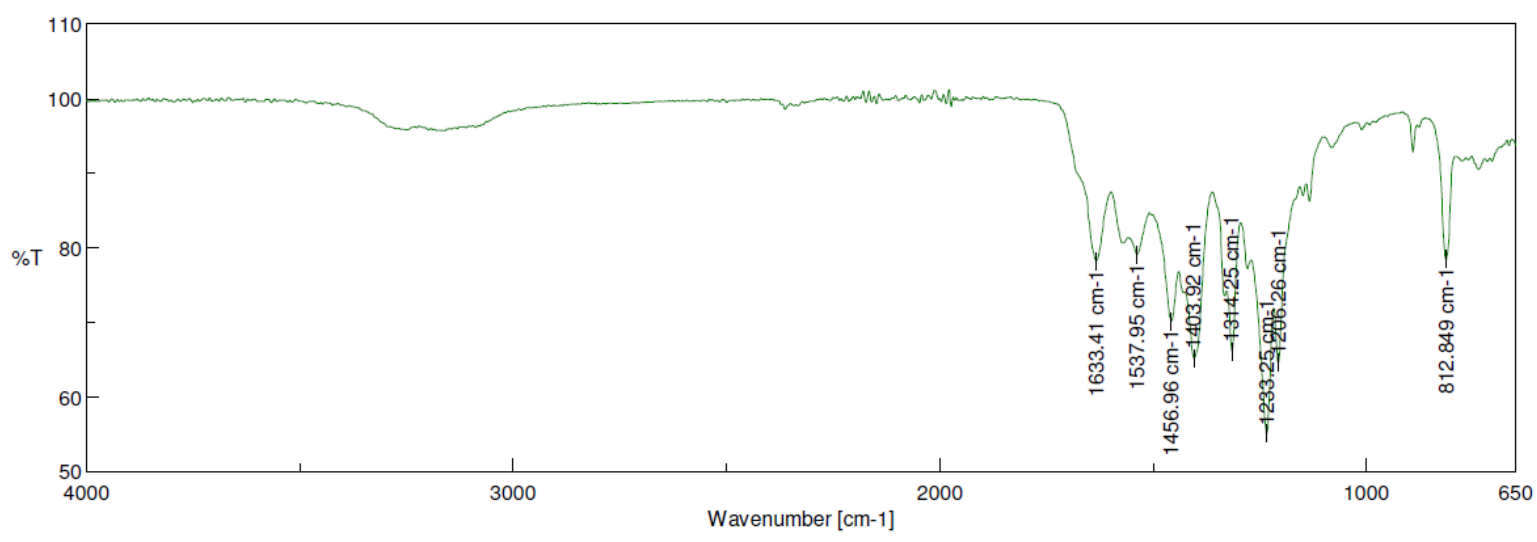

\begin{tabular}{|c|c|c|c|}
\hline \multicolumn{2}{|l|}{ [Comments] } & \multicolumn{2}{|c|}{ [Measurement Information] } \\
\hline Sample name & WW-gC3N4_urea & Model Name & FT/R-4100typ \\
\hline Comment & - & Serial Number & C188561016 \\
\hline User & WW & & \\
\hline Division & AK Das & Measurement Date & $05.12 .201816: 00$ \\
\hline Company & Univ. Göttingen Org. + Biomol. Chemie & Light Source & Standard \\
\hline Jue & & Detector & TGS \\
\hline Creation d & 05.12.2018 16:01 & $\begin{array}{l}\text { Accumulation } \\
\text { Resolution }\end{array}$ & $\begin{array}{l}32 \\
4 \mathrm{~cm}-1\end{array}$ \\
\hline Data array type & Linear data & Zero Filling & $\begin{array}{l}\text { On } \\
\text { Cosine }\end{array}$ \\
\hline $\begin{array}{l}\text { Horizontal axi } \\
\text { Vertical axis }\end{array}$ & $\begin{array}{l}\text { Wavenumber [cm-1] } \\
\% \mathrm{~T}\end{array}$ & $\begin{array}{l}\text { Apodizatıon } \\
\text { Gain }\end{array}$ & Auto (8) \\
\hline & $649.893 \mathrm{~cm}-1$ & Aperture & Auto $(7.1 \mathrm{~mm})$ \\
\hline & 4000.6 & Scanning Speed & Auto $(2 \mathrm{~mm} / \mathrm{sec})$ \\
\hline lata min & 0.964233 & Filter & Auto $(30000 \mathrm{~Hz})$ \\
\hline
\end{tabular}




\section{WW11 Thiourea Entry 3}

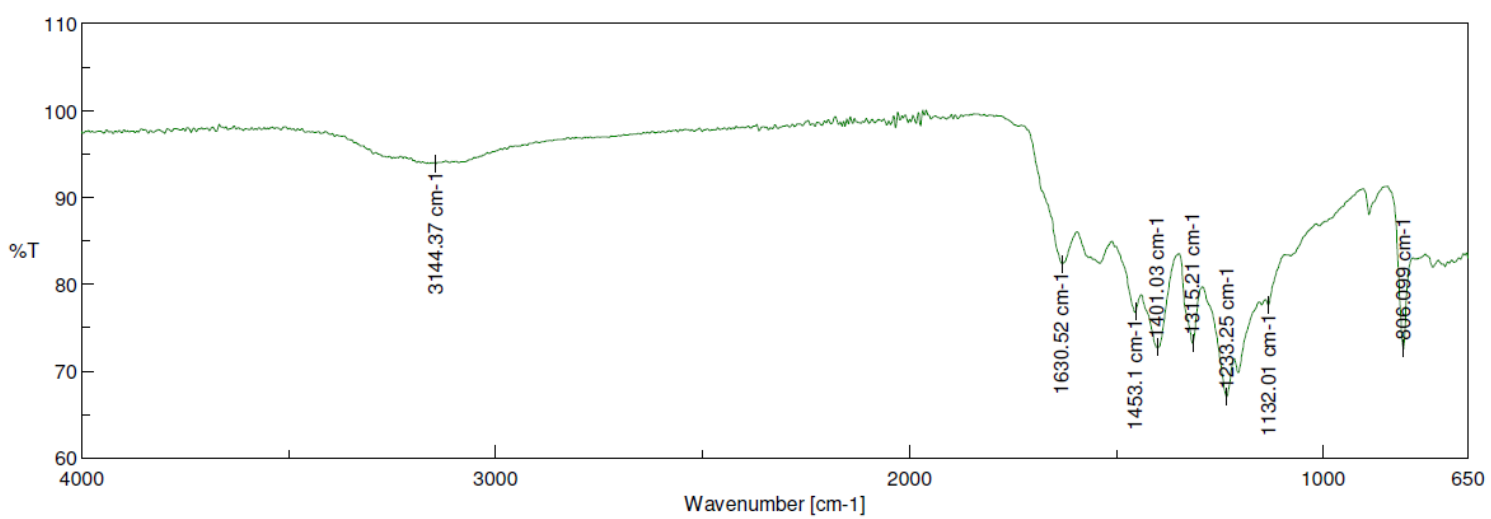

\begin{tabular}{|c|c|c|c|}
\hline [Comments] & & [Measurement Inforr & \\
\hline Sample name & g-C3N4_WW11_thiourea & Model Name & FT/IR-4100typeA \\
\hline Comment & & Serial Number & C188561016 \\
\hline User & WW & & \\
\hline Division & AK Das & Measurement Date & 05.12.2018 16:04 \\
\hline Company & Univ. Göttingen Org. + Biomol. Chemie & Light Source & Standard \\
\hline & & Detect & TGS \\
\hline Creation da & 05.12 .2018 16:05 & $\begin{array}{l}\text { Accumulation } \\
\text { Resolution }\end{array}$ & $\begin{array}{l}32 \\
4 \mathrm{~cm}-1\end{array}$ \\
\hline Data array type & Linear d & Zero Filling & \\
\hline Horizontal axis & Wavenumber & Apodization & Cosine \\
\hline Vertical axis & & Gain & Auto (8) \\
\hline & $649.893 \mathrm{~cm}-1$ & Aperture & Auto $(7.1 \mathrm{~mm})$ \\
\hline End & $4000.6 \mathrm{~cm}-1$ & Scanning Speed & Auto $(2 \mathrm{~mm} / \mathrm{sec})$ \\
\hline Data interval & $0.964233 \mathrm{~cm}-1$ & Filter & Auto $(30000 \mathrm{~Hz})$ \\
\hline
\end{tabular}

\section{WW11 Dicyandiamide Entry 4}

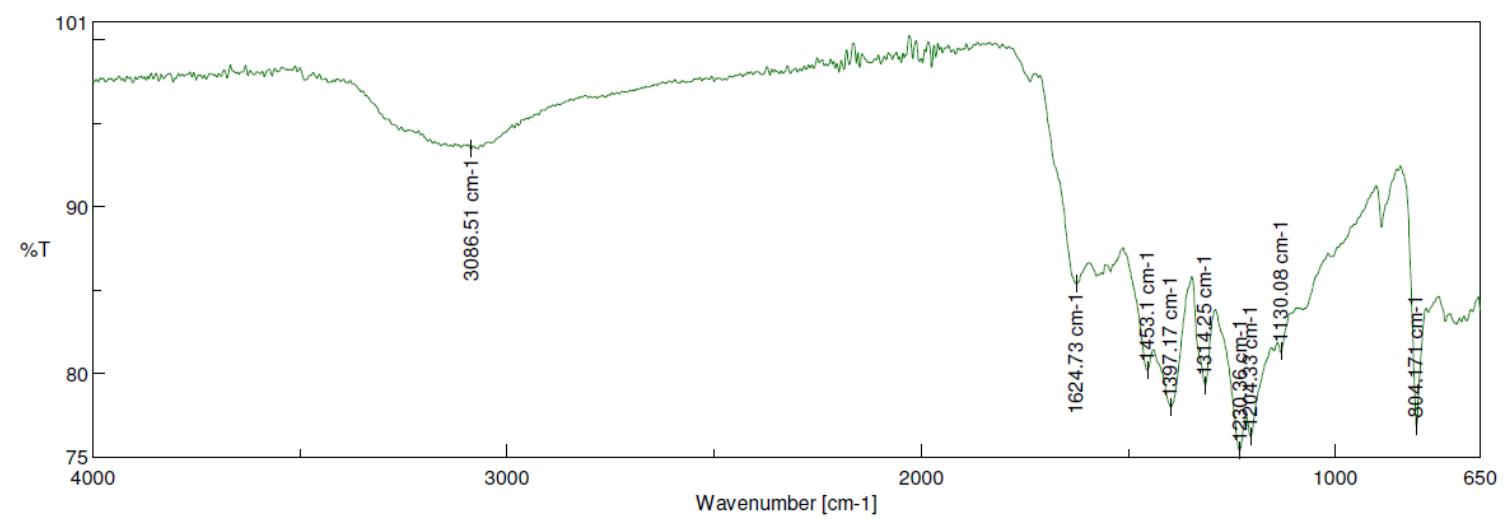

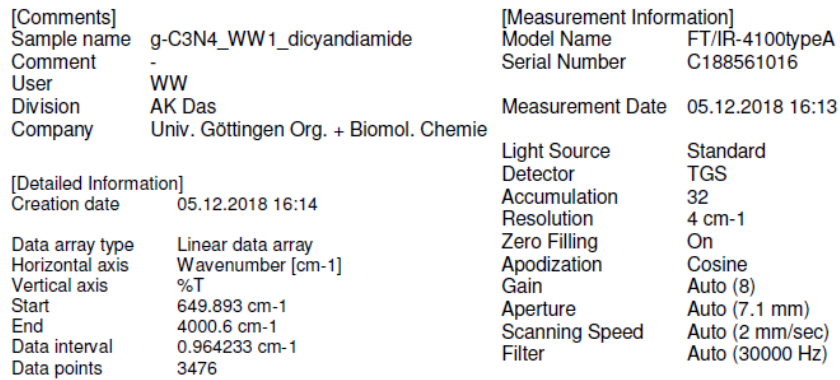


FWW2 Entry 5

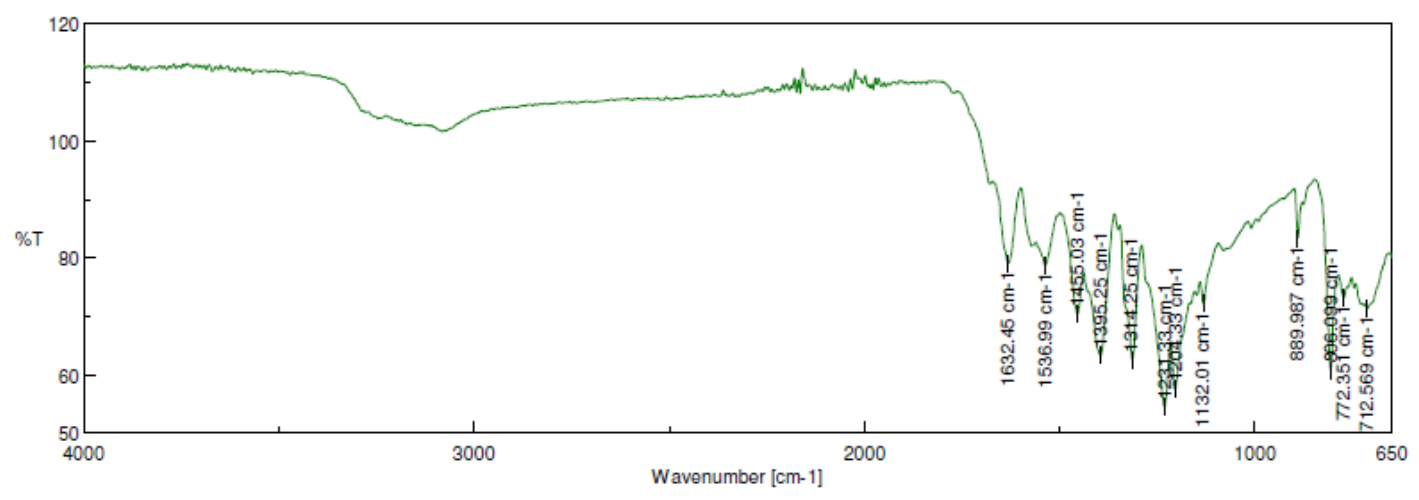

\begin{tabular}{|c|c|c|c|}
\hline Comments] & & [Measurement Infor & nation] \\
\hline Sample name & WW-g-C3N4 & Model Name & $\mathrm{FT} / \mathrm{IR}-41 \mathrm{C}$ \\
\hline $\begin{array}{l}\text { Comment } \\
\text { User }\end{array}$ & $\begin{array}{l}\text { Background } \\
\text { WW }\end{array}$ & Serial Number & C188561016 \\
\hline Division & AK Das & Measurement Date & 13.01 .202013 \\
\hline Company & Univ. Gottingen Org. + Biomol. Chemie & & \\
\hline & & $\begin{array}{l}\text { Light Source } \\
\text { Detector }\end{array}$ & $\begin{array}{l}\text { Standard } \\
\text { TGS }\end{array}$ \\
\hline $\begin{array}{l}\text { Detalled informm } \\
\text { Creation date }\end{array}$ & 13.01.2020 13:41 & Accumulation & \\
\hline & & Resoluti & $\begin{array}{l}4 \mathrm{~cm} \\
\text { On }\end{array}$ \\
\hline $\begin{array}{l}\text { Data array yppe } \\
\text { Horizontal axis }\end{array}$ & $\begin{array}{l}\text { Unear data array } \\
\text { Wavenumber [cm-1] }\end{array}$ & $\begin{array}{l}\text { Zero Filling } \\
\text { Apodization }\end{array}$ & $\begin{array}{l}\text { On } \\
\text { Cosine }\end{array}$ \\
\hline ittical axis & $\% \mathrm{~T}$ & Gain & Auto (16) \\
\hline & $649.893 \mathrm{~cm}-1$ & Aperture & Auto $(7.1 \mathrm{~mm})$ \\
\hline End & $4000.6 \mathrm{~cm}$ & Scanning Speed & Auto $(2 \mathrm{~mm} / \mathrm{sec})$ \\
\hline $\begin{array}{l}\text { ata interval } \\
\text { gatantol ponts }\end{array}$ & $\begin{array}{l}0.964233 \mathrm{~cm}-1 \\
3476\end{array}$ & & Auto $(30000 \mathrm{H}$ \\
\hline
\end{tabular}

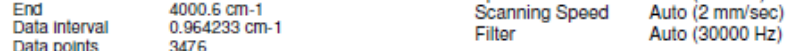

\section{FWW6 Entry 6}

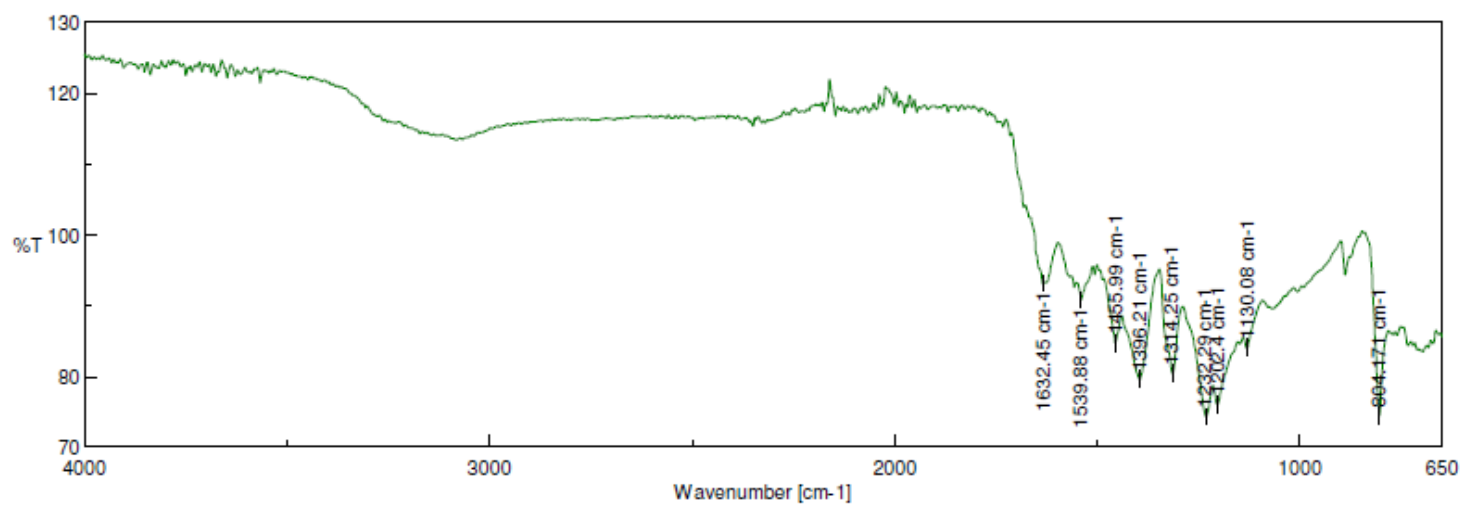

\begin{tabular}{|c|c|c|c|}
\hline & \multicolumn{2}{|c|}{ [Measurement Information] } \\
\hline \multirow{4}{*}{$\begin{array}{l}\text { Sample name } \\
\text { Comment } \\
\text { User } \\
\text { Division } \\
\text { Company }\end{array}$} & \multirow{2}{*}{$\begin{array}{l}\text { WW-g-C3N4-FWW6 } \\
\text { FWW6 } \\
\text { WW }\end{array}$} & \multirow{2}{*}{$\begin{array}{l}\text { Model Name } \\
\text { Serial Number }\end{array}$} & \multirow{2}{*}{$\begin{array}{l}\text { FT/IR-4100typeA } \\
\text { C188561016 }\end{array}$} \\
\hline & & & \\
\hline & & Measurement Date & 13.01 .2020 \\
\hline & Univ. Gottingen Org. + Biomol. Chemie & & \\
\hline & & Ligh & \\
\hline \multicolumn{2}{|c|}{ Detalled Intormation] } & on & \\
\hline eatio & 13 & & $4 \mathrm{~cm}$ \\
\hline ta & & & \\
\hline & Waven & Ap & \\
\hline & & & \\
\hline & 649.893 & & $\mathrm{Au}$ \\
\hline & 4000 & Spe & \\
\hline a interval & $0.964233 \mathrm{~cm}-1$ & Filter & Auto $(30000 \mathrm{~Hz})$ \\
\hline
\end{tabular}


FWW3 Entry 7

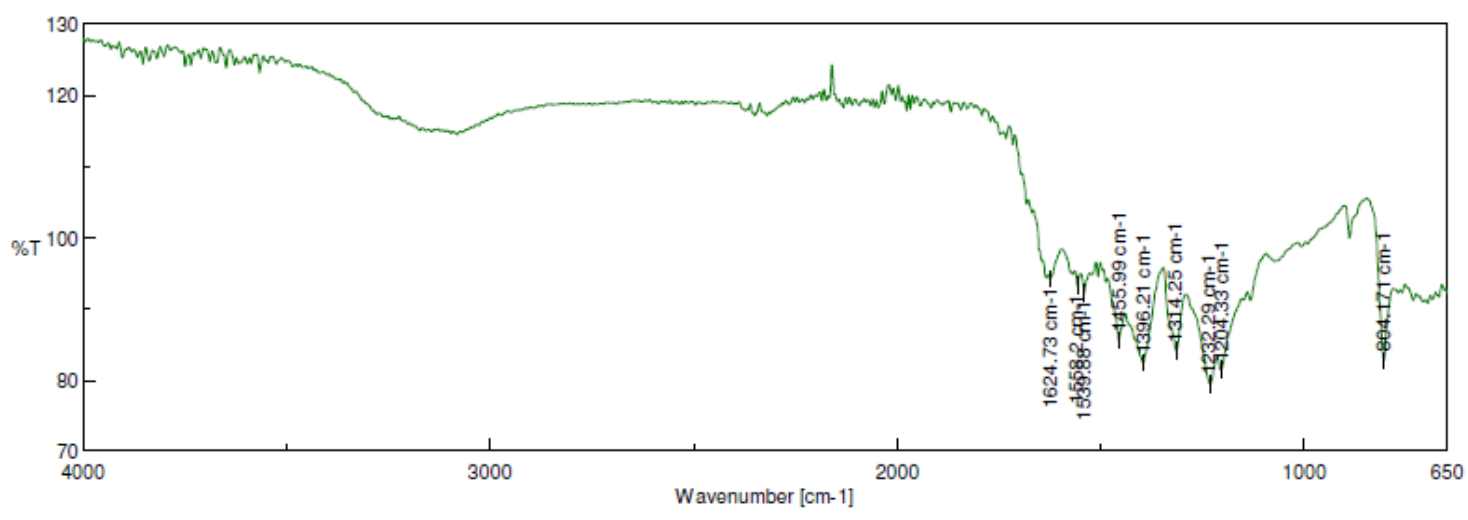

\begin{tabular}{|c|c|c|c|}
\hline \multicolumn{2}{|l|}{ [Comments] } & \multicolumn{2}{|c|}{ [Measurement Information] } \\
\hline Sample name & WW-g-C3N4-FWW3 & Model Name & $\mathrm{FT} / \mathrm{R}-4100 \mathrm{typ}$ \\
\hline Comment & FWW3 & Serial Number & C18856 \\
\hline User & Ww & & \\
\hline $\begin{array}{l}\text { ivision } \\
\text { ompany }\end{array}$ & $\begin{array}{l}\text { AK Das } \\
\text { Univ. Gottingen Org. + Biomol. Chemie }\end{array}$ & Measurement Date & $13.01 .202014:$ \\
\hline & & $\begin{array}{l}\text { Light } \\
\text { Detec }\end{array}$ & $\begin{array}{l}\text { Standard } \\
\text { TGS }\end{array}$ \\
\hline Creation & $13.01 .202014: 50$ & $\begin{array}{l}\text { Accumulation } \\
\text { Resolution }\end{array}$ & $\begin{array}{l}32 \\
4 \mathrm{~cm}-1\end{array}$ \\
\hline ata & Unea & & \\
\hline$\pi z 2$ & Wav & Ap & \\
\hline itica & $\% \mathrm{~T}$ & & \\
\hline & 649.893 & $\mathrm{Ap}$ & $\mathrm{Au}$ \\
\hline & 4000 & Speed & \\
\hline a interval & $0.964233 \mathrm{~cm}-1$ & Filter & Auto $(30000 \mathrm{~Hz})$ \\
\hline
\end{tabular}

\section{FWW4 Entry 8}

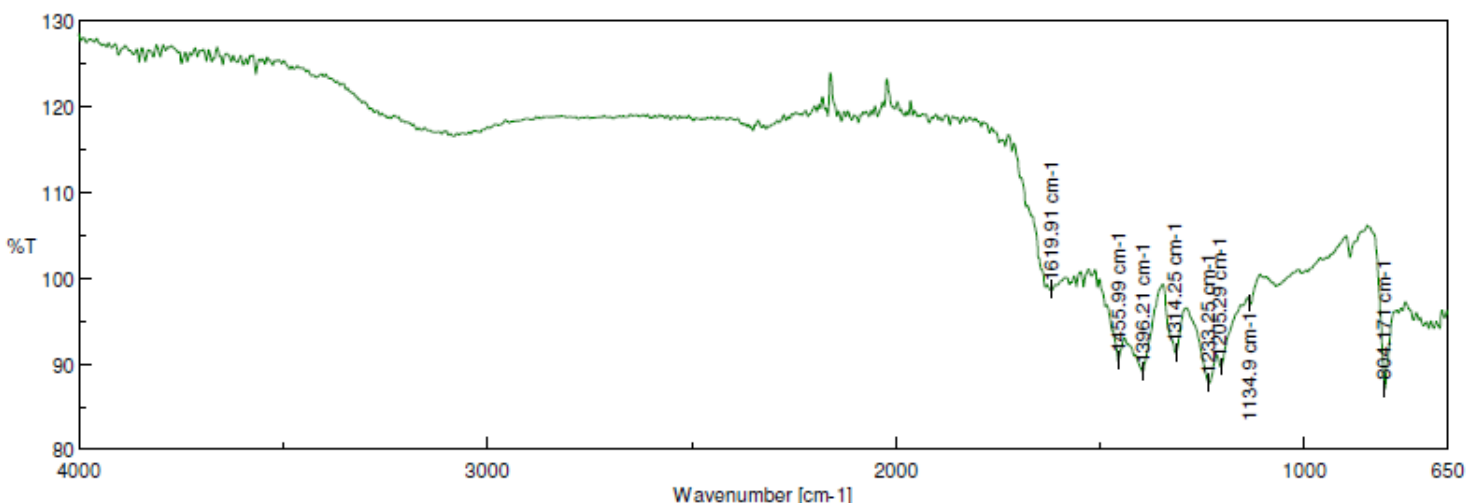

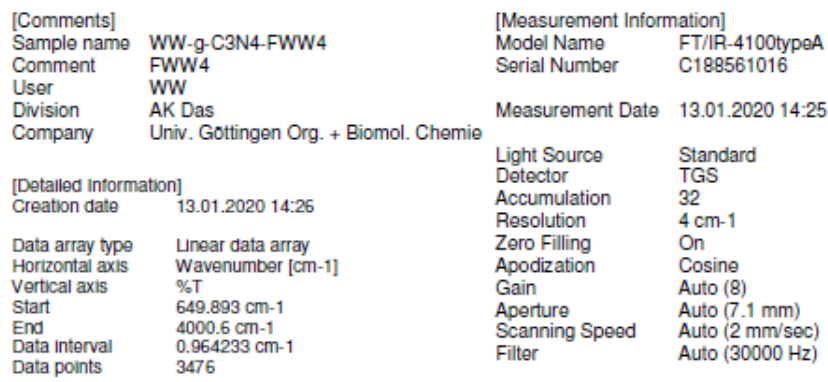


FWW5 Entry 9

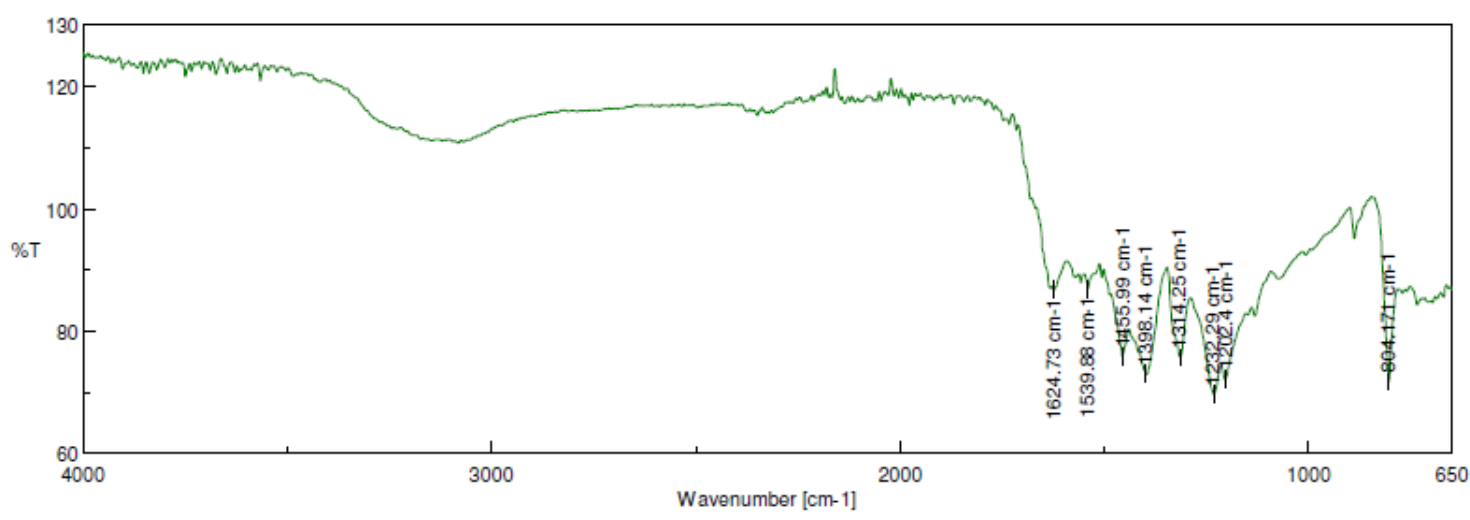

\begin{tabular}{|c|c|c|c|}
\hline \multicolumn{2}{|l|}{ [Comments] } & \multicolumn{2}{|c|}{ [Measurement Information] } \\
\hline Sample name & WW-g-C3N4-FWW5 & Model Name & $\mathrm{FT} / \mathrm{IR}$ - \\
\hline Comment & FWw5 & Serial Number & C188561016 \\
\hline User & WW & & \\
\hline Division & AK Das & Measurement Date & 13.01 .202014 .45 \\
\hline ompany & Univ. Gottingen Org. + Biomol. Chemie & Light Source & Standard \\
\hline [Detalled Intorme & ation! & Detector & TGS \\
\hline Creation date & $13.01 .202014: 46$ & $\begin{array}{l}\text { Accumulation } \\
\text { Resolution }\end{array}$ & $\begin{array}{l}32 \\
4 \mathrm{~cm}-1\end{array}$ \\
\hline ata & Unea & Zero Filling & On \\
\hline Horizontal axis & Wavenumber [cm-1] & Apodization & Cosine \\
\hline Vertical axis & $\% \mathrm{~T}$ & Gain & Auto (8) \\
\hline Start & $649.893 \mathrm{~cm}-1$ & Aperture & Auto $(7.1 \mathrm{~mm})$ \\
\hline & $4000.6 \mathrm{~cm}-1$ & Scanning Speed & Auto ( $2 \mathrm{~mm} / \mathrm{sec})$ \\
\hline Data interval & $\begin{array}{l}0.964233 \mathrm{~cm}-1 \\
3476\end{array}$ & Filter & Auto $(30000 \mathrm{~Hz})$ \\
\hline
\end{tabular}

\section{FWW15 Entry 10}

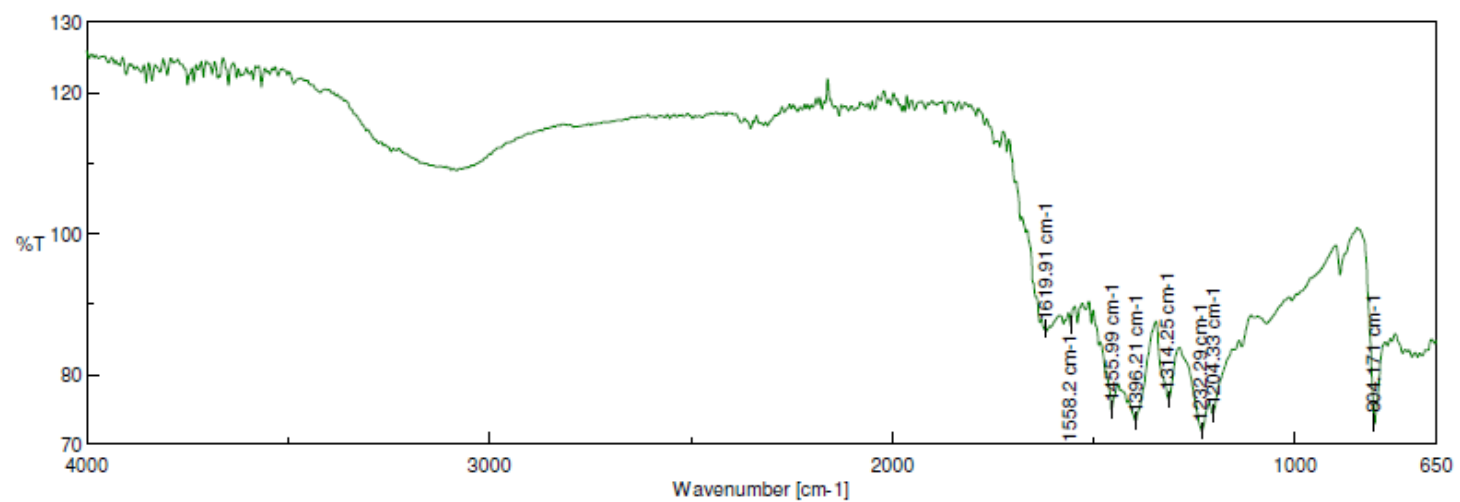

\begin{tabular}{|c|c|c|c|}
\hline \multirow{5}{*}{$\begin{array}{l}\text { [Comments] } \\
\text { Sample name } \\
\text { Comment } \\
\text { User } \\
\text { Division } \\
\text { Company }\end{array}$} & & \multicolumn{2}{|c|}{ [Measurement Information] } \\
\hline & & Model Name & $\mathrm{FT} / \mathrm{IR}$ \\
\hline & FWW 15 & Serial Number & C188561016 \\
\hline & WW & & \\
\hline & $\begin{array}{l}\text { AK Das } \\
\text { Univ. Gottingen Org. + Biomol. Chemie }\end{array}$ & Measurement Date & 13.01.2020 15:09 \\
\hline & & Light Source & Standard \\
\hline [Detalled Intorm: & ation] & Detector & ${ }_{32}$ \\
\hline Creation date & $13.01 .202015: 10$ & $\begin{array}{l}\text { Accumulation } \\
\text { Resolution }\end{array}$ & $4 \mathrm{~cm}-1$ \\
\hline Data array ty & Lnear & Zero Filling & \\
\hline Horizontal ax & Wavenum & Apodization & Cosin \\
\hline Vertical axis & & Gain & Auto (8) \\
\hline & $649.893 \mathrm{~cm}-1$ & Aperture & Auto $(7.1 \mathrm{~mm})$ \\
\hline & $4000.6 \mathrm{~cm}-1$ & Scanning Speed & Auto $(2 \mathrm{~mm} / \mathrm{sec})$ \\
\hline $\begin{array}{l}\text { Data interval } \\
\text { Data ponts }\end{array}$ & $0.964233 \mathrm{~cm}-1$ & Filter & Auto $(30000 \mathrm{~Hz}$ \\
\hline
\end{tabular}


FWW16 Entry 11

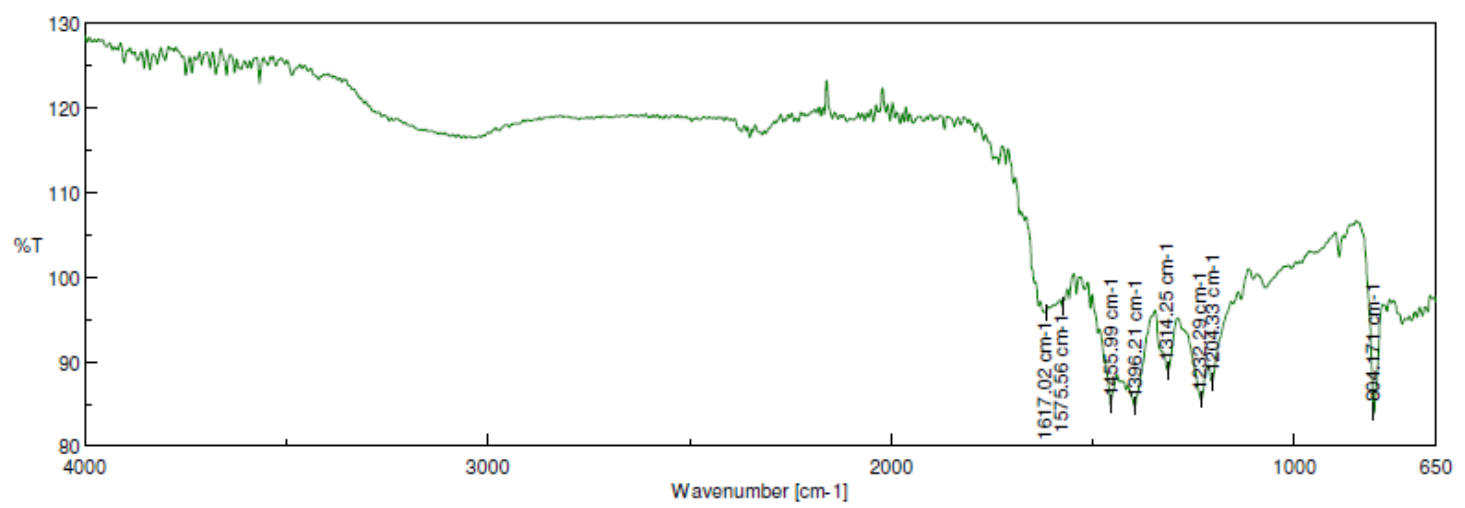

\begin{tabular}{|c|c|c|c|}
\hline \multicolumn{2}{|l|}{ [Comments] } & \multicolumn{2}{|c|}{ [Measurement Information] } \\
\hline Sample name & WW-g-C3N4-FWW 16 & Model Name & $\mathrm{FT} / \mathrm{RR}-4100 \mathrm{type}$ \\
\hline $\begin{array}{l}\text { Comment } \\
\text { User }\end{array}$ & $\begin{array}{l}\text { FWW16 } \\
\text { WW }\end{array}$ & Serial Number & C188561016 \\
\hline vision & AK Das & Measurement Date & 13.01.2020 14:56 \\
\hline & Stungen Urg. + Biomol. Chemie & $\begin{array}{l}\text { Light Source } \\
\text { Detector }\end{array}$ & $\begin{array}{l}\text { Standard } \\
\text { TGS }\end{array}$ \\
\hline $\begin{array}{l}\text { [Detalled Intorma } \\
\text { Creation date }\end{array}$ & ${ }_{13.01 .2020} 14: 58$ & $\begin{array}{l}\text { Decec } \\
\text { Accur } \\
\text { Resol }\end{array}$ & $\begin{array}{l}32 \\
4 \mathrm{~cm}-1\end{array}$ \\
\hline ta a & & Zero & On \\
\hline thol & Wav & Ap & $\operatorname{Cos}$ \\
\hline ertical ax & & & Auto (8) \\
\hline & $649.893 \mathrm{~cm}$ & Aperture & Auto $(7.1 \mathrm{~m}$ \\
\hline End & 4000 & Scanning Speed & (sec) \\
\hline point & $\begin{array}{l}0.9642 \\
3476\end{array}$ & Filter & Auto $(30000 \mathrm{~Hz})$ \\
\hline
\end{tabular}

\section{FWW17 Entry 12}

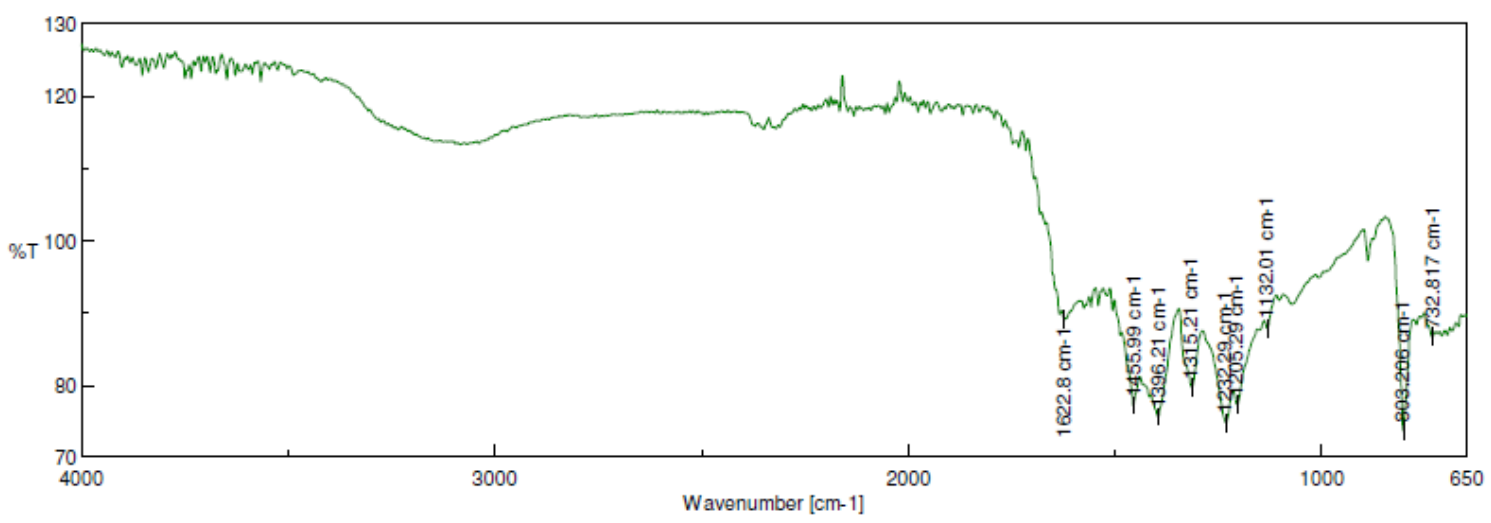

\begin{tabular}{|c|c|c|c|}
\hline \multicolumn{2}{|l|}{ [Comments] } & \multicolumn{2}{|c|}{ [Measurement Information] } \\
\hline Sample name & WW-g-C3N4-FWW17 & Model Name & $\mathrm{FT} / \mathrm{IR}$ \\
\hline Comment & FWW17 & Serial Number & $\mathrm{C} 188561016$ \\
\hline User & ww & & \\
\hline Division & AK Das & Measurement Date & 13.01.2020 15:02 \\
\hline Company & Univ. Gottingen Org. + Biomol. Chemie & & Standard \\
\hline [Detalled inform? & ation] & Detector & TGS \\
\hline Creation date & $13.01 .202015: 05$ & $\begin{array}{l}\text { Accumulation } \\
\text { Resolution }\end{array}$ & $\begin{array}{l}32 \\
4 \mathrm{~cm}-1\end{array}$ \\
\hline Data array type & Unear data & Zero Filling & \\
\hline Horizontal axis & Wavenumber [cm-1] & Apodization & Cosine \\
\hline Vertical axis & $\% \mathrm{~T}$ & Gain & Auto (8) \\
\hline & $649.893 \mathrm{~cm}-1$ & Aperture & Auto $(7.1 \mathrm{~mm})$ \\
\hline & $4000.6 \mathrm{~cm}-1$ & Scanning Speed & Auto $(2 \mathrm{~mm} / \mathrm{sec})$ \\
\hline Data interval & $0.964233 \mathrm{~cm}-1$ & Filter & Auto $(30000 \mathrm{~Hz})$ \\
\hline
\end{tabular}


FWW20 Entry 13

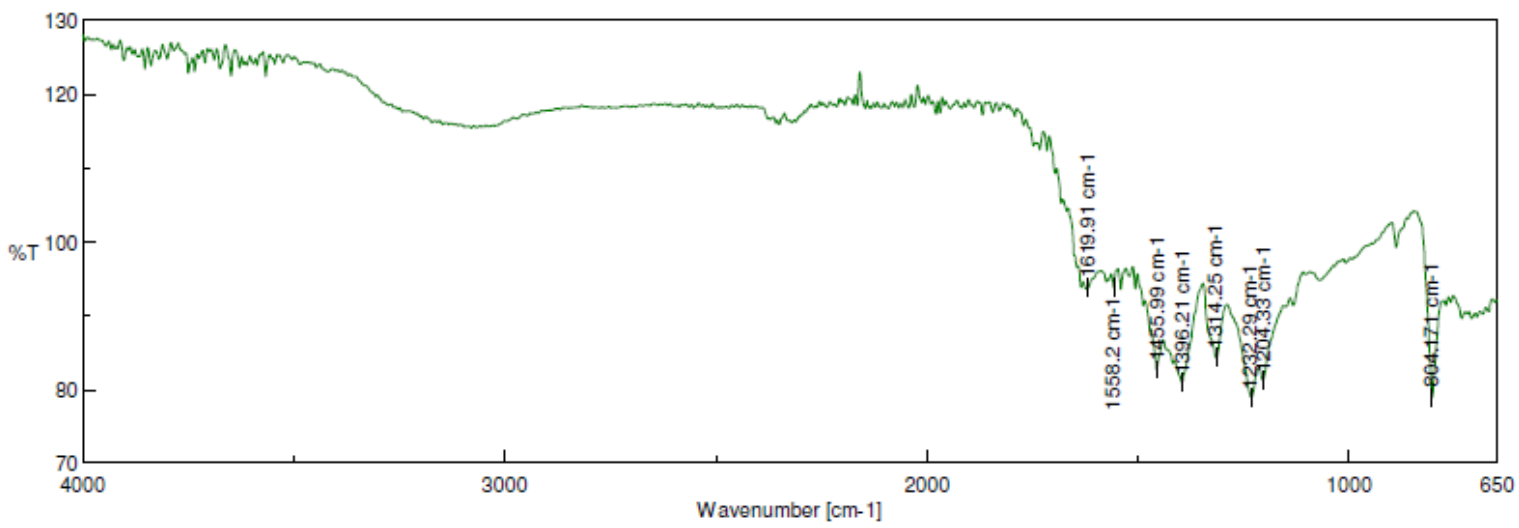

\begin{tabular}{|c|c|c|c|}
\hline & \multicolumn{2}{|c|}{ [Measurement Information] } \\
\hline \multirow{4}{*}{$\begin{array}{l}\text { Sample name } \\
\text { Comment } \\
\text { User } \\
\text { Division } \\
\text { Company }\end{array}$} & \multirow{3}{*}{$\begin{array}{l}\text { WW-g-C3N4-FWW20 } \\
\text { FWW20 } \\
\text { WW }\end{array}$} & \multirow{3}{*}{$\begin{array}{l}\text { Model Name } \\
\text { Serial Number }\end{array}$} & \multirow{3}{*}{$\begin{array}{l}\text { FT/IR-4100typeA } \\
\text { C188561016 }\end{array}$} \\
\hline & & & \\
\hline & & & \\
\hline & AK Das & Measurement Date & $13.01 .202015: 13$ \\
\hline & ingen Org. + Biomol. Cher & Light & Stand: \\
\hline \multicolumn{2}{|c|}{ [Detalled Intormation] } & Detec & TGS \\
\hline Creation date & $13.01 .202015: 14$ & $\begin{array}{l}\text { Accumulation } \\
\text { Resolution }\end{array}$ & 32 \\
\hline ata array t & Unear & Zero Fill & On \\
\hline orizontal & Waven & Apodization & $\cos$ \\
\hline ertical axis & $\% \mathrm{~T}$ & Gain & Auto (8) \\
\hline Start & $649.893 \mathrm{~cm}$ & Aperture & Auto $(7.1 \mathrm{~mm})$ \\
\hline & 4000.6 & Scanning Speed & Auto $(2 \mathrm{~mm} / \mathrm{sec})$ \\
\hline & $\mathrm{cm}-1$ & Filter & Auto $(30000 \mathrm{~Hz})$ \\
\hline Data points & 3476 & & \\
\hline
\end{tabular}

\section{FWW21 Entry 14}

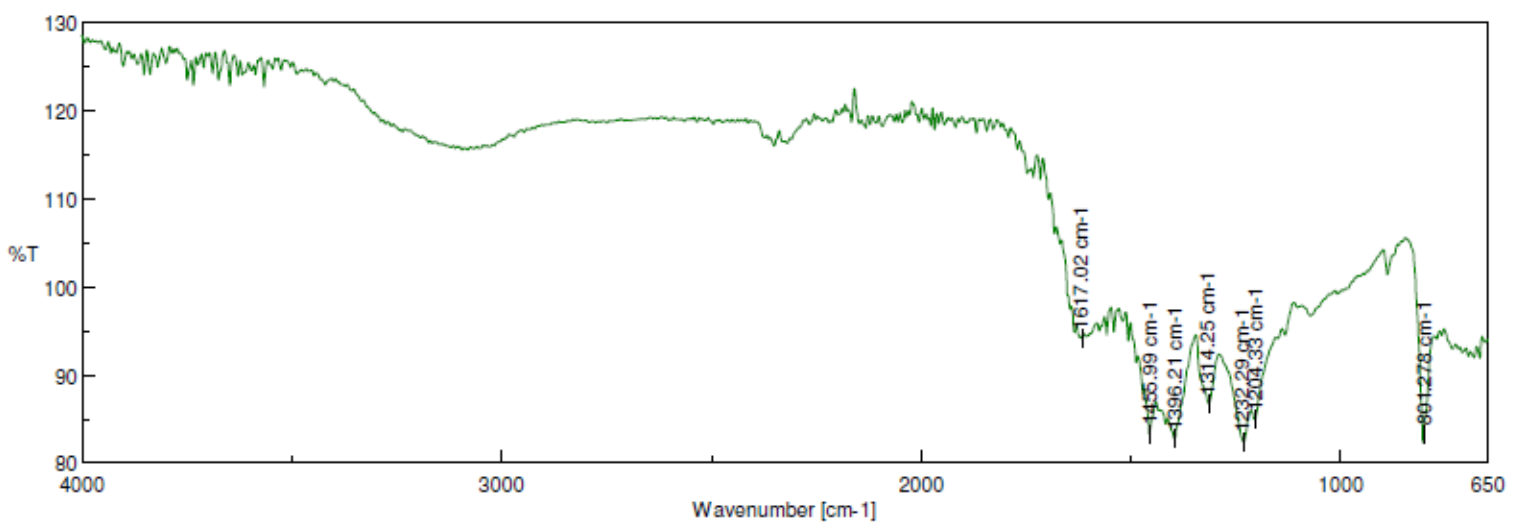

\begin{tabular}{|c|c|c|c|}
\hline [Comments] & & [Measurement Inforr & nation] \\
\hline Sample name & WW-g-C3N4-FWW21 & Model Name & FT/RR-4100typeA \\
\hline Comment & FWW21 & Serial Number & C188561016 \\
\hline User & WW & & \\
\hline Division & AK Das & Measurement Date & 13.01.2020 15:17 \\
\hline Comp & sottungen Urg. + Biomol. Chemie & Light Source & Standard \\
\hline [Detalled Intorm, & ation] & Detector & \\
\hline Creation date & $13.01 .202015: 18$ & $\begin{array}{l}\text { Accumulation } \\
\text { Resolution }\end{array}$ & $\begin{array}{l}32 \\
4 \mathrm{~cm}-1\end{array}$ \\
\hline Data array type & Unear data array & Zero Filling & \\
\hline Horizontal axis & Wavenumber [cm-1] & Apodization & Cosine \\
\hline Vertical axis & $\% \mathrm{~T}$ & Gain & Auto (8) \\
\hline Start & $649.893 \mathrm{~cm}-1$ & Aperture & Auto $(7.1 \mathrm{~mm})$ \\
\hline End & $4000.6 \mathrm{~cm}-1$ & Scanning Speed & Auto $(2 \mathrm{~mm} / \mathrm{sec})$ \\
\hline Data interval & $0.964233 \mathrm{~cm}-1$ & Filter & Auto $(30000 \mathrm{~Hz})$ \\
\hline Data points & 3476 & & \\
\hline
\end{tabular}


FWW22 Entry 15

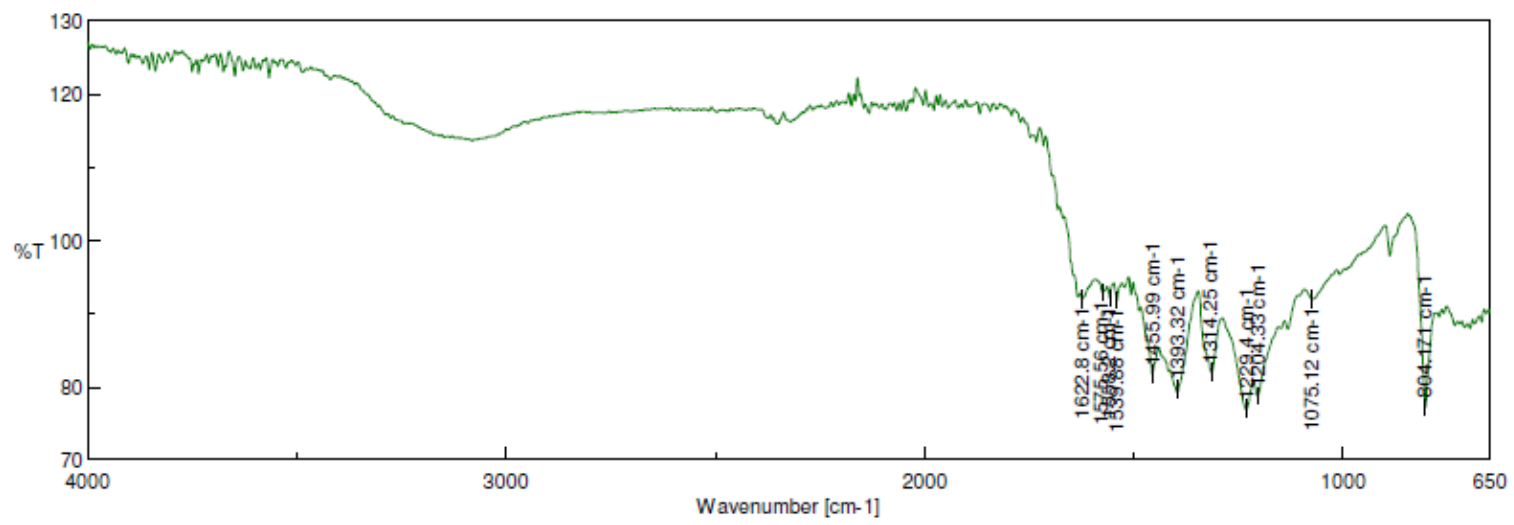

\begin{tabular}{|c|c|c|c|}
\hline \multicolumn{2}{|l|}{ [Comments] } & \multicolumn{2}{|c|}{ [Measurement Information] } \\
\hline Sample name & WW-g-C3N4-FWW22 & Model Name & FT/R-4100typeA \\
\hline Comment & FWW22 & Serial Number & C188561016 \\
\hline $\begin{array}{l}\text { User } \\
\text { Division }\end{array}$ & WW & & \\
\hline $\begin{array}{l}\text { Division } \\
\text { Company }\end{array}$ & AK Das & Measurement Date & 13.01.2020 14:52 \\
\hline Company & Univ. Gottingen Org. + Biomol. Chemie & $\begin{array}{l}\text { Light Source } \\
\text { Detector }\end{array}$ & $\begin{array}{l}\text { Standard } \\
\text { TGS }\end{array}$ \\
\hline $\begin{array}{l}\text { [Detalled Intorm } \\
\text { Creation date }\end{array}$ & $\begin{array}{l}\text { ation] } \\
{ }_{13.01 .2020} 14: 53\end{array}$ & $\begin{array}{l}\text { Accumulation } \\
\text { Resolution }\end{array}$ & \\
\hline Data array & Unear da & Zero Filling & On \\
\hline orizontal axis & Wavenumber [cm-1] & Apodization & Cosine \\
\hline Vertical axis & $\% \mathrm{~T}$ & Gain & Auto (8) \\
\hline Start & $649.893 \mathrm{~cm}-1$ & Aperture & Auto $(7.1 \mathrm{~mm})$ \\
\hline End & $4000.6 \mathrm{~cm}-1$ & Scanning Speed & Auto $(2 \mathrm{~mm} / \mathrm{sec})$ \\
\hline ta interval & $0.964233 \mathrm{~cm}-1$ & Filter & Auto $(30000 \mathrm{~Hz})$ \\
\hline Data points & 3476 & & \\
\hline
\end{tabular}

FWW23 Entry 16

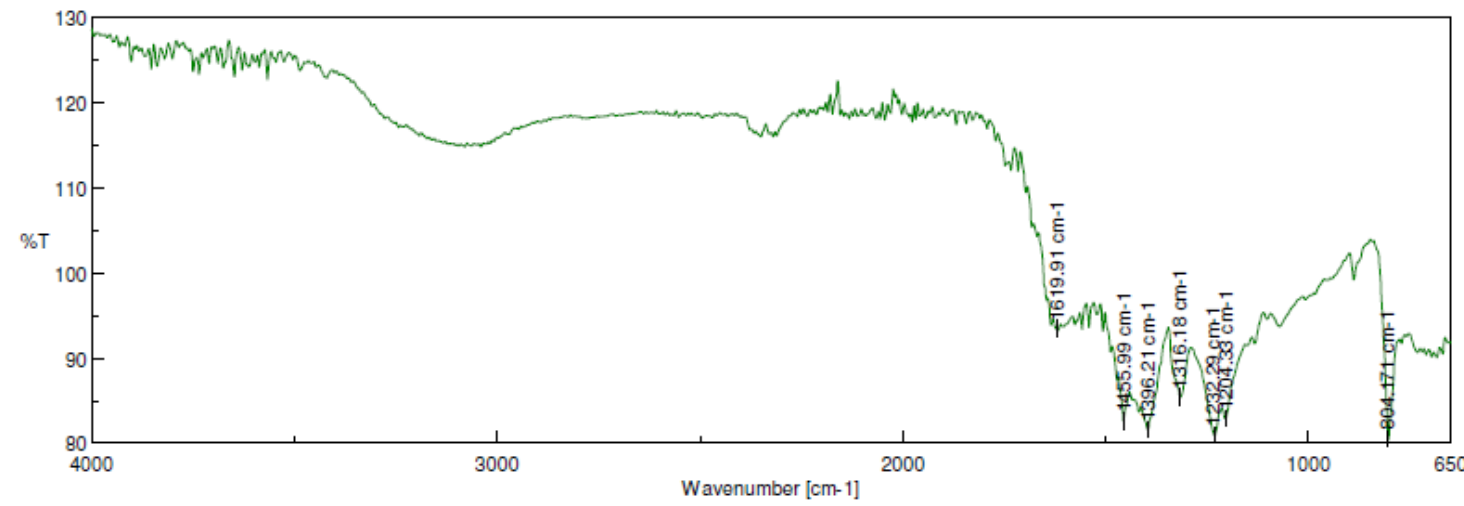

\begin{tabular}{|c|c|c|c|}
\hline \multicolumn{2}{|l|}{ [Comme } & \multicolumn{2}{|c|}{ [Measurement Information] } \\
\hline Sample name & WW-g-C3N4-FWW23 & Model Name & $\mathrm{FT} / \mathrm{IR}$ \\
\hline Comment & FWW 23 & Serial Number & C188561016 \\
\hline User & WW & & \\
\hline $\begin{array}{l}\text { Division } \\
\text { Company }\end{array}$ & AK Das & Measurement Date & $13.01 .202015-20$ \\
\hline & Ottingen Urg. + Blomol. Chemie & $\begin{array}{l}\text { Light Source } \\
\text { Detector }\end{array}$ & $\begin{array}{l}\text { Standard } \\
\text { TGS }\end{array}$ \\
\hline $\begin{array}{l}\text { [Detalled in } \\
\text { Creation da }\end{array}$ & ation] & Accumulation & \\
\hline Creation d & 13.01.2020 15:21 & Resc & $4 \mathrm{~cm}-1$ \\
\hline & & Zer & \\
\hline He & Wav & Apodiza & Cosine \\
\hline itical ax & $\% \mathrm{~T}$ & Gain & Auto (8) \\
\hline & 649.893 & Aperture & Auto $(7.1 \mathrm{~mm})$ \\
\hline & & Scanning Speed & Auto $(2 \mathrm{~mm} / \mathrm{sec})$ \\
\hline ata interval & $0.964233 \mathrm{~cm}-1$ & Filter & Auto $(30000 \mathrm{~Hz})$ \\
\hline
\end{tabular}


FWW24 Entry 17

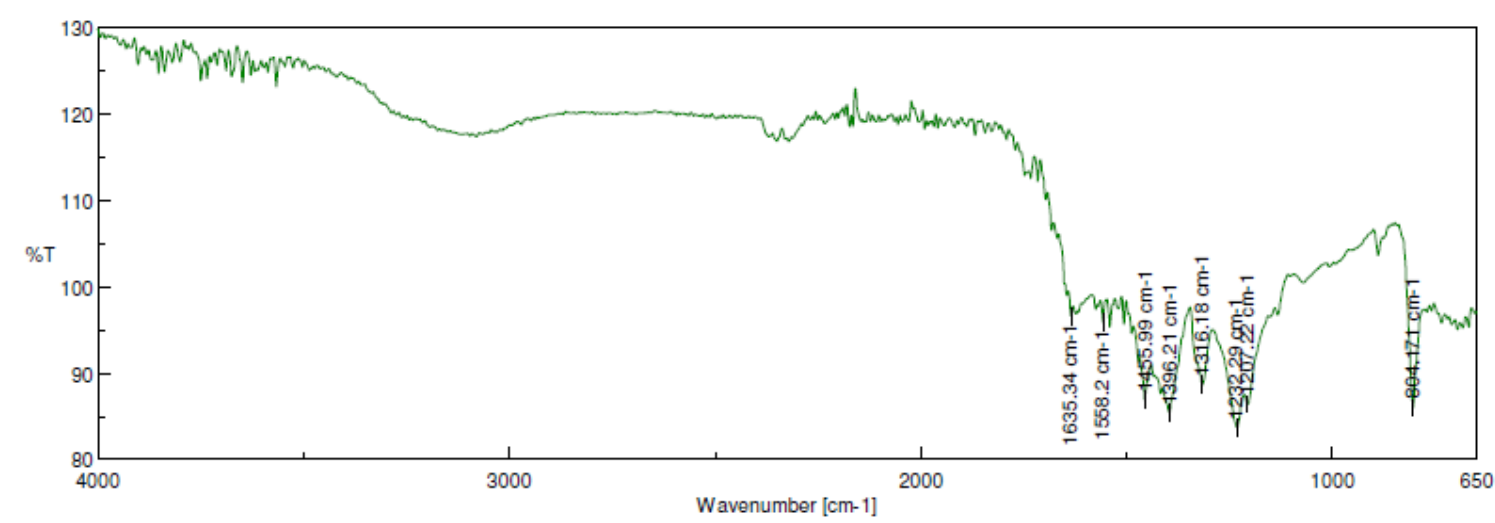

\begin{tabular}{|c|c|c|c|}
\hline Comments] & & Measurement Infor & nation] \\
\hline Sample name & WW-g-C3 & Model Name & FT/IR-4 \\
\hline iomment & FWW & Serial & C1885 \\
\hline Jser & WW & Monsuram & \\
\hline & $\begin{array}{l}\text { AK Uas } \\
\text { Univ. Gottingen Org. + Biomol. Chemie }\end{array}$ & Measurement Date & 13.01 .2020 \\
\hline & & $\begin{array}{l}\text { Light } \\
\text { Detec }\end{array}$ & $\begin{array}{l}\text { Ste } \\
\text { TG }\end{array}$ \\
\hline $\begin{array}{l}\text { Detalle } \\
\text { reatio }\end{array}$ & & ation & \\
\hline & & & \\
\hline & Never & & \\
\hline as & Waven & zation & \\
\hline & $\begin{array}{l}\% 1 \\
649.893 \mathrm{~cm}-1\end{array}$ & $A p$ & Auto 7.1 \\
\hline & & Scanning Speed & Auto $(2 \mathrm{mn}$ \\
\hline ata points & & Filter & Auto $(30000 \mathrm{~Hz})$ \\
\hline
\end{tabular}

\section{FWW13 Entry 18}

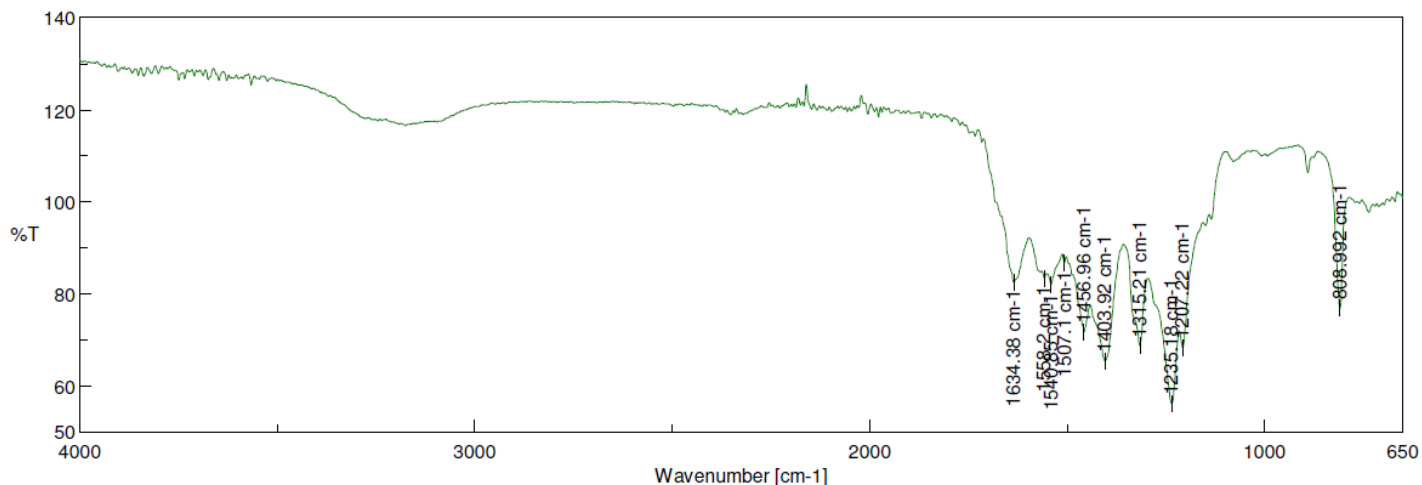

\begin{tabular}{|c|c|c|c|}
\hline & \\
\hline Sample name & WW-g-C3N4-FWW 13 & Model Name & FT/R-4100typeA \\
\hline Comment & FWW13 & Serial Number & C188561016 \\
\hline Division & AK Das & Measurement Date & 13.01.2020 14:41 \\
\hline Company & Univ. Göttingen Org. + Biomol. Chemie & & \\
\hline & & Light Source & Standard \\
\hline |L & & Detector & TGS \\
\hline Creation date & 13.01.2020 14:41 & $\begin{array}{l}\text { Accumulation } \\
\text { Resolution }\end{array}$ & 32 \\
\hline & Line & Zero Filling & \\
\hline Horizontal axis & Wavenumber [cm-1] & Apodization & Cosine \\
\hline Vertical axis & & Gain & Auto (8) \\
\hline & $649.893 \mathrm{~cm}-1$ & Aperture & Auto $(7.1 \mathrm{~mm})$ \\
\hline & $4000.6 \mathrm{~cm}-1$ & Scanning Speed & Auto $(2 \mathrm{~mm} / \mathrm{sec})$ \\
\hline Data interval & $0.964233 \mathrm{~cm}-1$ & Filter & Auto $(30000 \mathrm{~Hz})$ \\
\hline
\end{tabular}


FWW8 Entry 19

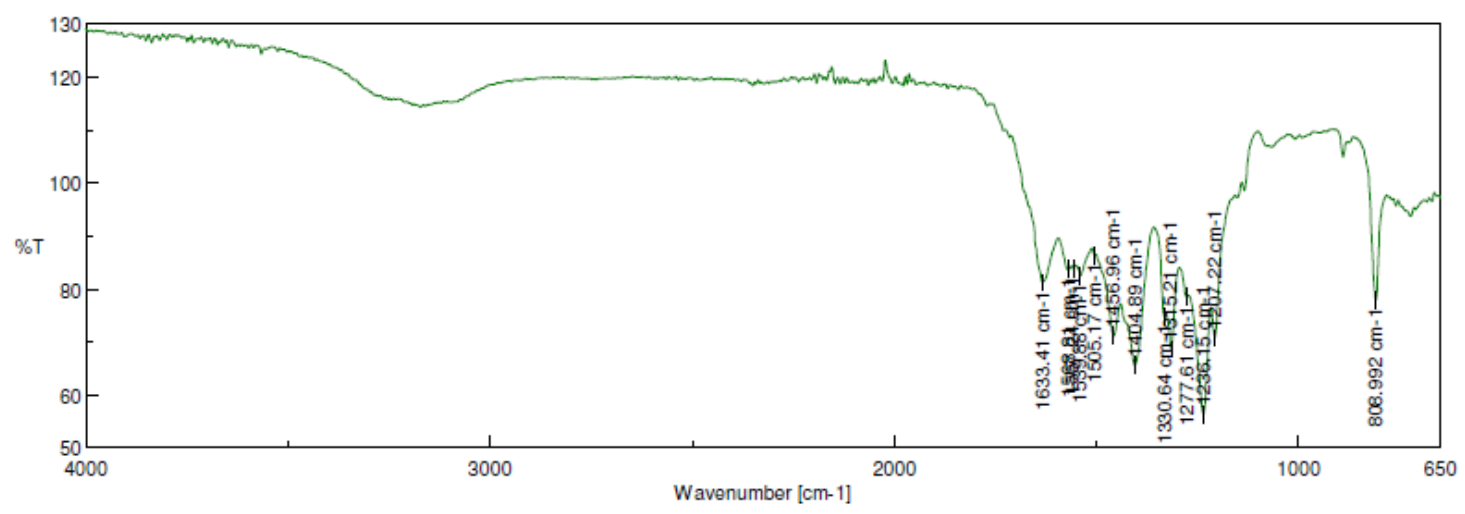

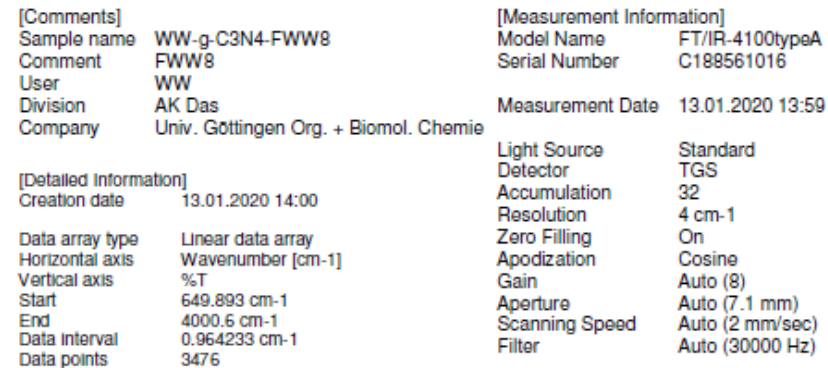

FWW9 Entry 20

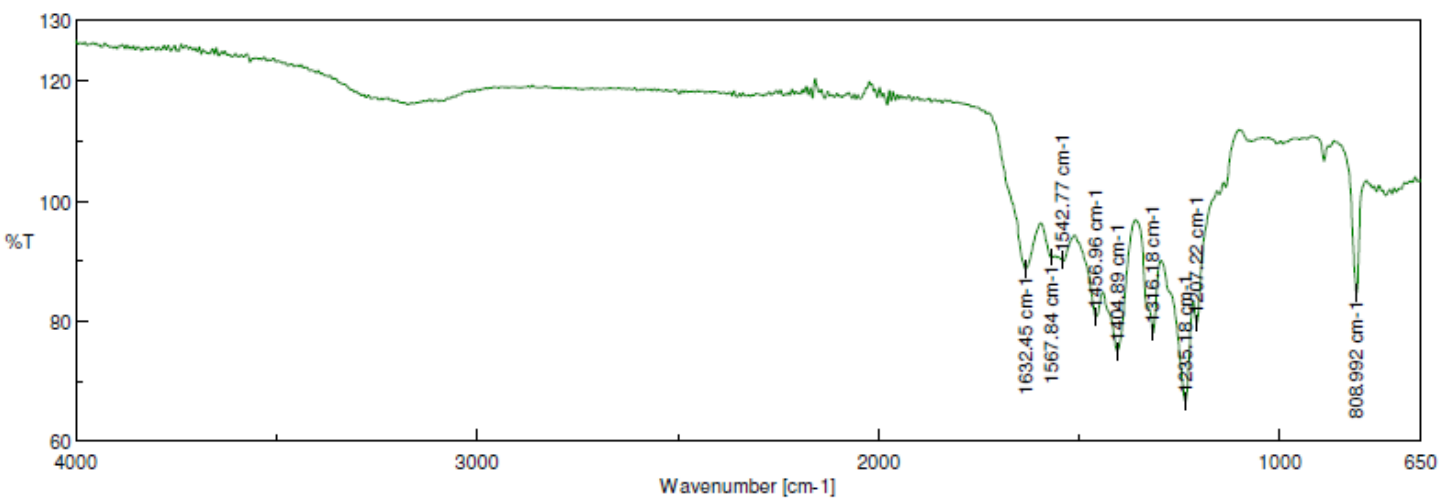

\begin{tabular}{|c|c|c|c|}
\hline Comments] & & [Measurement Inform & nation] \\
\hline Sample name & WW-g-C3N4-FWW9 & Model Name & FT/IR-4100typeA \\
\hline $\begin{array}{l}\text { Comment } \\
\text { User }\end{array}$ & $\begin{array}{l}\text { FWW9 } \\
\text { WW }\end{array}$ & Serial Number & C188561016 \\
\hline Division & AK Das & Measurement Date & 13.01.2020 13:44 \\
\hline Company & Univ. Gottingen Org. + Biomol. Chemie & & \\
\hline [Detalled Intorm & & Detec & \\
\hline Creation date & $13.01 .202013: 45$ & Accumulation & \\
\hline & & Resolution & $4 \mathrm{~cm}-$ \\
\hline $\begin{array}{l}\text { ta array type } \\
\text { prizontal axis }\end{array}$ & $\begin{array}{l}\text { Unear } \\
\text { Waven }\end{array}$ & Zero Filling & $\begin{array}{l}\text { On } \\
\text { Cosine }\end{array}$ \\
\hline rizontal & $\begin{array}{l}\text { Waven } \\
\text { \% }\end{array}$ & $\begin{array}{l}\text { Apodization } \\
\text { Gain }\end{array}$ & $\begin{array}{l}\text { Cosine } \\
\text { Auto (8) }\end{array}$ \\
\hline & $649.893 \mathrm{cr}$ & Aperture & Auto $(7.1 \mathrm{~mm})$ \\
\hline & 4000 & Scanning Speed & Auto $(2 \mathrm{~mm} / \mathrm{sec})$ \\
\hline Data Interval & 0.9642 & Filter & Auto $(30000 \mathrm{~Hz})$ \\
\hline Data points & & & \\
\hline
\end{tabular}


FWW10 Entry 21

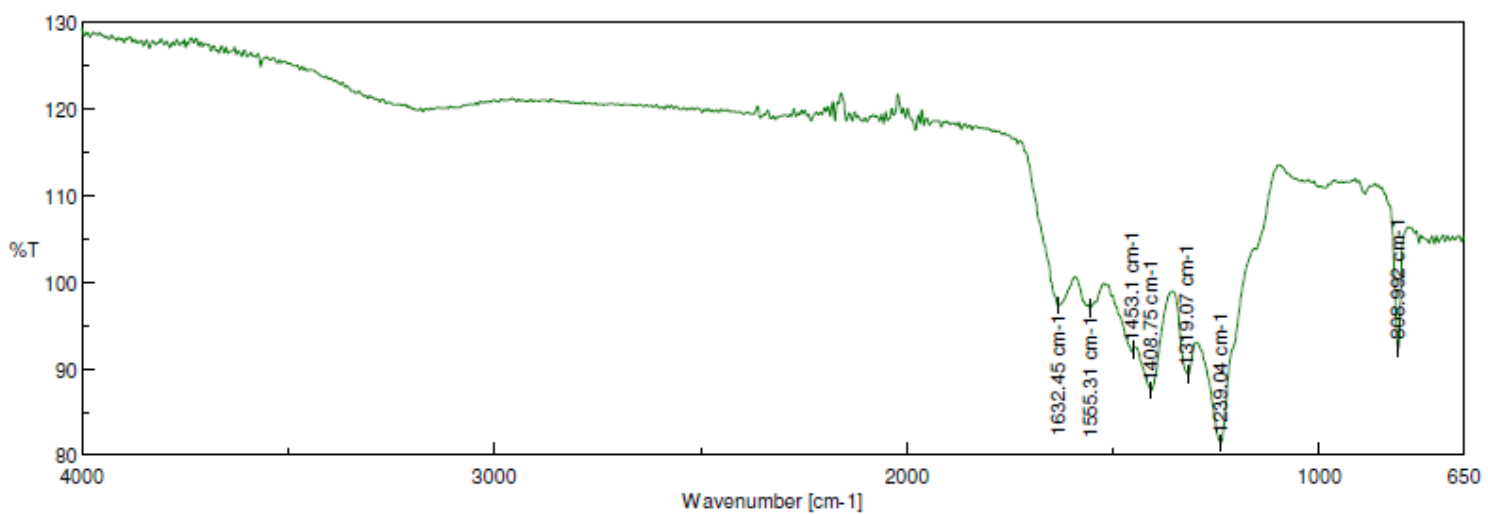

\begin{tabular}{|c|c|c|c|}
\hline [Comments] & & [Measurement Infort & nation] \\
\hline Sample name & WW-g-C3N4-FWW 10 & Model Name & FT/IR-4100typeA \\
\hline $\begin{array}{l}\text { Comment } \\
\text { User }\end{array}$ & FWW 10 no2 & Serial Number & C188561016 \\
\hline $\begin{array}{l}\text { User } \\
\text { Division }\end{array}$ & $\begin{array}{l}\text { WW } \\
\text { AK Das }\end{array}$ & & \\
\hline $\begin{array}{l}\text { Division } \\
\text { Company }\end{array}$ & Aniv. Gottingen Org. + Biomol. Chemie & Measurement Date & $13.01 .202013 .5 \mathrm{C}$ \\
\hline & & Light Source & idard \\
\hline [Detalled Informe & ation] & Detect & \\
\hline Creation date & 13.01 .202013 .51 & $\begin{array}{l}\text { Accumulation } \\
\text { Resolution }\end{array}$ & $\begin{array}{l}32 \\
4 \mathrm{~cm}-1\end{array}$ \\
\hline Data array type & Lnear data & Zero Filling & \\
\hline Horizontal axis & Wavenumber [cm-1] & Apodization & Cosine \\
\hline Vertical axis & & Gai & Auto (8) \\
\hline & $649.893 \mathrm{~cm}-1$ & Aperture & Auto $(7.1 \mathrm{~mm})$ \\
\hline & $4000.6 \mathrm{~cm}-1$ & Scanning Speed & Auto $(2 \mathrm{~mm} / \mathrm{sec})$ \\
\hline Data in & $0.964233 \mathrm{~cm}-1$ & Filter & Auto $(30000 \mathrm{~Hz})$ \\
\hline lata & & & \\
\hline
\end{tabular}

\section{FWW11 Entry 22}

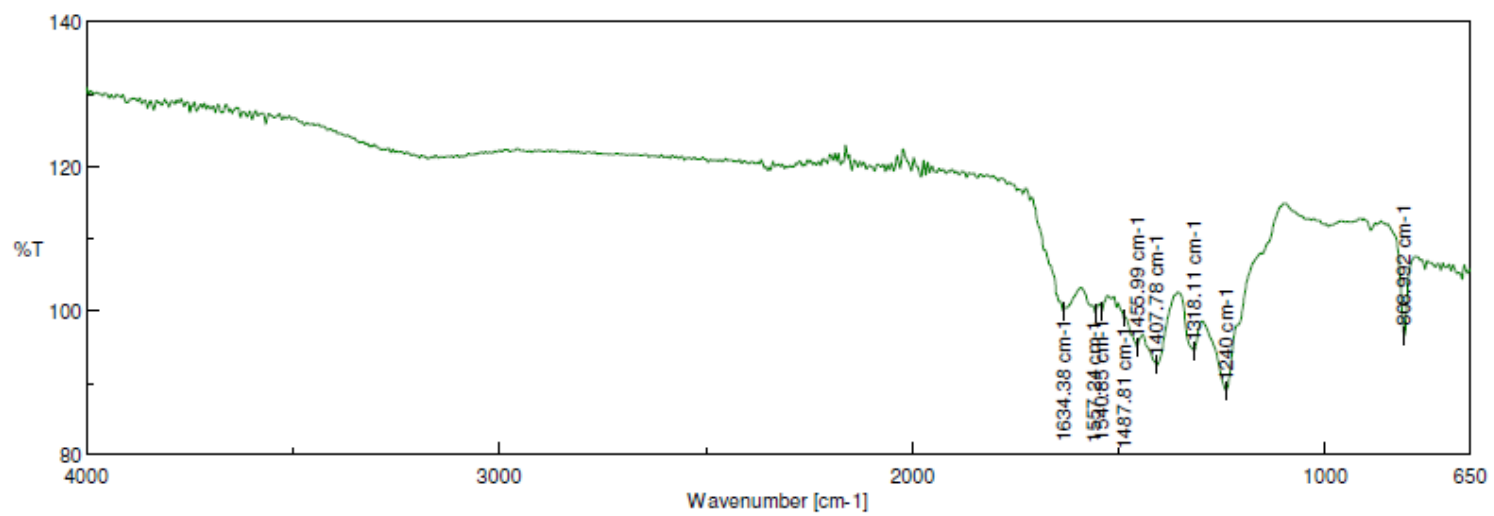

\begin{tabular}{|c|c|c|c|}
\hline [Comments] & & [Measurement Infor] & nation] \\
\hline Sample name & WW-g-C3N4-FWW11 & Model Name & FT/IR-4100typeA \\
\hline Comment & FWW11 & Serial Number & C188561016 \\
\hline User & WW & & \\
\hline $\begin{array}{l}\text { Division } \\
\text { Company }\end{array}$ & $\begin{array}{l}\text { AK Das } \\
\text { Univ. Gottingen Org. + Biomol. Chemie }\end{array}$ & Measurement Date & $13.01 .202014: 03$ \\
\hline & & Light Source & Standard \\
\hline [Detalled & ion] & & \\
\hline Creatio & $13.01 .202014: 03$ & $\begin{array}{l}\text { Accumulation } \\
\text { Resolution }\end{array}$ & $\begin{array}{l}32 \\
4 \mathrm{~cm}-1\end{array}$ \\
\hline Data array type & Unear data & Zero Filling & \\
\hline rizontal axis & Wavenumber [cm-1] & Apodization & $\operatorname{Cos}$ \\
\hline Vertical axis & & Gain & Auto (8) \\
\hline & $649.893 \mathrm{~cm}$ - & Aperture & Auto $(7.1 \mathrm{~mm})$ \\
\hline & $4000.6 \mathrm{~cm}-1$ & Scanning Speed & Auto $(2 \mathrm{~mm} / \mathrm{sec})$ \\
\hline Data interval & $0.964233 \mathrm{~cm}$ & Filter & Auto $(30000 \mathrm{~Hz})$ \\
\hline
\end{tabular}




\section{REFERENCES}

[1] J. F. Bourgeois, F. Barja, Arch. Sci. 2009, 62, 147-160.

[2] H. Taylor, "Classification of catalysts", can be found under https://www.britannica.com/science/catalysis/Classification-of-catalysts ; retrieved $20^{\text {th }}$ July 2020.

[3] S. A. Matlin, B. M. Abegaz, Chemistry for Development in: The Chemical Element: Chemistry's Contribution in our global Future, $1^{\text {st }}$ Ed. (Eds.: J. Gracia-Martinez, E. Serrano-Torregrosa), Wiley-VCH, Weinheim, 2011, pp. 1-13.

[4] a) The Core Writing Team, R. K. Pachauri, L. A. Meyer, Climate Change 2014. Synthesis Report Summary for Policymakers, IPCC, Geneva, Switzerland, 2014, pp. 151; b) A. J. McMichael, D. H. Campbell-Lendrum, C. F. Corvalán, K. L. Ebi, A. K. Githeko, J. D. Scheraga, A. Woodward, Climate change and human health, risks and responses, World Health Organization, Geneva, 2003; c) P. Neal, The greenhouse effect and ozone layer, Dryad, London, 1989.

[5] AG Energiebilanz, Verband der Chemischen Industrie e.V., 2015.

[6] A. Brunning, Chem. Eng. News, 2019, 97, 23.

[7] a) P. T. Anastas, M. M. Kirchhoff, Acc. Chem. Res. 2002, 35, 686-694; b) P. T. Anastas, J. C. Warner, Green Chemistry: Theory and Practice, Oxford University Press, 1998.

[8] Clarivate Analytics, 2019 Journal Citation Reports, Clarivate Analytics, can be found under: https://www.rsc.org/journals-books-databases/about-journals/impactfactor/, retrieved $20^{\text {th }}$ July 2020.

[9] J. M. Coronado, F. Fresno, M. D. Hernández-Alonso, R. Portela, Design of Advanced Photocatalytic Materials for Energy and Environmental Applications. Green Energy and Technology, Springer Verlag, London, 2013.

[10] a) D. Prat, J. Hayler, A. Wells, Green Chem. 2014, 16, 4546-4551; b) C. J. Clarke, W.-C. Tu, O. Levers, A. Bröhl, J. P. Hallett, Chem. Rev. 2018, 118, 747-800; c) P. G. Jessop, Green Chem. 2011, 13, 1391-1398.

[11] F. P. Byrne, S. Jin, G. Paggiola, T. H. M. Petchey, J. H. Clark, T. J. Farmer, A. J. Hunt, C. R. McElroy, J. Sherwood, Sustain. chem. process. 2016, 4, article number 7.

[12] a) B. M. Trost, Science 1991, 254, 1471-1477; b) R. A. Sheldon, Green Chem. 2017, 17, 18-43.

[13] R. A. Sheldon, J. Chem Technol Biotechnol., 1997, 68, 381-388.

[14] M. A. Scibioh, B. Viswanathan, Carbon Dioxide to fine chemicals and Fuels, Elsevier, Amsterdam, 2018.

[15] N. Gunasekaran, Adv. Synth. Catal. 2015, 357, 1990-2010.

[16] D. Ravelli, D. Dondi, M. Fagnoni A. Albini, Chem. Soc. Rev. 2009, 38, 1999-2011.

[17] a) M. C. DeRosa, R. J. Crutchley, Coord. Chem. Rev. 2002, 233-234, 351-371;

b) M. M. Montemore, M. A. van Spronsen, R. J. Madix, C. M. Friend, Chem. Rev. 2018, 118, 2816-2862; c) D. M. Schultz, T. P. Yoon, Science 2014, 343, 6174, 1239176, DOI: 10.1126/science.1239176; d) U. Ulmer, T. Dingle, P. N. Duchesne, R. H. Morris, 
A. Tavasoli, T. Wood, G. A. Ozin, Nat. Comm. 2019, 10, 3169; e) Y. Bo, C. Gao, Y. Xiong, Nanoscale 2020, 12, 12196-12209; f) V. Etacheri, C. Di Valentin, J. Schneider, D. Bahnemann, S. C. Pillai, J. Photochem. Photobiol. C 2015, 25, 1-29; g) L. Liu, Y. Li, Aerosol Air Qual. Res. 2014, 14, 453-469.

[18] Digital Science. (2018-) Dimensions [Software] available from https://app.dimensions.ai. Accessed on (25.11.2020), under licence agreement. Search parameters "Green Chemistry", "Photocatalysis" sorted by Publications with citations, Total $>1$.

[19] J. M. R. Narayanam, J. W. Tucker, C. R. J. Stephenson, J. Am. Chem. Soc. 2009, 131, 8756-8757.

[20] H. D. Roth Angew. Chem. Int. Ed. Engl. 1989, 28, 1193-1207.

[21] A. Albini, Photochemistry: Past, Present and Future, Springer Verlag, Berlin Heidelberg, 2015.

[22] J. M. Coronado, F. Fresno, M. D. Hernández-Alonso, R. Portela, Design of Advanced Photocatalytic Materials for Energy and Environmental Applications. Green Energy and Technology, Springer Verlag, London, 2013.

[23] G. Dong, Y. Zhang, Q. Pan, J. Qiu, J. Photochem. Photobiol. C. 2014, 20, 33-50.

[24] D. Cambié, T. Noël, Solar Photochemistry in Flow, in: Accounts on Sustainable Flow Chemistry (eds.: T. Noël, R. Luque), Springer Internation Publishing, 2020, pp. 1-15.

[25] G. Laudadio, S. Govaerts, Y. Wang, D. Ravelli, H. F. Koolman, M. Fagnoni, S. W. Djuric, T. Noël, Angew. Chem. Int. Ed. 2018, 57, 4078-4082.

[26] a) A. Ambrosi, M. Pumera, Chem. Soc. Rev. 2016, 45, 2740-2755; b) M. D. Symes, P. J. Kitson, J. Yan, C. J. Richmond, G. J. T. Cooper, R. W. Bowman, T. Vilbrandt, L. Cronin, Nat. Chem. 2012, 4, 349-354; c) P. J. Kitson, G. Marie, J.-P. Francoia, S. S. Zalesskiy, R. C. Sigerson, J. S. Mathieson, L. Cronin, Science 2018, 359, 314-319.

[27] L. Buzzetti, G. E. M. Crisenza, P. Melchiorre, Angew. Chem. Int. Ed. 2019, 58, 3730-3747.

[28] Handbook pf synthetic Photochemistry (Eds.: A. Albini, M. Fagnoni), Wiley-VCH, Weinheim, 2010, pp 2-10.

[29] A. Albini, Photochem. Photobiol. Sci. 2016, 15, 319-324.

[30] V. Balzani, P. Ceroni, A. Juris, Photochemistry and Photophysics: Concepts, Research, Applications, Wiley-VCH, Weinheim, 2014.

[31] a) T. Noël, J. Flow Chem. 2017, 7, 87-93; b) D. Cambié, F. Zhao, V. Hessel, M. G. Debije, T. Noël, Angew. Chem. Int. Ed. 2017, 56, 1050-1054.

[32] C. Le, M. K. Wismer, Z.-C. Shi, D. V. Conway, G. Li, P. Vachel, I. W. Davies, D. W. C. Macmillan, ACS Cent. Sci. 2017, 3, 647-653.

[33] a) D. Cambié, C. Bottecchia, N. J. W. Straathof, V. Hessel, T. Noël, Chem. Rev. 2016, 116, 10276-10341; b) O. Shvydkiv, K. Jähnisch, N. Steinfeldt, A. Yavorskyy, M. Oelgemöller, Catal. Today 2018, 308, 102-118; c) H. Seo, M. H. Katcher, T. F. Jamison, Nat. Chem. 2017, 9, 453-456; d) J. W. Beatty, J. J. Douglas, R. Miller, R. C. McAtee, K. P. Cole, C. R. J. Stephenson, Chem 2016, 1, 456-472. 
[34] S. Kumar, D. Aand, S. Pabbaraja, D.-P. Kim, A. K. Singh, ACS Sustainable Chem. Eng. 2019, 7, 19605-19611.

[35] K. P. L. Kuijpers, M. A. H. van Dijk, Q. G. Rumeur, V. Hessel, Y. Su, T. Noël, React. Chem. Eng. 2017, 2, 109-115.

[36] a) T. Koike, M. Akita, Inorg. Chem. Front. 2014, 1, 562-576; b) K. S. Schanze, J. Am. Chem. Soc. 1988, 110, 1180-1180; c) Z. Wang, A. G. Herraiz, A. M. Del Hoyo, M. G. Suero, Nature 2018, 554, 86-91; d) A. Noble, R. S. Mega, D. Pflästerer, E. L. Myers, V. K. Aggarwal, Angew. Chem. Int. Ed. 2018, 57, 2155-2159.

[37] a) K. Teegardin, J. I. Day, J. Chan, J. Weaver, Org. Process Rev. Dev. 2016, 20, 1156-1163; b) Z.-Y. Xu, D.-W. Zhang, H. Wang, X.-W. Sun, T.-T. Li, Green Chem. 2020, 22, 136-143; c) J. Lalevée, M. Peter, F. Dumur, D. Gigmes, N. Blanchard, M. A. Tehfe, F. Morlet-Savary, J. P. Subtle, Chem. Eur. J. 2011, 17,15027-15031; d) Y. Chen, Z. Hu, D. Xu, Y. Yu, X. Tang, H. Guo, Macromol. Chem. Phys. 2015, 216, 10551060; e) A. Singh, J. J. Kubik, J. D. Weaver, Chem. Sci. 2015, 6, 7206-7212.

[38] a) M. A. Tehfe, L. Ma, B. Graff, F. Morlet-Savary, J. P. Fouassier, J. Zhao, J. Lalevée, Macromol. Chem. Phys. 2012, 213, 2282-2286; b) Y. Chen, Z. U. Wang, H. Wang, J. Lu, S. Yu, H. Jiang, J. Am. Chem. Soc. 2017, 139, 2035-2044; c) B. Cao, J. Zhao, Y. Yu, W. Li, S. Xu, R. Sun, X. Wang, J. Guo, C. Zhou. J. Zhang, Catal. Today, 2020, 340, 183-187.

[39] a) S. Yurdakal, G. Palmisano, V. Loddo, V. Augugliaro, L. Palmisano, J. Am. Chem. Soc. 2008,130, 1568-1569; b) D. Tsukamoto, Y. Shiarishi, Y. Sugano, S. Ichikawa, S. Tanaka, T. Hirai, J. Am. Chem. Soc. 2012, 134, 6309-6315; c) X. Lang, W. Ma, C. Chen, J. Ji, J. Zhao, Acc. Chem Res. 2014, 47, 355-363; d) Y. Tamaki, A. Furube, M. Murai, K. Hara, R. Katoh, M. Tachiya J. Am. Chem. Soc. 2006, 128, 416417.

[40] a) S. Furukawa, T. Shishido, K. Teramura, T. Tanaka, ACS. Catal. 2012, 2, 175179; b) C. Meng, K. Yang, X. Fu, R. Yuan, ACS Catal. 2015, 5, 3760-3766; c) J. Zhang, D. Campolo, F. Dumer, P. Xiao, J. P. Fouassier, D. Gigmes, J. Lalevée, ChemCatChem 2016, 8, 227-2233.

[41] N. A. Romero, D. A. Nicewicz, Chem. Rev. 2016, 116, 10075-10166.

[42] a) K. Ohkubo, K. Suga, K. Morikawa, S. Fukuzumi, J. Am. Chem. Soc. 2003, 125, 12850-12859; b) D. Mangion, J. Kendall, D. R. Arnold, Org. Lett. 2001, 3, 45-48; c) Y. Wang, O. Haze, J. P. Dinnocenzo, S. Farid, R. S. Farid, I. R. Gould, J. Org. Chem. 2007, 72, 6970-6981; d) H. Görner, K.-D. Warzecha, M. Demuth, J. Phys. Chem. A 1997, 101, 9964-9973; e) I. R. Gould, D. Ege, J. E. Moser, S. Farid, J. Am. Chem. Soc. 1990, 112, 4290-4301; f) A. P. Darmanyan, Chem. Phys. Lett. 1984, 110, 89-94.

[43] a) H. C. Heller, J. Am. Chem. Soc. 1967, 89, 4288-4294; b) H.-J. Timpe, K.-P. Dronfeld, U. Lammel, J.-P. Fouassier, D.-J. Lougnot, J. Photochem. Photobiol. A, 1990, 52, 111-122; c) P. Supan, J. Chem. Soc., Faraday Trans. 1 1975, 71, 539-547; d) I. Ghosh, A. Mukhopadhyay, A. L. Koner, S. Samanta, W. M. Nau, J. N. Moorthy, Phys. Chem. Chem. Phys. 2014, 16, 16436-16445; e) H.-J. Timpe, K.-P. Kronfeld, J. Photochem. Photobio. A 1989, 46, 253-267; f) J.-B. Xia, C. Zhu, C. Hen, J. Am. Chem. Soc. 2013, 135, 17494-17500; g) J.-P. Malval, M. Jin, F. Morlet-Savary, H. Chaunmeil, A. Defoin, O. Soppera, T. Scheul, M. Bouriau, P. L. Baldeck, Chem. Mater. 2011, 23, 3411-3420. 
[44] a) M. R. Reta, R. Catana, J. D. Anunziata, J. J. Silber, Spectrochim. Acta Part Mol. Spectrosc. 1993, 49, 903-912; b) C. Serpa, L. G. Arnaut, J. Physl. Chem. A 2000, 104, 11075-11086; c) K. Ohkubo, A. Fujimoto, S. Fukuzimi, J. Am. Chem. Soc. 2013, 135, 5368-5371; d) S. M. hubig, J. K. Kochi, J. Am. Chem. Soc. 1999, 121, 1688-1694; e) T. Del Giacoo, E. Vaciocchi, O. Langzalunga, F. Elisei, Chem. Eur. J. 2001, 7, 30053013.

[45] a) S. P. Pitre, C. D. McTiernan, H. Ismaili, J. C. Scaiano, J. Am. Chem. Soc. 2013, 135, 13286-13289; b) H.-J. Timpe, S. Neuenfeld, J. Chem. Soc., Faraday Trans. 1992, 88, 2329-2336.

[46] a) G. Weber, F. W. J. Teale, Trans. Faraday Soc. 1957, 53, 646-655; b) S. Fukuzumi, M. Fujita, S. Noura, K. Ohkubo, T. Suenobu, Y. Araki, O. Ito, J. Phys. Chem. A 2001, 105, 1857-1868; c) R. W. Chambers, D. R. Kearns, Photochem. Photobiol. $1969,10,215-219$.

[47] a) X.-F. Zhang, I. Zhang, L. Liu, J. Photochem. Photobiol. 2010, 86, 492-498; b) T. Shen, Z.-G. Zhao, Q. Yu, H.-J. Xu, J. Photochem. Photobiol. A 1989, 47, 203-212; c) K. Fidaly, C. Ceballos, A. Falguières, M. S.-I. Veitia, A. Guy, C. Ferround, Green Chem. 2012, 14, 1293-1297; d) E. Yoshioka, S. Kohtani, T. Jichu, T. Fukazawa, T. Nagai, Y. Takemoto, H. Miyabe, Synlett 2015, 26, 265-270; e) L. Yuan, W. Lin, Y. Yang, H. Chen, J. Am. Chem. Soc. 2012, 13, 1200-1211; f) D. Magde, G. E. Rojas, P. G. Seybold, Photochem. Photobiol. 1999, 70, 737-744; g) D. Magde, R. Wong, P. G. Seybold, Photochem. Photobiol. 2002, 75, 327-334; h) V. E. Korobov, V. V. Shubin, A. K. Chibisov, Chem. Phys. Lett. 1977, 45, 498-501; i) M. M. Martin, L. Lindqvist, J. Lumin. 1975, 10, 381-390.

[48] M. Majek, F. Filace, A. J. von Wangelin, Beilstein J. Org. Chem. 2014, 10, 981 989.

[49] B. G. McCarthy, R. M. Pearson, C.-H. Lim, S. M. Sartor, N. H. Damrauer, G. M. Miyake, J. Am. Chem. Soc. 2018, 140, 5088-5101.

[50] E. Speckmeier, T. G. Fischer, K. Zeitler, J. Am. Chem. Soc. 2018, 140, 1535315365.

[51] a) N. Rono, J. K. Kibet, B. S. Martincigh, V. O. Nyamori, Crit. Rev. Solid State Mater. Sci. 2020, DOI: 10.1080/10408436.2019.1709414; b) Q. Hao, G. Jia, W. Wei, A. Vinu, Y. Wang, H. Arandiyan, B.-J. Ni, Nano Research 2020, 13, 18-37; c) C. Zhang, Y. Li, Y. Shen, W. Xiong, L. Wang, Chemosphere 2019, 214, 462-479; d) L. Zhou, H. Zhang, H. Sun, S. Liu, M. O. Tade, S. Wang, W. Jin, Catal. Sci. Technol. 2016, 6, 7002-7023.

[52] a) B. A. Bhanvase, T. P. Shende, S. H. Sonawane, Environ. Technol. Rev. 2017, 6, 1-14; b) X. Li, S. Wageh, A. A. Al-Ghamdi, J. Xie, Small 2016, 12, 6640-6696; c) L. K. Putri, W.-J. Ong, W. S. Chang, S.-P. Chai, Appl. Surf. Sci. 2015, 358, 2-14; d) N. N. Rosman, R. M. Yunas, L. J. Minggu, K. Arifin, M. N. I. Salehmin, M. A. Mohamed, M. B. Kassim, Int. J. Hydrog. Energy 2018, 43, 18925-18945.

[53] a) S.-N. Zhao, G. Wang, D. Poelman, P. Van Der Voort, Molecules 2018, 23, 2947; b) N. Huber, R. Li, C. T. J. Ferguson, D. W. Gehrig, C. Ramanan, P. W. M. Blom, K. Landfester, K. A. I. Zhang, Catal. Sci. Technol. 2020, 10, 2092-2099; c) M. A. Nasalevich, M. van der Veen, F. Kapteijn, J. Gascon, CrystEngComm. 2014, 16, 49194926; d) L. Zeng, X. Guo, C. He, C. Duan, ACS Catal. 2016, 6, 7935-7947.

[54] Y. Qu, X. Duan, Chem. Soc. Rev. 2013, 42, 2568-2580. 
[55] a) M. Ismael, Y. Wu, D. H. Taffa, P. Bottke, M. Wark, New. J. Chem. 2019, 43, 6909-6920; b) V. Devthade, D. Kulhari, S. S. Umare, Mater. Today-Proc. 2018, 5, 9203-9210.

[56] Z. Zhao, Y. Sun, F. Dong, Nanscale 2015, 7, 15-37.

[57] A. Mishra, A. Mehta, S. Basu, N. P. Shetti, K. R. Reddy, T. M. Aminabhavi, Carbon 2019, 149, 693-721.

[58] J. Huang, J. Huang, X. Liu, C. Li, L. Ding, H. Yu, Sci. Bull. 2018, 63, 1180-1186.

[59] H. H. Binder, Lexikon der chemischen Elemente, S. Hirzel Verlag, Stuttgart, 1999.

[60] D. T. Sawyer, Oxygen Chemistry, Oxford university Press, New York, 1991.

[61] Y. Nosaka, A. Y. Nosaka, Chem. Rev. 2017, 117, 11302-11336.

[62] a) M. Wrzaczek, M. Brosche, J. Kangasjarvi, Curr. Opin. Plant Biol. 2013, 16, 575582; b) P. D. Ray, B.-W. Huang, Y. Tsuji, Cell. Signalling 2012, 24, 981-990.

[63] U. Langematz, ChemTexts 2019, 5, https://doi.org/10.1007/s40828-019-0082-7.

[64] I. Gately, Drink: A Cultural History of Alcohol, Gotham Books, New York, 2008.

[65] a) D. Kralisch, D. Ott, D. Gericke, Green Chem. 2015, 17, 123-145; b) P. Sengupta, B. Chatterjee, R. K. Tekade, Int. J. Pharm. 2018, 543, 328-344; c) J.-M. Kauffmann, J.-C. Viré, Anal. Chim. Acta 1993, 273, 329-334.

[66] H. Becker, W. Berger, G. Domschke, E. Fanghänel, Organikum - Organisch Chemisches Praktikum, 22th ed., Wiley-VCH, Weinheim, 2004.

[67] a) G. Tojo, M. Fernández, Oxidation of Alcohols to Aldehyde and Ketones, Springer, New York, 2006; b) K. E. Harding, L. M. May, K. F. Dick, J. Org. Chem. 1975, 40, 1664-1665.

[68] K. Omura, D. Swern, Tetrahedron 1978, 34, 1651-1660.

[69] D. B. Dess, J. C. Martin, J. Org. Chem. 1983, 48, 4155-4156.

[70] J. C. Collin, W. W. Hess, F. J. Frank, Tetrahedron 1968, 9, 3363-3366.

[71] E. J. Corey, J. W. Suggs, Tetrahedron Lett. 1975, 16, 2647-2650.

[72] E. J. Corey, G. Schmidt, Tetrahedron Lett. 1979, 20, 399-402.

[73] K. E. Pfitzner, J. G. Moffatt, J. Am. Chem. Soc. 1963, 85, 3027-3028.

[74] a) D. A. Blackaddar, H. M. Schleinitz Nature 1964, 202, 179-181; b) R. J. Highet, W. C. Wildman, J. Am. Chem. Soc. 1955, 77, 4399-4401; c) R. Hänsel, T. L. Su, J. Schulz, Chem. Ber. 1977, 110, 3664-3671; d) E. Hayashi, Y. Yamaguchi, K. Katama, N. Tsunoda, Y. Kumagai, F. Oba, M. Hara, J. Am. Chem. Soc. 2019, 141, 890-900; e) J. Wang, H. Luo, P. Liu, Catal. Commun. 2020, https://doi.org/10.1016/j.catcom.2020.106030.

[75] P. Gamez, I. W. C. E. Arends, J. Reedijk, R. A. Sheldon, Chem. Commun. 2003, 19, 2414-2415.

[76] A. Das, S. S. Stahl, Angew. Chem. Int. Ed. 2017, 56, 8892-8897.

[77] J. M. Hoover B. L. Ryland, S. S. Stahl, ACS Catal. 2013, 3, 2599-2605.

[78] M. Königsmann, N. Donati, D. Stein, H. Schönberg, H. Harmer, A. Sreekanth, H. Grützmacher, Angew. Chem. Int. Ed. 2007, 46, 3567-3570. 
[79] R. Liu, X. Liang, C. Dong, X. Hu, J. Am. Chem. Soc. 2004, 126, 4112-4113.

[80] D. Riemer, B. Mandaviya, W. Schilling, A. C. Götz, T. Kühl, M. Finger, S. Das, ACS Catal. 2018, 8, 3030-3034.

[81] F. Su, S. C. Mathew, G. Lipner, X. Fu, M. Antonietti, S. Blechert, X. Wang, J. Am. Chem. Soc. 2010, 132, 16299-16301.

[82] a) J. Zhu, P. Xiao, H. Li, S. A. C. Carabinerio, ACS Appl. Mater. Interfaces 2014, 6, 16449-16465; b) Q. Li, Y. He, R. Peng, RSC Adv. 2015, 5, 24507-24512.

[83] Y.-Z. Chen, Z. U. Wang, H. Wang, J. Lu, S.-H. Yu, H.-L. Jiang, J. Am. Chem. Soc. 2017, 139, 2035-2044.

[84] W. Huang, B. C. Ma, H. Lu, R. Li, L. Wang, K. Landfester. K. A. I. Zhang, ACS Catal. 2017, 7, 5438-5442.

[85] a) T. Eicher, S. Hauptmann, A. Speicher, The Chemistry of Heterocycles: Structures, Reactions, Synthesis, and Applications, 3rd, Completely Revised and Enlarged Edition, Wiley-VCH, Weinheim, 2012; b) N. Chadha, O. Silakari, Eur. J. Med. Chem. 2017, 134, 159-184; c) E. Abele, R. Abele, O. Dzenitis, E. Lukevics, Chem. Heterocycl. Com. 2003, 39, 3-35; d) G. Mathur, S. Nain, Med. Chem. 2014, 4, 417427; e) P. A. Szijj, C. Bahou, V. Chudasama, Drug Discov. Today Technol. 2018, $27-$ 34; f) E. Baltazzi, L. I. Krimen, Chem. Rev. 1963, 63, 511-556; g) W. C. Sumpter, Chem. Rev. 1944, 34, 393-434.

[86] Olfactory Perception in Humans in Neuroscience (Eds.: D. Purves, G. J. Augustine, D. Fritzpatrick et al.), $2^{\text {nd }}$ ed., Sinauer Associates, Sunderland, 2001.

[87] L. Kürti, B. Czakó, Strategic Applications of Named Reactions in Organic Synthesis, $1^{\text {st }}$ ed., Elsivier Academic Press, Burlingtion-San Diego-London, 2005.

[88] G. Bartoli, G. Palmieri, M. Bosco, R. Dalpozzo, Tetrahedron Lett. 1989, 30, 2129 2132.

[89] J. J. Li, Bischler-Möhlau indole synthesis, in: Name Reactions, Springer, Berlin, Heidelberg, 2002.

[90] a) H. Tokuyama, T. Yamashita, M. T. Reding, Y. Kaburagi, T. Fukuyama, J. Am. Chem. Soc. 1999, 121, 3791-3792; b) T. Fukuyama, X. Chen, G. Peng, J. Am Chem. Soc. 1994, 116, 3127-3128.

[91] a) P. G. Gassman, T. J. Van Bergen, G. Grützmacher, J. Am. Chem Soc. 1973, 95, 6508-6509; b) P. G. Gassman, T. J. Van Gergen, D. P. Gilbert, B. W. Cue Jr., J. Am. Chem. Soc. 1974, 96, 5495-5508; c) P. G. Gassman, T. J. Van Bergen, J. Am. Chem. Soc. 1974, 96, 5508-5512.

[92] H. Hemetsberger, D. Knittel, Monatsh, Chem. 1972, 103, 194-204.

[93] R. C. Larock, E. K. Yum, J. Am. Chem. Soc. 1991, 113, 6689-6690.

[94] a) W. Madelung, Ber. Dtsch. Chem. Ges. 1912, 146, 1128; b) W. J. Houlihan, Y. Uike, V. A. Parrino, J. Org. Chem. 1981, 46, 4515-4517.

[95] C. D. Nenitzescu, Bull. Soc. Chim. Romania 1929, 11, 37-43.

[96] a) A. Reissert, Ber. Dtsch. Chem. Ges. 1897, 30, 1030-1053; b) J. R. Johnson, R. B. Hasbrouck, J. D. Dutcher, W. F. Bruce, J. Am. Chem. Soc. 1945, 67, 423-430. 
[97] a) A. Baeyer, A. Emmerling, Ber. Dtsch. Chem. Ges. 1869, 2, 679-682; b) H. Foster, D. Hein, J. Org. Chem. 1961, 26, 2539-2541.

[98] a) E. C. Creencia, M. Tsukamoto, T. Horaguchi, J. Heterocyclic Chem. 2011, 48, 1095-1102; b) V. Sridharan, S. Perumal, C. Avendño, J. C. Menédez, Synlett 2006, 1, 91-95; c) S. A. Patil, R. Patil, D. D. Miller, Curr. Med. Chem. 2011, 18, 615-637; d) F. Petronijevic, C. Timmons, A. Cuzzupe, P. Wipf, Chem. Commun. 2008, 104-106; e) G. Bratulescu, Tetrahedron Lett. 2008, 49, 984.

[99] a) A. D. Batcho, W. Leimgruber, US. Patent 3,732,245, 1975; b) A. D. Batcho, W. Leimgruber, U.S. Patent 3,976,639, 1975; c) A. D. Batcho, W. Leimgruber, Org. Synth. 1985, 63, 214-220.

[100] a) G. W. Gribble, Indole Ring Synthesis: From Natrual Products to Drug Discovery, $1^{\text {st }}$ ed., Wiley-VCH, New York, 2016; b) M. Inman, C. J. Moody, Chem. Sci. 2013, 4, 29-41; c) M. M. Heravi, S. Rohani, V. Zadsirjan, N. Zahedi, RSC Adv. 2017, 7, 52852-52887.

[101] G. Collins, H. Höke, Indole in: Ullmann's Encyclopedia of Industrial Chemistry, Wiley-VCH, Weinheim, 2000.

[102] A. Chandra, N. R. Yadav, J. N. Moorthy, Tetrahedron 2019, 75, 2169-2174.

[103] M. Muneer, M. Saquib, M. Qamar, D. Bahnemann, Res. Chem. Intermed. 2010, $36,121-125$.

[104] C. Zhang, S. Li, F. Bureš, R. Lee, X. Ye, Z. Jiang, ACS. Catal. 2016, 6, 68536860.

[105] a) H. Nakano, S. Umio, K. Kariyone, K. Tanaka, T. Kishimoto, H. Noguchi, I. Ueda, H. Nakamura, T. Morimoto, Tetrahedron Lett. 1966, 7, 737-740; b) R. Kaur, V. Rani, V. Abbot, Y. Kappor, D. Konar, K. Kumar, J. Pharm. Chem. Chem. Sci. 2017, 1, 17-32; c) S. D. Joshi, U. A. More, V. H. Kulkarni, T. M. Aminabhavi, Cur. Org. Chem. 2013, 17, 2279-2304.

[106] a) A. Hantzsch, Ber. Dtsch. Chem. Ges. 1890, 23, 1474-1476; b) F. Freist, Ber. Dtsch. Chem. Ges. 1902, 35, 1537-1544.

[107] a) L. Knorr, Ber. Dtsch. Chem. Ges. 1884, 17, 1635-1642; b) L. Knorr, H. Lange, Ber. Dtsch. Chem. Ges. 1902, 35, 2998-3000.

[108] a) C. Paal, Ber. Dtsch. Chem. Ges. 1884, 17, 2756-2767; b) L. Knorr, Ber. Dtsch. Chem. Ges. 1884, 17, 2863-2870; c) V. Amarnath, K. Amarnath, J. Org. Chem. 1995, 60, 301-307; d) V. Amarnath, D. C. Anthony, K. Amarnath, W. M. Valentine, L. A. Wetterau, D. G. Graham, J. Org. Chem. 1991, 56, 6924-6931.

[109] D. Van Leusen, O. Oldenziel, A. Van Leusen, J. Org. Chem. Am. Chem. Soc. 1977, 42, 3114-3118.

[110] D. H. R. Barton, S. Z. Zard, J. Chem. Soc., Chem. Commun. 1985, 1098-1100.

[111] a) O. Piloty, Ber. Dtsch. Chem. Ges. 1910, 43, 489-498; b) G. M. Robinson, R. Robinson, J. Chem. Soc., Trans. 1918, 113, 639-645.

[112] a) W. Lubell, D. Saint-Cyr, J. Dufour-Gallant, R. Hopewell, N. Boutard, T. Kassem, A. Dörr, R. Zelli, Sci. Synth. 2013, 1, 157-388; b) C. T. Walsh, S. GarneauTsodikova, A. R. Howard-Jones, Nat. Prod. Rep. 2006, 23, 517-531; c) S. Maiti, S. Biswas, U. Jana, J. Org. Chem., 2010, 75, 1674-1683. 
[113] a) P. A. Szijj, C. Bahou, V. Chudasama, Drug. Discov. Today Technol. 2018, 30, 27-34; b) P. O. Tawney, R. H. Snyder, R. P. Conger, K. A. Leibbrand, C. H. Stiteler, A. R. Williams, J. Org. Chem. 1961, 26, 15-21; c) E. Dolci, V. Froidevaux, C. JolyDuhamel, R. Auvergne, B. Boutevin, S. Caillol, Polym. Rev. 2016, 56, 512-556; d) T. Li, S. Takeoka, Int. J. Nanomedicine 2013, 8, 3855-3866.

[114] a) K. Jin, E. K. Leitsch, X. Chen, W. H. Heath, J. M. Torkelson, Macromolecules 2018, 51, 3620-3631; b) Y. S. Patel, H. S. Patel, Arab. J. Chem. 2017, 10, S1373S1380; c) O. Konuray, X. Fernádez-Francos, S. De la Flor, X. Ramis, À. Serra, Polymers 2020, 12, 1084-1128; d) H. Yamamoto, H. Okamura, K. Matsukawa, A. Matsumoto, J. Photopolym. Sci. Technol. 2014, 27, 151-154.

[115] a) C. La Sann, Nat. Prod. Rep. 2006, 23, 357-367; b) J. J. M. Ravasco, H. Faustino, A. Trindade, P. M. P. Gois, Chem. Eur. J. 2019, 25, 43-59; c) K. Renault, J. W. Fredy, P.-Y. Renard, C. Sabot, Bioconjugate Chem. 2018, 29, 2497-2513.

[116] a) M. Oelgemöller, Chem. Rev. 2016, 116, 9664-9664; b) K.-H. Funken, Nachr. Chem. Tech. Lab. 1992, 40, 793-800; c) A. Kriefer-Liszkay, J. Exp. Bot. 2005, 56, 337346; d) J. Park, D. Feng, S. Yuan, H.-C. Zhou, Angew. Chem. Int. Ed. 2015, 54, $430-$ 445; e) A. Krieger-Liszkay, C. Fufezan, A. Trebst, Photosynth. Res. 2008, 98, 551564; f) M. Trytek, E. Janik, W. Maksymiec, J. Fiedurek, A. Lipke, M. Majdan, J. Photochem. Photobiol. A 2011, 223, 14-24; g) A. Y. Rybkin, A. Y. Belik, O. A. Kraevaya, E. A. Khakina, A. V. Zhilenkov, N. S. Goryachev, D. Volyniuk, J. V. Grazulevicius, P. A. Troshin, A. I. Kotelnikov, Dyes Pigm. 2019, 160, 457-466; h) A. Harriman, B. G. Maiya, T. Murai, G. Hemmi, J. L. Sessler, J. Chem. Soc., Chem. Commun. 1989, 314-316; i) P. Klán, J. Wirz, Photochemistry of Organic Compounds: From Concepts to Practice, Wiley, Chichester, 2009; j) K. Schaffner, Angew. Chem. Int. Ed. 2003, 42, 2932-2933.

[117] a) B. F. Minaev, H. Ågren, Faraday Trans. 1997, 93, 2231-2239; b) K. Krukiewicz, Chemik 2011, 11, 1190-1192.

[118] a) M. DeRosa, Coord. Chem. Rev. 2002, 233, 351-371; b) A. Talla, B. Driessen, N. J. W. Straathof, L.-G. Milroy, L. Brunsveld, V. Hessl, T. Noël, Adv. Synth. Catal. 2015, 357, 2180-2186; c) S. Mazur, C. S. Foote, J. Am. Chem. Soc. 1970, 92, 32253226; d) D. B. Ushakov, K. Gilmore, D. Kopetzki, D. T. McQuade, P. H. Seeberger, Angew. Chem. Int. Ed. Engl. 2014, 53, 557-561; e) G. O. Schenck, Naturwissenschaften 1948, 35, 28-29.

[119] a) M. Rein, W. Adam, Angew. Chem. Int. Ed. 1996, 35, 477-494; b) P. Bayer, R. Pérez-Ruiz, A. J. von Wangelin, ChemPhotoChem 2018, 2, 559-570.

[120] W. Ando, T. Saiki, T. Migita, J. Am. Chem. Soc. 1975, 97, 5028-5029.

[121] a) H. H. Wasserman, J. L. Ives, J. Am. Chem. Soc. 1976, 98, 7868-7869; b) H. H. Wasserman, S. Terao, Tetrahedron Lett. 1975, 16, 1735-1738; c) H. H. Wasserman, J. L. Ives, Tetrahedron 1981, 10, 1825-1852.

[122] W. Fan, P. Li, Angew. Chem. Int. Ed. 2014, 53, 12201-12204.

[123] Q.-Y. Meng, T. Lei, L.-M. Zhao, C.-J. Wu, J.-J. Zhong, X.-W. Gao, C.-H. Tung, L.-Z. Wu, Org. Lett. 2014, 16, 5968-5971.

[124] a) Y. Li, X. Wu, T. B. Lee, E. K. Eleanor, E. J. Parish, A. E. V. Gordon, J. Org. Chem. 2010, 75, 1807-1810; b) J. M. Brunel, C. Loncle, N. Vidal, M. Dherbomeu, Y. Letourneux, Steroids 2005, 70, 907-912. 
[125] P. K. Walia, M. Sharma, M. Kumar, V. Bhalla, RSC Adv. 2019, 9, 36198-36203. [126] a) Y.-P. Lam, Y.-Y. Yeung, Chem. Asian J. 2018, 13, 2369-2372; b) J. F. S. Carvalho, M. M. C. Silva, J. N. Moreira, S. Simoes, M. L. Sa e Melo, J. Med. Chem. 2010, 53, 7632-7638.

[127] L. E. L. Rasmussen, J. Chem. Ecol. 1990, 16, 2167-2181.

[128] S. Annen, T. Zweifel, F. Ricatto, H. Grützmacher, ChemCatChem 2010, 2, 12861295.

[129] a) G. Mereni, J. S. Lind, J. Am. Chem. Soc. 1980, 102, 5830-5835; b) E. Warm, G. G. Laties, Phytochemistry 1982, 21, 827-831.

[130] a) A. K. Sharipov, Russ. J. Appl. Chem. 2003, 76, 108-113; b) Y. Imada, T. Ohno, T. Naota, Tetrahedron Lett. 2007, 48, 937-939; c) A. F. Choban, I. R. Yurchuk, A. S. Lyavinets, Russ. J. Gen. Chem. 2008, 78, 2071-2074.

[131] J. Schachtner, P. Bayer, A. J. von Wangelin, Beilstein J. Org. Chem. 2016, 12, 1798-1811.

[132] W. Schilling, D. Riemer, Y. Zhang, N. Hatami, S. Das, ACS Catal. 2018, 8, 54255430.

[133] Y. Zhang, W. Schilling, D. Riemer, S. Das, Nat. Protoc. 2020, 15, 822-839.

[134] S. Rekulapally, R. Jarapula, K. Gangarapu, S. Manda, J. R. Vaidya, Med. Chem. Res. 2015, 24, 3412-3422.

[135] K. L. Vine, J. M. Locke, M. Ranson, S. G. Pyne, J. B. Bremmer, Bioorg. Med. Chem. 2007, 15, 931-938.

[136] B. Suresh, G. Brahmeshwary, T. Swamy, I. Gopi, V. Ravindar, Russ. J. Gen. Chem. 2016, 96, 1144-1150.

[137] N. Karali, Ö. Güzel, N. Özsoy, N. Özbey, A. Salman, J. Med. Chem. 2010, 45, 1068-1077.

[138] Y.-L. Zhang, Y.-F. Li, J.-W. Wang, B. Yu, Y.-K. Shi, H.-M. Liu, Steroids 2016, 109, $22-28$.

[139] W. Schilling, Y. Zhang, D. Riemer, S. Das, Chem. Eur. J. 2020, 26, 390-395.

[140] V. T. Kamble, N. S. Joshi, S. T. Atkore, J. Iran. Chem. Soc. 2011, 8, 616-621.

[141] W. Schilling, Y. Zhang, P. K. Sahoo, S. K. Sarkar, S. Gandhi, H. W. Roesky, S. Das, Green Chem. 2021, 23, 379-387.

[142] R. J. Abraham, M. Mobli, R. J. Smith. Magn. Reson. Chem. 2003, 41, 26-36.

[143] J. O. Bauer, G. Leitus, Y. Ben-David, D. Milstein, ACS Catal. 2016, 6, 84158419.

[144] P. Xiao, Z. Tang, K. Wang, H. Chen, Q. Guo, Y. Chu, L. Gao, Z. Song, J. Org. Chem. 2018, 83, 1687-1700.

[145] P. D. Giorgi, N. Elizarov, S. Antoniotti, ChemCatChem, 2017, 9, 1830-1836.

[146] S. Gu, J. Du, J. Huang, Y. Guo, L. Yang, W. Xu, W. Chen, Dalton Trans. 2017, 46, 586-594.

[147] L. Li, R. Matsuda, I. Tanaka, H. Sato, P. Kanoo, H. J. Jeon, M. L. Foo, A. Wakamiya, Y. Murata, S. Kitagawa, J. Am. Chem. Soc. 2014, 136, 7543-7546. 
[148] J. Katla, H. Bhat, P. C. Jha, D. S. Ghalsasi, S. Kanvah, Chemistry Select 2017, 2, $1902-1910$.

[149] G. Pelletier, W. S. Bachara, A. B. Charette, J. Am. Chem. Soc. 2010, 132, 1281712819.

[150] N. Duarte, M.-J. U. Ferreura, Org. Lett. 2007, 9, 489-492.

[151] P. V. Podea, M. I. Tosa, C. Paizs, F. D. Irimie, Tetrahedron 2008, 19, 500-511.

[152] S. Velusamy, M. Ahamed, T. Punniyamurthy, Org, Lett. 2004, 6, 4821-4824.

[153] P. Gautam, M. Dhiman, V. Polshettiwar, B. M. Bhanage, Green Chem. 2016, 18, 5890-5899.

[154] K. J. Jeon, J. H. Jun, J. S. Yu, C. K. Lee, J. Heterocyclic Chem. 2003, 40, 763771.

[155] M. Cai, J. Peng, W. Hao, G. Ding, Green Chem. 2011, 13, 190-196.

[156] M. Baranac-Stojanovic, R. Marković, M. Stojanović, Tetrahedron 2011, 67, 8000 8008.

[157] Y. Yuan, X. Shi, W. Liu, Synlett. 2011, 559-564.

[158] B. Liu, F. Jin, T. Wang, X. Yuan, W. Han, Angew. Chem. Int. Ed. 2017, 56, $12712-12717$.

[159] S.-I. Inaba, R. D. Rieke, J. Org. Chem. 1985, 1373-1381.

[160] A. Goel, V. Kumar, S. Chaurasia, M. Rawat, R. Prasad, R. S. Anand, J. Org. Chem. 2010, 75, 3656-3662.

[161] B. Xin, Y. Zahng, K. Cheng, J. Org. Chem. 2006, 71, 5725-5731.

[162] M. M. Hossain, S.-G. Shyu, Tetrahedron 2016, 72, 4252-4257.

[163] C. Metallinos, J. Zaifman, L. Van Belle, L. Dodge, M. Pilkington, Organometallics 2009, 28, 4534-4543.

[164] F. M. Piller, P. Appukkuttan, A. Gavryushin, M. Helm, P. Knochel, Angew. Chem. Int. Ed. 2008, 47, 6802-6806.

[165] K. Tanaka, S. Matsui, A. Kaji, Bull. Chem. Soc. Jpn. 1980, 53, 3619-3622.

[166] C. Peppe, E. Schulz Lang, G. N. Ledesma, L. Borges de Castro, O. Soares do Rego Barros, P. de Azevedo Mello, Synlett. 2005, 20, 3091-3094.

[167] J.-L. Wang, L.-N. He, C.-X. Miao, Y.-N. Li, Green Chem. 2009, 11, 1317-1320.

[168] K. Mitsudo, T. Kaide, E. Nakamoto, K. Yoshida, H. Tanaka, J. Am. Chem. Soc. 2007, 129, 2246-2247.

[169] A. A. Ponaras, O. Zaim, Y. Pazo, L. Ohannesian, J. Org. Chem. 1988, 53, 11101112.

[170] J. Sun, Y. Dong, L. Cao, X. Wang, Y. Hu, J. Org. Chem. 2014, 69, 8932-8934.

[171] G. E. Hawkes, K. Herwig, J. D. Roberts, J. Org. Chem. 1974, 39, 1017-1028.

[172] A. Le Pera, A. Leggio, C. Siciliano, M. L. Di Dioia, A. Napoli, G. Sindona, A. Liguori, Steroids 2003, 68, 139-142. 
[173] M.-K. Wong, N.-W. Chung, L. He, X.-C. Wang, T. Yan, Y. C. Tang, D. Yang, J. Org. Chem. 2003, 68, 6321-6328.

[174] S. M. Huber, A. Henning, F. G. Pühlhofer, R. Weiss, J. Heterocyclic Chem. 2009, 46, $421-427$.

[175] B. C. G. Soederberg, S. P. Gorugantula, C. R. Howerton, J. L. Petersen, S. W. Dantale, Tetrahedron, 2009, 65, 7357-7363.

[176] H. A. Radhy, G. F. Fadhil, A. Perjessy, E. Kolehmainen, W. M. F. Fabian, M. Samalikova, K. Laihia, Z. Sustekova, Heterocycl. Commun. 2001, 7, 387-392.

[177] P. J. Montoya-Pelaez, Y.-S. Uh, C. Lata, M. P. Thompson, R. P. Lemieux, C. M. Crudden, J. Org. Chem. 2006, 71, 7921-5929.

[178] M. E. Matheus, F. D. A. Violante, S. J. Garden, A. C. Pinto, P. D. Fernandes, Eur. J. Pharmacol. 2007, 556, 200-206.

[179] W.-B. Chen, X.-L. Du, L.-F. Cun, X.-M. Zhang, W.-C. Yuan, Tetrahedron 2010, $66,1441-1446$.

[180] F. Ablondi, S. Gordon, J. II. Morton, J. H. Williams, J. Org. Chem. 1952, 17, 149156.

[181] E. Ziegler, R. Wolf, T. Kappe, Monatsh. Chem. 1965, 96, 418-422.

[182] J. S. Yadav, B. V. Subba Reddy, C. Suresh Reddy, A. D. Krishna, Synthesis 2007, 5, 693-696.

[183] L. A. McAllister, R. A. McCormick, K. M. James, S. Brand, N. Willetts, D. J. Procter, Chem. Eur. J. 2007, 13, 1032-1046.

[184] Q. Gui, F. Dai, J. Liu, P. Chen, Z. Yang, X. Chen, Z. Tan, Org. Biomol. Chem. 2014, 12, 3349-3353.

[185] P. Diaz, J. Xu, F. Astruc-Diaz, H.-M. Pan, D. L. Brown, M. Naguib, J. Med. Chem. 2008, 51, 4932-4947.

[186] M. Akkurt, S. Tuerktekin, A. A. Jarrahpour, D. Khalili, O. Buyukgungor, Acta Cryst. Section E 2006, 62, 1575-1577.

[187] H. Wittmann, F. Guenzl, Z. Naturforsch. B, 1978, 33B, 1540-1546.

[188] A. Verma, S. Kumar, Org. Lett. 2016, 18, 4388-4391.

[189] M. R. Mutra, G. K. Dhandabani, J.-J. Wang, Adv. Synth. Catal. 2018, 360, 39603968.

[190] L. Zhang, X. Zheng, J. Chen, K. Cheng, L. Jin, X. Jiang, C. Yu, Org. Chem. Front. 2018, 5, 2115-2119.

[191] X. Ji, D. Li, Z. Wang, M. Tan, H. Huang, G.-J. Deng, Eur. J. Org. Chem. 2017, 45, 6652-6659.

[192] M. Broering, F. Bregier, C. Kleeberg, Acta Cryst. Section C 2007, 63, 225-227.

[193] Z. Han, P. Li, Z. Zhang, C. Chen, Q. Wang, X.-Q. Dong, X. Zhang, ACS Catal. 2016, 6, 6214-6218.

[194] H. S. Lee, J. S. Yu, C. K. Lee, Magn. Reson. Chem. 2009, 47, 711-715.

[195] N. Matuszak, G. G. Muccioli, G. Labar, D. M. Lambert, J. Med. Chem. 2009, 52, 7410-7420. 
[196] J. Trujillo-Ferrara, R. Santillan, H. I. Beltran, N. Farfan, H. Hopfl, Mag. Reson. Chem. 1999, 9, 682-686.

[197] M. Abass, A. S. Mayas, J. Chem. Res. 2009, 2, 93-94.

[198] M. Pandey, D. S. Raghuvanshi, K. N. J. Singh, Heterocyclic Chem. 2009, 46, 49-53.

[199] N. Karali, O. Guzel, N. Ozsoy, S. Ozbey, A. Salman, Eur. J. Med. Chem. 2010, 45, 1068-1077.

[200] K. Rad-Moghadam, M. Sharifi-Kiasaraie, H. Taheri-Amlashi, Tetrahedron 2010, 13, 2316-2321.

[201] Y.-L. Zhang, Y.-F. Li, J.-W. Wang, B. Yu, Y.-K. Shi, Liu, H.-M. Steroids 2016, $109,22-28$.

[202] V. T. Kamble, N. S. Joshi, S. T. Atkore, J. Iran. Chem. Soc. 2011, 8, 616-621.

[203] W. Chen, Y. Wang, X. Mi, S. Luo, Org. Lett. 2019, 21, 8178-8182.

[204] S. A. Patil, P. A. Medina, D. Gonzalez-Flores, J. K. Vohs, S. Dever, L. W. Pineda, M. L. Montero, B. D. Fahlmann, Syn. Commun. 2013, 43, 2349-2364.

[205] C. K. Z. Andrade, A. d. F. S. Barreto, W. A. Silva, ARKIVOC 2008, 12, 226-232. [206] L. Wu et al. CN104163768, 2014.

[207] D. R. Moore, M. Cheng, E. B. Lobkovsky, G. F. Coates, J. Am. Chem. Soc. 2003, $125,11911-11924$.

[208] J. Vincente, M. T. Chicote, A. J. Martinez-Martinez, P. G. Jones, D. Bautista, Organometallics 2008, 27, 3254-3271.

[209] D.-H. Lee, J.-Y. Jung, I.-M. M.-J. Jin, Eur. J. Org. Chem. 2008, 2, 356-360.

[210] R. K. Vohra, J.-L. Renaud, C. Bruneau, Synthesis 2007, 5, 731-738.

[211] M. Takani, T. Takeda, T. Yajima, O. Yamauchi, Inorg. Chem. 2006, 45, 59385946.

[212] G. Bartoli, M. Bosco, M. Locatelli, E. Marcantoni, P. Melchiorre, L. Sambri, Synlett 2004, 2, 239-242. 


\section{APPENDIX}

\subsection{ADDITIONAL DATA FROM COAUTHORS OF PUBLICATIONS}

Supplementary Figure 1: TEM image of synthesized graphitic carbon nitride.

SEM of synthesized of PCN. (left) unused catalyst; (right) recycled catalyst. (Das et al.)
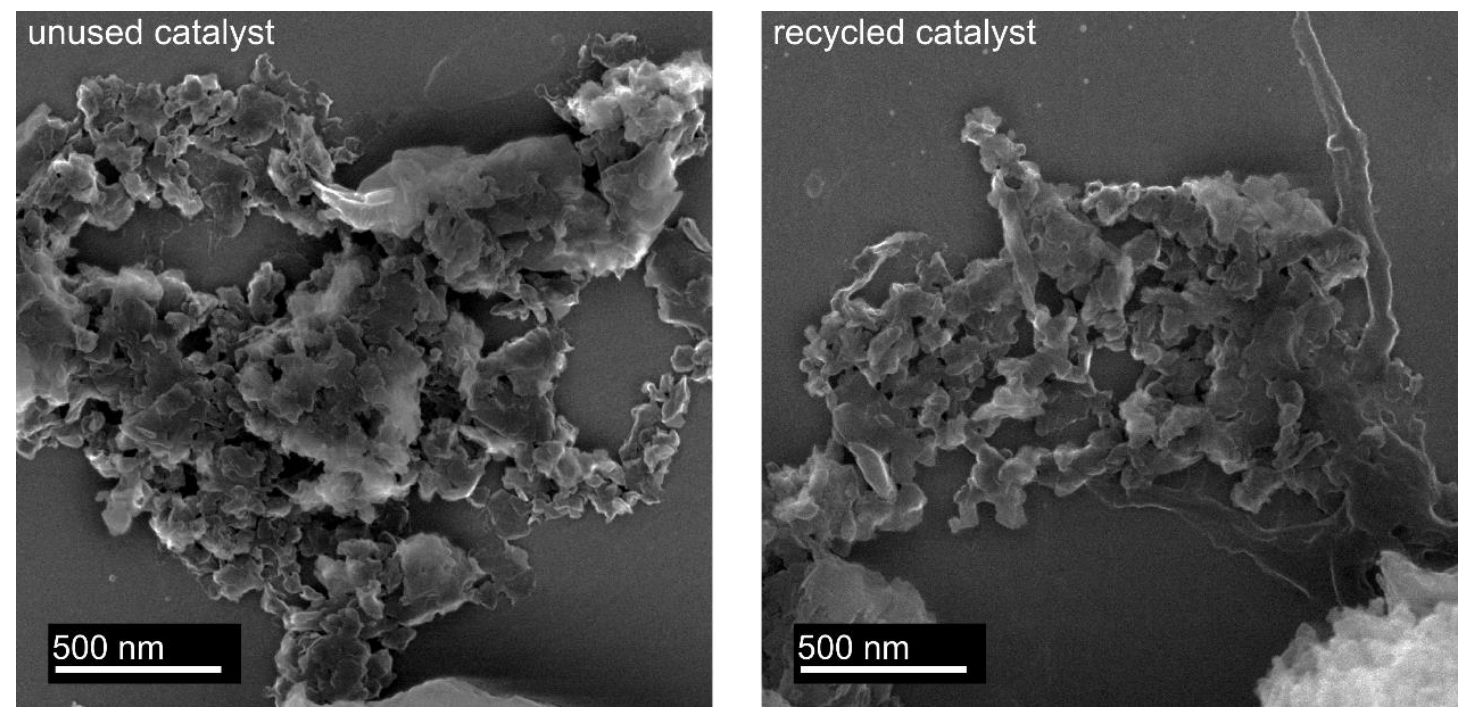

Table S1: Element analysis of fresh and reused catalyst have no difference.

\begin{tabular}{|l|l|l|l|}
\hline Name & $\mathrm{C}(\%)$ & $\mathrm{H}(\%)$ & $\mathrm{N}(\%)$ \\
\hline New catalyst & 35.18 & 2.00 & 62.00 \\
\hline Reused catalyst & 35.26 & 2.01 & 61.36 \\
\hline
\end{tabular}


Supplementary Figure 2 XRD analysis of synthesized graphitic carbon nitiride.

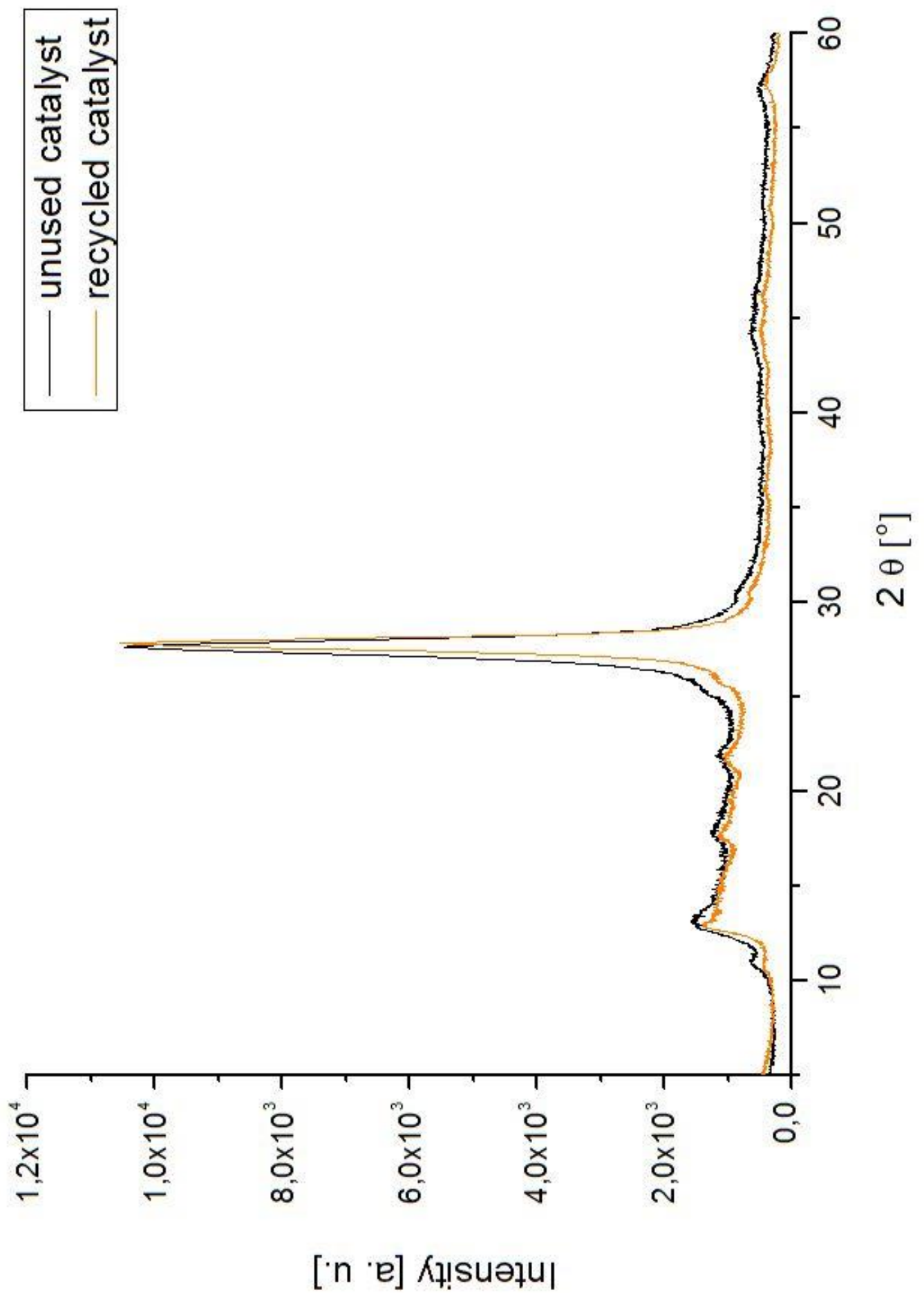


Supplementary Figure 3 HR-TEM, IR, etc. of synthesized graphitic carbon nitiride.
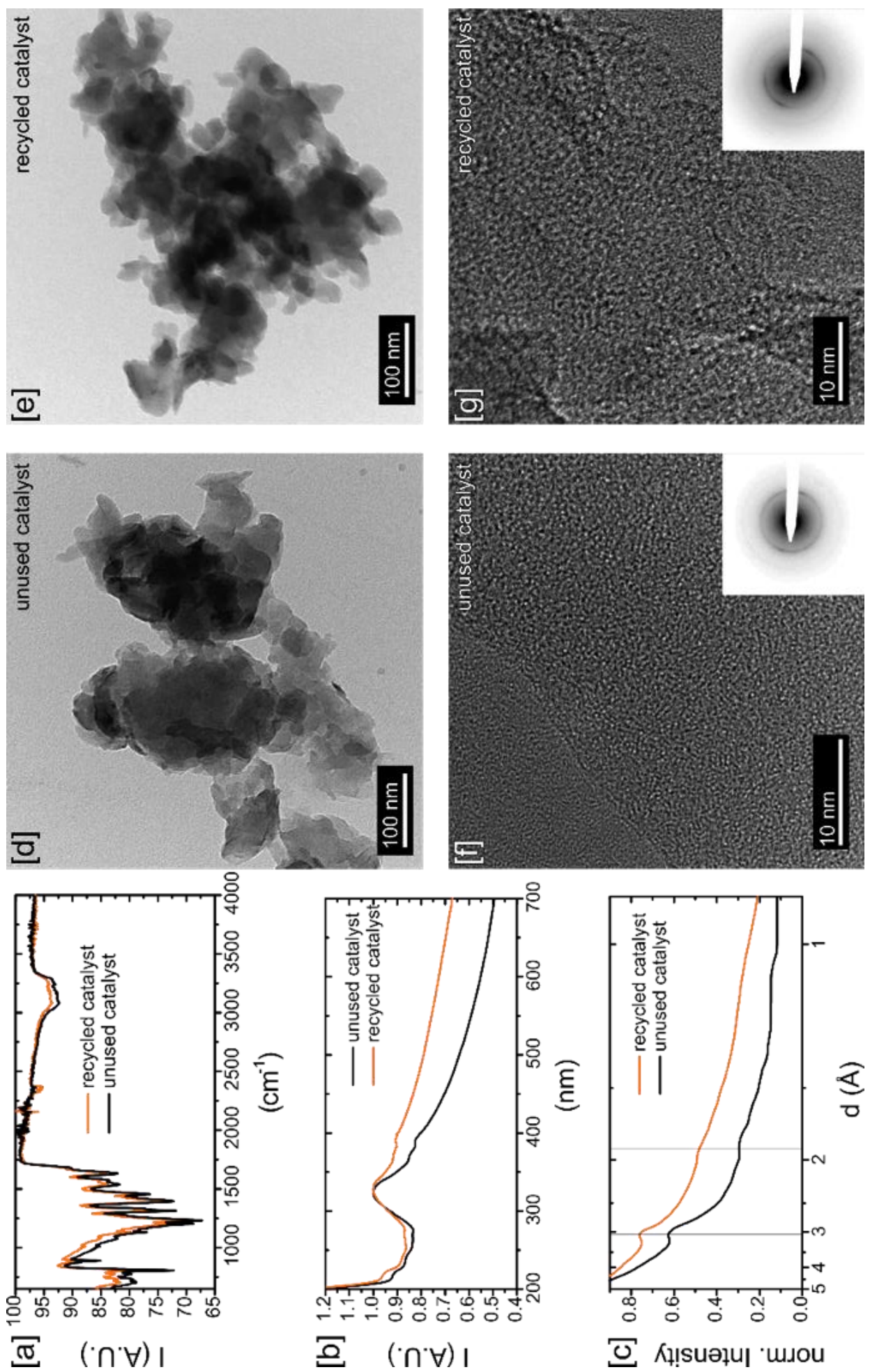


\section{Associated information of DFT calculations}

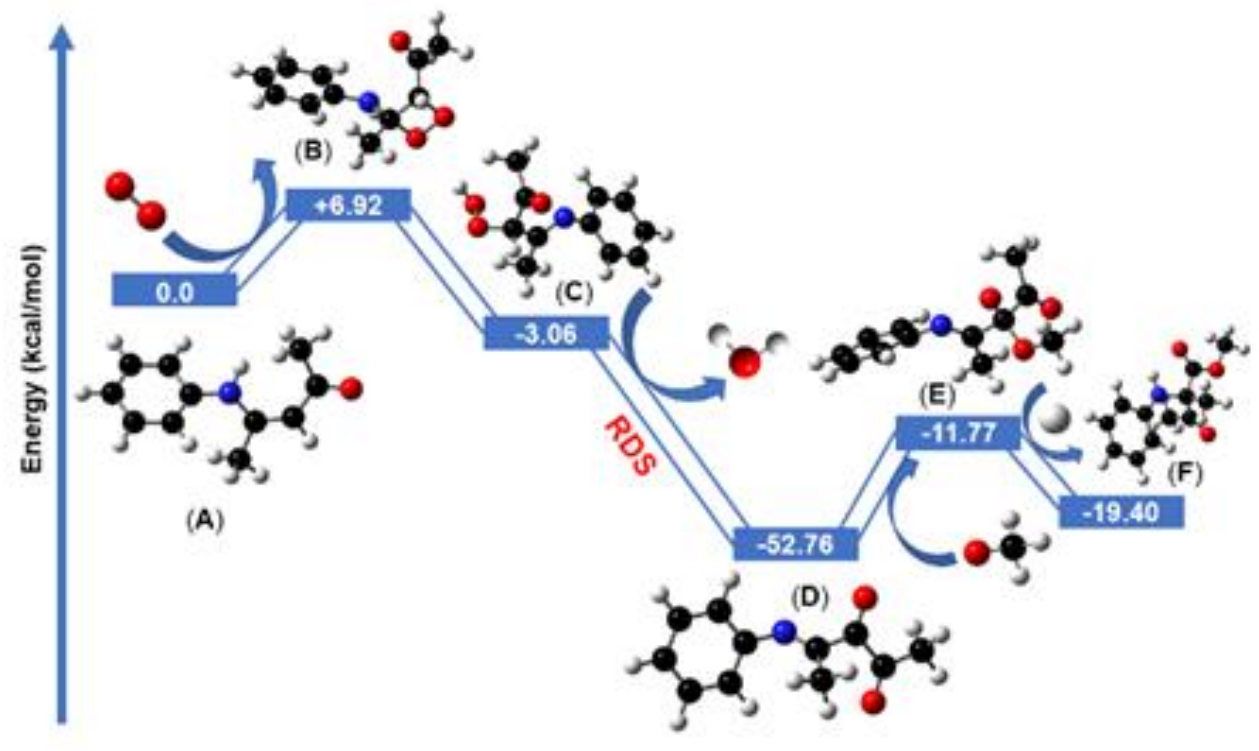

Fig. S4: The hybrid UB3LYP ${ }^{1}$ functional has been used in all calculations as incorporated in Gaussian 09 package $^{2}$, mixing the exact Hartree-Fock-type exchange with Becke's exchange functional ${ }^{3}$ and that proposed by Lee-Yang-Parr for the correlation contribution. ${ }^{4}$ The $6-311++g(d, p)$ basis set was used for all the atoms which provides reasonably high quality results in moderate timescales. All ground state geometry optimizations were followed by subsequent frequency test to ascertain stationary points.

For $\mathbf{A}$

Zero-point correction $=$

Thermal correction to Energy=

Thermal correction to Enthalpy=

Thermal correction to Gibbs Free Energy=

Sum of electronic and zero-point Energies=

Sum of electronic and thermal Energies=

Sum of electronic and thermal Enthalpies=

Sum of electronic and thermal Free Energies=

C

C

C

C

C

C

$\mathrm{H}$

$\mathrm{H}$

$\mathrm{H}$

$\mathrm{H}$

$\mathrm{H}$

$\mathrm{N}$ $\begin{array}{lll}-1.98077 & 0.57226 & -1.46055\end{array}$

$\begin{array}{llll}-0.98768 & 1.26957 & -2.16159\end{array}$

$\begin{array}{lll}-0.82824 & 2.64797 & -1.9654\end{array}$

$\begin{array}{lll}-1.6619 & 3.32908 & -1.06816\end{array}$

$\begin{array}{lll}-2.65499 & 2.63178 & -0.36712\end{array}$

$\begin{array}{llll}-2.81443 & 1.25337 & -0.56331\end{array}$

$\begin{array}{lll}-2.10251 & -0.48018 & -1.61035\end{array}$

$\begin{array}{lll}-0.35117 & 0.74953 & -2.84666\end{array}$

$\begin{array}{lll}-0.07 & 3.18038 & -2.50066\end{array}$

$\begin{array}{lll}-3.2915 & 3.15182 & 0.31795\end{array}$

$\begin{array}{llll}-3.57267 & 0.72096 & -0.02805\end{array}$

$\begin{array}{llll}-1.49466 & 4.77496 & -0.86236\end{array}$ 


$\begin{array}{llll}\mathrm{H} & -0.5365 & 5.02329 & -1.00473 \\ \mathrm{C} & -1.89275 & 5.12399 & 0.50899 \\ \mathrm{C} & -1.30586 & 6.17027 & 1.13941 \\ \mathrm{H} & -1.65875 & 7.15592 & 0.91841 \\ \mathrm{C} & 0.07279 & 6.02469 & 1.81004 \\ \mathrm{O} & 0.15199 & 5.84412 & 3.0529 \\ \mathrm{C} & 1.35451 & 6.10008 & 0.95969 \\ \mathrm{H} & 2.11668 & 5.49847 & 1.40922 \\ \mathrm{H} & 1.15008 & 5.73859 & -0.02643 \\ \mathrm{H} & 1.68733 & 7.11555 & 0.90544 \\ \mathrm{C} & -2.97673 & 4.30069 & 1.22925 \\ \mathrm{H} & -3.50109 & 4.92788 & 1.9196 \\ \mathrm{H} & -3.66478 & 3.90951 & 0.50921 \\ \mathrm{H} & -2.51746 & 3.49265 & 1.75939\end{array}$

For B

Zero-point correction=

Thermal correction to Energy=

0.224857 (Hartree/Particle)

Thermal correction to Enthalpy=

0.239236

Thermal correction to Gibbs Free Energy=

0.240180

Sum of electronic and zero-point Energies=

0.182573

Sum of electronic and thermal Energies=

$-707.066345$

$-707.051966$

Sum of electronic and thermal Enthalpies=

$-707.051022$

Sum of electronic and thermal Free Energies=

$-707.108629$
C
$\begin{array}{lll}-0.97795 & -1.25805 & 0.75447\end{array}$
$\begin{array}{llll}\text { C } & 0.23014 & -0.7735 & 0.24599\end{array}$
$\begin{array}{llll}\text { C } & 0.40866 & 0.60046 & 0.04418\end{array}$
$\begin{array}{llll}\text { C } & -0.63222 & 1.49227 & 0.33333\end{array}$
$\begin{array}{llll}\text { C } & -1.8431 & 1.00645 & 0.84259\end{array}$
$\begin{array}{llll}\text { C } & -2.01334 & -0.3677 & 1.0568\end{array}$
$\begin{array}{llll}\mathrm{H} & -1.10947 & -2.30888 & 0.91327\end{array}$
$\mathrm{H} \quad 1.02118-1.452750 .01121$
$\begin{array}{llll}\mathrm{H} & -0.50331 & 2.54086 & 0.16728\end{array}$
$\begin{array}{llll}\mathrm{H} & -2.63913 & 1.68404 & 1.07008\end{array}$
$\begin{array}{llll}\mathrm{H} & -2.93361 & -0.7358 & 1.45135\end{array}$
$\begin{array}{llll}\mathrm{N} & 1.69705 & 1.08745 & -0.46797\end{array}$
$\mathrm{H} \quad 2.11144 \quad 0.38045-1.04133$
$\begin{array}{llll}\text { C } & 2.58501 & 1.38244 & 0.66866\end{array}$
$\begin{array}{llll}\mathrm{C} & 2.31154 & 2.51507 & 1.47761\end{array}$
$\begin{array}{llll}\mathrm{H} & 1.5871 & 2.42687 & 2.26183\end{array}$
$\begin{array}{llll}\text { C } & 2.65646 & 0.07296 & 1.47841\end{array}$
$\begin{array}{llll}\mathrm{H} & 1.67064 & -0.19913 & 1.79635\end{array}$
$\begin{array}{llll}\mathrm{H} & 3.06188 & -0.70704 & 0.86633\end{array}$
$\begin{array}{llll}\mathrm{H} & 3.28485 & 0.21519 & 2.33674\end{array}$
$\begin{array}{llll}\mathrm{O} & 3.88396 & 1.92692 & 0.46277\end{array}$
$\begin{array}{llll}O & 3.71042 & 2.53532 & 1.76649\end{array}$

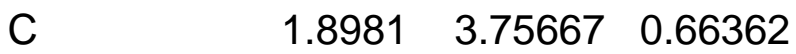
$\begin{array}{lllll}C & 2.97306 & 4.57344 & -0.07518\end{array}$
$\begin{array}{lllll}\mathrm{H} & 2.54233 & 5.02912 & -0.94327\end{array}$ 


$\begin{array}{llrr}\mathrm{H} & 3.35488 & 5.33306 & 0.57533 \\ \mathrm{H} & 3.77067 & 3.92368 & -0.37371 \\ \mathrm{O} & 0.68728 & 4.10225 & 0.60115\end{array}$

For $\mathbf{C}$

Zero-point correction=

Thermal correction to Energy=

0.222947 (Hartree/Particle)

Thermal correction to Enthalpy=

0.238509

Thermal correction to Gibbs Free Energy=

Sum of electronic and zero-point Energies=

0.239453

Sum of electronic and thermal Energies $=$

0.178293

$-707.073147$

$-707.057585$

Sum of electronic and thermal Enthalpies=

$-707.056641$

Sum of electronic and thermal Free Energies=

$-707.117802$

$\begin{array}{llll}\mathrm{C} & -2.27555 & 0.58264 & -1.76506 \\ \mathrm{C} & -1.3239 & 1.30906 & -2.49348 \\ \mathrm{C} & -0.95671 & 2.59617 & -2.07824 \\ \mathrm{C} & -1.54116 & 3.15687 & -0.93458 \\ \mathrm{C} & -2.49281 & 2.43045 & -0.20616 \\ \mathrm{C} & -2.86001 & 1.14333 & -0.6214 \\ \mathrm{H} & -2.55592 & -0.40011 & -2.0821 \\ \mathrm{H} & -0.87766 & 0.88095 & -3.36669 \\ \mathrm{H} & -0.2301 & 3.15081 & -2.63441 \\ \mathrm{H} & -2.93906 & 2.85855 & 0.66705 \\ \mathrm{H} & -3.58662 & 0.58869 & -0.06524 \\ \mathrm{~N} & -1.15599 & 4.50699 & -0.49902 \\ \mathrm{C} & -1.6955 & 5.02456 & 0.55666 \\ \mathrm{C} & -1.29199 & 6.43897 & 1.01296 \\ \mathrm{C} & -0.09962 & 6.34478 & 1.98301 \\ \mathrm{O} & -0.30213 & 6.23189 & 3.21987 \\ \mathrm{C} & 1.34058 & 6.38874 & 1.43942 \\ \mathrm{H} & 1.9814 & 5.82054 & 2.08082 \\ \mathrm{H} & 1.36266 & 5.97274 & 0.45384 \\ \mathrm{H} & 1.67834 & 7.40348 & 1.4059 \\ \mathrm{C} & -2.74126 & 4.22629 & 1.35713 \\ \mathrm{H} & -3.42917 & 4.90301 & 1.81945 \\ \mathrm{H} & -3.27277 & 3.57285 & 0.69727 \\ \mathrm{H} & -2.24846 & 3.64838 & 2.11083 \\ \mathrm{H} & -2.11893 & 6.90342 & 1.5083 \\ \mathrm{O} & -0.91934 & 7.21911 & -0.12607 \\ \mathrm{O} & 0.10081 & 6.64615 & -0.73714 \\ \mathrm{H} & 0.84411 & 6.58743 & -0.13244\end{array}$

For D

Zero-point correction=

Thermal correction to Energy=

0.196407 (Hartree/Particle)

Thermal correction to Enthalpy=

0.210218

Thermal correction to Gibbs Free Energy=

Sum of electronic and zero-point Energies=

0.211162

Sum of electronic and thermal Energies=

0.153292

$-630.764034$

$-630.750223$ 
Sum of electronic and thermal Enthalpies=

Sum of electronic and thermal Free Energies=

$-630.749278$

C

$\begin{array}{lll}-3.26223 & 0.60257 & -1.80557\end{array}$

$\begin{array}{llll}\text { C } & -2.55545 & 1.22542 & -2.84308\end{array}$

$\begin{array}{llll}\text { C } & -1.77512 & 2.35878 & -2.57756\end{array}$

$\begin{array}{llll}\text { C } & -1.70157 & 2.86929 & -1.27452\end{array}$

$\begin{array}{llll}C & -2.40835 & 2.24643 & -0.23701\end{array}$

$\begin{array}{lllll}\text { C } & -3.18868 & 1.11307 & -0.50253\end{array}$

$\mathrm{H} \quad-3.85803-0.26278-2.0083$

$\mathrm{H} \quad \begin{array}{llll}\mathrm{H} & -2.61161 & 0.83564 & -3.83798\end{array}$

$\mathrm{H} \quad-1.23548 \quad 2.83435-3.36972$

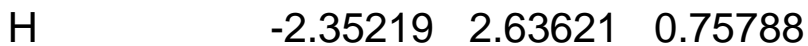

$\begin{array}{llll}H & -3.72832 & 0.6375 & 0.28963\end{array}$

$\begin{array}{llll}\mathrm{N} & -0.88304 & 4.05813 & -0.99601\end{array}$

$\begin{array}{llll}\text { C } & -0.81516 & 4.52936 & 0.20679\end{array}$

$\begin{array}{llll}\text { C } & 0.04234 & 5.77482 & 0.49857\end{array}$

$\begin{array}{llll}C & 0.12316 & 6.33582 & 1.93048\end{array}$

$\begin{array}{llll}\text { O } & -0.5115 & 5.77652 & 2.86212\end{array}$

$\begin{array}{llll}\text { C } & 0.98066 & 7.58127 & 2.22226\end{array}$

$\begin{array}{llll}\mathrm{H} & 1.34911 & 7.53353 & 3.22569\end{array}$

$\begin{array}{llll}\mathrm{H} & 1.80495 & 7.6144 & 1.54083\end{array}$

$\begin{array}{llll}\mathrm{H} & 0.38371 & 8.46123 & 2.10299\end{array}$

$\begin{array}{llll}\text { C } & -1.59183 & 3.8449 & 1.34692\end{array}$

$\begin{array}{llll}\mathrm{H} & -1.86216 & 4.57381 & 2.08212\end{array}$

$\begin{array}{llll}\mathrm{H} & -2.47682 & 3.39046 & 0.95299\end{array}$

$\begin{array}{llll}\mathrm{H} & -0.97617 & 3.09487 & 1.79781\end{array}$

$\begin{array}{llll}O & 0.677 & 6.33412 & -0.43307\end{array}$

For $\mathbf{E}$

Zero-point correction $=$

Thermal correction to Energy=

0.252734 (Hartree/Particle)

Thermal correction to Enthalpy=

0.269208

Thermal correction to Gibbs Free Energy=

0.270152

Sum of electronic and zero-point Energies=

0.207444

$-746.450429$

$-746.433956$

$-746.433012$

Sum of electronic and thermal Enthalpies=

$-746.495720$
Sum
$-2.29845$
$0.70765-1.93927$
C
$\begin{array}{lll}-1.26919 & 1.42373 & -2.56519\end{array}$
C
$\begin{array}{llll}-0.86893 & 2.66594 & -2.05467\end{array}$
C
$\begin{array}{llll}-1.49792 & 3.19207 & -0.91824\end{array}$
C
$\begin{array}{llll}-2.52718 & 2.47598 & -0.29232\end{array}$
$\begin{array}{llll}\text { C } & -2.92744 & 1.23377 & -0.80284\end{array}$
$\mathrm{H} \quad-2.60406-0.24081-2.32906$
$\begin{array}{llll}H & -0.78894 & 1.02203 & -3.43289\end{array}$
$\begin{array}{llll}H & -0.08307 & 3.21269 & -2.53258\end{array}$
$\begin{array}{llll}H & -3.00743 & 2.87769 & 0.57537\end{array}$
$\begin{array}{lllll}\mathrm{H} & & -3.7133 & 0.68702 & -0.32494\end{array}$
$\begin{array}{llll}\mathrm{N} & -1.07806 & 4.49508 & -0.38273\end{array}$

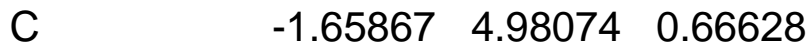




$\begin{array}{lcll}\mathrm{C} & -1.21882 & 6.3458 & 1.22729 \\ \mathrm{C} & -0.09638 & 6.13838 & 2.26108 \\ \mathrm{O} & -0.38231 & 5.96835 & 3.47471 \\ \mathrm{C} & 1.37597 & 6.13905 & 1.80965 \\ \mathrm{H} & 1.94717 & 5.50486 & 2.45497 \\ \mathrm{H} & 1.44111 & 5.77704 & 0.80485 \\ \mathrm{H} & 1.76262 & 7.13569 & 1.85546 \\ \mathrm{C} & -2.78973 & 4.19383 & 1.3541 \\ \mathrm{H} & -3.47301 & 4.87819 & 1.81201 \\ \mathrm{H} & -3.30836 & 3.60524 & 0.62644 \\ \mathrm{H} & -2.37368 & 3.55132 & 2.10175 \\ \mathrm{O} & -0.73807 & 7.16725 & 0.16006 \\ \mathrm{O} & -2.3334 & 6.98451 & 1.85552 \\ \mathrm{C} & -1.92496 & 8.25206 & 2.37646 \\ \mathrm{H} & -1.56524 & 8.86672 & 1.5779 \\ \mathrm{H} & -2.75895 & 8.72998 & 2.84654 \\ \mathrm{H} & -1.14509 & 8.10795 & 3.09474 \\ \mathrm{H} & 0.01018 & 6.73847 & -0.26169\end{array}$

For $\mathbf{F}$

Zero-point correction $=$

Thermal correction to Energy=

0.254362 (Hartree/Particle)

Thermal correction to Enthalpy=

0.270800

Thermal correction to Gibbs Free Energy=

Sum of electronic and zero-point Energies=

0.271744

Sum of electronic and thermal Energies=

Sum of electronic and thermal Enthalpies=

0.209869

$-746.480894$

$-746.464456$

$-746.463512$

Sum of electronic and thermal Free Energies=

$-746.525387$
C
$\begin{array}{lll}-2.46076 & 0.46647 & -1.89045\end{array}$
$-2.15603 \quad 1.35397-2.93131$

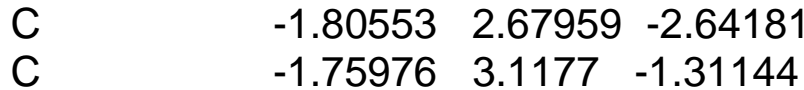
C $\quad-2.06449 \quad 2.2302 \quad-0.27057$
C $\quad-2.414990 .90458-0.56008$
$\mathrm{H} \quad-2.72837-0.54567 \quad-2.11149$
$\mathrm{H} \quad-2.19098 \quad 1.01946-3.94708$
$\mathrm{H} \quad-1.57287 \quad 3.35722 \quad-3.43653$
$\begin{array}{llll}\mathrm{H} & -2.02954 & 2.56471 & 0.7452\end{array}$
$\begin{array}{llll}\mathrm{H} & -2.64765 & 0.22695 & 0.23465\end{array}$
N $\quad \begin{array}{llll}-1.39211 & 4.50821 & -1.00776\end{array}$

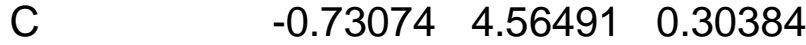
$\begin{array}{llll}\text { C } & -0.34558 & 6.02164 & 0.62197\end{array}$
$\begin{array}{llll}\mathrm{C} & 0.68058 & 7.50271 & 2.10739\end{array}$
$\begin{array}{llll}\mathrm{H} & 0.71553 & 7.83722 & 3.12315\end{array}$
$\begin{array}{llll}\mathrm{H} & 1.63973 & 7.1225 & 1.82389\end{array}$
$\begin{array}{llll}\mathrm{H} & 0.41937 & 8.32213 & 1.47082\end{array}$
$\begin{array}{llll}\text { C } & -1.69189 & 4.03992 & 1.38651\end{array}$

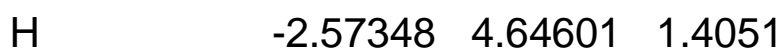
$\begin{array}{llll}\mathrm{H} & -1.9595 & 3.02778 & 1.16547\end{array}$

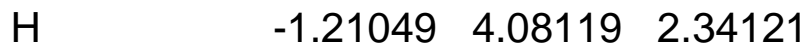
$\begin{array}{lllll}\mathrm{O} & -0.07195 & 6.81858 & -0.31268\end{array}$ 


\begin{tabular}{|c|c|c|c|}
\hline 0 & -0.29887 & 6.46869 & 1.97949 \\
\hline $\mathrm{H}$ & -0.76799 & 4.84912 & -1.7108 \\
\hline C & 0.53809 & 3.69259 & 0.27708 \\
\hline 0 & 1.52366 & 3.99321 & 0.99947 \\
\hline C & 0.6008 & 2.45239 & -0.63372 \\
\hline $\mathrm{H}$ & 1.61293 & 2.28333 & -0.9369 \\
\hline $\mathrm{H}$ & 0.24138 & 1.59835 & -0.09861 \\
\hline $\mathrm{H}$ & -0.00834 & 2.61377 & -1.49848 \\
\hline
\end{tabular}




\section{DFT calculations for the photocatalytic behavior of chlorophyll and Intrinsic}

\section{Reaction Coordinate (IRC) calculations}

DFT calculations were carried out with the Gaussian09 program package. Ground state geometry optimization and frequency calculations were performed at the gradient-corrected DFT level using B3LYP correlation functional sets and LANL2DZ sub sets. The excited state geometries were optimized using Time dependent -DFT (TDDFT) using aforementioned functional basis sets. Intrinsic reaction coordinate (IRC) calculations were carried out to confirm that the transition state structures connect the reactants and the respective products.

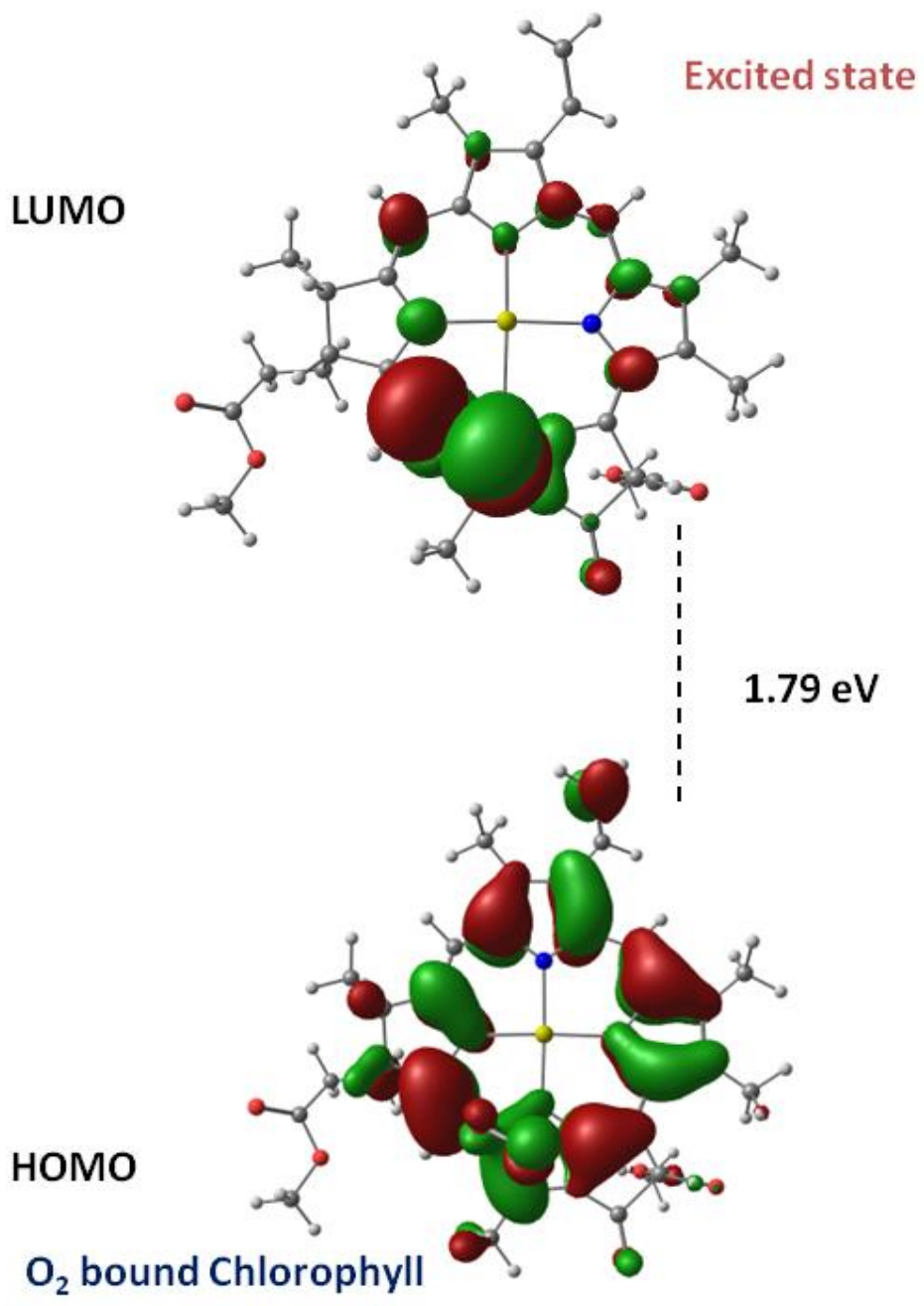

Fig S5: Frontier molecular orbitals of $\mathrm{O}_{2}$ bound chlorophyll-A in Excited state. 


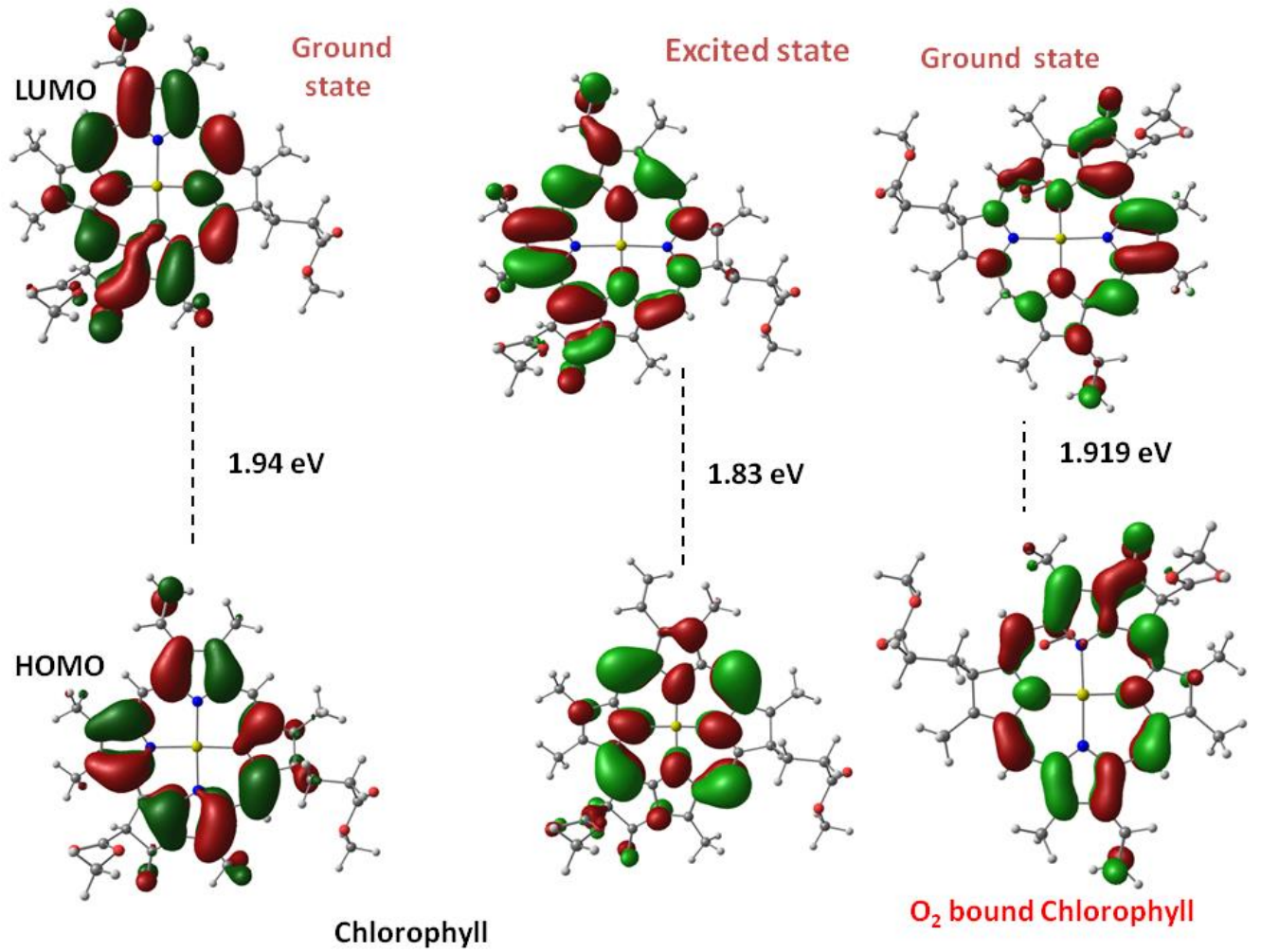

Fig S6: Frontier molecular orbitals of chlorophyll and $\mathrm{O}_{2}$ bound chlorophyll in ground state and Excited state. 
Structure of Transition state $-\mathrm{I}$ :

Structure of Transition state-II:

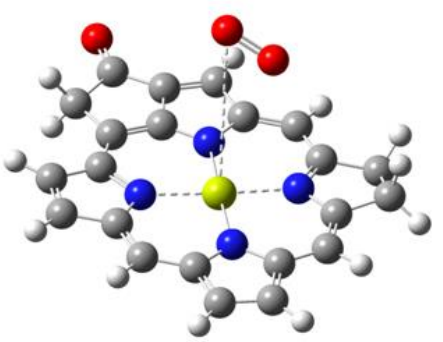

Molecular Oxygen bound

Structure of Transition state-III:
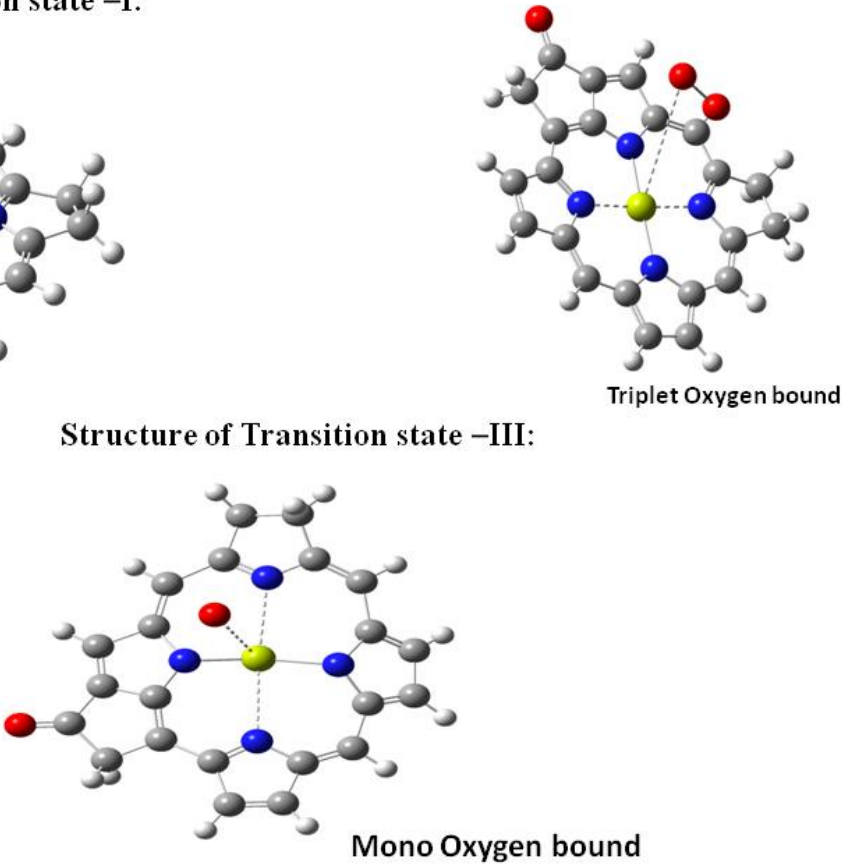

Fig S7: Structure of the transition states for the conversion of $\mathrm{O}_{2}$ into singlet oxygen. 


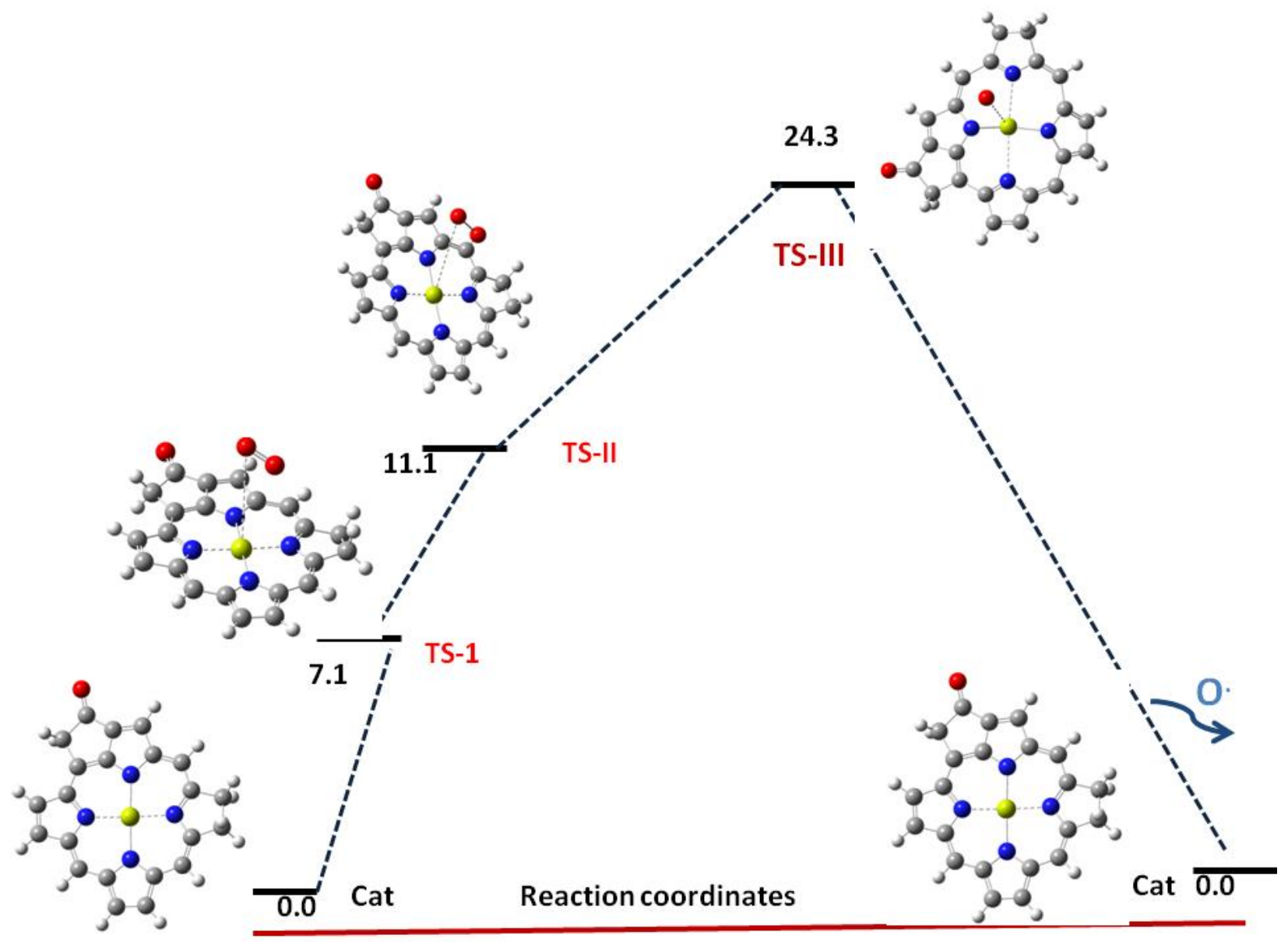

Fig S8: Molecular oxygen conversion into singlet oxygen mechanism by chlorophyll calculated using B3LYP level. Relative free energies are given in $\mathrm{kcal} / \mathrm{mol}$. 


\subsection{NMR SPECTRA OF PRODUCTS}

$1 \mathrm{~b}$
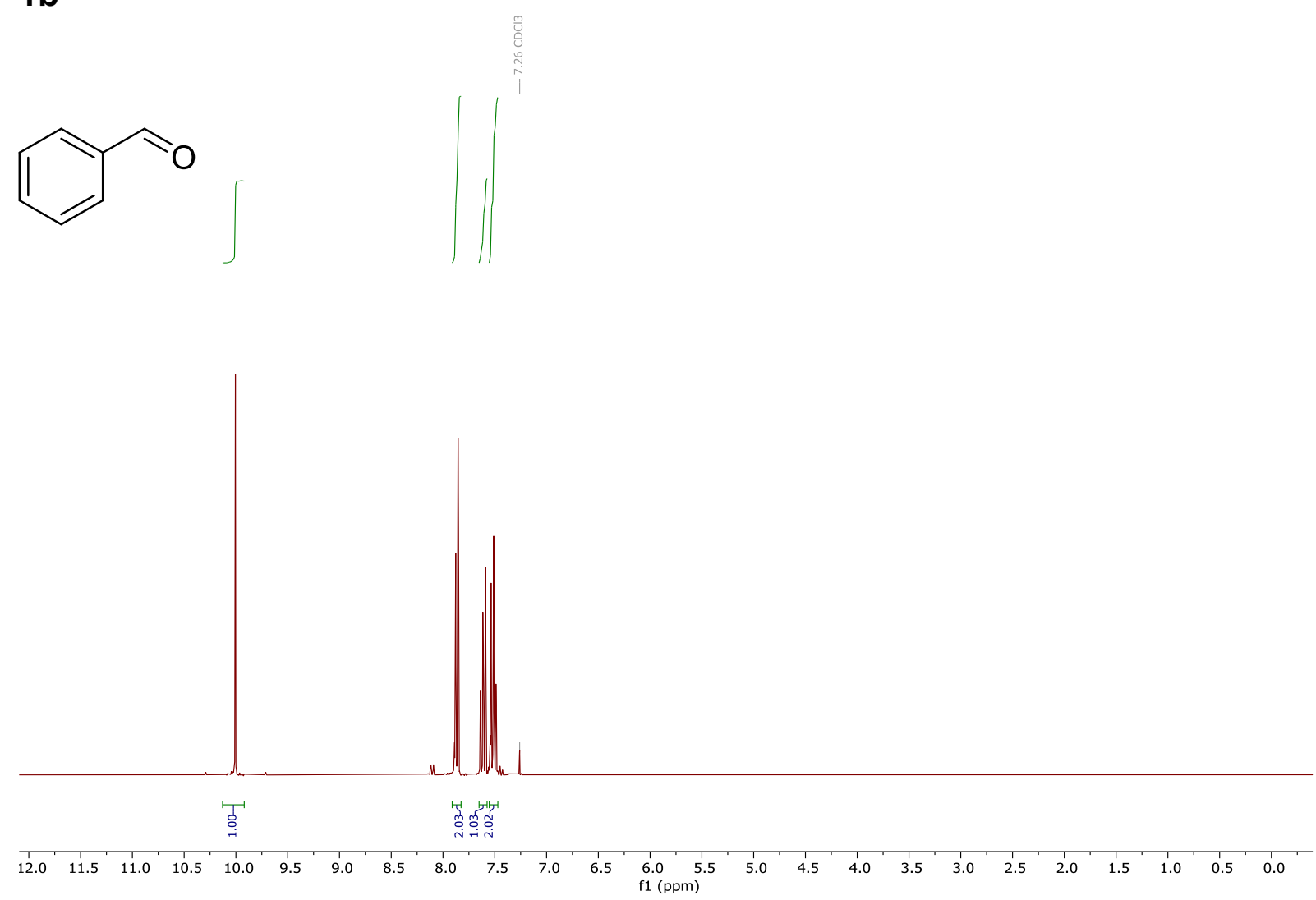

${ }^{1} \mathrm{H}$ NMR spectrum in $\mathrm{CDCl}_{3}$.

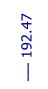

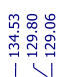<smiles>O=Cc1ccccc1</smiles>

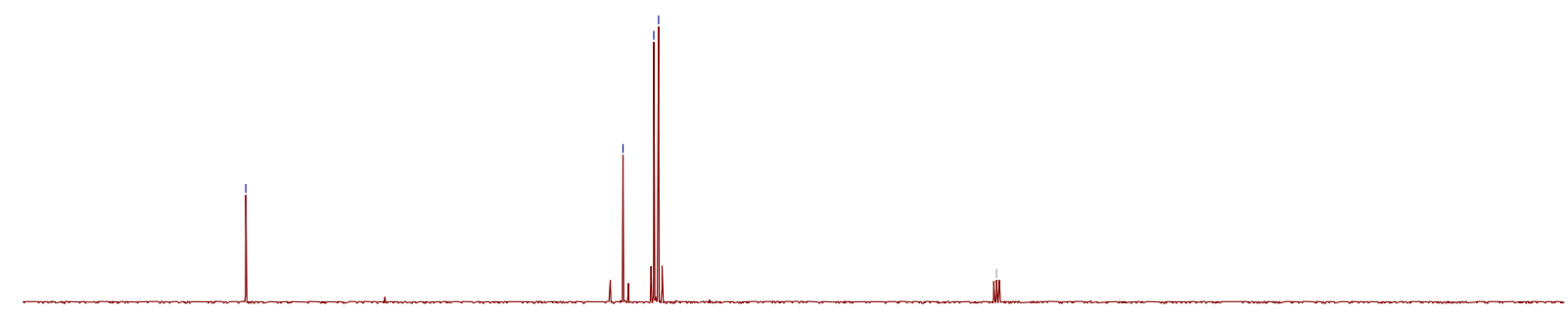

$\begin{array}{llllllll}220 & 210 & 200 & 190 & 180 & 170 & 160 & 150\end{array}$

${ }^{13} \mathrm{C}$ NMR spectrum in $\mathrm{CDCl}_{3}$. 
$2 b$

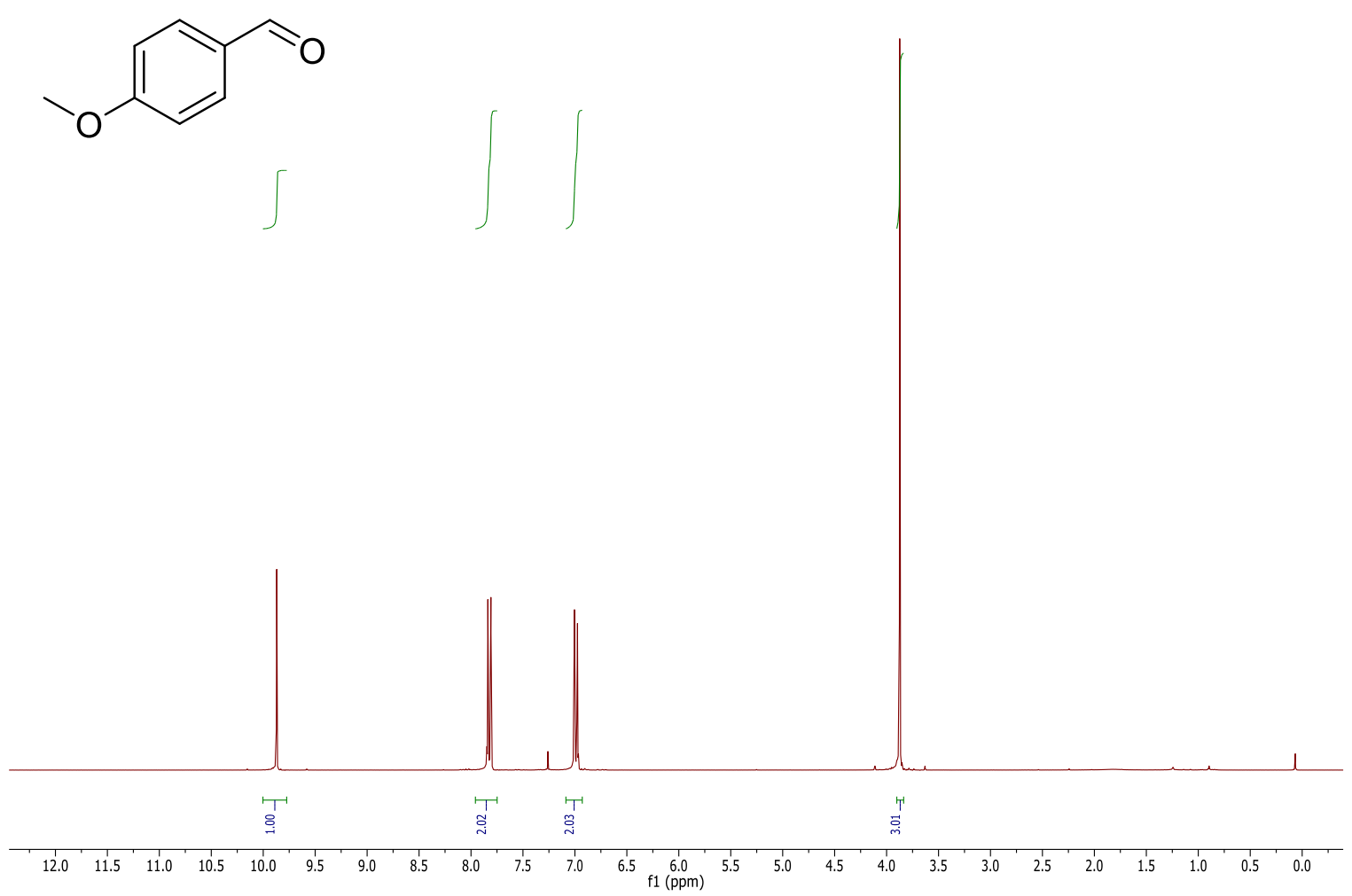

${ }^{1} \mathrm{H}$ NMR spectrum in $\mathrm{CDCl}_{3}$.

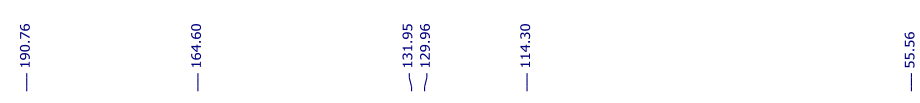<smiles>COc1ccc(C=O)cc1</smiles>

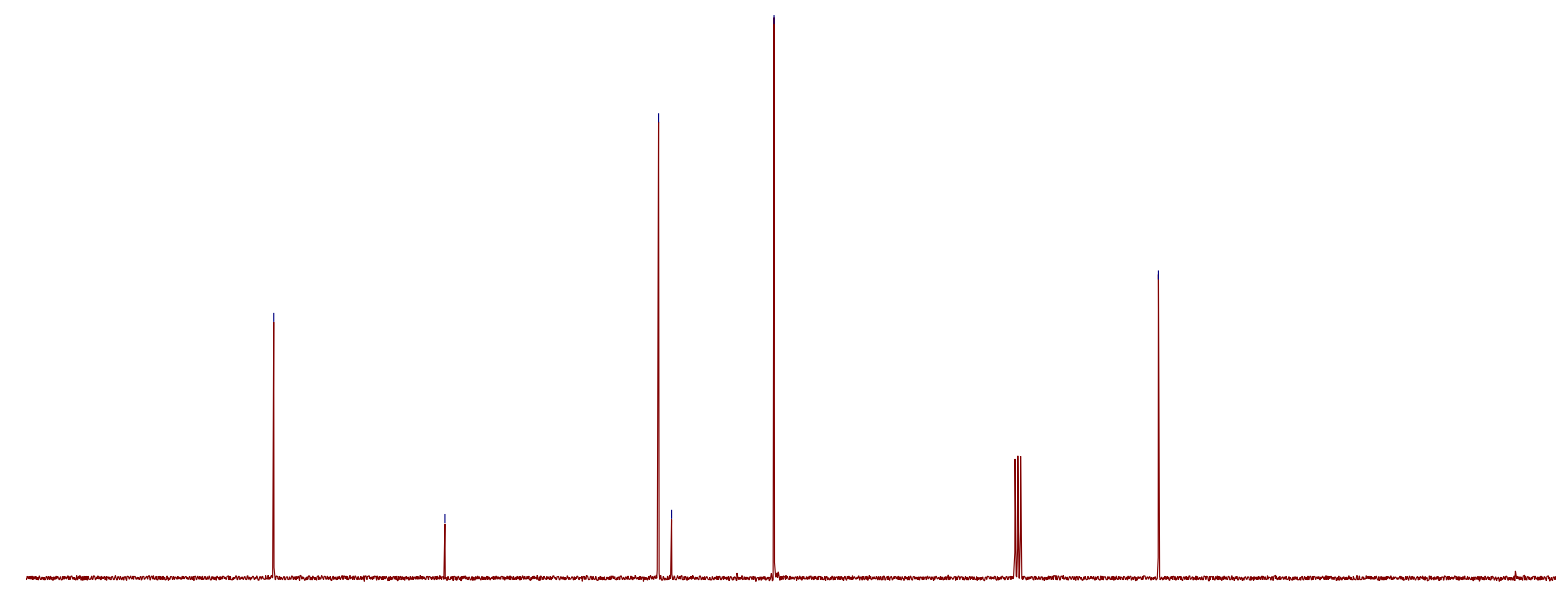

$\begin{array}{llllllllllllllllllllllllll}220 & 210 & 200 & 190 & 180 & 170 & 160 & 150 & 140 & 130 & 120 & 110 & 100 & 90 & 80 & 70 & 60 & 50 & 40 & 30 & 20 & 10 & 0\end{array}$ ${ }^{13} \mathrm{C}$ NMR spectrum in $\mathrm{CDCl}_{3}$. 


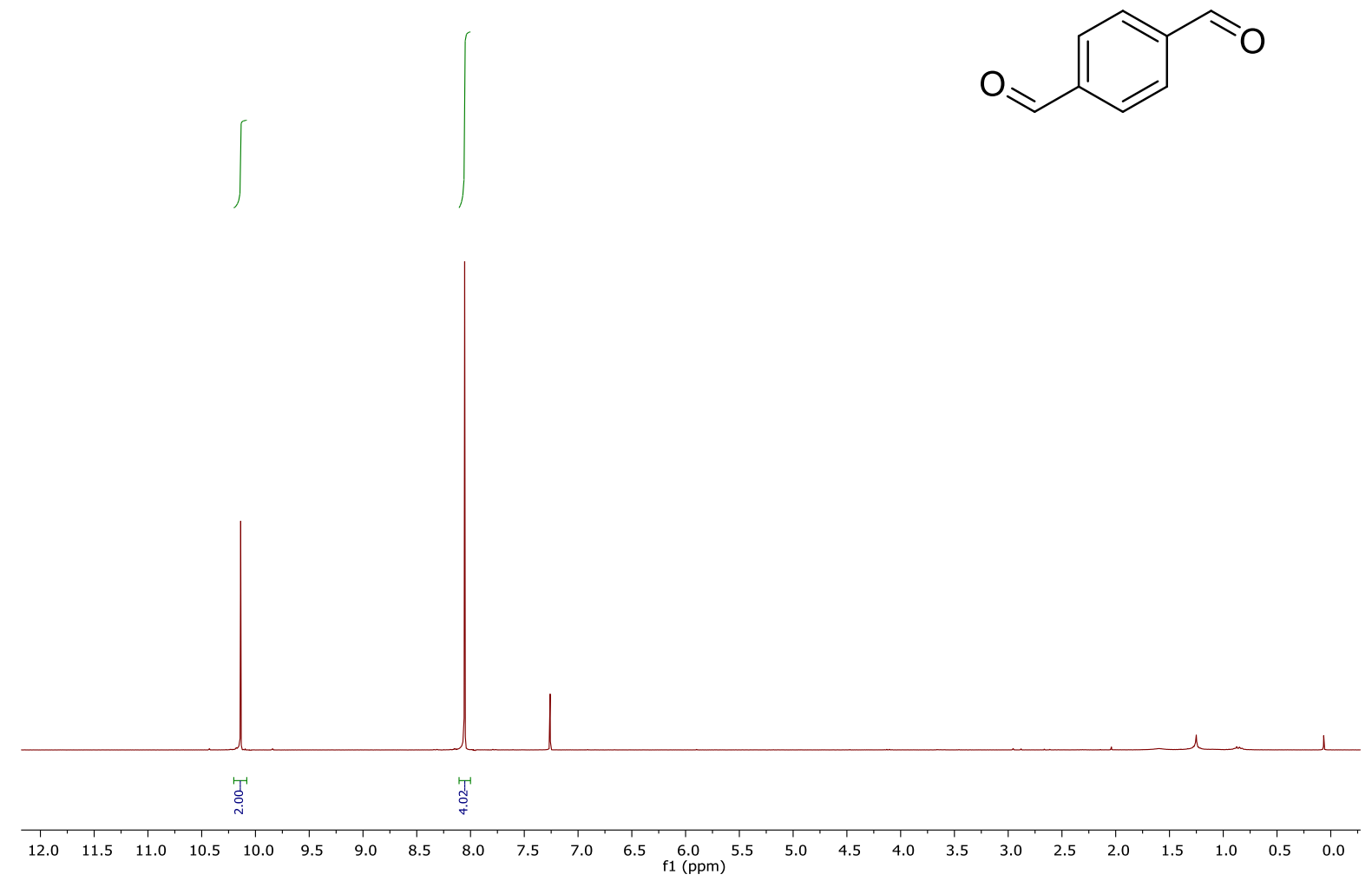

${ }^{1} \mathrm{H}$ NMR spectrum in $\mathrm{CDCl}_{3}$.

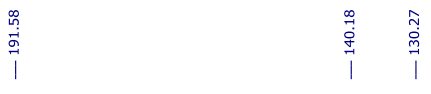<smiles>O=Cc1ccc(C=O)cc1</smiles>

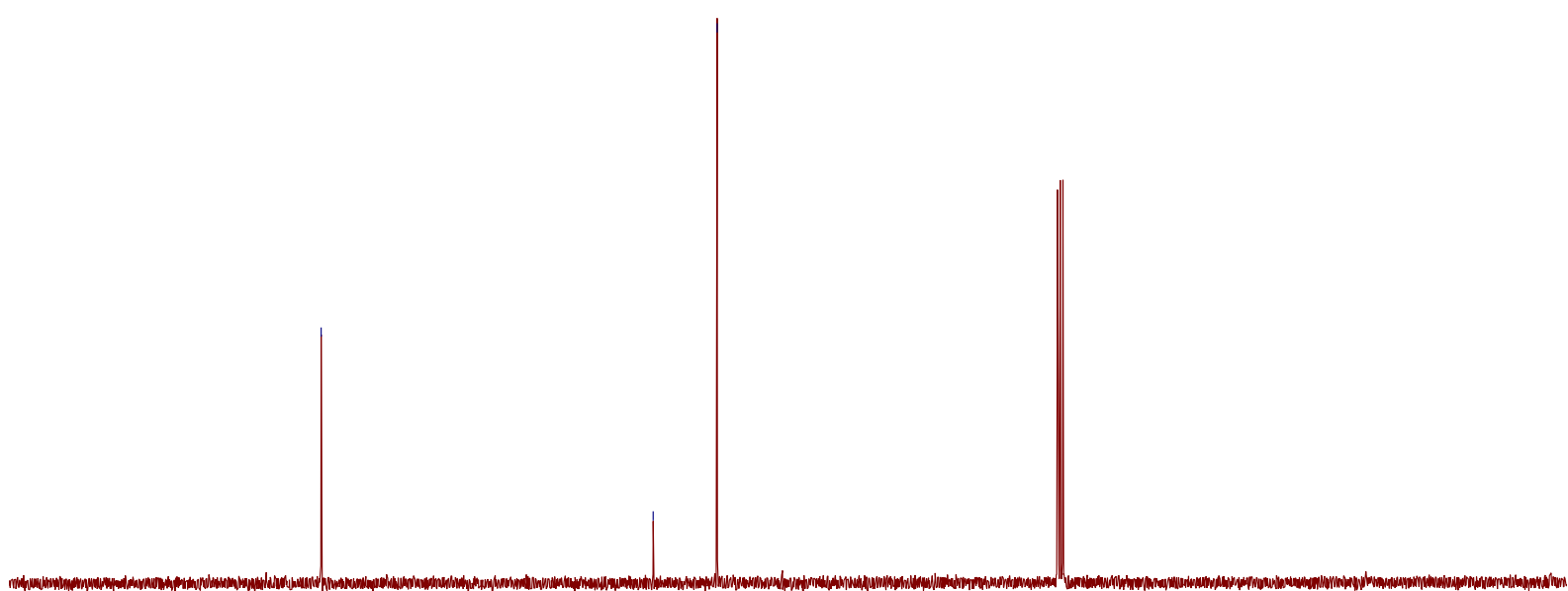

$\begin{array}{llllllllllllllllllllllll}230 & 220 & 210 & 200 & 190 & 180 & 170 & 160 & 150 & 140 & 130 & \begin{array}{l}120 \\ \mathrm{f} 1(\mathrm{ppm})\end{array} & 110 & 100 & 90 & 80 & 70 & 60 & 50 & 40 & 30 & 20 & 10 & 0\end{array}$ ${ }^{13} \mathrm{C}$ NMR spectrum in $\mathrm{CDCl}_{3}$. 


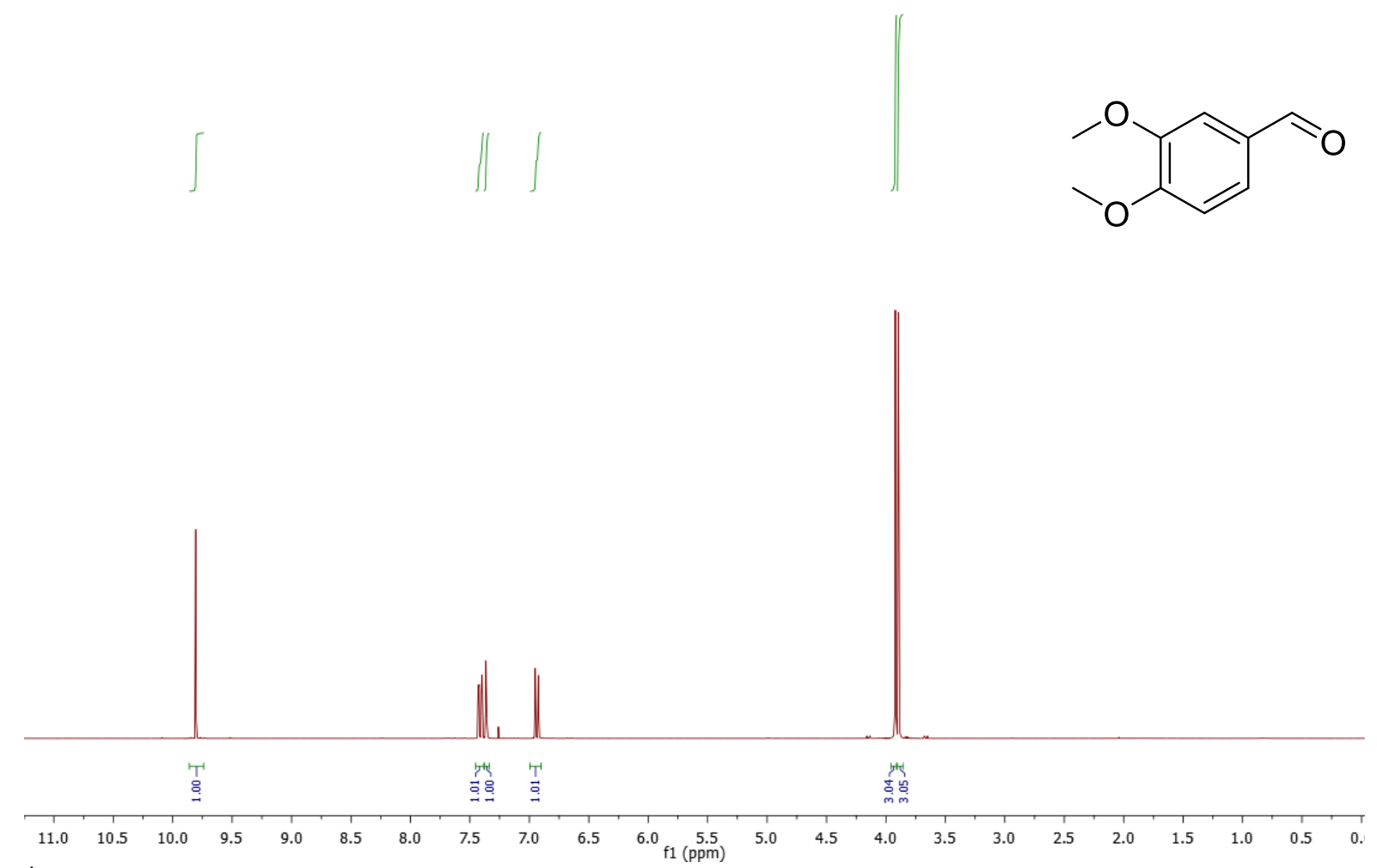

${ }^{1} \mathrm{H}$ NMR spectrum in $\mathrm{CDCl}_{3}$.

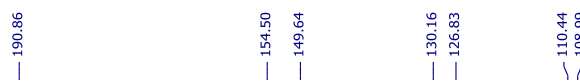

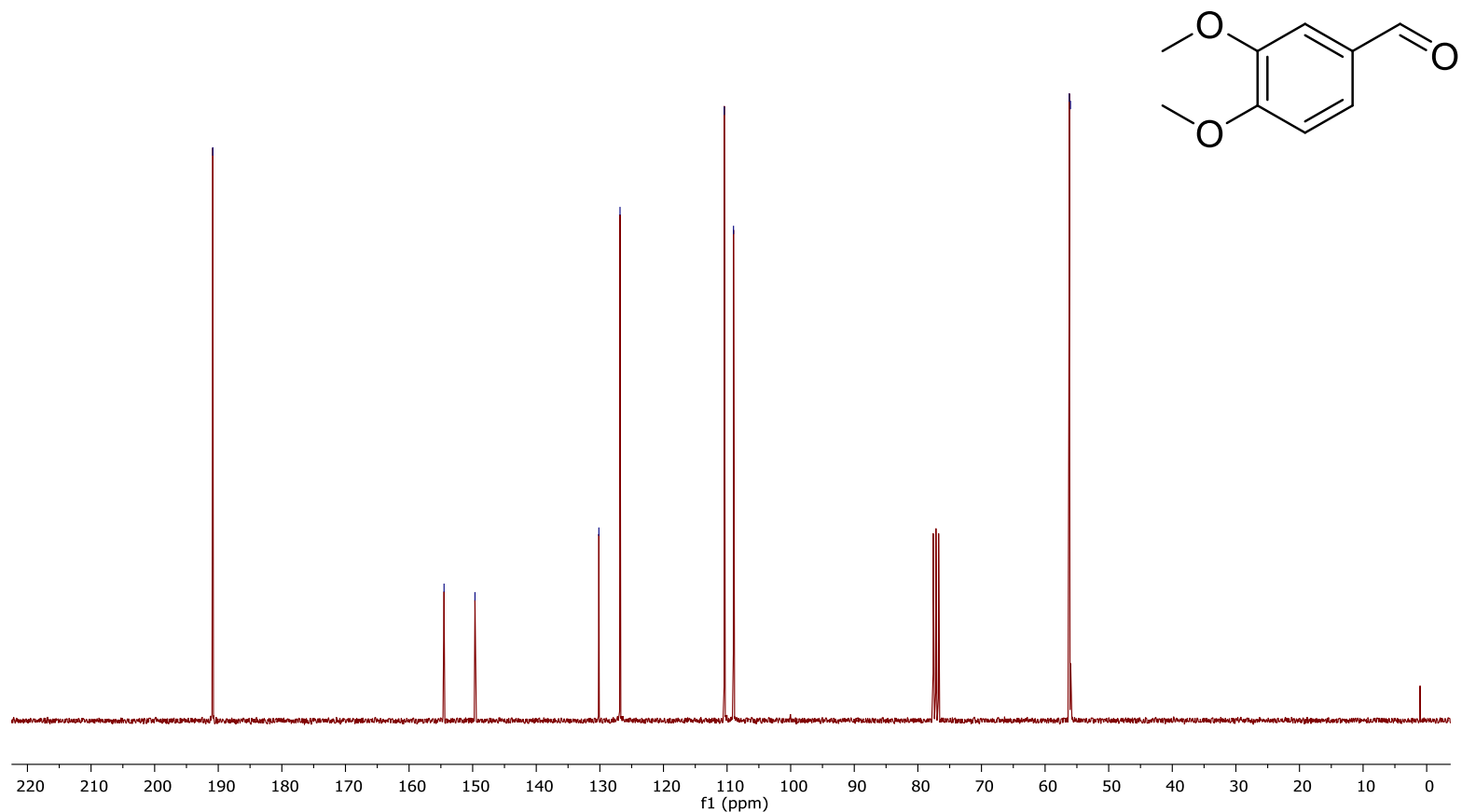

${ }^{13} \mathrm{C}$ NMR spectrum in $\mathrm{CDCl}_{3}$. 
$5 b$

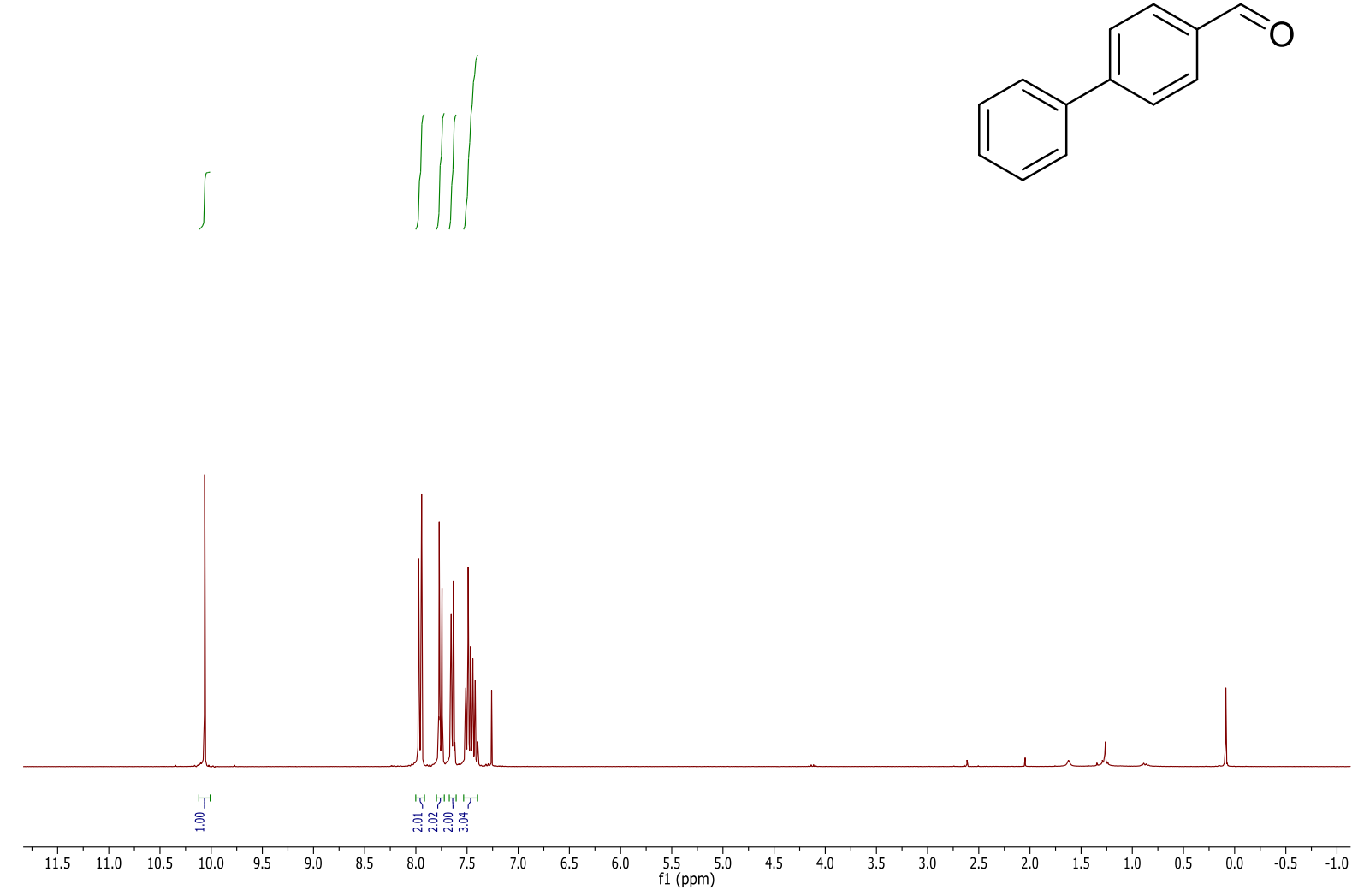

${ }^{1} \mathrm{H}$ NMR spectrum in $\mathrm{CDCl}_{3}$.

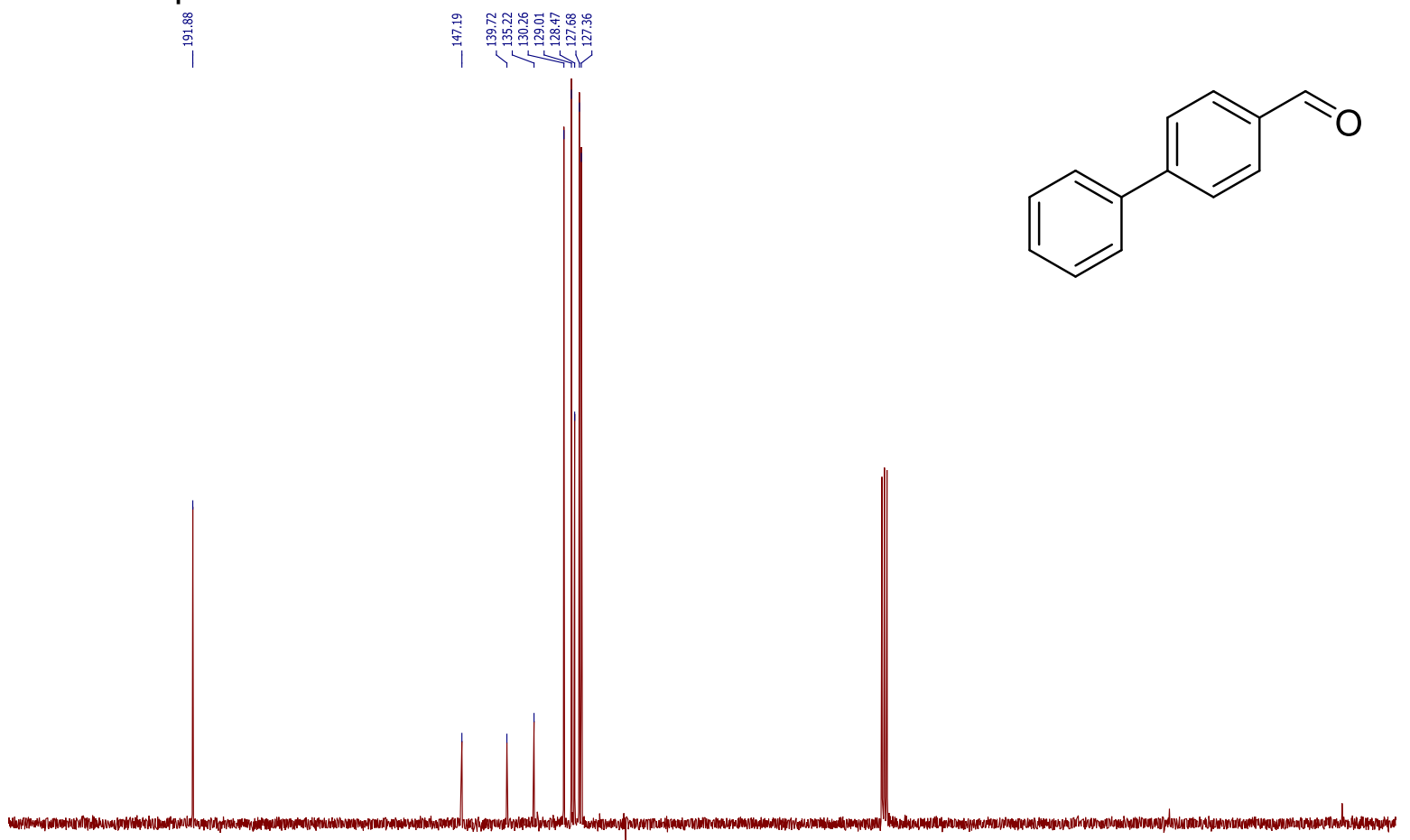

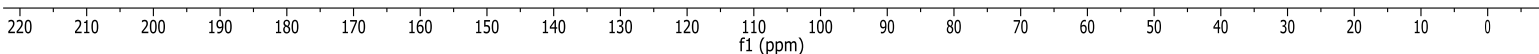
${ }^{13} \mathrm{C}$ NMR spectrum in $\mathrm{CDCl}_{3}$. 
$6 b$<smiles>COc1cc(C=O)cc(OC)c1OC</smiles>
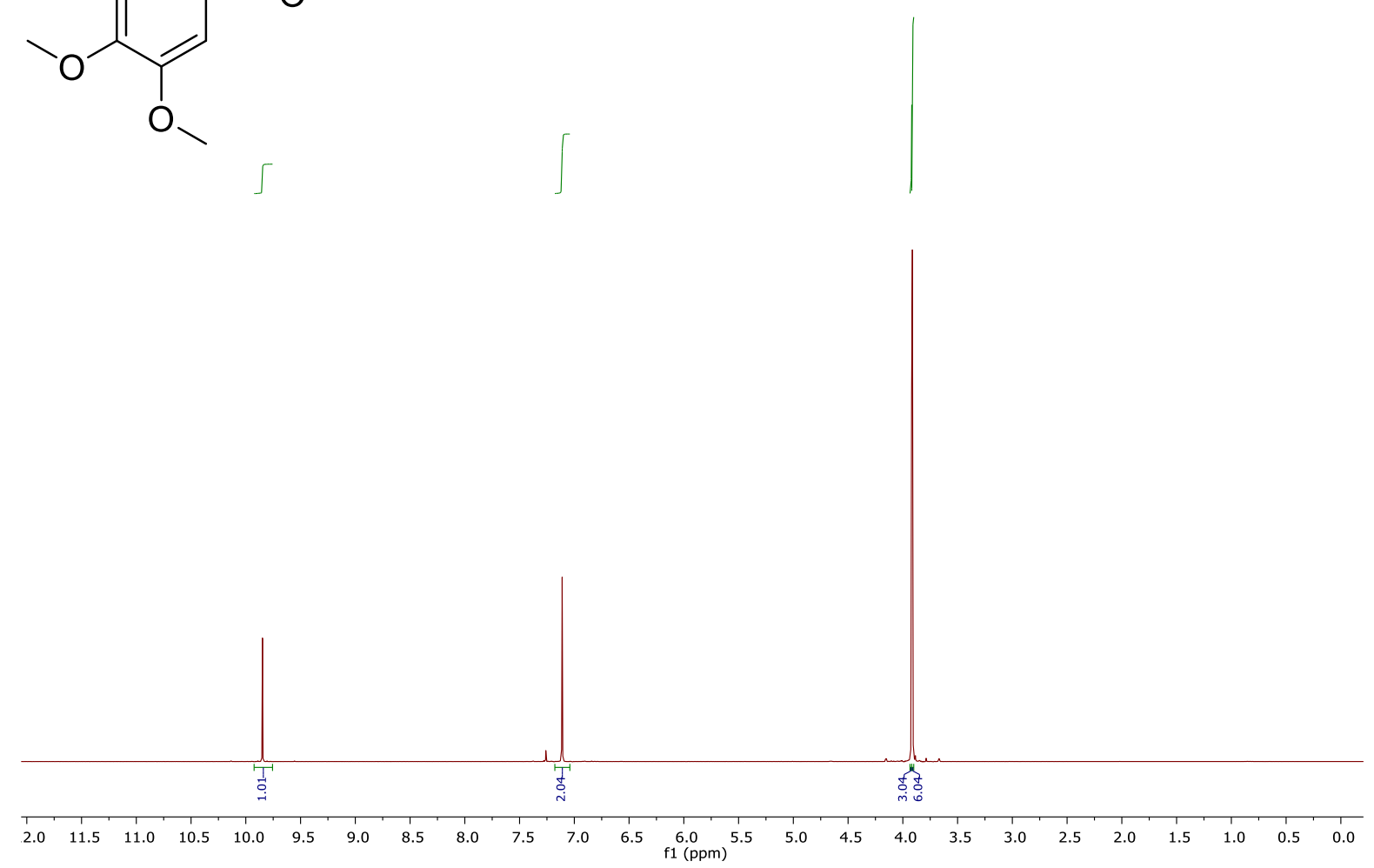

${ }^{1} \mathrm{H}$ NMR spectrum in $\mathrm{CDCl}_{3}$.

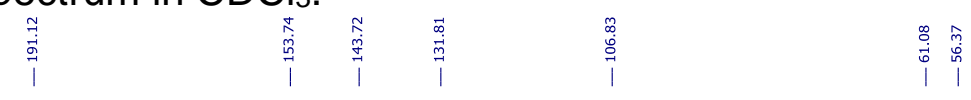<smiles>COc1cc(C=O)cc(OC)c1OC</smiles>

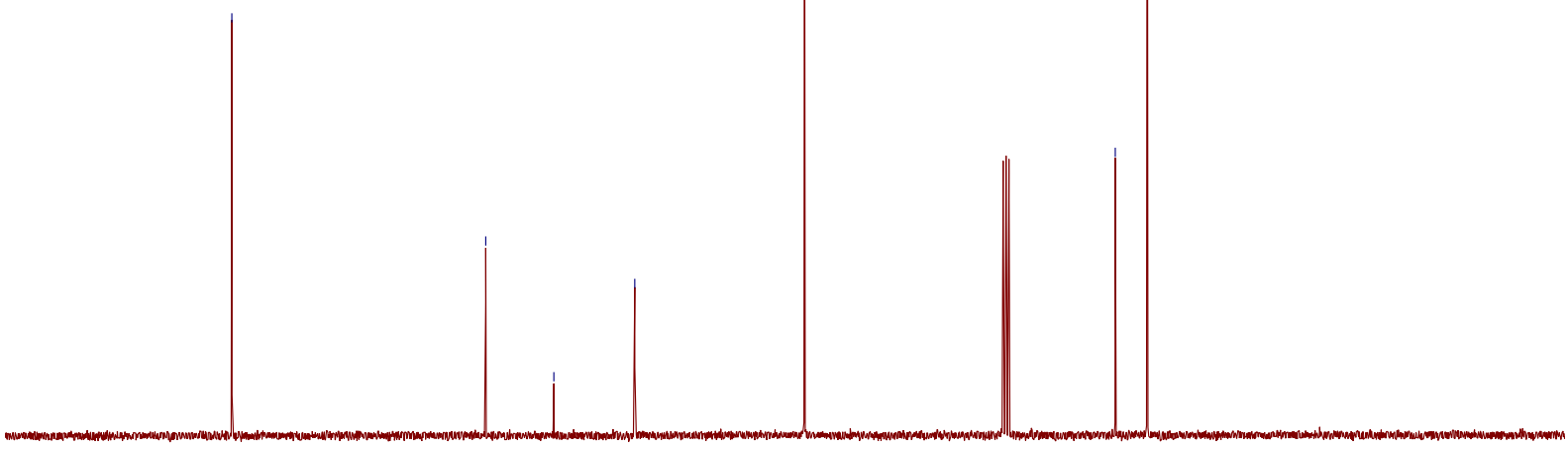

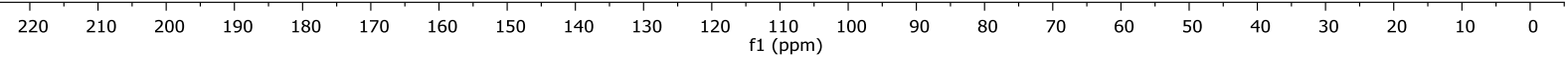
${ }^{13} \mathrm{C}$ NMR spectrum in $\mathrm{CDCl}_{3}$. 
$7 b$<smiles>CC1CCCCC1C=O</smiles>

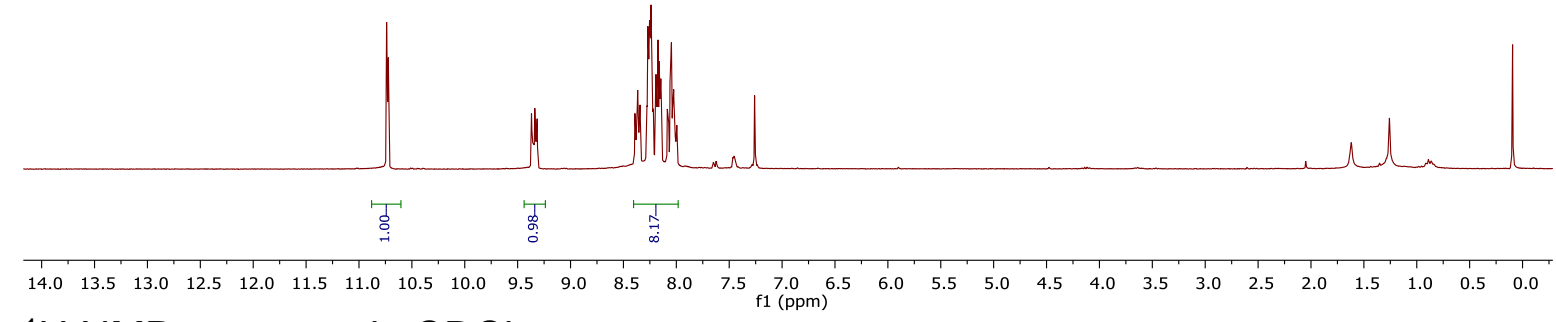

${ }^{1} \mathrm{H}$ NMR spectrum in $\mathrm{CDCl}_{3}$.

ํำ

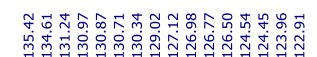

等<smiles>O=Cc1ccc2ccc3cccc4ccc1c2c34</smiles>

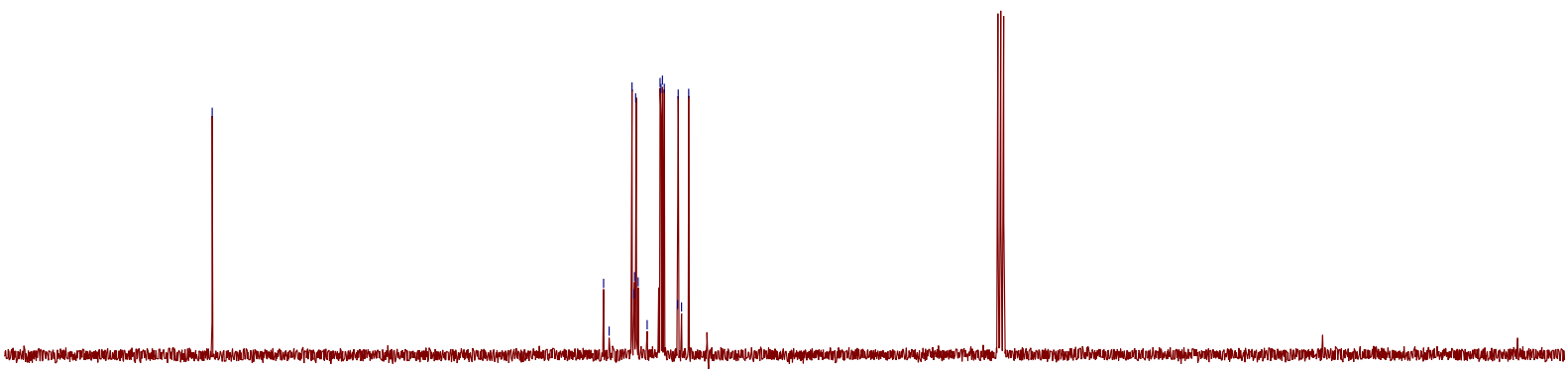

\begin{tabular}{lllllllllllllllllllllllll}
\hline & 220 & 210 & 200 & 190 & 180 & 170 & 160 & 150 & 140 & 130 & 120 & 110 & 100 & 90 & 80 & 70 & 60 & 50 & 40 & 30 & 20 & 10 & 0
\end{tabular} ${ }^{13} \mathrm{C}$ NMR spectrum in $\mathrm{CDCl}_{3}$. 


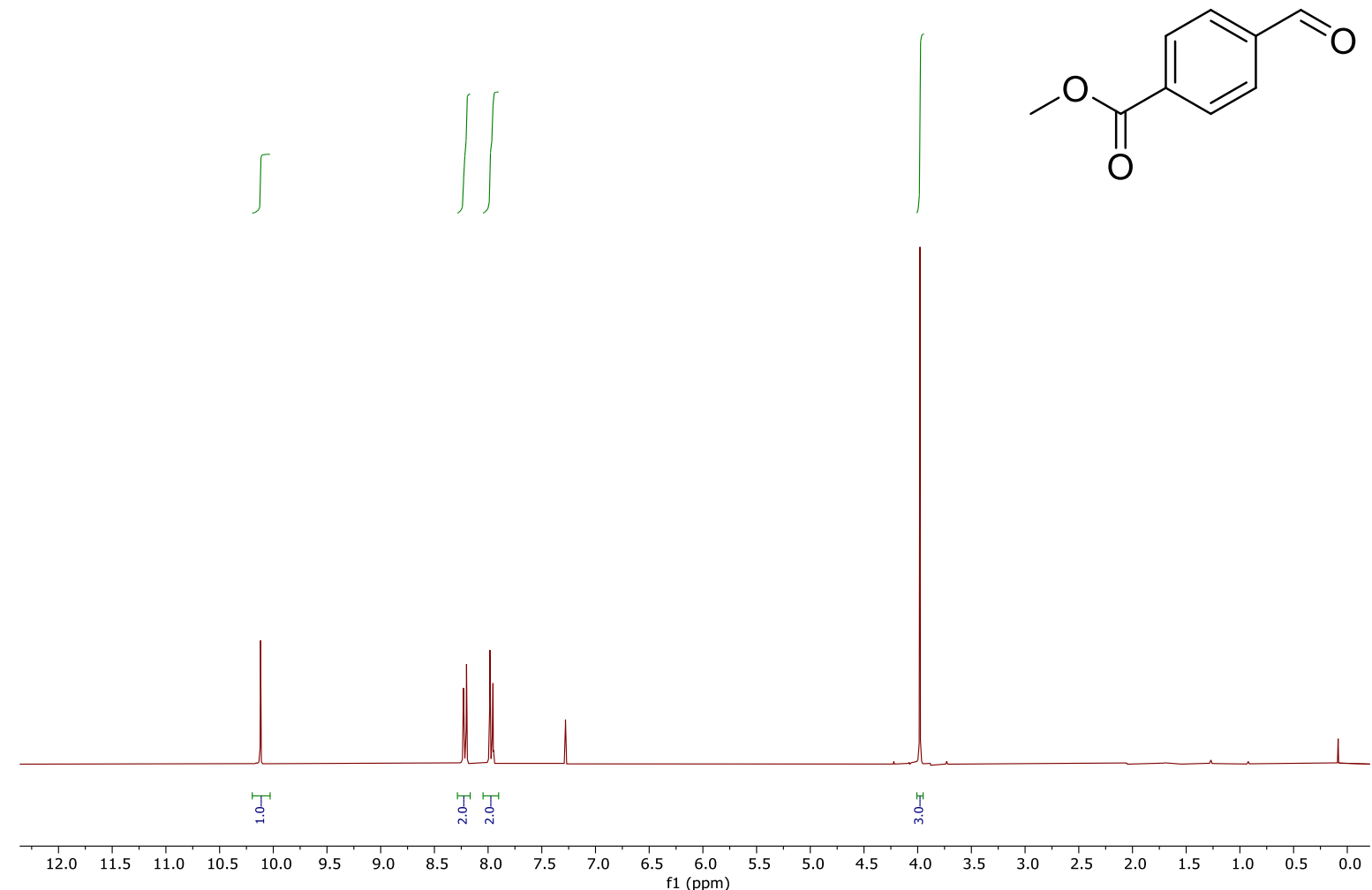

${ }^{1} \mathrm{H}$ NMR spectrum in $\mathrm{CDCl}_{3}$.
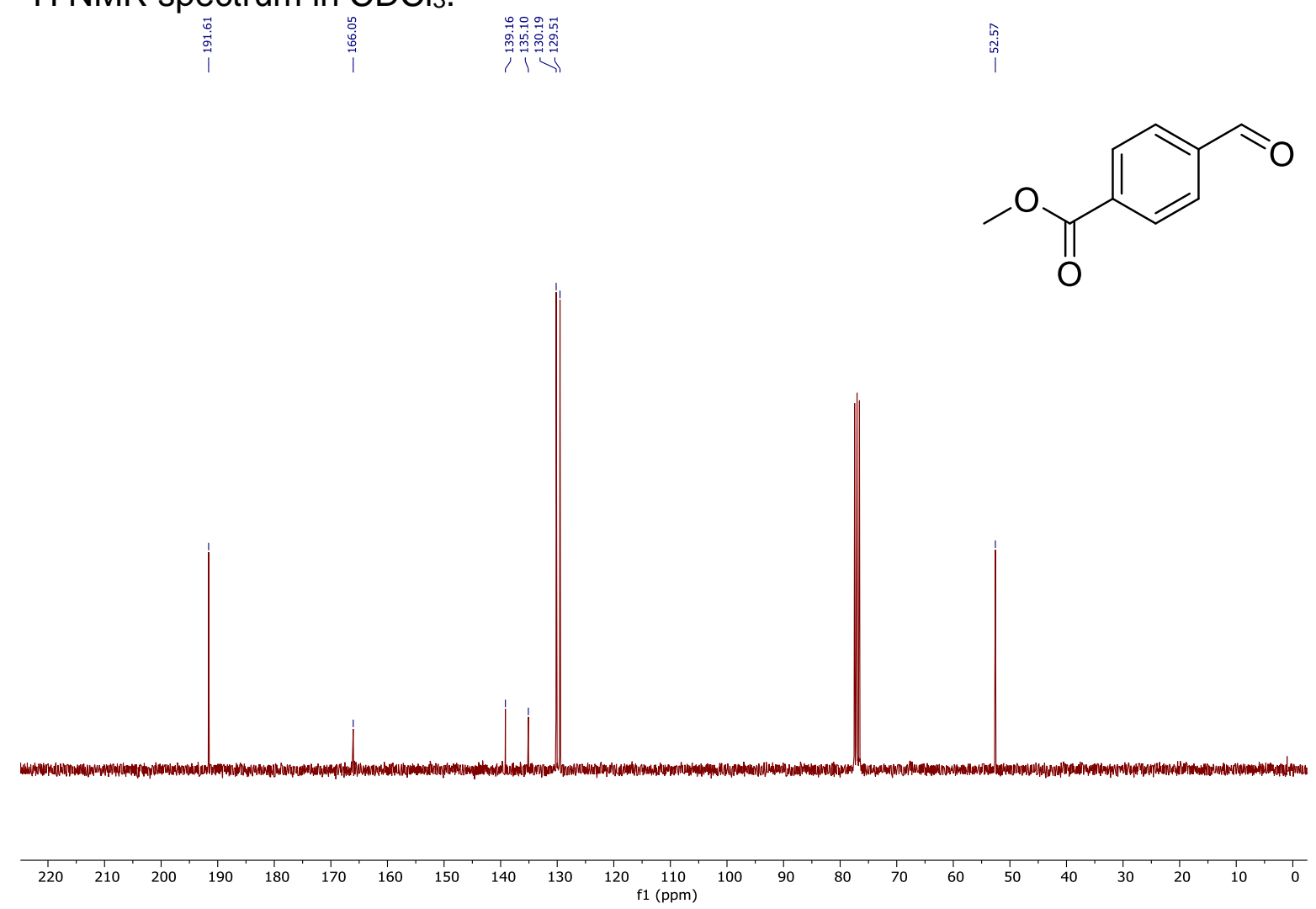

${ }^{13} \mathrm{C}$ NMR spectrum in $\mathrm{CDCl}_{3}$. 
$18 b$
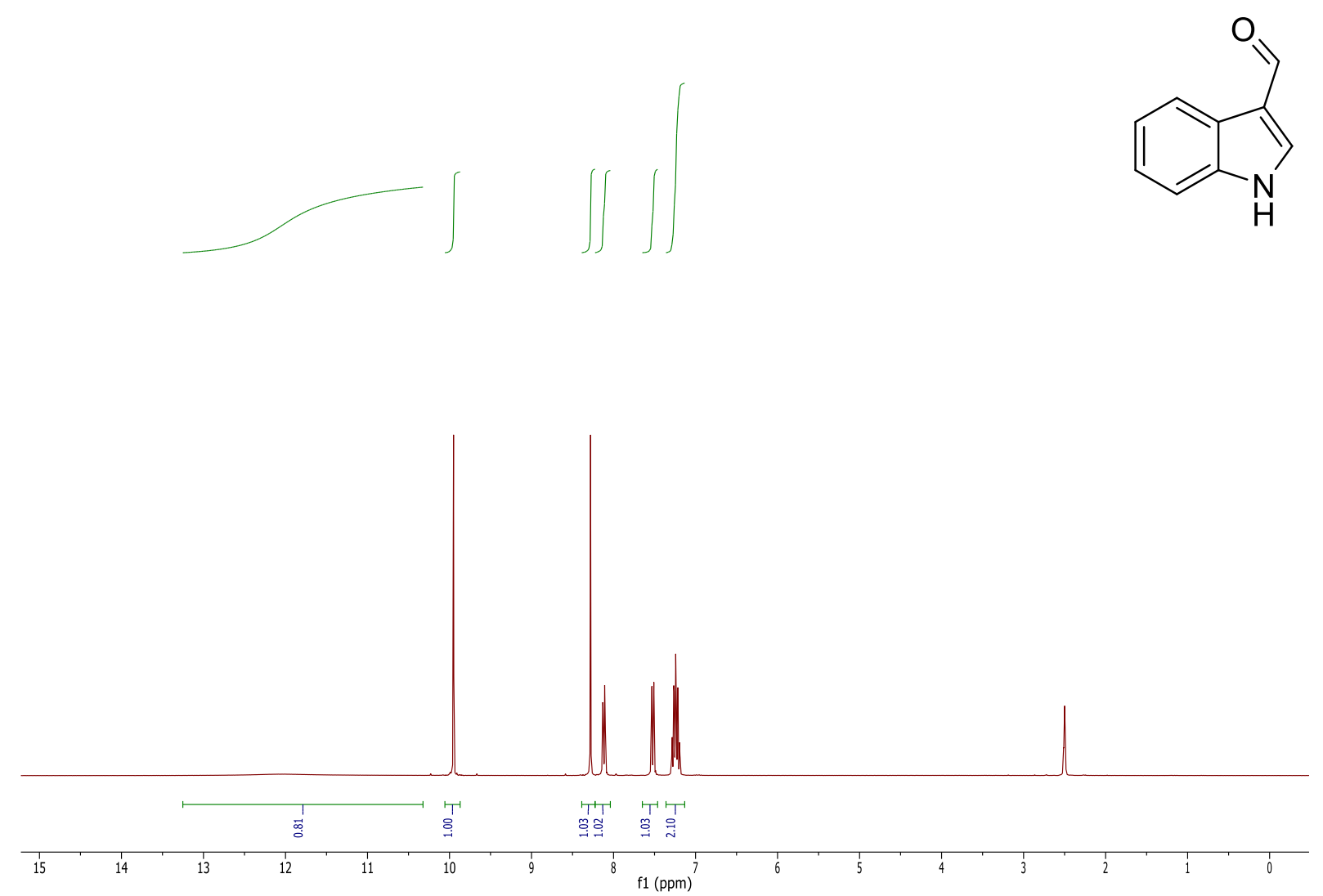

${ }^{1} \mathrm{H}$ NMR spectrum in DMSO-d6.
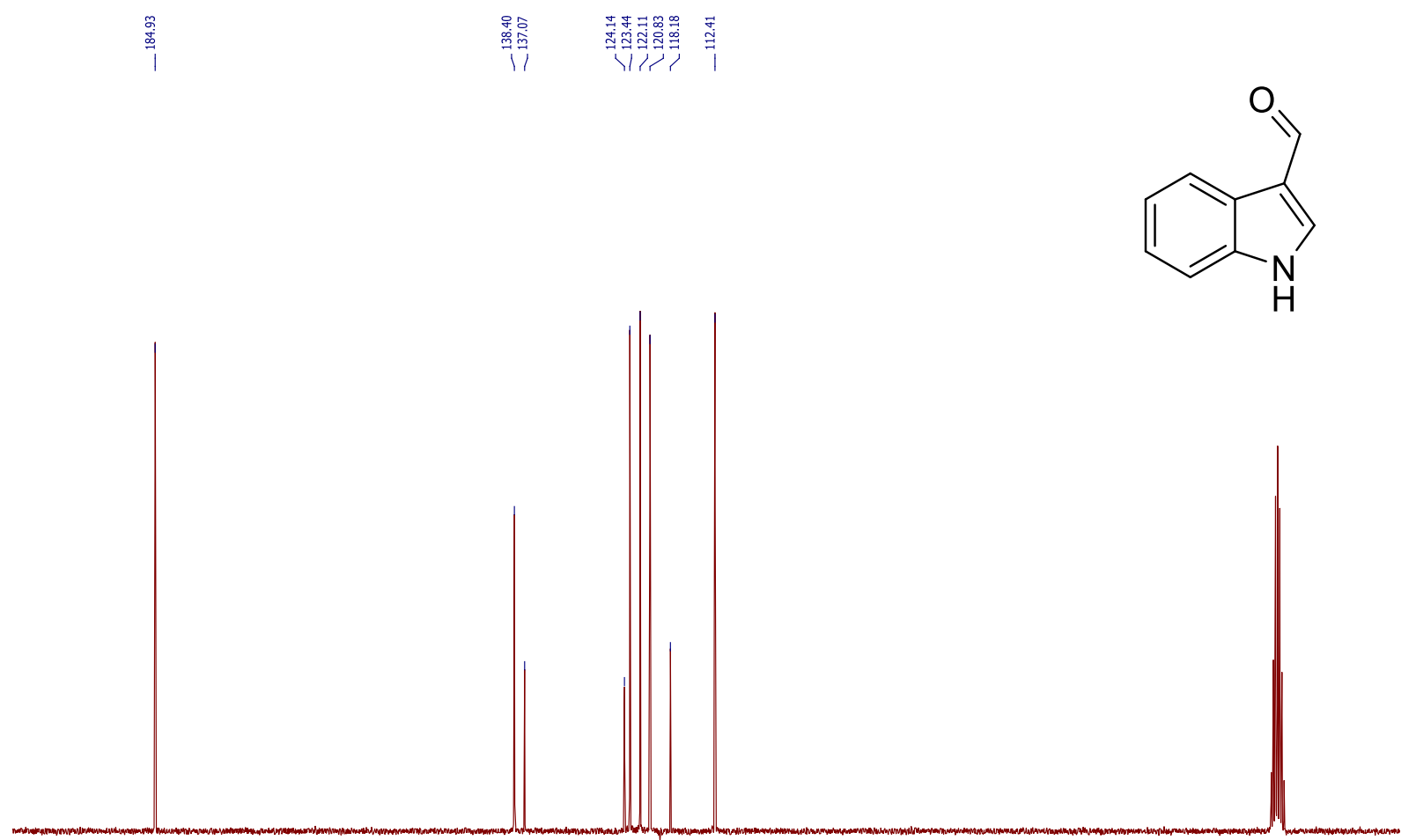

${ }^{13} \mathrm{C}$ NMR spectrum in DMSO-d6. 
$19 b$

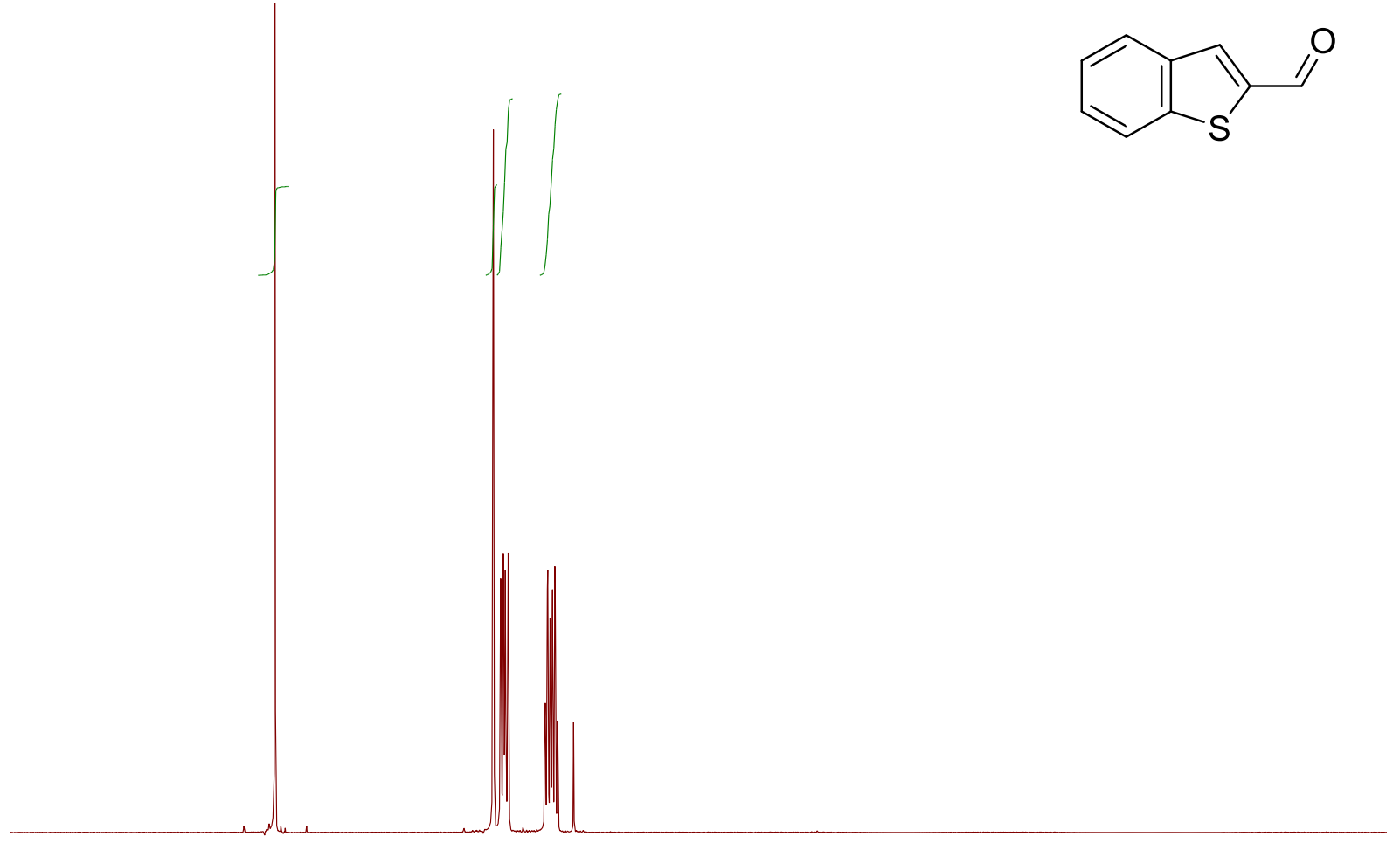

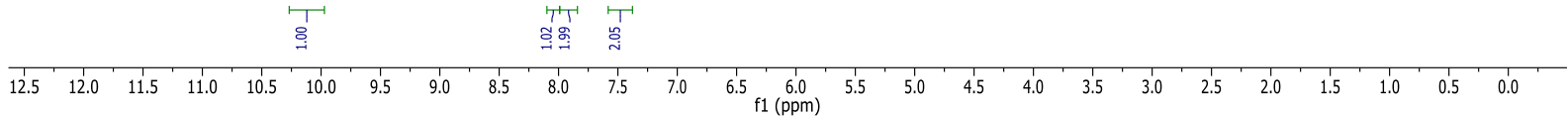

${ }^{1} \mathrm{H}$ NMR spectrum in $\mathrm{CDCl}_{3}$.

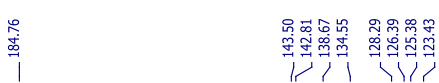

$\overbrace{\mathrm{s}}^{\mathrm{O}}$

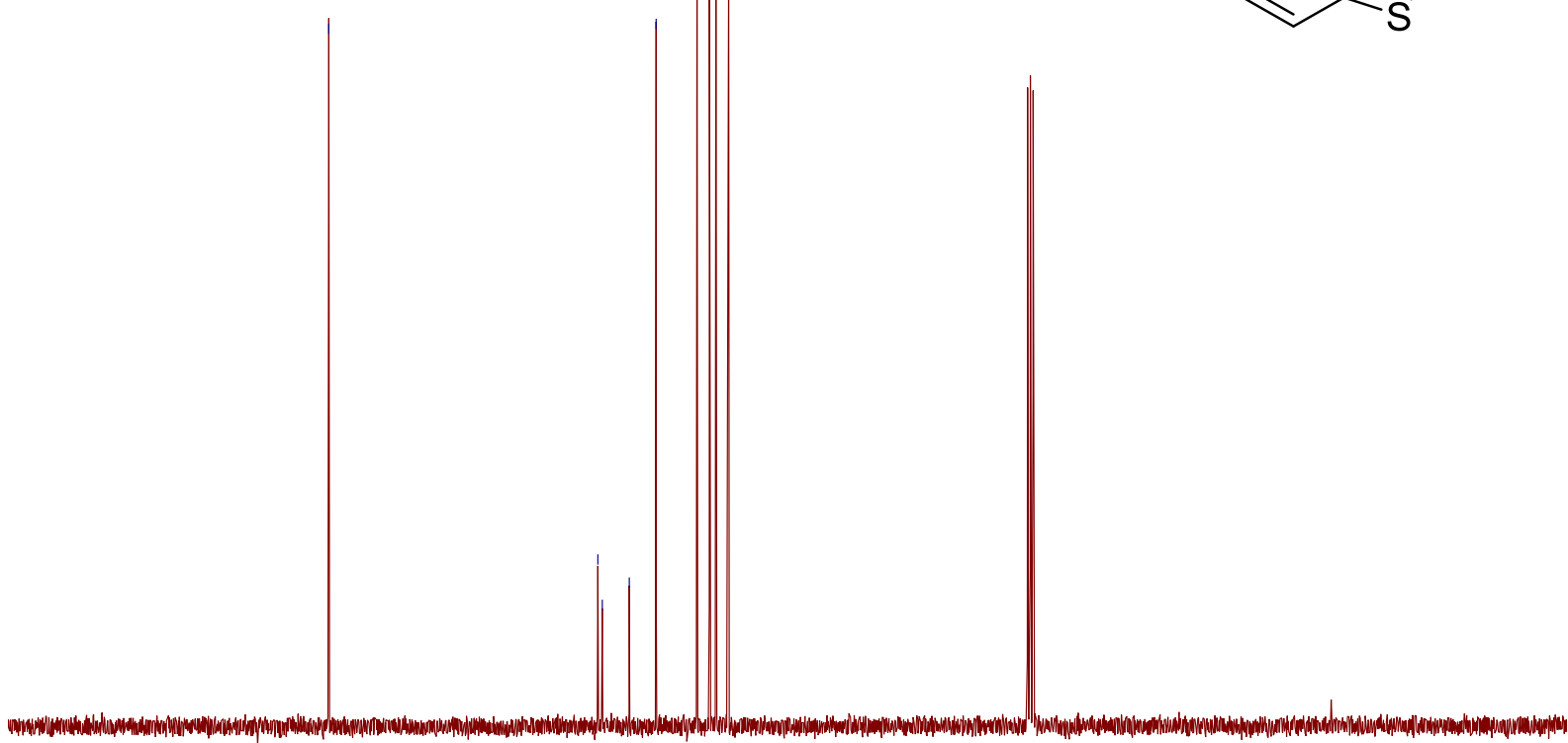

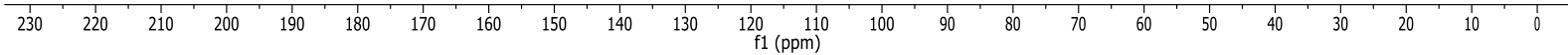
${ }^{13} \mathrm{C}$ NMR spectrum in $\mathrm{CDCl}_{3}$. 


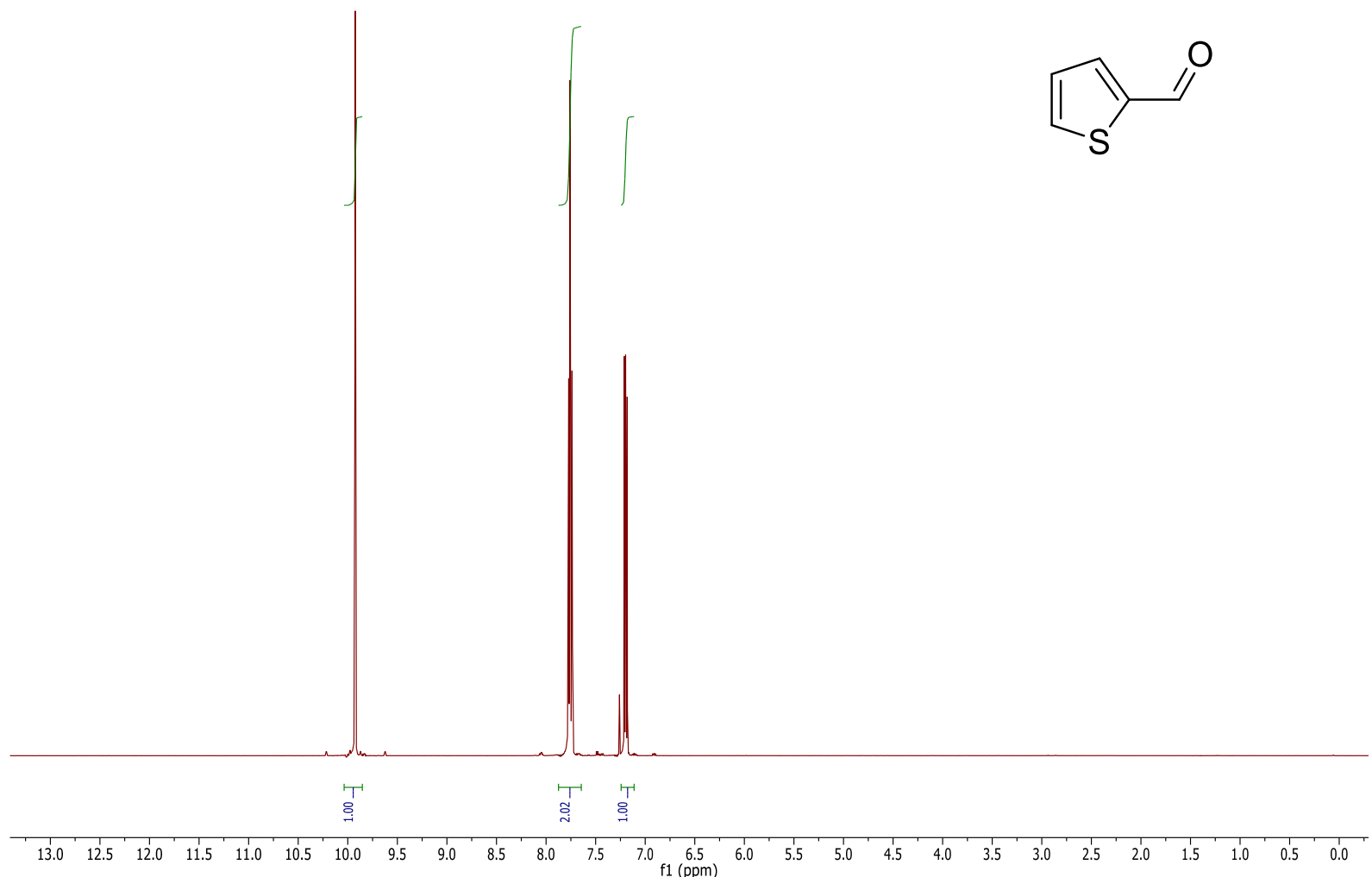

${ }^{1} \mathrm{H}$ NMR spectrum in $\mathrm{CDCl}_{3}$.

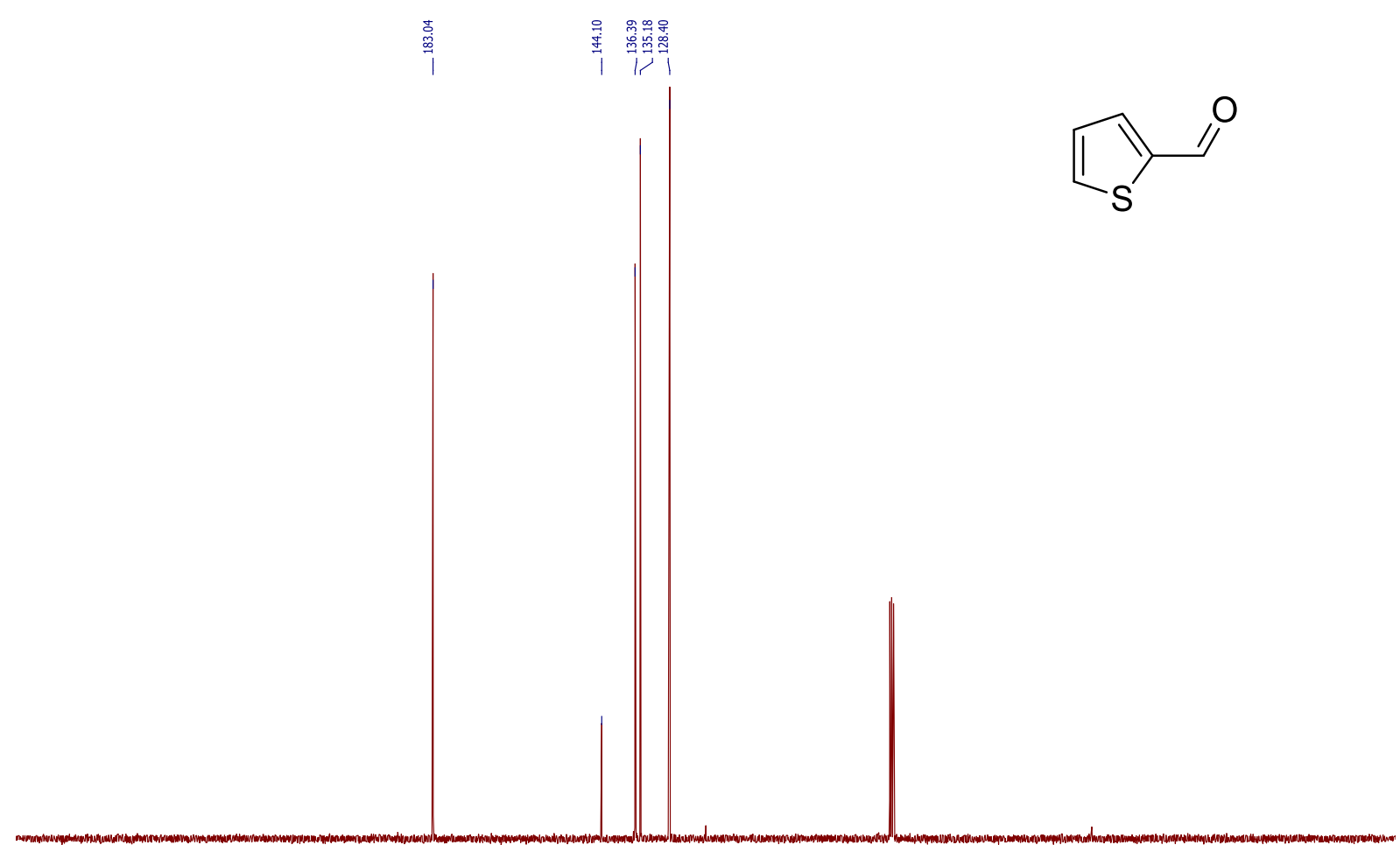

$\begin{array}{lllllllllllllllllllllllllllllllllllllllllll}270 & 260 & 250 & 240 & 230 & 220 & 210 & 200 & 190 & 180 & 170 & 160 & 150 & 140 & 130 & 120 & 110 & 100 & 90 & 80 & 70 & 60 & 50 & 40 & 30 & 20 & 10 & 0 & -10 & -20 & -30\end{array}$ ${ }^{13} \mathrm{C}$ NMR spectrum in $\mathrm{CDCl}_{3}$. 
<smiles>C#CCCCC#C</smiles>

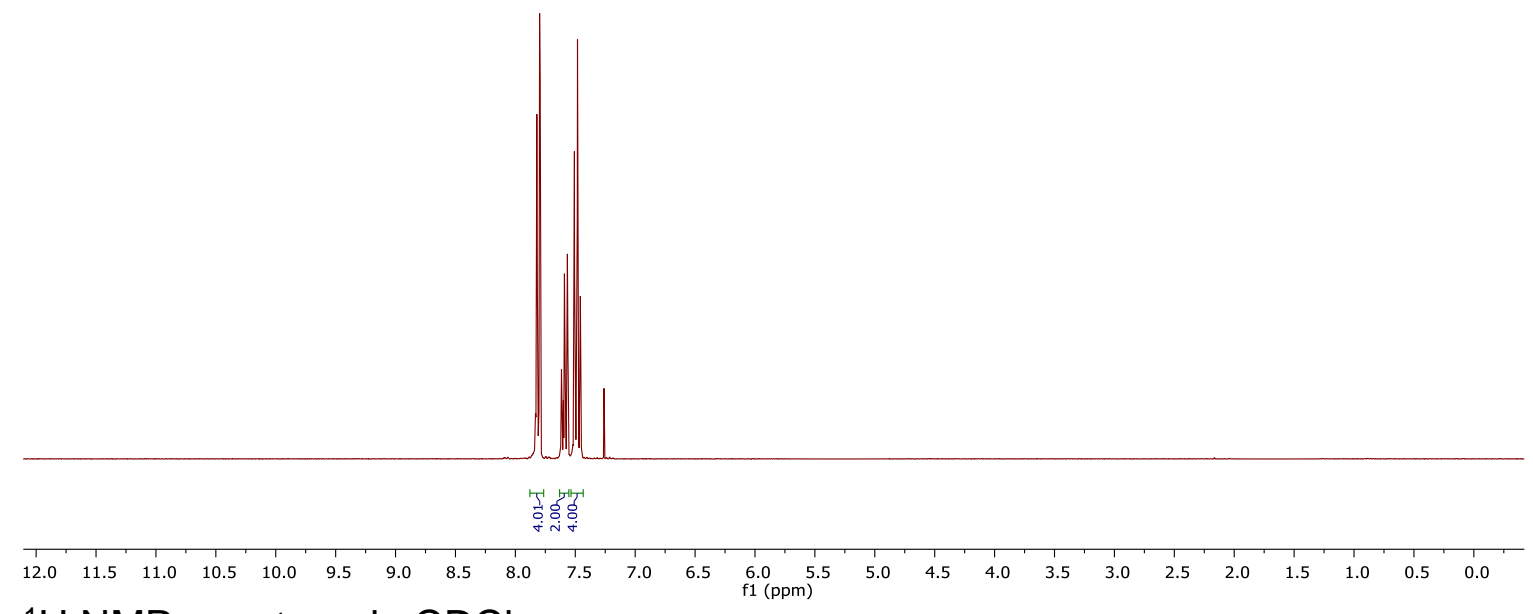

${ }^{1} \mathrm{H}$ NMR spectrum in $\mathrm{CDCl}_{3}$.

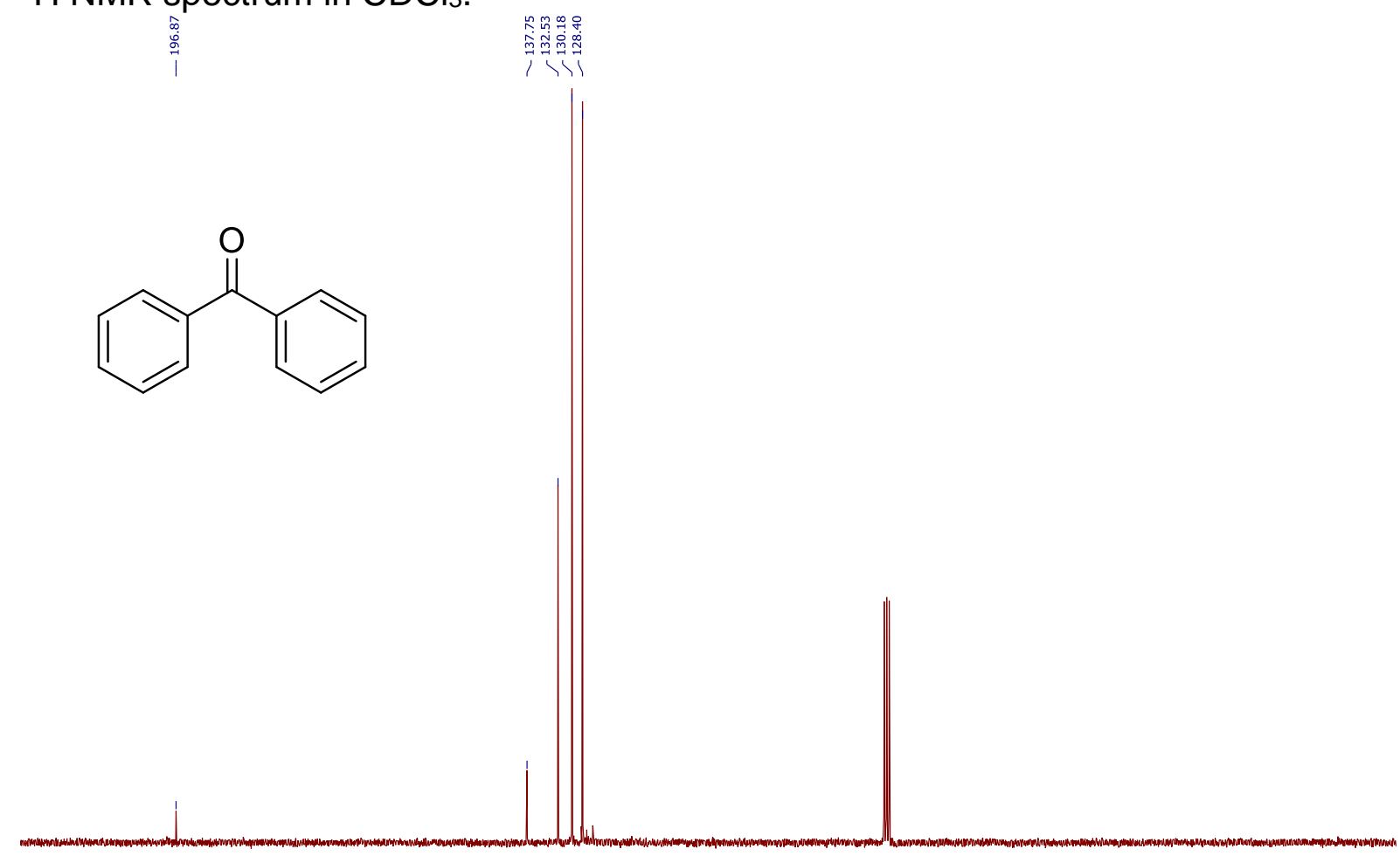

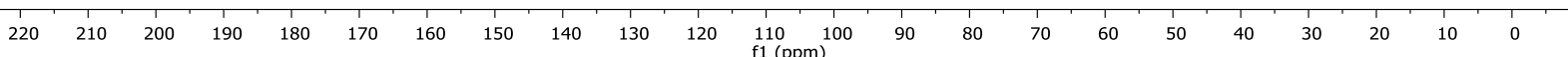
${ }^{13} \mathrm{C}$ NMR spectrum in $\mathrm{CDCl}_{3}$. 
<smiles>O=C(c1ccccc1)c1ccc(Br)cc1</smiles>

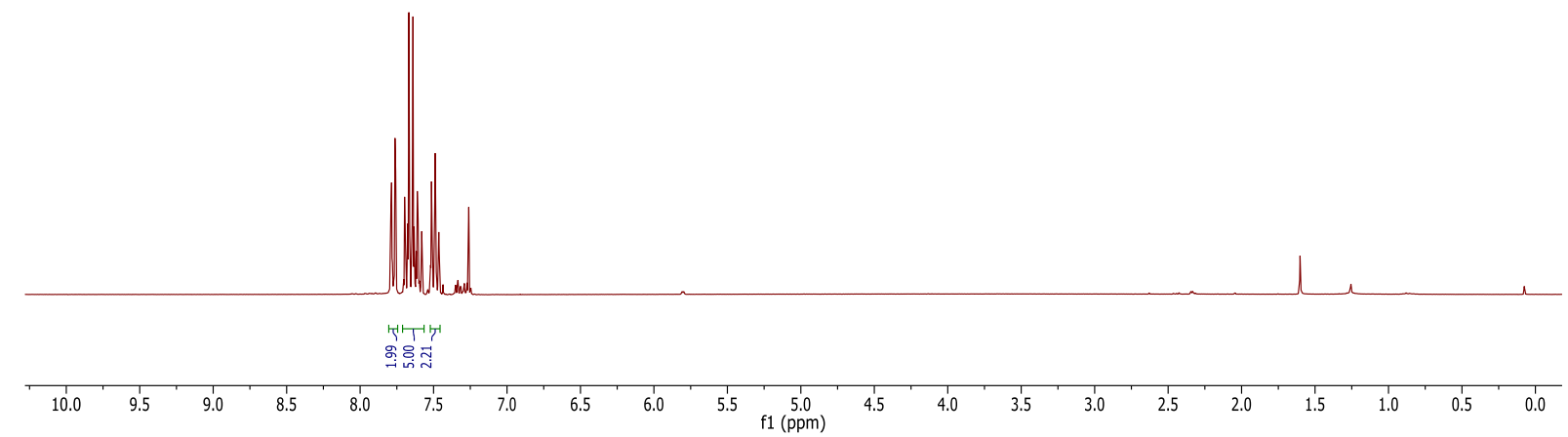

${ }^{1} \mathrm{H}$ NMR spectrum in $\mathrm{CDCl}_{3}$.

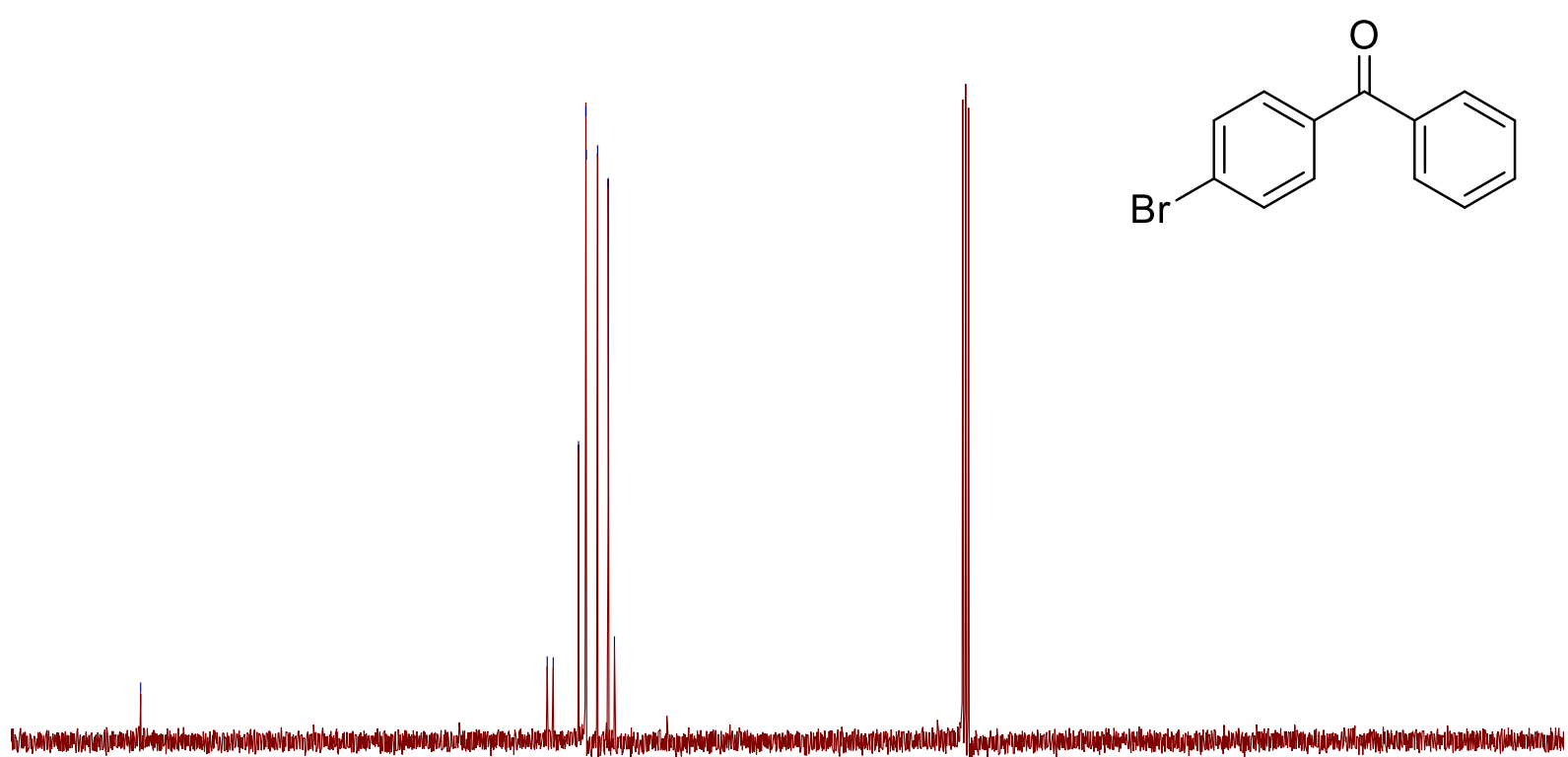

$\begin{array}{lllllllllll}210 & 200 & 190 & 180 & 170 & 160 & 150 & 140 & 130 & 120 & 110 \\ \mathrm{f} 1(\mathrm{ppm}) & 1\end{array}$

${ }^{13} \mathrm{C}$ NMR spectrum in $\mathrm{CDCl}_{3}$. 
$28 b$<smiles>O=C(c1ccc(Cl)cc1)c1ccc(Cl)cc1</smiles>

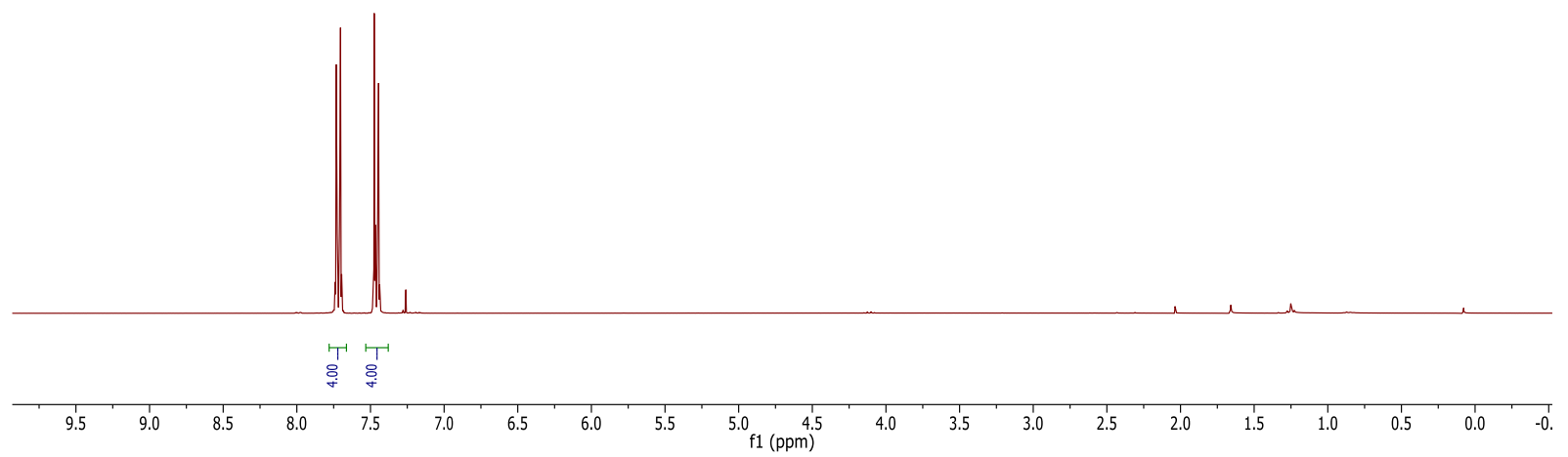

${ }^{1} \mathrm{H}$ NMR spectrum in $\mathrm{CDCl}_{3}$.

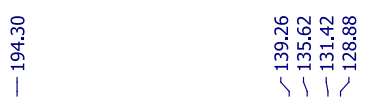

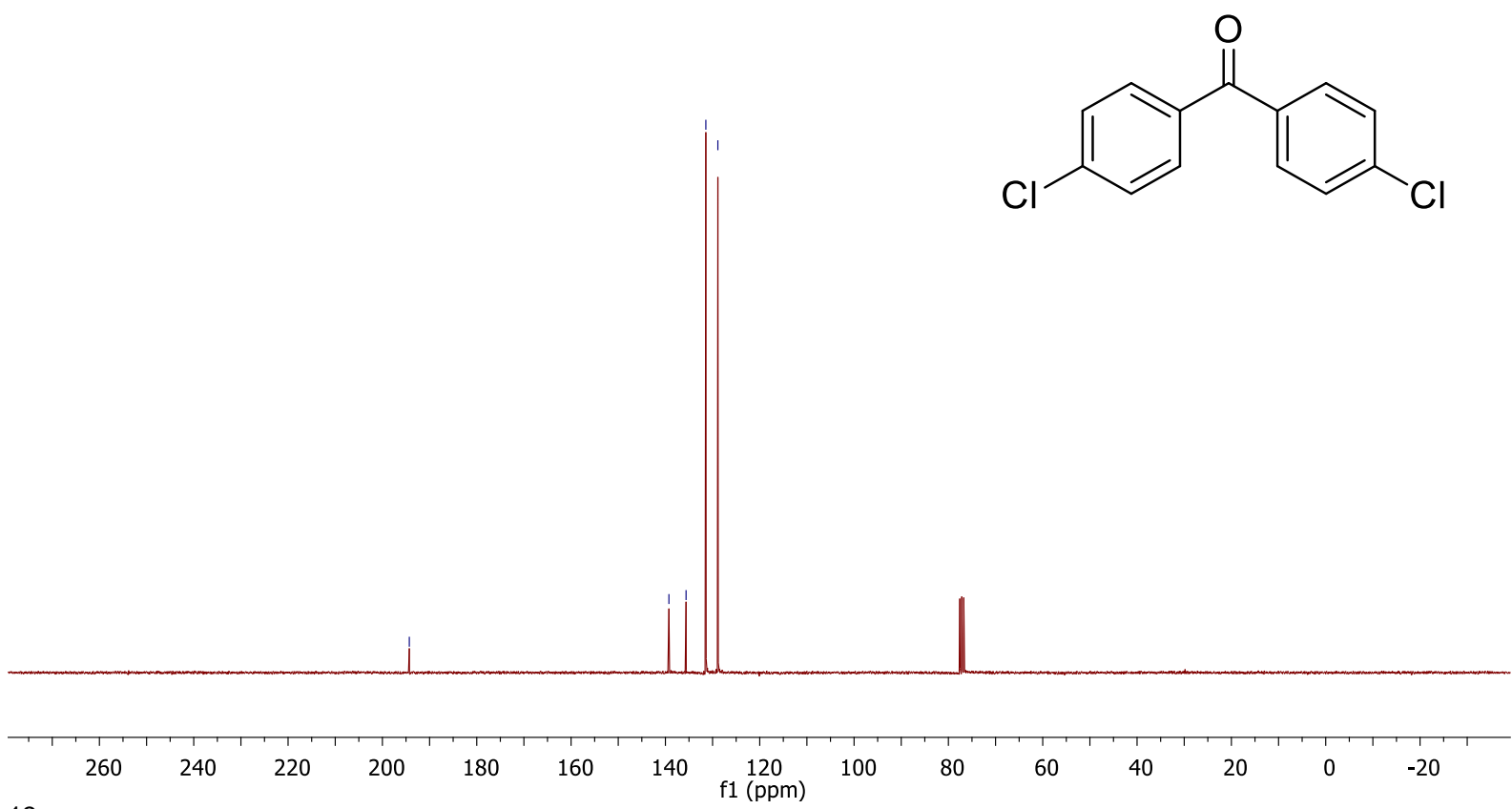

${ }^{13} \mathrm{C}$ NMR spectrum in $\mathrm{CDCl}_{3}$. 


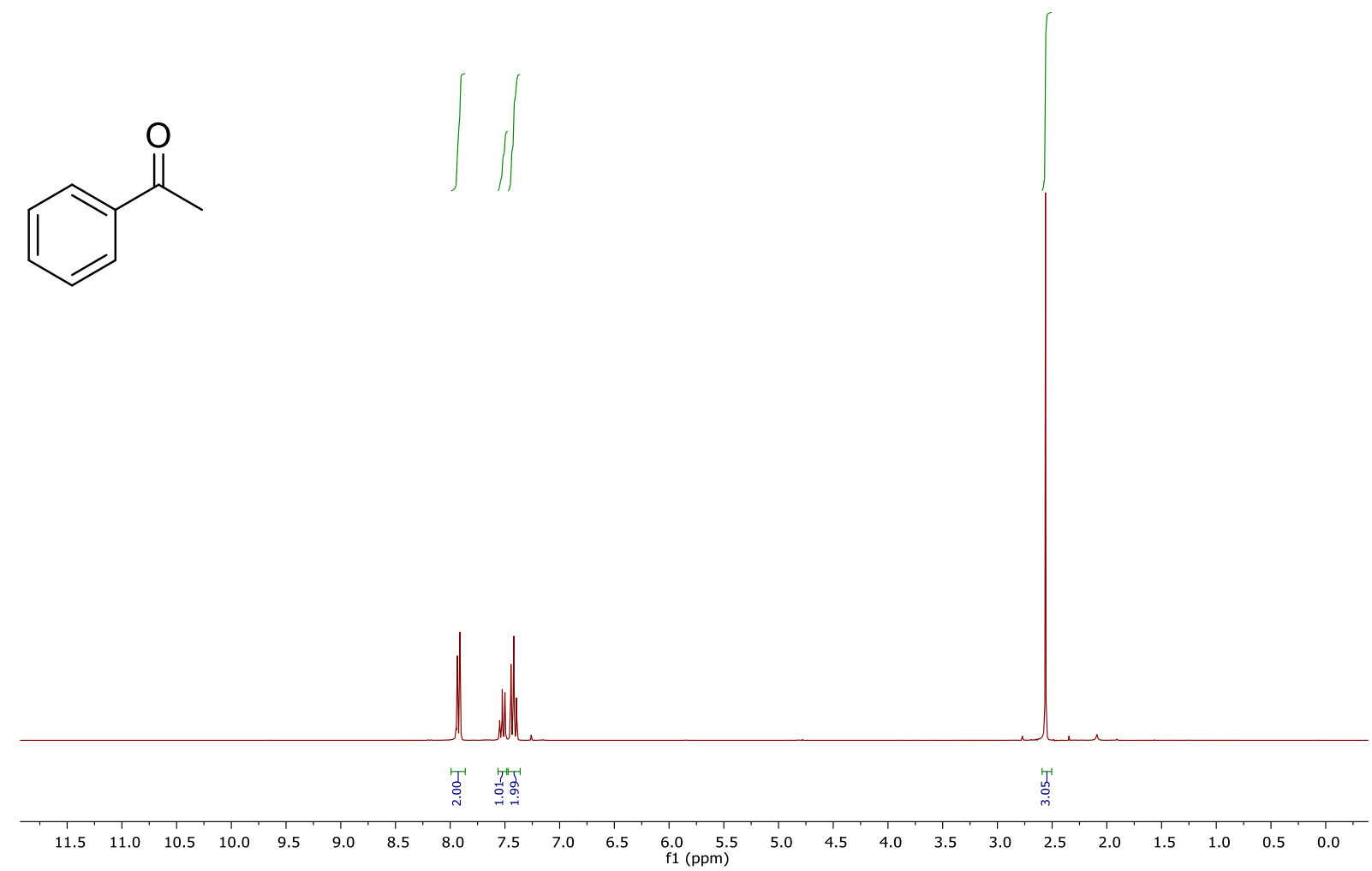

${ }^{1} \mathrm{H}$ NMR spectrum in $\mathrm{CDCl}_{3}$.

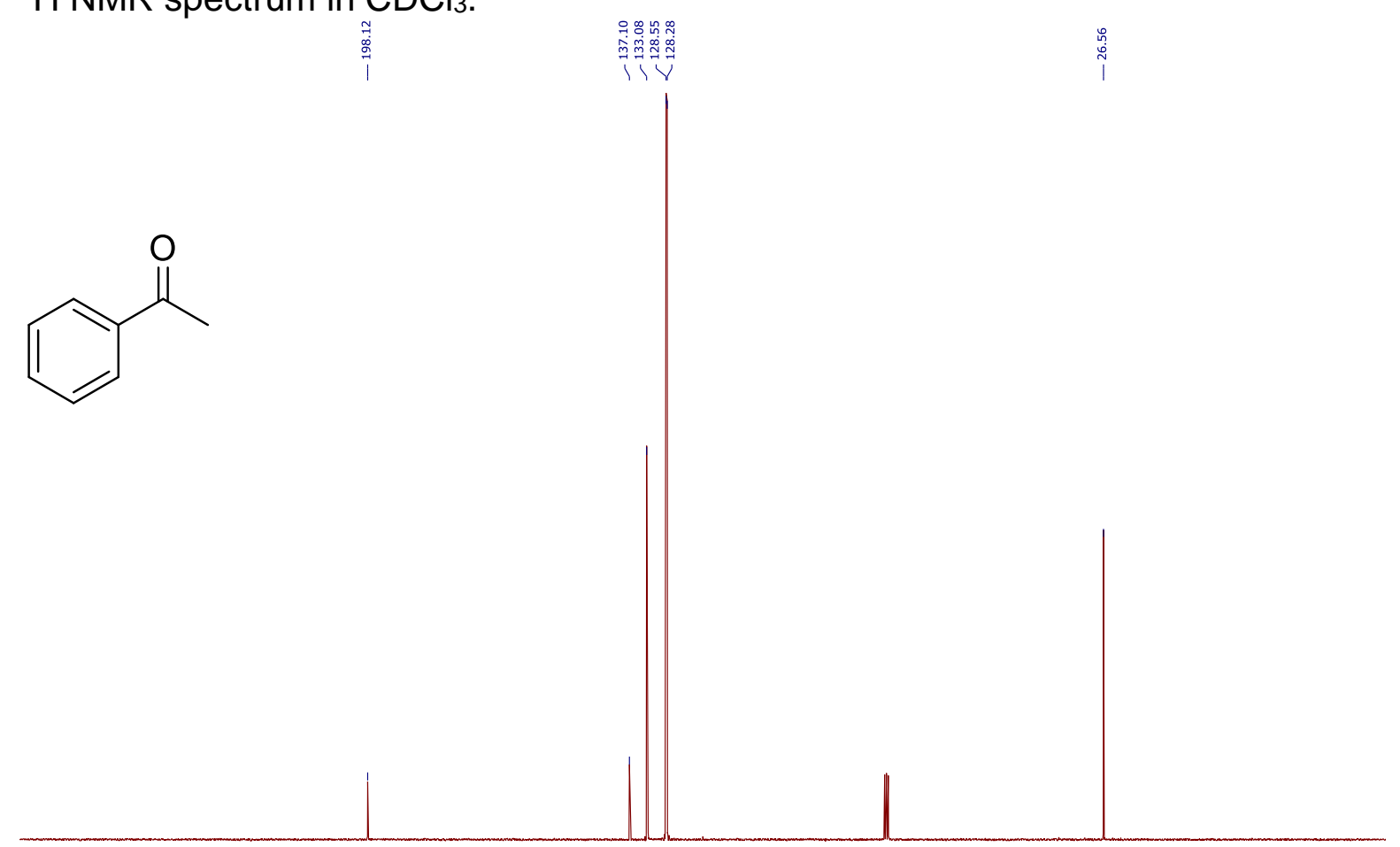

$\begin{array}{llllllllllllllllllllllllllllllll}270 & 260 & 250 & 240 & 230 & 220 & 210 & 200 & 190 & 180 & 170 & 160 & 150 & 140 & 130 & 120 & 110 & 100 & 90 & 80 & 70 & 60 & 50 & 40 & 30 & 20 & 10 & 0 & -10 & -20 & -30\end{array}$ ${ }^{13} \mathrm{C}$ NMR spectrum in $\mathrm{CDCl}_{3}$. 
<smiles>O=C1CCCc2ccccc21</smiles>
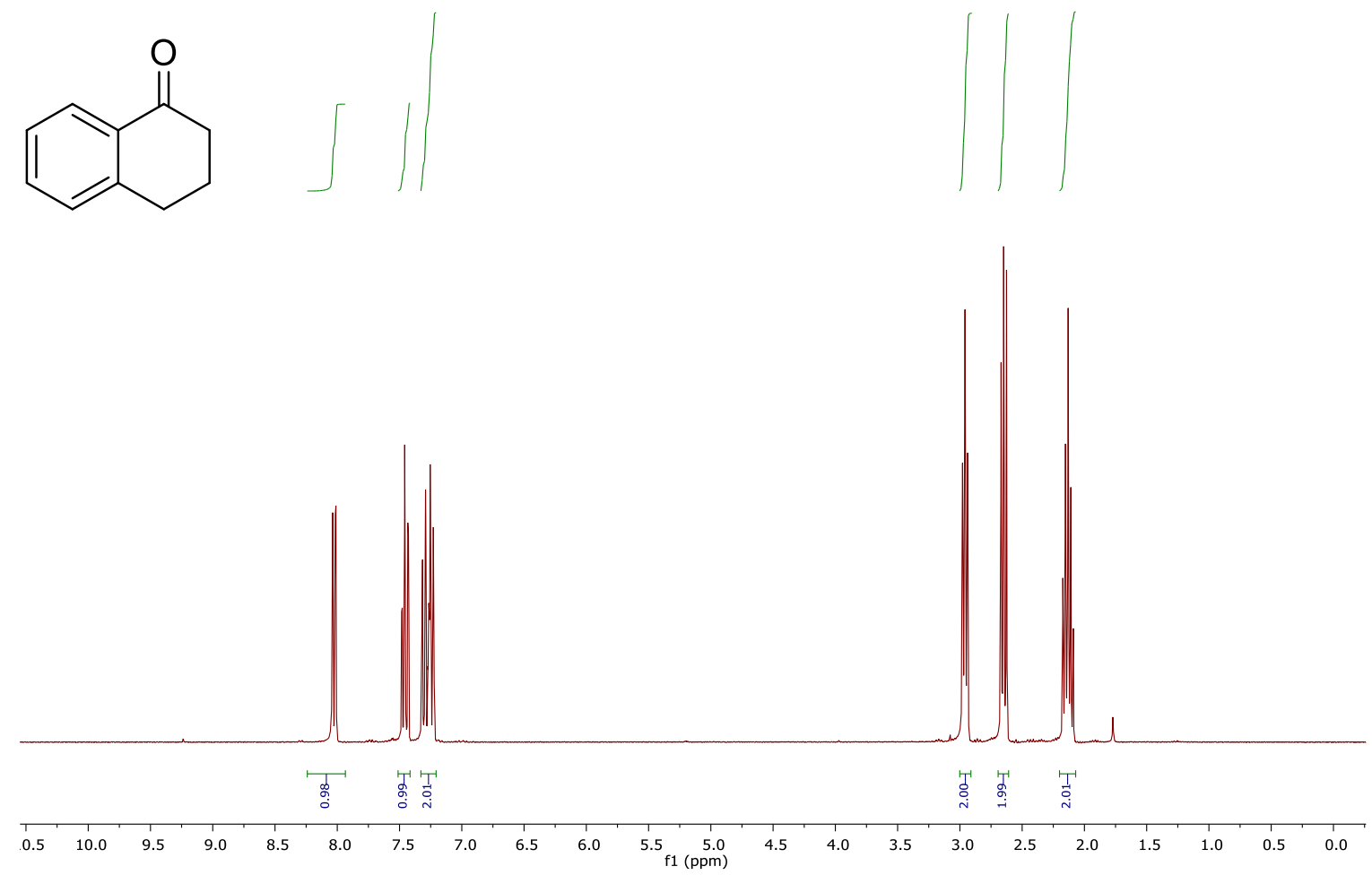

${ }^{1} \mathrm{H}$ NMR spectrum in $\mathrm{CDCl}_{3}$.<smiles>O=C1CCCc2ccccc21</smiles>

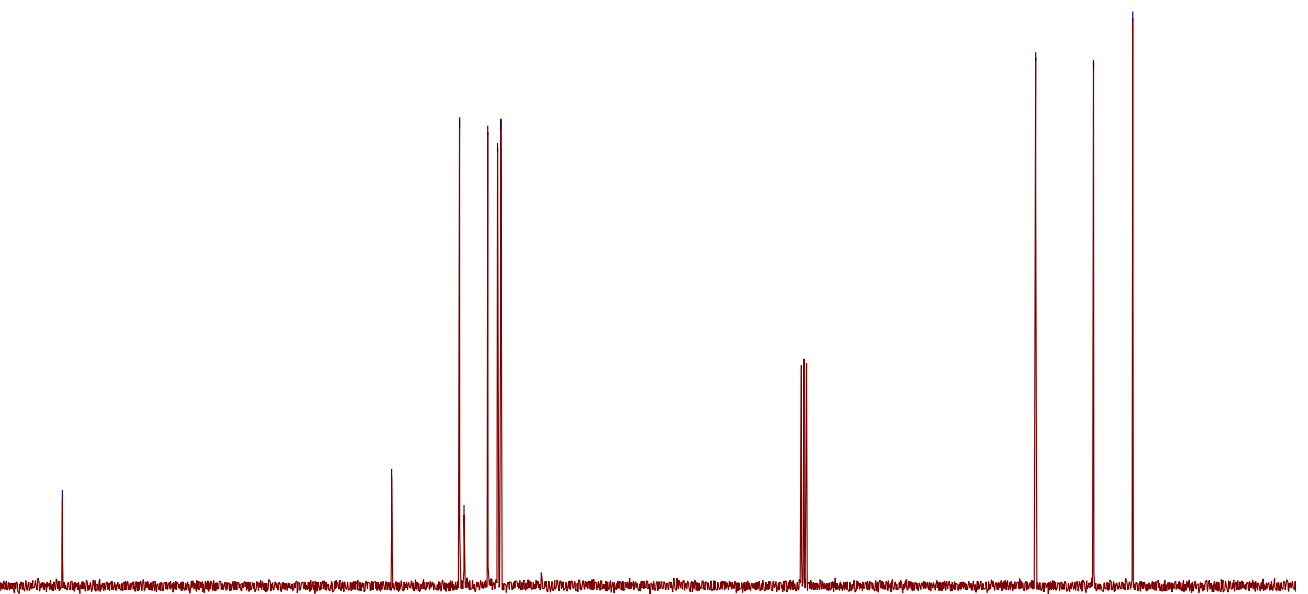

$\begin{array}{llllllllllllllllllllllllll}240 & 230 & 220 & 210 & 200 & 190 & 180 & 170 & 160 & 150 & 140 & 130 & 120 & 110 & 100 & 90 & 80 & 70 & 60 & 50 & 40 & 30 & 20 & 10 & 0\end{array}$ ${ }^{13} \mathrm{C}$ NMR spectrum in $\mathrm{CDCl}_{3}$. 
$32 b$

10

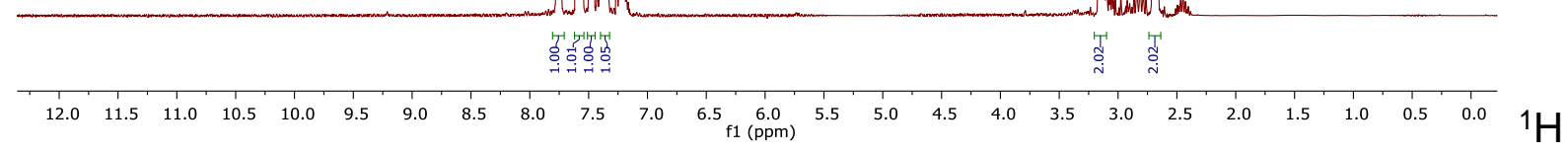

NMR spectrum in $\mathrm{CDCl}_{3}$.

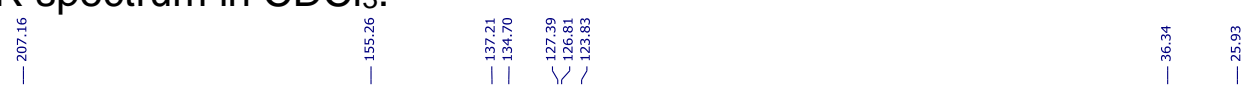<smiles>O=C1CCc2ccccc21</smiles>

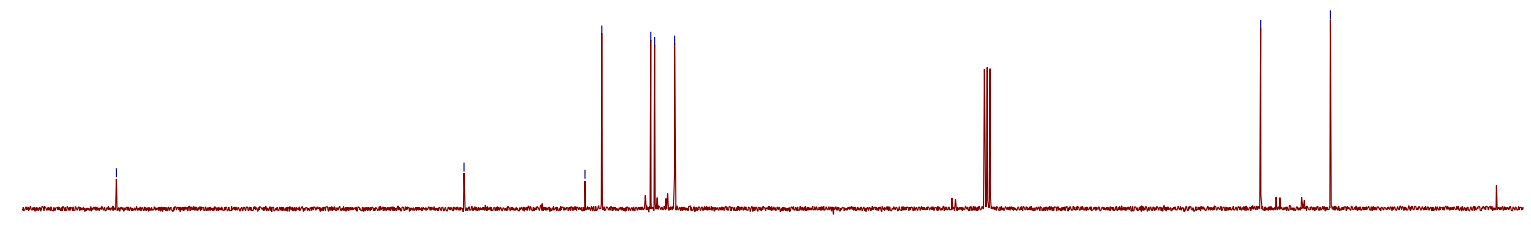

$\begin{array}{llllllllllllllllllllllll}220 & 210 & 200 & 190 & 180 & 170 & 160 & 150 & 140 & 130 & 120 & \begin{array}{c}110 \\ \mathrm{fi}(\mathrm{ppm})\end{array} & 100 & 90 & 80 & 70 & 60 & 50 & 40 & 30 & 20 & 10 & { }^{1} & 13 \mathrm{C}\end{array}$ NMR spectrum in $\mathrm{CDCl}_{3}$. 
$33 b$

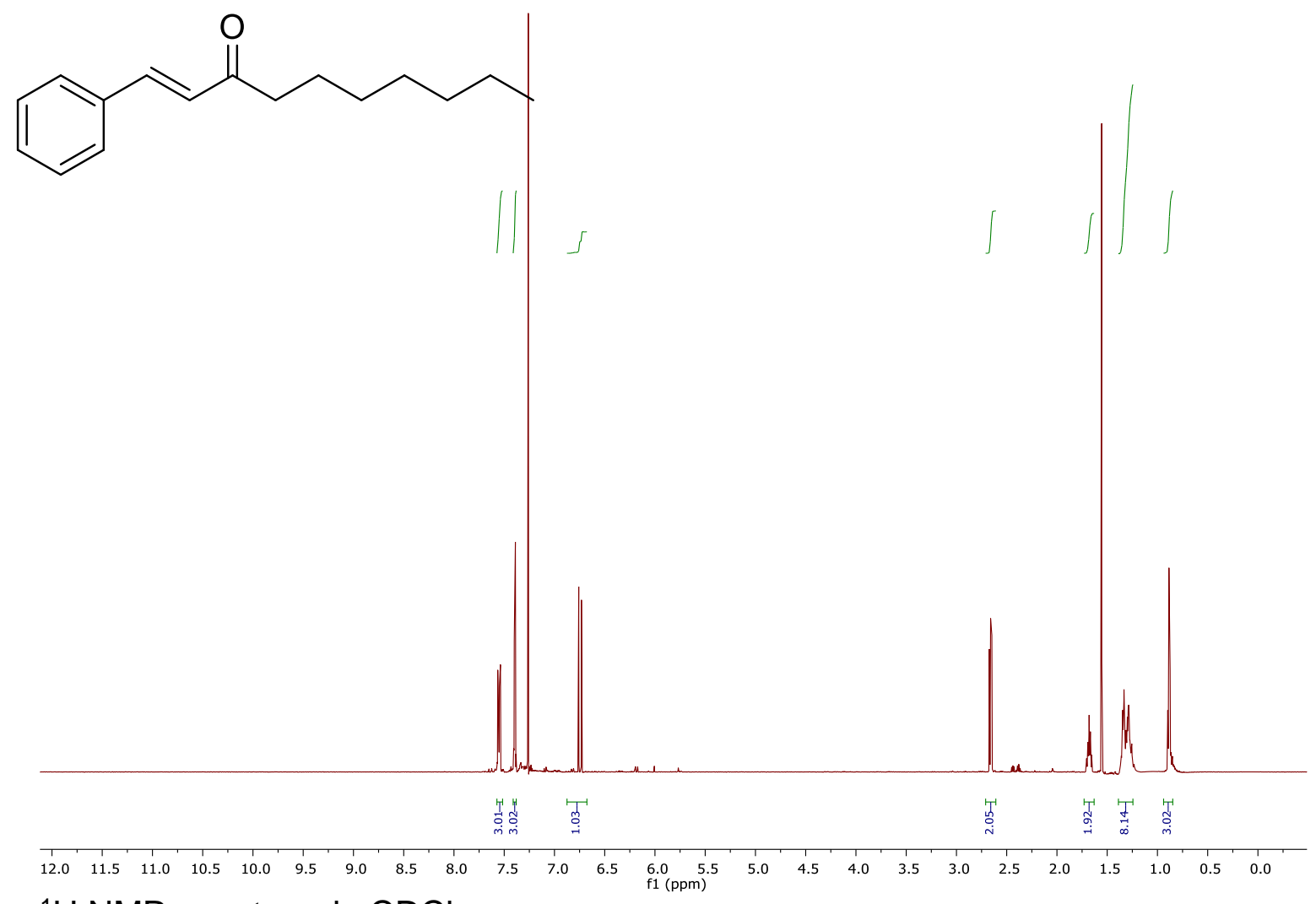

${ }^{1} \mathrm{H}$ NMR spectrum in $\mathrm{CDCl}_{3}$.

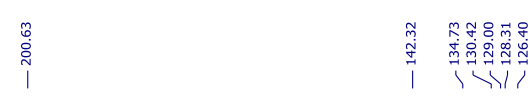

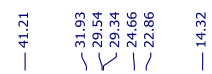<smiles>CCCCCCCC(=O)/C=C/c1ccccc1</smiles>

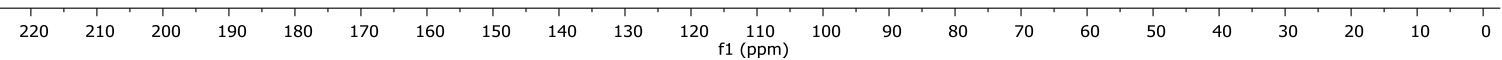
${ }^{13} \mathrm{C}$ NMR spectrum in $\mathrm{CDCl}_{3}$. 
<smiles>O=C1Cc2cccc3cccc1c23</smiles>

\section{$\iint\left\|\int\right\| \int$}

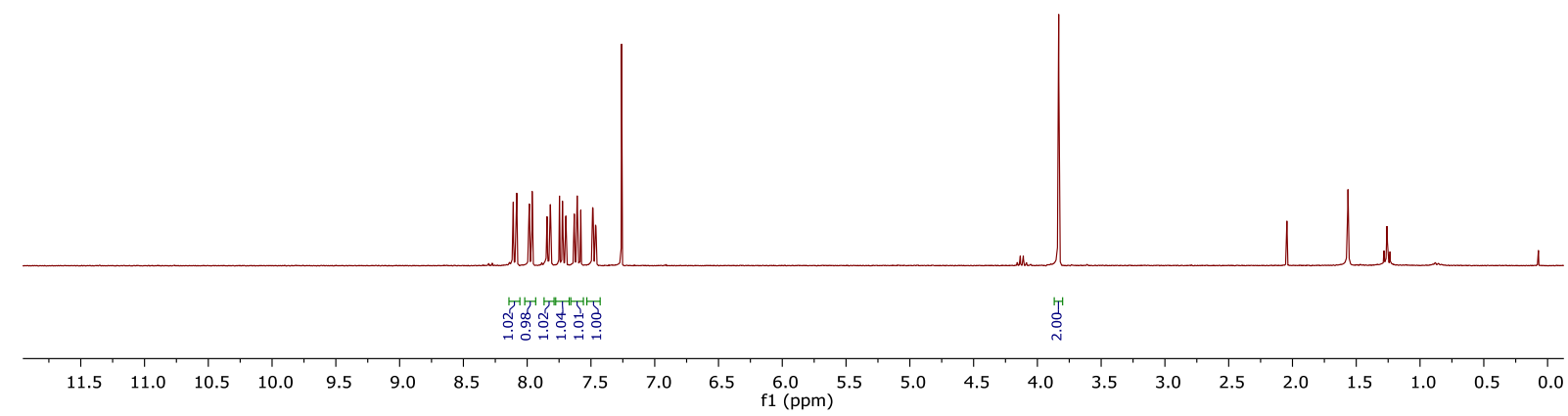

${ }^{1} \mathrm{H}$ NMR spectrum in $\mathrm{CDCl}_{3}$.

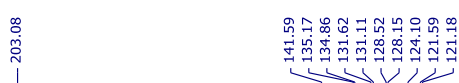<smiles>O=C1Cc2cccc3cccc1c23</smiles>
${ }^{13} \mathrm{C}$ NMR spectrum in $\mathrm{CDCl}_{3}$. 


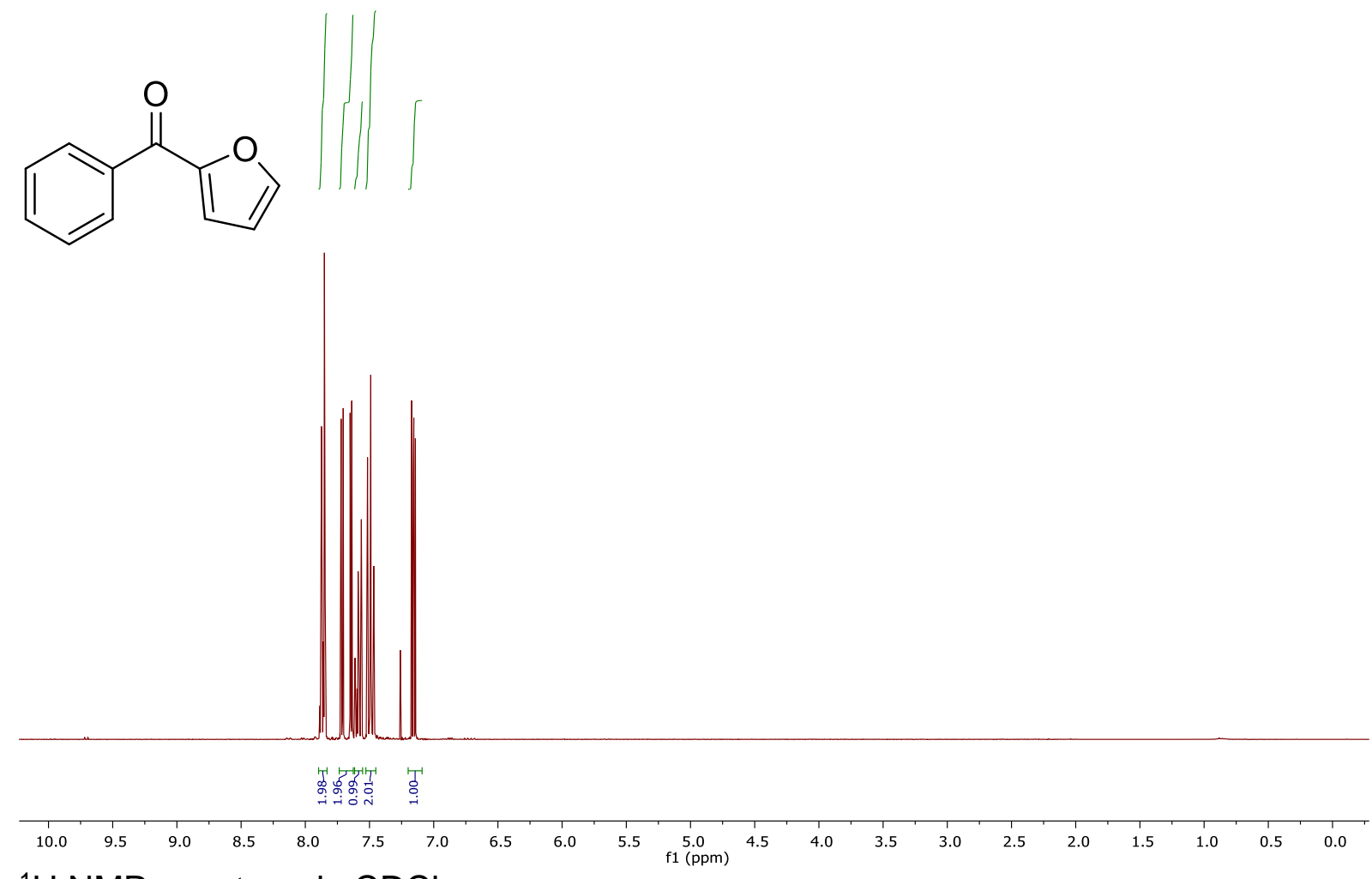

${ }^{1} \mathrm{H}$ NMR spectrum in $\mathrm{CDCl}_{3}$.
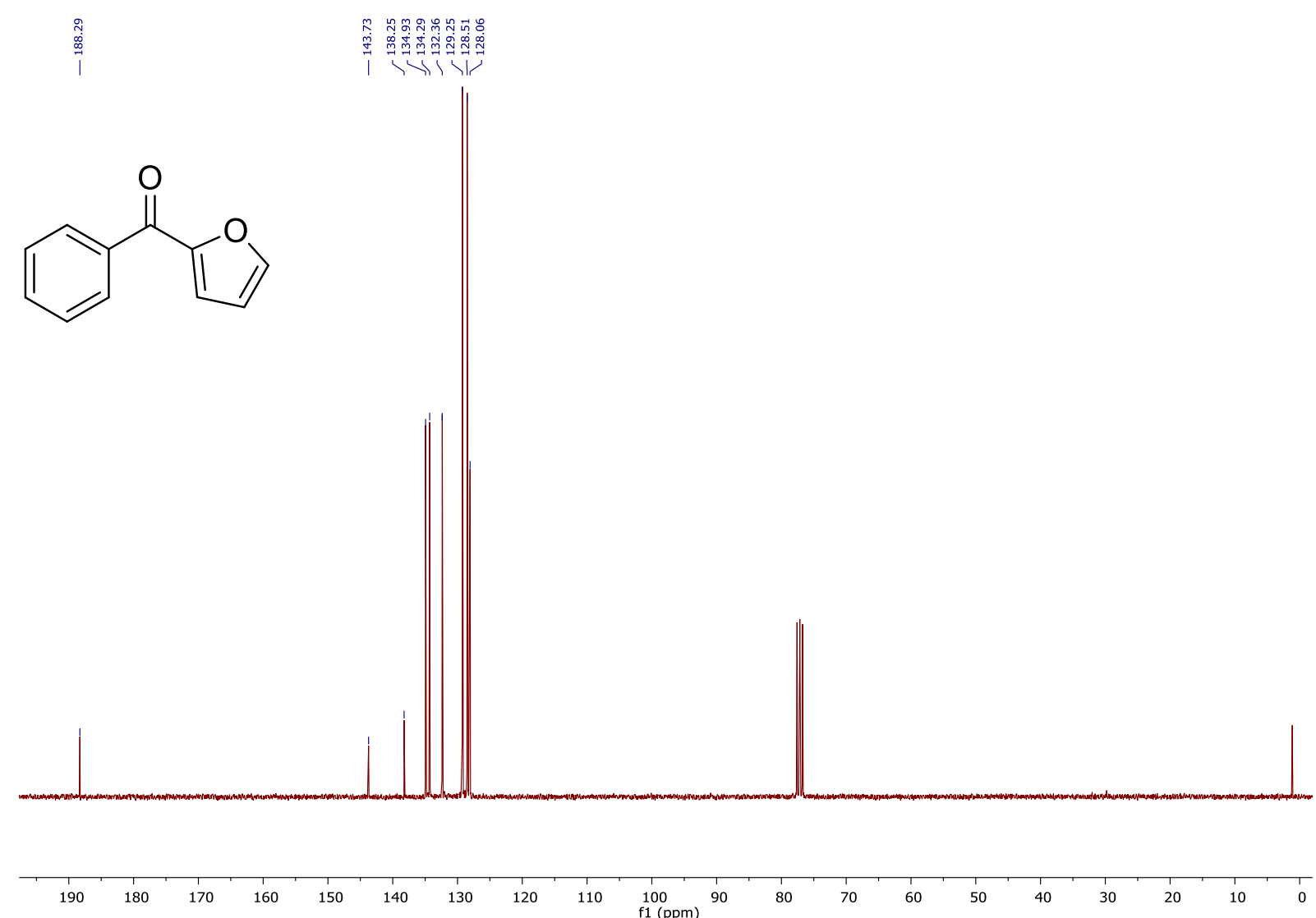
${ }^{13} \mathrm{C}$ NMR spectrum in $\mathrm{CDCl}_{3}$. 
$36 b$

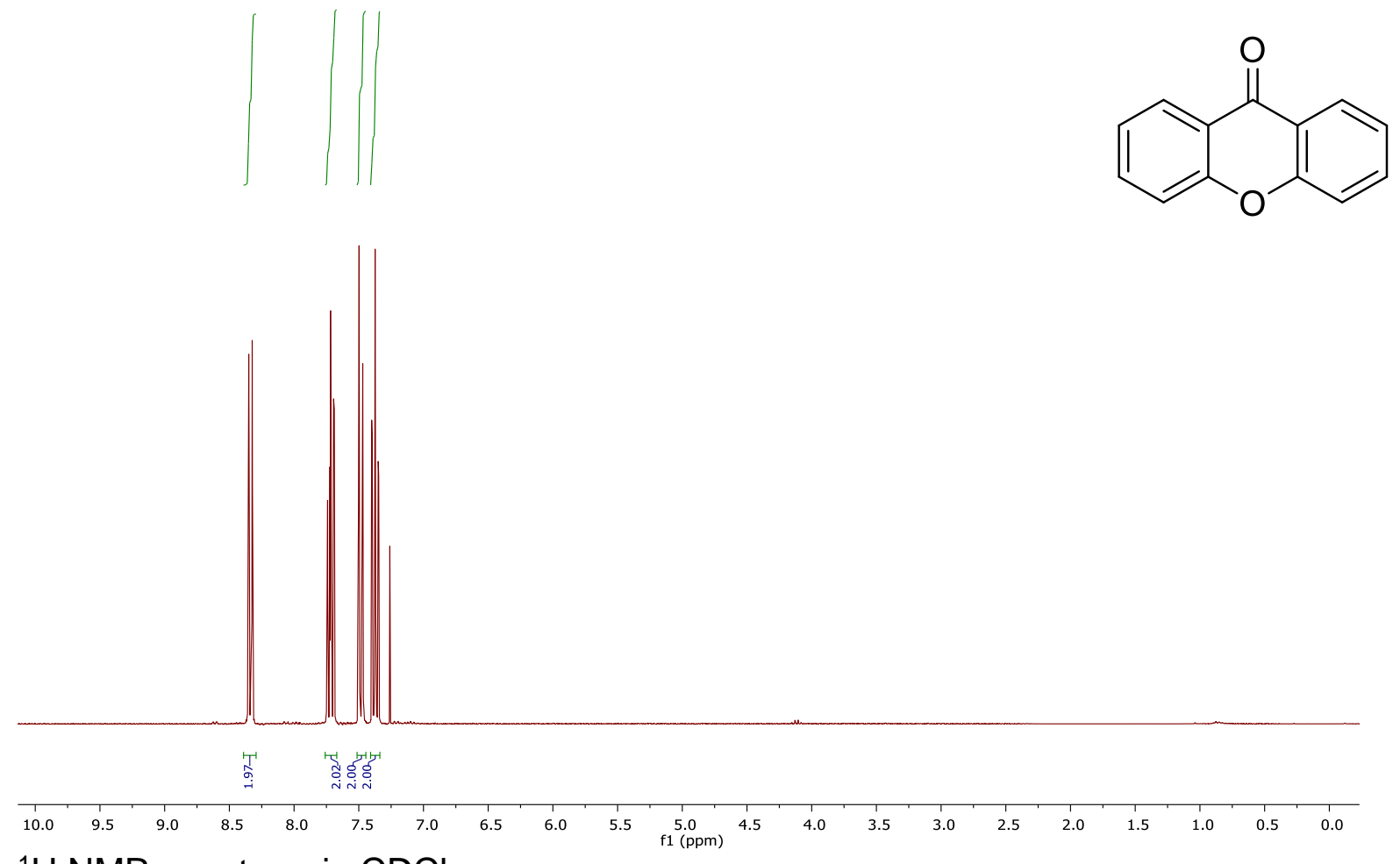

${ }^{1} \mathrm{H}$ NMR spectrum in $\mathrm{CDCl}_{3}$.

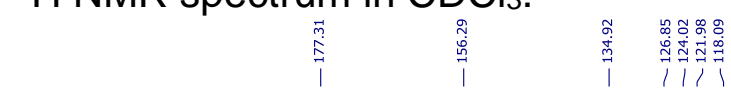

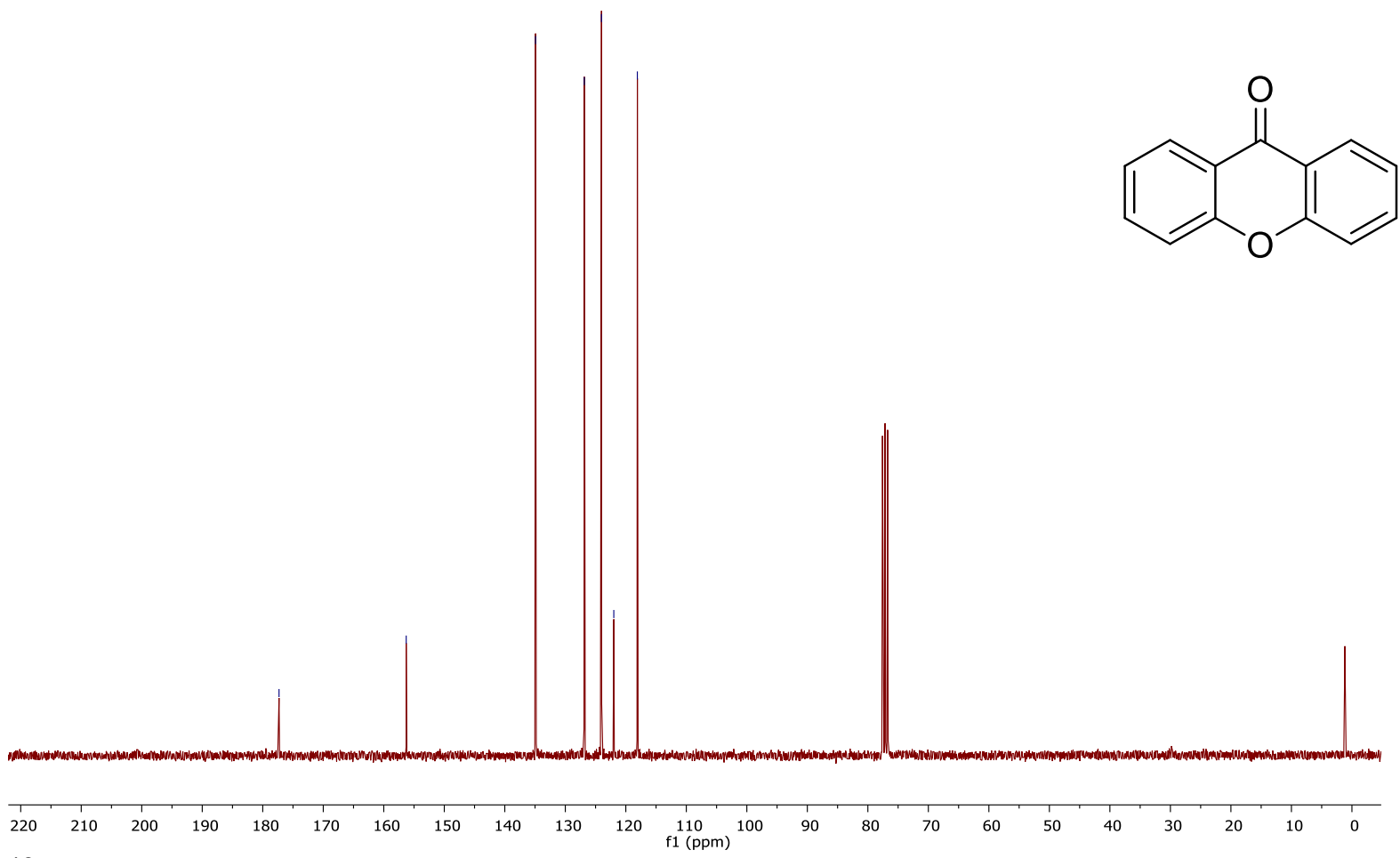

${ }^{13} \mathrm{C}$ NMR spectrum in $\mathrm{CDCl}_{3}$. 
<smiles>O=C1CCOc2ccccc21</smiles>
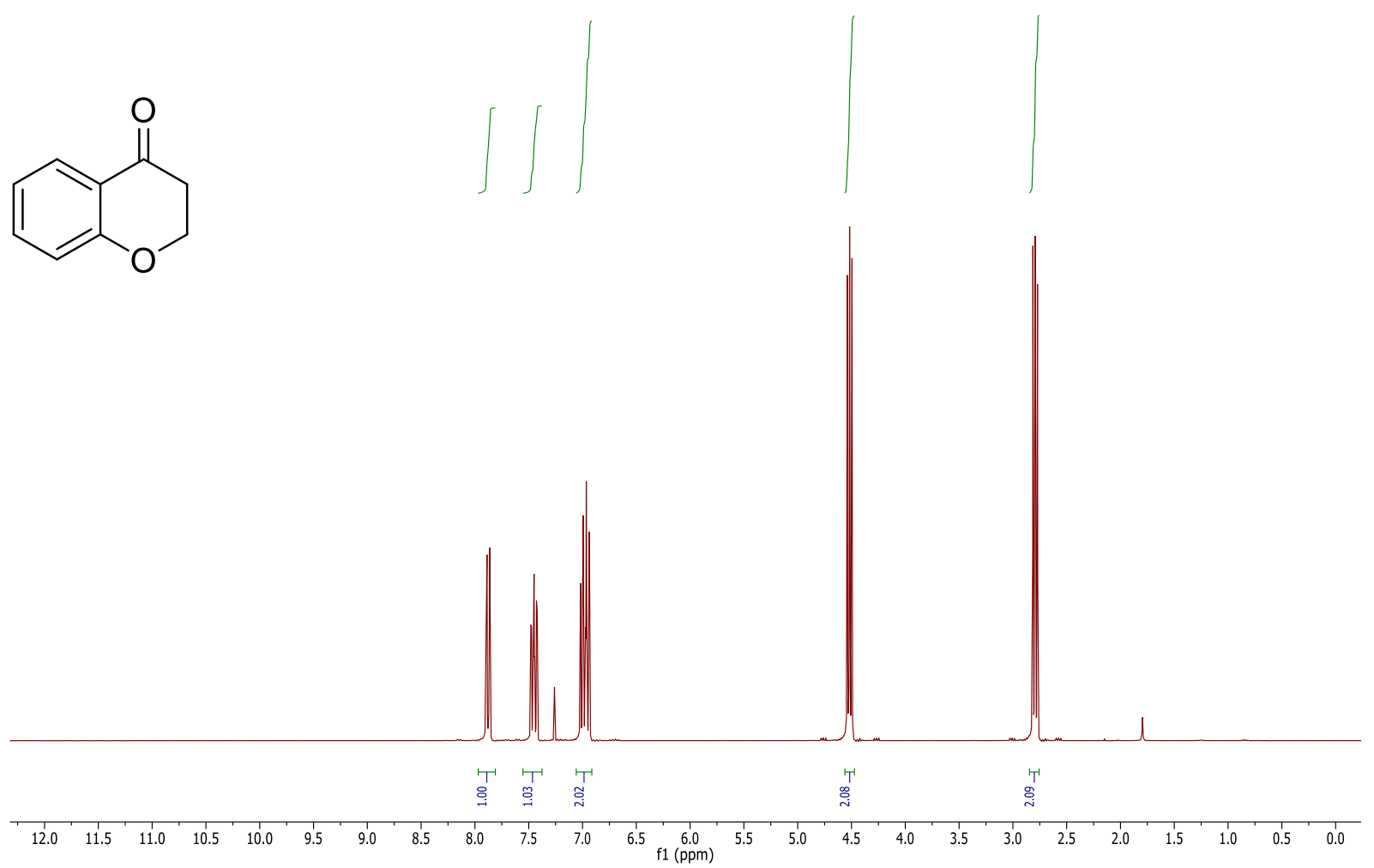

${ }^{1} \mathrm{H}$ NMR spectrum in $\mathrm{CDCl}_{3}$.<smiles>O=C1CCOc2ccccc21</smiles>

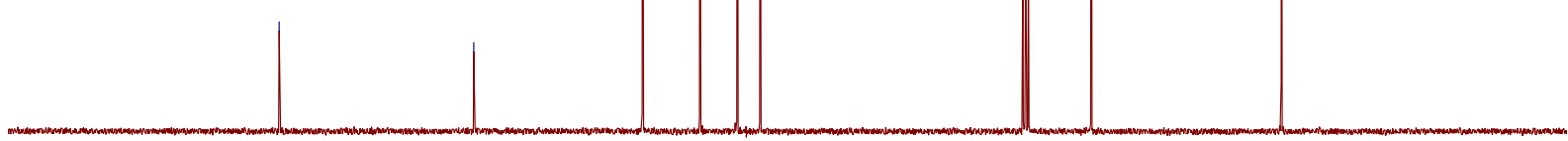

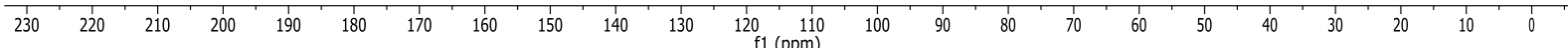
${ }^{13} \mathrm{C}$ NMR spectrum in $\mathrm{CDCl}_{3}$. 
$\prod_{\mathrm{O}} \int \mathrm{f} T$

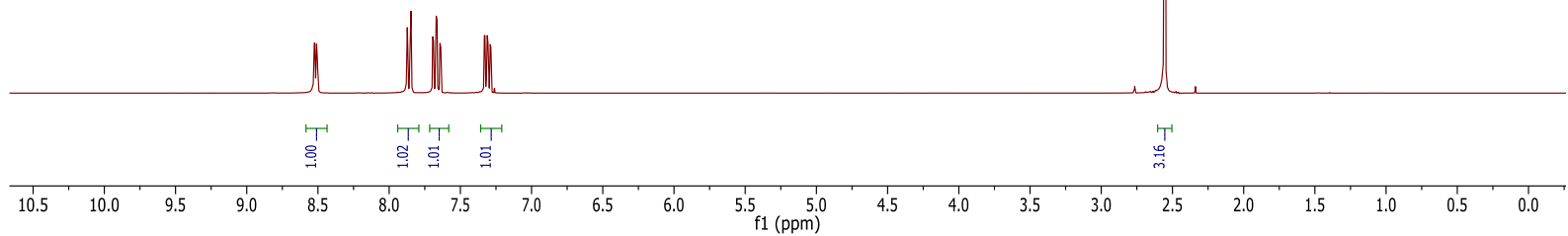

${ }^{1} \mathrm{H}$ NMR spectrum in $\mathrm{CDCl}_{3}$.<smiles>CC(=O)c1ccccn1</smiles>

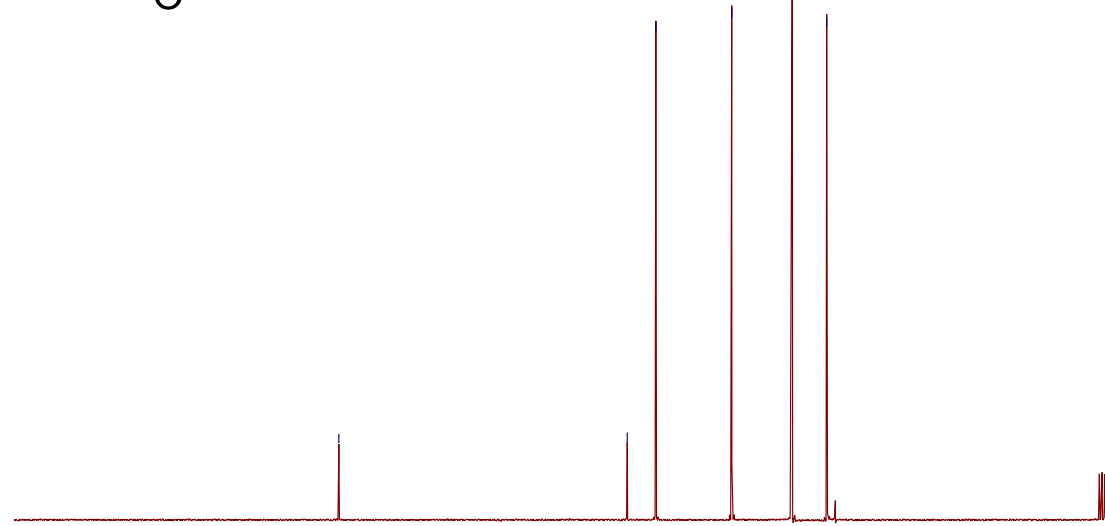

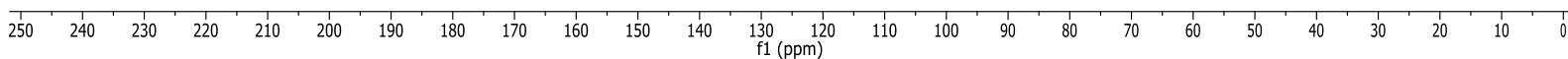
${ }^{13} \mathrm{C}$ NMR spectrum in $\mathrm{CDCl}_{3}$. 
<smiles>CCCCC(=O)CCCC</smiles>
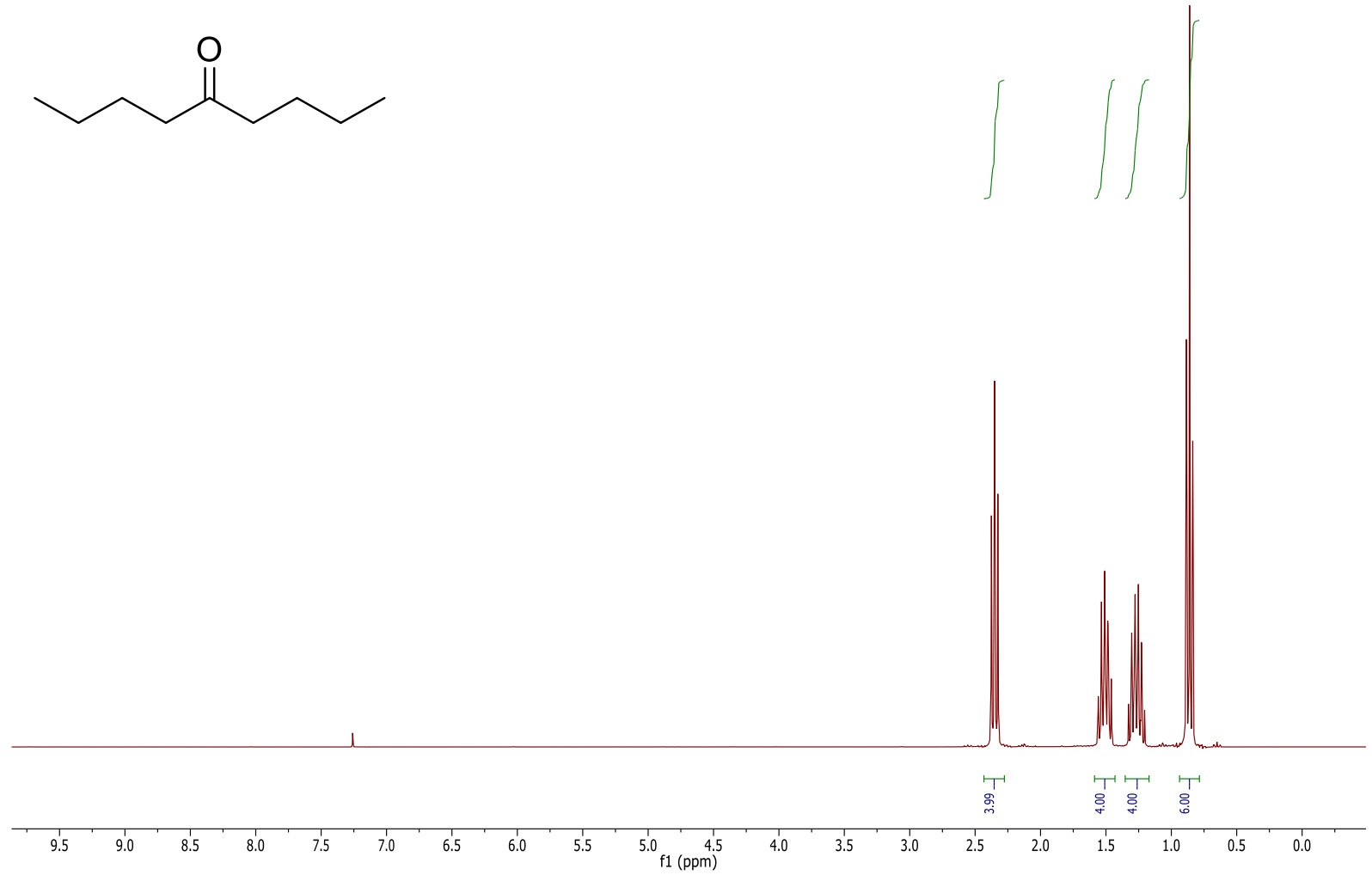

${ }^{1} \mathrm{H}$ NMR spectrum in $\mathrm{CDCl}_{3}$.

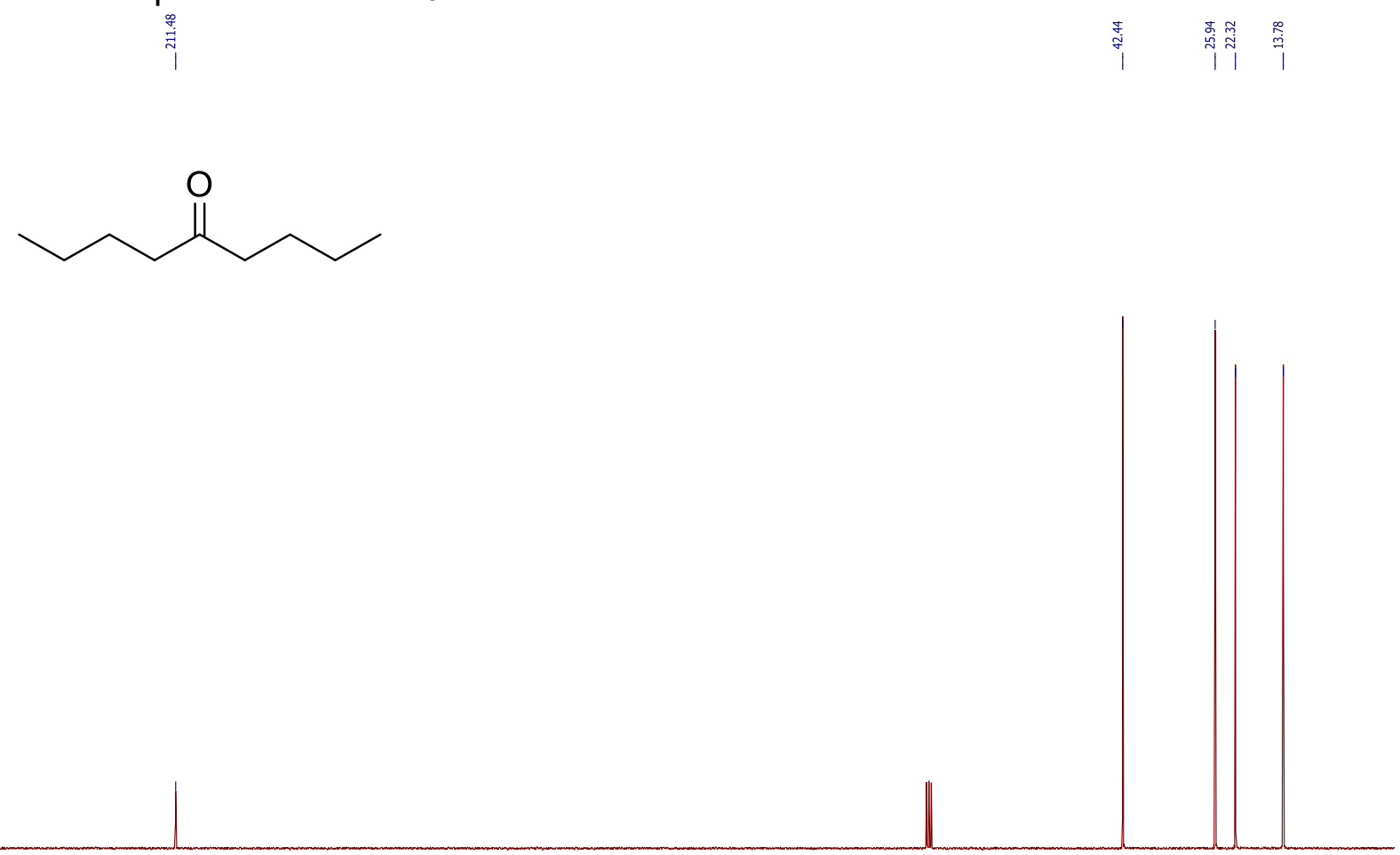

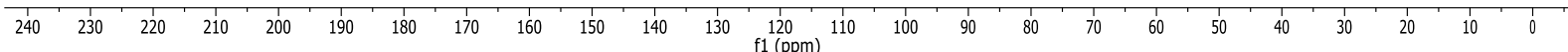
${ }^{13} \mathrm{C}$ NMR spectrum in $\mathrm{CDCl}_{3}$. 


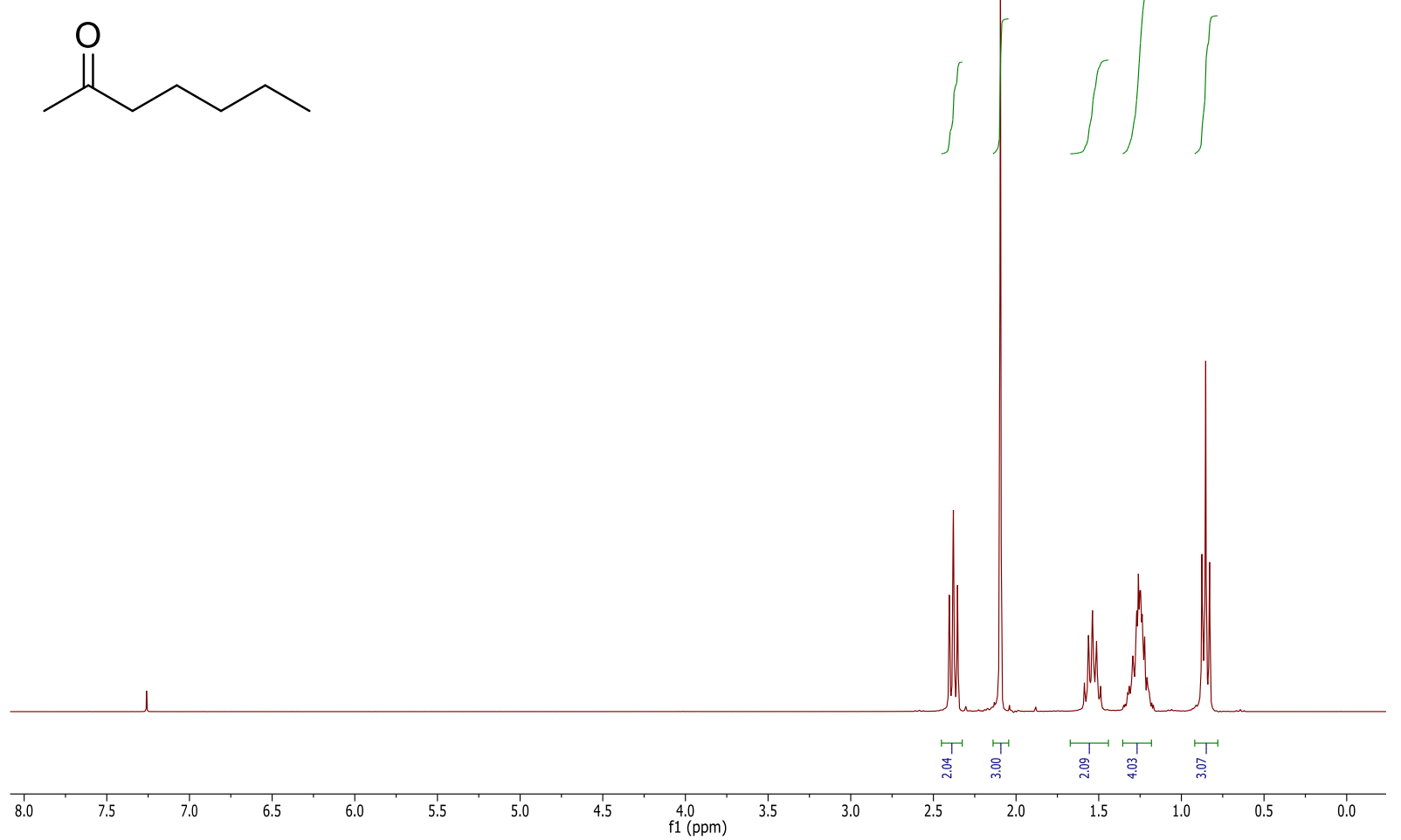

${ }^{1} \mathrm{H}$ NMR spectrum in $\mathrm{CDCl}_{3}$.<smiles>CCCCCC(C)=O</smiles>

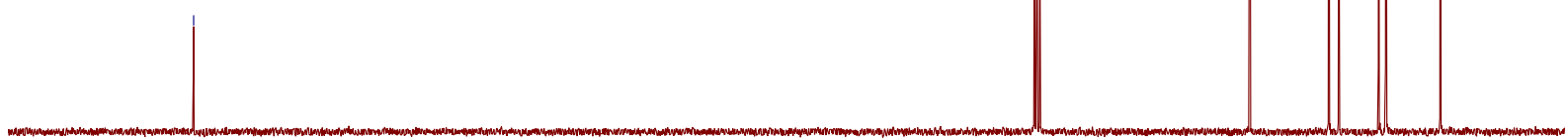

$\begin{array}{lllllllllllllllllllllllllll}230 & 220 & 210 & 200 & 190 & 180 & 170 & 160 & 150 & 140 & 130 & 120 & 110 & 100 & 90 & 80 & 70 & 60 & 50 & 40 & 30 & 20 & 10 & 0\end{array}$ ${ }^{13} \mathrm{C}$ NMR spectrum in $\mathrm{CDCl}_{3}$. 


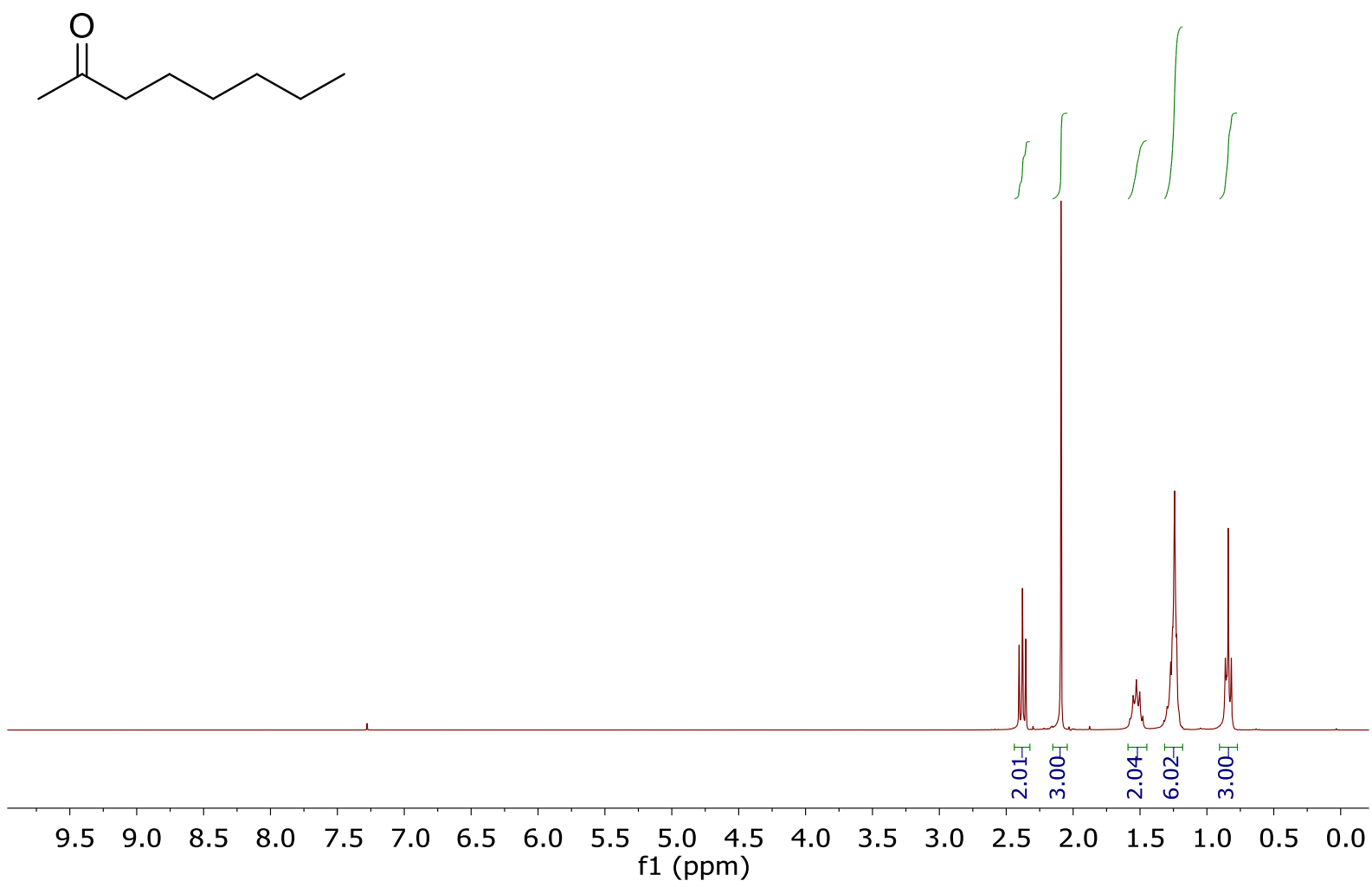
${ }^{1} \mathrm{H}$ NMR spectrum in $\mathrm{CDCl}_{3}$.<smiles>CCCCCCC(C)=O</smiles>

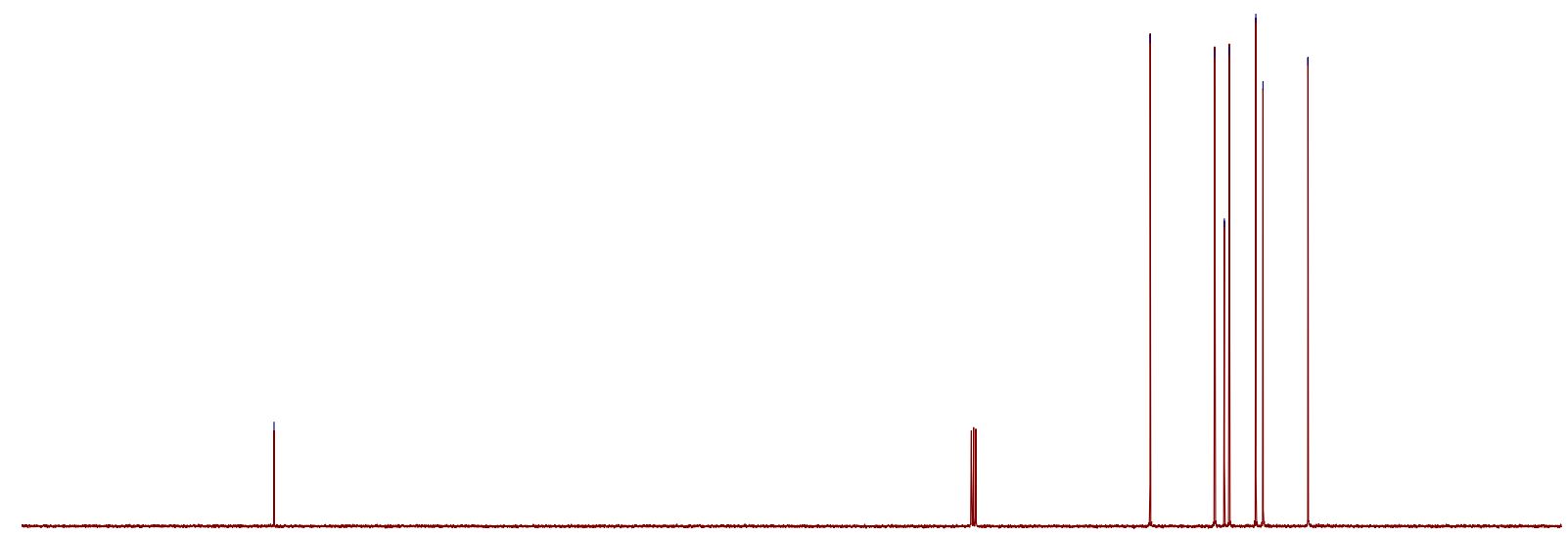

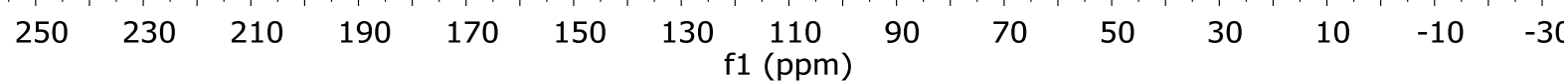
${ }^{13} \mathrm{C}$ NMR spectrum in $\mathrm{CDCl}_{3}$. 
$42 b$

$\sim^{\sim}$

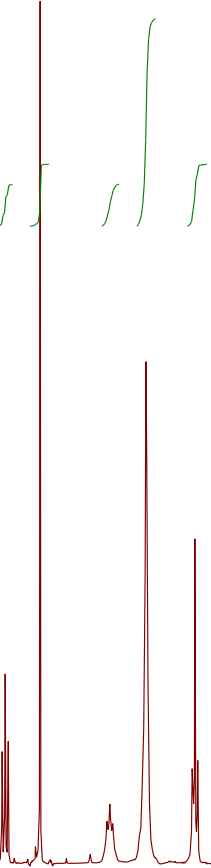

TT TT T T

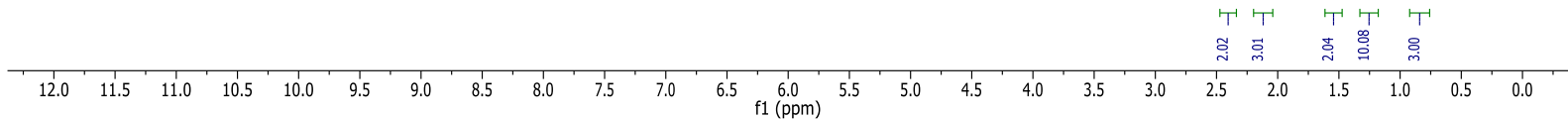

${ }^{1} \mathrm{H}$ NMR spectrum in $\mathrm{CDCl}_{3}$.

素

事

|

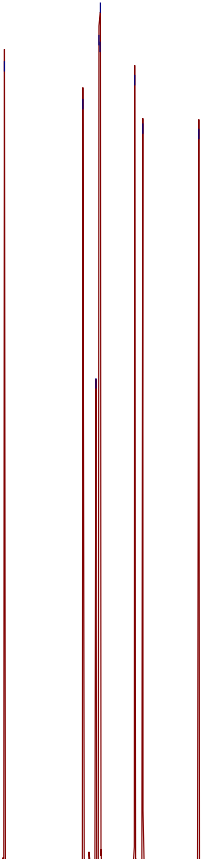

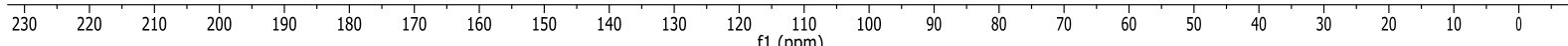
${ }^{13} \mathrm{C}$ NMR spectrum in $\mathrm{CDCl}_{3}$. 
$43 b$

$\overbrace{}^{1}$

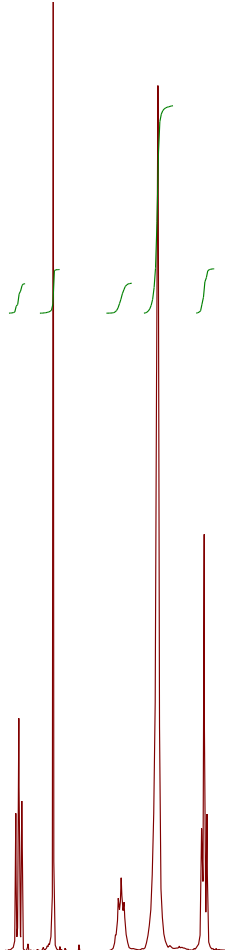

TT TT TT

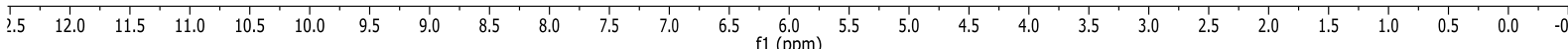
${ }^{1} \mathrm{H}$ NMR spectrum in $\mathrm{CDCl}_{3}$.

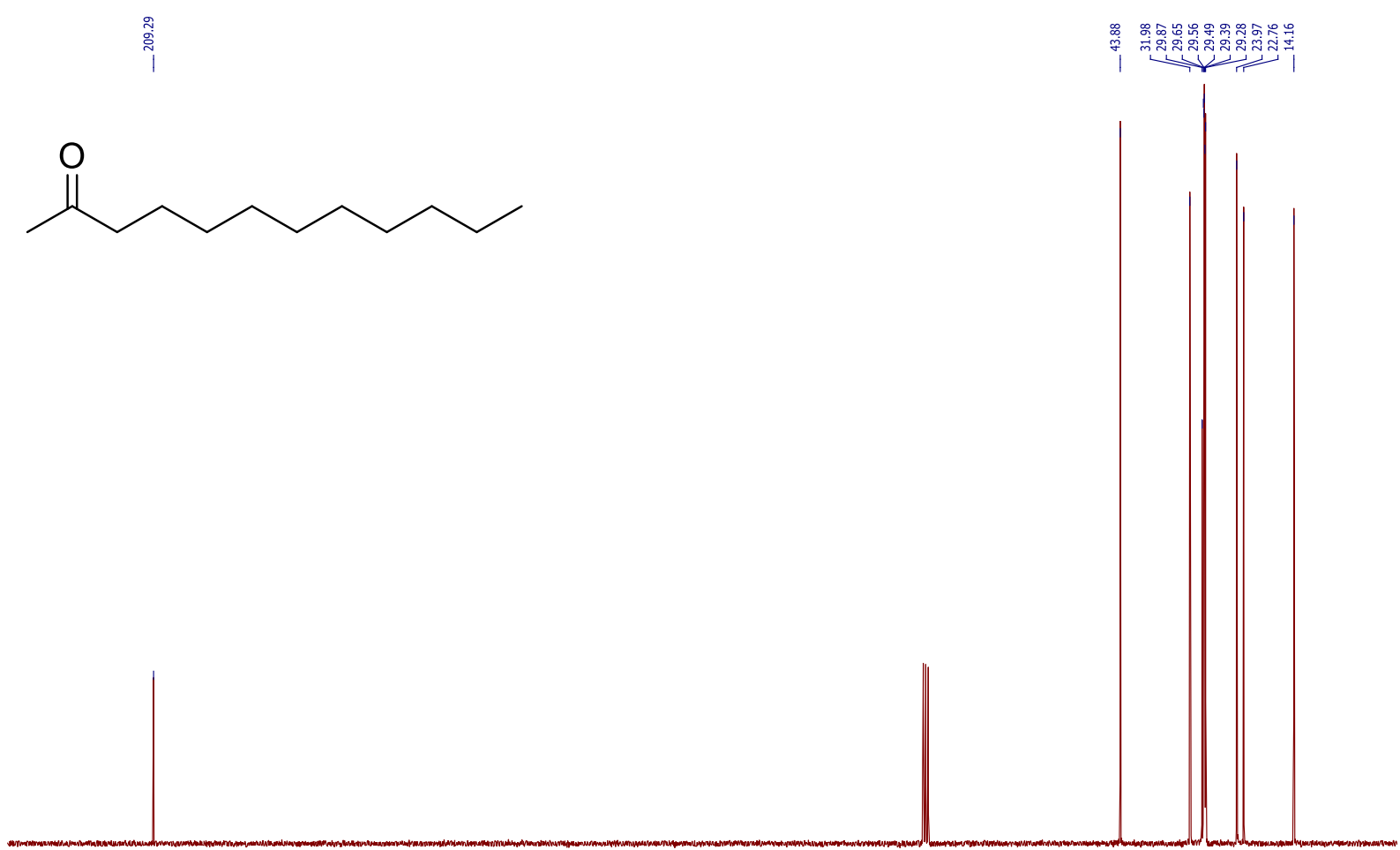

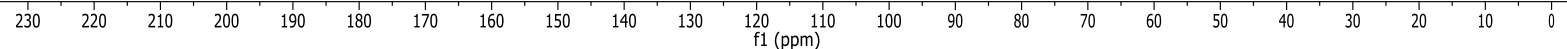
${ }^{13} \mathrm{C}$ NMR spectrum in $\mathrm{CDCl}_{3}$. 
45b
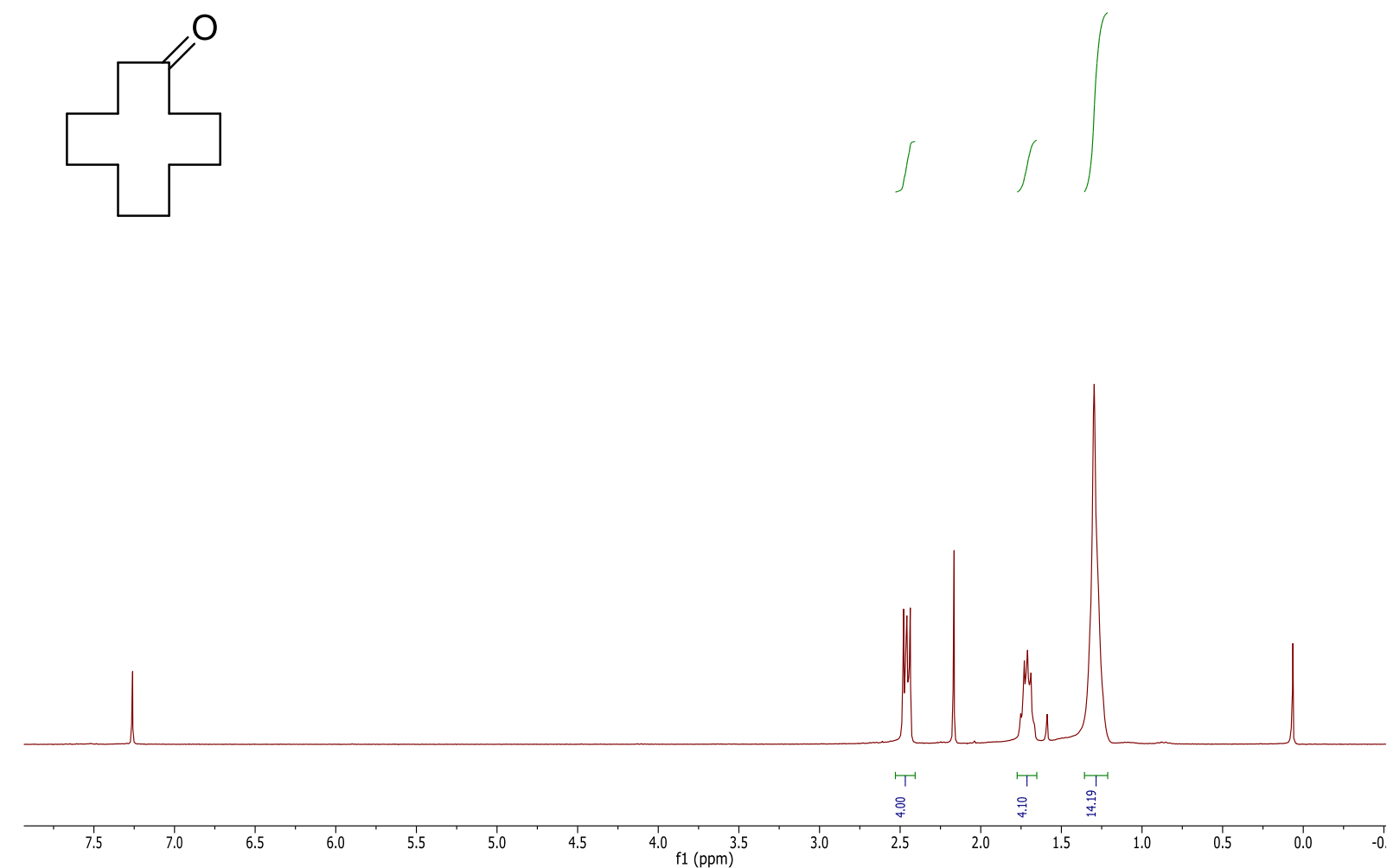

${ }^{1} \mathrm{H}$ NMR spectrum in $\mathrm{CDCl}_{3}$.

$\stackrel{\stackrel{0}{\pi}}{\sim}$
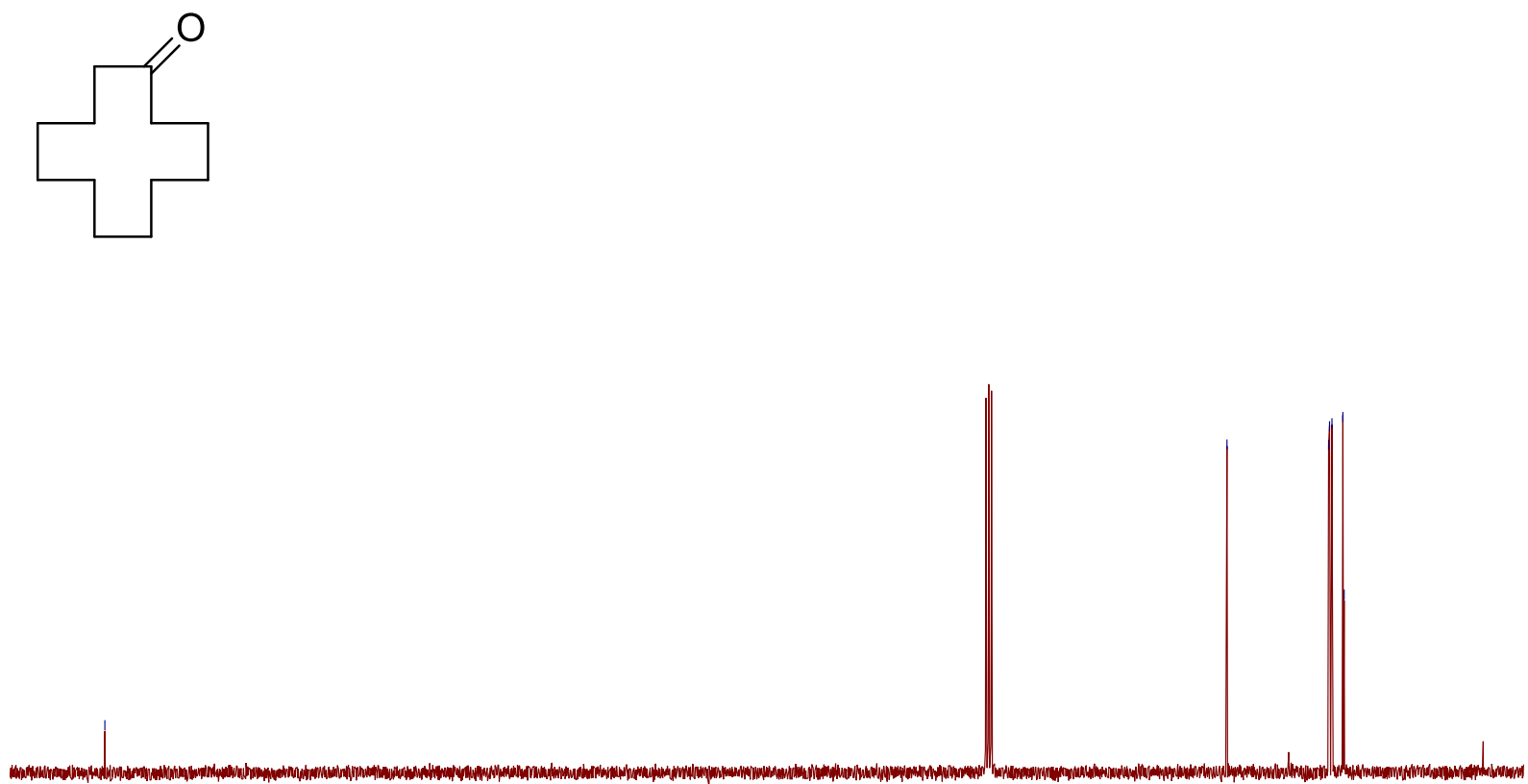

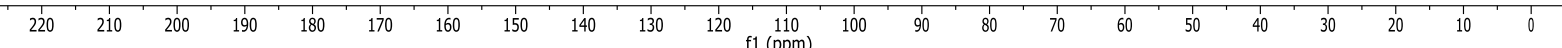
${ }^{13} \mathrm{C}$ NMR spectrum in $\mathrm{CDCl}_{3}$. 
46b

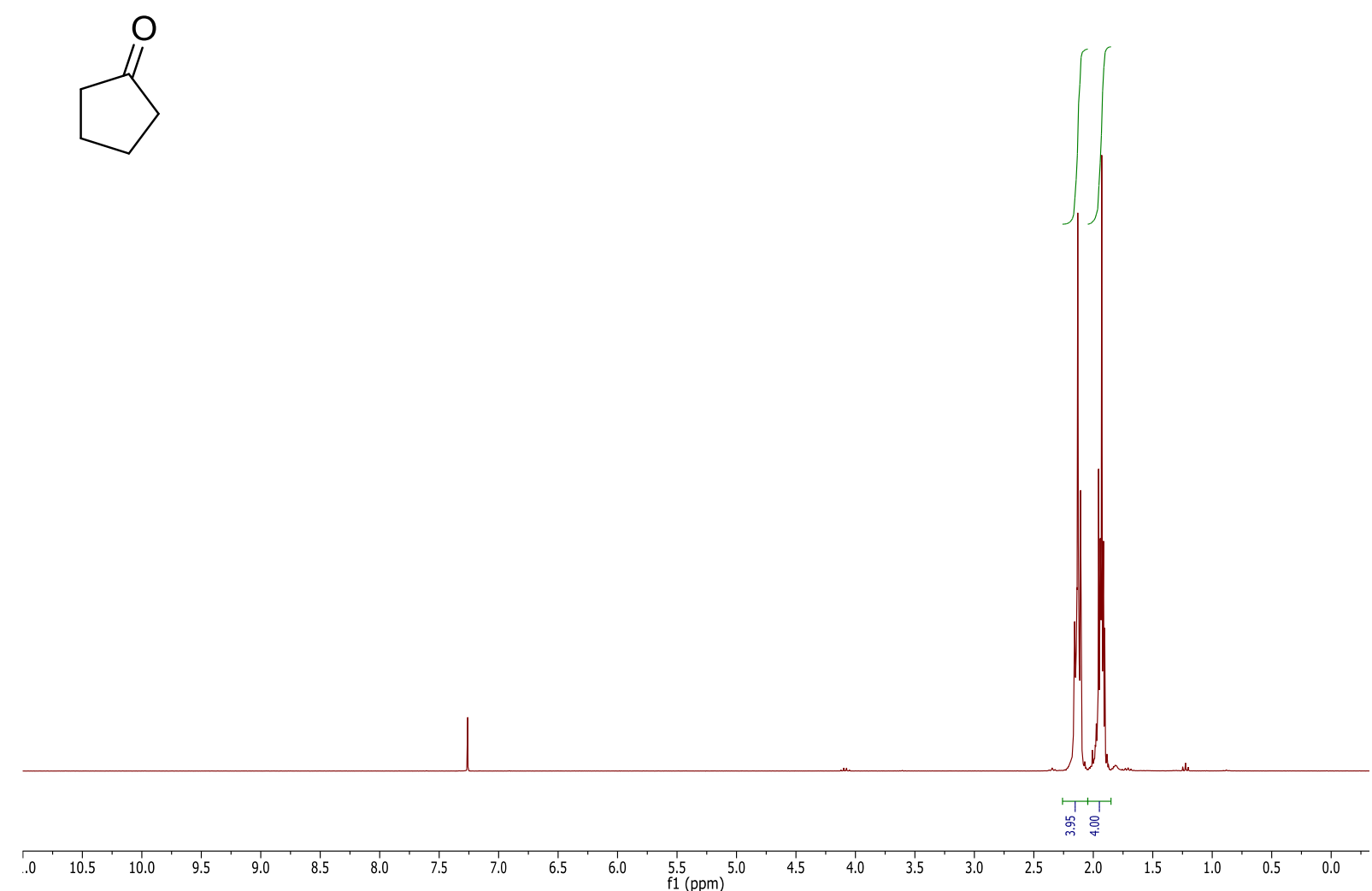

${ }^{1} \mathrm{H}$ NMR spectrum in $\mathrm{CDCl}_{3}$.

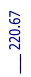

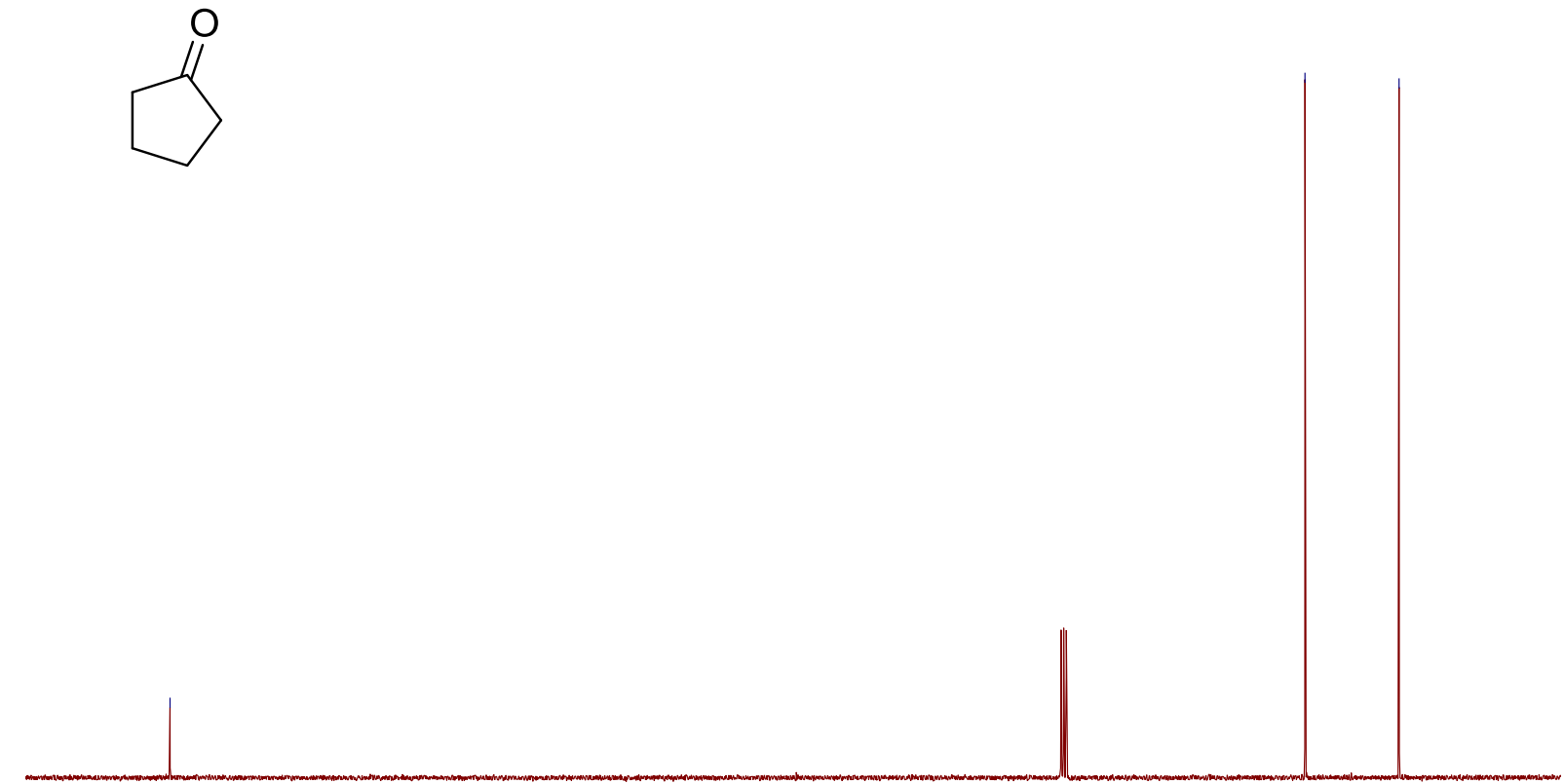

\begin{tabular}{lllllllllllllllllllllllllll}
\hline 240 & 230 & 220 & 210 & 200 & 190 & 180 & 170 & 160 & 150 & 140 & 130 & 120 & 110 & 100 & 90 & 80 & 70 & 60 & 50 & 40 & 30 & 20 & 10 & 0
\end{tabular} ${ }^{13} \mathrm{C}$ NMR spectrum in $\mathrm{CDCl}_{3}$. 
47b

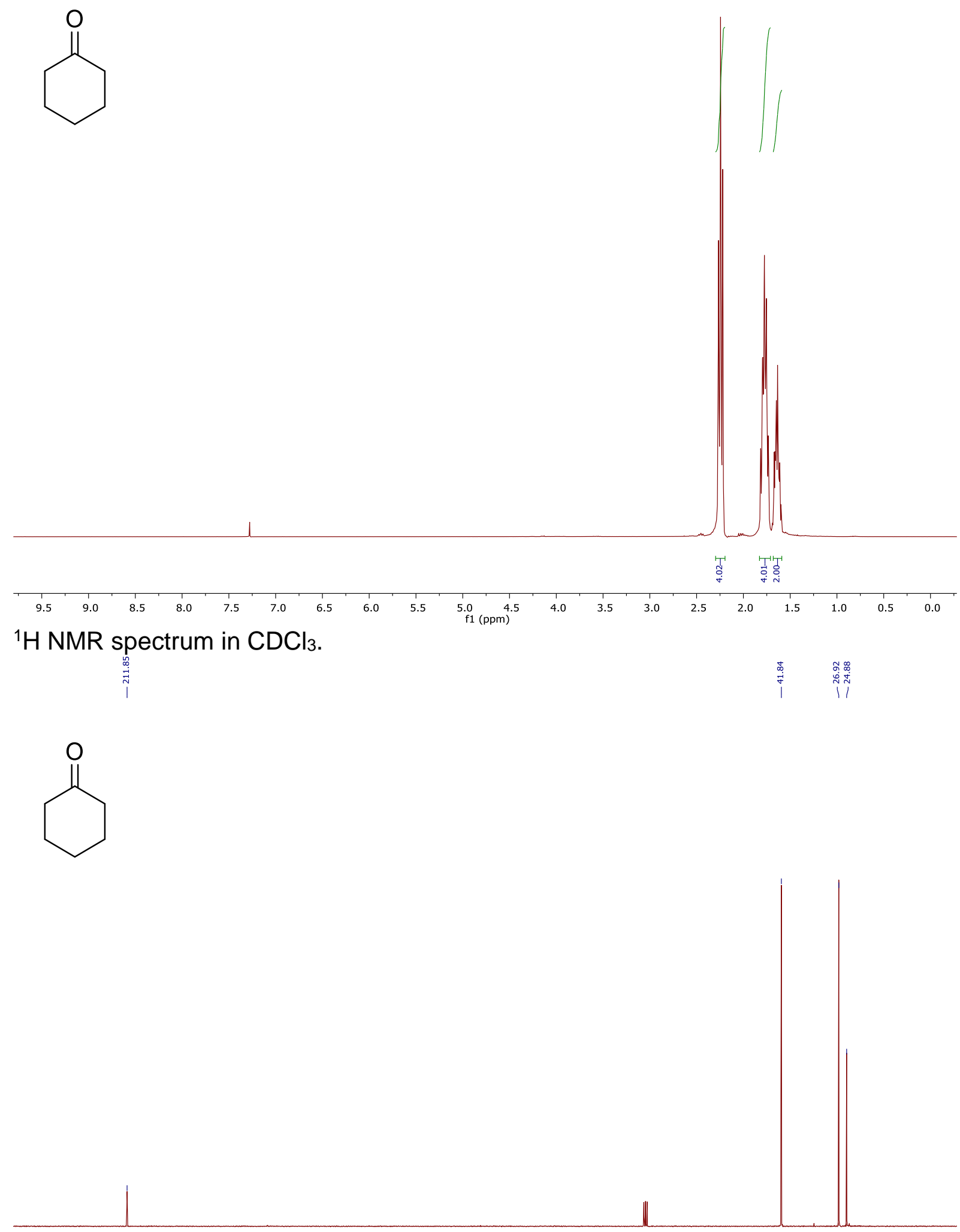

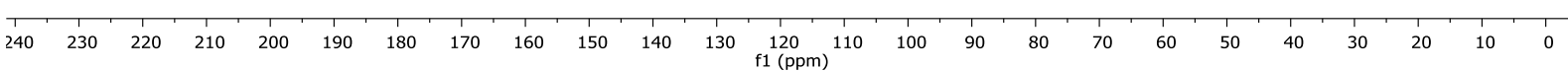
${ }^{13} \mathrm{C}$ NMR spectrum in $\mathrm{CDCl}_{3}$. 
<smiles>C[C@]12CC[C@H]3[C@@H](CCC4=CC(=O)CC[C@]43C)[C@@H]1CCC2=O</smiles>
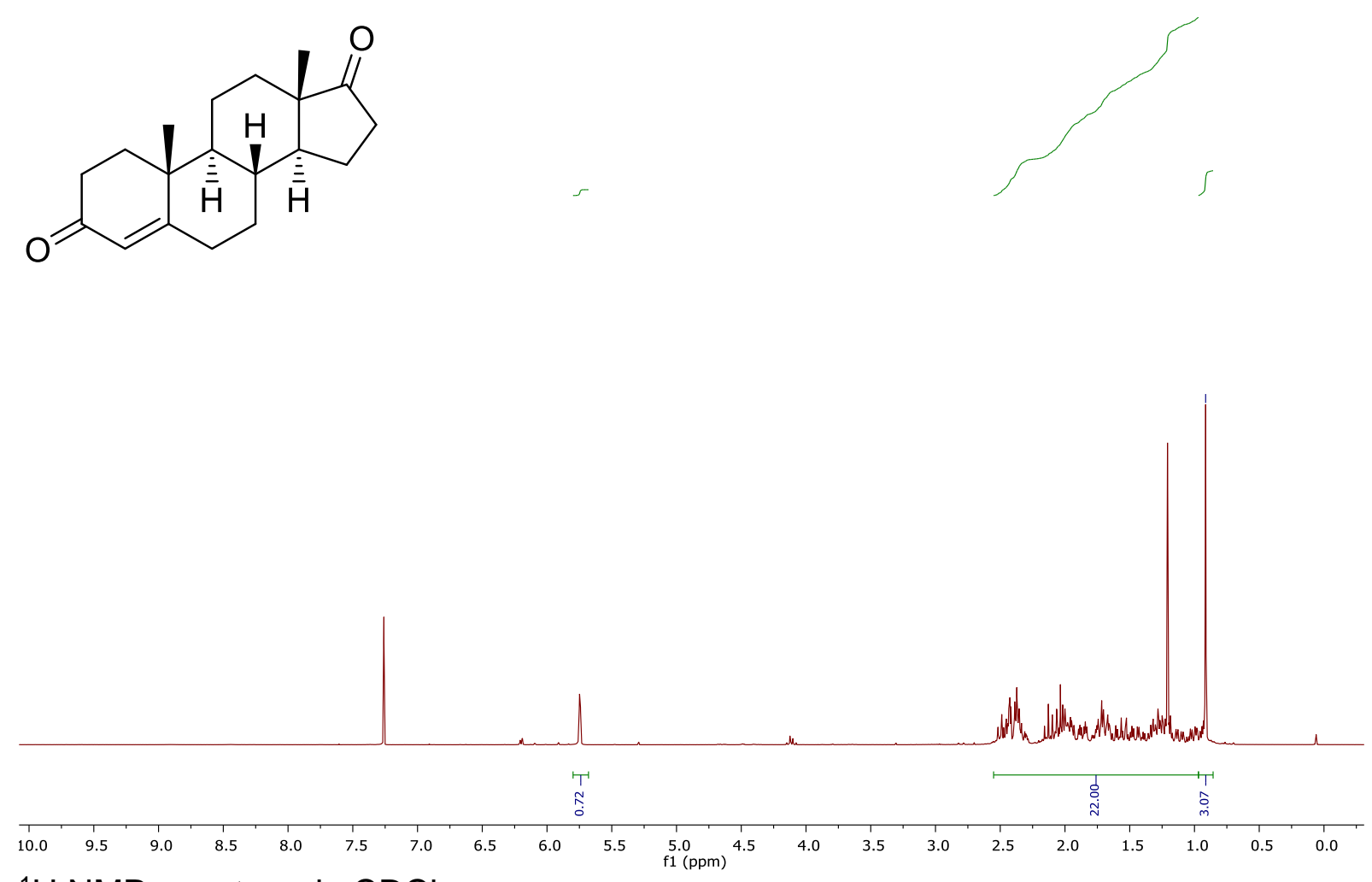

${ }^{1} \mathrm{H}$ NMR spectrum in $\mathrm{CDCl}_{3}$.

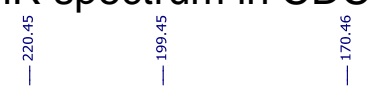

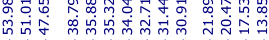

111 सोik 111<smiles>C[C@]12CC[C@H]3[C@@H](CCC4=CC(=O)CC[C@]43C)[C@@H]1CCC2=O</smiles>
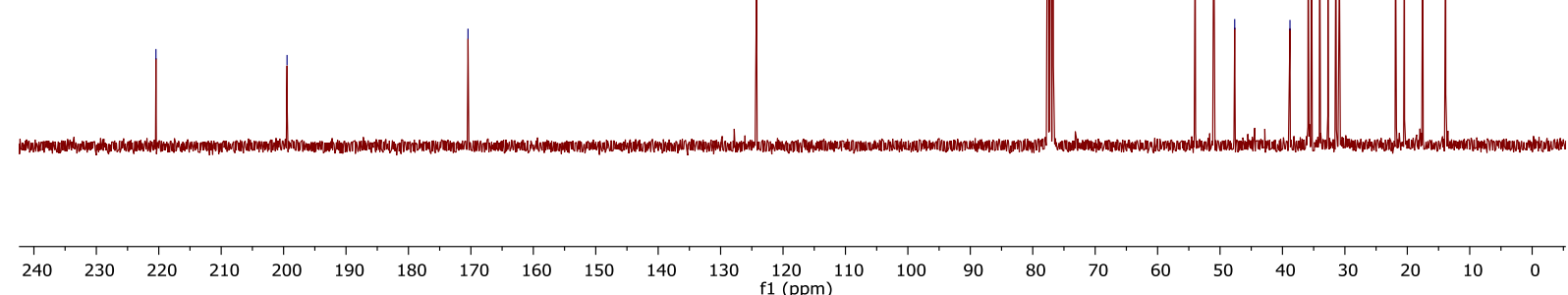
${ }^{13} \mathrm{C}$ NMR spectrum in $\mathrm{CDCl}_{3}$. 
$48 b$<smiles>C[C@]12CC[C@H]3[C@@H](CCC4=CC(=O)CC[C@]43C)[C@@H]1CCC2=O</smiles>

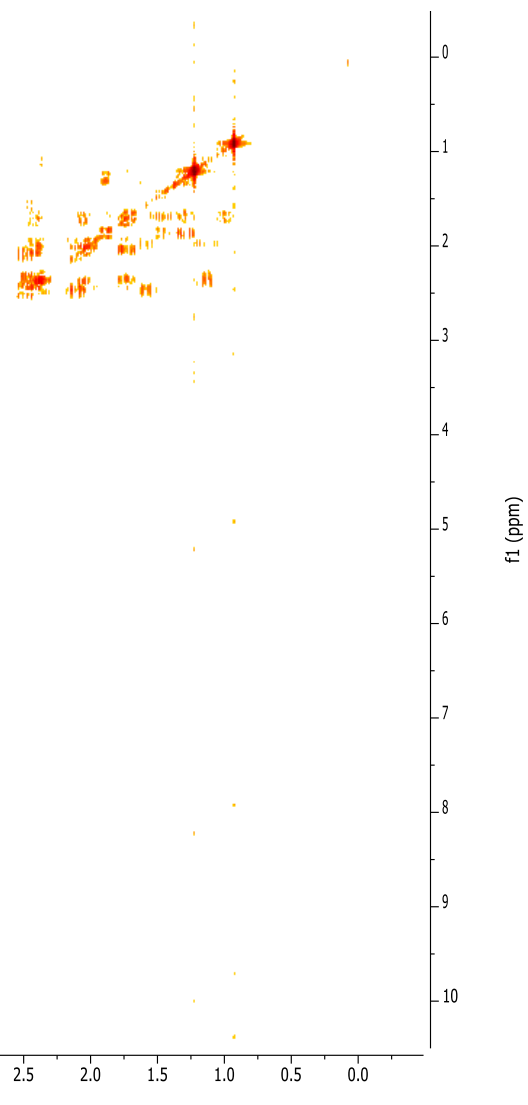

COSY spectrum in $\mathrm{CDCl}_{3}$.<smiles>C[C@]12CC[C@H]3[C@@H](CCC4=CC(=O)CC[C@]43C)[C@@H]1CCC2=O</smiles>

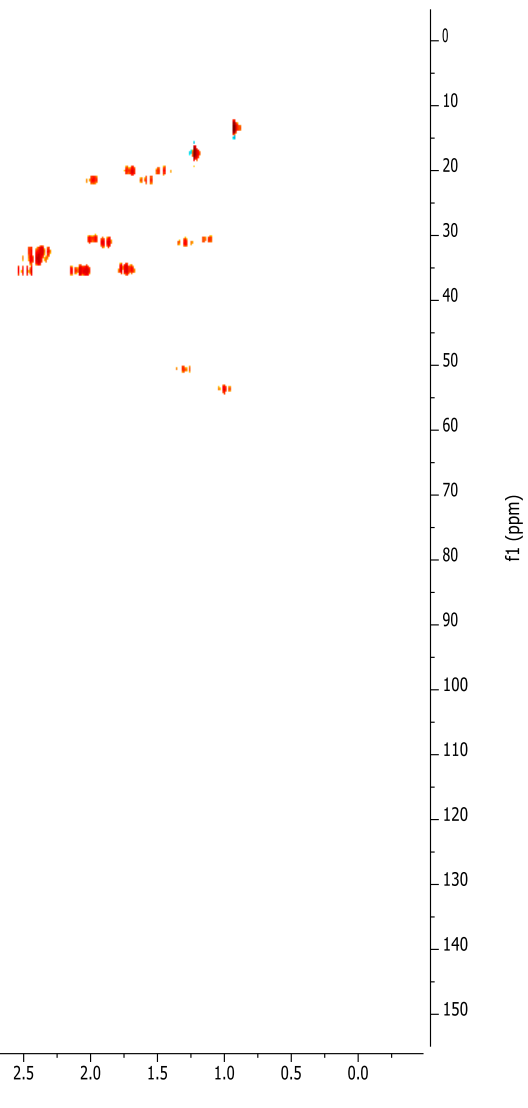

HSQC spectrum in $\mathrm{CDCl}_{3}$. 

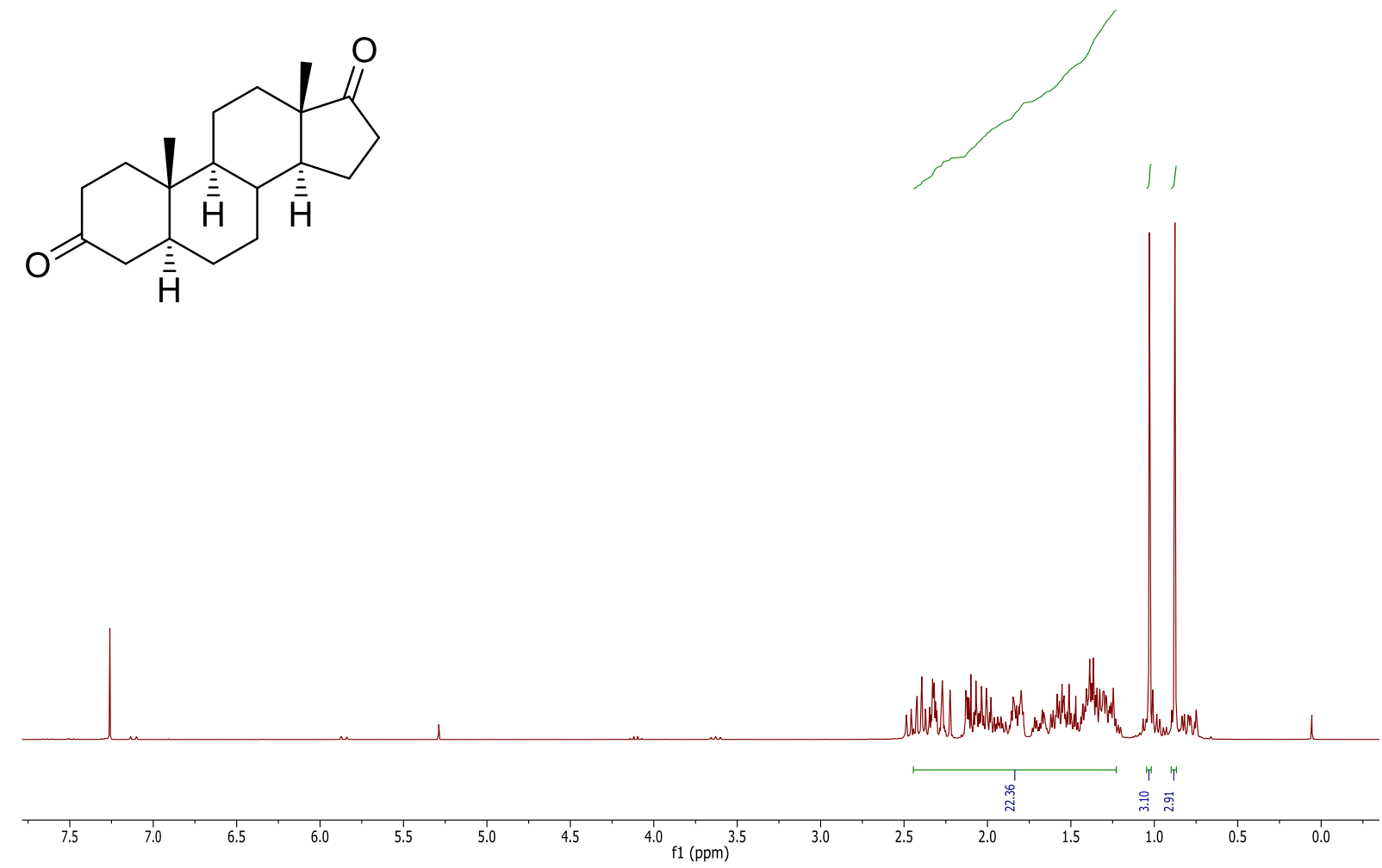

${ }^{1} \mathrm{H}$ NMR spectrum in $\mathrm{CDCl}_{3}$.
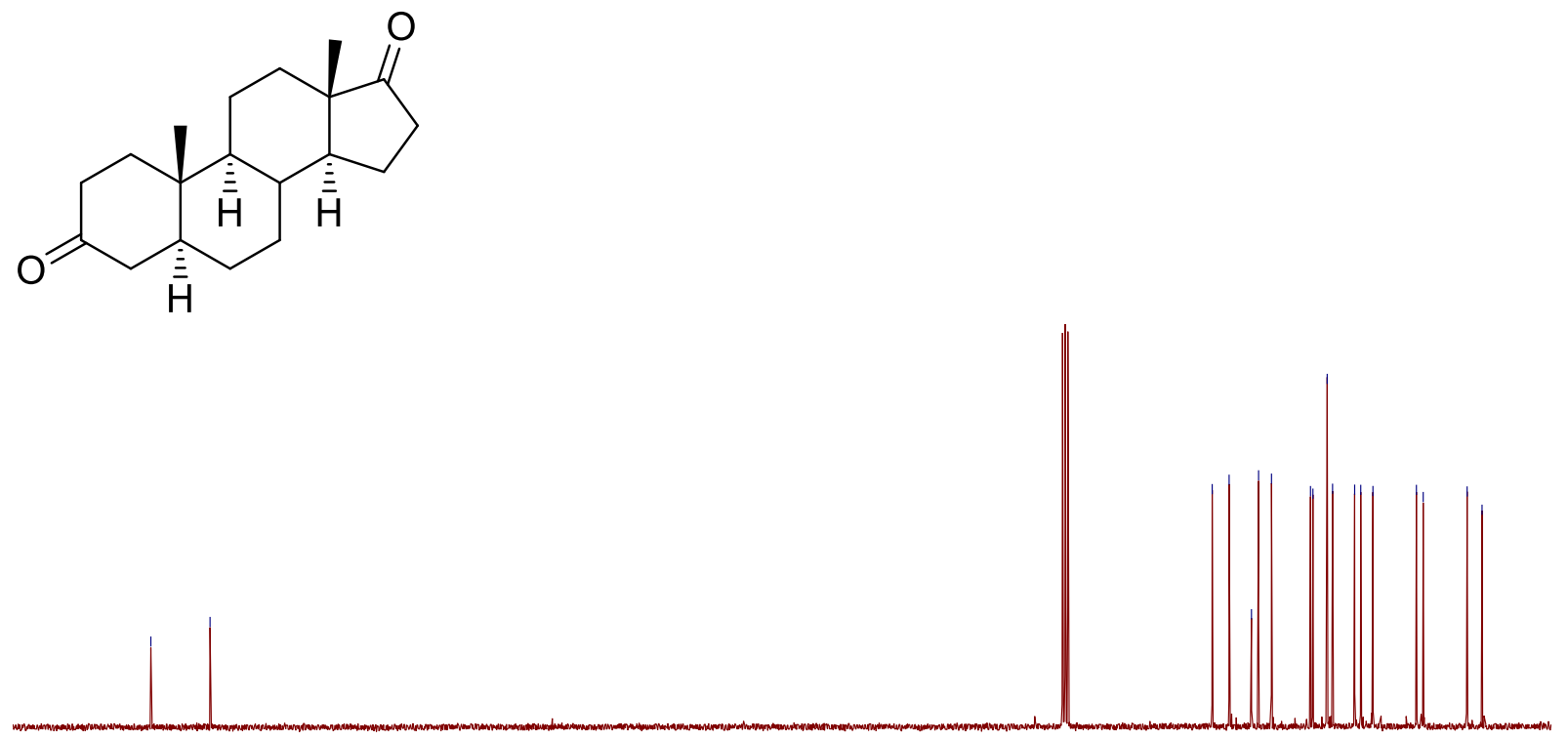

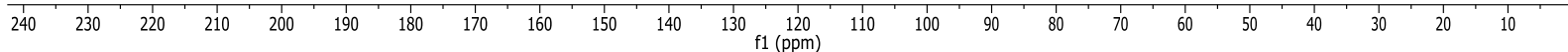
${ }^{13} \mathrm{C}$ NMR spectrum in $\mathrm{CDCl}_{3}$. 
49/50b<smiles>C[C@]12CC[C@H]3C(CC[C@@H]4CC(=O)CC[C@]34C)[C@@H]1CCC2=O</smiles>

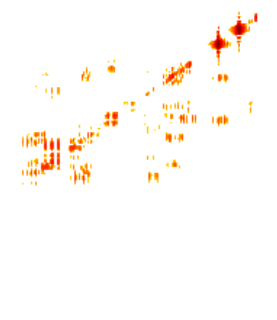<smiles>C[C@]12CC[C@H]3C(CC[C@@H]4CC(=O)CC[C@@H]43)[C@@H]1CCC2=O</smiles>

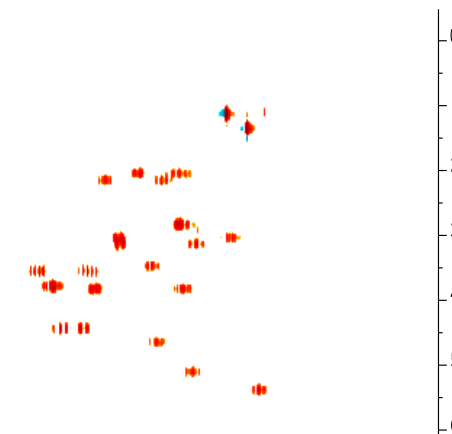

COSY spectrum in $\mathrm{CDCl}_{3}$.

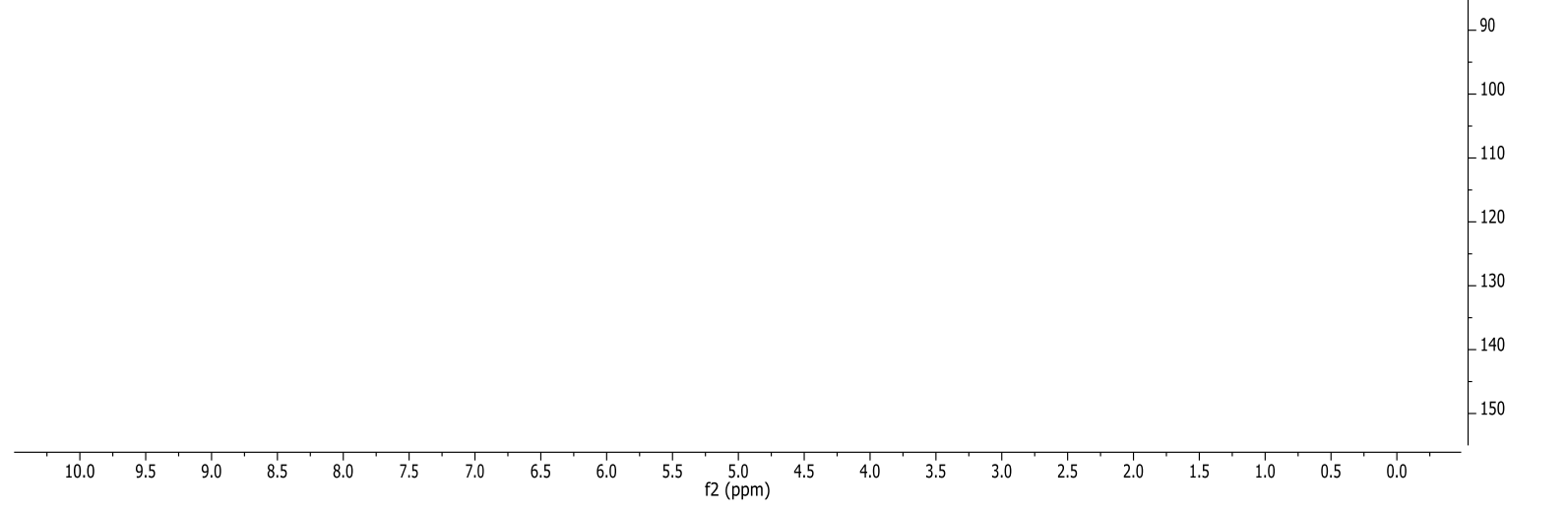
$\mathrm{HSQC}$ spectrum in $\mathrm{CDCl}_{3}$. 

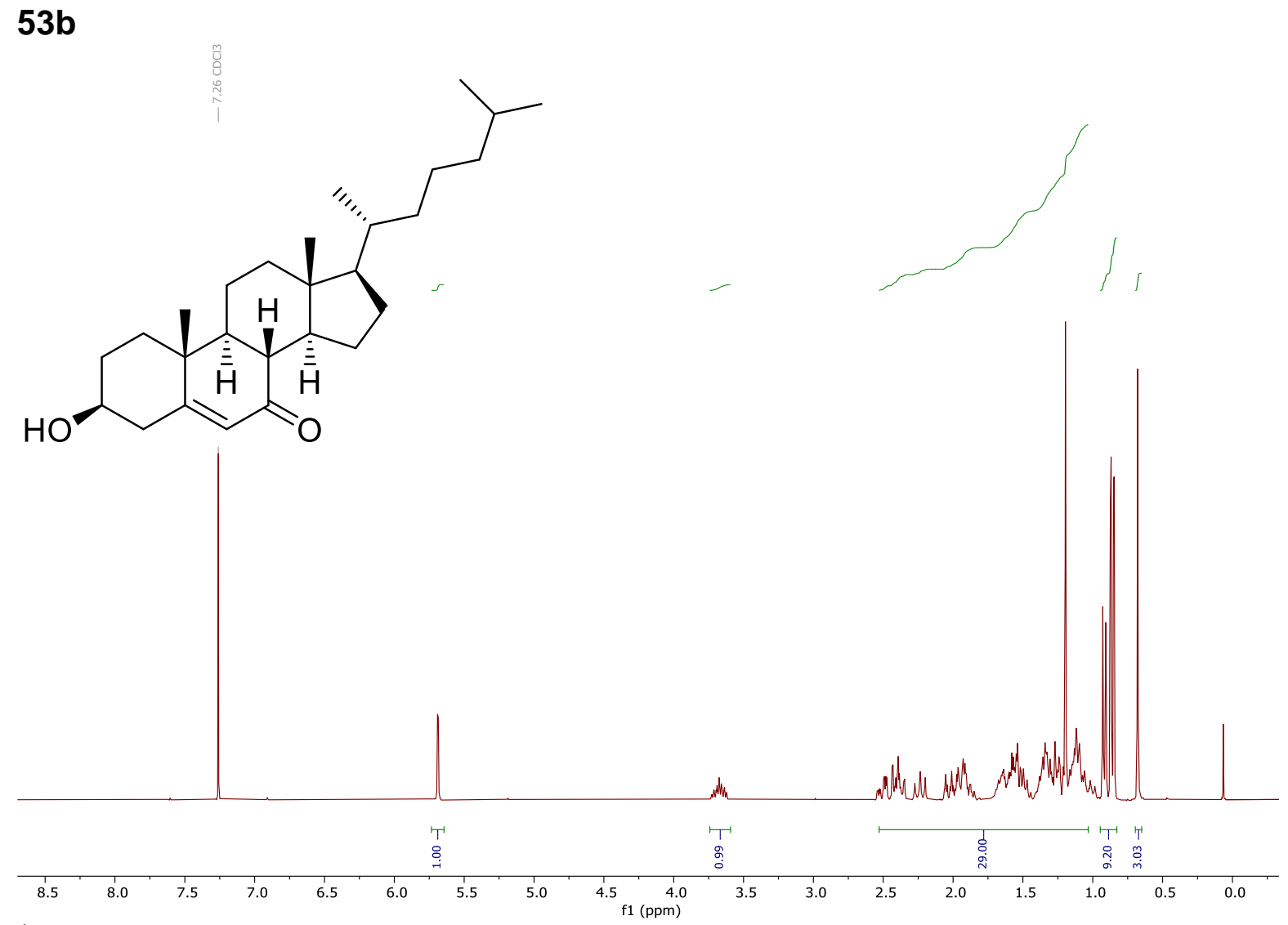

${ }^{1} \mathrm{H}$ NMR spectrum in $\mathrm{CDCl}_{3}$.

嘀

商

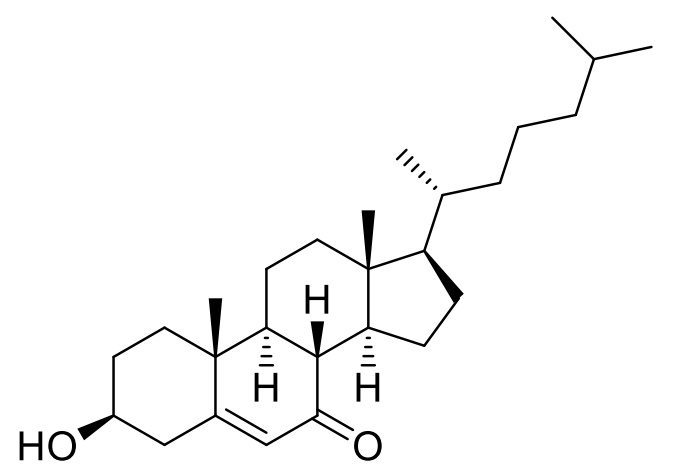

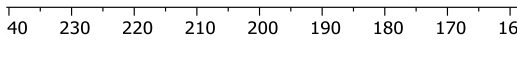

${ }^{13} \mathrm{C}$ NMR spectrum in $\mathrm{CDCl}_{3}$. 
$53 b$

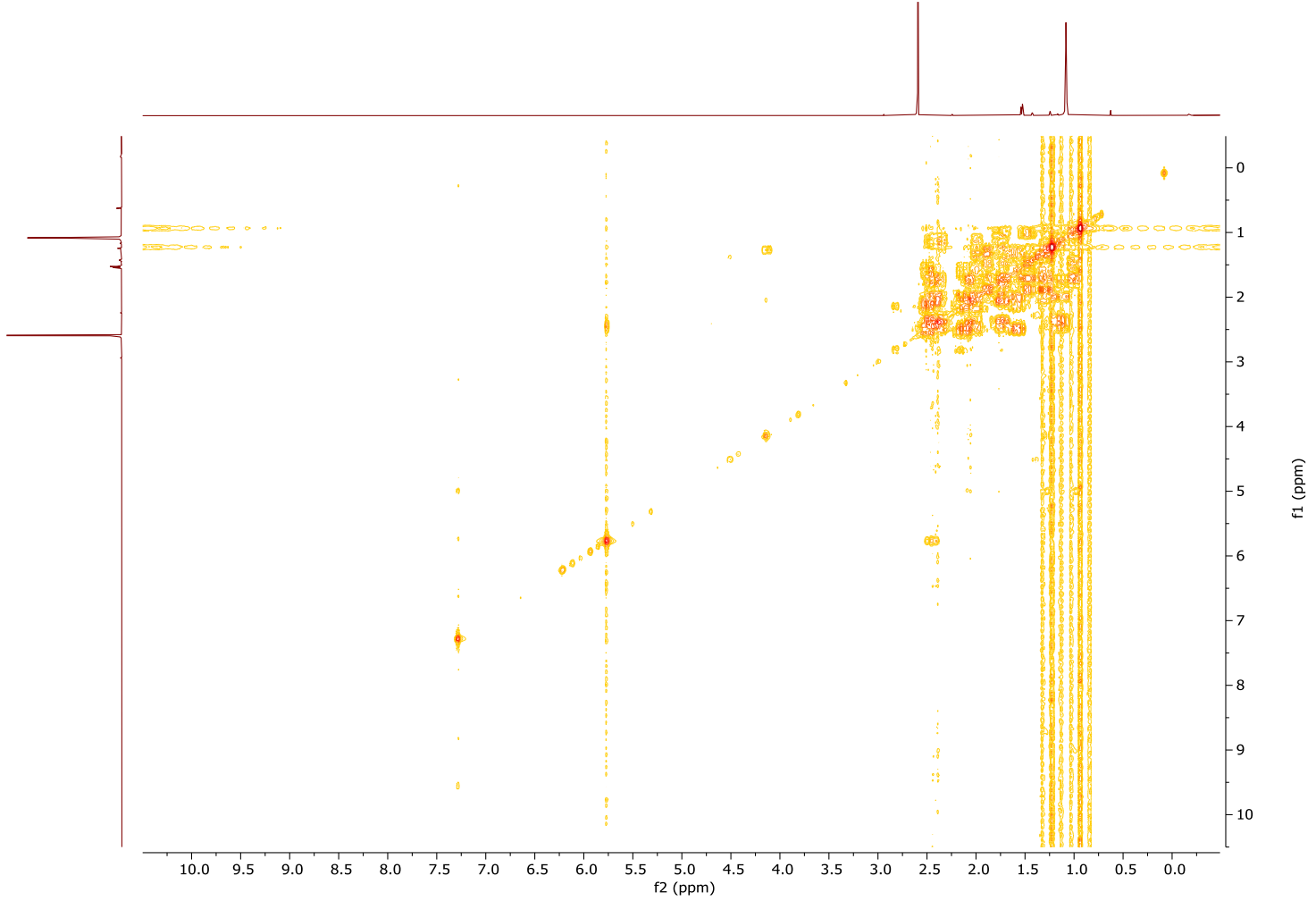

COSY spectrum in $\mathrm{CDCl}_{3}$.

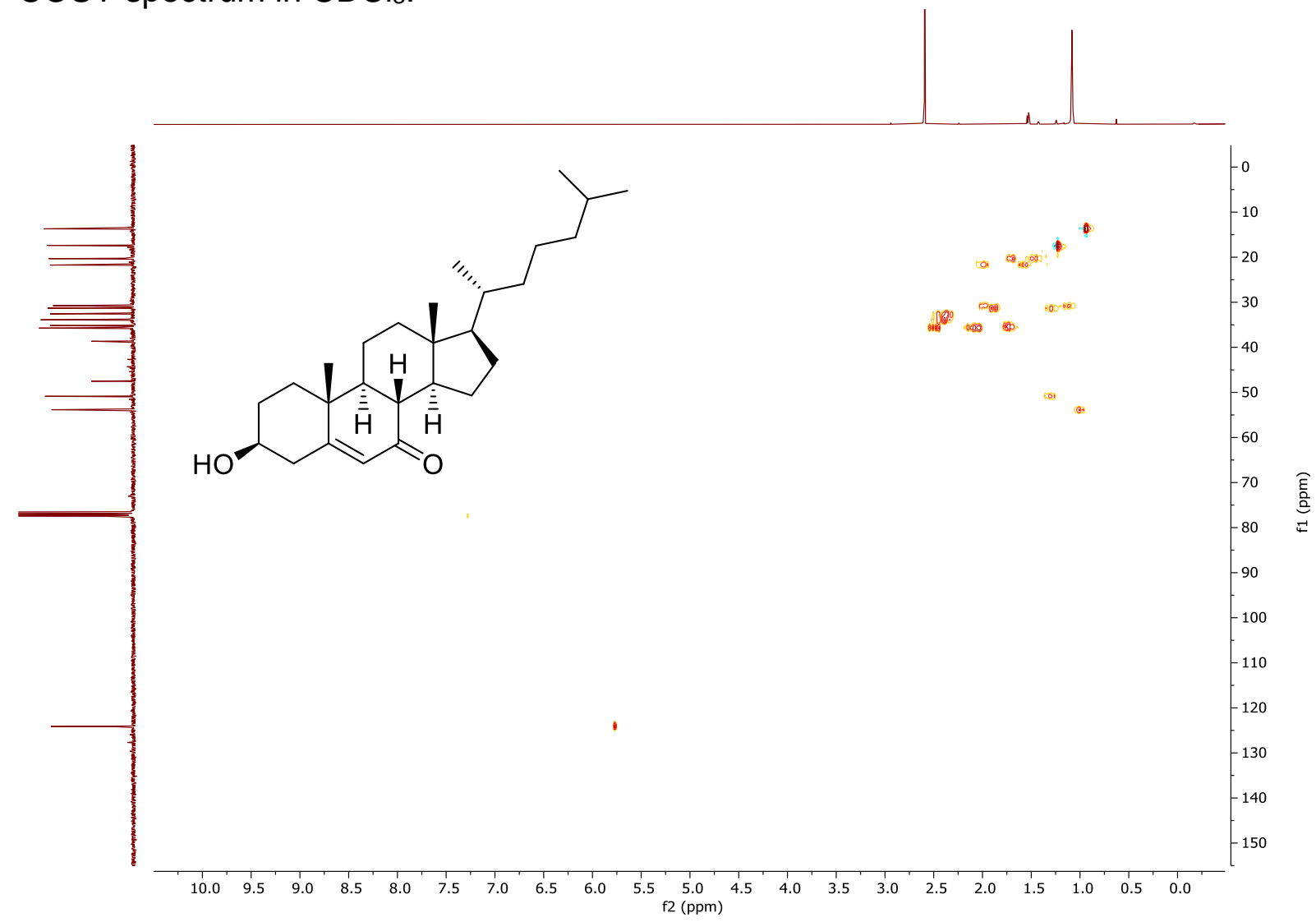

$\mathrm{HSQC}$ spectrum in $\mathrm{CDCl}_{3}$. 
$54 b$

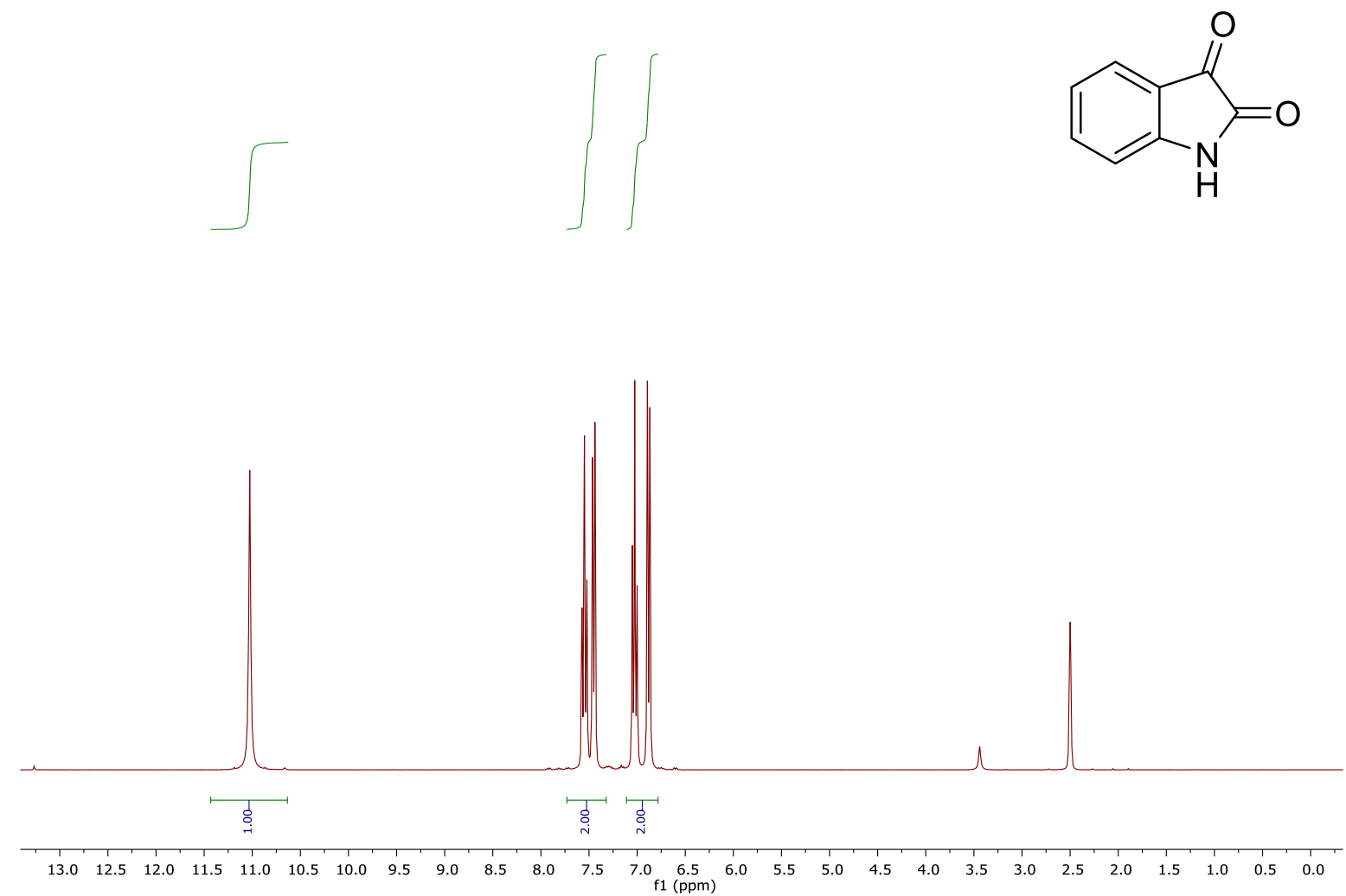

${ }^{1} \mathrm{H}$ NMR spectrum in DMSO- $d_{6}$.

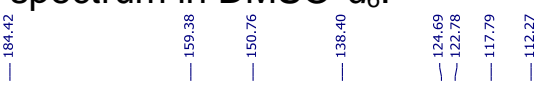

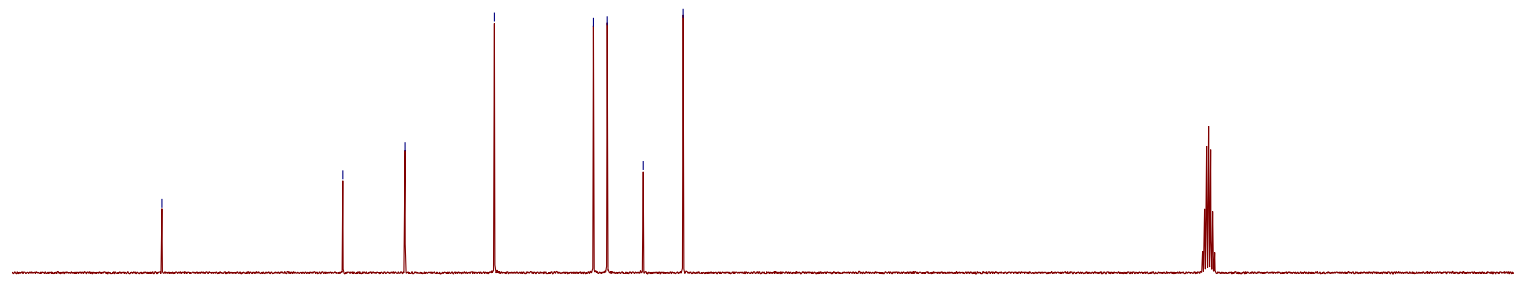<smiles>O=C1Nc2ccccc2C1=O</smiles>

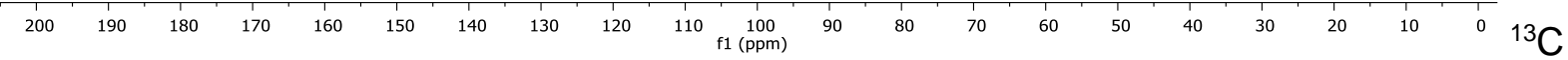
NMR spectrum in DMSO- $d_{6}$. 
$55 b$<smiles>Cc1cccc2c1C(=O)C(=O)N2</smiles>
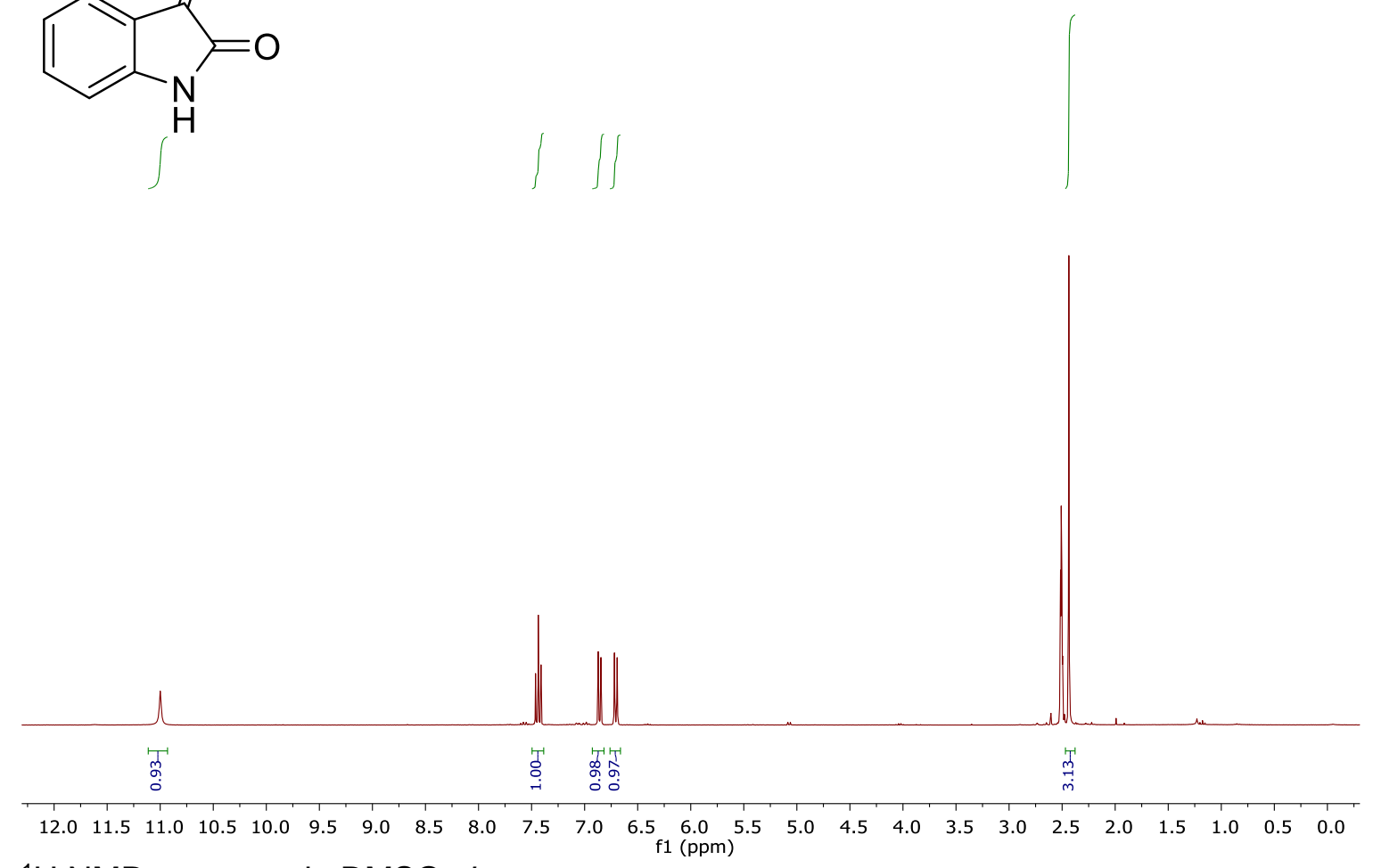

${ }^{1} \mathrm{H}$ NMR spectrum in DMSO- $d_{6}$.

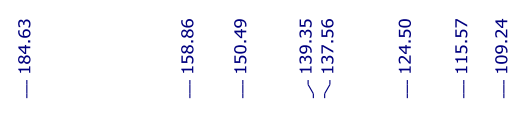<smiles>Cc1cccc2c1C(=O)C(=O)N2</smiles>

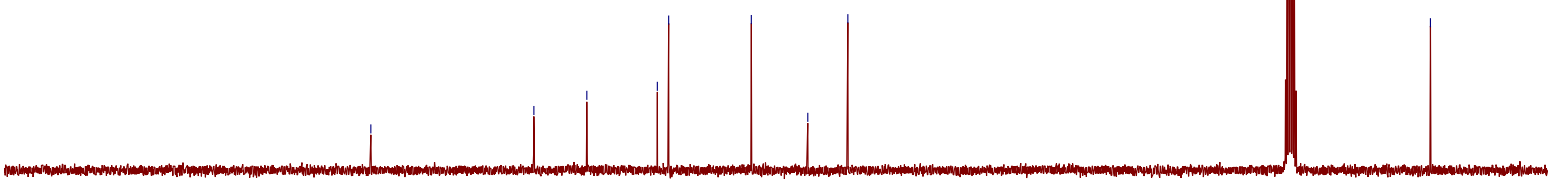

$\begin{array}{lllllllllllllllllllllllll}240 & 230 & 220 & 210 & 200 & 190 & 180 & 170 & 160 & 150 & 140 & 130 & 120 & 110 & 100 & 90 & 80 & 70 & 60 & 50 & 40 & 30 & 20 & 10 & 0\end{array}$ ${ }^{13} \mathrm{C}$ NMR spectrum in DMSO- $d_{6}$. 
$56 b$<smiles>Cc1ccc2c(c1)C(=O)C(=O)N2</smiles>

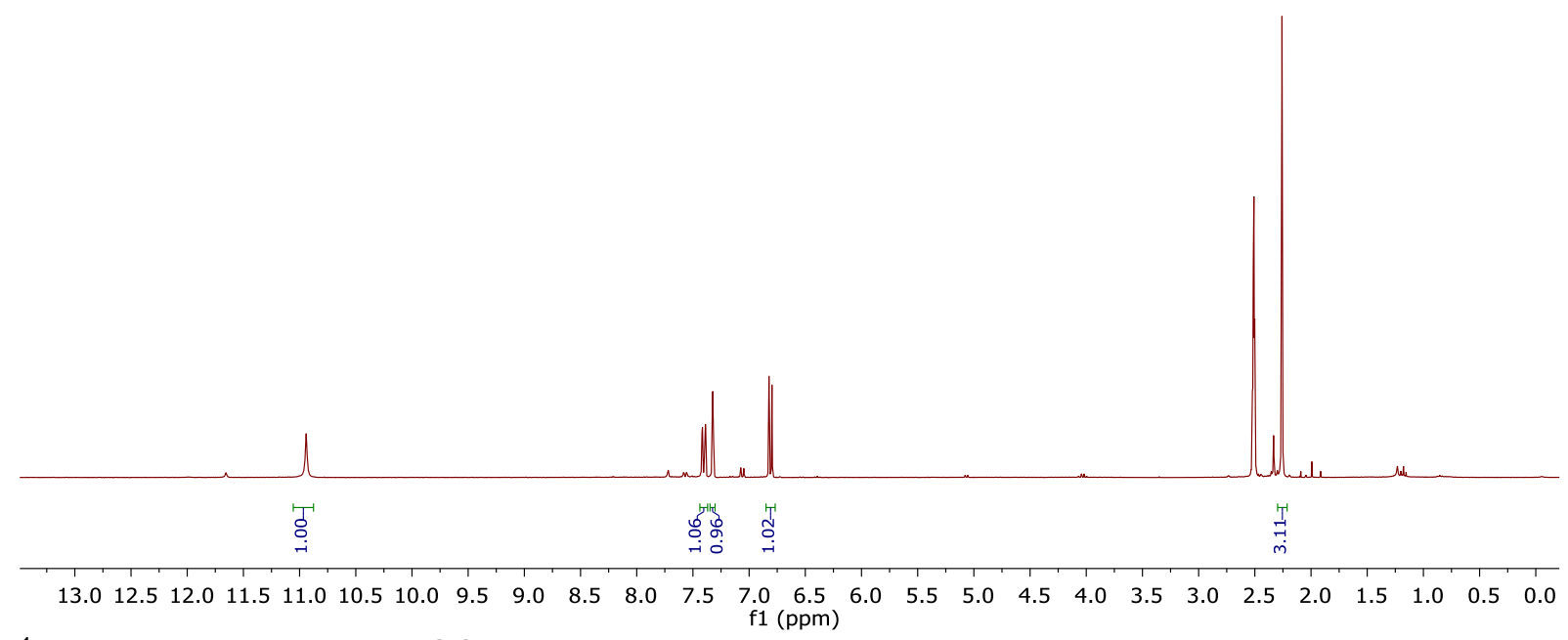

${ }^{1} \mathrm{H}$ NMR spectrum in DMSO- $d_{6}$.

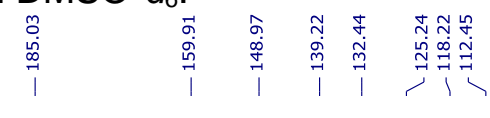<smiles>Cc1ccc2c(c1)C(=O)C(=O)N2</smiles>

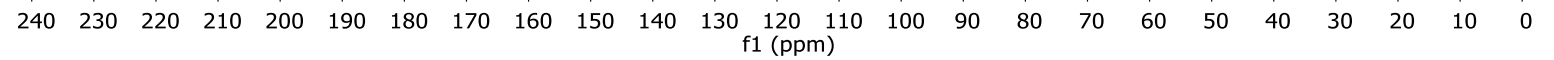
${ }^{13} \mathrm{C}$ NMR spectrum in DMSO- $d_{6}$. 


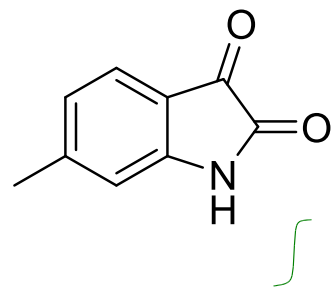

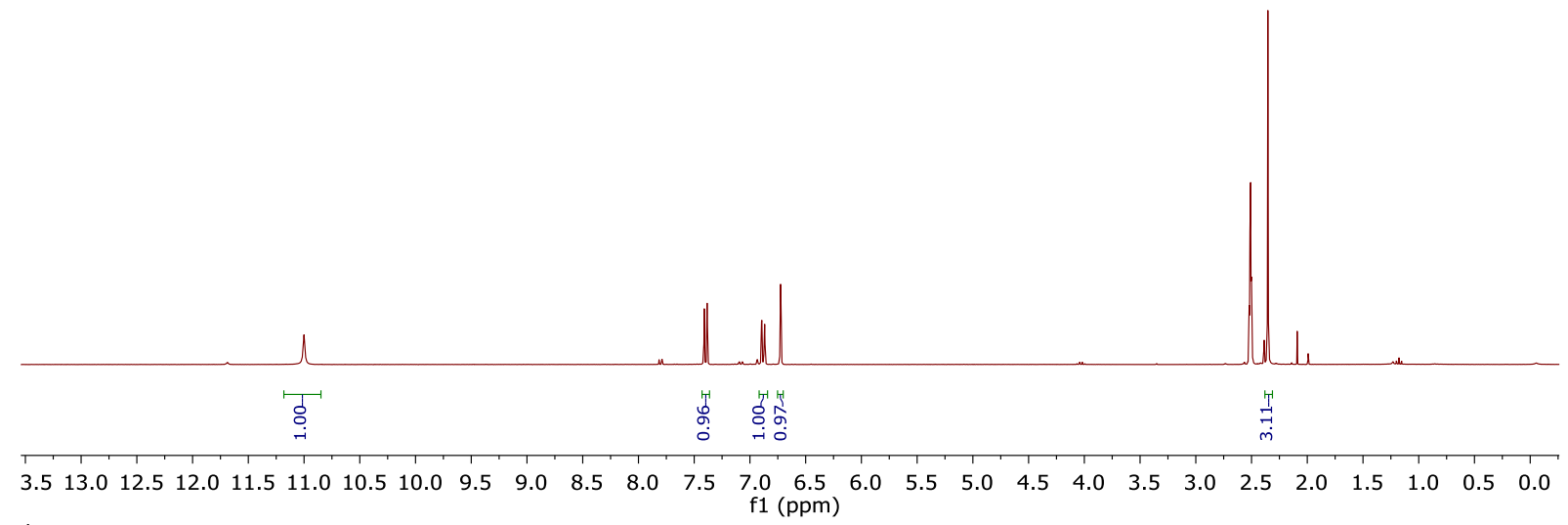

${ }^{1} \mathrm{H}$ NMR spectrum in DMSO- $d_{6}$.

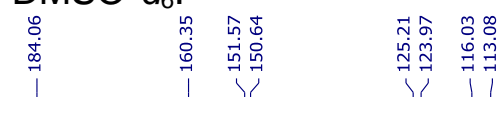<smiles>Cc1ccc2c(c1)NC(=O)C2=O</smiles>
${ }^{13} \mathrm{C}$ NMR spectrum in DMSO- $d_{6}$. 
$58 b$<smiles>Cc1cccc2c1NC(=O)C2=O</smiles>

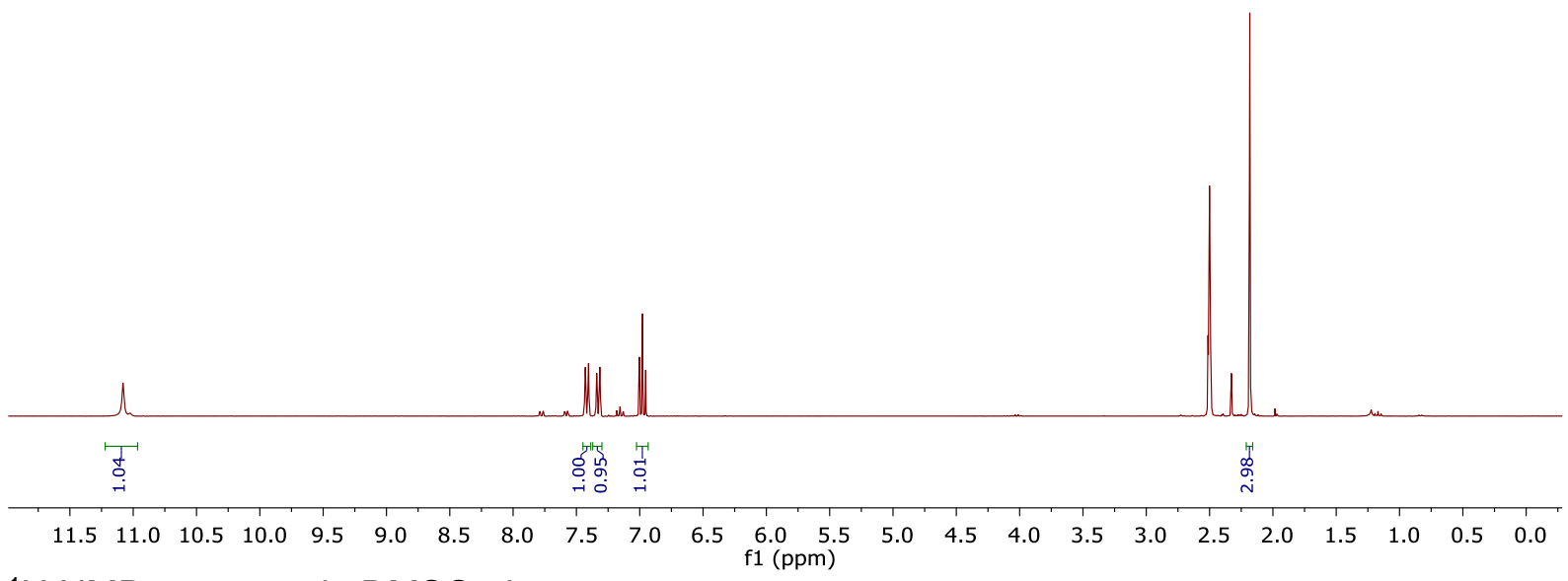
${ }^{1} \mathrm{H}$ NMR spectrum in DMSO- $d_{6}$.<smiles>Cc1cccc2c1NC(=O)C2=O</smiles>

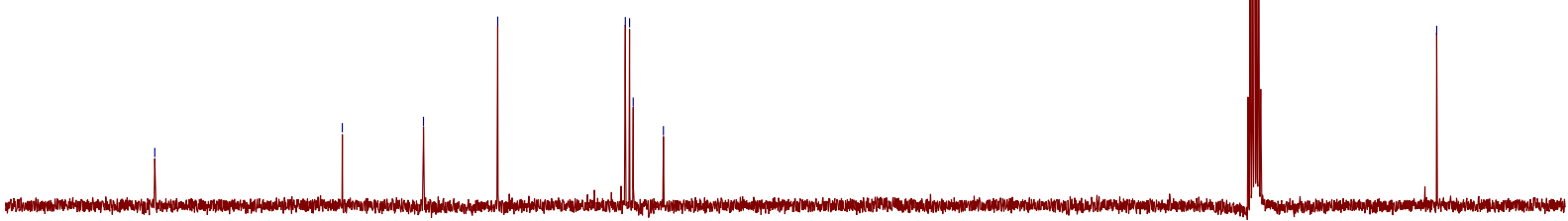

$\begin{array}{lllllllllllllllllllll}200 & 190 & 180 & 170 & 160 & 150 & 140 & 130 & 120 & 110 \begin{array}{l}100 \\ \mathrm{f} 1(\mathrm{ppm})\end{array} & 90 & 80 & 70 & 60 & 50 & 40 & 30 & 20 & 10 & 0\end{array}$ ${ }^{13} \mathrm{C}$ NMR spectrum in DMSO- $d_{6}$. 
$59 b$
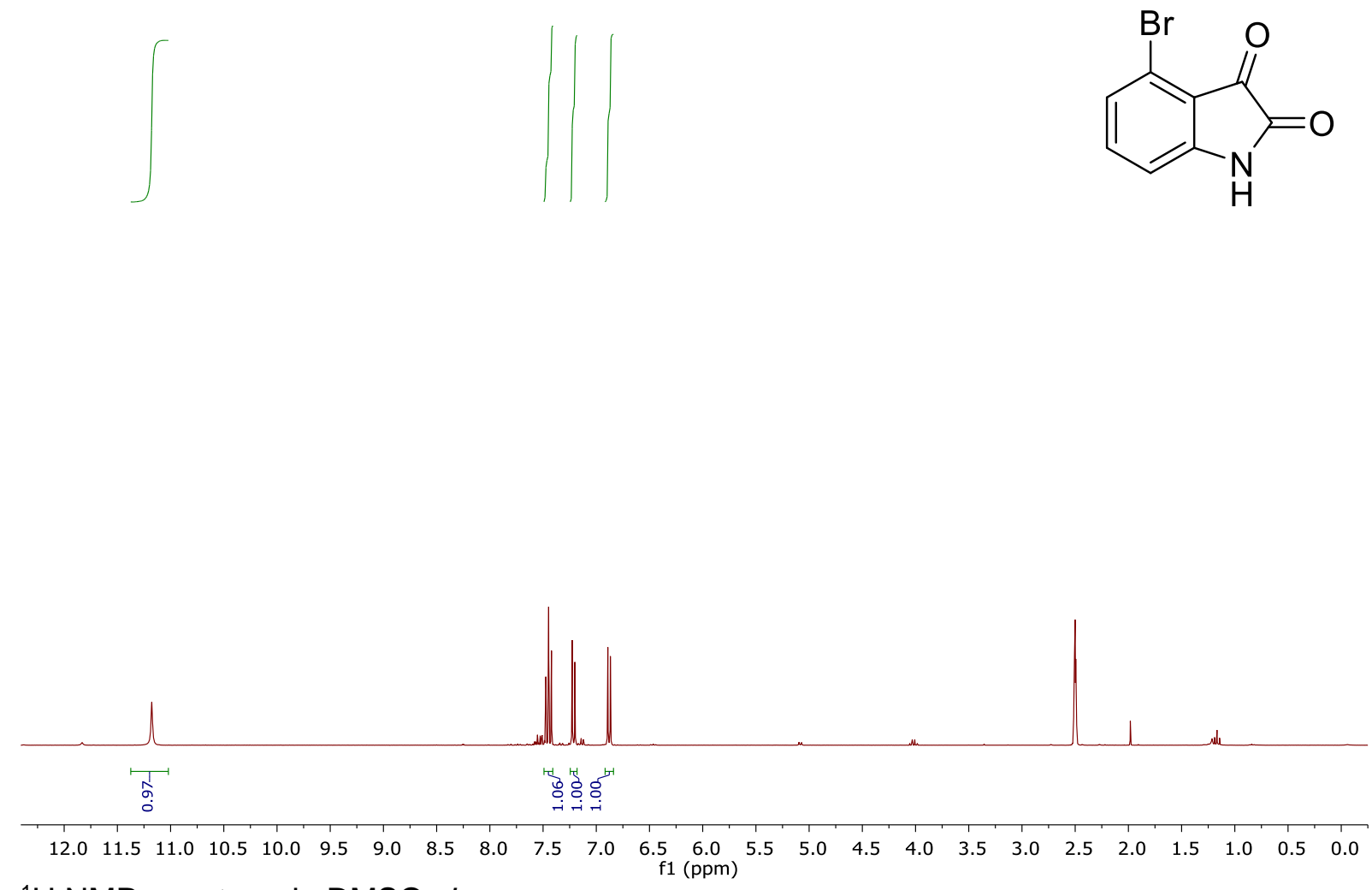

${ }^{1} \mathrm{H}$ NMR spectrum in DMSO- $d_{6}$.

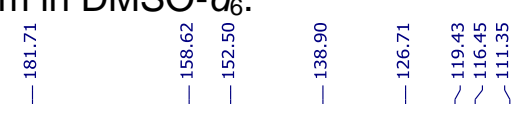<smiles>O=C1Nc2cccc(Br)c2C1=O</smiles>

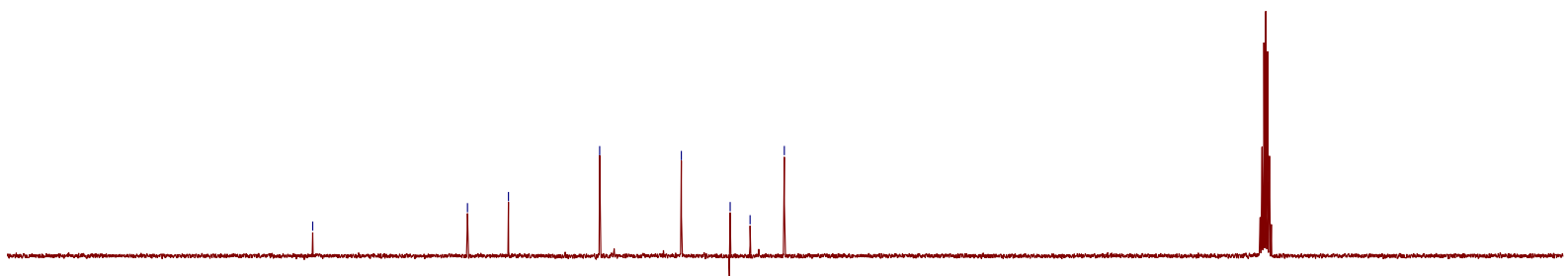

$\begin{array}{lllllllllllllllllllllll}220 & 210 & 200 & 190 & 180 & 170 & 160 & 150 & 140 & 130 & \begin{array}{c}120 \\ \mathrm{f} 1(\mathrm{ppm})\end{array} & 100 & 90 & 80 & 70 & 60 & 50 & 40 & 30 & 20 & 10 & 0\end{array}$ ${ }^{13} \mathrm{C}$ NMR spectrum in DMSO- $d_{6}$. 
<smiles>O=C1Nc2cc(Br)cc(Br)c2C1=O</smiles>

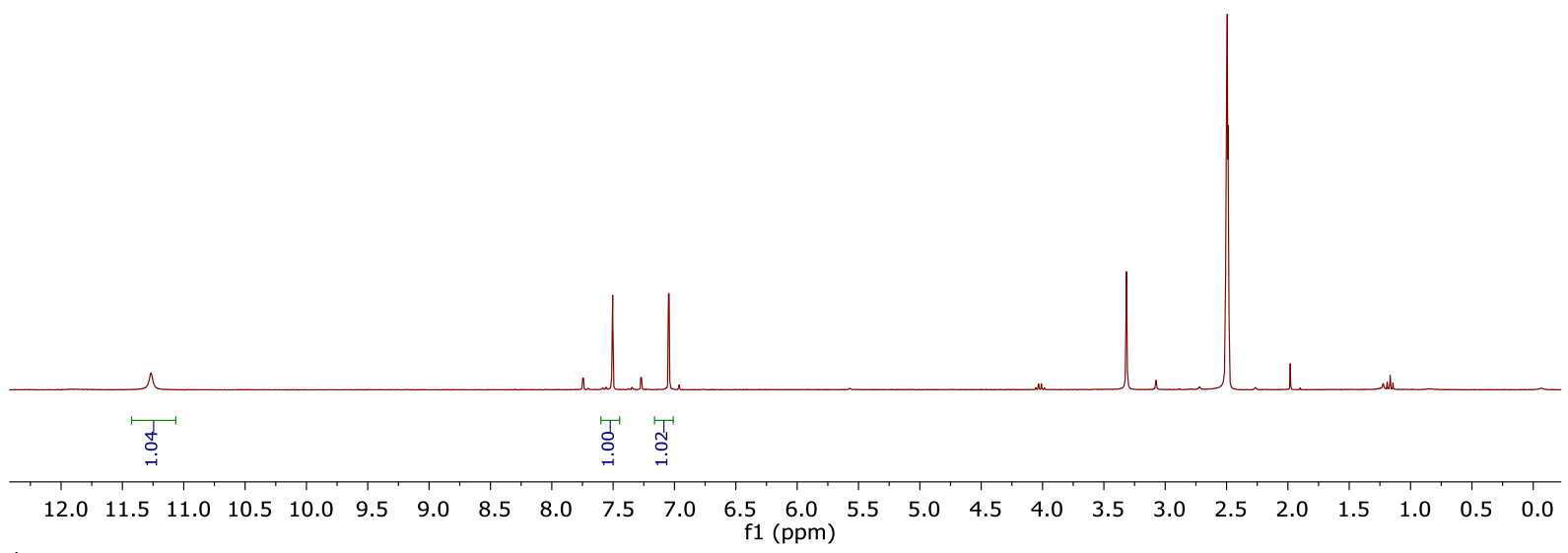

${ }^{1} \mathrm{H}$ NMR spectrum in DMSO- $d_{6}$.

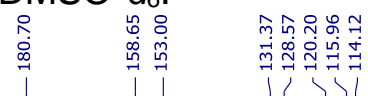<smiles>O=C1Nc2cc(Br)cc(Br)c2C1=O</smiles>

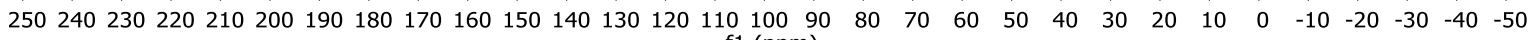
${ }^{13} \mathrm{C}$ NMR spectrum in DMSO- $d_{6}$. 
$62 b$

$\underset{7}{\stackrel{m}{ت}}$
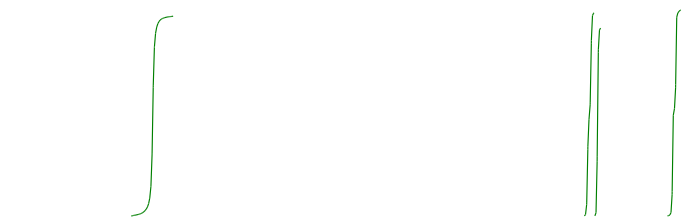<smiles>O=C1Nc2ccc(Cl)cc2C1=O</smiles>

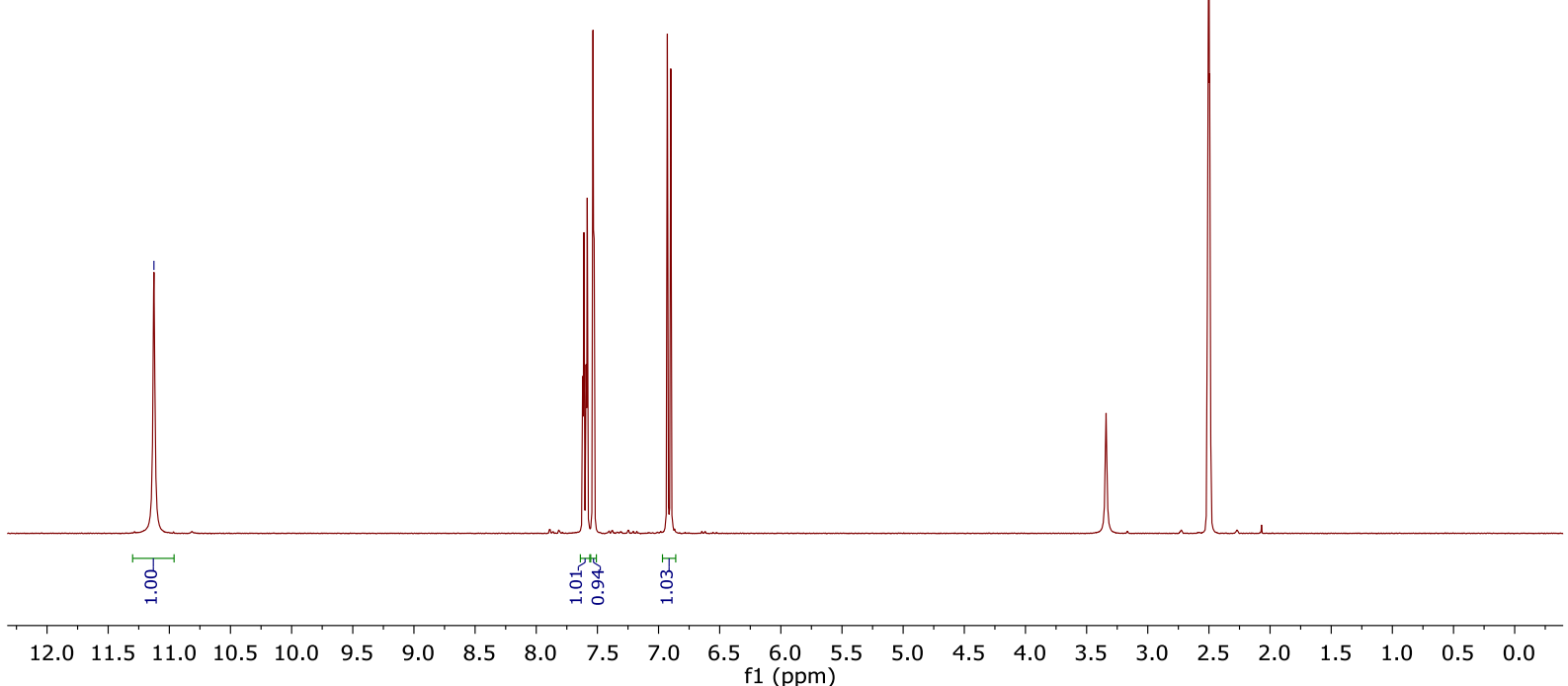

${ }^{1} \mathrm{H}$ NMR spectrum in DMSO- $d_{6}$.

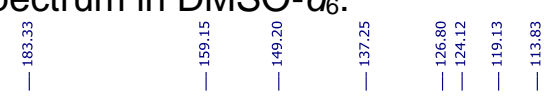<smiles>O=C1Nc2ccc(Cl)cc2C1=O</smiles>
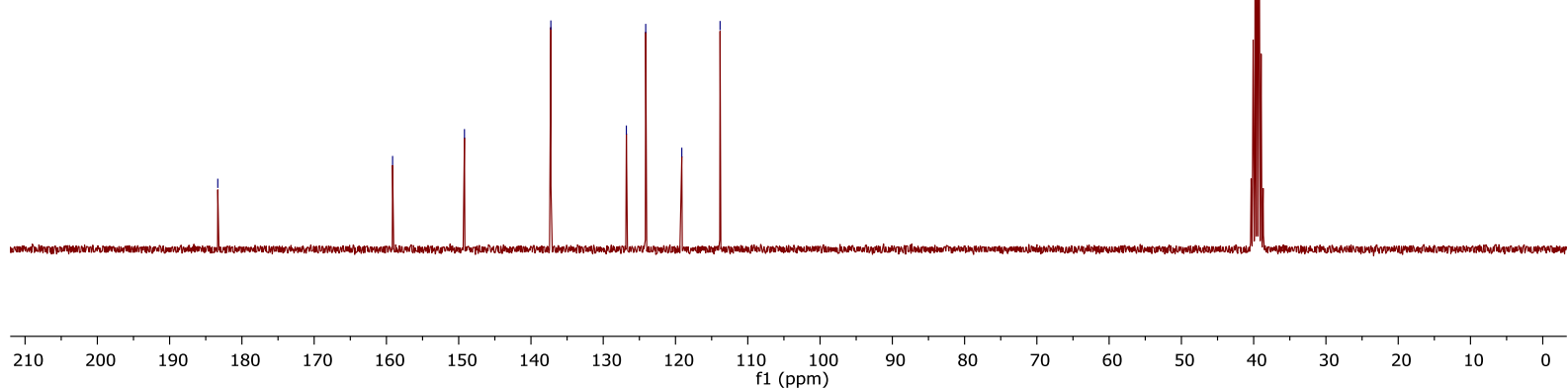

${ }^{13} \mathrm{C}$ NMR spectrum in DMSO- $d_{6}$. 
$63 b$<smiles>O=C1Nc2cc(Cl)c(Cl)cc2C1=O</smiles>

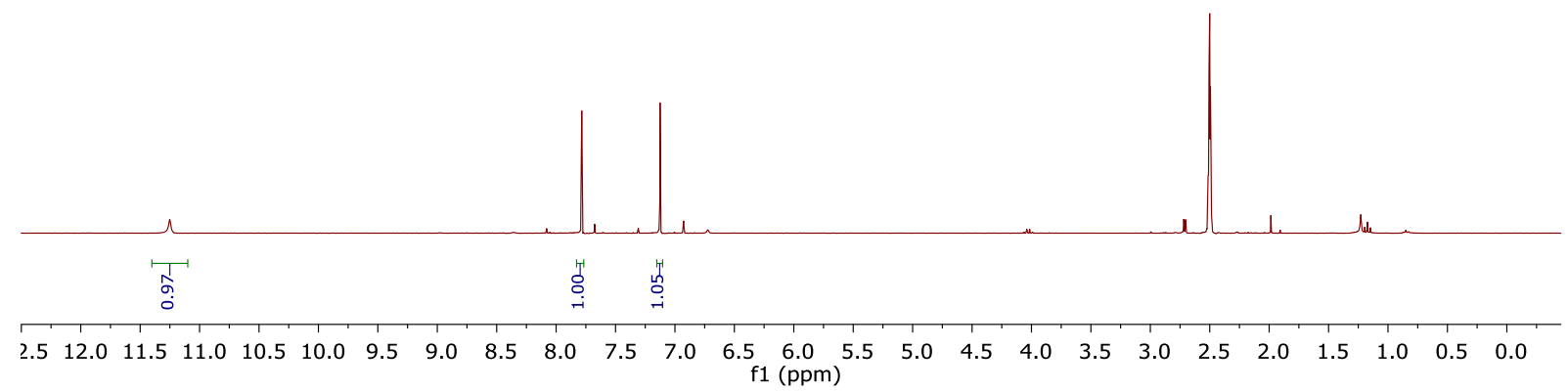

${ }^{1} \mathrm{H}$ NMR spectrum in DMSO- $d_{6}$.

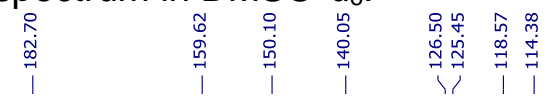

$\begin{array}{lllllllllllllllllllll}200 & 190 & 180 & 170 & 160 & 150 & 140 & 130 & 120 & 110 \begin{array}{c}100 \\ \mathrm{f} 1(\mathrm{ppm})\end{array} & 90 & 80 & 70 & 60 & 50 & 40 & 30 & 20 & 10 & 0\end{array}$ ${ }^{13} \mathrm{C}$ NMR spectrum in DMSO- $d_{6}$.<smiles>O=C1Nc2cc(Cl)c(Cl)cc2C1=O</smiles>

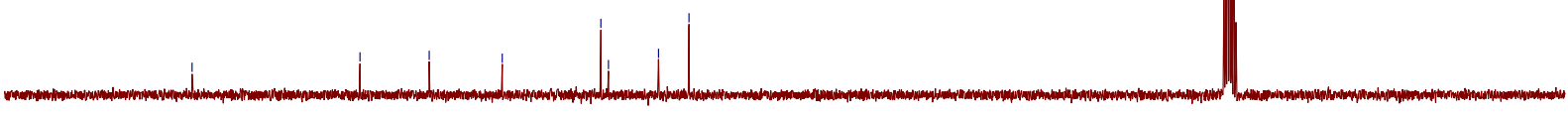




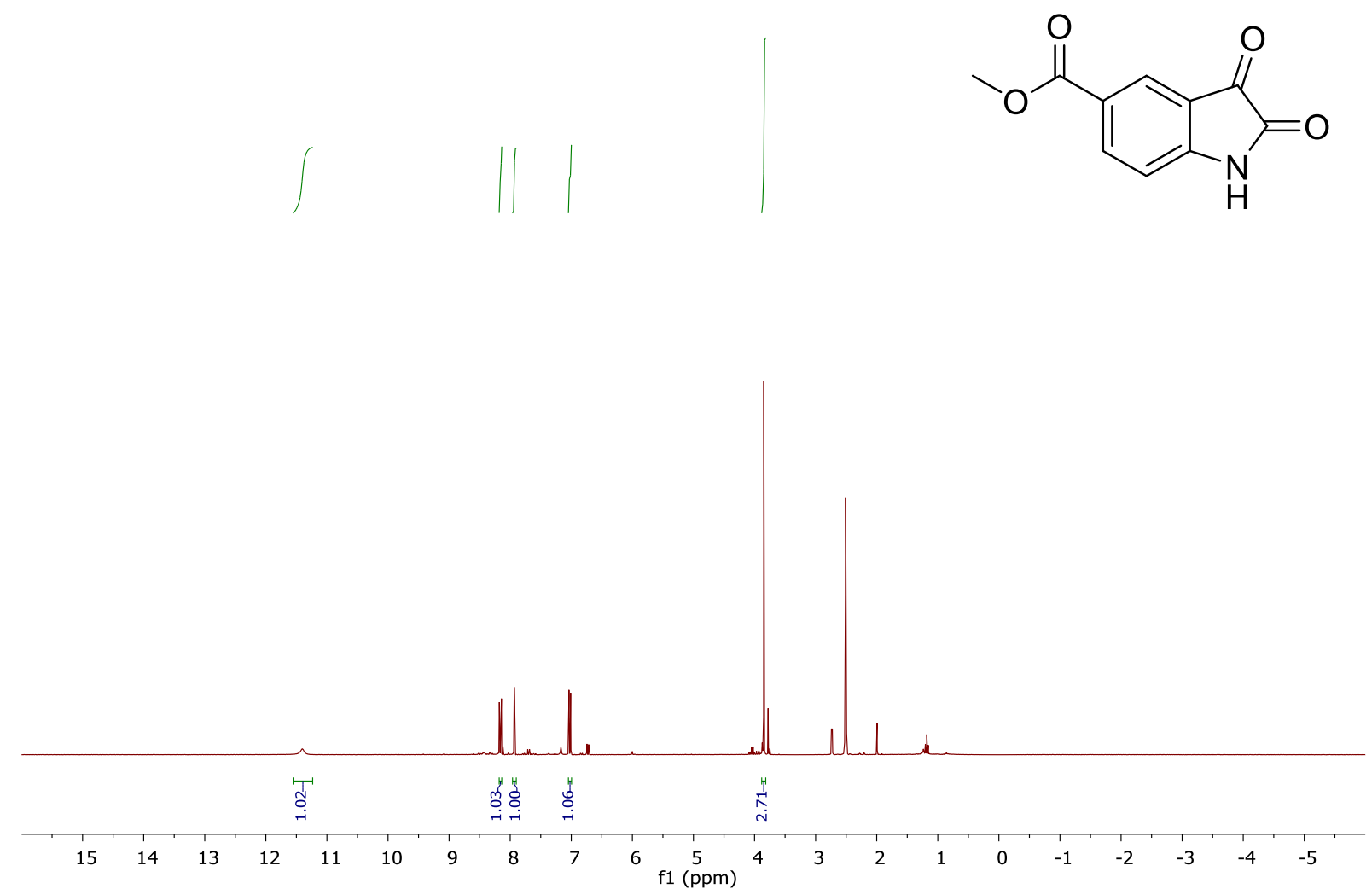

${ }^{1} \mathrm{H}$ NMR spectrum in DMSO- $d_{6}$.

垔

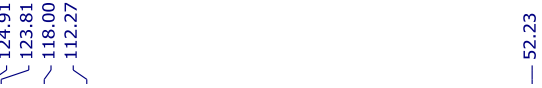<smiles>COC(=O)c1ccc2c(c1)C(=O)C(=O)N2</smiles>

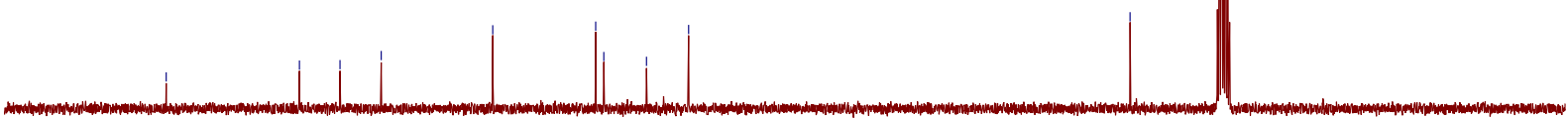

$\begin{array}{lllllllllllllllllllll}200 & 190 & 180 & 170 & 160 & 150 & 140 & 130 & 120 & 110 \underset{\mathrm{f} 1(\mathrm{ppm})}{100} & 90 & 80 & 70 & 60 & 50 & 40 & 30 & 20 & 10 & 0\end{array}$ ${ }^{13} \mathrm{C}$ NMR spectrum in DMSO- $d_{6}$. 


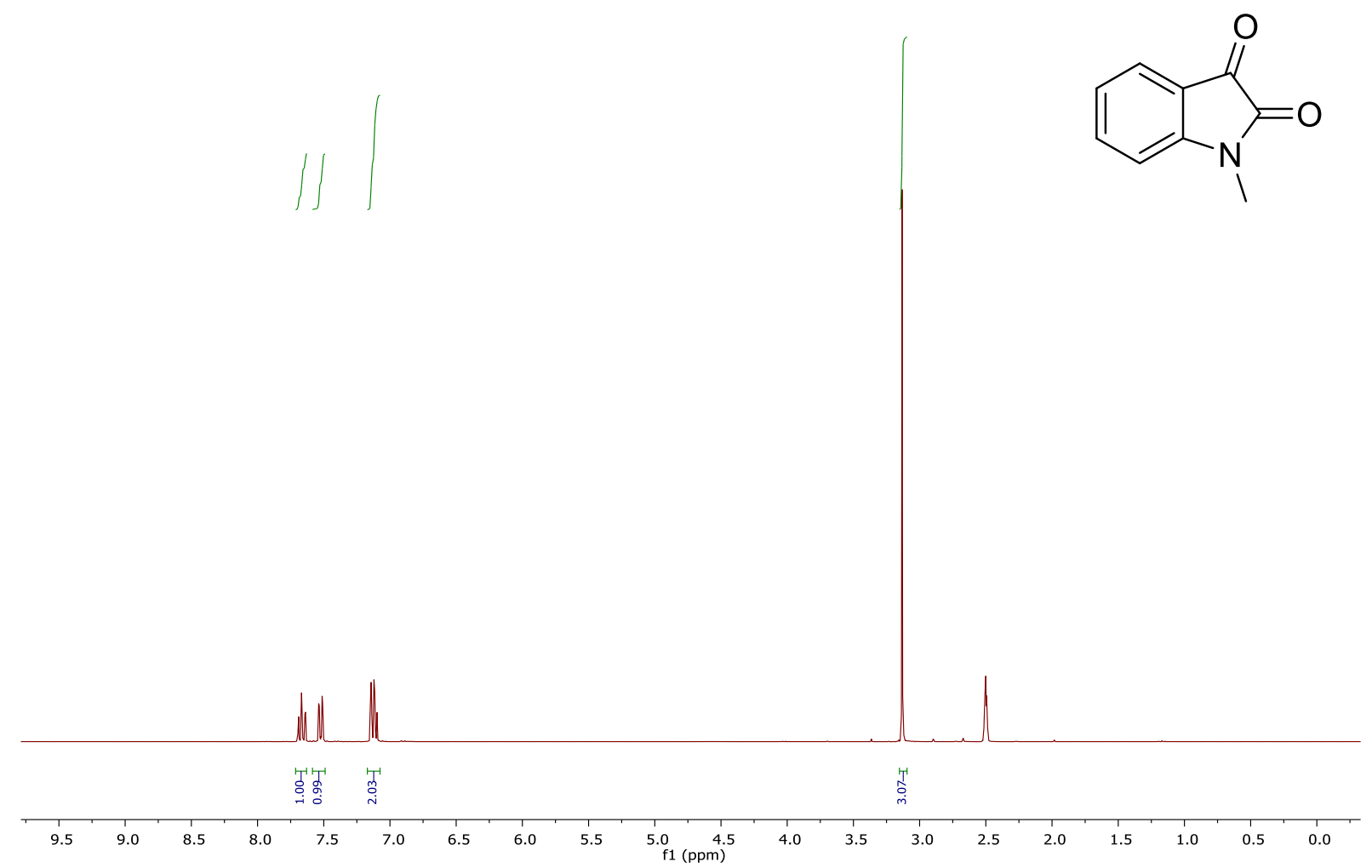

${ }^{1} \mathrm{H}$ NMR spectrum in DMSO- $d_{6}$.

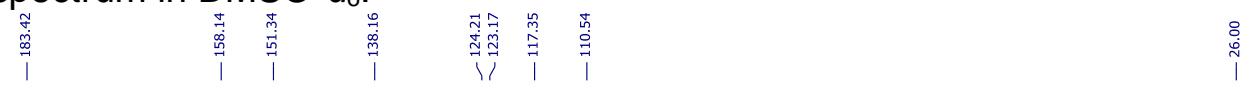<smiles>CN1C(=O)C(=O)c2ccccc21</smiles>

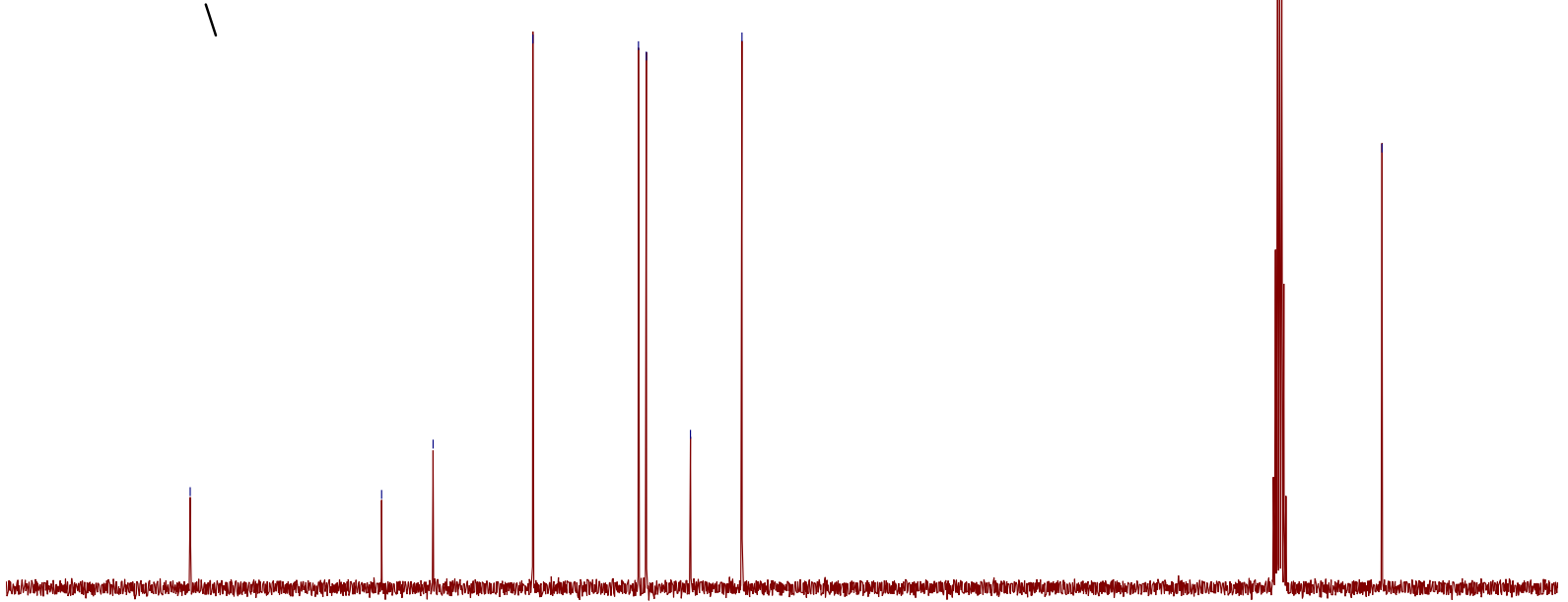

$\begin{array}{llllllllll}200 & 190 & 180 & 170 & 160 & 150 & 140 & 130 & 120 & 110 \\ \mathrm{f} 1(\mathrm{ppm}) & 100\end{array}$

${ }^{13} \mathrm{C}$ NMR spectrum in DMSO- $d_{6}$. 
$75 b$<smiles>Cc1ccc2c(c1)N(C(C)C)C(=O)C2=O</smiles>
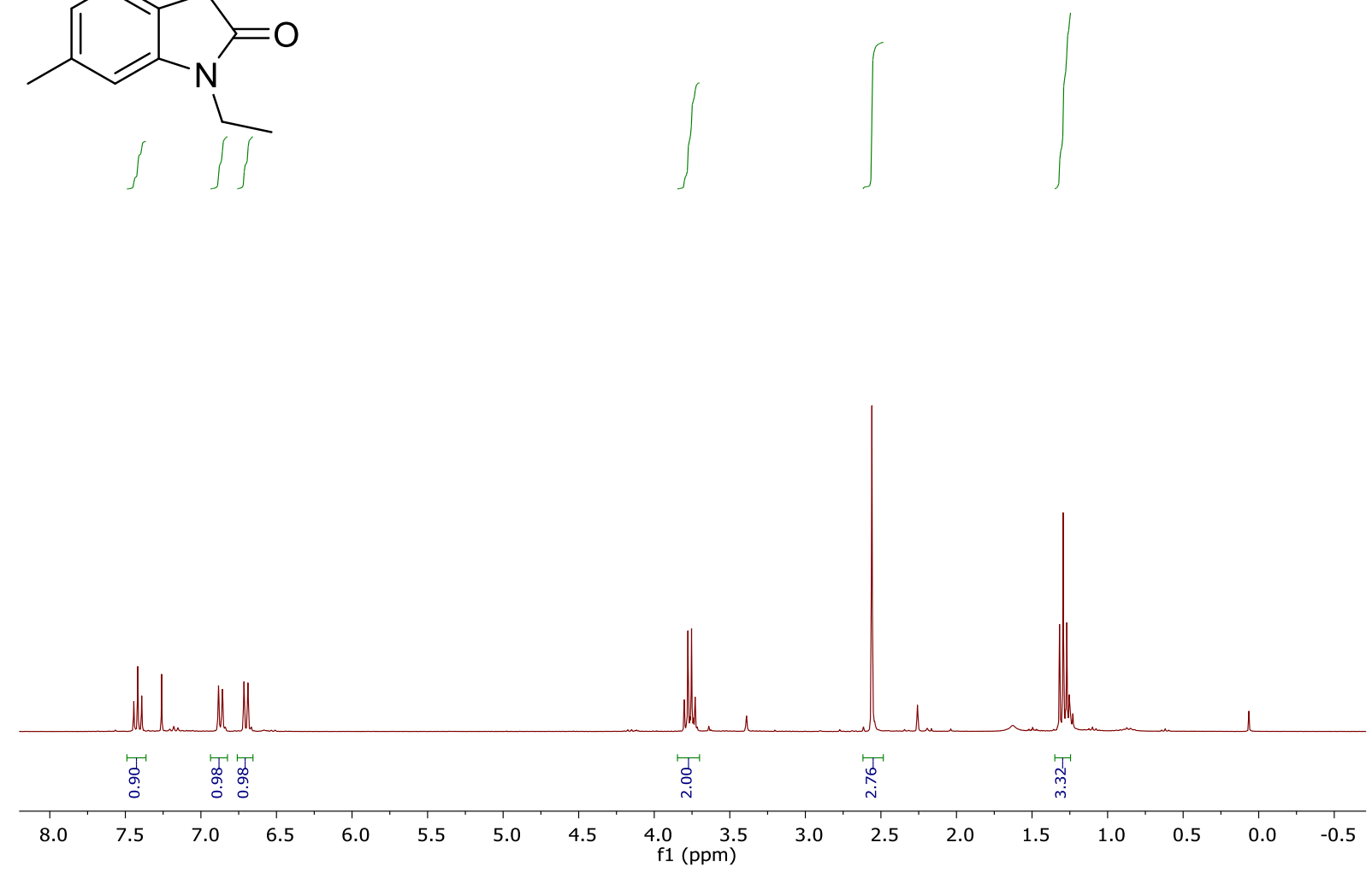

${ }^{1} \mathrm{H}$ NMR spectrum in $\mathrm{CDCl}_{3}$.

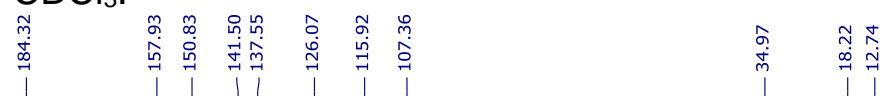<smiles>CCN1C(=O)C(=O)c2ccc(C)cc21</smiles>

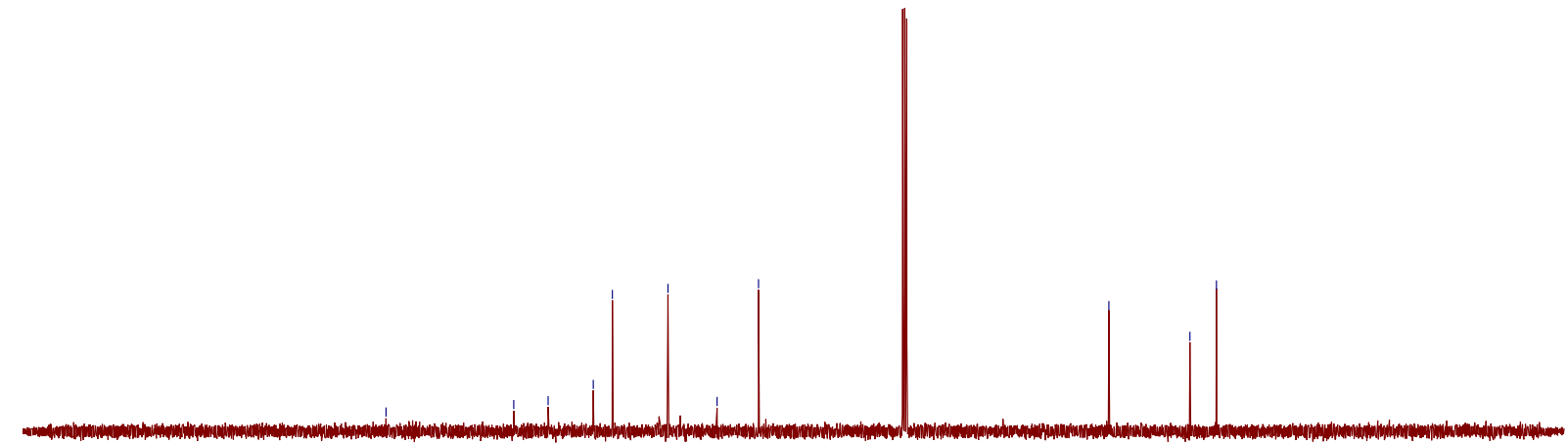

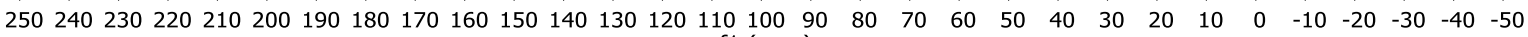
${ }^{13} \mathrm{C}$ NMR spectrum in $\mathrm{CDCl}_{3}$. 
<smiles>CCCCCCN1C(=O)C(=O)c2ccccc21</smiles>

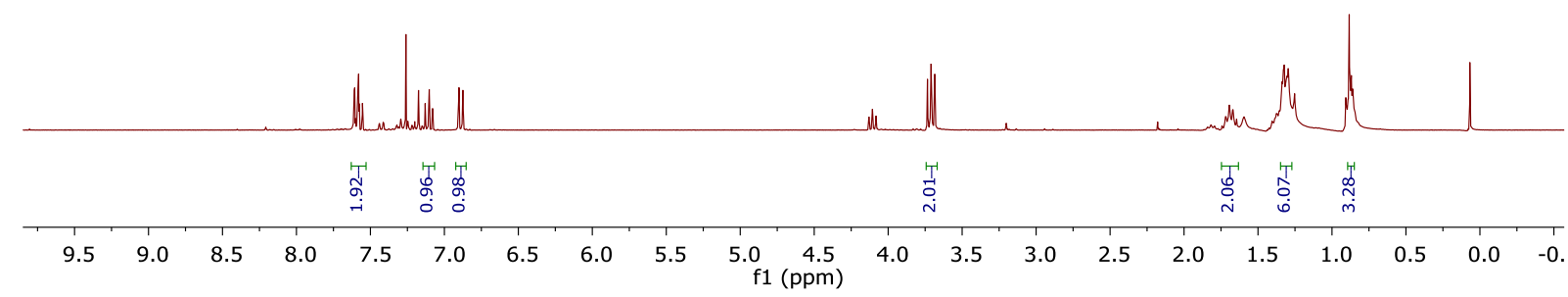

${ }^{1} \mathrm{H}$ NMR spectrum in $\mathrm{CDCl}_{3}$.

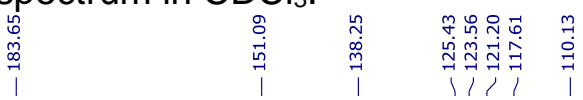

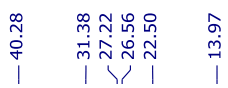<smiles>CCCCCCN1C(=O)C(=O)c2ccccc21</smiles>

$\begin{array}{lllllllllllllllllllll}200 & 190 & 180 & 170 & 160 & 150 & 140 & 130 & 120 & 110 \underset{\mathrm{f} 1(\mathrm{ppm})}{100} & 90 & 80 & 70 & 60 & 50 & 40 & 30 & 20 & 10 & 0\end{array}$ ${ }^{13} \mathrm{C}$ NMR spectrum in $\mathrm{CDCl}_{3}$. 


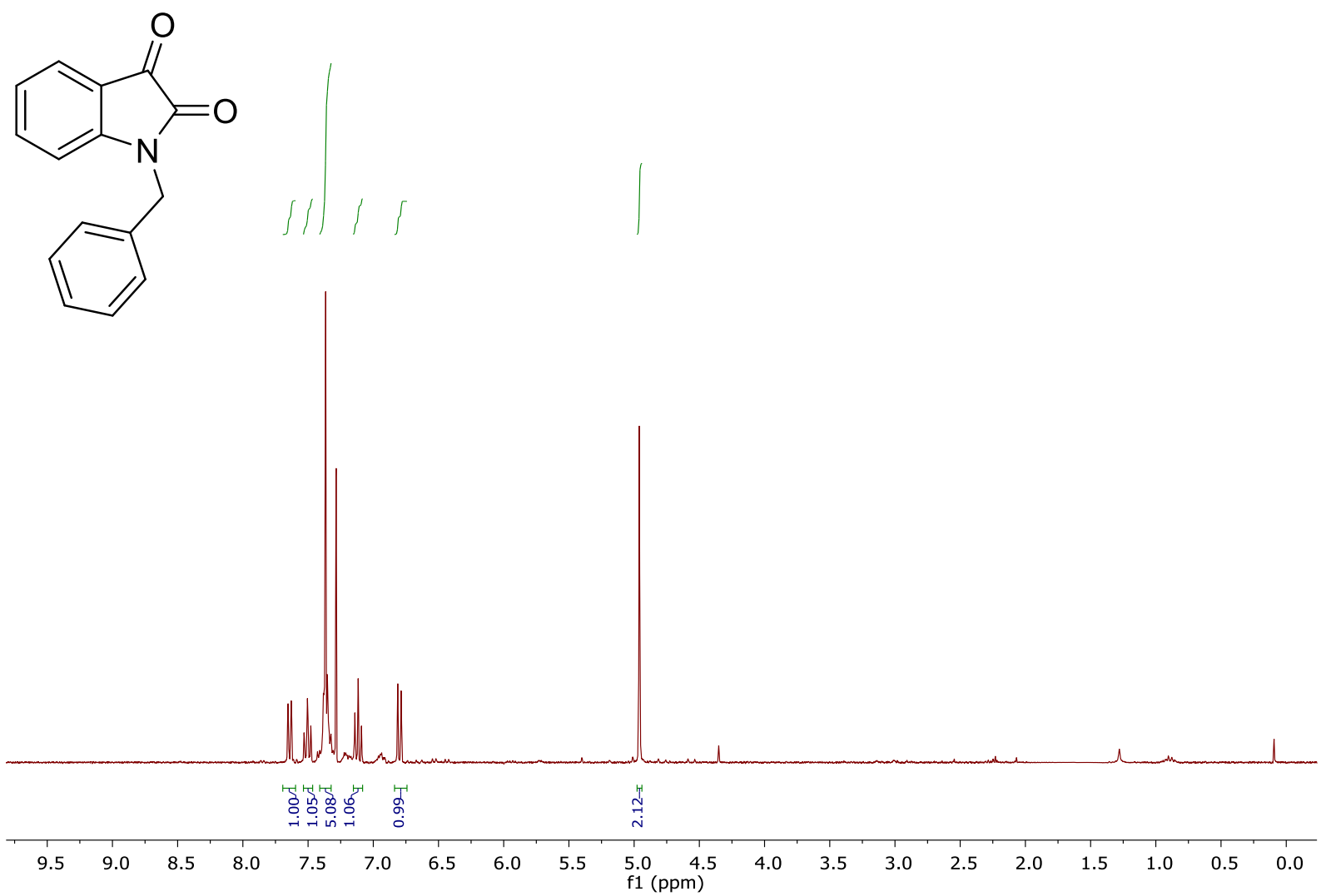

${ }^{1} \mathrm{H}$ NMR spectrum in $\mathrm{CDCl}_{3}$.

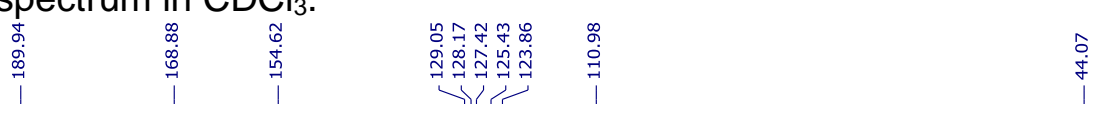<smiles>O=C1C(=O)N(Cc2ccccc2)c2ccccc21</smiles>

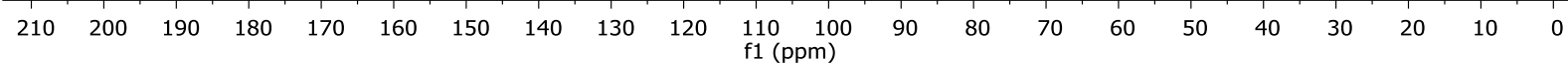

${ }^{13} \mathrm{C}$ NMR spectrum in $\mathrm{CDCl}_{3}$. 
<smiles>CCCCCCCCCCCC(C)=O</smiles>
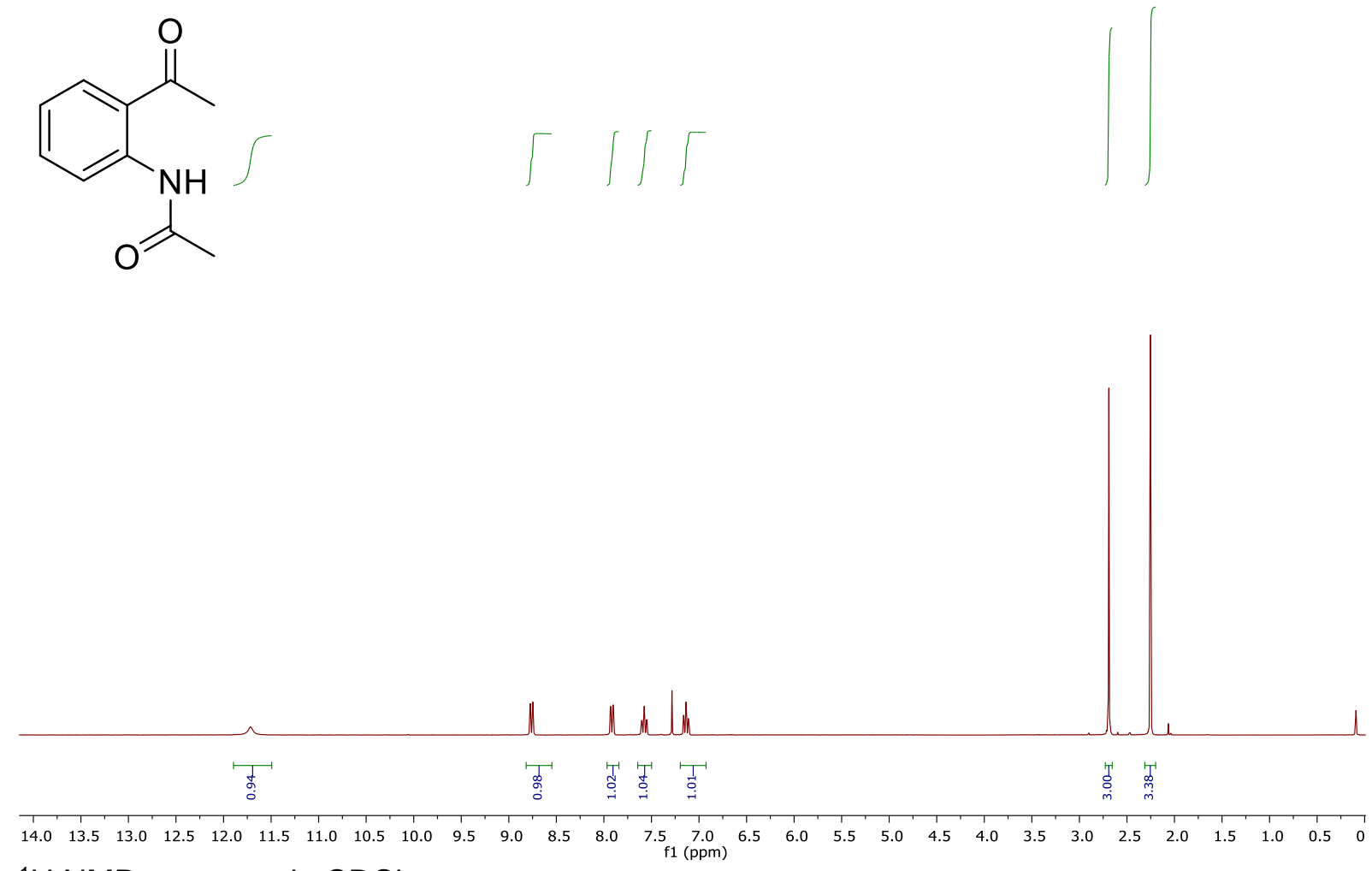

${ }^{1} \mathrm{H}$ NMR spectrum in $\mathrm{CDCl}_{3}$.

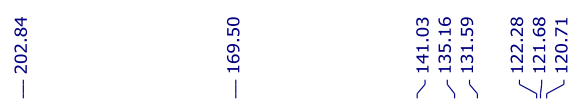<smiles>CC(=O)Nc1ccccc1C(C)=O</smiles>

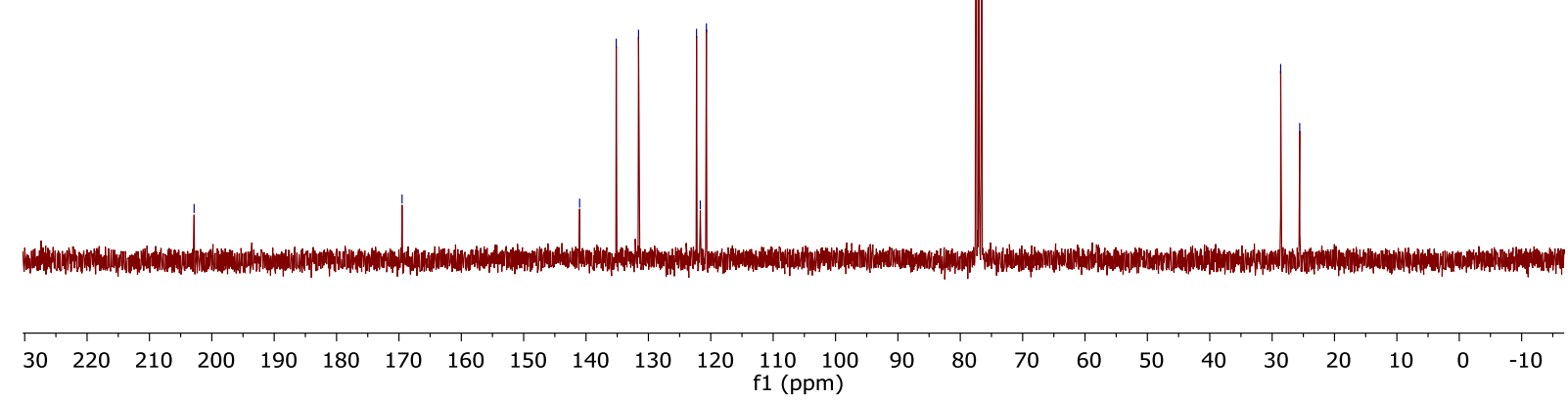
${ }^{13} \mathrm{C}$ NMR spectrum in $\mathrm{CDCl}_{3}$. 


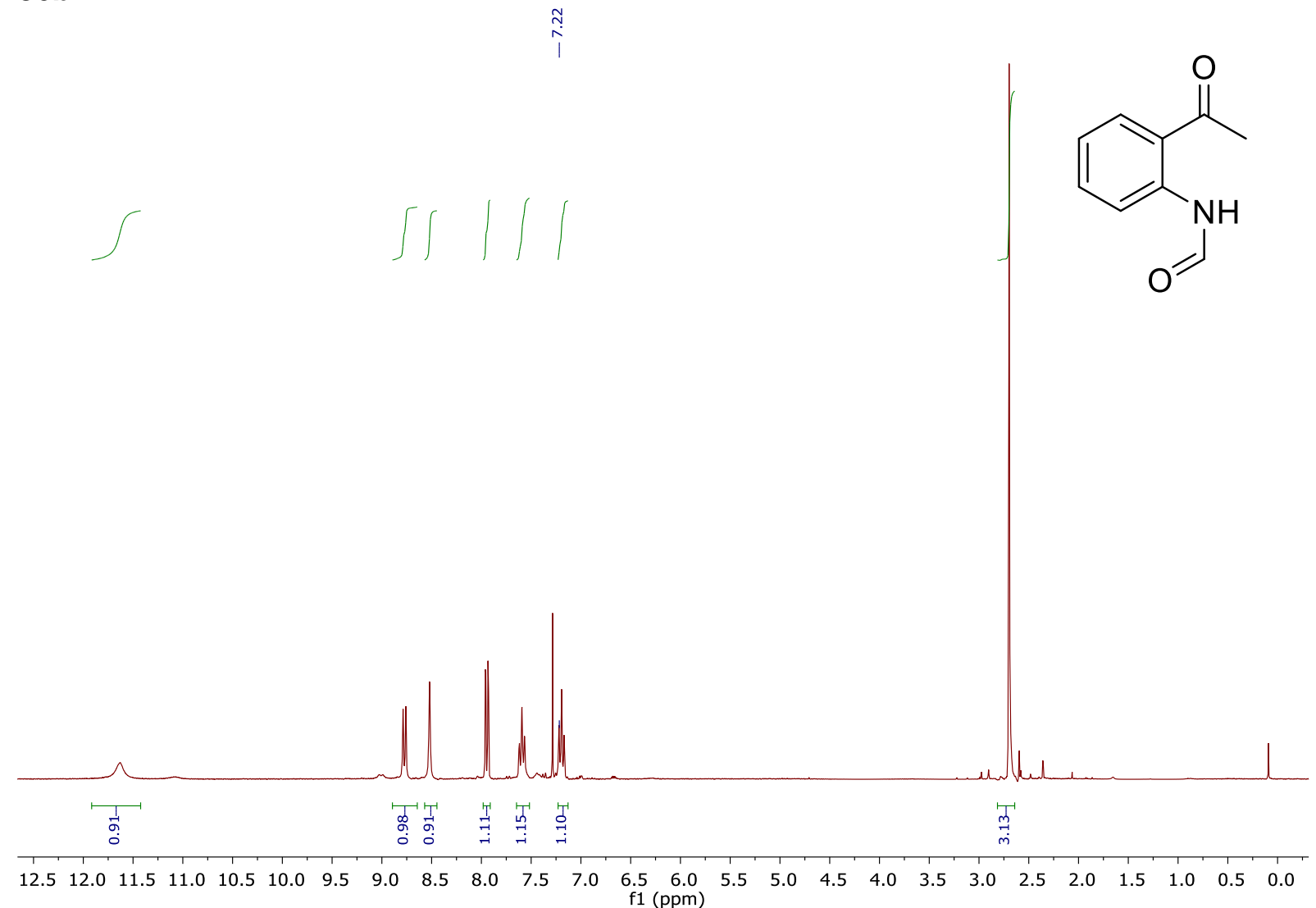

${ }^{1} \mathrm{H}$ NMR spectrum in $\mathrm{CDCl}_{3}$.
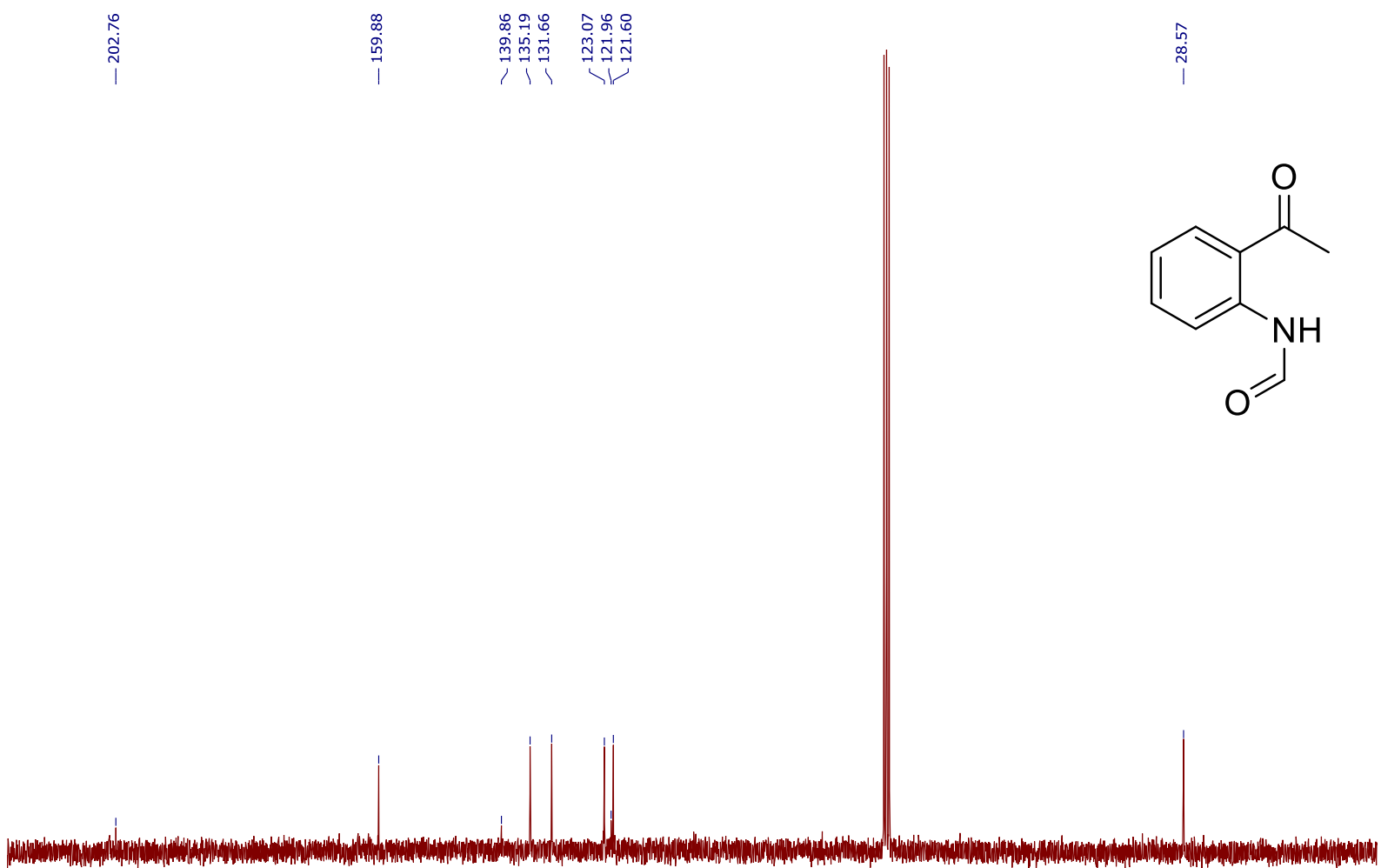
${ }^{13} \mathrm{C}$ NMR spectrum in $\mathrm{CDCl}_{3}$. 


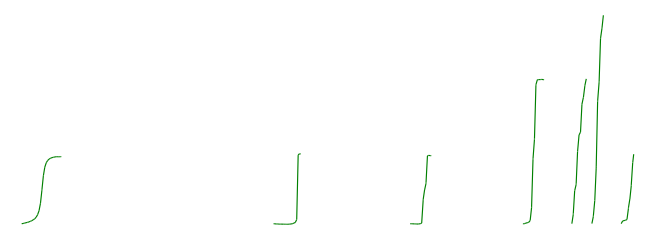<smiles>O=Cc1ccccc1NC(=O)c1ccccc1</smiles>

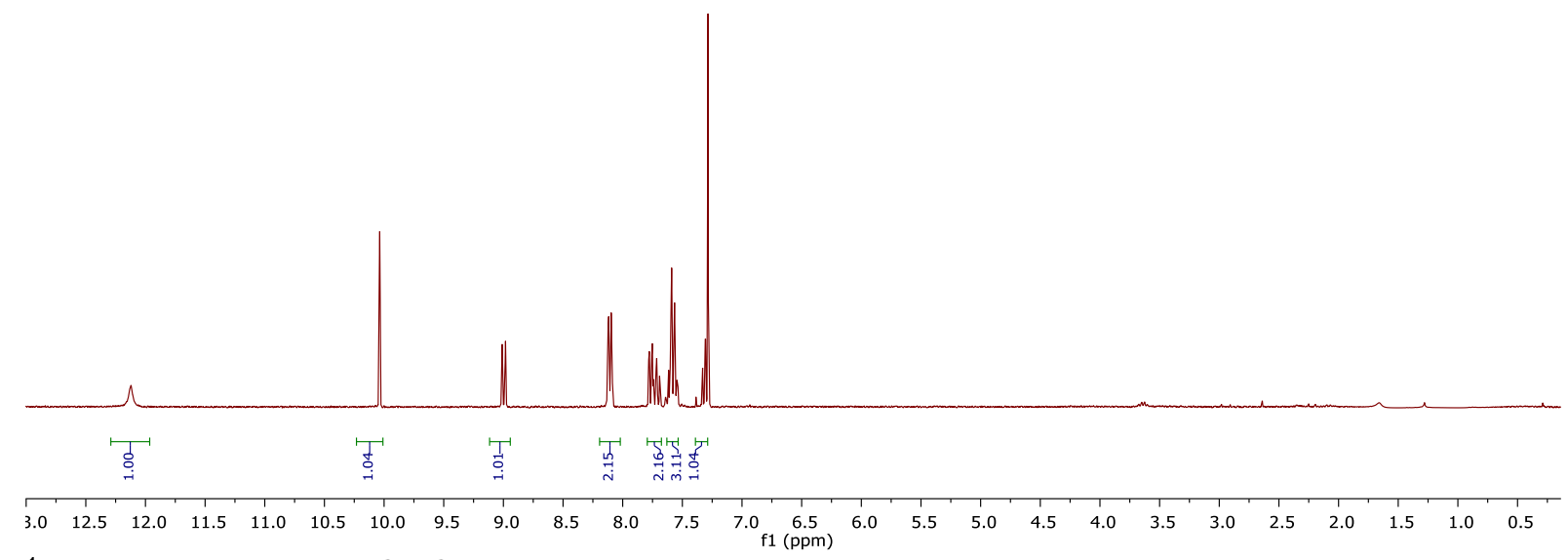

${ }^{1} \mathrm{H}$ NMR spectrum in $\mathrm{CDCl}_{3}$.

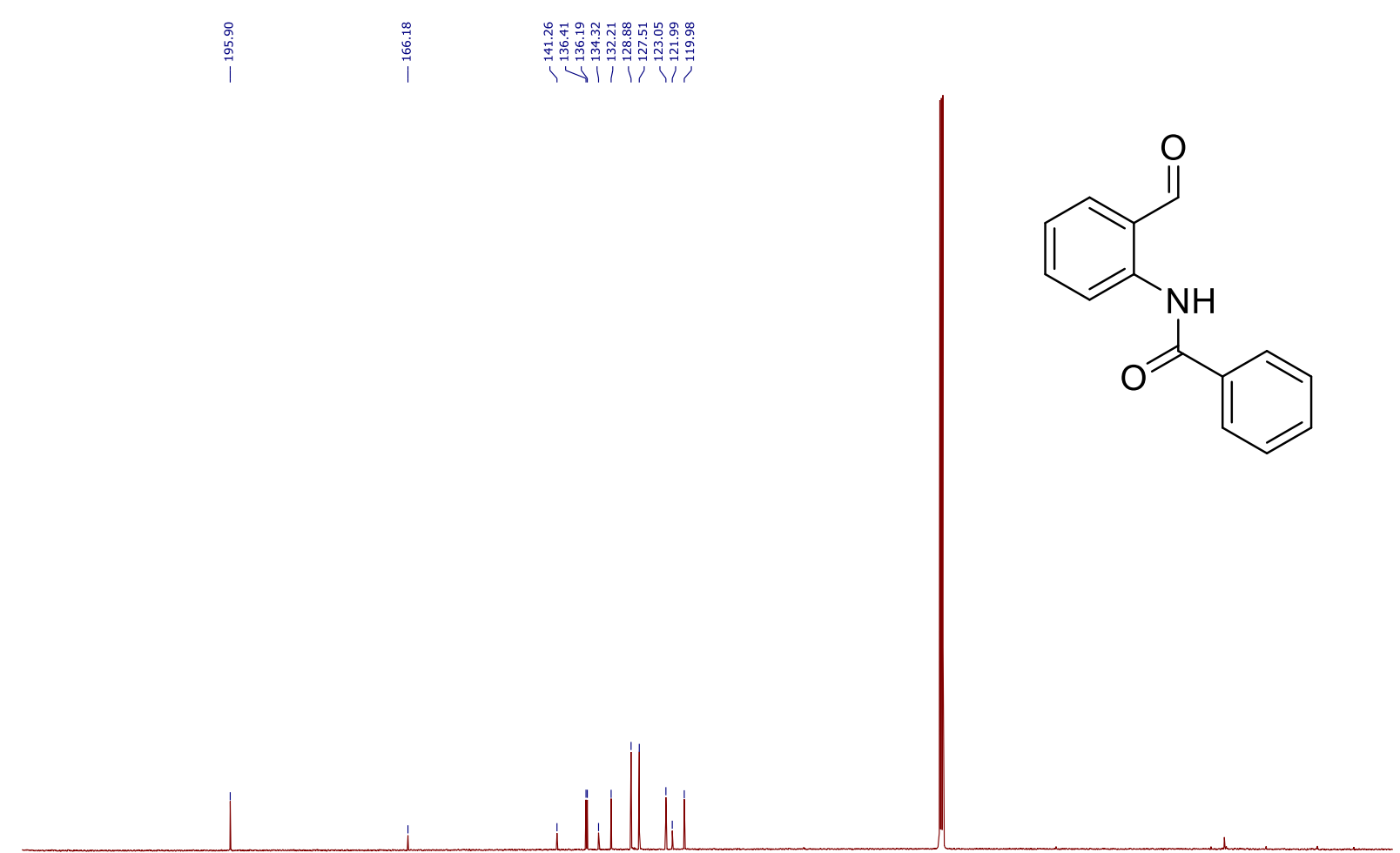

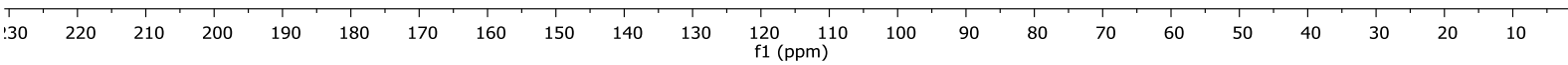
${ }^{13} \mathrm{C}$ NMR spectrum in $\mathrm{CDCl}_{3}$. 
$88 b$

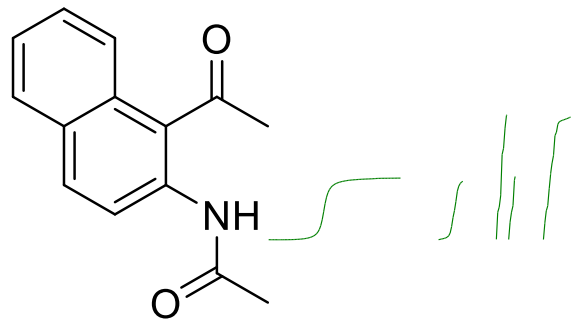

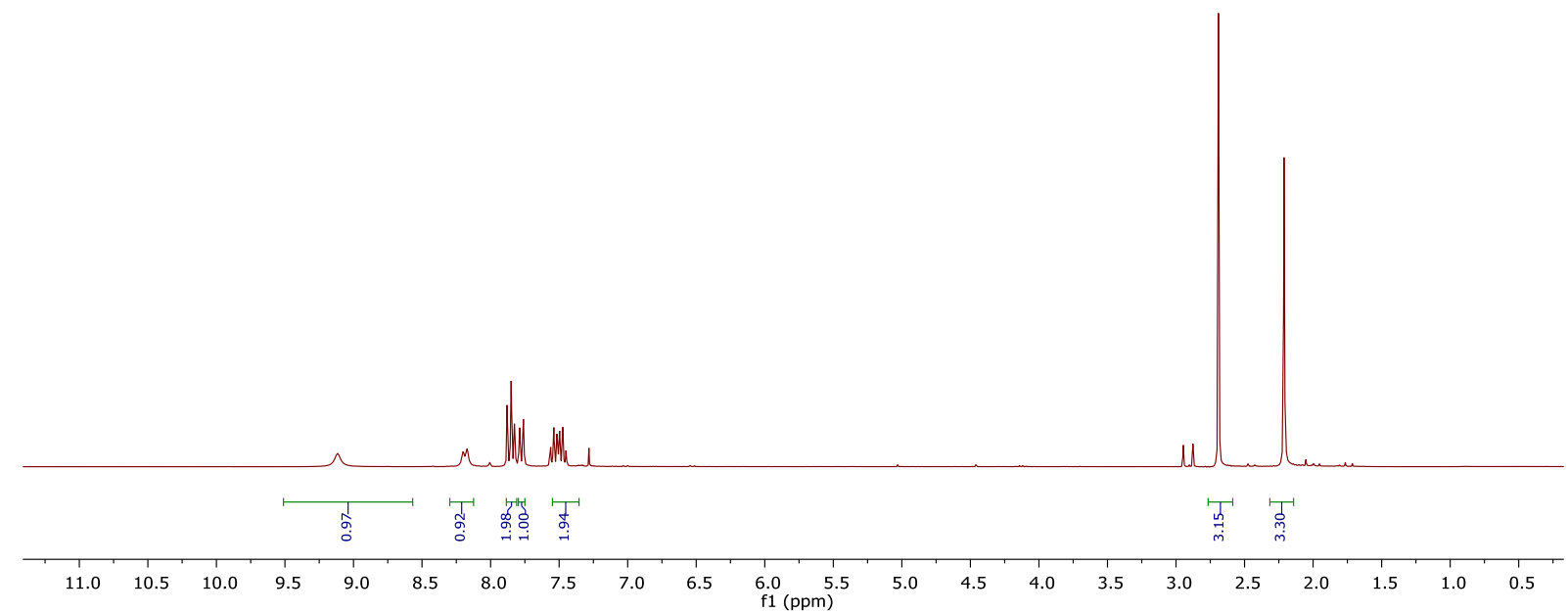

${ }^{1} \mathrm{H}$ NMR spectrum in $\mathrm{CDCl}_{3}$.

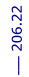

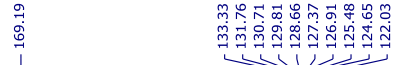

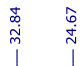<smiles>CC(=O)Nc1ccc2ccccc2c1C(C)=O</smiles>

$\begin{array}{lllllll}220 & 210 & 200 & 190 & 180 & 170 & 160\end{array}$

${ }^{13} \mathrm{C}$ NMR spectrum in $\mathrm{CDCl}_{3}$. 
<smiles>CC(=O)N(C)c1ccccc1C=O</smiles>
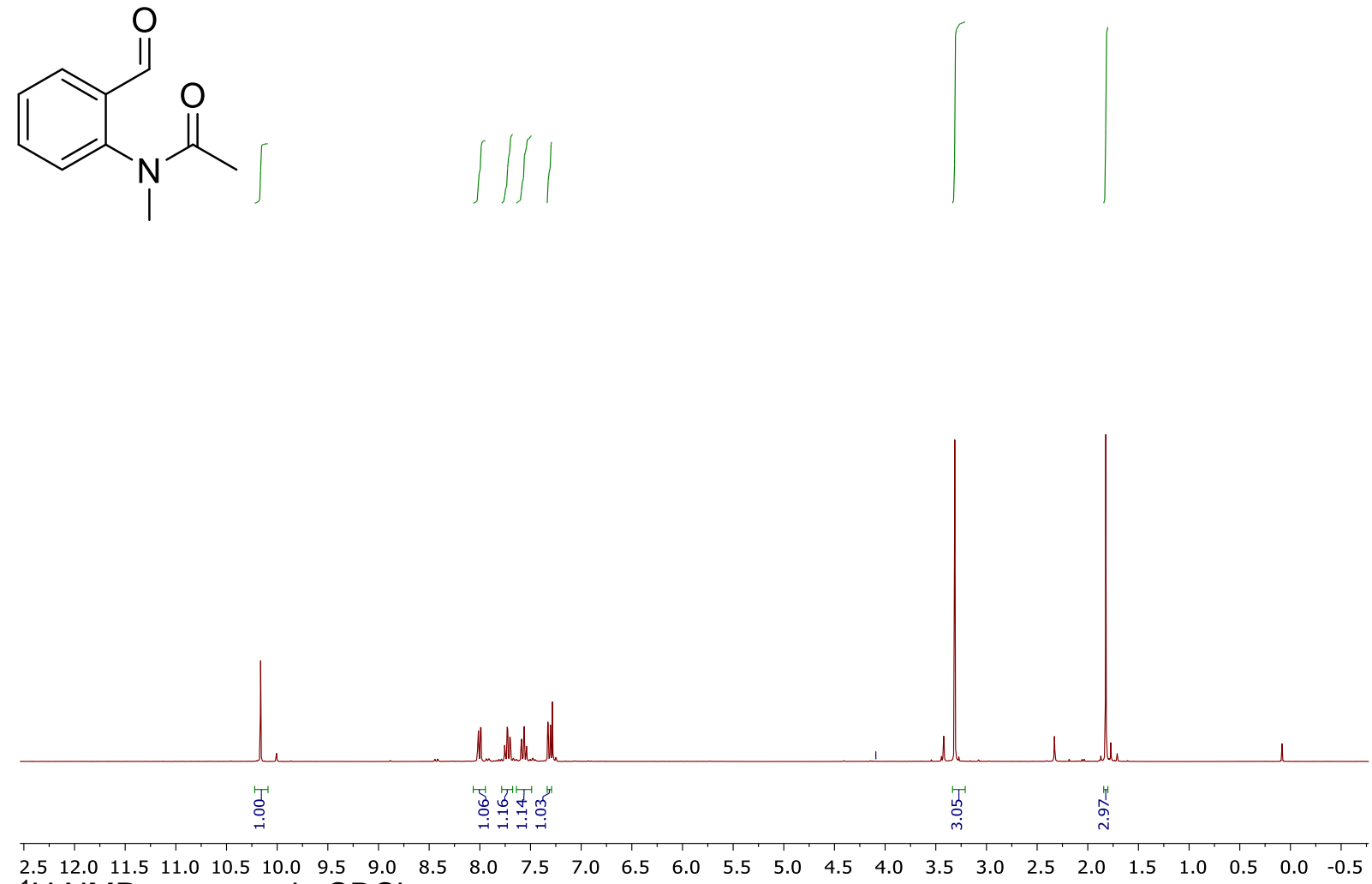
${ }^{1} \mathrm{H}$ NMR spectrum in $\mathrm{CDCl}_{3}$.

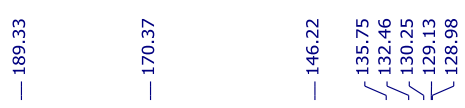<smiles>CC(=O)N(C)c1ccccc1C=O</smiles>

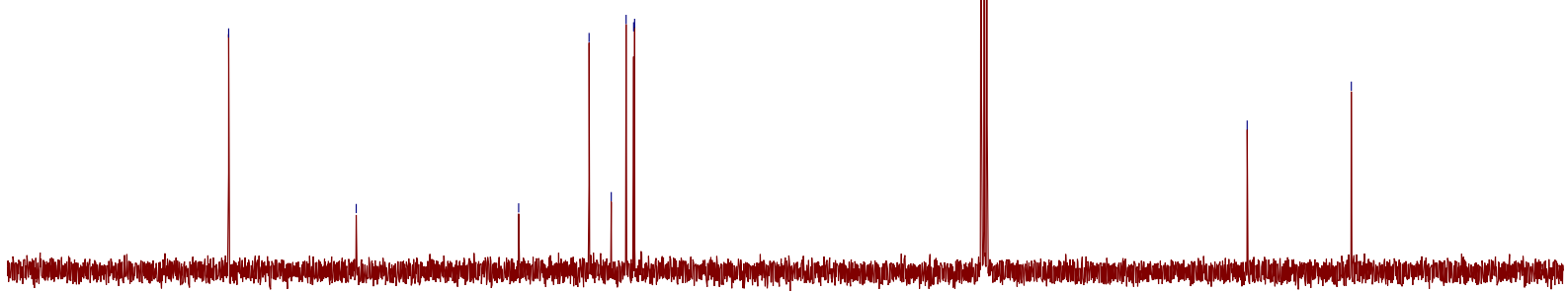

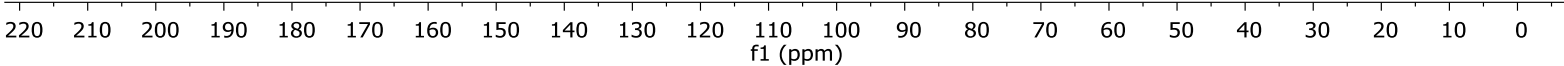
${ }^{13} \mathrm{C}$ NMR spectrum in $\mathrm{CDCl}_{3}$. 
$90 \mathrm{~b}$
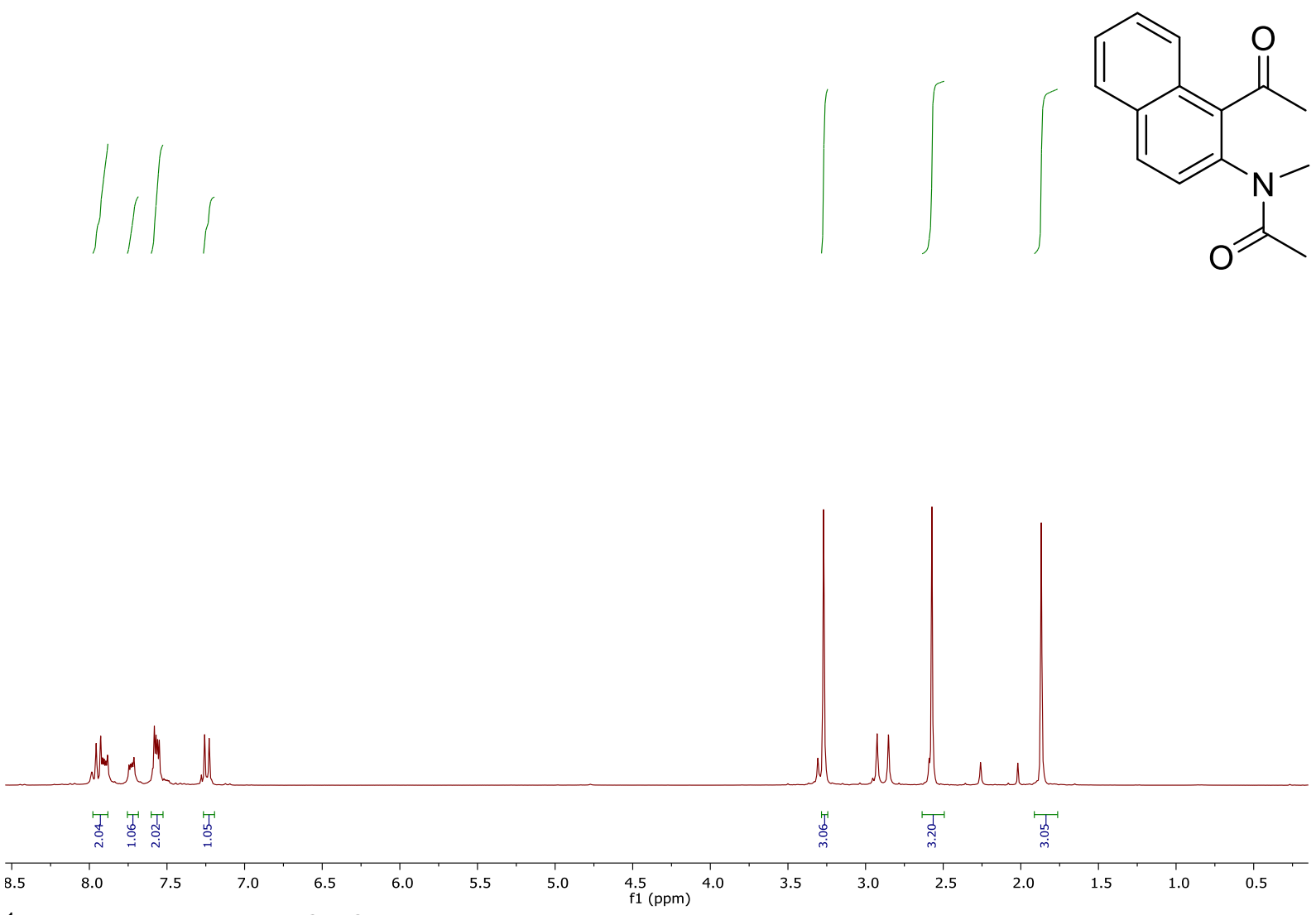

${ }^{1} \mathrm{H}$ NMR spectrum in $\mathrm{CDCl}_{3}$.

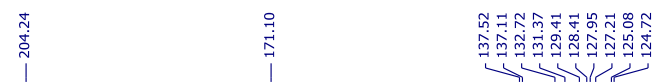

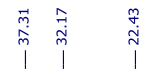
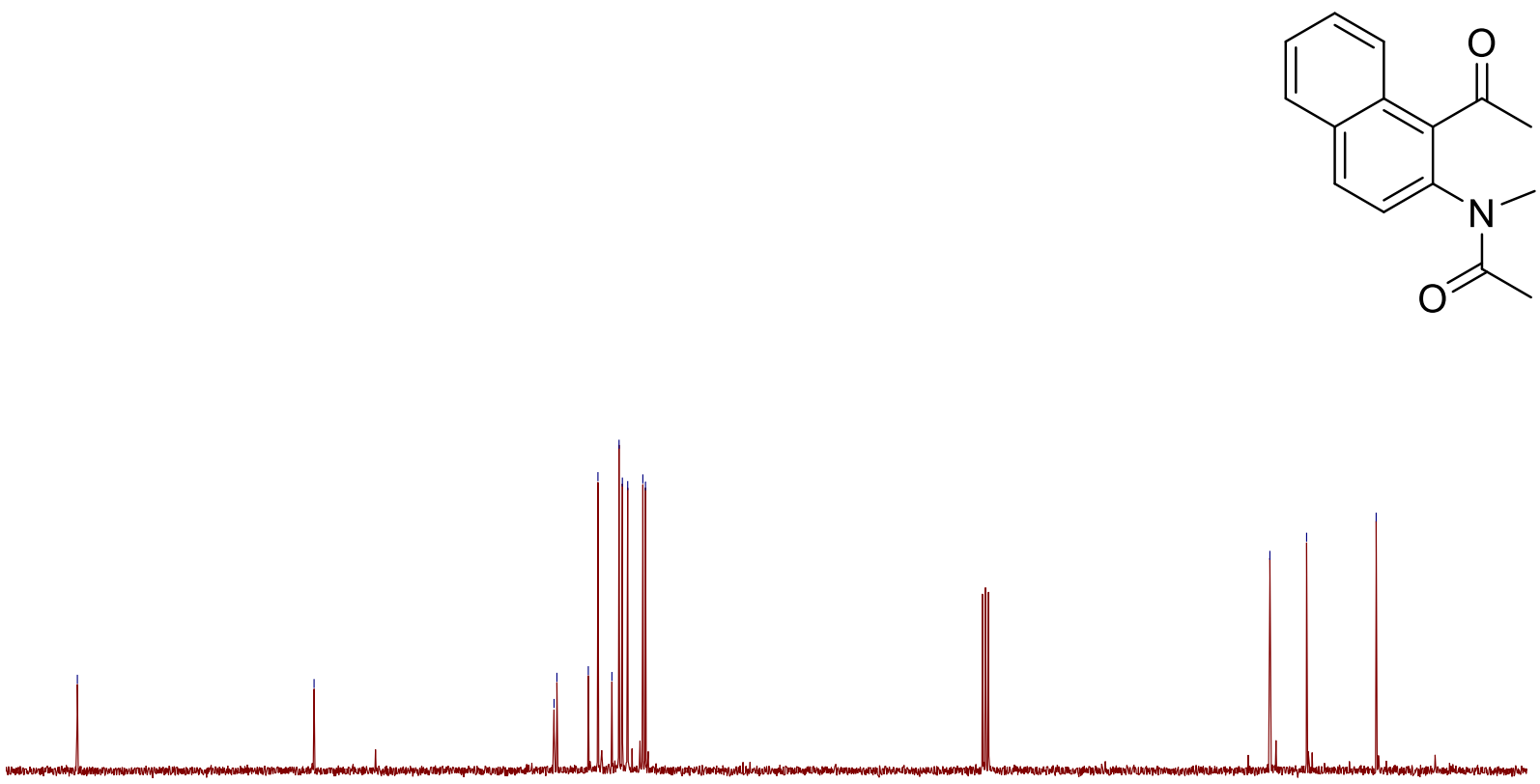

$\begin{array}{llllllllll}210 & 200 & 190 & 180 & 170 & 160 & 150 & 140 & 130 & 120\end{array}$ 110100 ${ }^{13} \mathrm{C}$ NMR spectrum in $\mathrm{CDCl}_{3}$. 


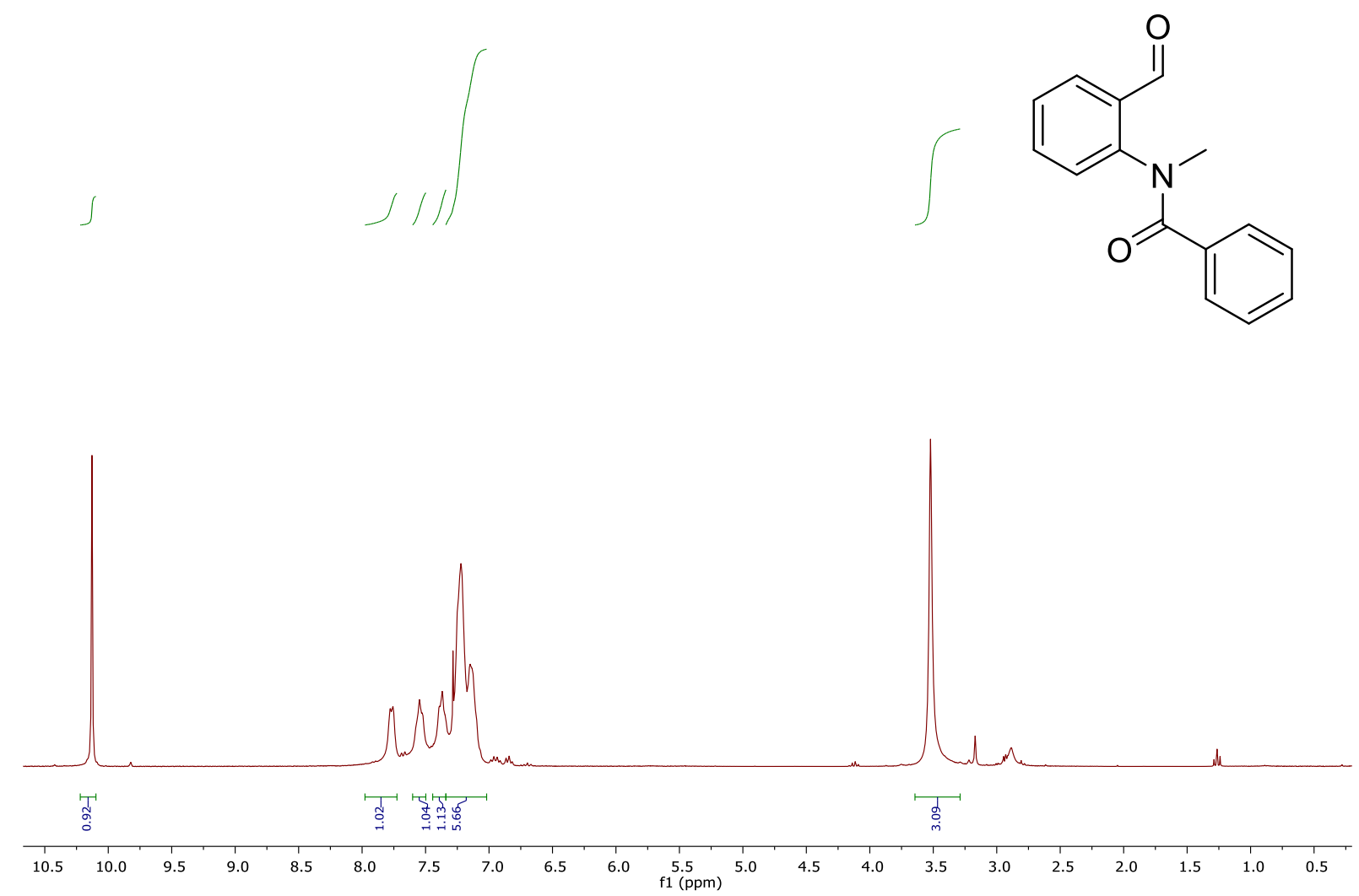

${ }^{1} \mathrm{H}$ NMR spectrum in $\mathrm{CDCl}_{3}$.
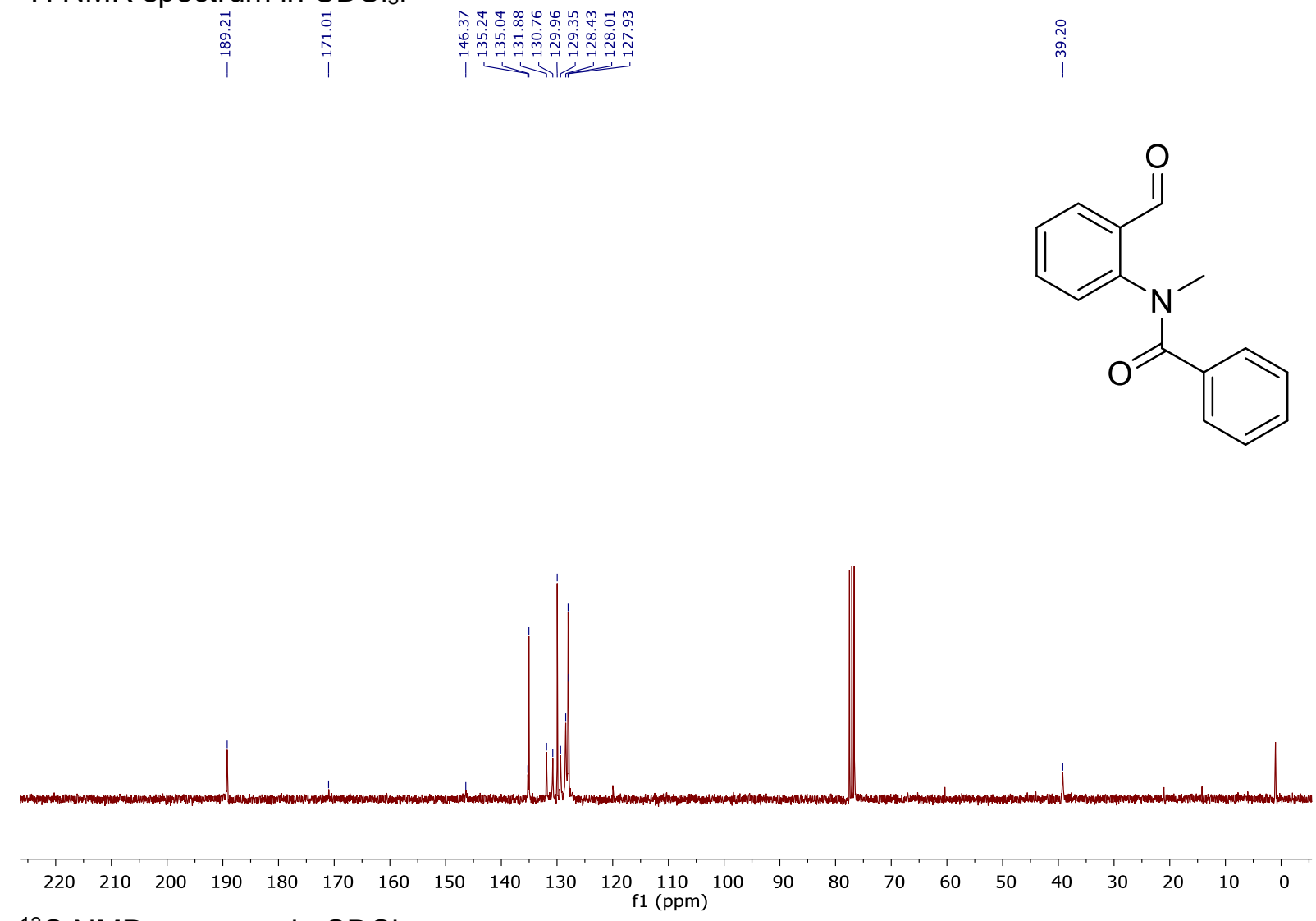

${ }^{13} \mathrm{C}$ NMR spectrum in $\mathrm{CDCl}_{3}$. 
$93 b$<smiles>CCC1=C(CC)C(=O)NC1=O</smiles>
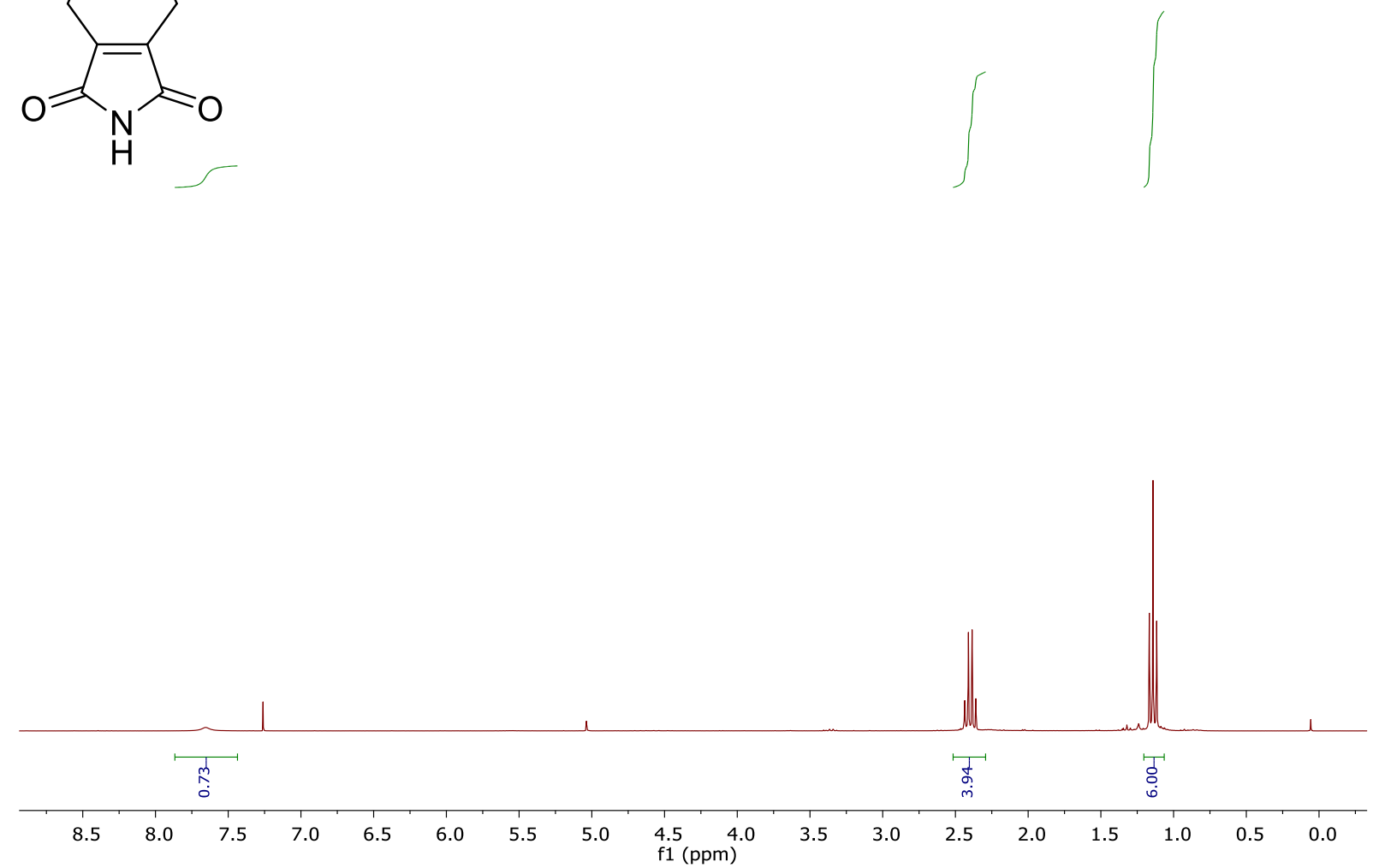

${ }^{1} \mathrm{H}$ NMR spectrum in $\mathrm{CDCl}_{3}$.

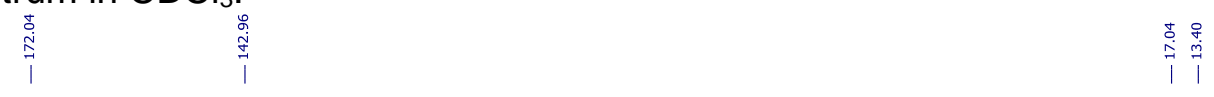<smiles>CCC1=C(CC)C(=O)NC1=O</smiles>
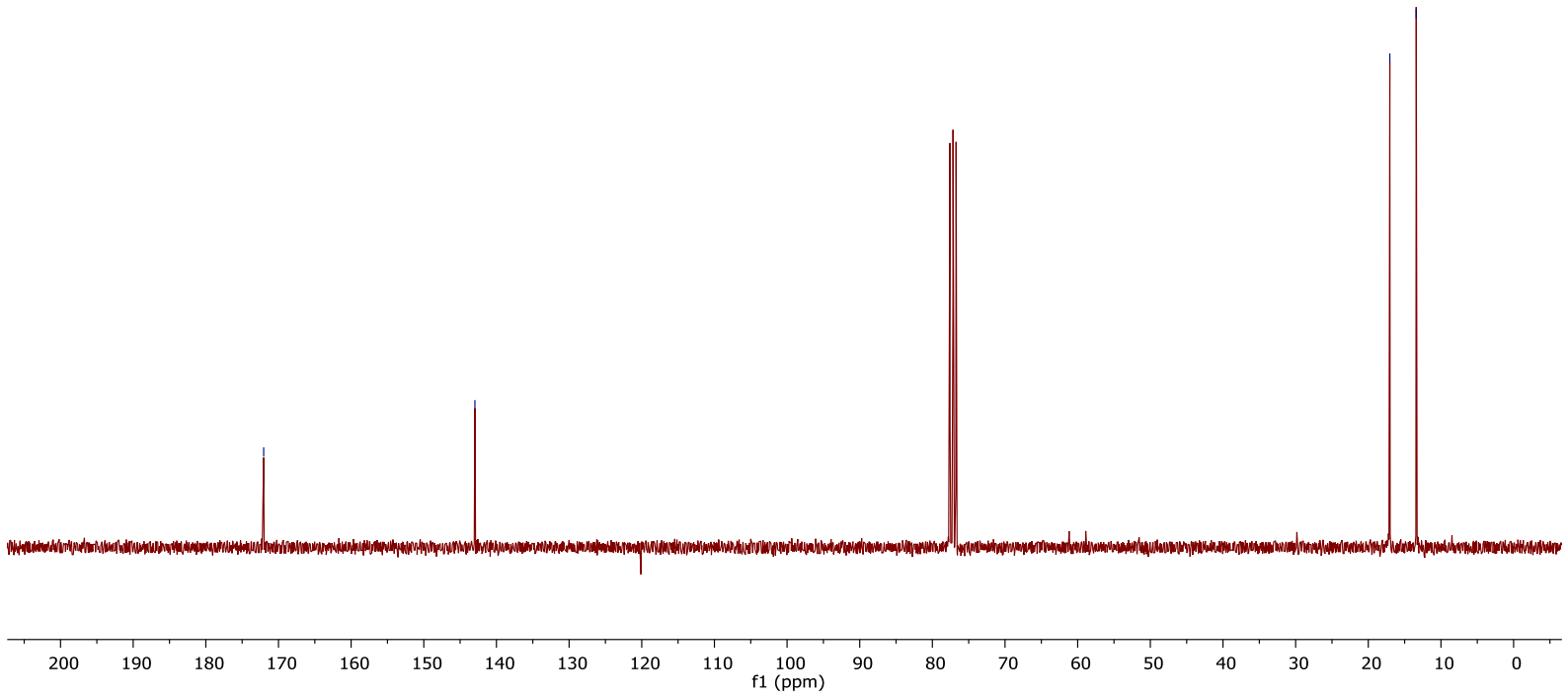

${ }^{13} \mathrm{C}$ NMR spectrum in $\mathrm{CDCl}_{3}$. 
$94 b$<smiles>CCOC(=O)C1=C(c2ccccc2)C(=O)NC1=O</smiles>
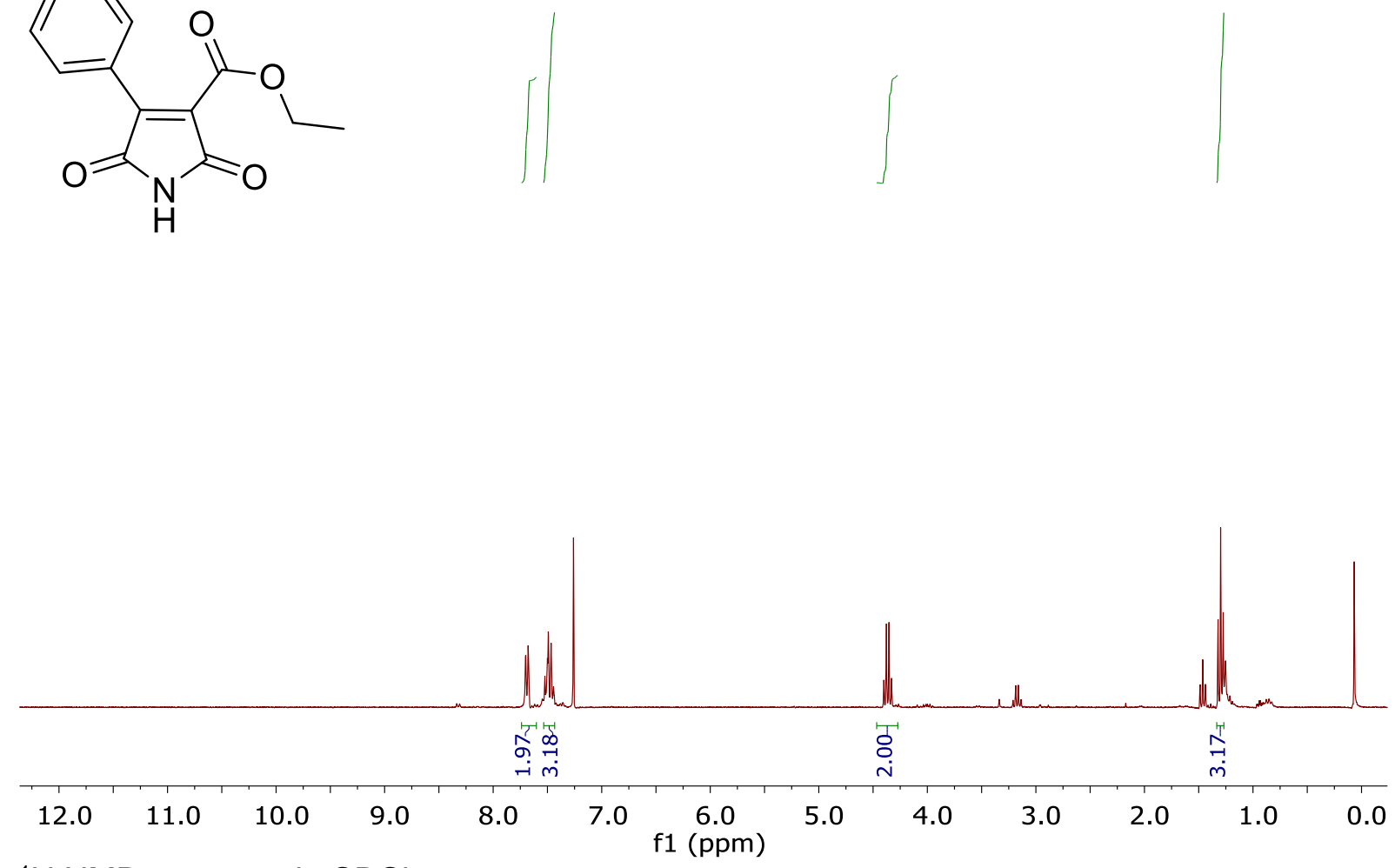

${ }^{1} \mathrm{H}$ NMR spectrum in $\mathrm{CDCl}_{3}$.

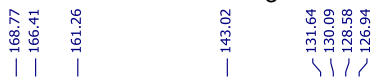<smiles>CCOC(=O)C1=C(c2ccccc2)C(=O)NC1=O</smiles>
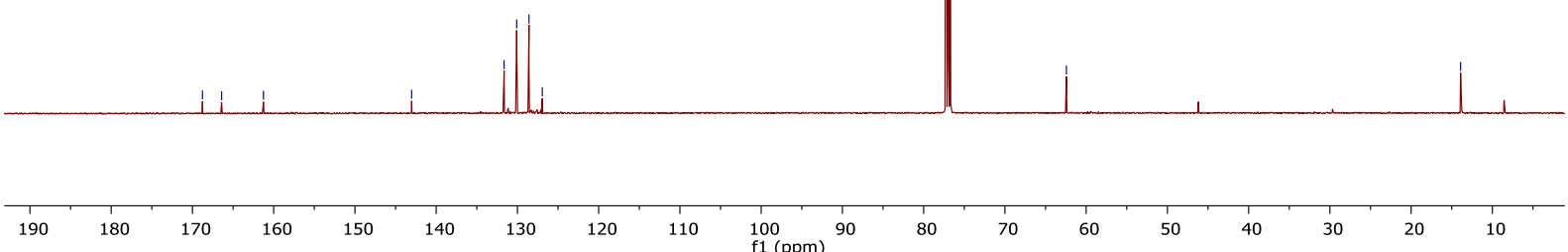

${ }^{13} \mathrm{C}$ NMR spectrum in $\mathrm{CDCl}_{3}$. 
95b<smiles>O=C1C=C(c2ccccc2)C(=O)N1</smiles>
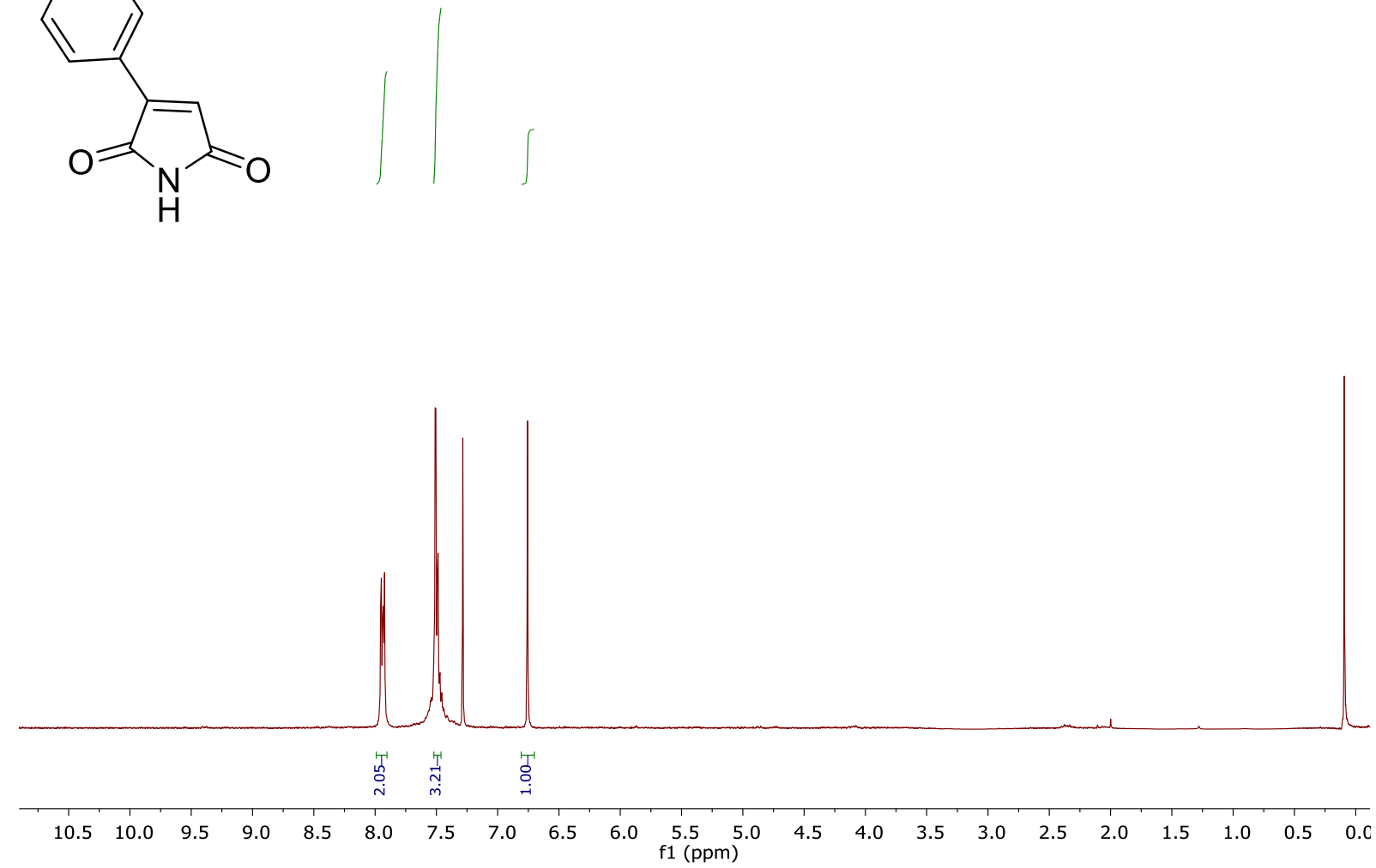

${ }^{1} \mathrm{H}$ NMR spectrum in $\mathrm{CDCl}_{3}$.
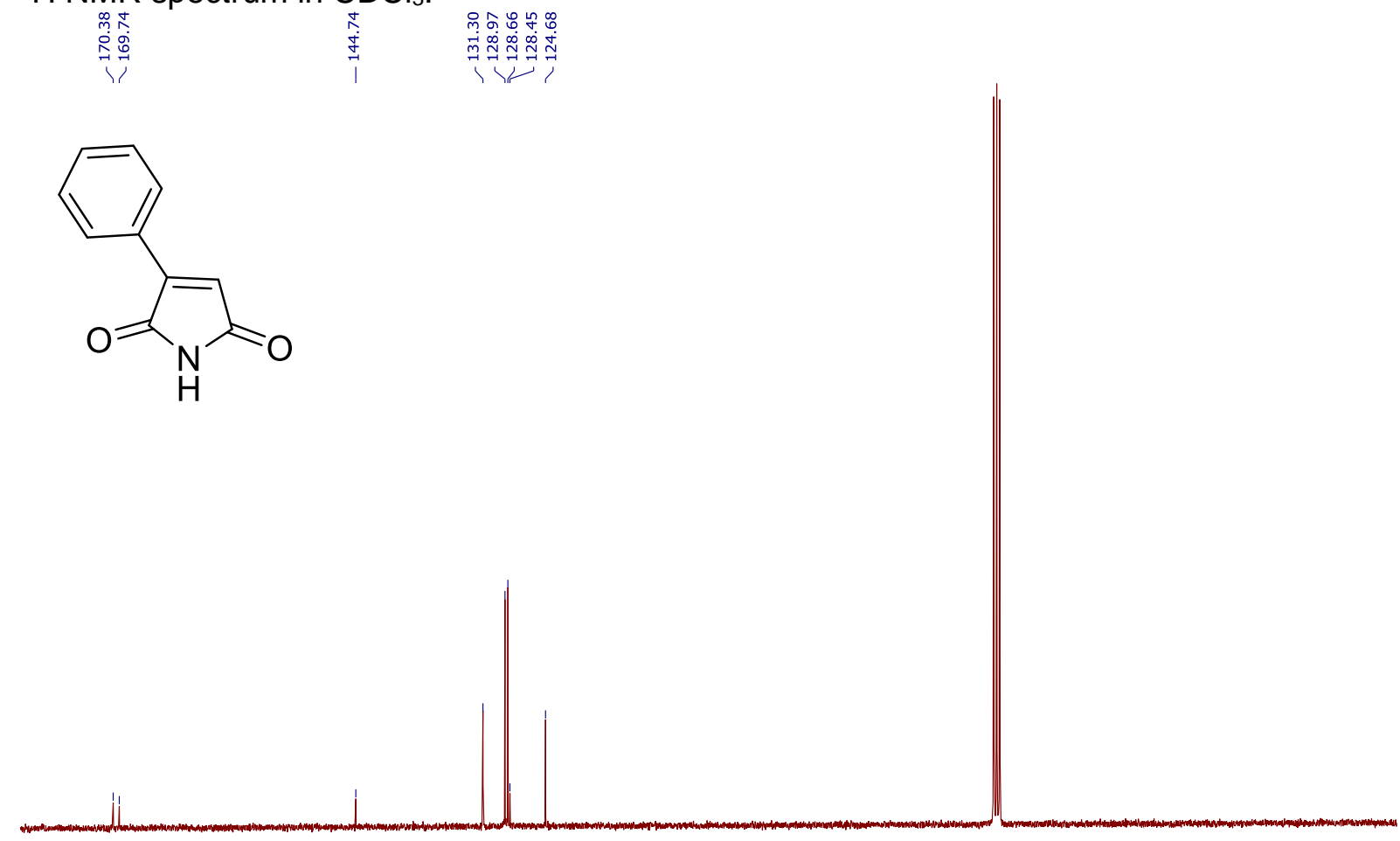

$\begin{array}{llllllllllllllllllllllllllllll}80 & 175 & 170 & 165 & 160 & 155 & 150 & 145 & 140 & 135 & 130 & 125 & 120 & 115 & 110 & 105 & 100 & 95 & 90 & 85 & 80 & 75 & 70 & 65 & 60 & 55 & 50 & 45 & 40 & 3\end{array}$ ${ }^{13} \mathrm{C}$ NMR spectrum in $\mathrm{CDCl}_{3}$. 
$96 b$<smiles>O=C1C=CC(=O)N1c1ccccc1</smiles>
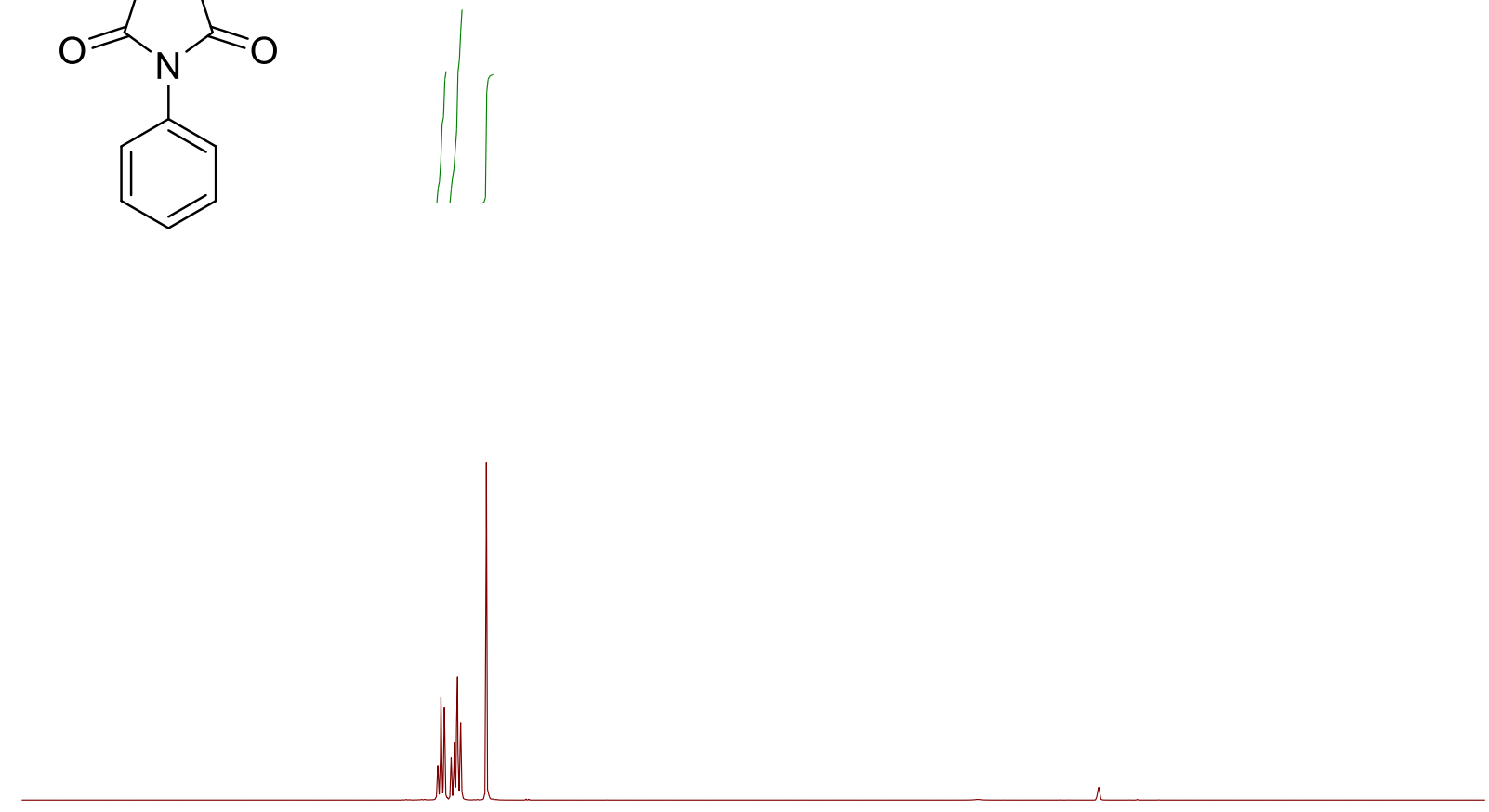

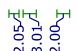

$\begin{array}{llllllllllllllllllllllllll}10.5 & 10.0 & 9.5 & 9.0 & 8.5 & 8.0 & 7.5 & 7.0 & 6.5 & 6.0 & 5.5 & 5.0 & 4.5 & 4.0 & 3.5 & 3.0 & 2.5 & 2.0 & 1.5 & 1.0 & 0.5 & 0.0\end{array}$

${ }^{1} \mathrm{H}$ NMR spectrum in DMSO- $d_{6}$.

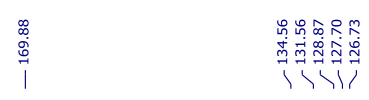<smiles>O=C1C=CC(=O)N1c1ccccc1</smiles>

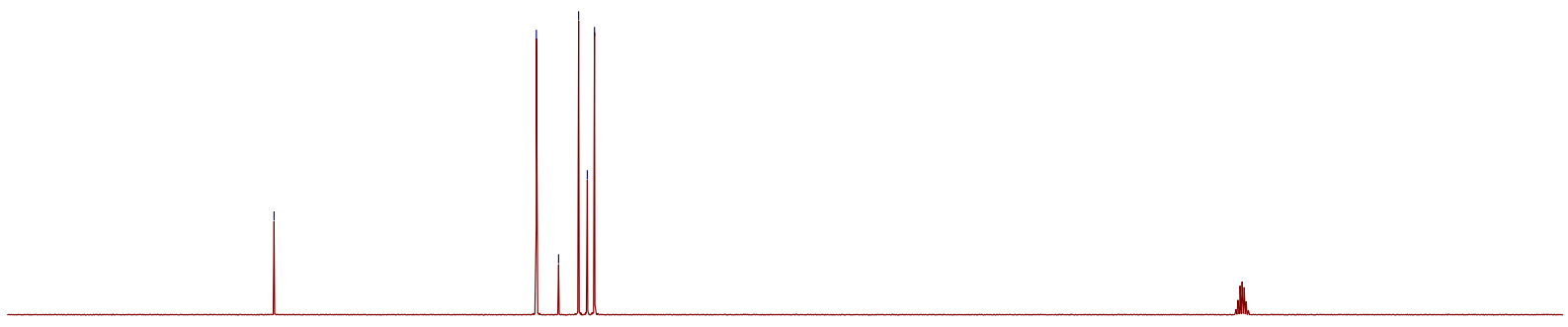

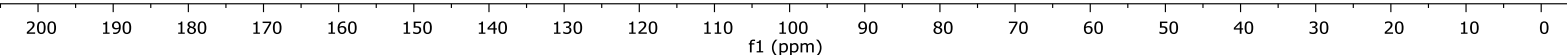

${ }^{13} \mathrm{C}$ NMR spectrum in DMSO- $d_{6}$. 
97b<smiles>O=C1C=CC(=O)N1c1ccc(I)cc1</smiles>

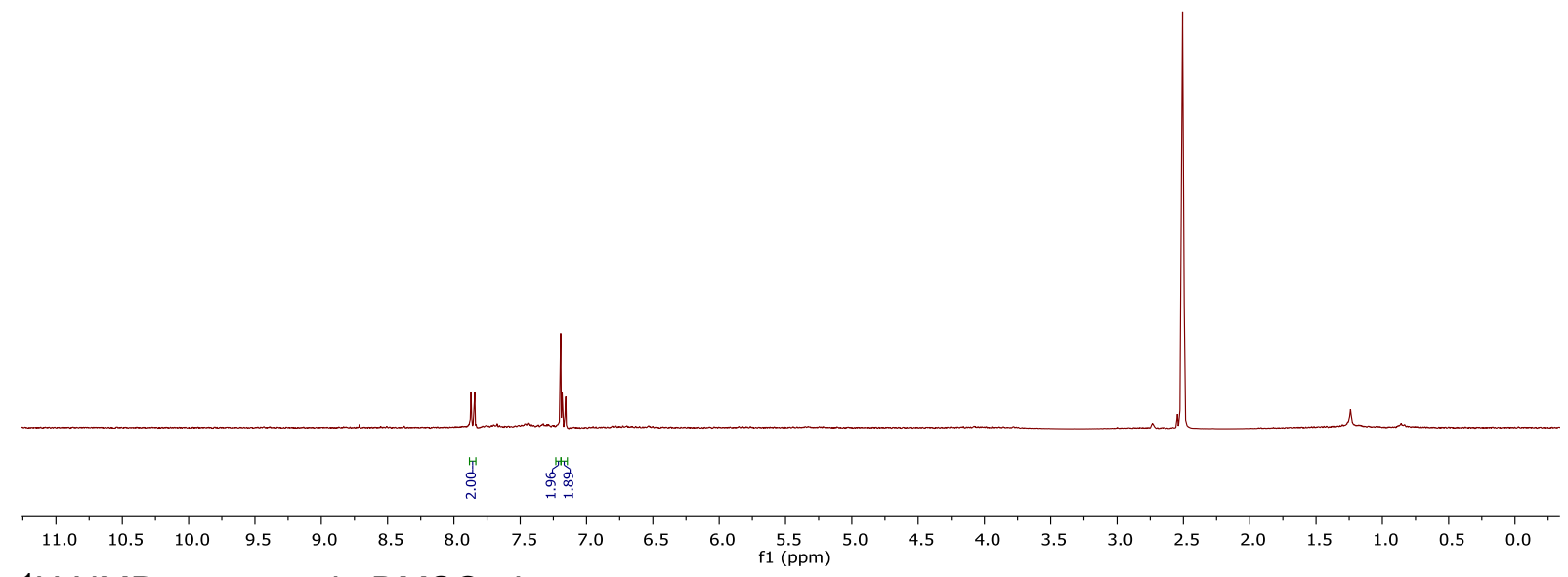

${ }^{1} \mathrm{H}$ NMR spectrum in DMSO- $d_{6}$.

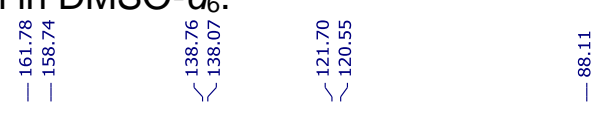<smiles>O=C1C=CC(=O)N1c1ccc(I)cc1</smiles>

$\begin{array}{lllllllllllllllllllll}200 & 190 & 180 & 170 & 160 & 150 & 140 & 130 & 120 & 110 & 100 & 90 & 80 & 70 & 60 & 50 & 40 & 30 & 20 & 10 & 0\end{array}$ ${ }^{13} \mathrm{C}$ NMR spectrum in $\mathrm{CDCl}_{3}$. 
$98 b$<smiles>COc1ccc(N2C(=O)C=CC2=O)cc1</smiles>
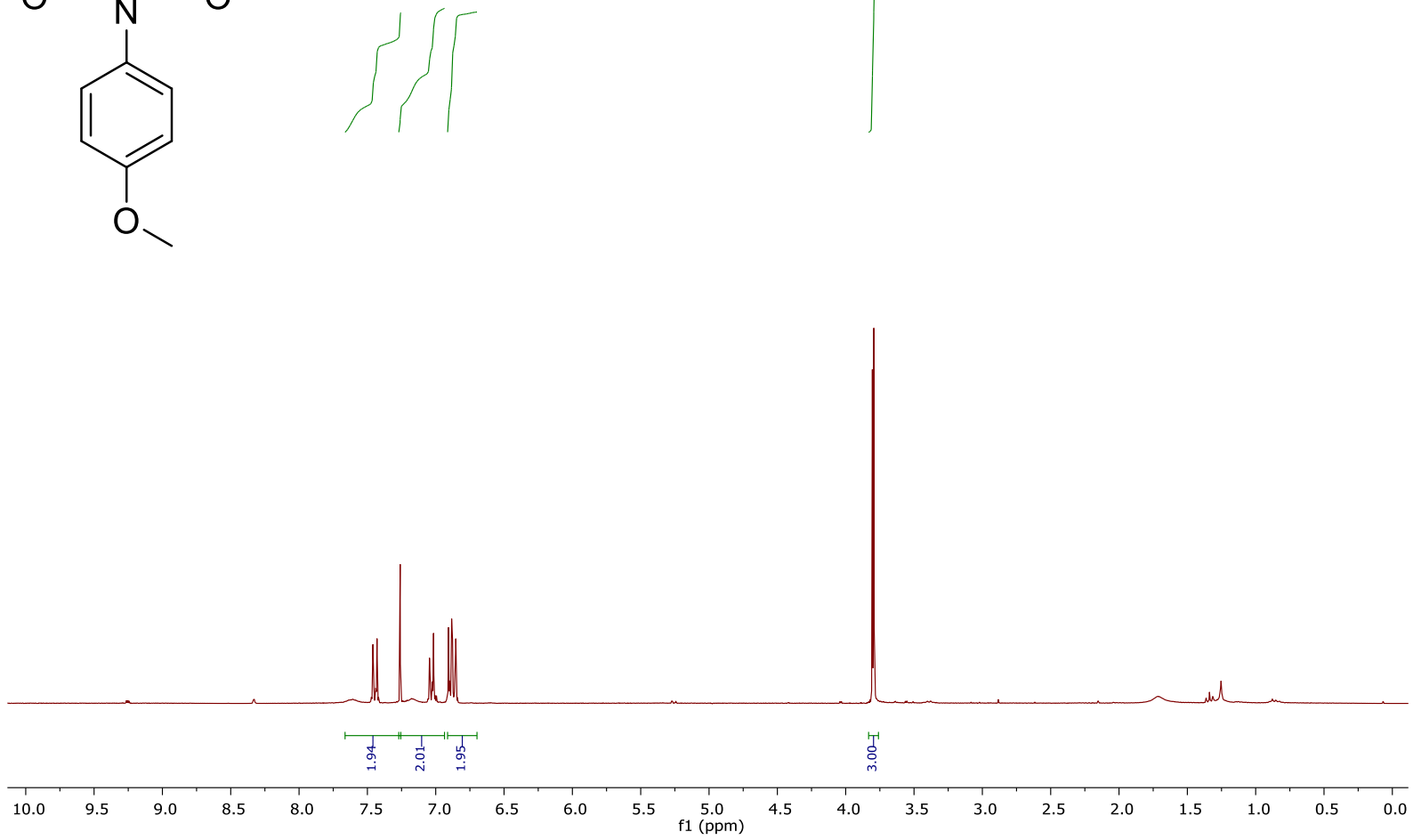

${ }^{1} \mathrm{H}$ NMR spectrum in $\mathrm{CDCl}_{3}$.

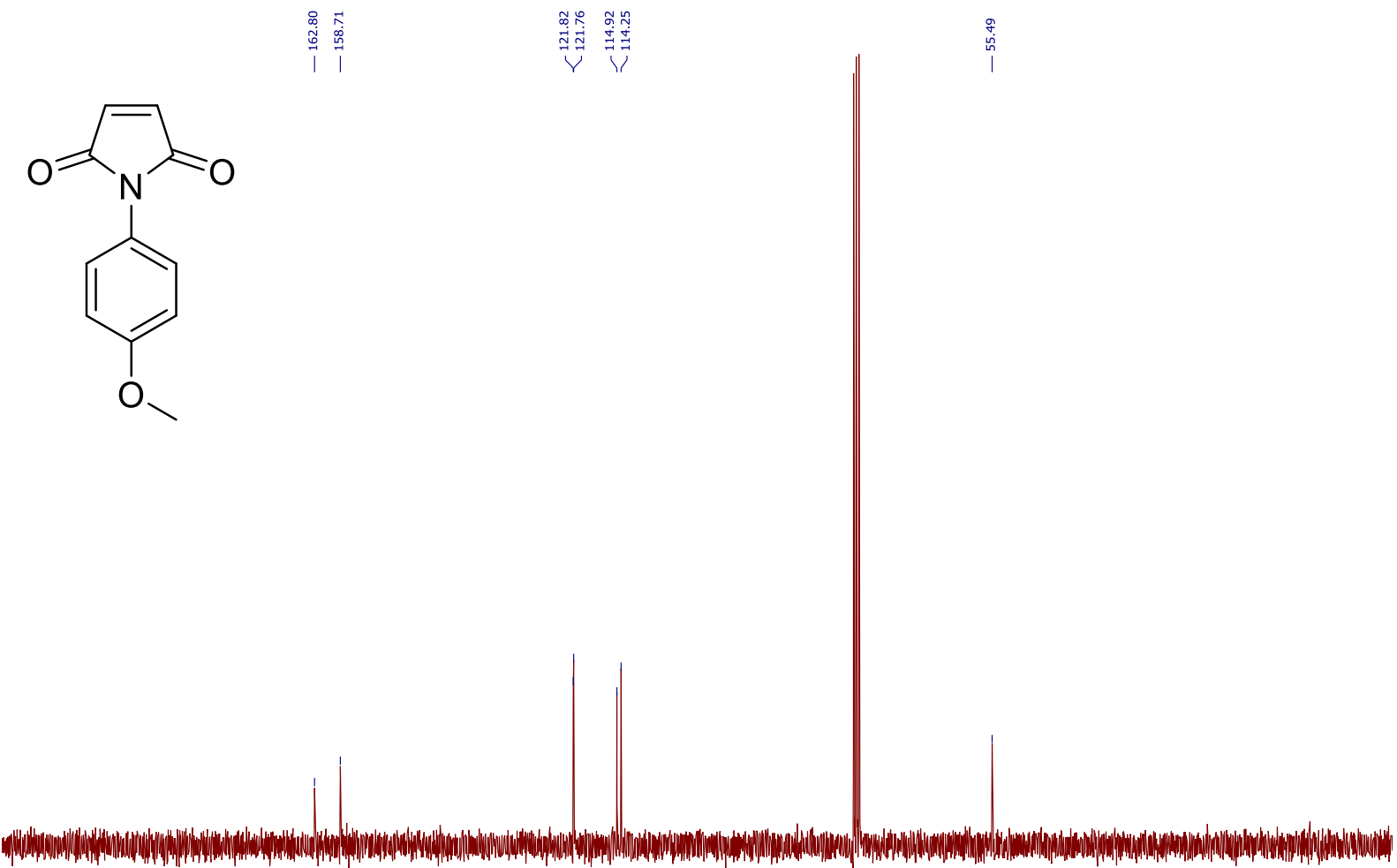

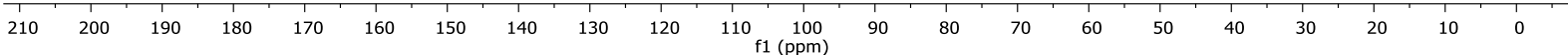
${ }^{13} \mathrm{C}$ NMR spectrum in $\mathrm{CDCl}_{3}$. 

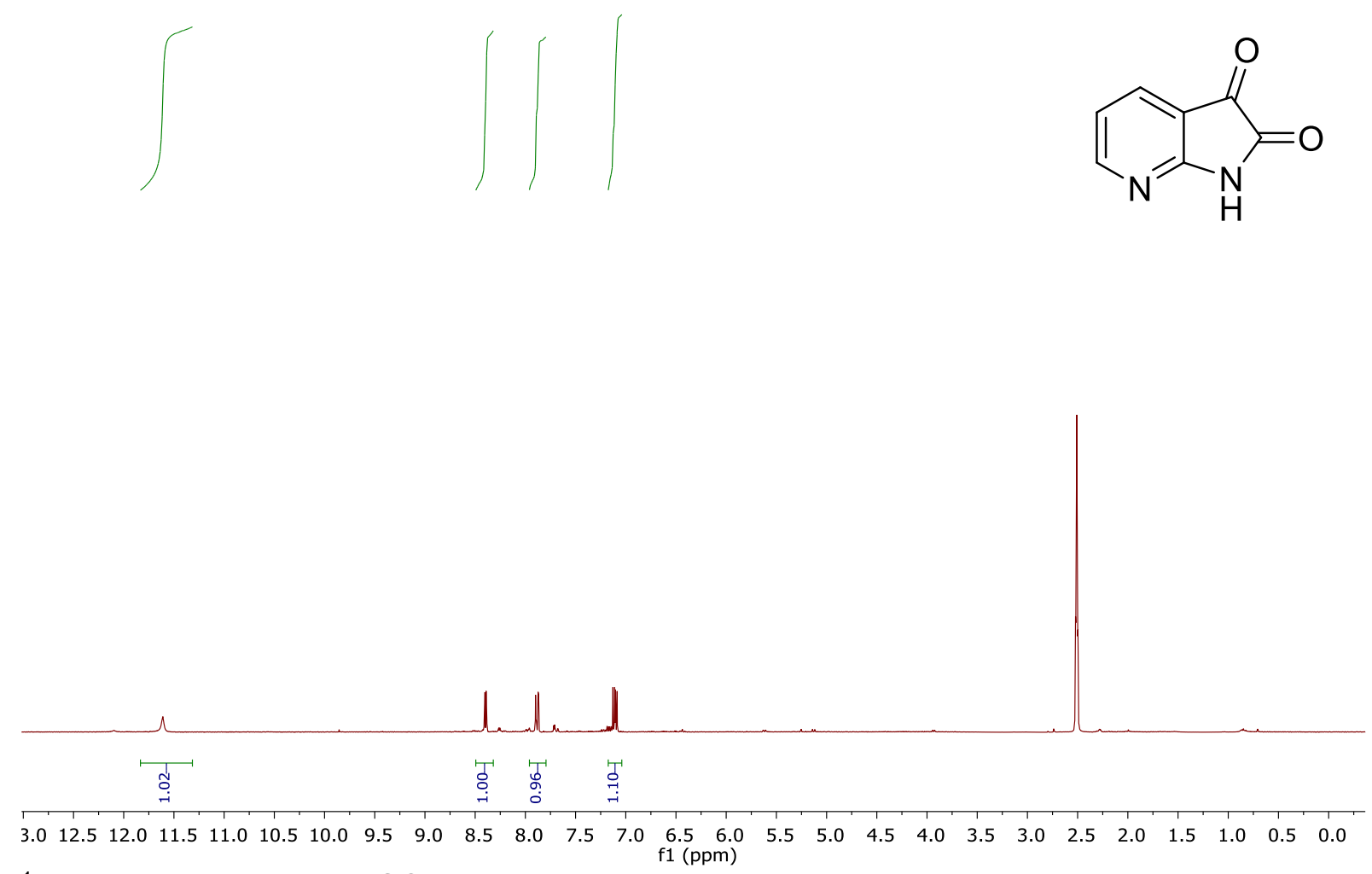

${ }^{1} \mathrm{H}$ NMR spectrum in DMSO- $d_{6}$.

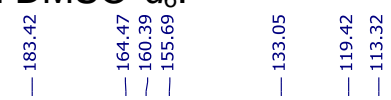

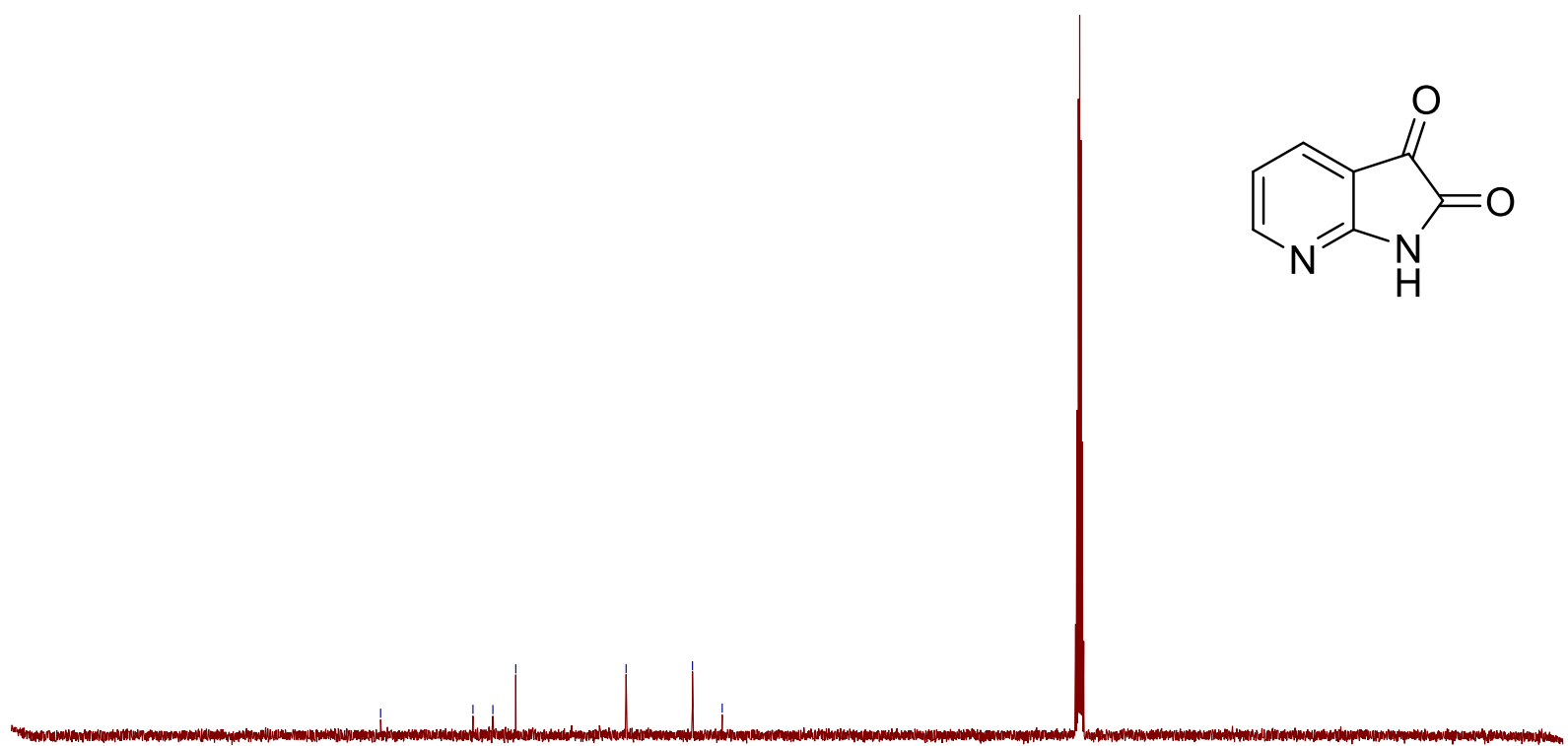

$250240230220210200190180170160150140130120110 \quad 10090 \quad 80 \quad 70 \quad 60 \quad 50 \quad 40 \quad 30 \quad 20 \begin{array}{lllllllll}0 & 0 & -10 & -20 & -30 & -40 & -50\end{array}$ ${ }^{13} \mathrm{C}$ NMR spectrum in DMSO- $d_{6}$. 


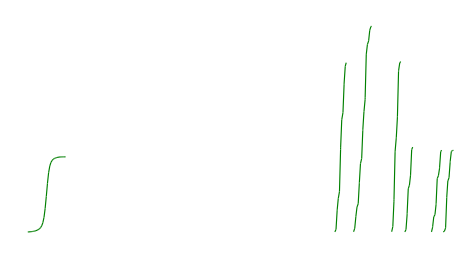<smiles>O=C1Nc2ccccc2/C1=N/c1ccccc1</smiles>

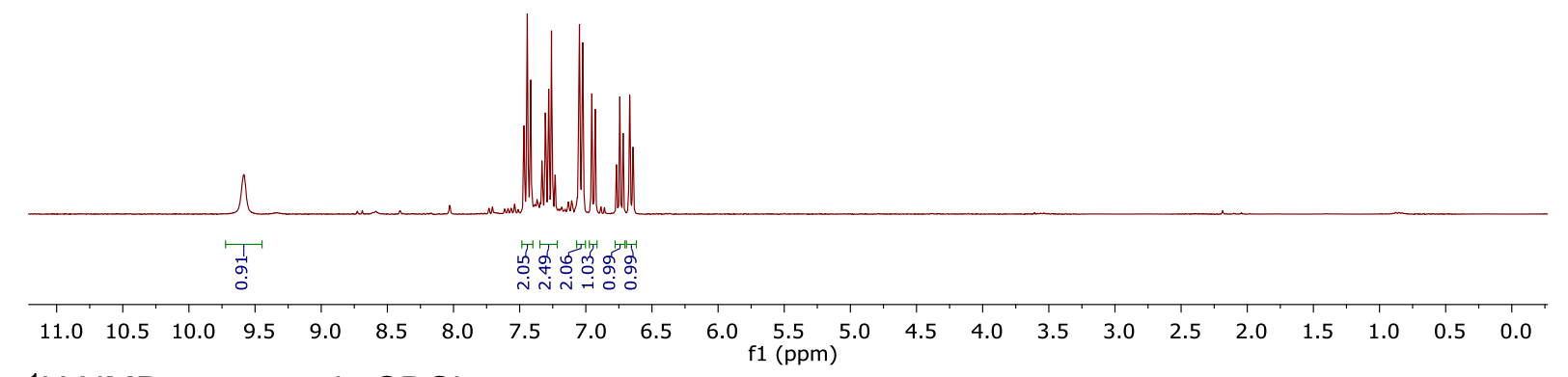

${ }^{1} \mathrm{H}$ NMR spectrum in $\mathrm{CDCl}_{3}$.

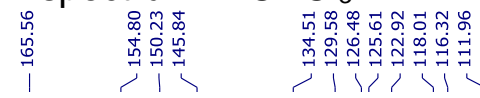

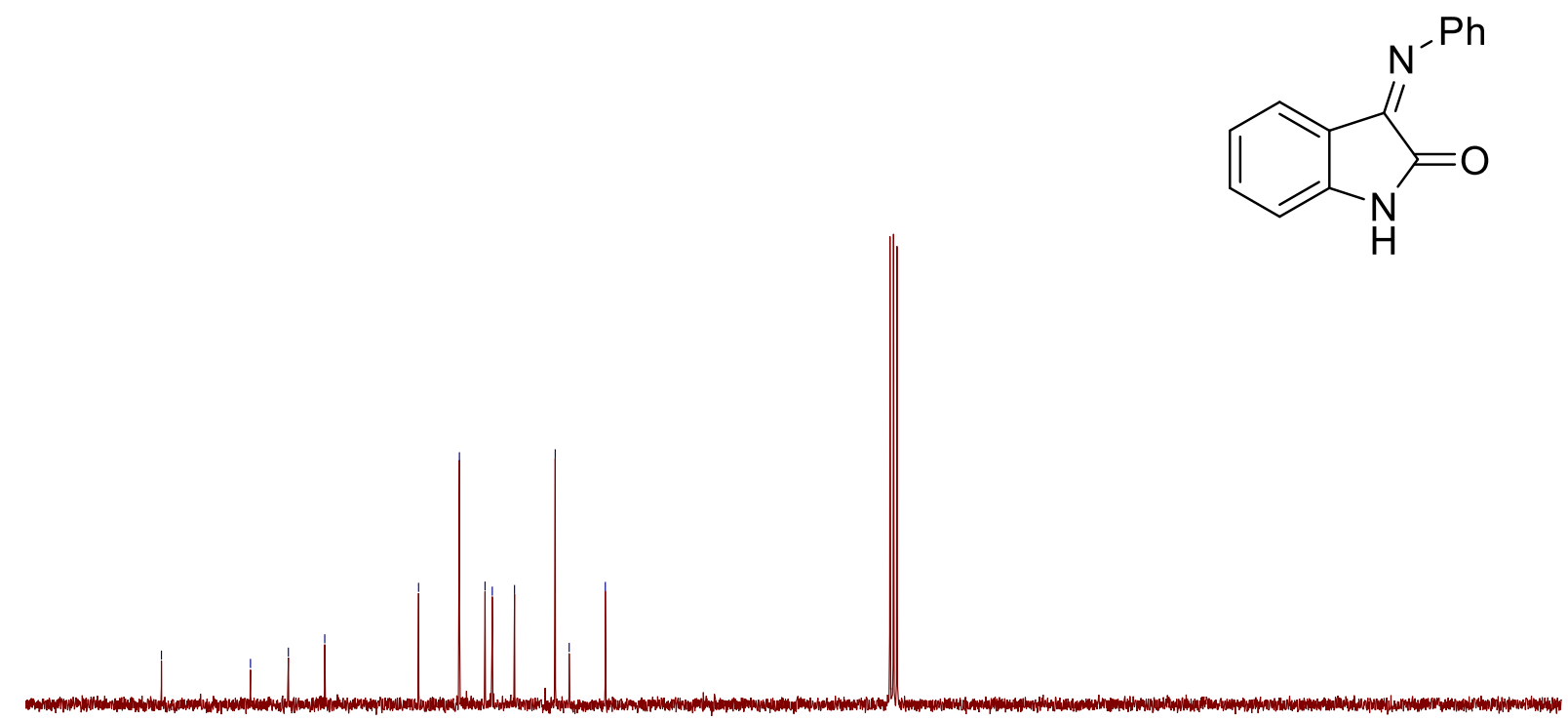

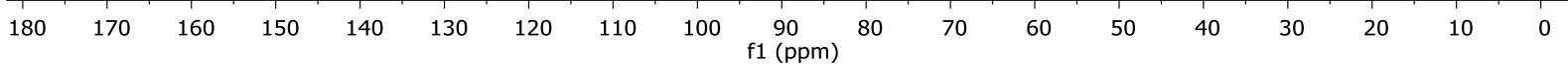

${ }^{13} \mathrm{C}$ NMR spectrum in $\mathrm{CDCl}_{3}$. 

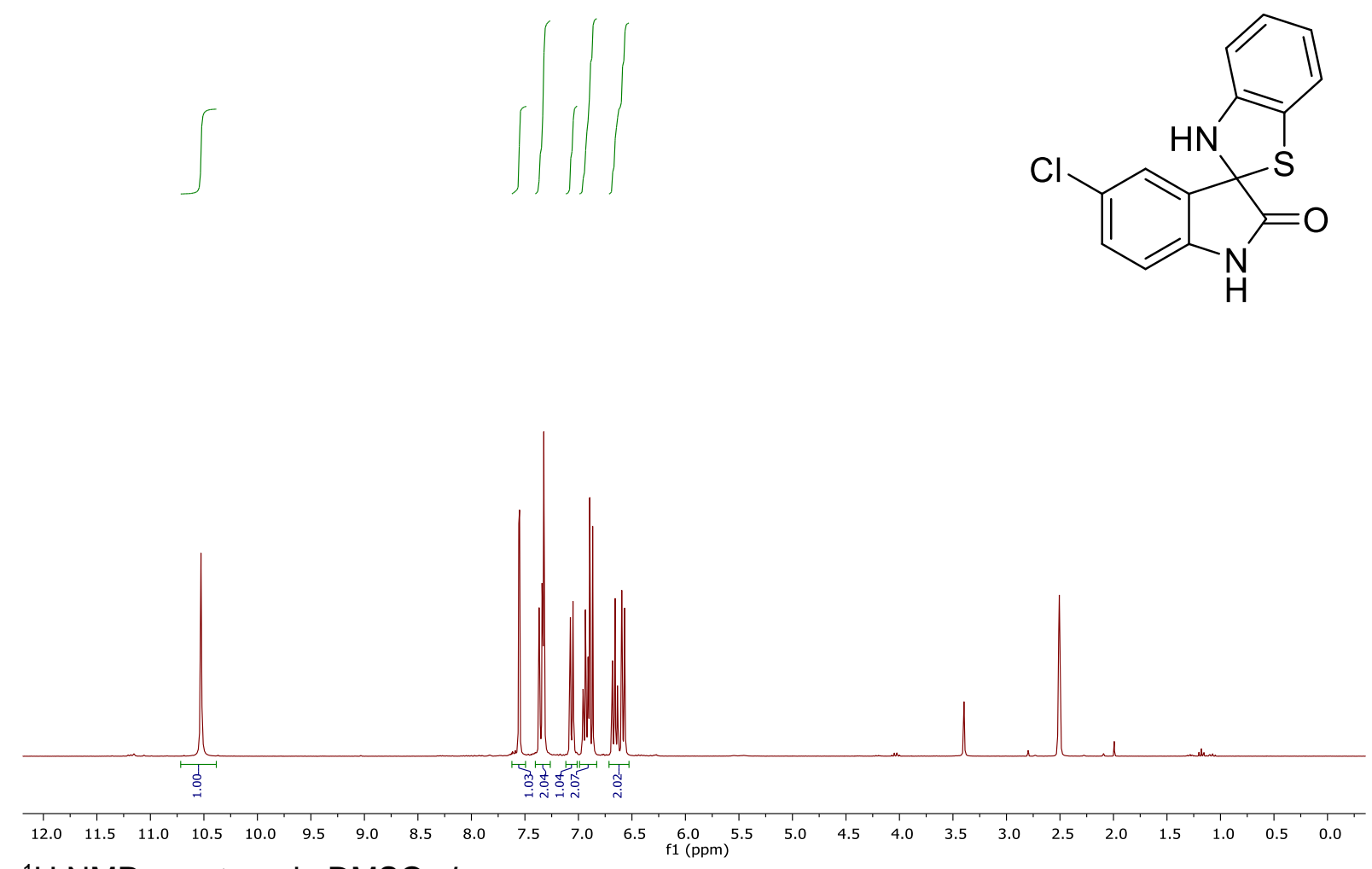

${ }^{1} \mathrm{H}$ NMR spectrum in DMSO- $d_{6}$.
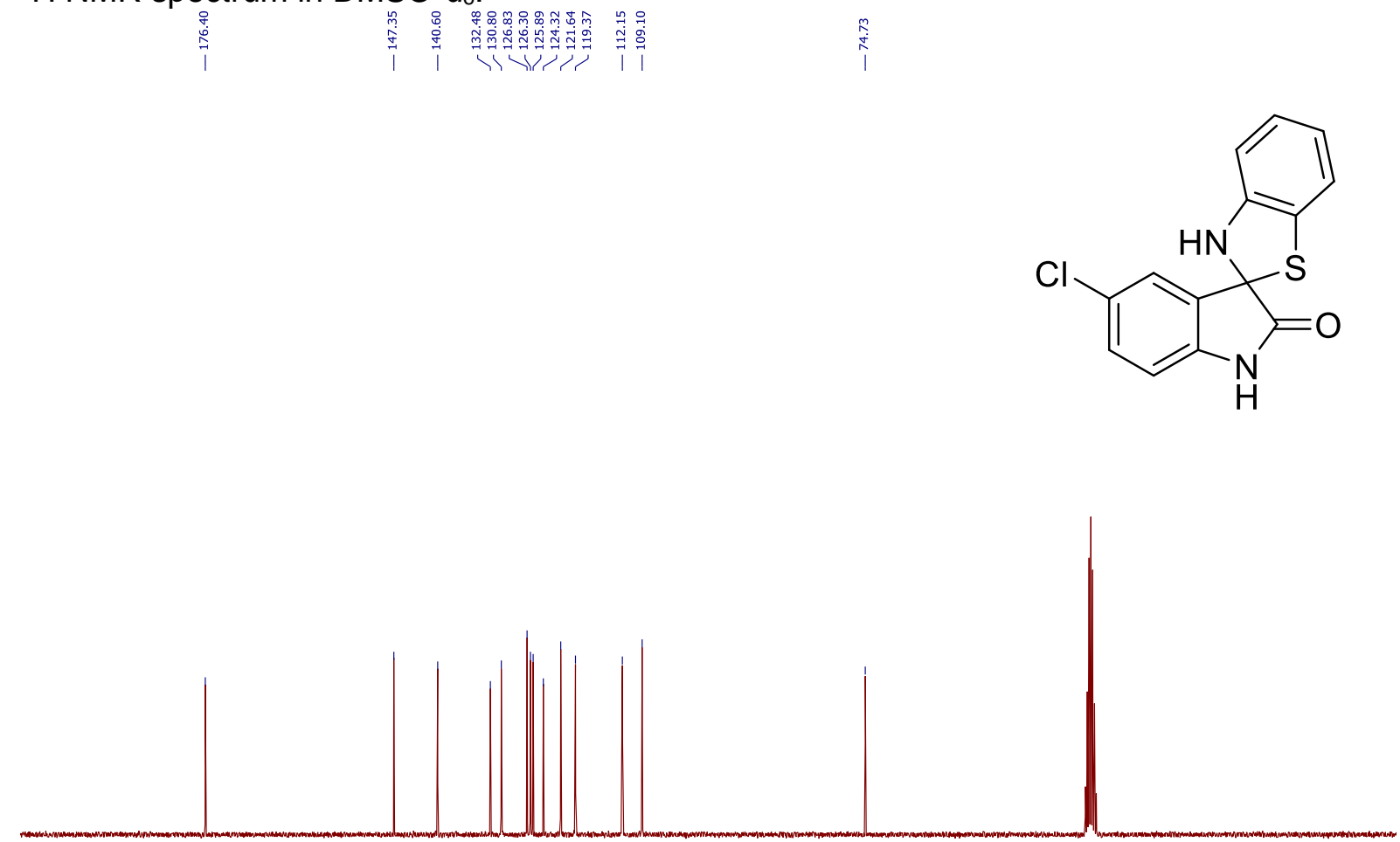

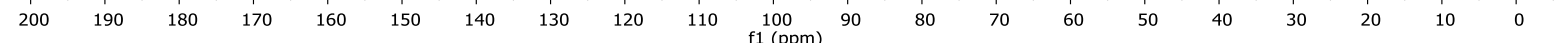
${ }^{13} \mathrm{C}$ NMR spectrum in DMSO- $d_{6}$. 


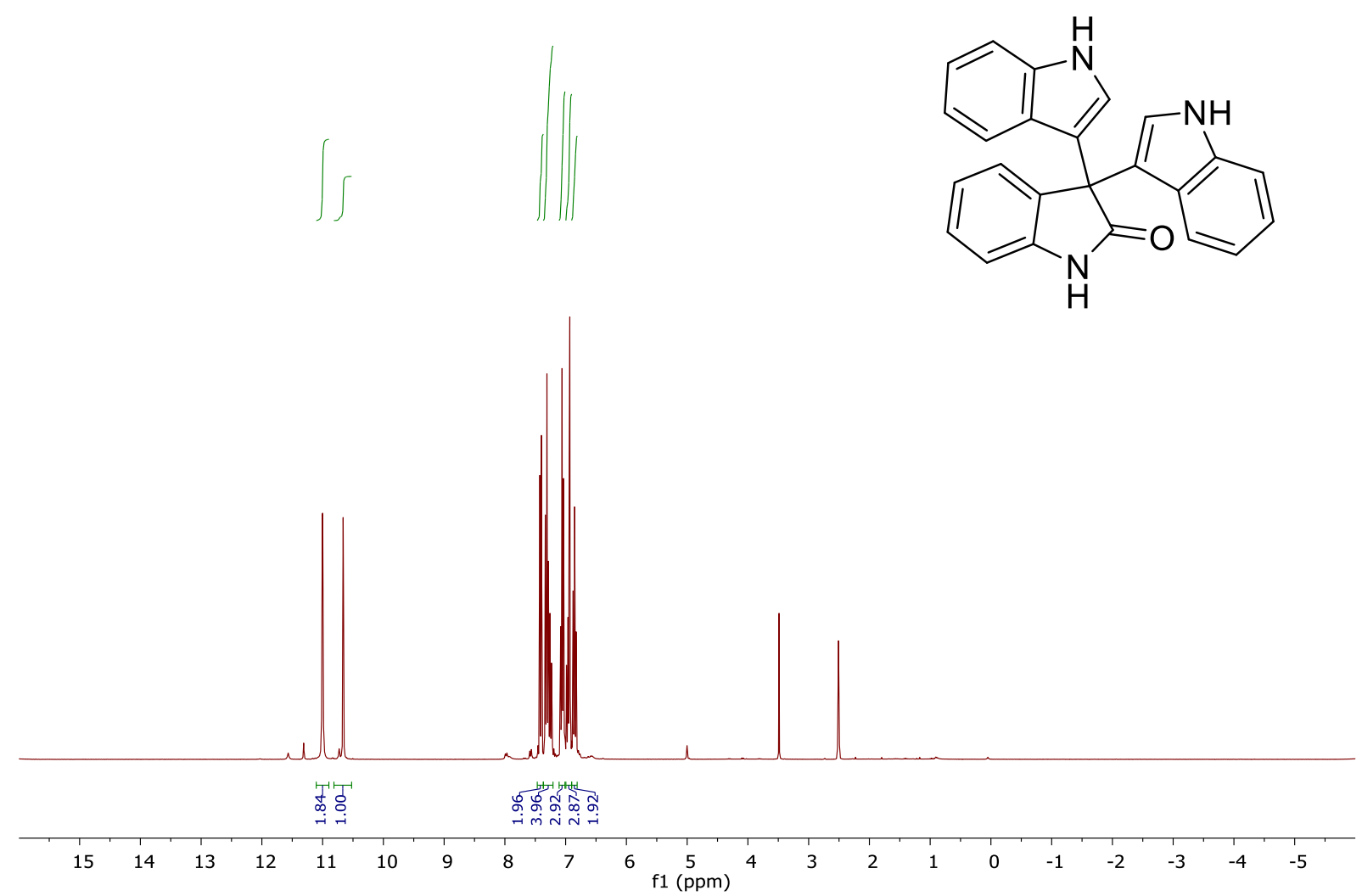

${ }^{1} \mathrm{H}$ NMR spectrum in DMSO- $d_{6}$.

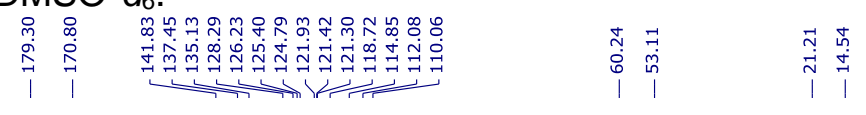

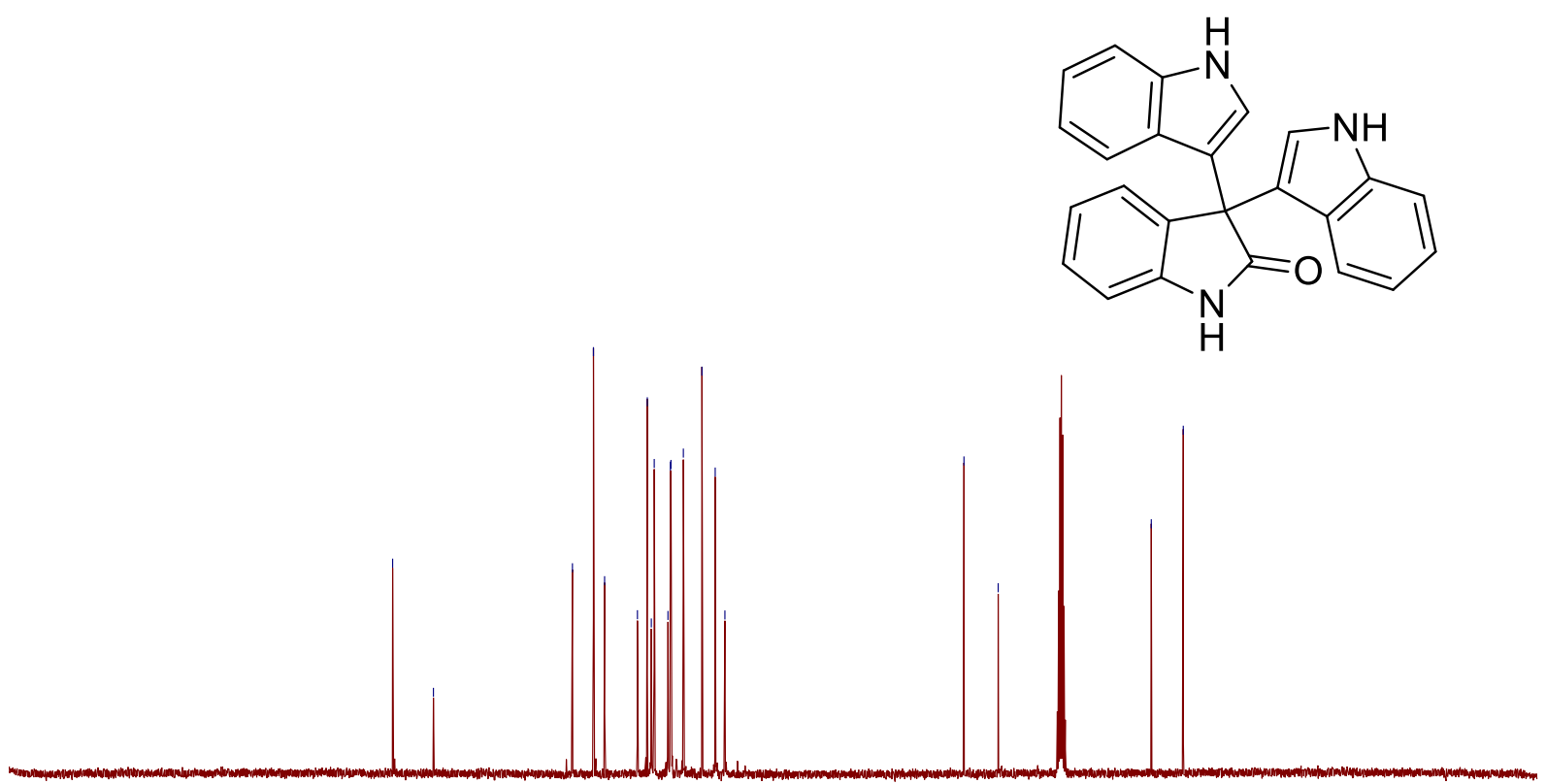

$250240230220210200190180170160150140130120110100 \quad 90 \quad 80 \quad 70 \quad 60 \begin{array}{lllllllllll}40 & 40 & 20 & 10 & 0 & -10 & -20 & -30 & -40 & -50\end{array}$ ${ }^{13} \mathrm{C}$ NMR spectrum in DMSO- $d_{6}$. 


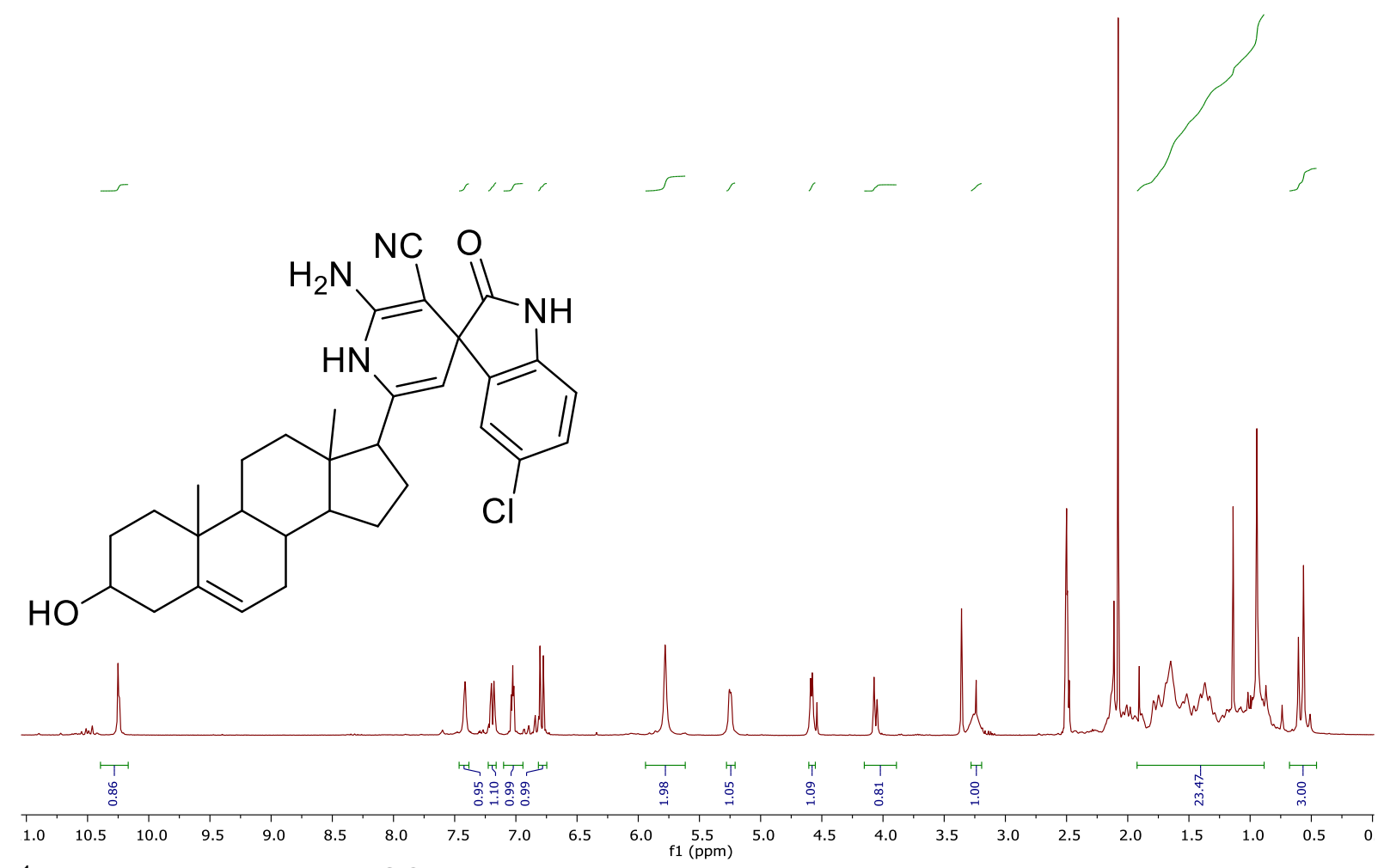

${ }^{1} \mathrm{H}$ NMR spectrum in DMSO- $d_{6}$.

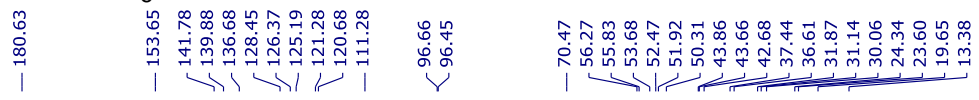<smiles>CC12CCC(O)CC1=CCC1C2CCC2(C)C(C3=CC4(C(=O)Nc5ccc(Cl)cc54)C(C#N)=C(N)N3)CCC12</smiles>

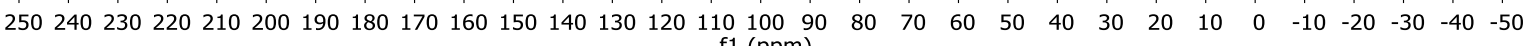
${ }^{13} \mathrm{C}$ NMR spectrum in DMSO- $d_{6}$. 
$106 a$<smiles>CCCCCCCCCCCCCCC(C)=O</smiles>
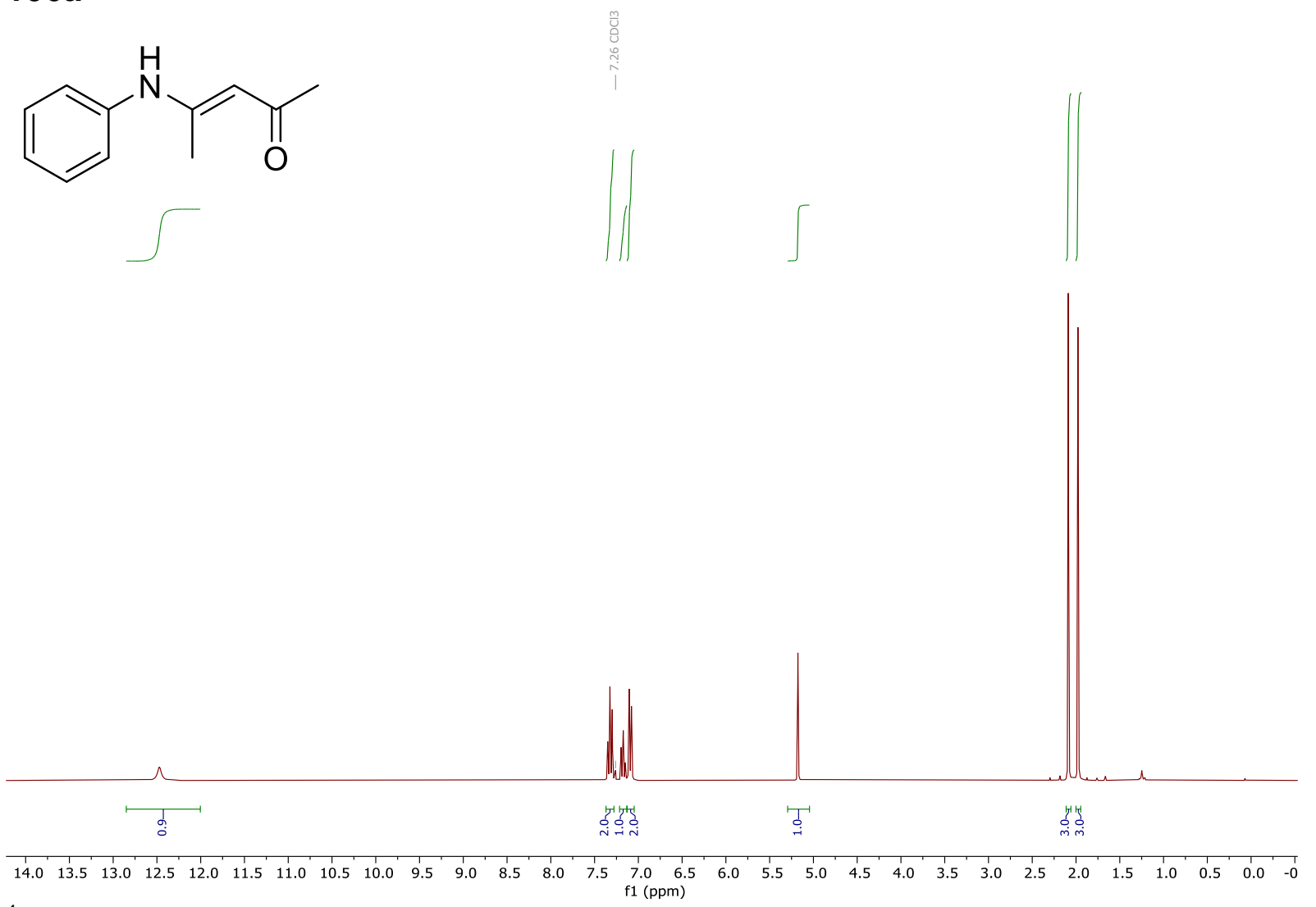
${ }^{1} \mathrm{H}-\mathrm{NMR}$ in $\mathrm{CDCl}_{3}$.

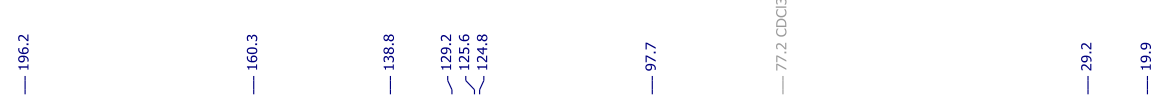<smiles>CC(=O)/C=C(\C)Nc1ccccc1</smiles>

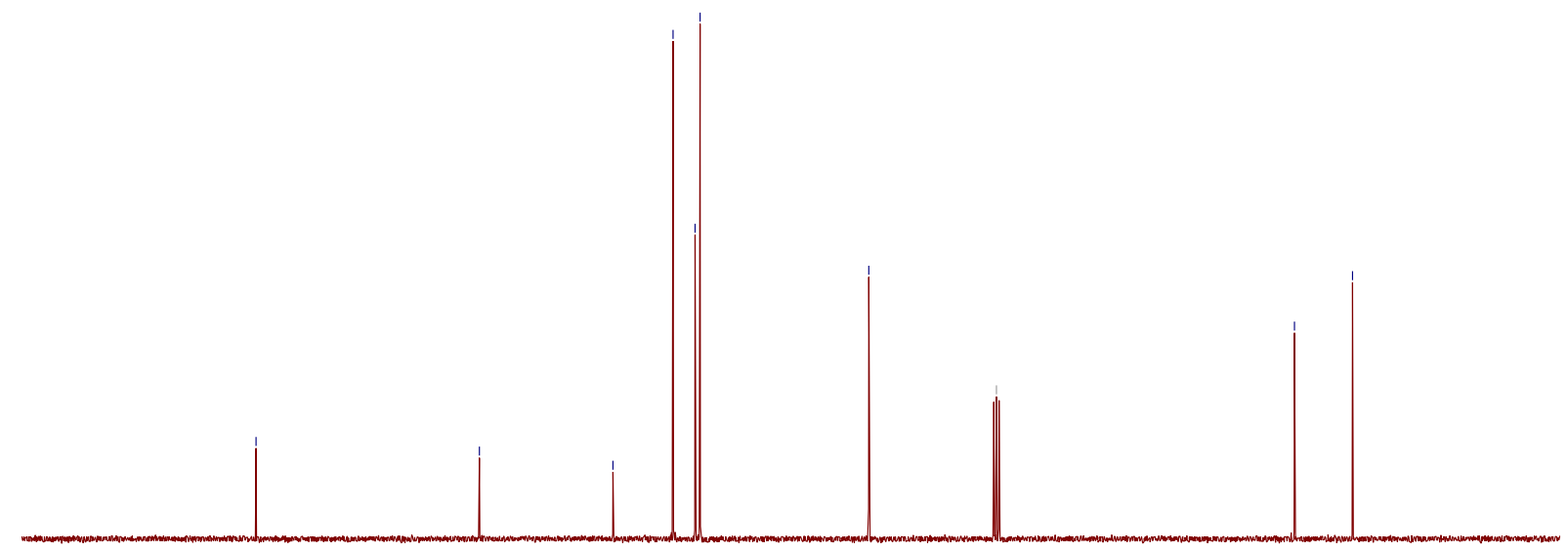

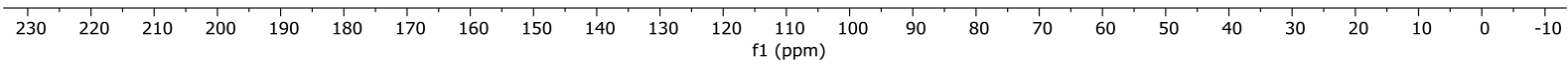
${ }^{13} \mathrm{C}-\mathrm{NMR}$ in $\mathrm{CDCl}_{3}$. 
106b<smiles>COC(=O)C(C)(Nc1ccccc1)C(C)=O</smiles>
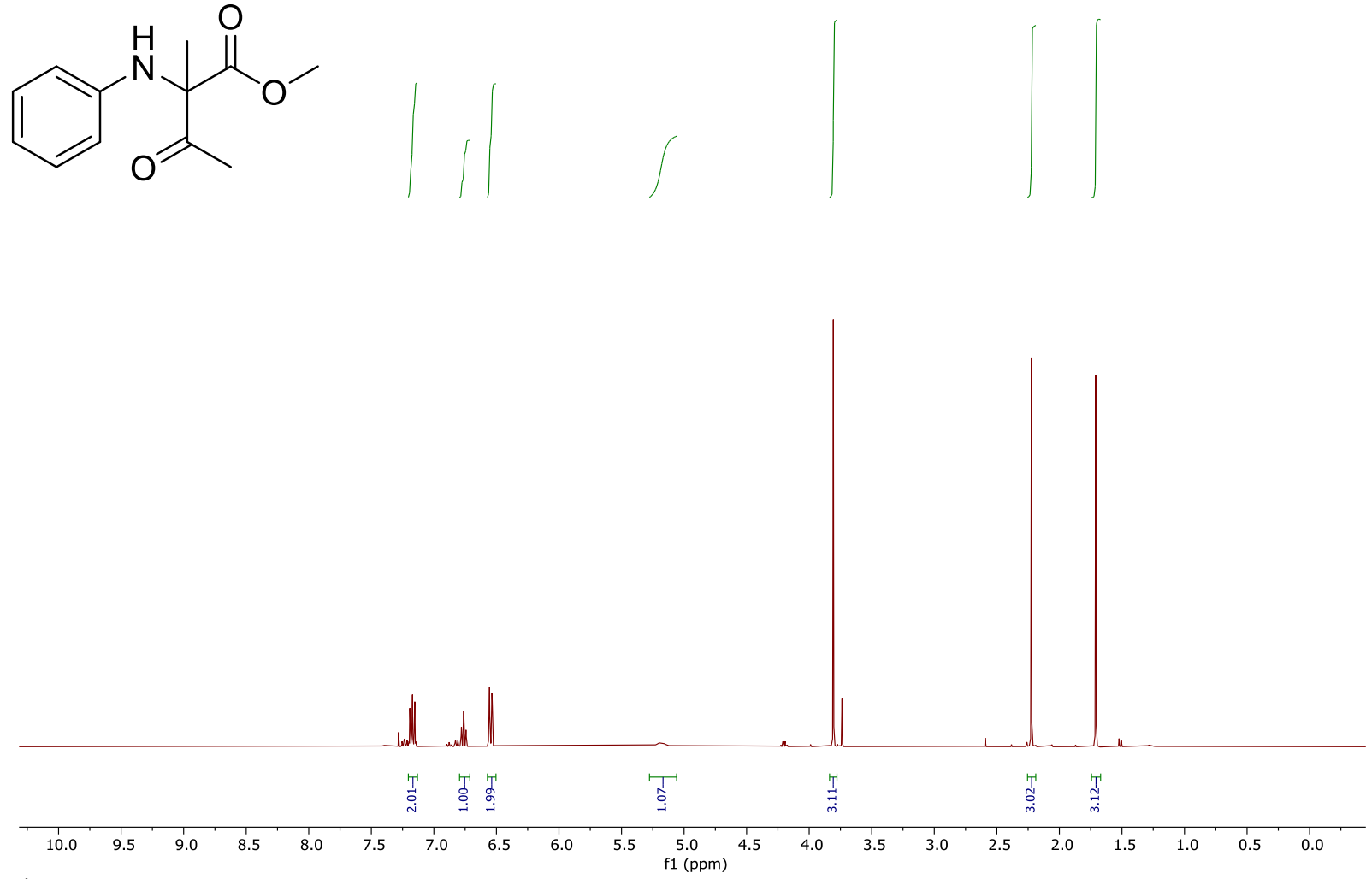

${ }^{1} \mathrm{H}-\mathrm{NMR}$ in $\mathrm{CDCl}_{3}$.

每<smiles>COC(=O)C(C)(Nc1ccccc1)C(C)=O</smiles>
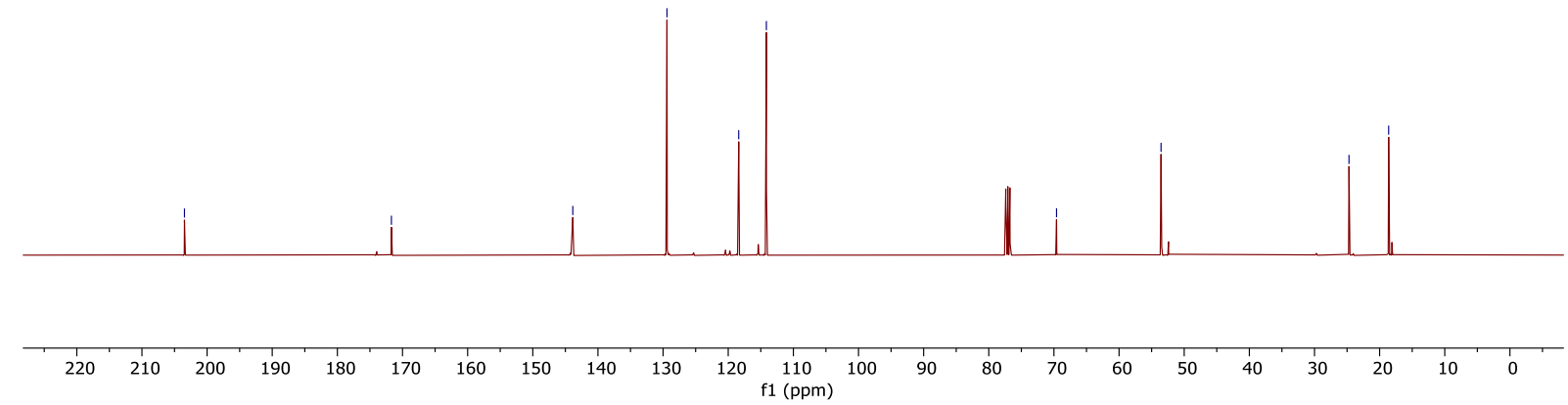
${ }^{13} \mathrm{C}-\mathrm{NMR}$ in $\mathrm{CDCl}_{3}$. 
107b<smiles>CCOC(=O)C(C)(Nc1ccccc1)C(C)=O</smiles>
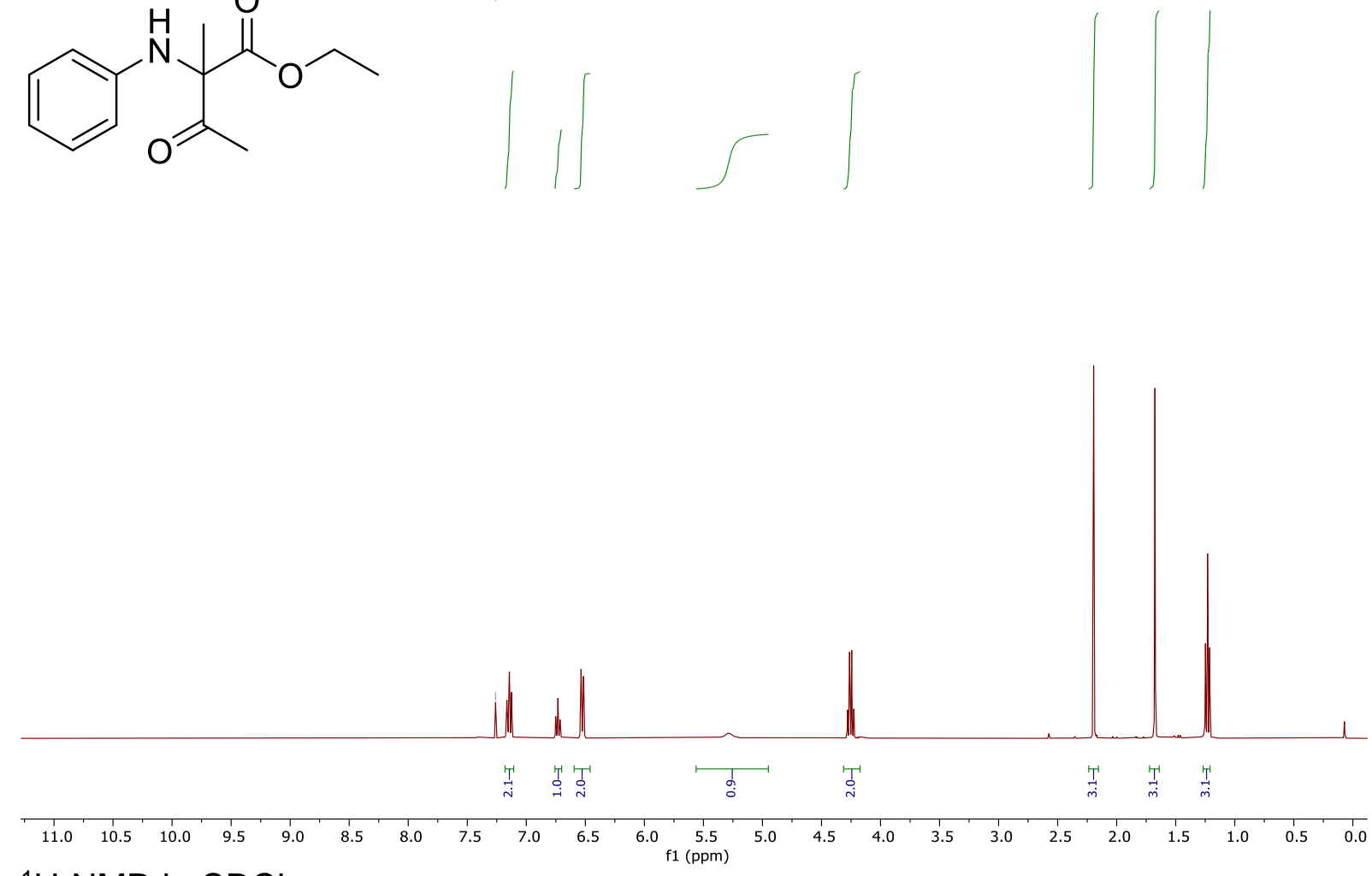

${ }^{1} \mathrm{H}-\mathrm{NMR}$ in $\mathrm{CDCl}_{3}$.

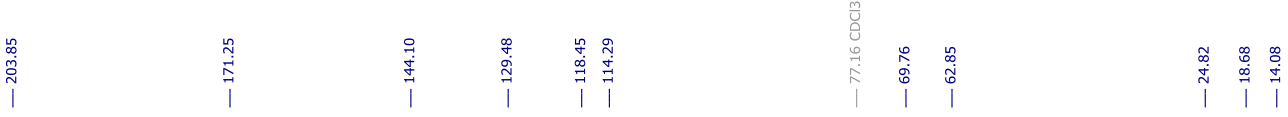<smiles>CCOC(=O)C(C)(Nc1ccccc1)C(C)=O</smiles>

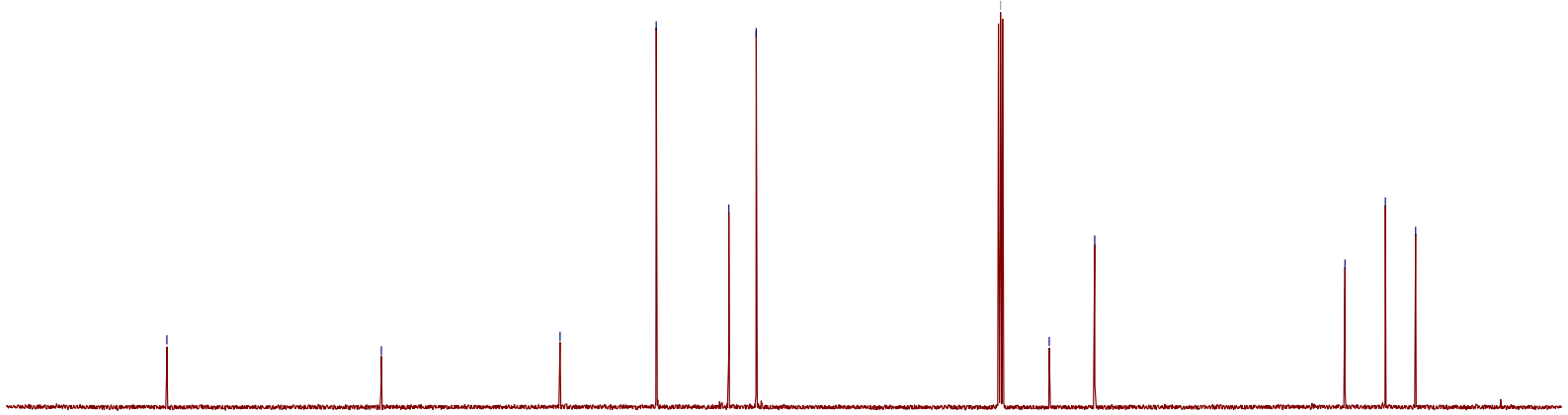

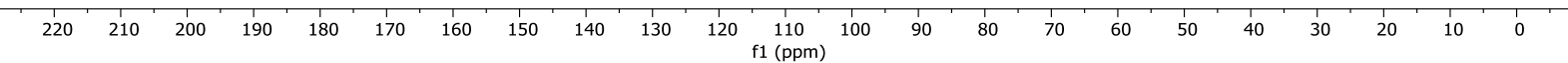
${ }^{13} \mathrm{C}-\mathrm{NMR}$ in $\mathrm{CDCl}_{3}$. 
$108 b$
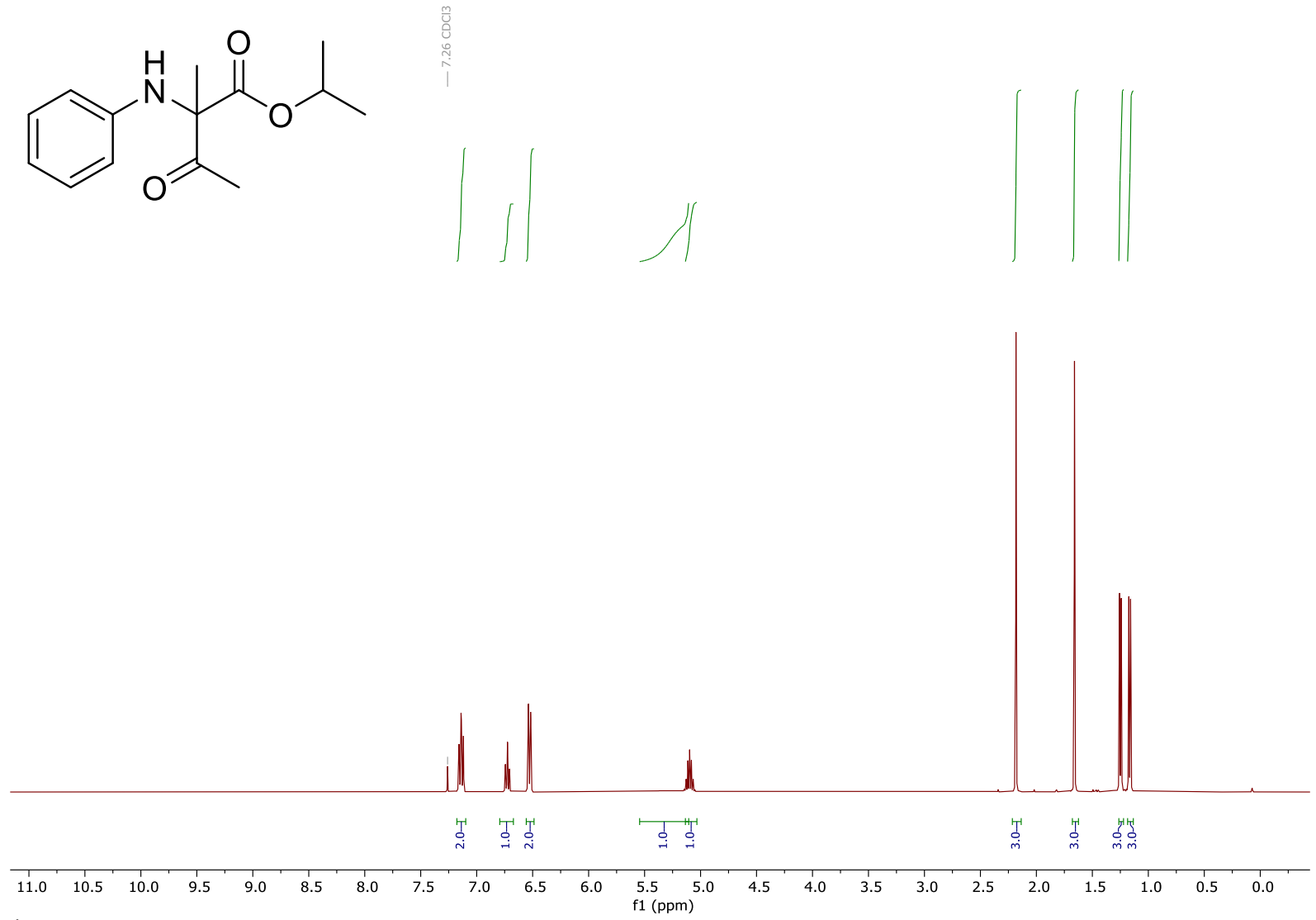

${ }^{1} \mathrm{H}-\mathrm{NMR}$ in $\mathrm{CDCl}_{3}$.

ָั.

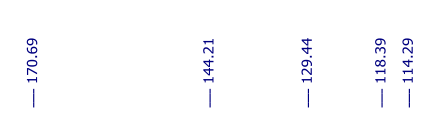

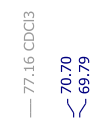

\\}
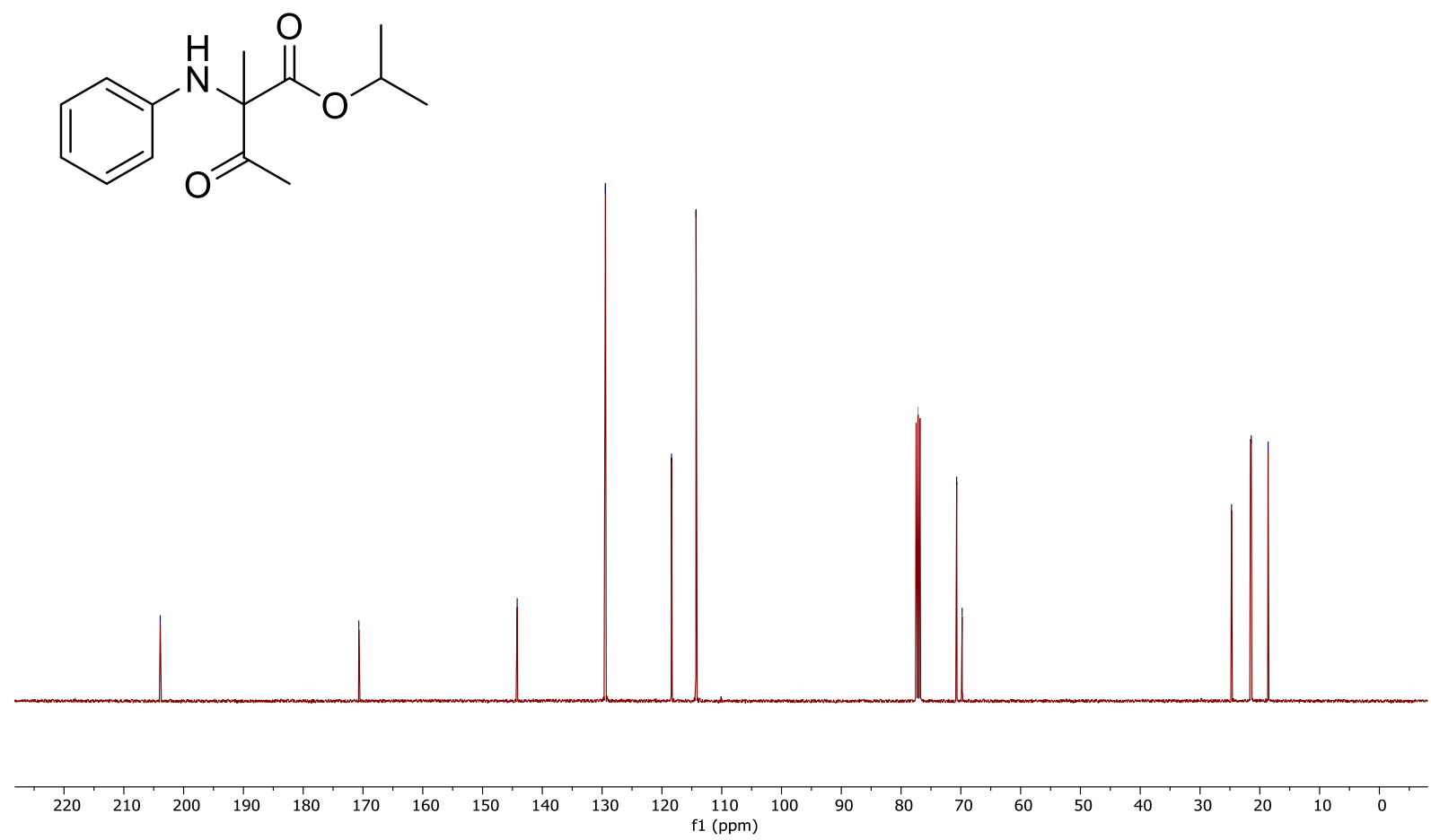

${ }^{13} \mathrm{C}-\mathrm{NMR}$ in $\mathrm{CDCl}_{3}$.

312 


\section{9b}<smiles>CC(=O)C(C)(Nc1ccccc1)C(=O)OCC(F)(F)F</smiles>
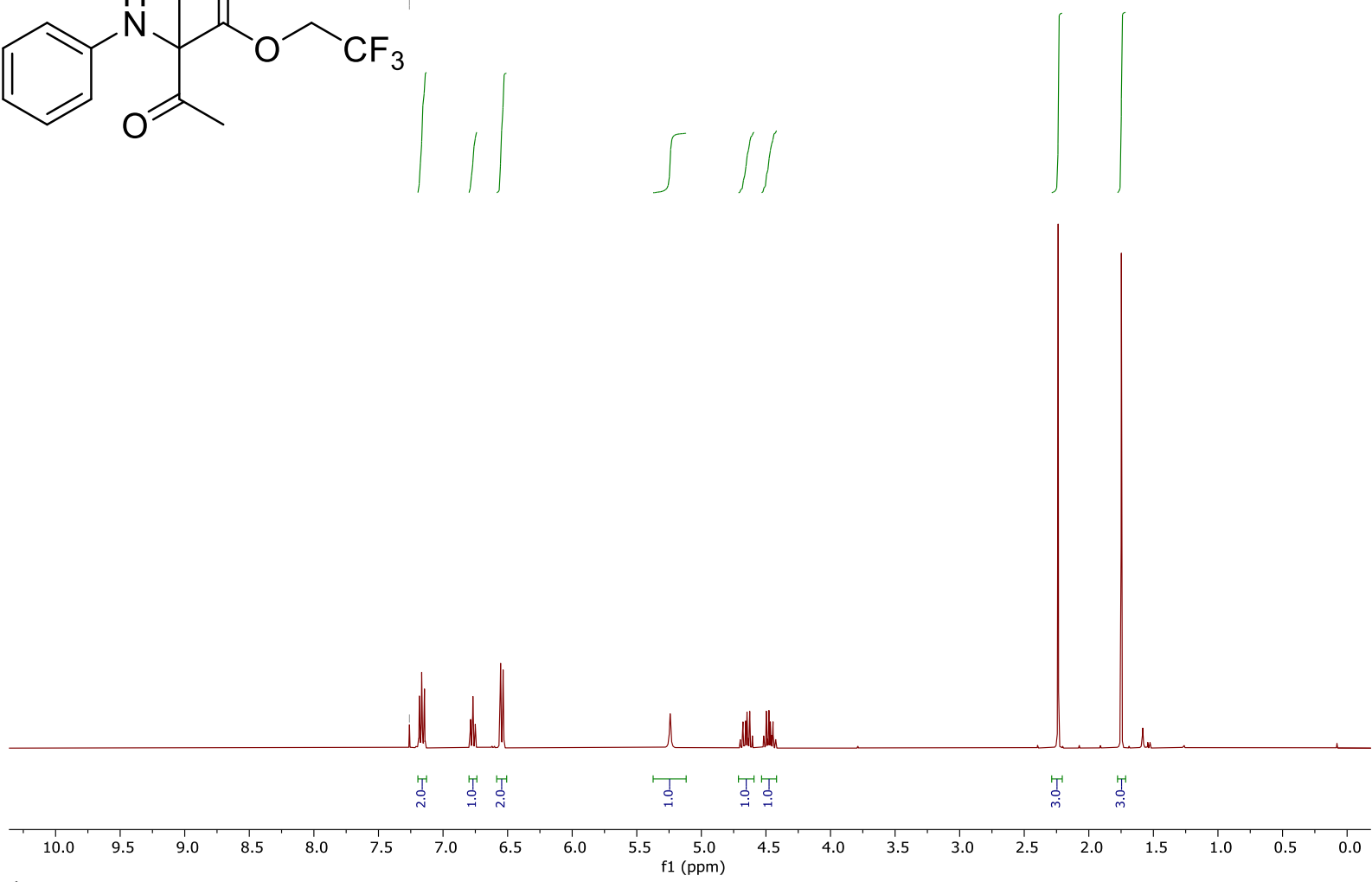

${ }^{1} \mathrm{H}-\mathrm{NMR}$ in $\mathrm{CDCl}_{3}$.

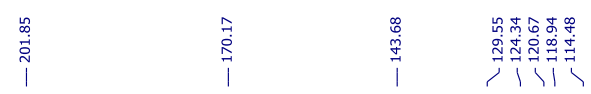<smiles>CC(=O)C(C)(Nc1ccccc1)C(=O)OCC(F)(F)F</smiles>

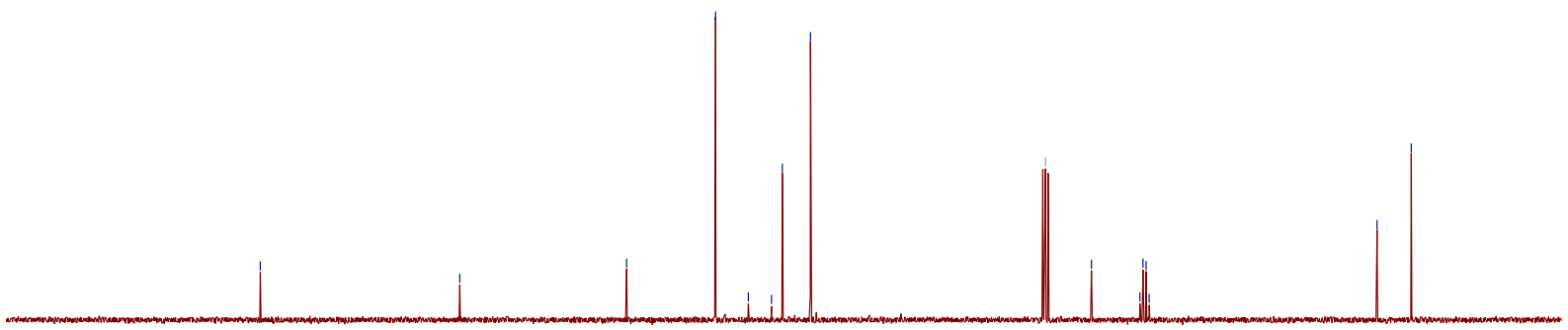

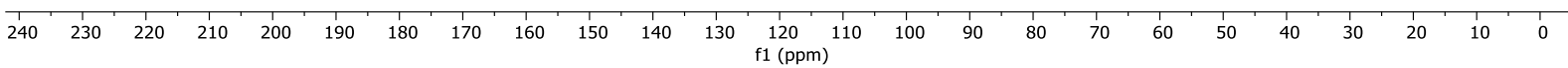
${ }^{13} \mathrm{C}-\mathrm{NMR}$ in $\mathrm{CDCl}_{3}$. 
110b<smiles>CCCCCCCCOC(=O)C(C)(Nc1ccccc1)C(C)=O</smiles>

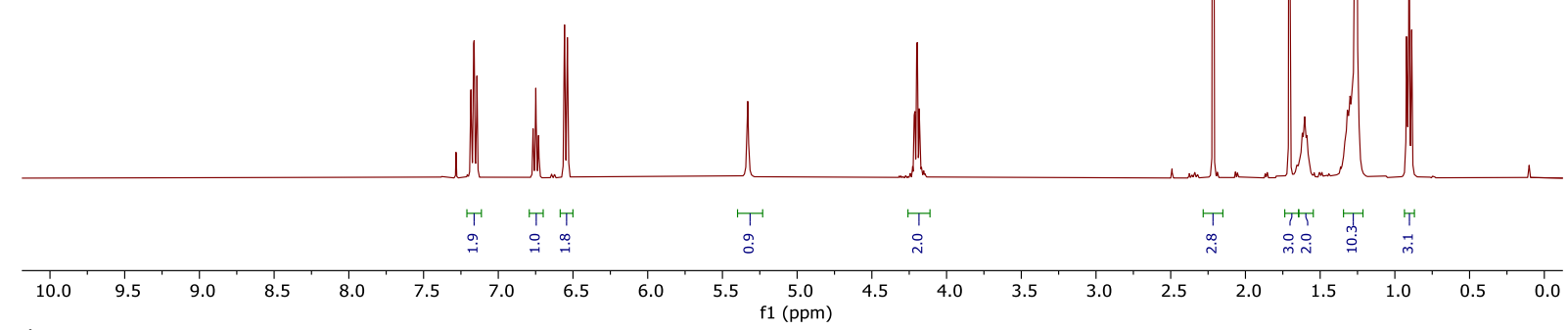

${ }^{1} \mathrm{H}-\mathrm{NMR}$ in $\mathrm{CDCl}_{3}$.

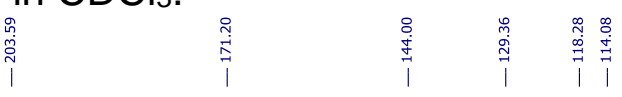

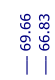

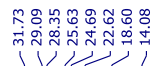

$\mathrm{N}_{\mathrm{O}}^{\mathrm{N}}$

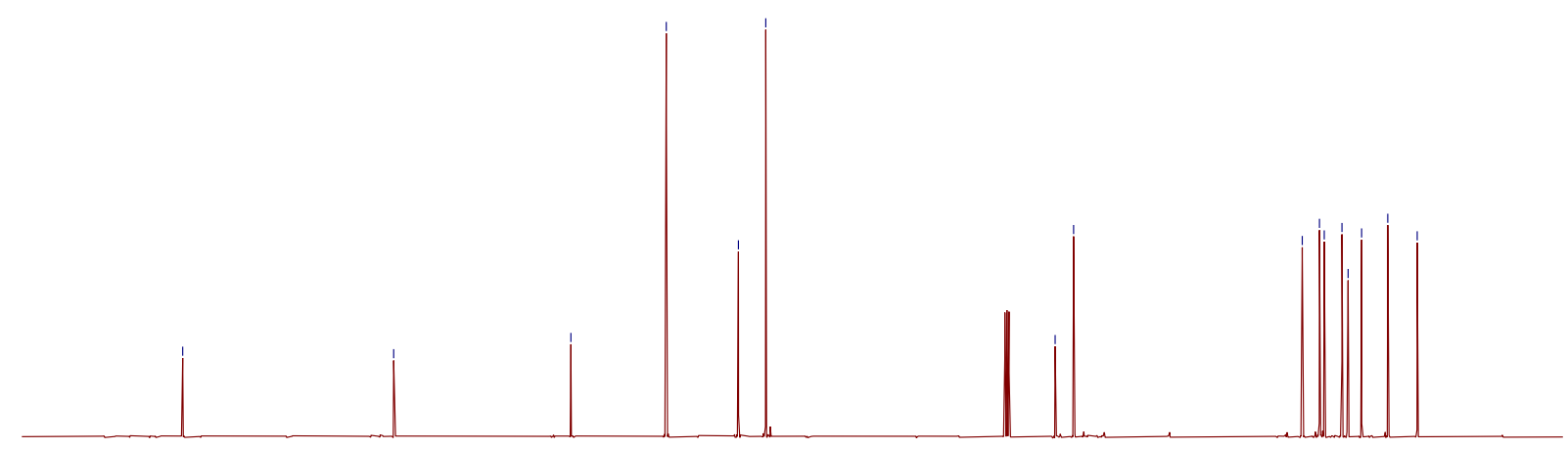

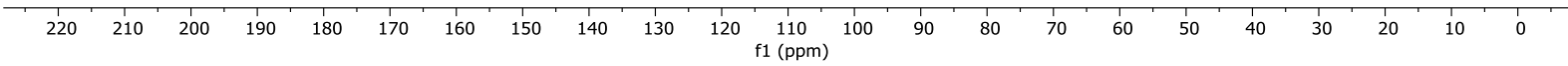
${ }^{13} \mathrm{C}-\mathrm{NMR}$ in $\mathrm{CDCl}_{3}$. 
111b
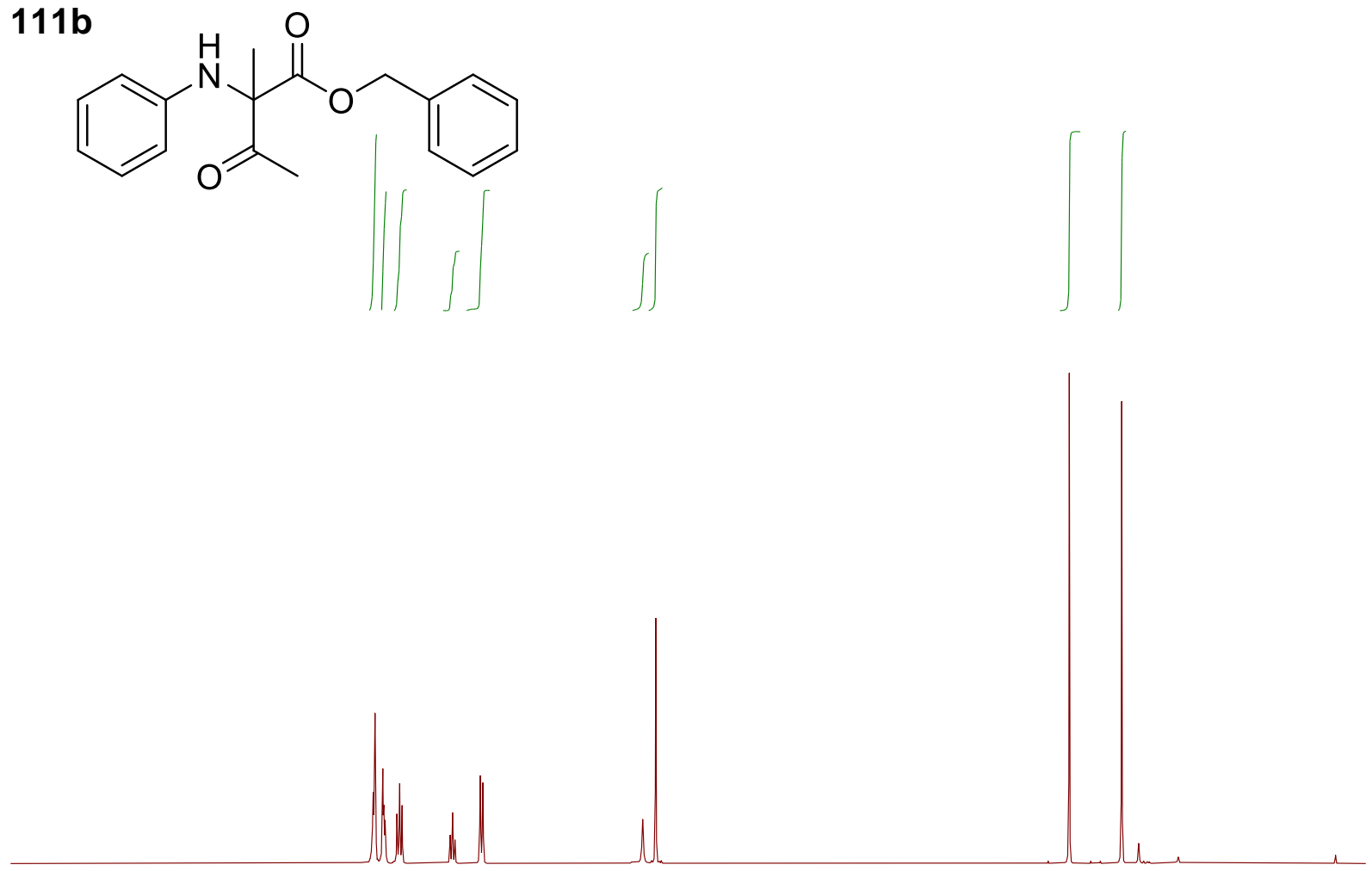

㗞芴

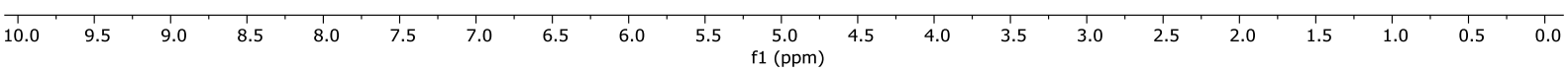

${ }^{1} \mathrm{H}-\mathrm{NMR}$ in $\mathrm{CDCl}_{3}$.

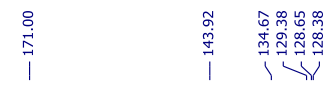

\11

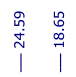
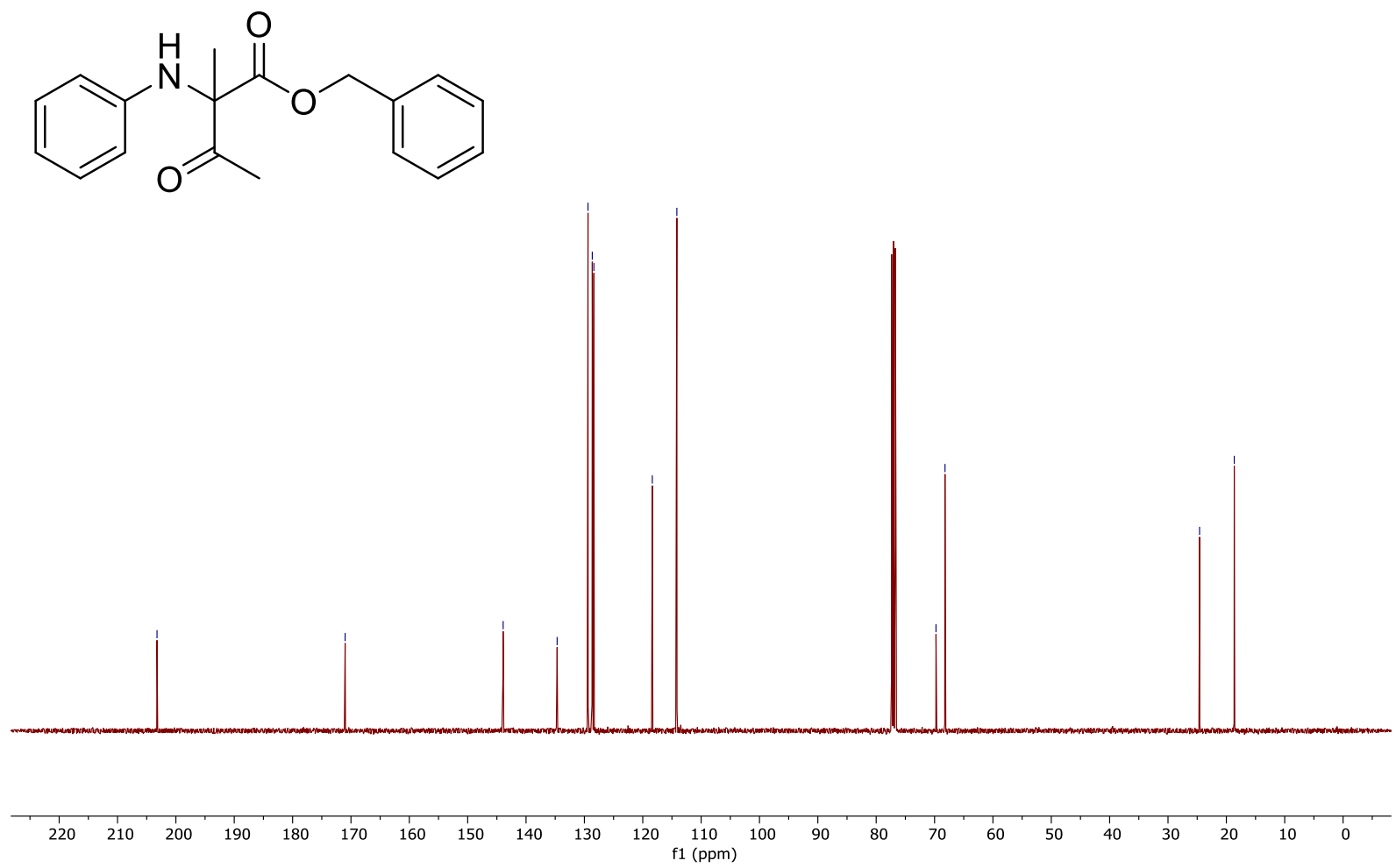

${ }^{13} \mathrm{C}-\mathrm{NMR}$ in $\mathrm{CDCl}_{3}$.

315 
$112 a$<smiles>CCCCCCCCC</smiles>
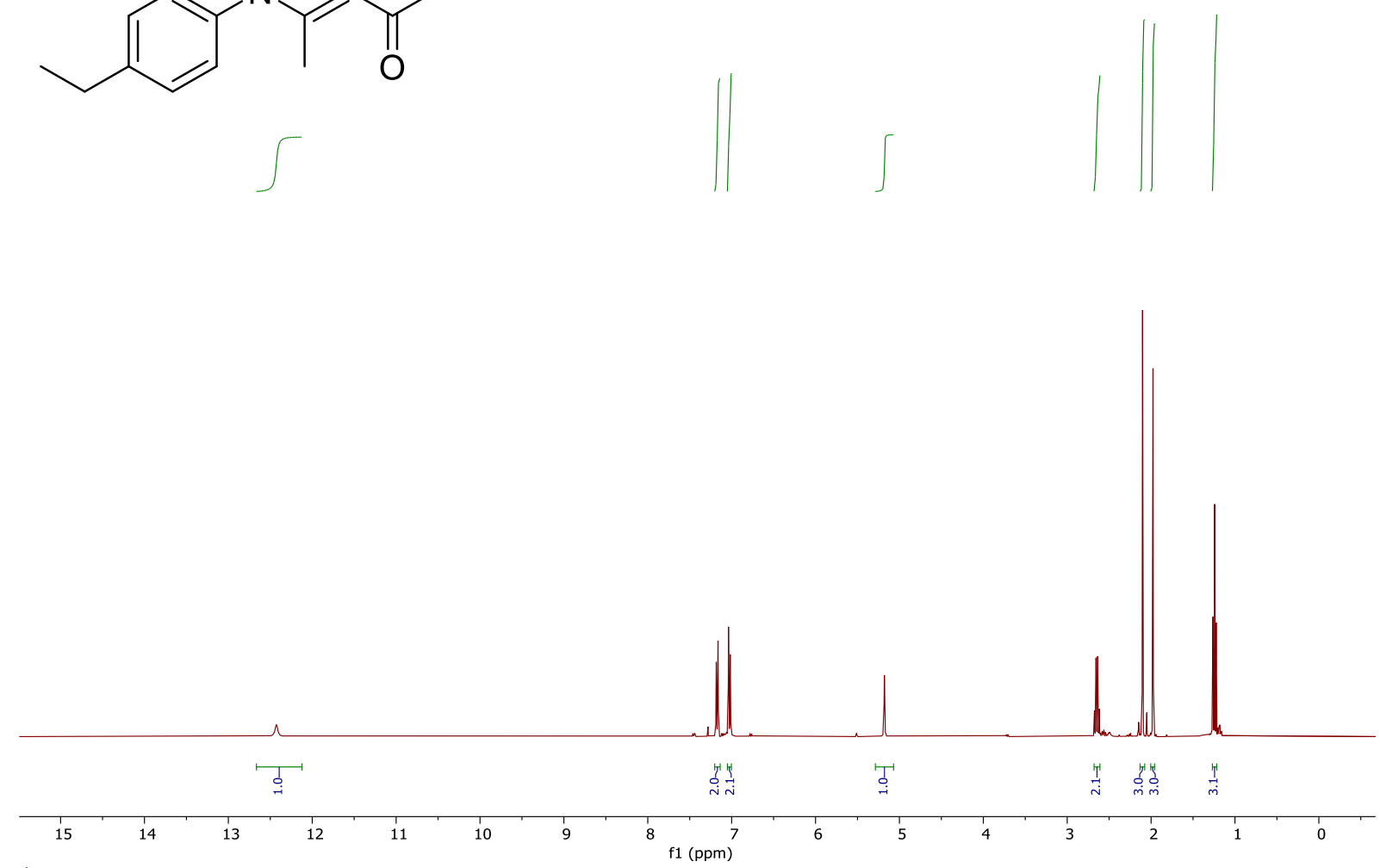

${ }^{1} \mathrm{H}-\mathrm{NMR}$ in $\mathrm{CDCl}_{3}$.

象

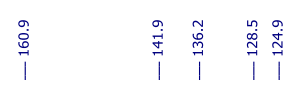

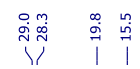<smiles>CCc1ccc(N/C(C)=C/C(C)=O)cc1</smiles>
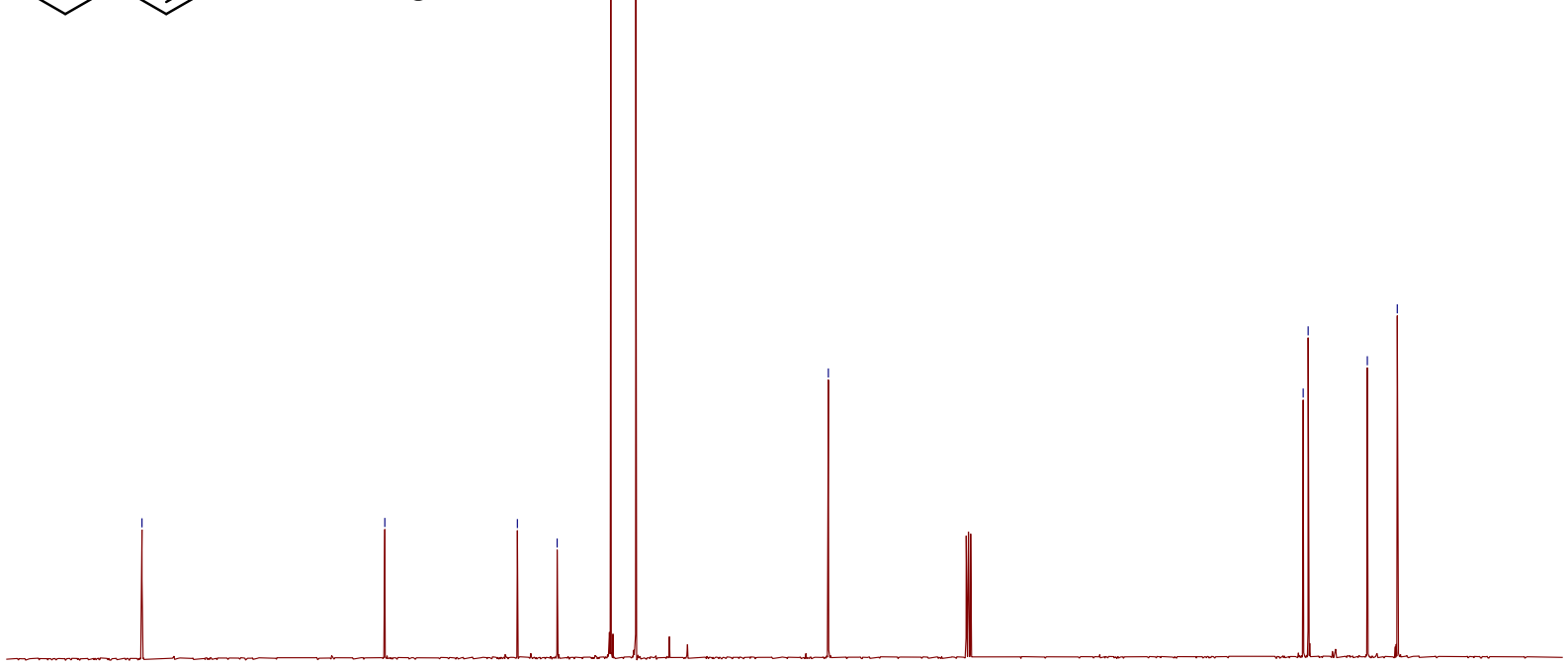

\begin{tabular}{lllllll}
\hline 210 & 200 & 190 & 180 & 170 & 160
\end{tabular}

${ }^{13} \mathrm{C}$-NMR in $\mathrm{CDCl}_{3}$. 
$112 b$<smiles>CCc1ccc(NC(C)(C(C)=O)C(=O)OC)cc1</smiles>
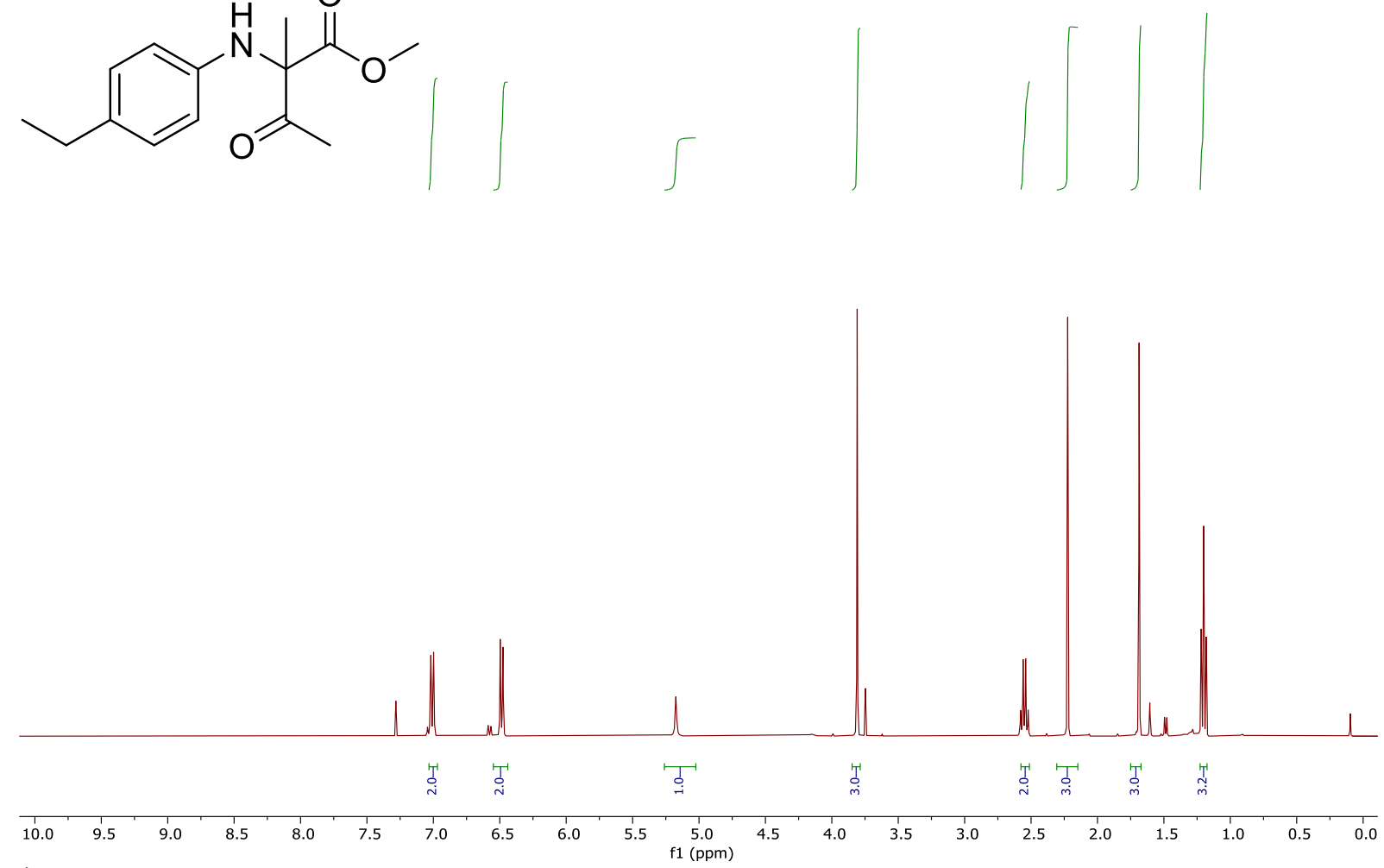

${ }^{1} \mathrm{H}-\mathrm{NMR}$ in $\mathrm{CDCl}_{3}$. 离<smiles>CCc1ccc(NC(C)(C(C)=O)C(=O)OC)cc1</smiles>

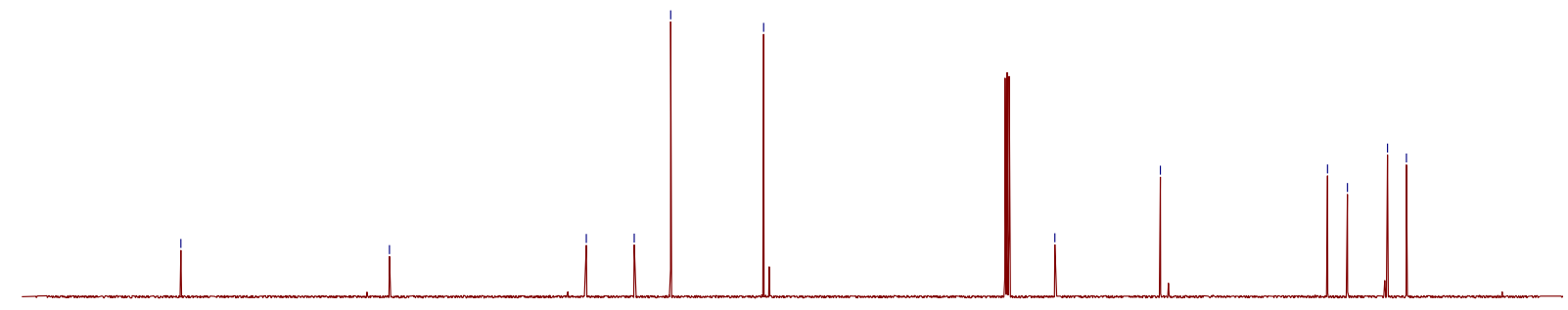

\begin{tabular}{rlllllllllllllllllllllllll}
\hline 220 & 210 & 200 & 190 & 180 & 170 & 160 & 150 & 140 & 130 & 120 & 110 & 100 & 90 & 80 & 70 & 60 & 50 & 40 & 30 & 20 & 10 & 0
\end{tabular} ${ }^{13} \mathrm{C}-\mathrm{NMR}$ in $\mathrm{CDCl}_{3}$. 


\section{3a}<smiles>CC(=O)/C=C(\C)Nc1ccc(C(C)(C)C)cc1</smiles>

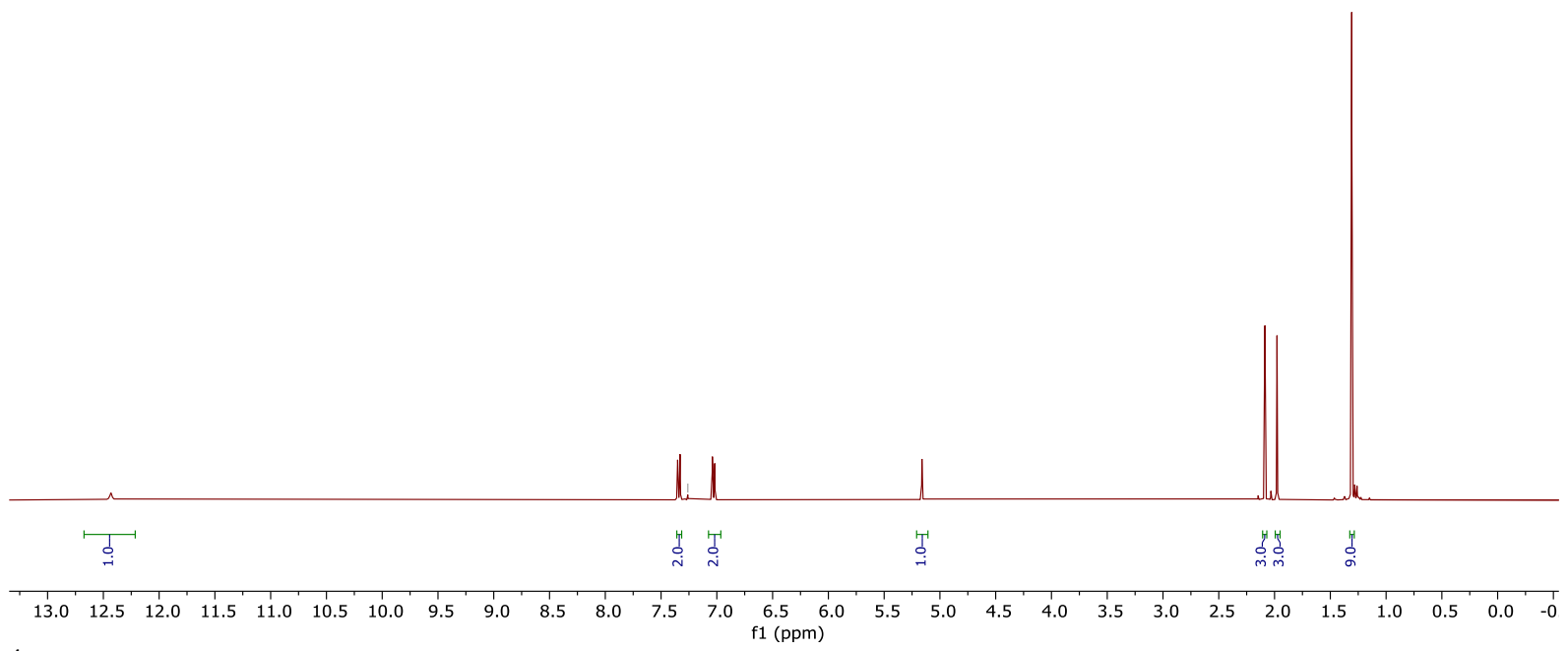

${ }^{1} \mathrm{H}-\mathrm{NMR}$ in $\mathrm{CDCl}_{3}$.
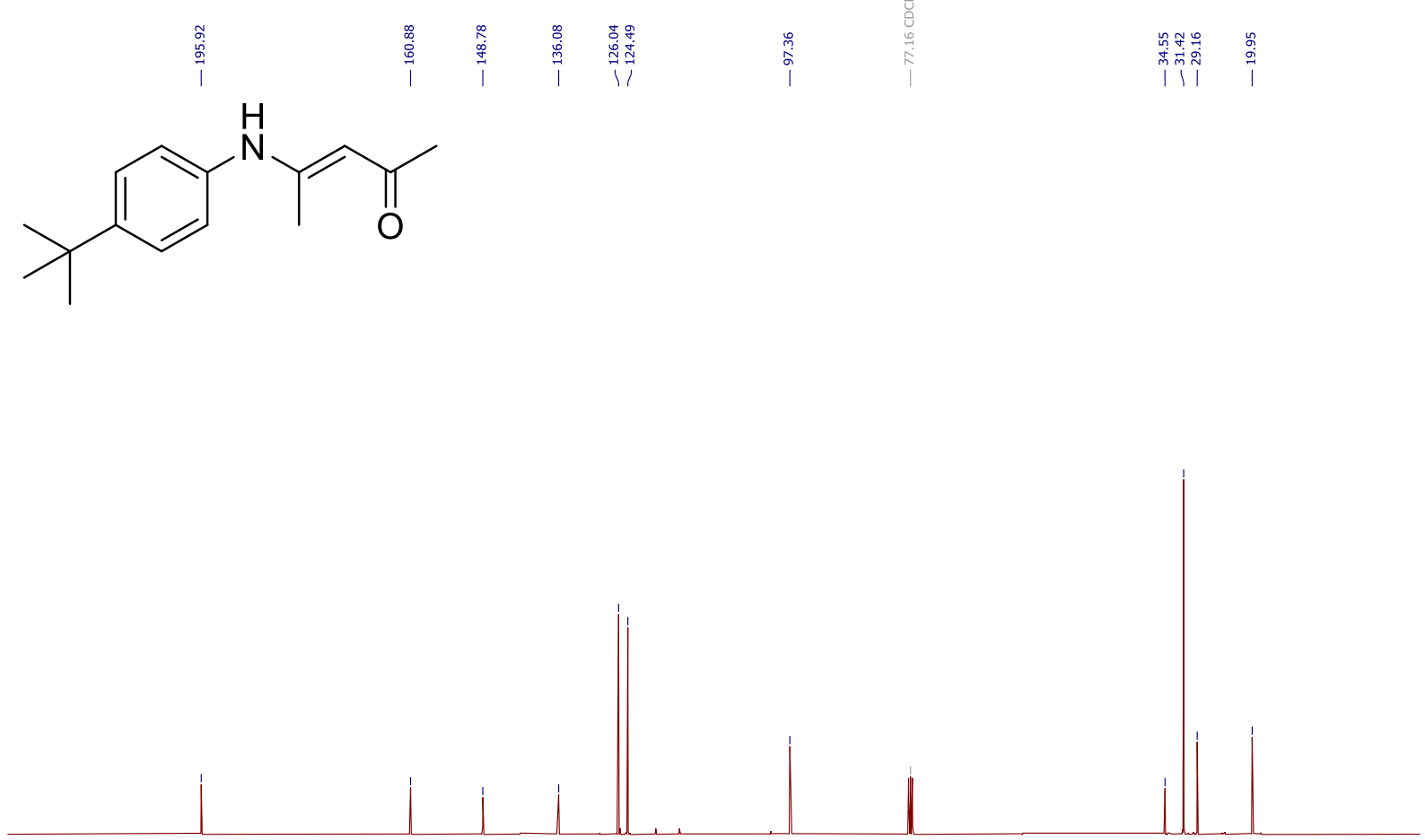

$\begin{array}{lllllllllllllllllllllllllllll}220 & 210 & 200 & 190 & 180 & 170 & 160 & 150 & 140 & 130 & 120 & 110 & 100 & 90 & 80 & 70 & 60 & 50 & 40 & 30 & 20 & 10 & 0\end{array}$ ${ }^{13} \mathrm{C}-\mathrm{NMR}$ in $\mathrm{CDCl}_{3}$. 
113b<smiles>COC(=O)C(C)(Nc1ccc(C(C)(C)C)cc1)C(C)=O</smiles>
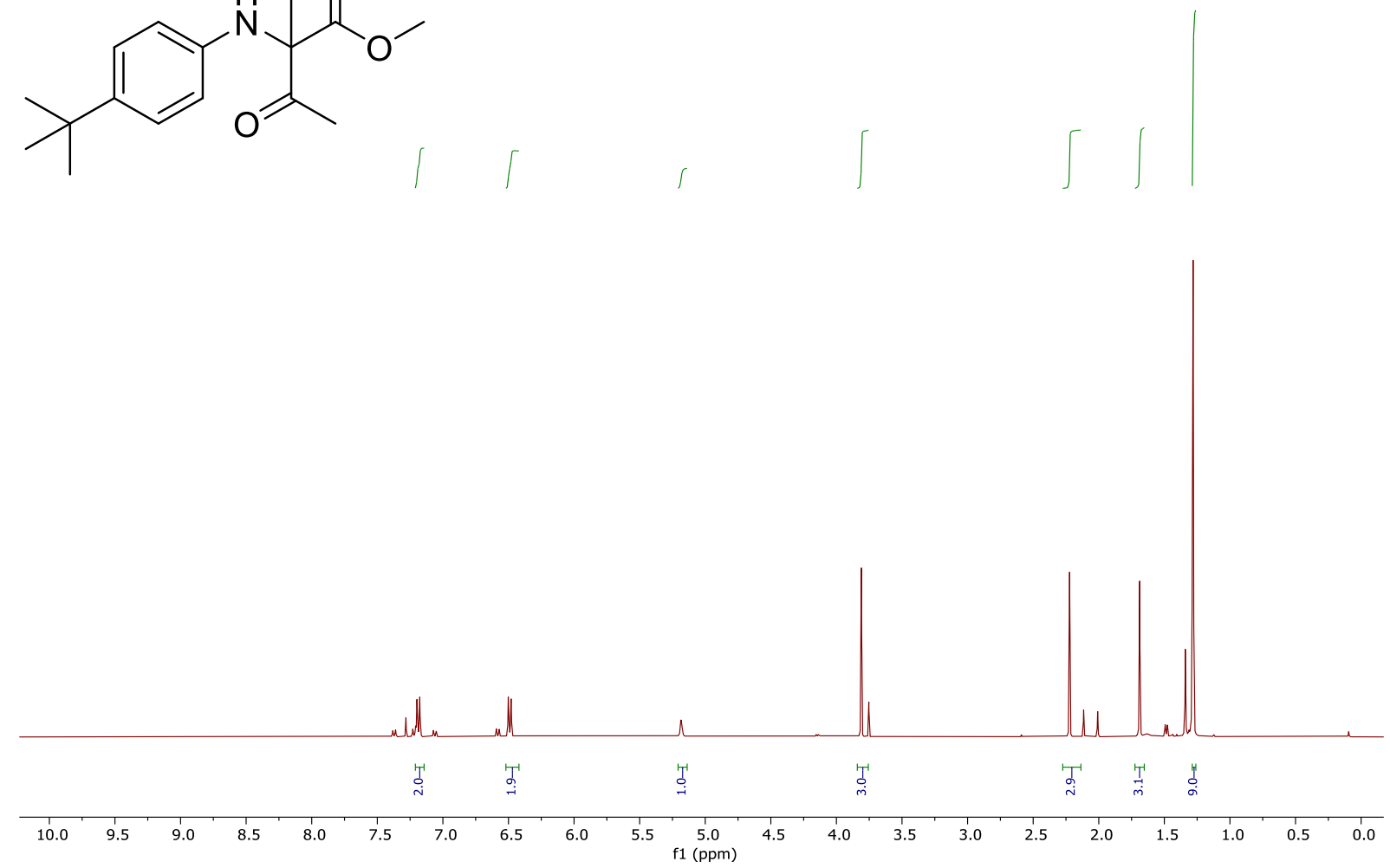

${ }^{1} \mathrm{H}-\mathrm{NMR}$ in $\mathrm{CDCl}_{3}$.

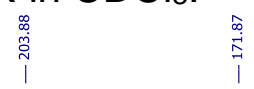

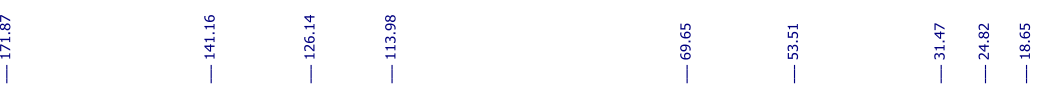<smiles>COC(=O)C(C)(Nc1ccc(C(C)(C)C)cc1)C(C)=O</smiles>

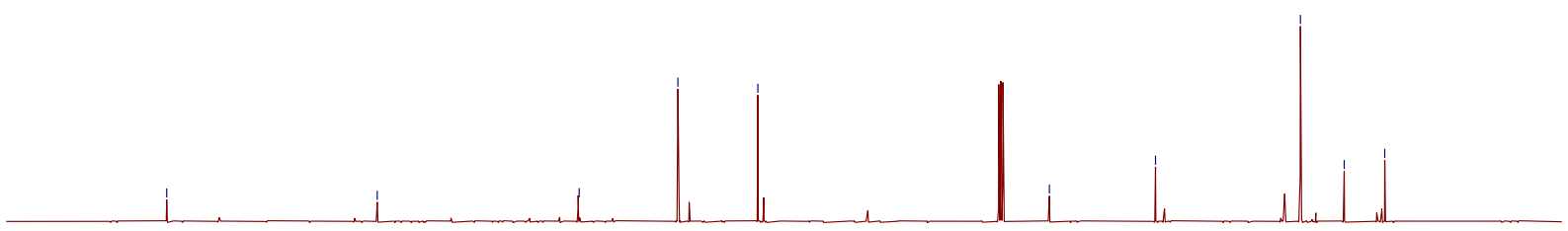

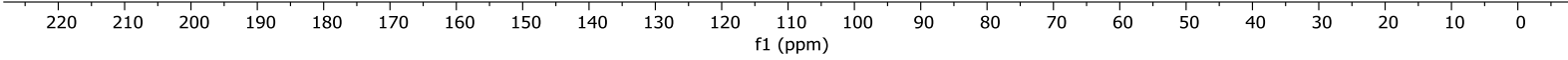
${ }^{13} \mathrm{C}-\mathrm{NMR}$ in $\mathrm{CDCl}_{3}$. 


\section{$114 a$}<smiles>CC(=O)/C=C(\C)Nc1c(C)cccc1C</smiles>
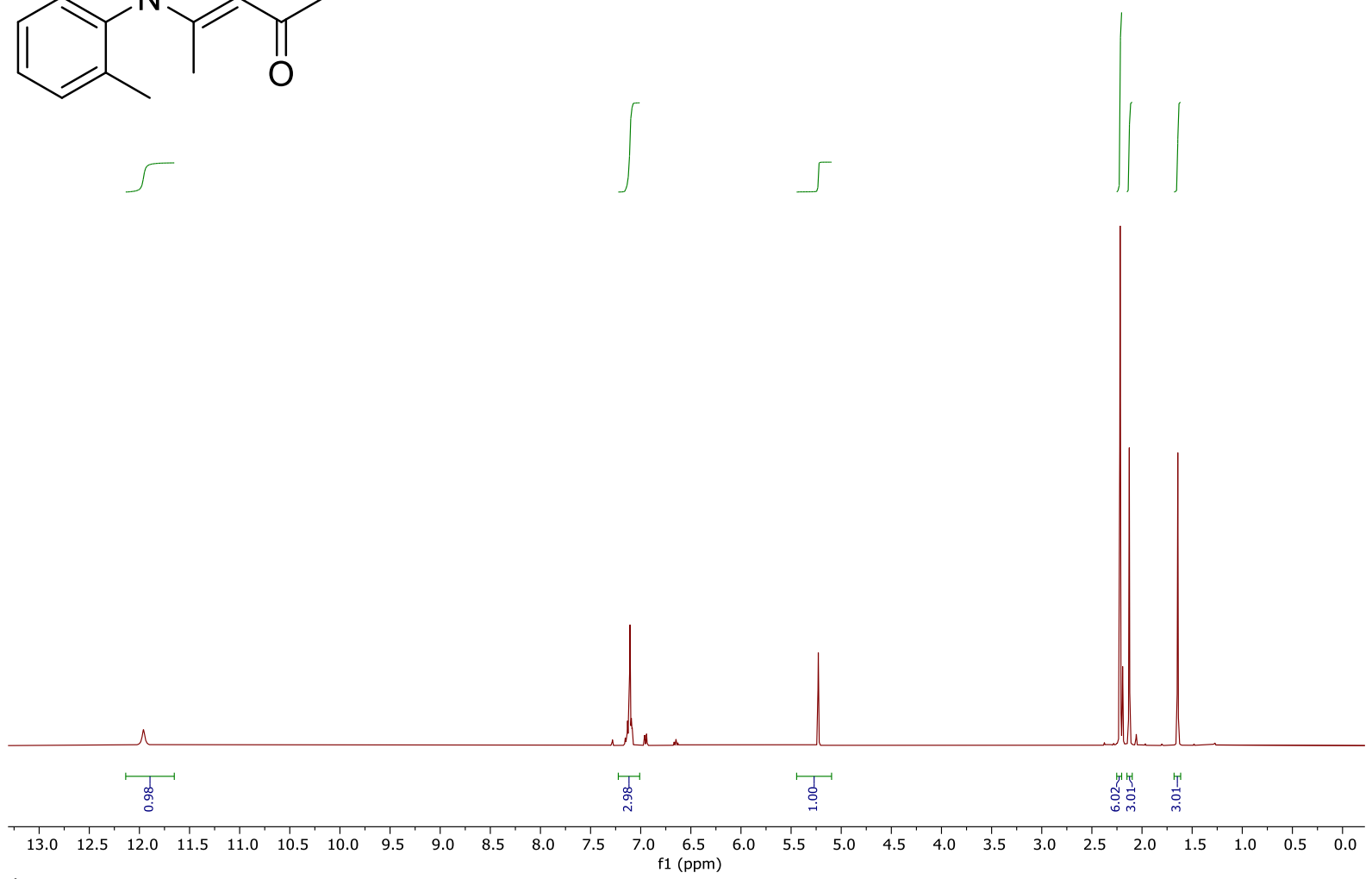

${ }^{1} \mathrm{H}-\mathrm{NMR}$ in $\mathrm{CDCl}_{3}$.<smiles>CC(=O)/C=C(\C)Nc1c(C)cccc1C</smiles>

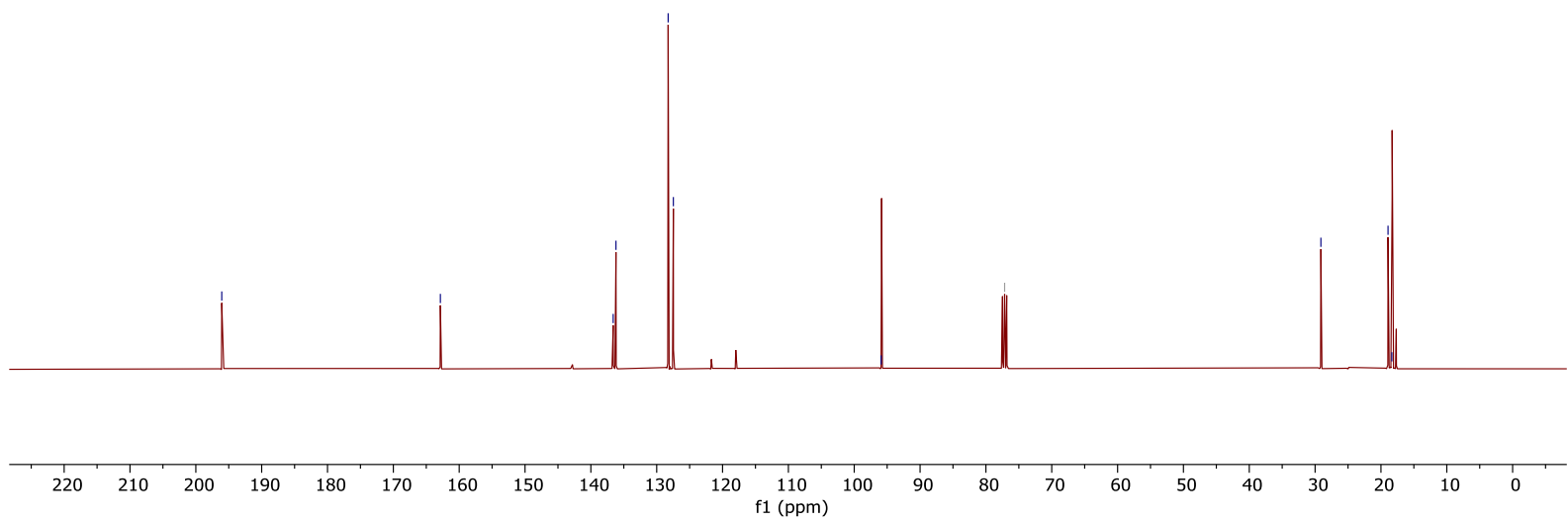

${ }^{13} \mathrm{C}-\mathrm{NMR}$ in $\mathrm{CDCl}_{3}$. 


\section{4b}<smiles>COC(=O)C(C)(Nc1c(C)cccc1C)C(C)=O</smiles>

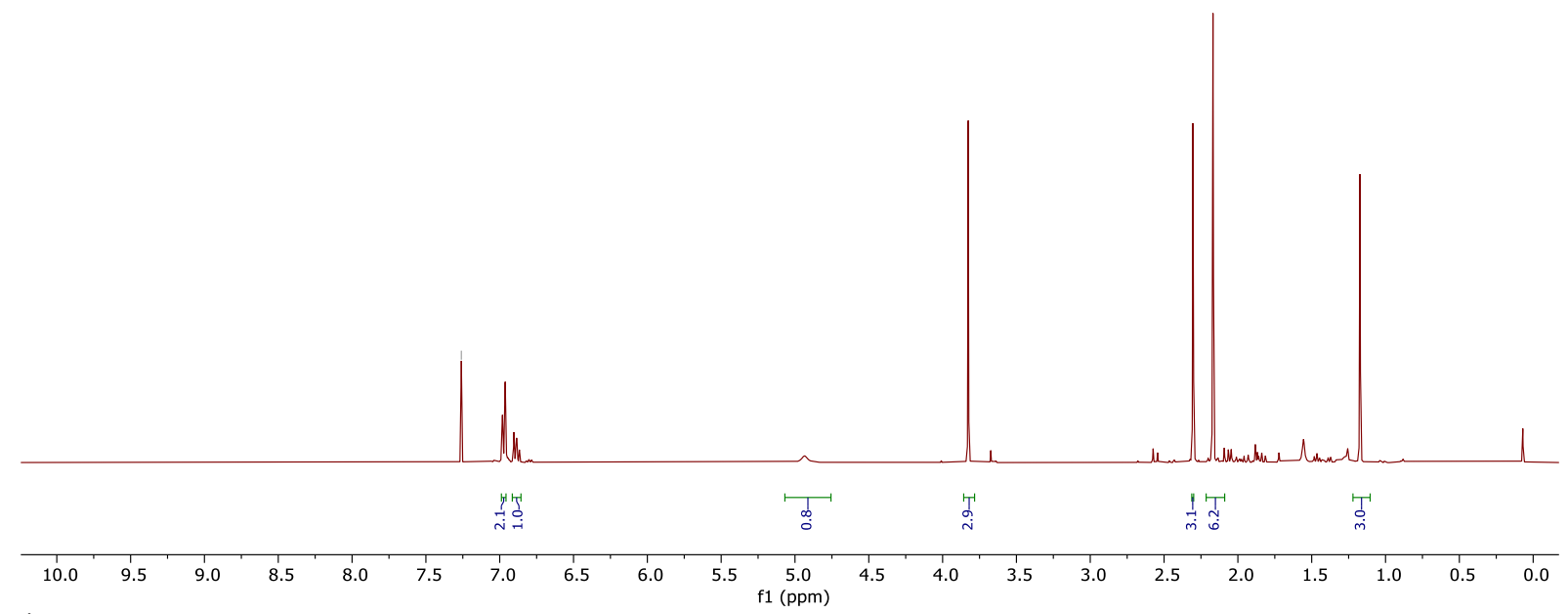

${ }^{1} \mathrm{H}-\mathrm{NMR}$ in $\mathrm{CDCl}_{3}$.<smiles>COC(=O)C(C)(Nc1c(C)cccc1C)C(C)=O</smiles>

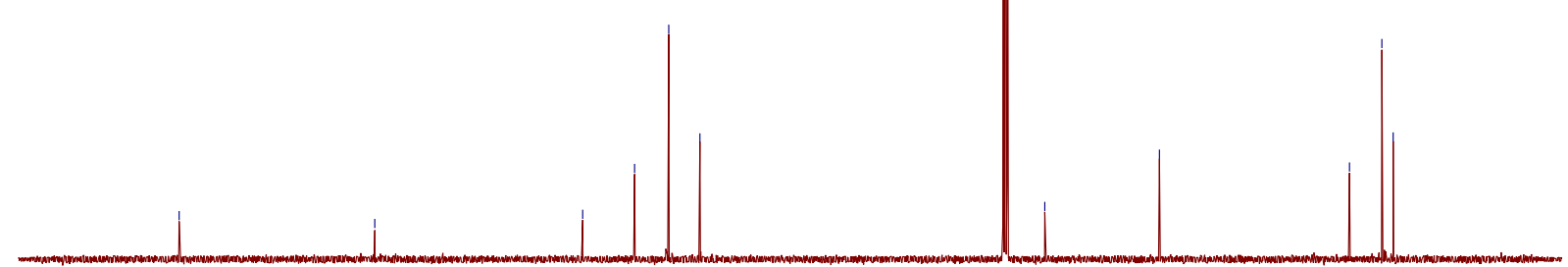

$\begin{array}{llllllllllll}220 & 210 & 200 & 190 & 180 & 170 & 160 & 150 & 140 & 130 & 120 \underset{\mathrm{f} 1(\mathrm{ppm})}{110} 100\end{array}$

${ }^{13} \mathrm{C}-\mathrm{NMR}$ in $\mathrm{CDCl}_{3}$. 
115a

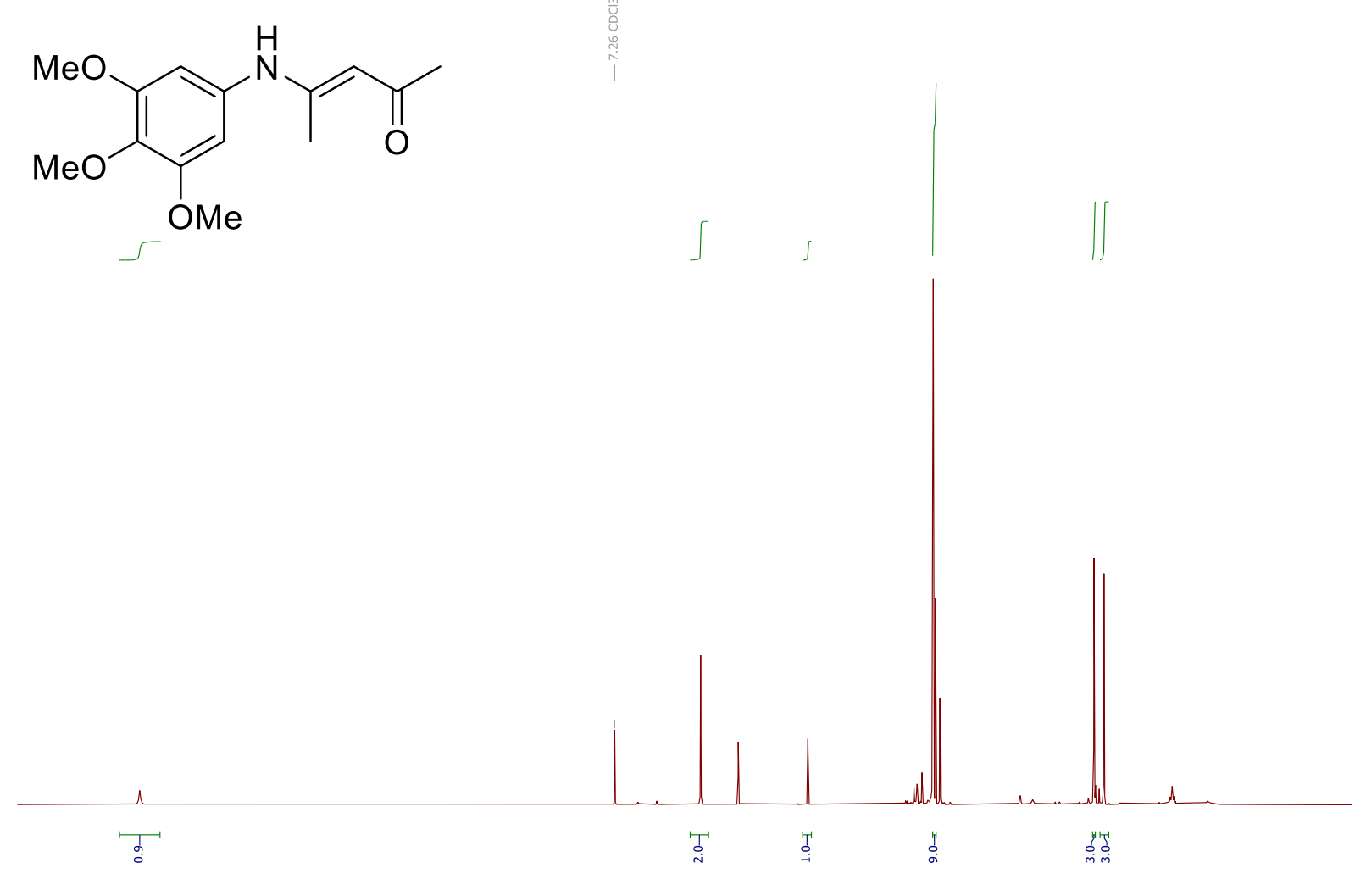

\begin{tabular}{lllllllllllllllllllllllllllll}
\hline 13.5 & 13.0 & 12.5 & 12.0 & 11.5 & 11.0 & 10.5 & 10.0 & 9.5 & 9.0 & 8.5 & 8.0 & 7.5 & 7.0 & 6.5 & 6.0 & 5.5 & 5.0 & 4.5 & 4.0 & 3.5 & 3.0 & 2.5 & 2.0 & 1.5 & 1.0 & 0.5 & 0.0 & -0.5
\end{tabular} ${ }^{1} \mathrm{H}-\mathrm{NMR}$ in $\mathrm{CDCl}_{3}$.

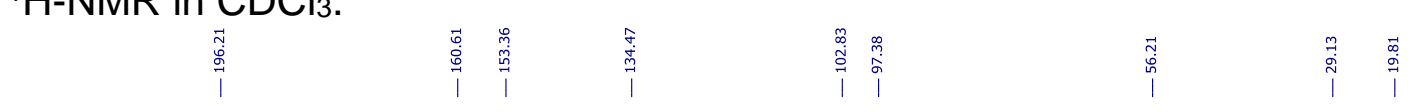<smiles>COc1cc(N/C(C)=C/C(C)=O)cc(OC)c1OC</smiles>

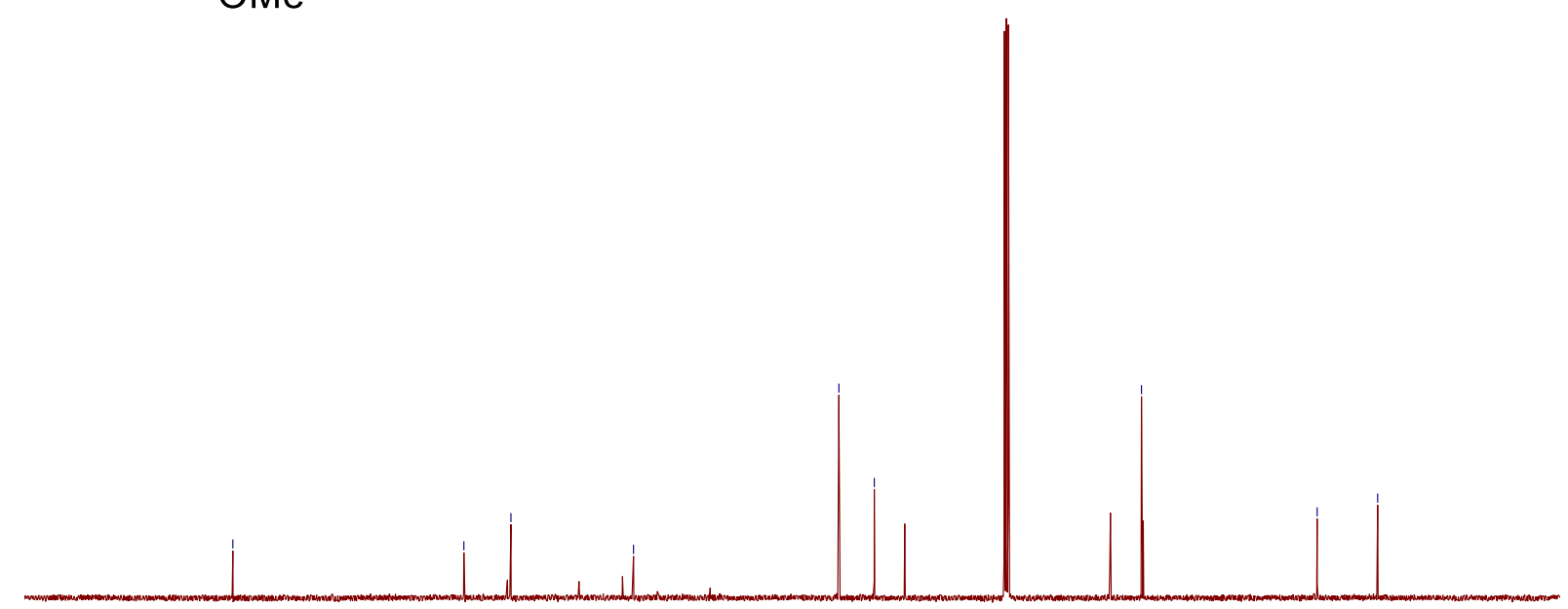

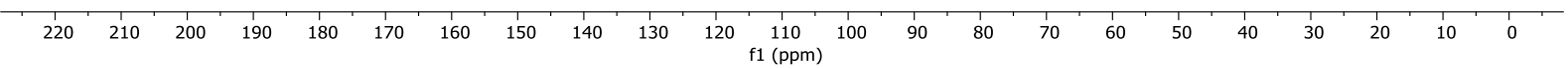
${ }^{13} \mathrm{C}-\mathrm{NMR}$ in $\mathrm{CDCl}_{3}$. 


\section{5b}<smiles>COC(=O)C(C)(Nc1cc(OC)c(OC)c(OC)c1)C(C)=O</smiles>

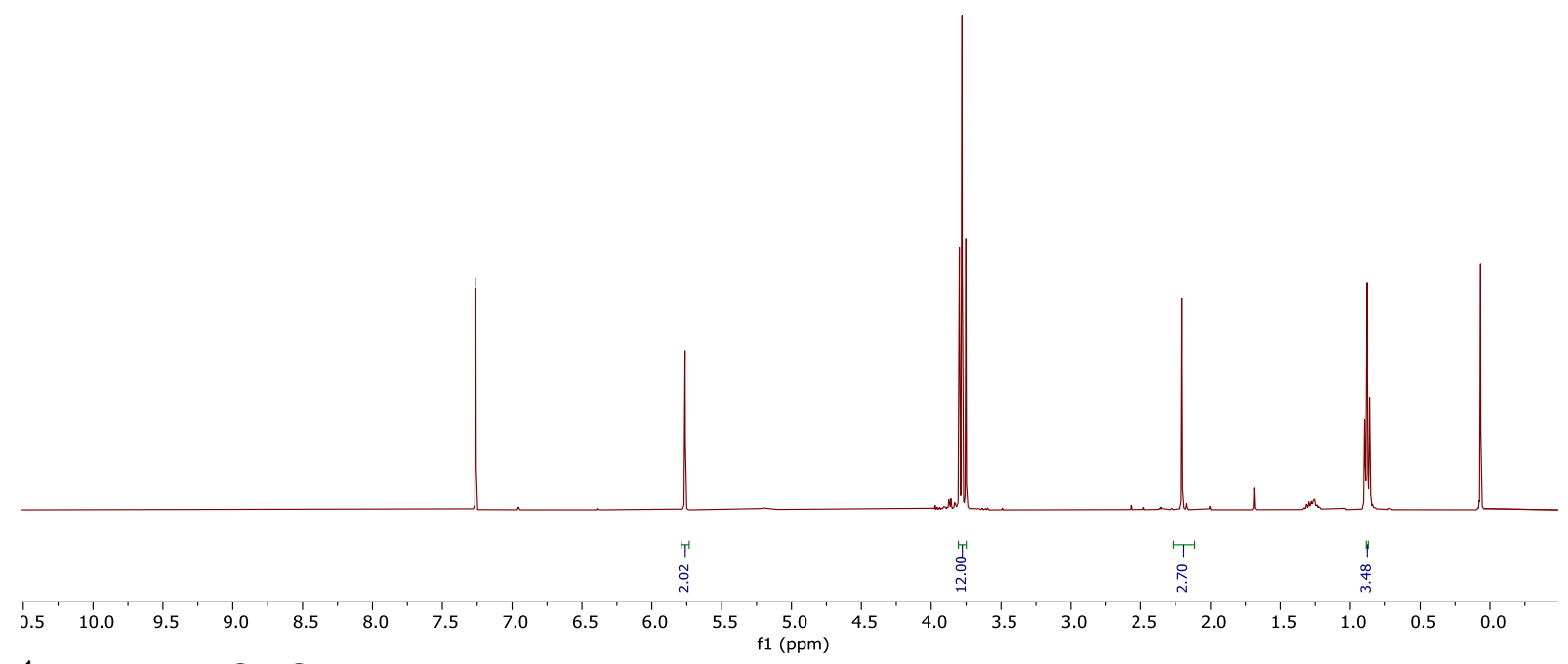

${ }^{1} \mathrm{H}-\mathrm{NMR}$ in $\mathrm{CDCl}_{3}$.

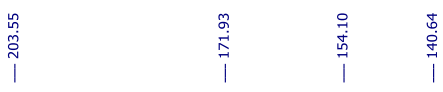

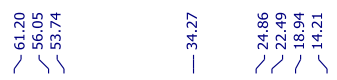<smiles>COC(=O)C(C)(Nc1cc(OC)c(OC)c(OC)c1)C(C)=O</smiles>

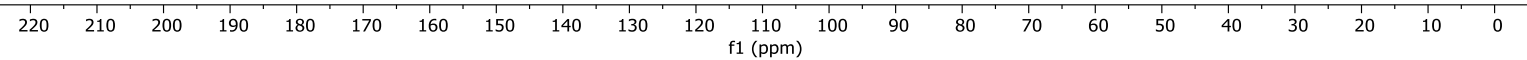
${ }^{13} \mathrm{C}-\mathrm{NMR}$ in $\mathrm{CDCl}_{3}$. 
$116 a$<smiles>CC(=O)/C=C(\C)Nc1ccc(Br)cc1</smiles>
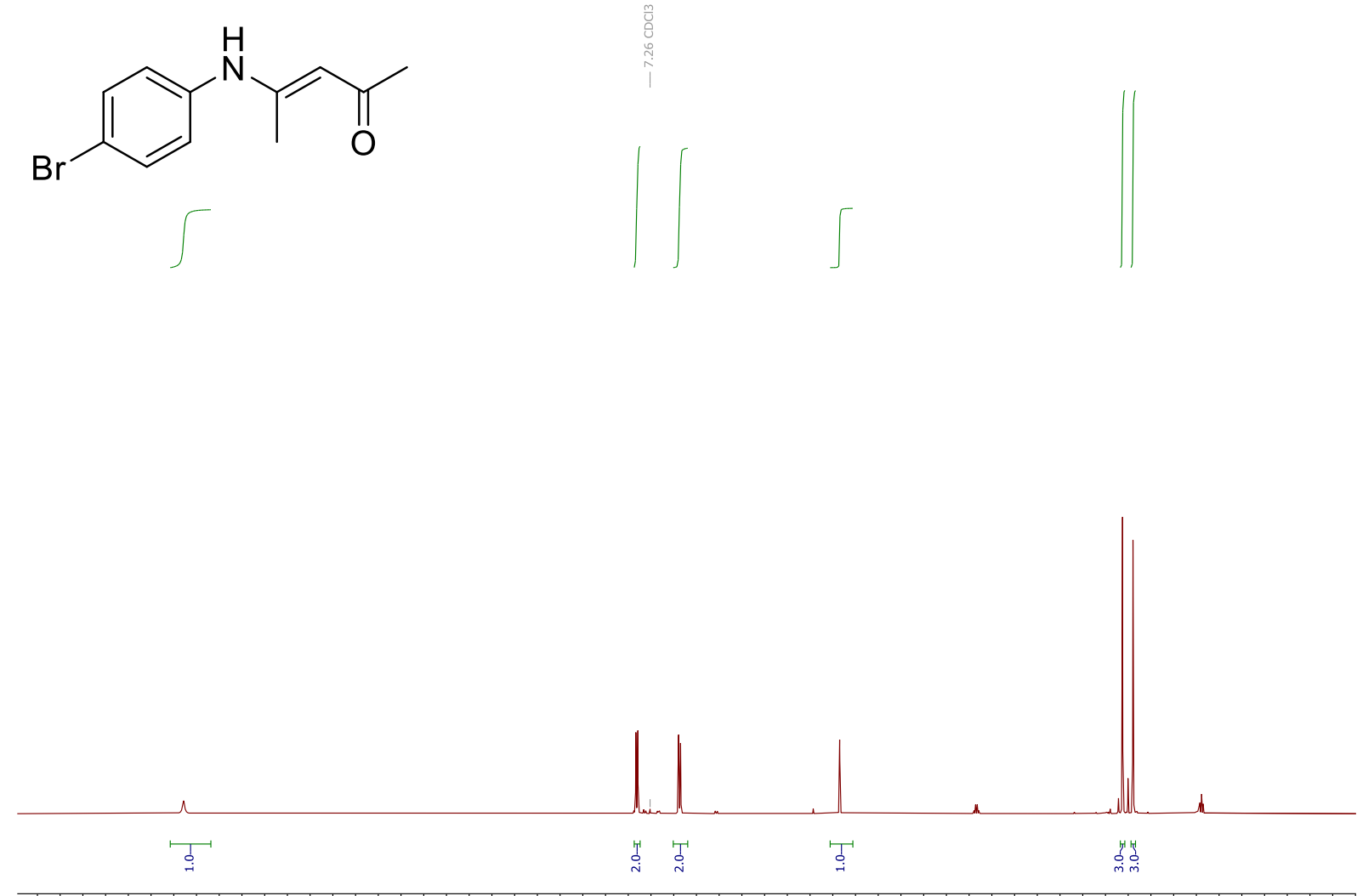

\begin{tabular}{llllllllllllllllllllllllllllll}
\hline 14.0 & 13.5 & 13.0 & 12.5 & 12.0 & 11.5 & 11.0 & 10.5 & 10.0 & 9.5 & 9.0 & 8.5 & 8.0 & 7.5 & $\begin{array}{c}7.0 \\
\mathrm{f} 1(\mathrm{ppm})\end{array}$ & 6.5 & 6.0 & 5.5 & 5.0 & 4.5 & 4.0 & 3.5 & 3.0 & 2.5 & 2.0 & 1.5 & 1.0 & 0.5 & 0.0 & $-\mathrm{C}$
\end{tabular} ${ }^{1} \mathrm{H}-\mathrm{NMR}$ in $\mathrm{CDCl}_{3}$.<smiles>CC(=O)/C=C(\C)Nc1ccc(Br)cc1</smiles>

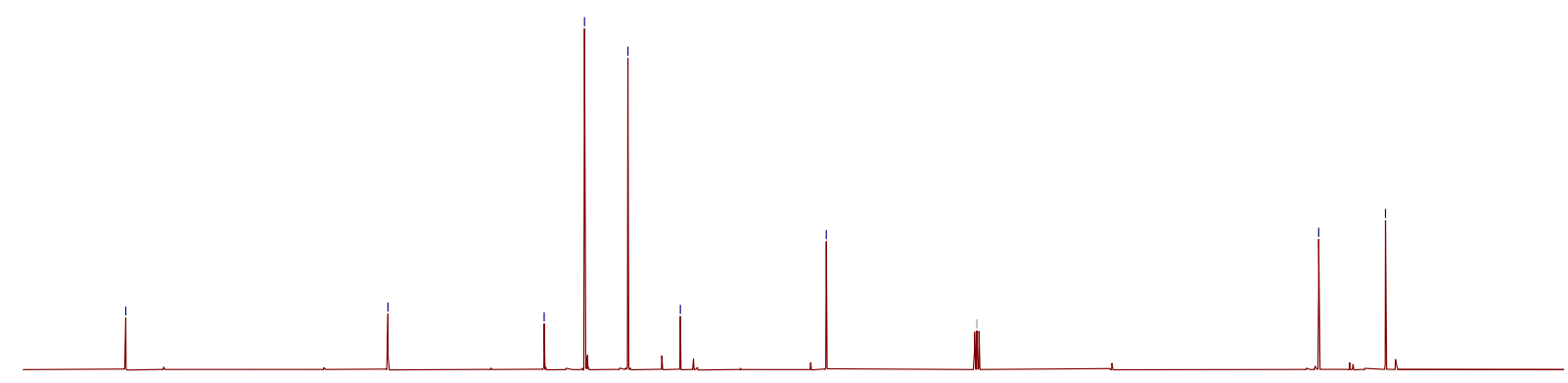

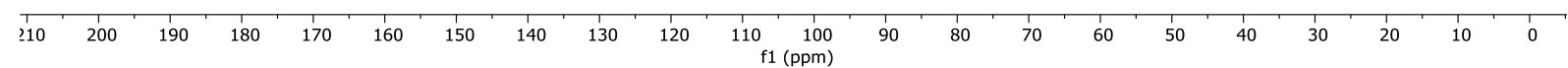
${ }^{13} \mathrm{C}-\mathrm{NMR}$ in $\mathrm{CDCl}_{3}$. 
$116 b$<smiles>COC(=O)C(C)(Nc1ccc(Br)cc1)C(C)=O</smiles>
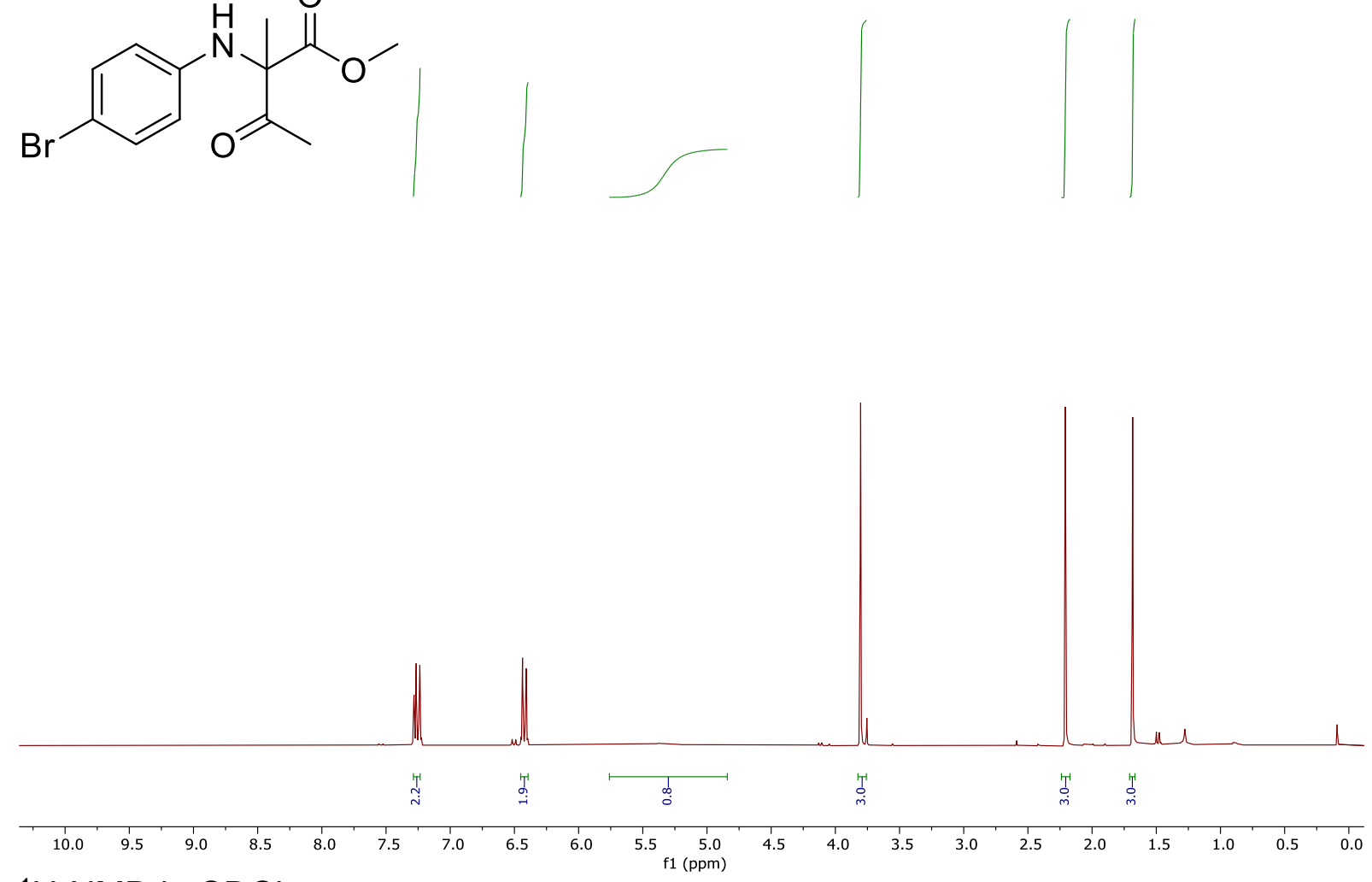

${ }^{1} \mathrm{H}-\mathrm{NMR}$ in $\mathrm{CDCl}_{3}$. 筩<smiles>COC(=O)C(C)(Nc1ccc(Br)cc1)C(C)=O</smiles>

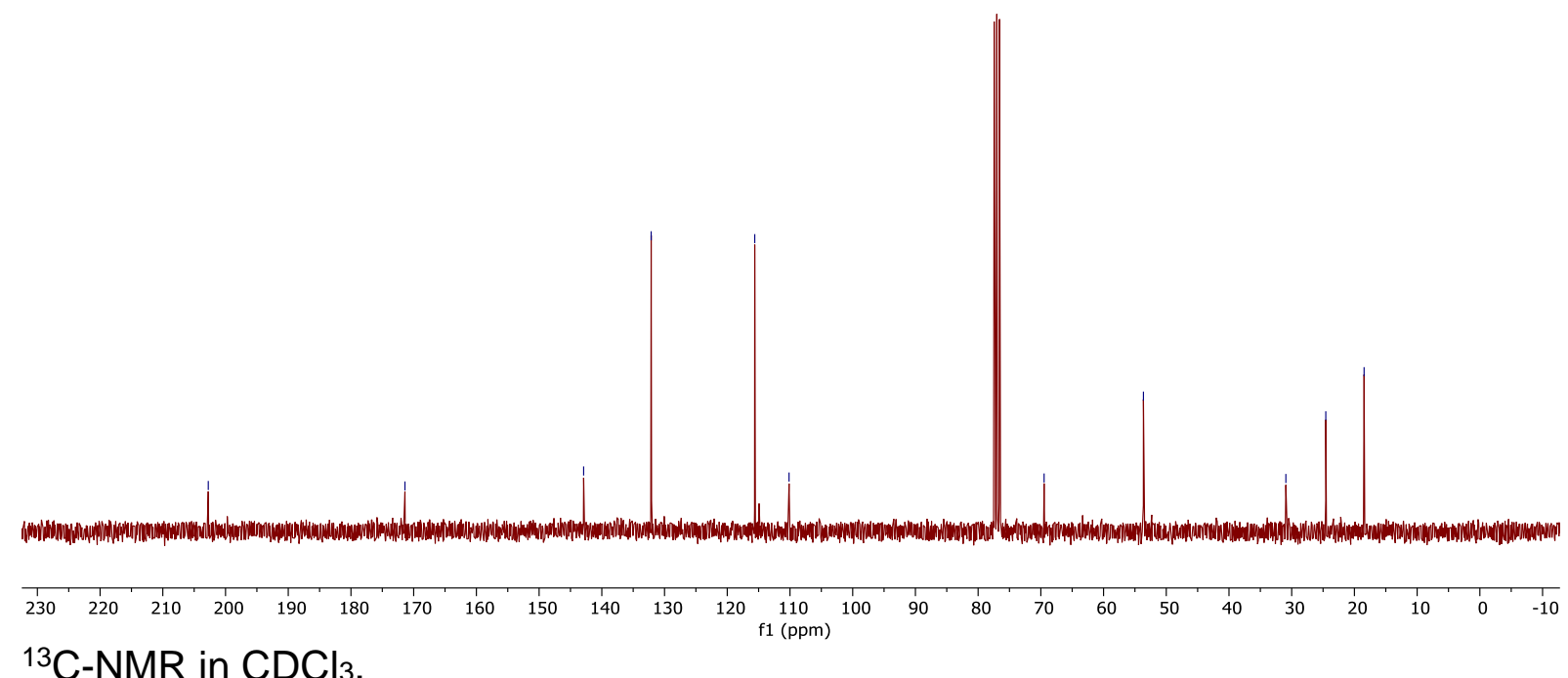
${ }^{13} \mathrm{C}-\mathrm{NMR}$ in $\mathrm{CDCl}_{3}$. 


\section{$117 a$}

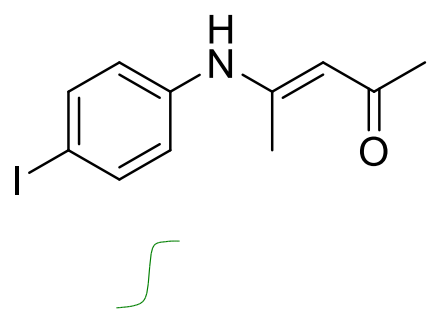
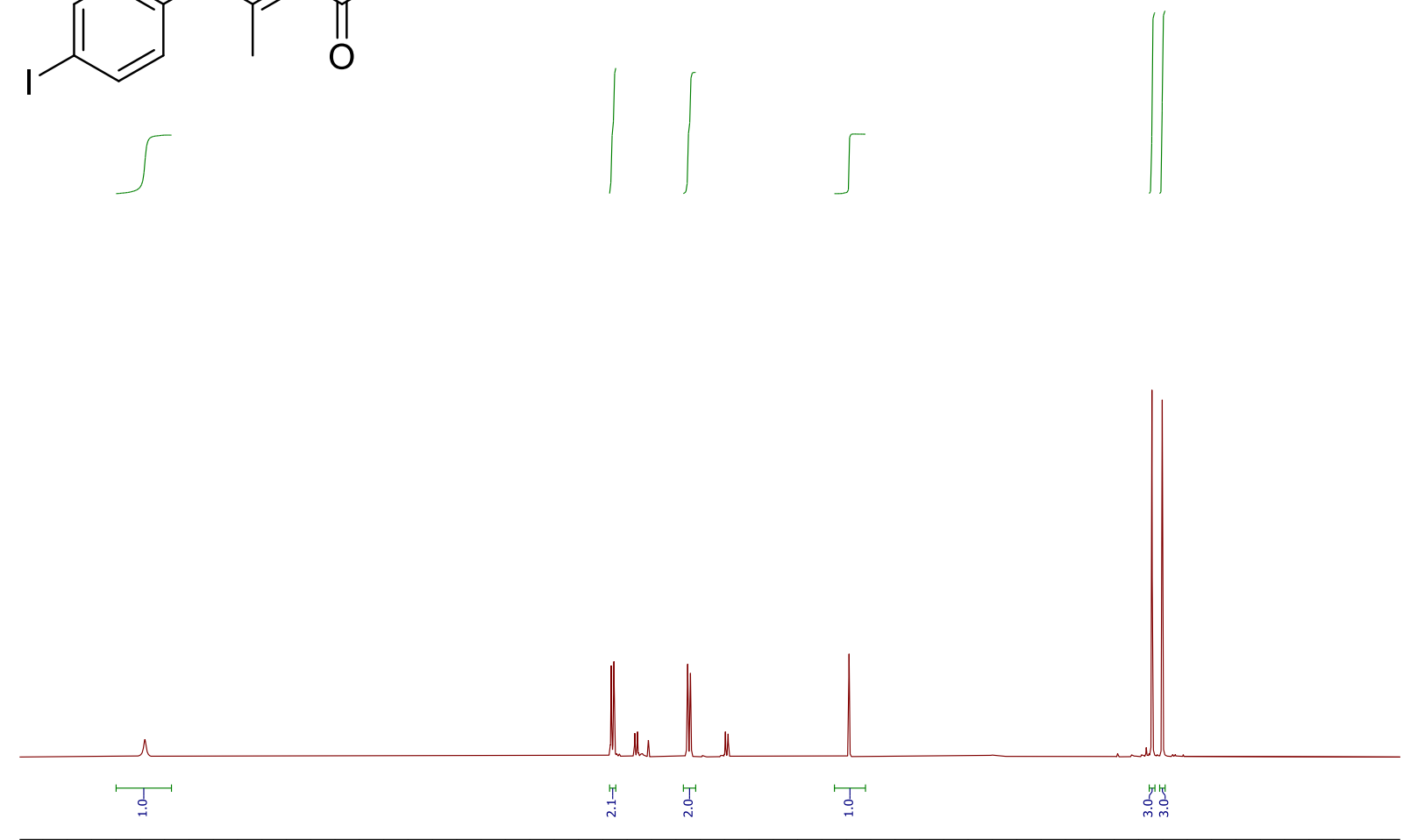

\begin{tabular}{llllllllllllllllllllllllllllll}
\hline 13.5 & 13.0 & 12.5 & 12.0 & 11.5 & 11.0 & 10.5 & 10.0 & 9.5 & 9.0 & 8.5 & 8.0 & 7.5 & 7.0 & 6.5 & 6.0 & 5.5 & 5.0 & 4.5 & 4.0 & 3.5 & 3.0 & 2.5 & 2.0 & 1.5 & 1.0 & 0.5 & 0.0
\end{tabular} ${ }^{1} \mathrm{H}-\mathrm{NMR}$ in $\mathrm{CDCl}_{3}$.<smiles>CC(=O)/C=C(\C)Nc1ccc(I)cc1</smiles>

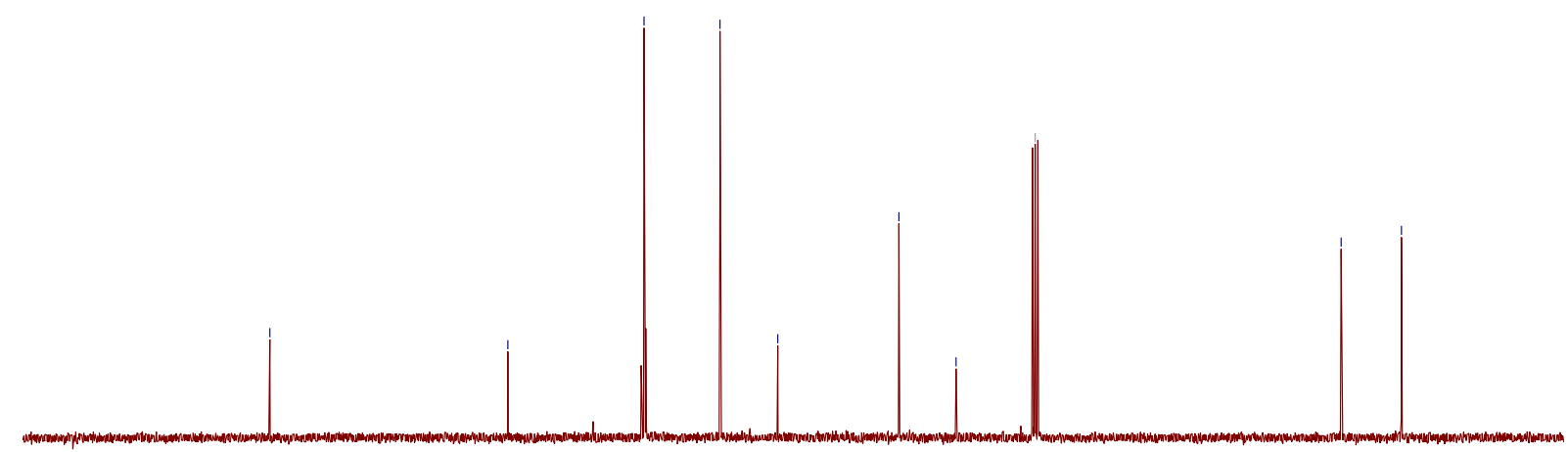

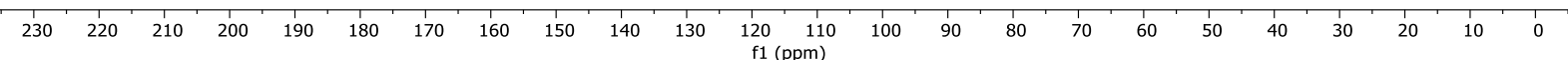
${ }^{13} \mathrm{C}-\mathrm{NMR}$ in $\mathrm{CDCl}_{3}$. 
<smiles>COC(=O)C(C)(Nc1ccc(I)cc1)C(C)=O</smiles>
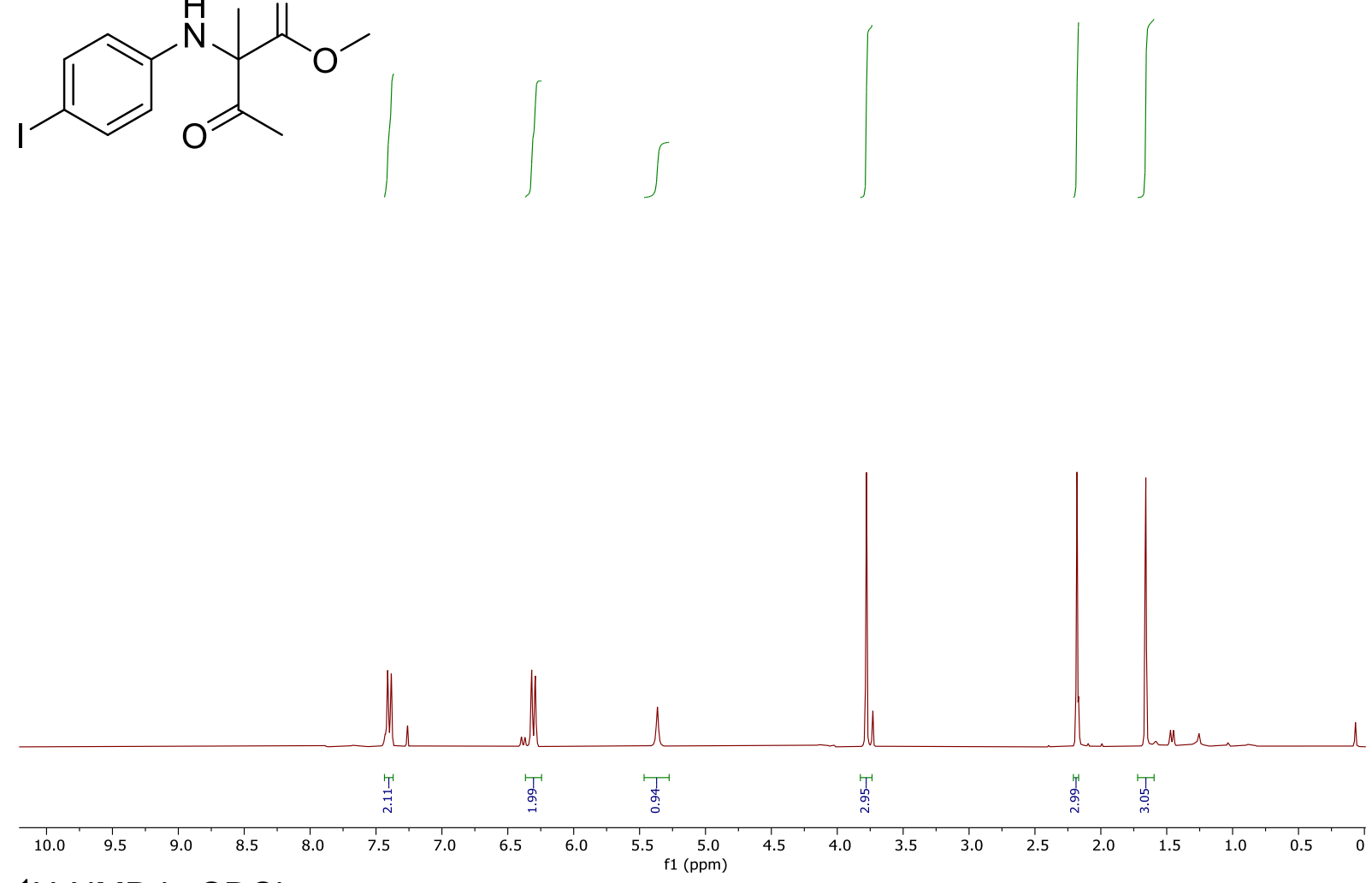

${ }^{1} \mathrm{H}-\mathrm{NMR}$ in $\mathrm{CDCl}_{3}$.

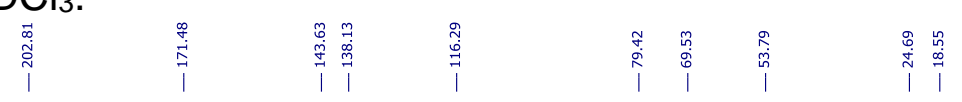<smiles>COC(=O)C(C)(Nc1ccc(I)cc1)C(C)=O</smiles>

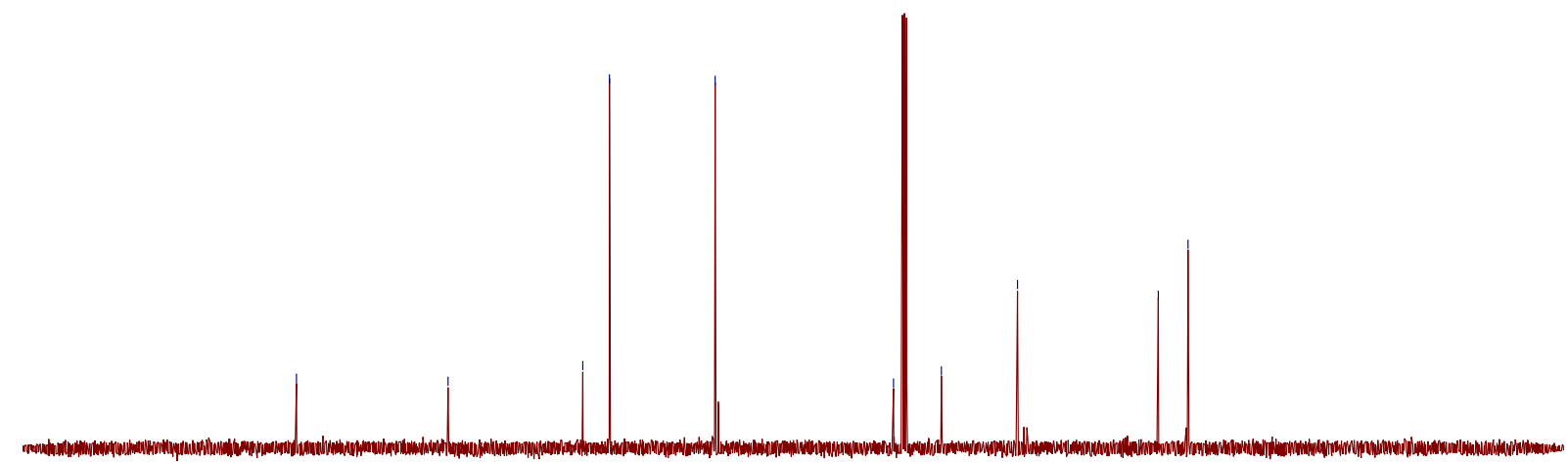

$\begin{array}{lllllllllllllllllllllllllllllllllll}250 & 240 & 230 & 220 & 210 & 200 & 190 & 180 & 170 & 160 & 150 & 140 & 130 & 120 & 110 & 100 & 90 & 80 & 70 & 60 & 50 & 40 & 30 & 20 & 10 & 0 & -10 & -20 & -30 & -40 & -50\end{array}$ ${ }^{13} \mathrm{C}-\mathrm{NMR}$ in $\mathrm{CDCl}_{3}$. 
$118 a$

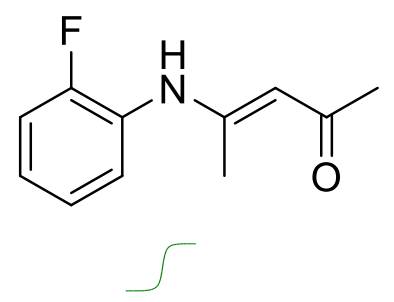
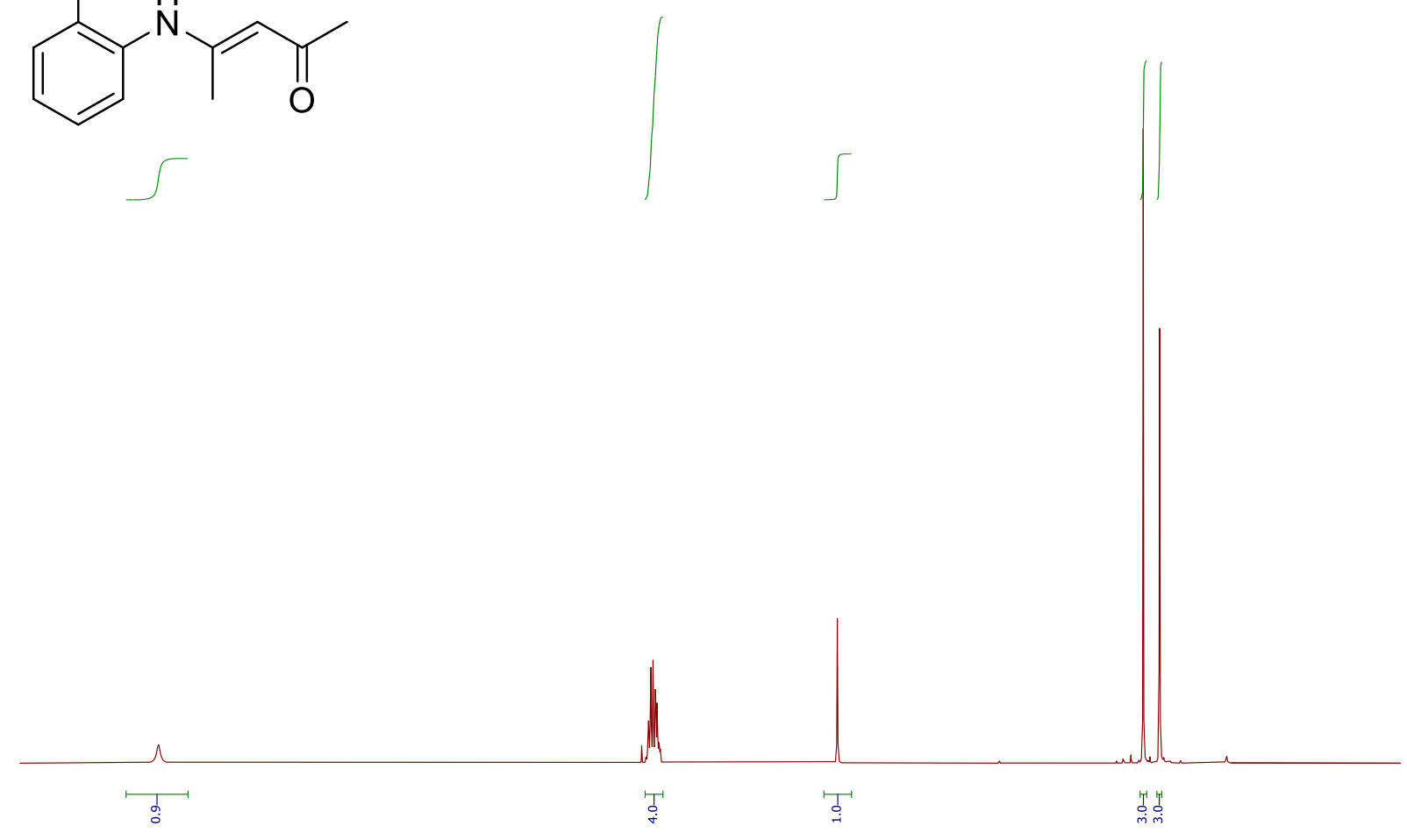

\begin{tabular}{llllllllllllllllllllllllllllll}
\hline 13.5 & 13.0 & 12.5 & 12.0 & 11.5 & 11.0 & 10.5 & 10.0 & 9.5 & 9.0 & 8.5 & 8.0 & 7.5 & 7.0 & 6.5 & 6.0 & 5.5 & 5.0 & 4.5 & 4.0 & 3.5 & 3.0 & 2.5 & 2.0 & 1.5 & 1.0 & 0.5 & 0.0 & -0
\end{tabular} ${ }^{1} \mathrm{H}-\mathrm{NMR}$ in $\mathrm{CDCl}_{3}$.<smiles>CC(=O)/C=C(\C)Nc1ccccc1F</smiles>

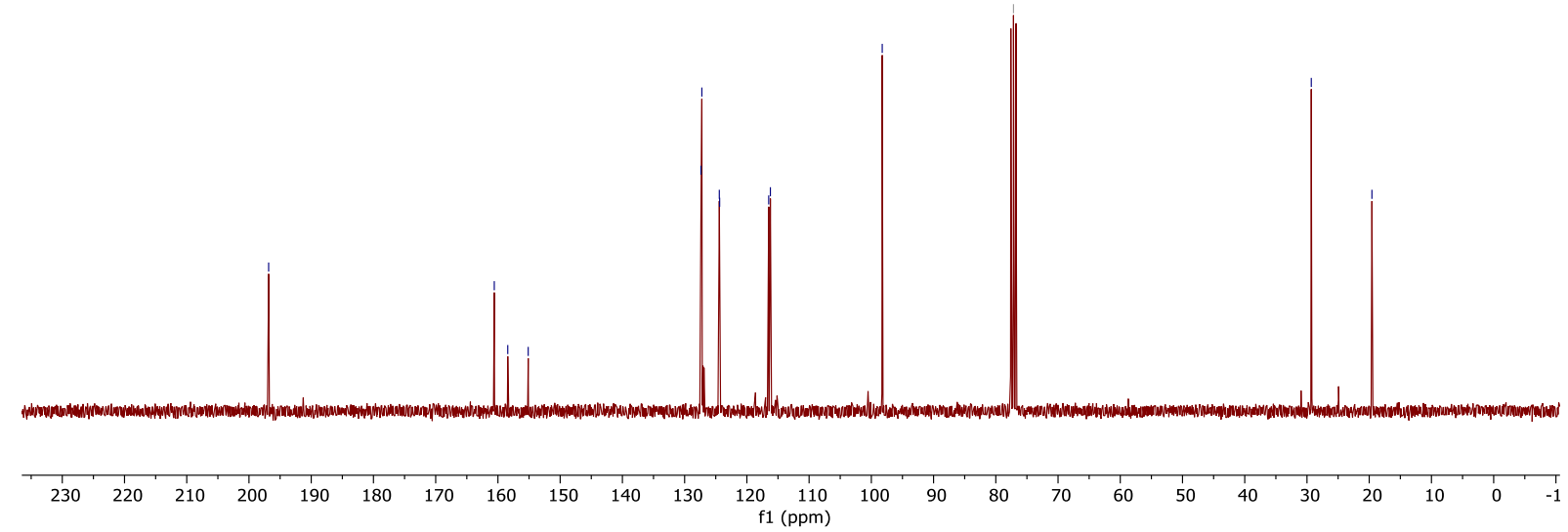
${ }^{13} \mathrm{C}-\mathrm{NMR}$ in $\mathrm{CDCl}_{3}$. 


\section{$118 b$}<smiles>COC(=O)C(C)(Nc1ccccc1F)C(C)=O</smiles>
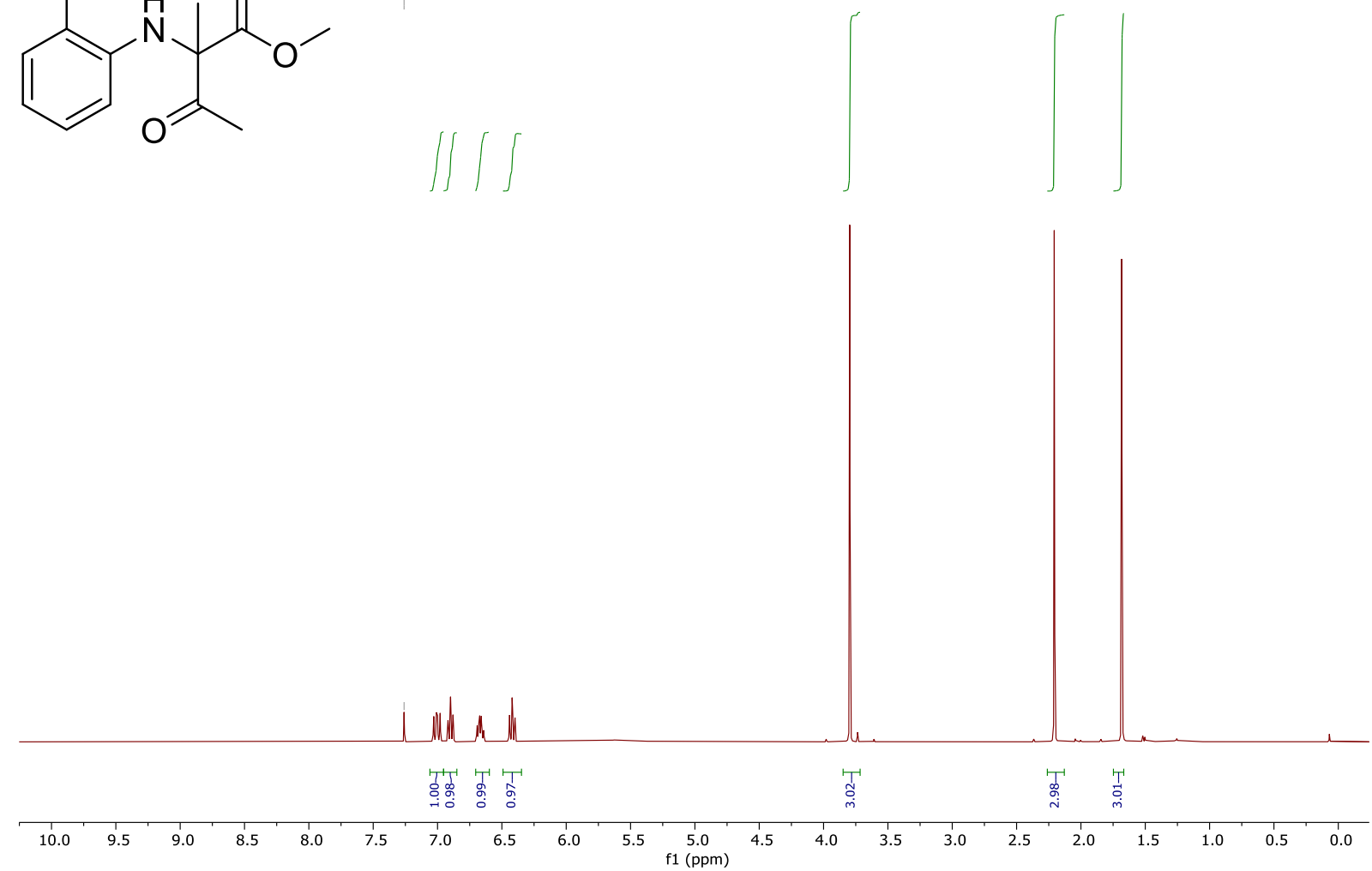

${ }^{1} \mathrm{H}-\mathrm{NMR}$ in $\mathrm{CDCl}_{3}$.

|<smiles>COC(=O)C(C)(Nc1ccccc1F)C(C)=O</smiles>

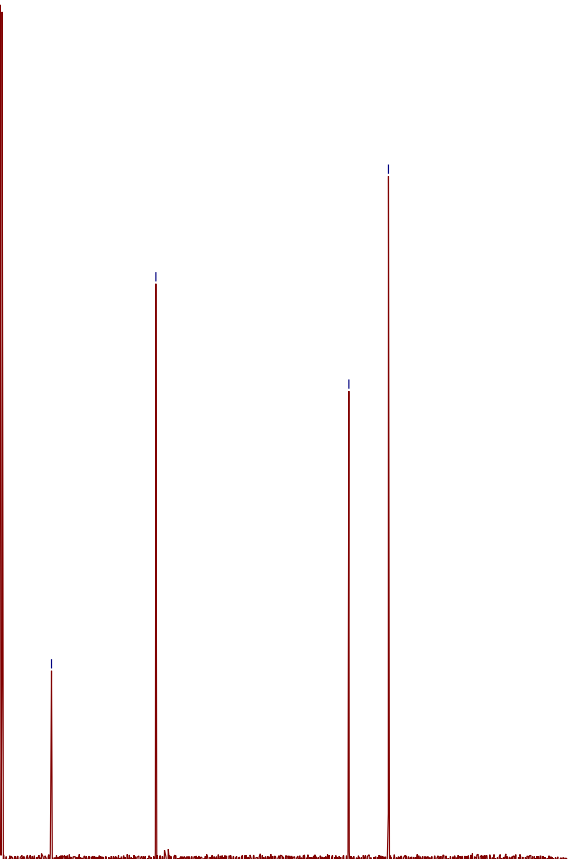

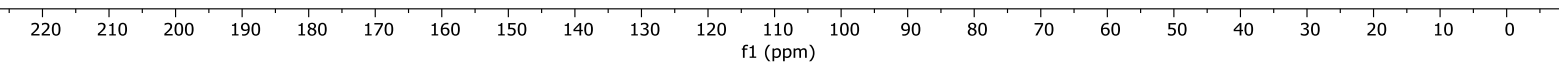
${ }^{13} \mathrm{C}-\mathrm{NMR}$ in $\mathrm{CDCl}_{3}$. 
$119 a$<smiles>CC(=O)/C=C(\C)Nc1ccc(Cl)c(C)c1</smiles>
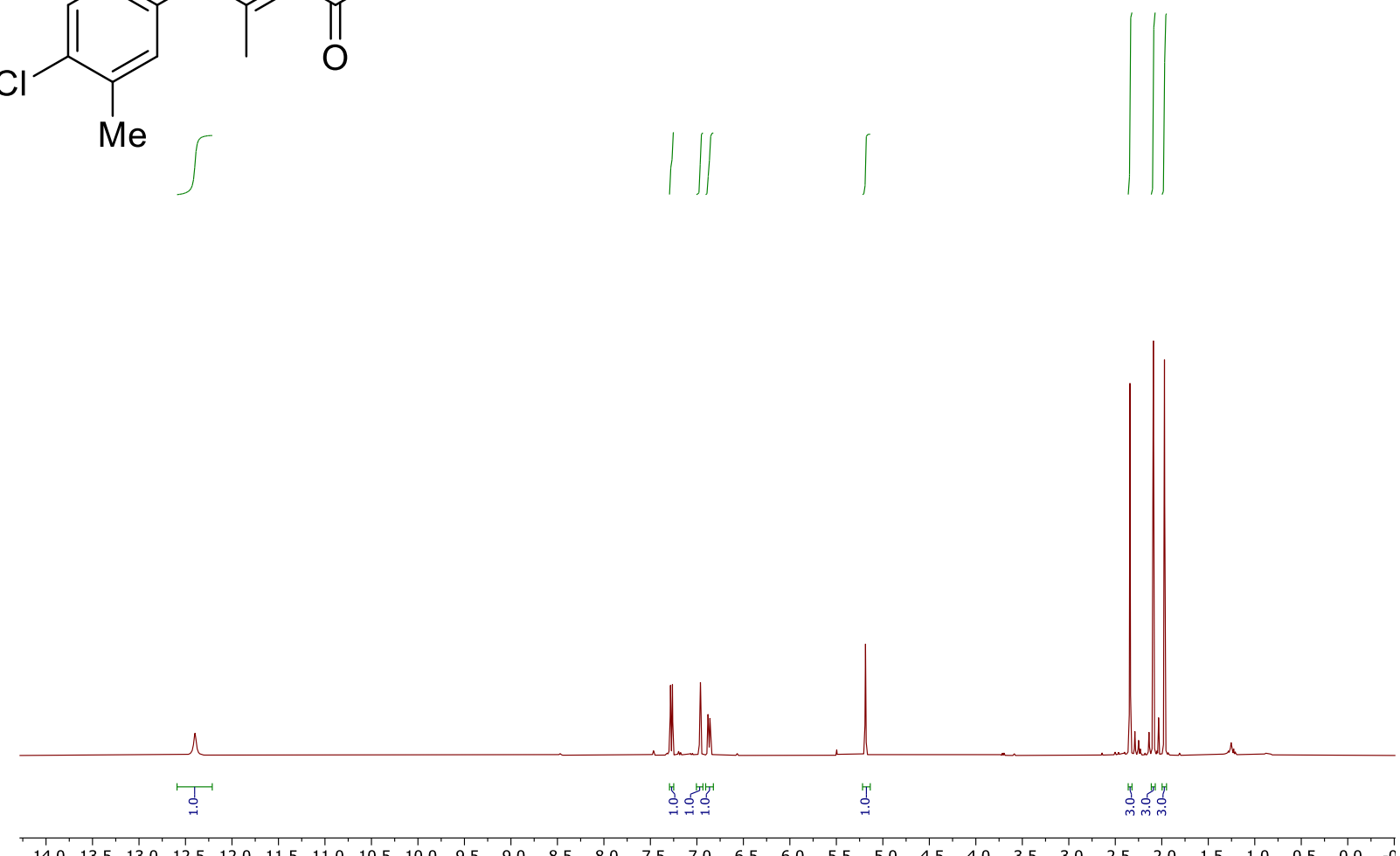

\begin{tabular}{lllllllllllllllllllllllllllllll}
\hline 14.0 & 13.5 & 13.0 & 12.5 & 12.0 & 11.5 & 11.0 & 10.5 & 10.0 & 9.5 & 9.0 & 8.5 & 8.0 & 7.5 & 7.0 & 6.5 & 6.0 & 5.5 & 5.0 & 4.5 & 4.0 & 3.5 & 3.0 & 2.5 & 2.0 & 1.5 & 1.0 & 0.5 & 0.0 & -0
\end{tabular} ${ }^{1} \mathrm{H}-\mathrm{NMR}$ in $\mathrm{CDCl}_{3}$.
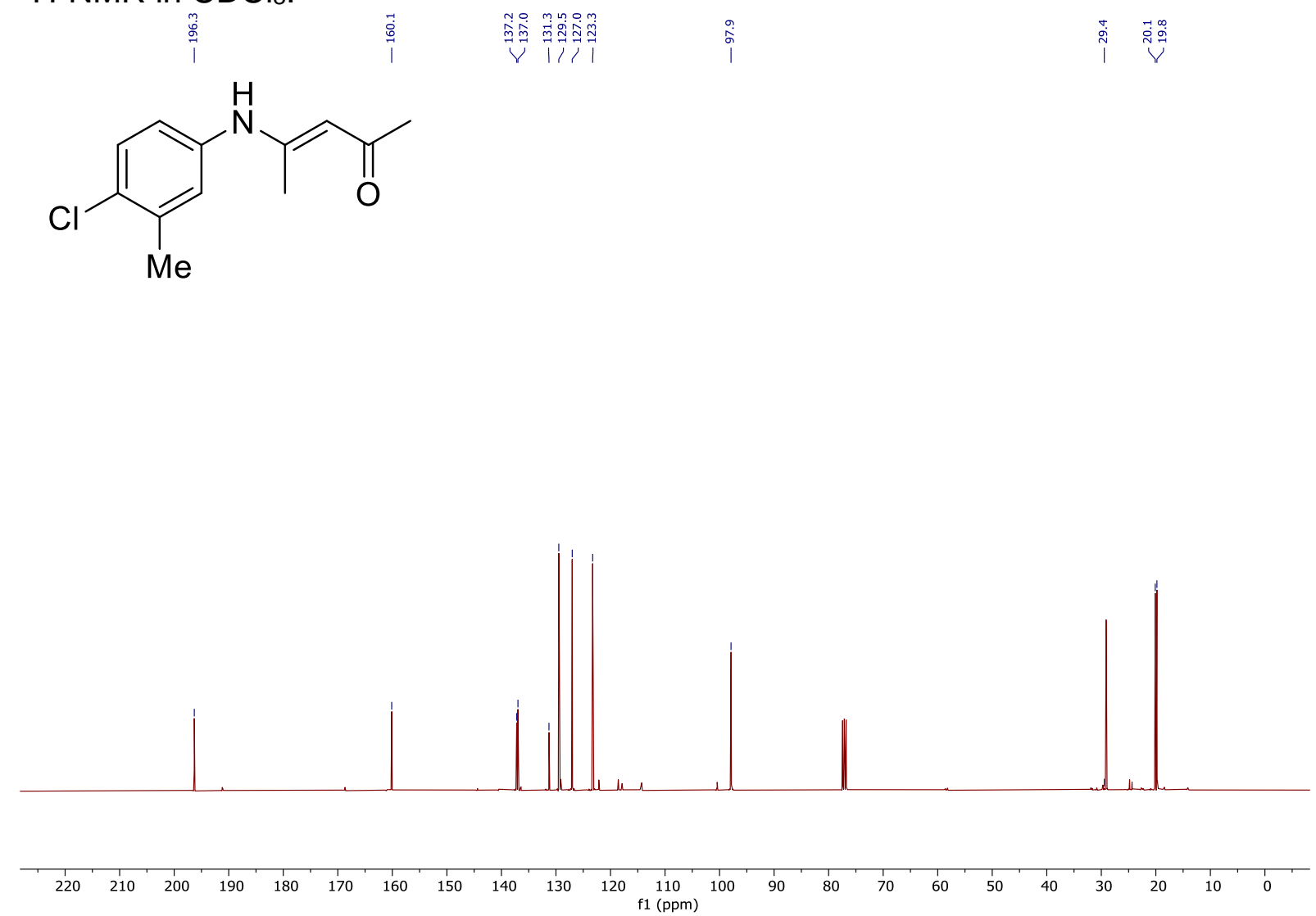
${ }^{13} \mathrm{C}-\mathrm{NMR}$ in $\mathrm{CDCl}_{3}$. 


\section{9b}<smiles>COC(=O)C(C)(Nc1ccc(Cl)c(C)c1)C(C)=O</smiles>

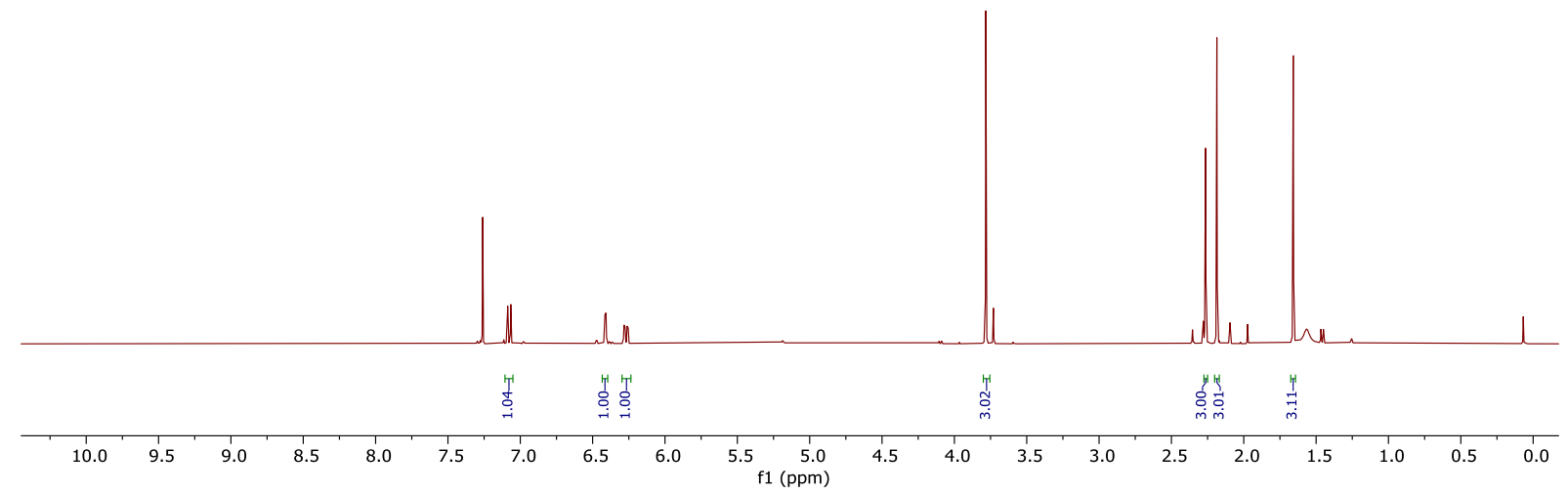

${ }^{1} \mathrm{H}-\mathrm{NMR}$ in $\mathrm{CDCl}_{3}$.

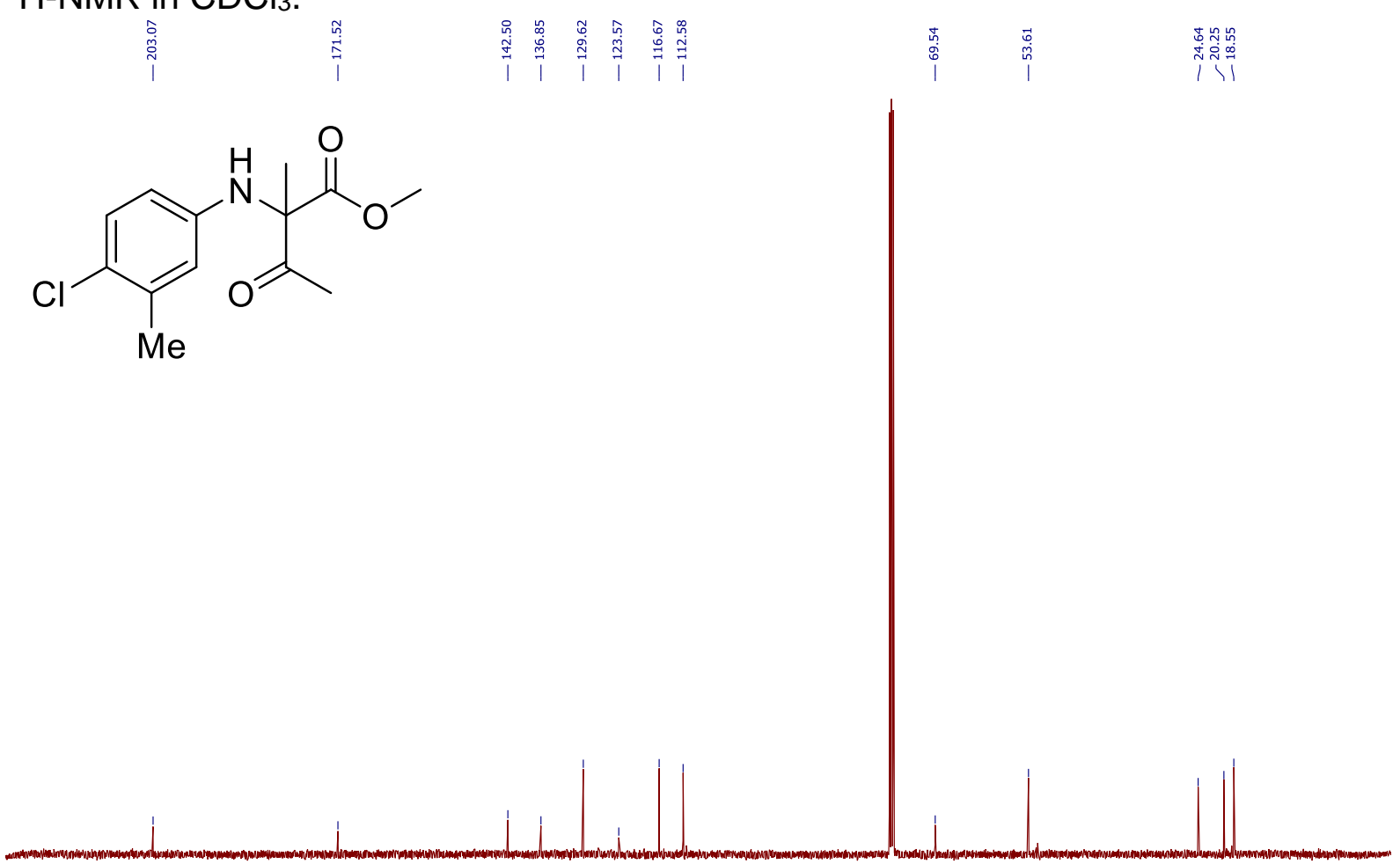

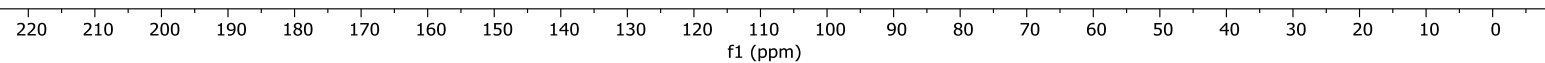
${ }^{13} \mathrm{C}-\mathrm{NMR}$ in $\mathrm{CDCl}_{3}$. 


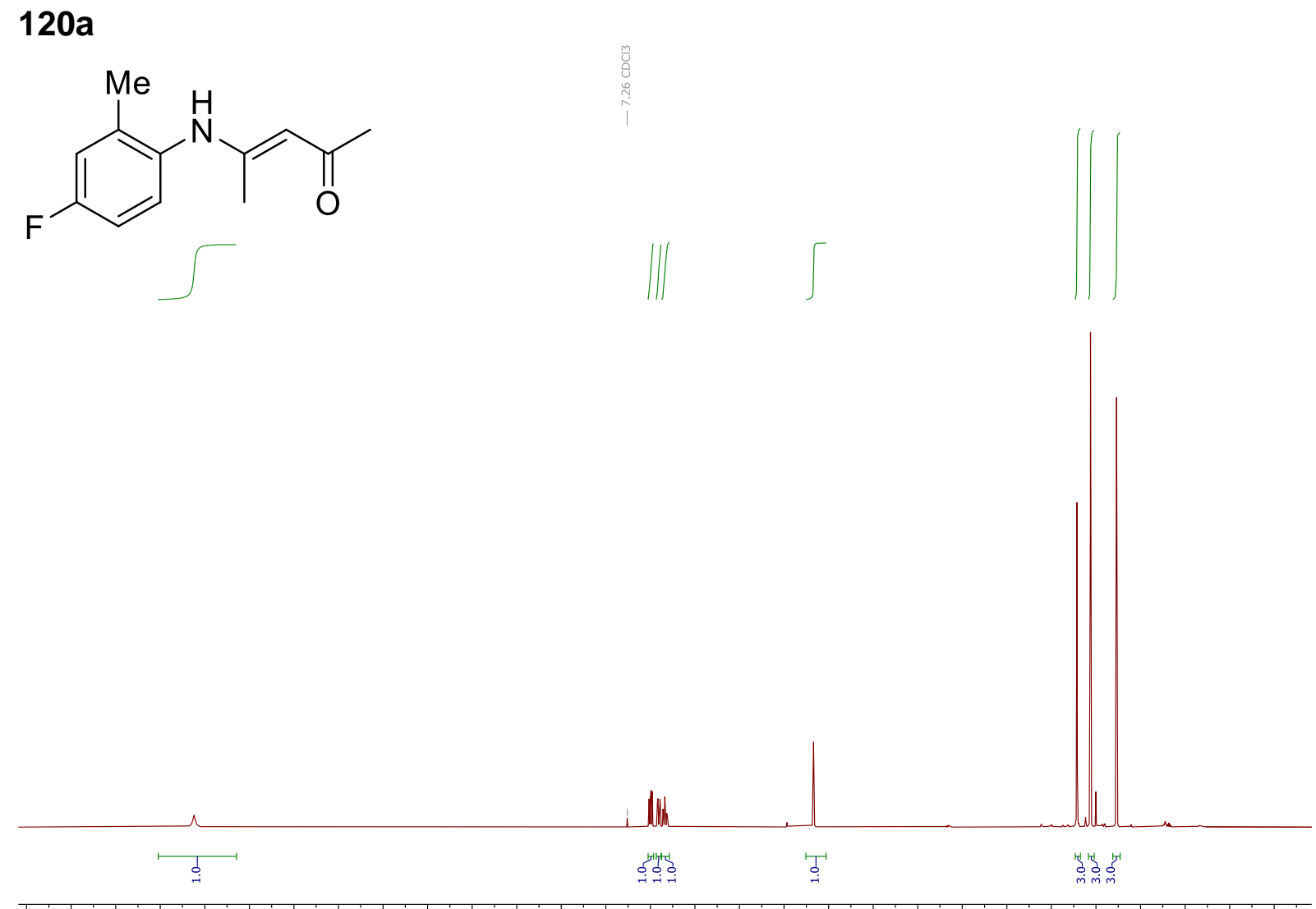

\begin{tabular}{lllllllllllllllllllllllllllllll}
\hline 4.0 & 13.5 & 13.0 & 12.5 & 12.0 & 11.5 & 11.0 & 10.5 & 10.0 & 9.5 & 9.0 & 8.5 & 8.0 & 7.5 & 7.0 & 6.5 & 6.0 & 5.5 & 5.0 & 4.5 & 4.0 & 3.5 & 3.0 & 2.5 & 2.0 & 1.5 & 1.0 & 0.5 & 0.0
\end{tabular} ${ }^{1} \mathrm{H}-\mathrm{NMR}$ in $\mathrm{CDCl}_{3}$.

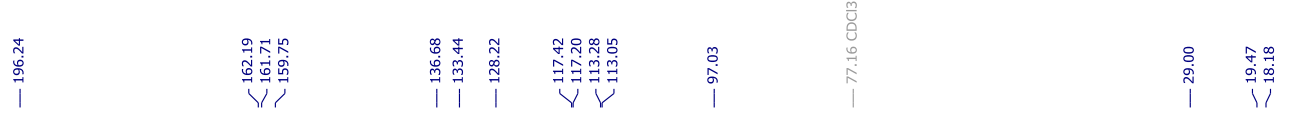<smiles>CC(=O)/C=C(\C)Nc1ccc(F)cc1C</smiles>

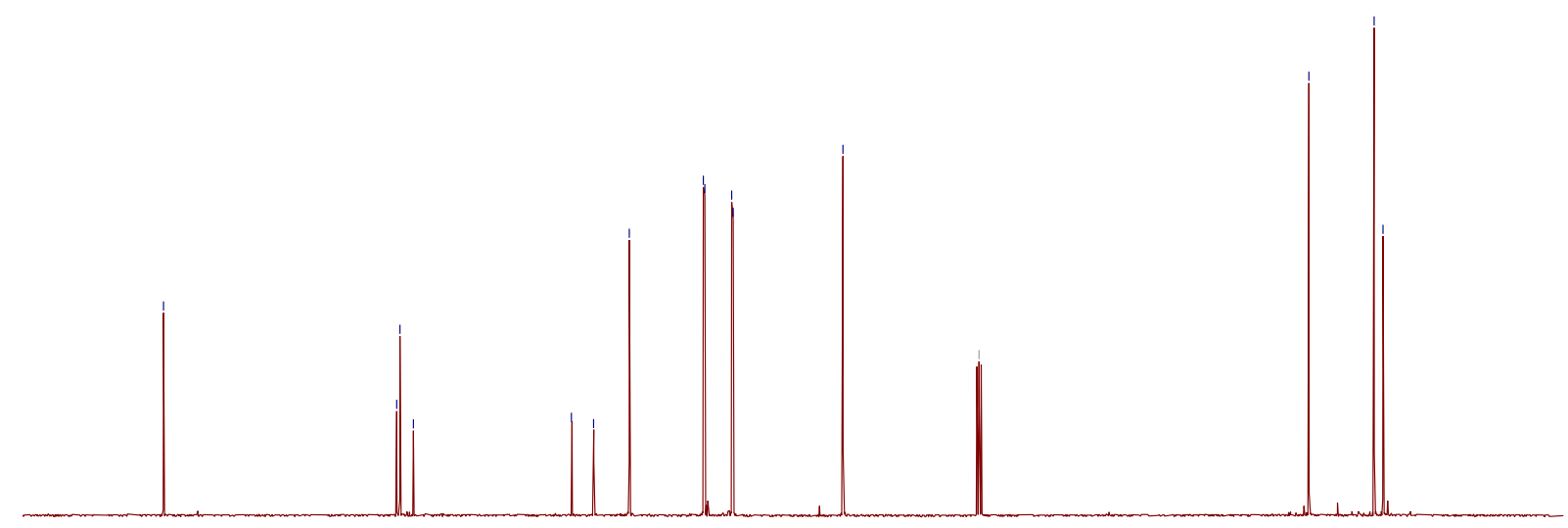

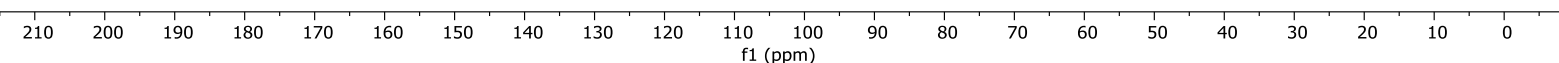
${ }^{13} \mathrm{C}-\mathrm{NMR}$ in $\mathrm{CDCl}_{3}$. 
120b<smiles>COC(=O)C(C)(Nc1ccc(F)cc1C)C(C)=O</smiles>
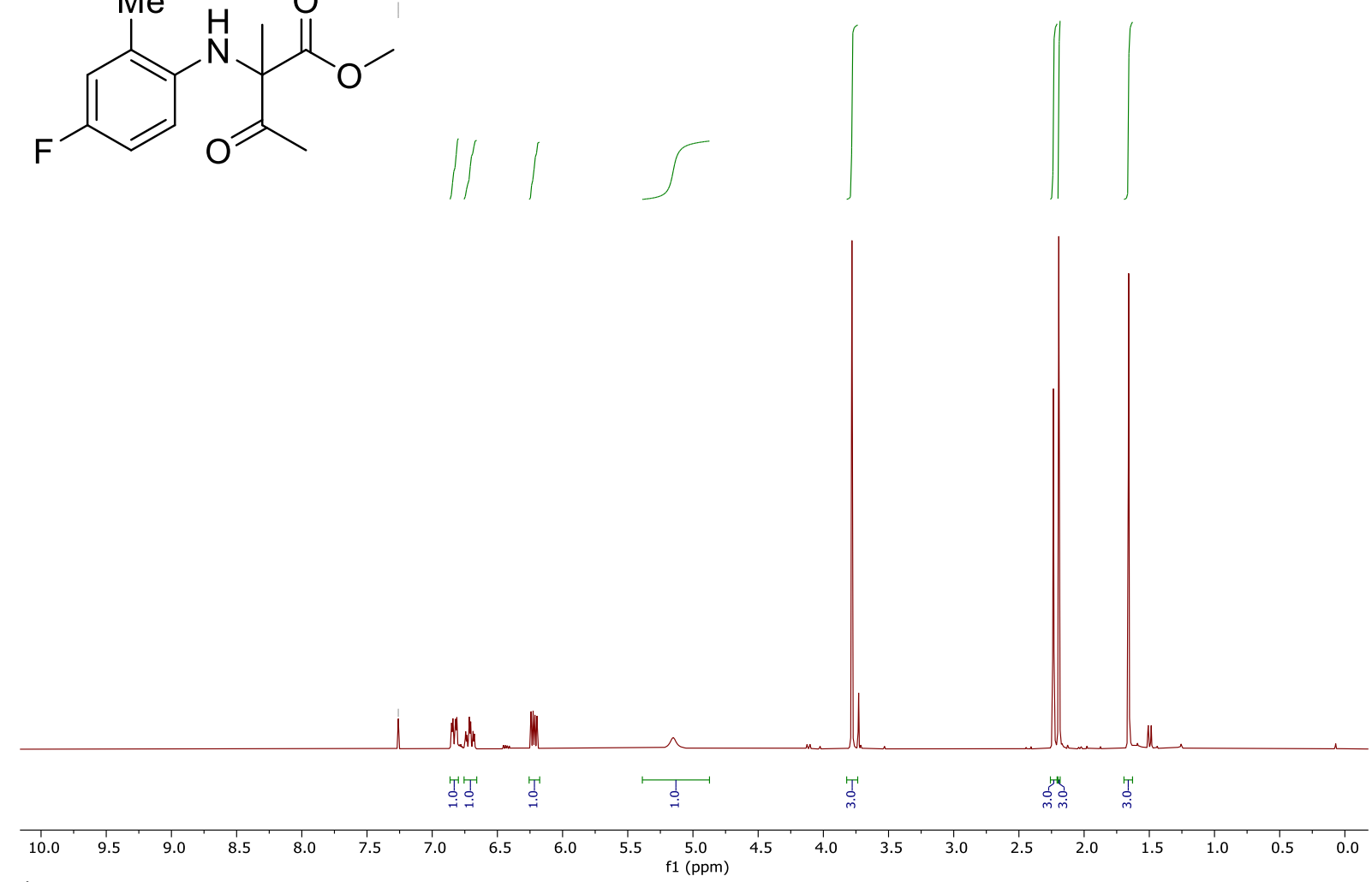

${ }^{1} \mathrm{H}-\mathrm{NMR}$ in $\mathrm{CDCl}_{3}$.

| l<smiles>COC(=O)C(C)(Nc1ccc(F)cc1C)C(C)=O</smiles>

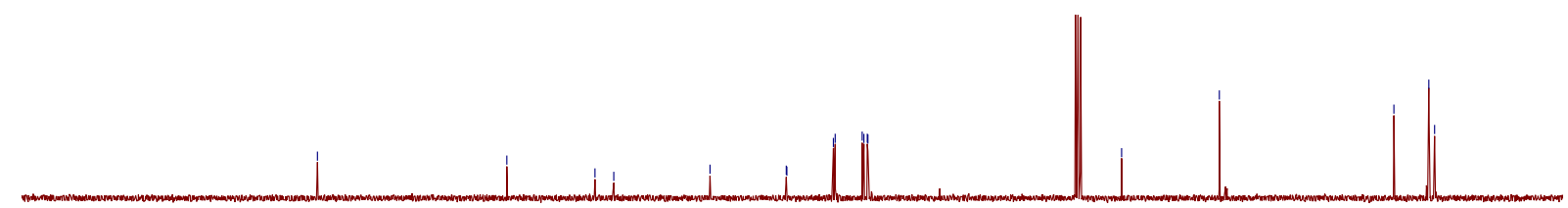

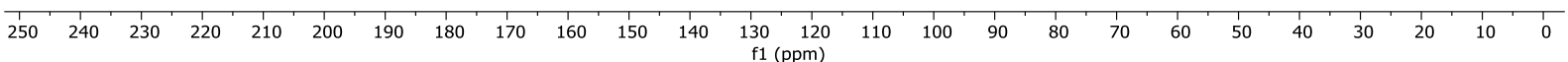
${ }^{13} \mathrm{C}-\mathrm{NMR}$ in $\mathrm{CDCl}_{3}$. 
$121 a$<smiles>CC(=O)/C=C(\C)NCc1ccccc1</smiles>
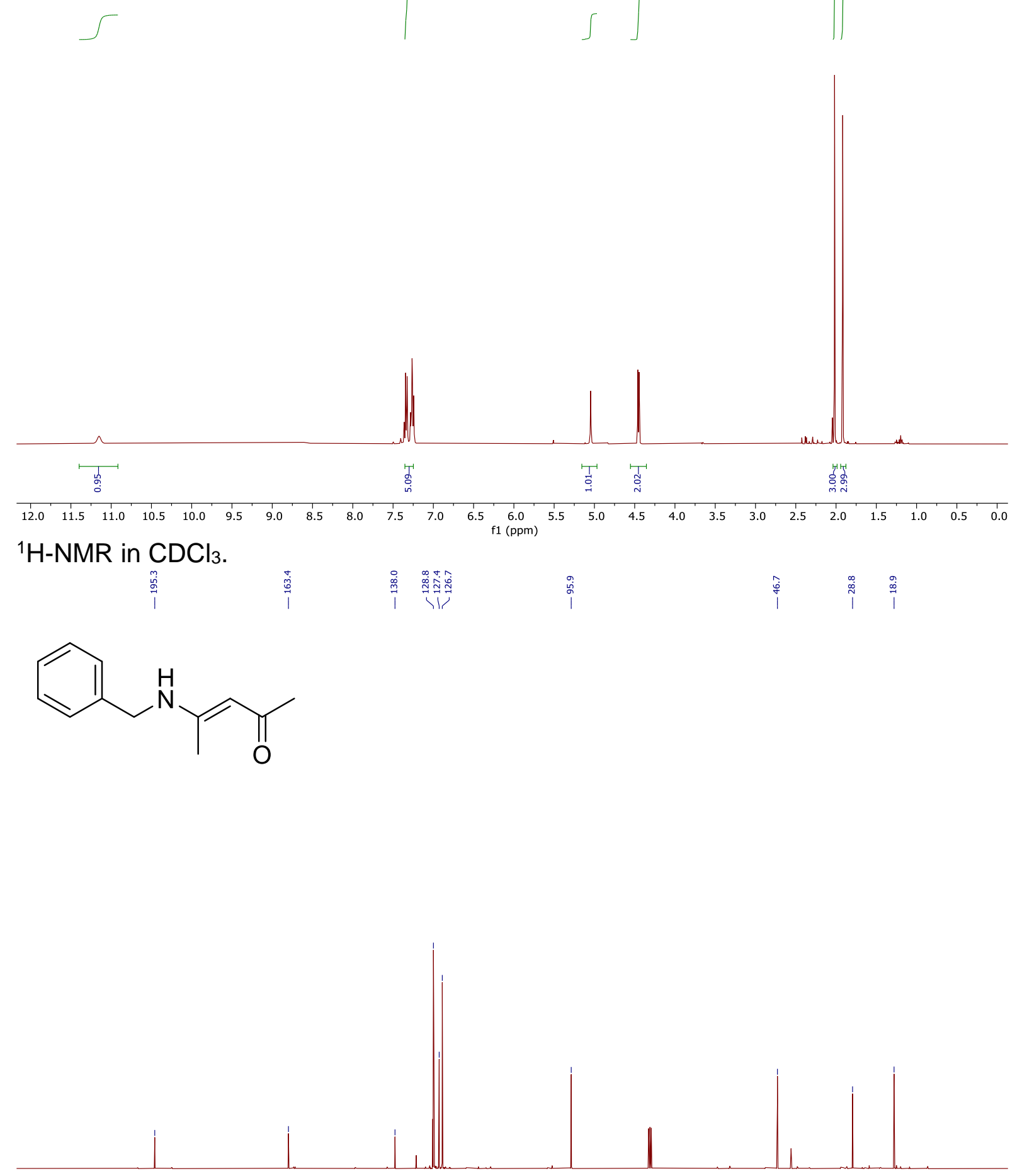

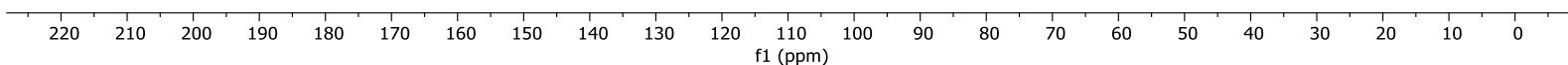
${ }^{13} \mathrm{C}-\mathrm{NMR}$ in $\mathrm{CDCl}_{3}$. 
121b<smiles>COC(=O)C(C)(NCc1ccccc1)C(C)=O</smiles>
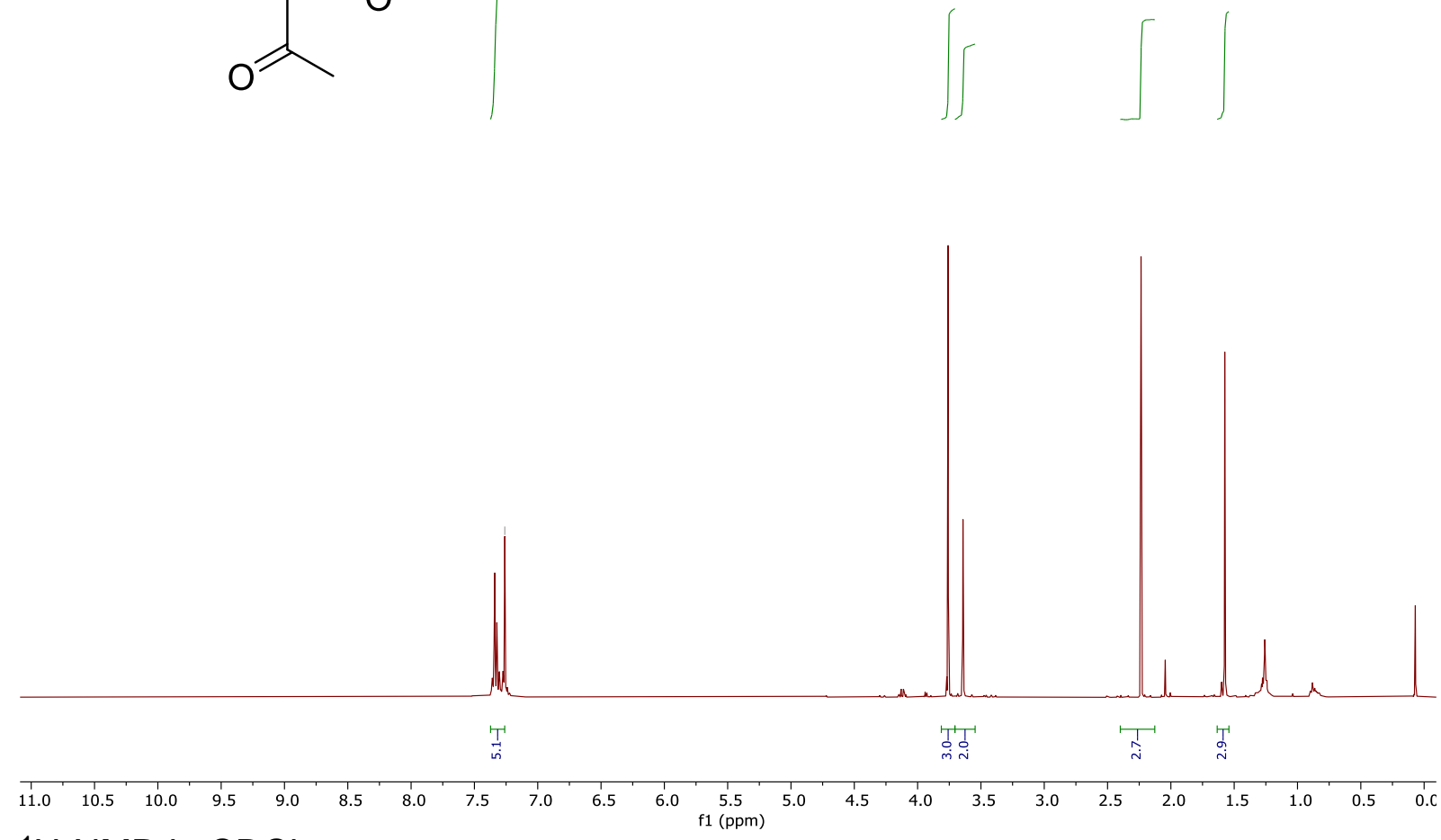

${ }^{1} \mathrm{H}-\mathrm{NMR}$ in $\mathrm{CDCl}_{3}$.<smiles>COC(=O)C(C)(NCc1ccccc1)C(C)=O</smiles>

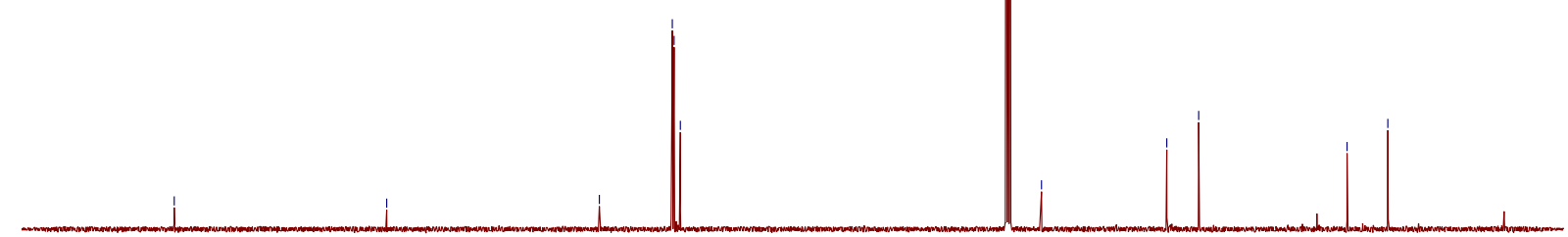

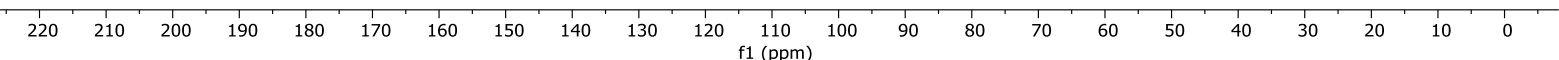
${ }^{13} \mathrm{C}-\mathrm{NMR}$ in $\mathrm{CDCl}_{3}$. 
$122 a$<smiles>CCc1cccc(CC)c1N/C(C)=C/C(C)=O</smiles>

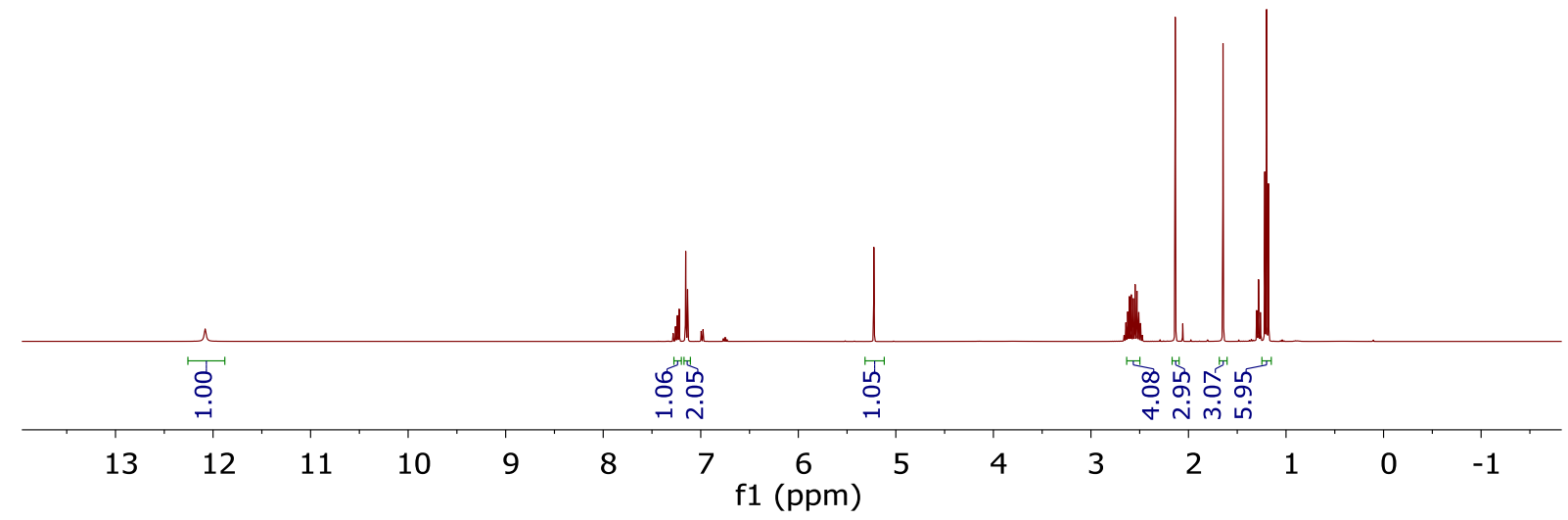

${ }^{1} \mathrm{H}-\mathrm{NMR}$ in $\mathrm{CDCl}_{3}$.

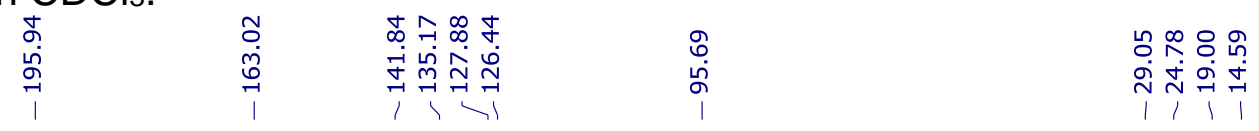<smiles>CCc1cccc(CC)c1N/C(C)=C/C(C)=O</smiles>

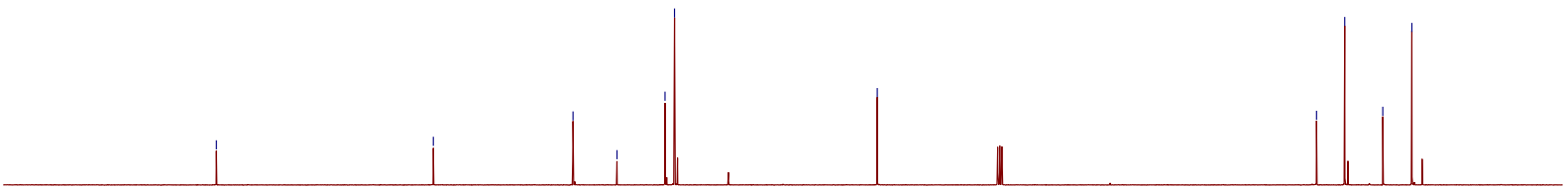

$220210200190180170160150140130120110100 \quad 90 \quad 80 \quad 70 \quad 60 \quad 50 \quad 40 \quad 30 \quad 20 \quad 10 \quad 0$ ${ }^{13} \mathrm{C}-\mathrm{NMR}$ in $\mathrm{CDCl}_{3}$. 
$123 a$<smiles>CC(=O)/C=C(\C)Nc1ccccc1I</smiles>

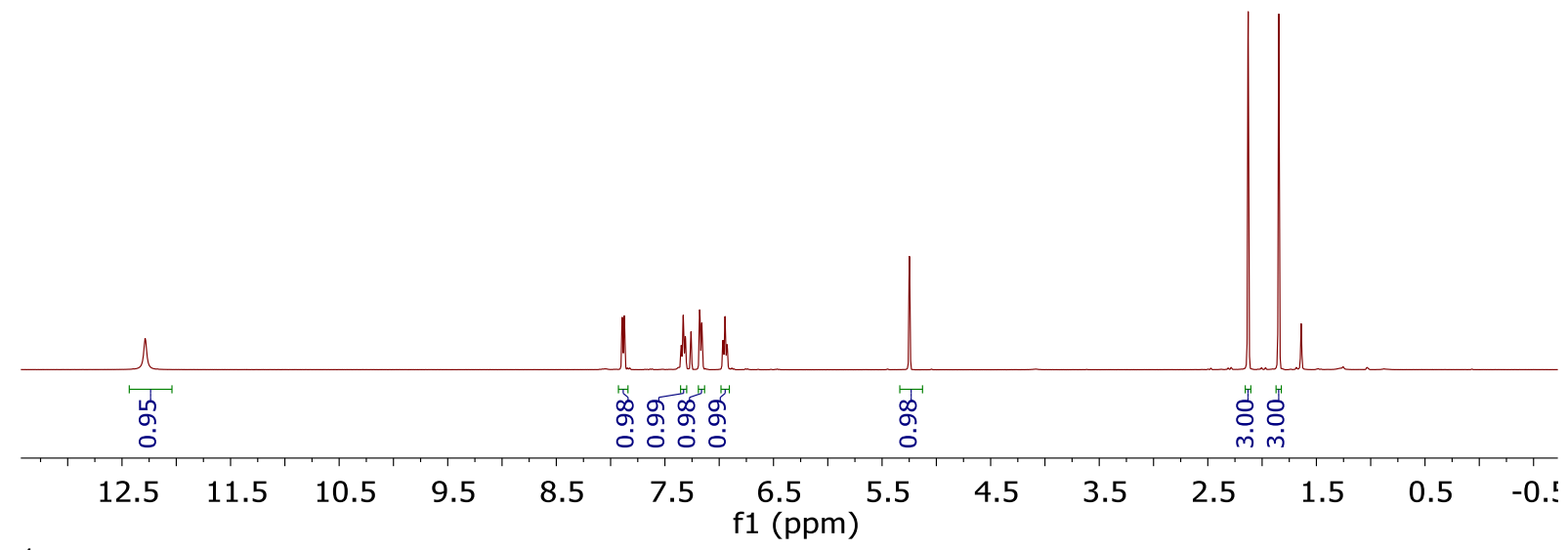

${ }^{1} \mathrm{H}-\mathrm{NMR}$ in $\mathrm{CDCl}_{3}$.

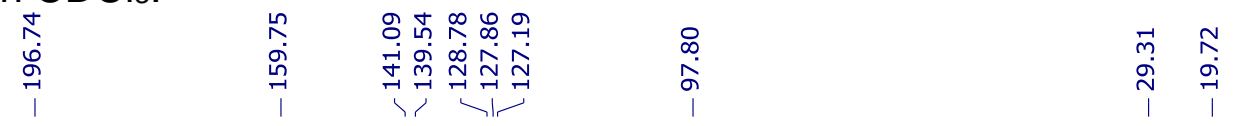<smiles>CC(=O)/C=C(\C)Nc1ccccc1I</smiles>

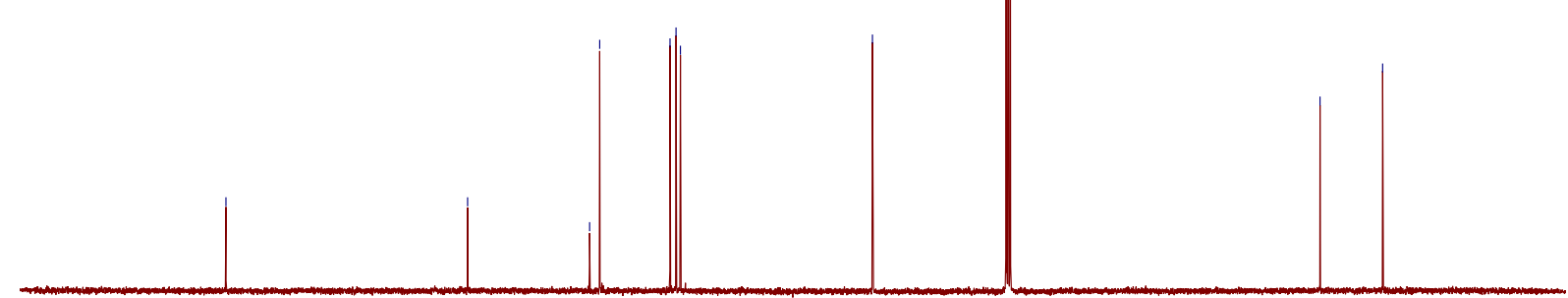

$220210200190180170160150140130120110100 \quad 90 \quad 80 \quad 70 \quad 60 \quad 50 \quad 40 \quad 30 \quad 20 \quad 10 \quad 0$ ${ }^{13} \mathrm{C}-\mathrm{NMR}$ in $\mathrm{CDCl}_{3}$. 
$124 a$

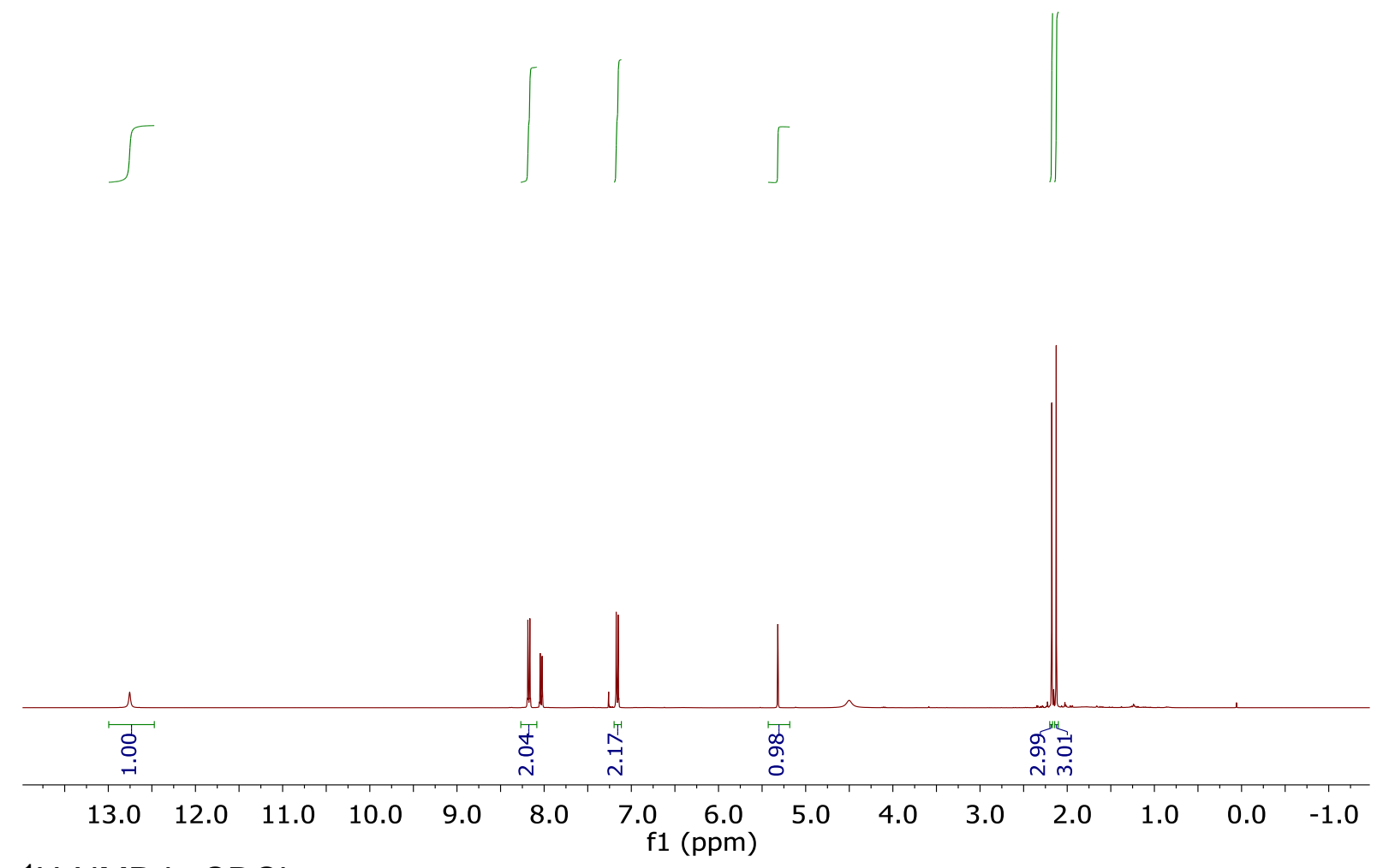

${ }^{1} \mathrm{H}-\mathrm{NMR}$ in $\mathrm{CDCl}_{3}$. 


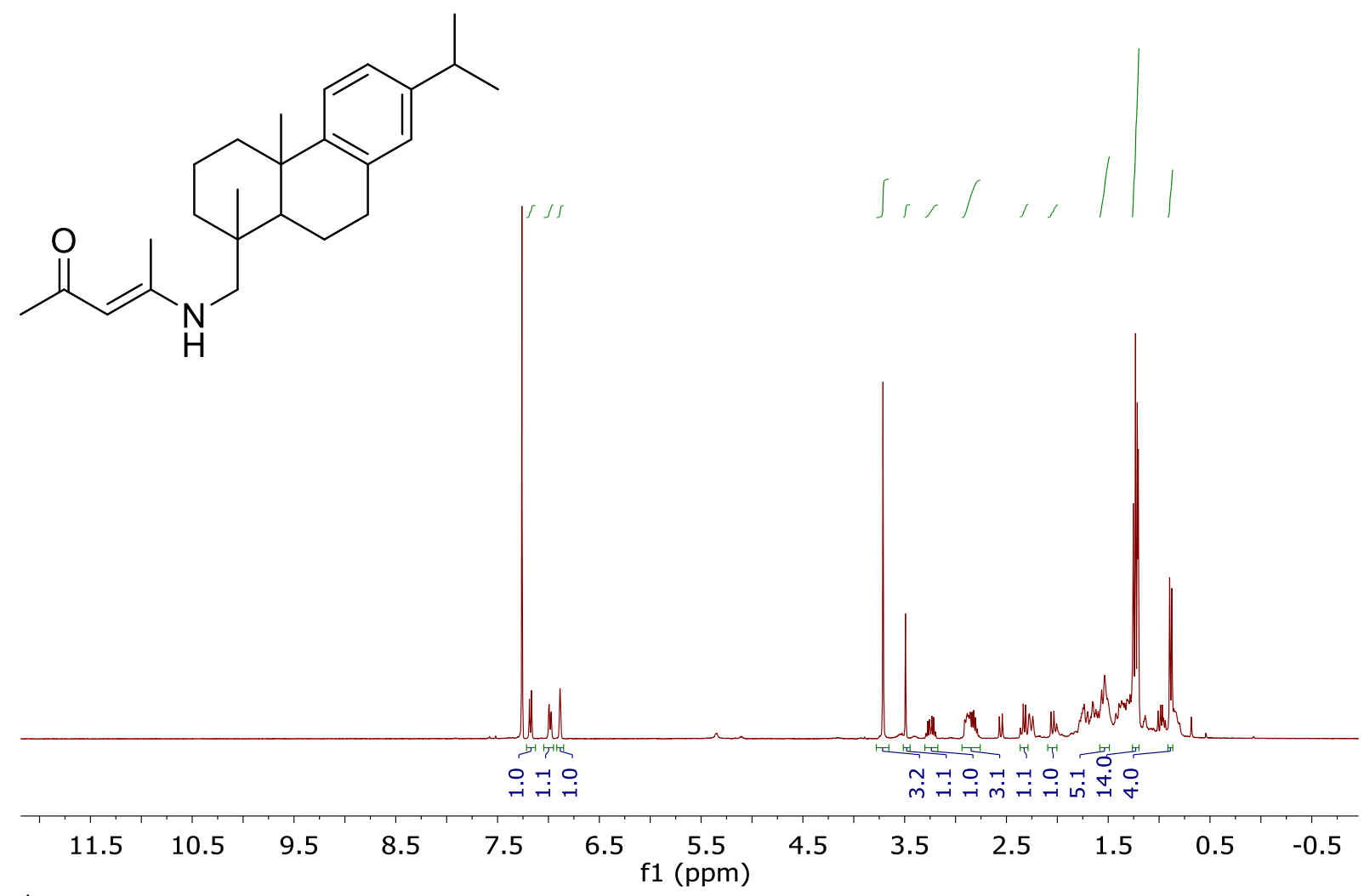

${ }^{1} \mathrm{H}-\mathrm{NMR}$ in $\mathrm{CDCl}_{3}$.

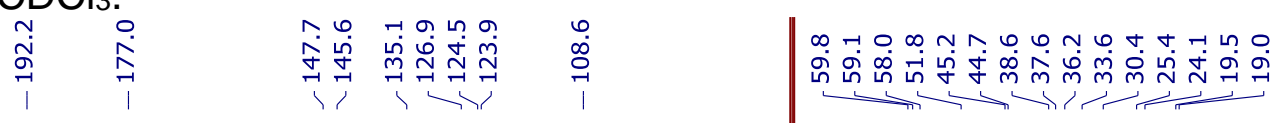<smiles>CC(=O)/C=C(\C)NCC1(C)CCCC2(C)c3ccc(C(C)C)cc3CCC12</smiles>

$220210200190180170160150140130120110100 \quad 90 \quad 80 \quad 70 \quad 60 \quad 50 \quad 40 \quad 30 \quad 20 \quad 10 \quad 0$ ${ }^{13} \mathrm{C}-\mathrm{NMR}$ in $\mathrm{CDCl}_{3}$. 
$128 \mathrm{a}$<smiles>CC(=O)/C=C(\C)NCCc1c[nH]c2ccccc12</smiles>

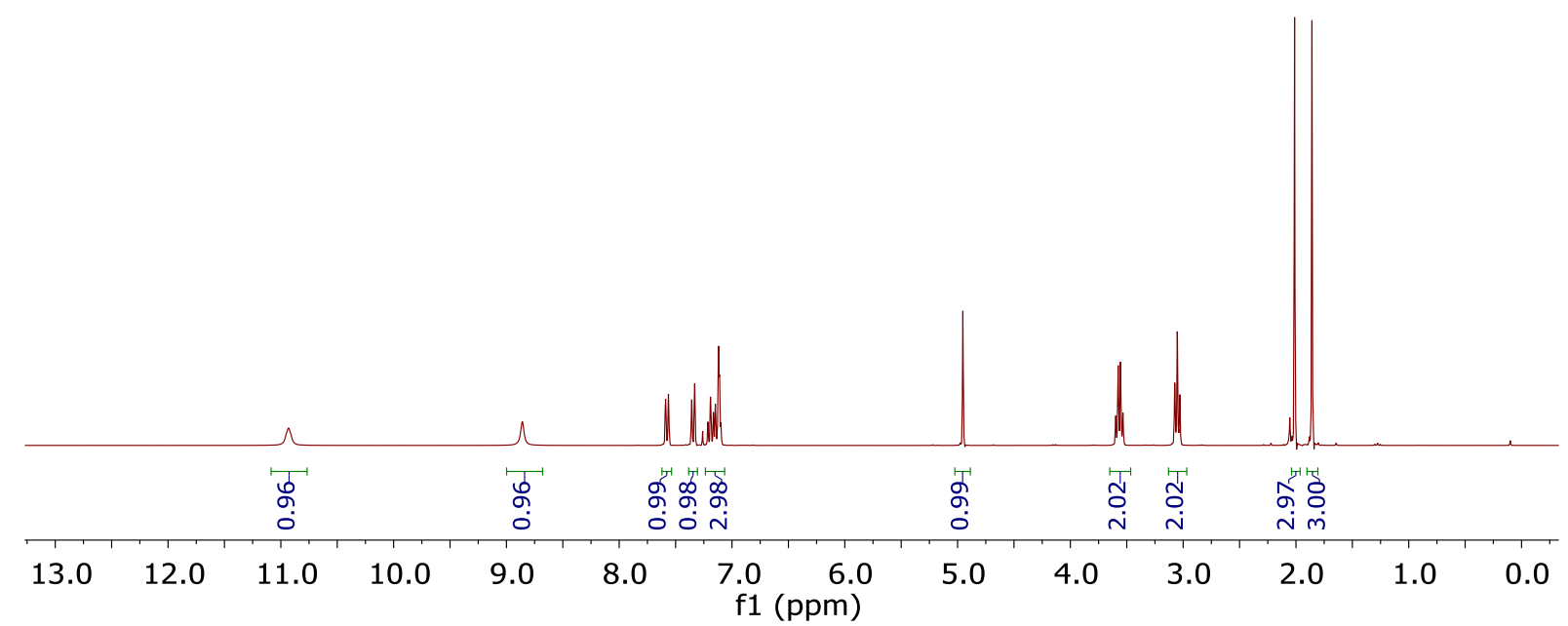
${ }^{1} \mathrm{H}-\mathrm{NMR}$ in $\mathrm{CDCl}_{3}$.

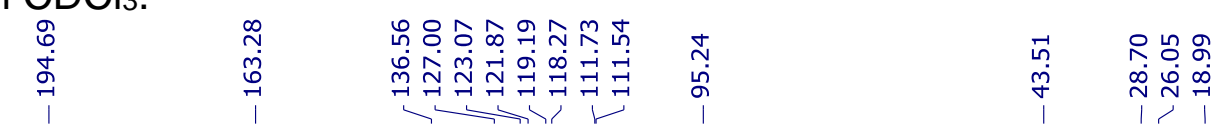<smiles>CC(=O)/C=C(\C)NCCc1c[nH]c2ccccc12</smiles>
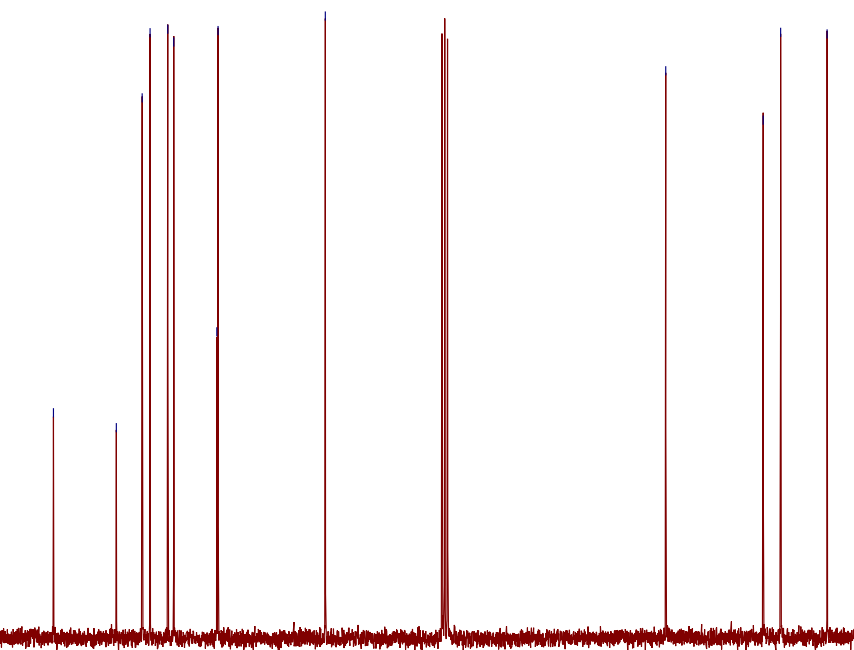

$30220210200190180170160150140130120110100 \quad 90 \quad 80 \quad 70 \quad 60 \quad 50 \quad 40 \quad 30 \quad 20 \quad 10 \quad 0$ ${ }^{13} \mathrm{C}-\mathrm{NMR}$ in $\mathrm{CDCl}_{3}$. f1 (ppm) 
$129 a$

$\mathrm{HO}$<smiles>CC(=O)/C=C(\C)N1CCC(C)CC1</smiles>
$\int$

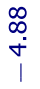

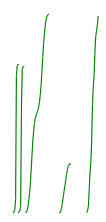

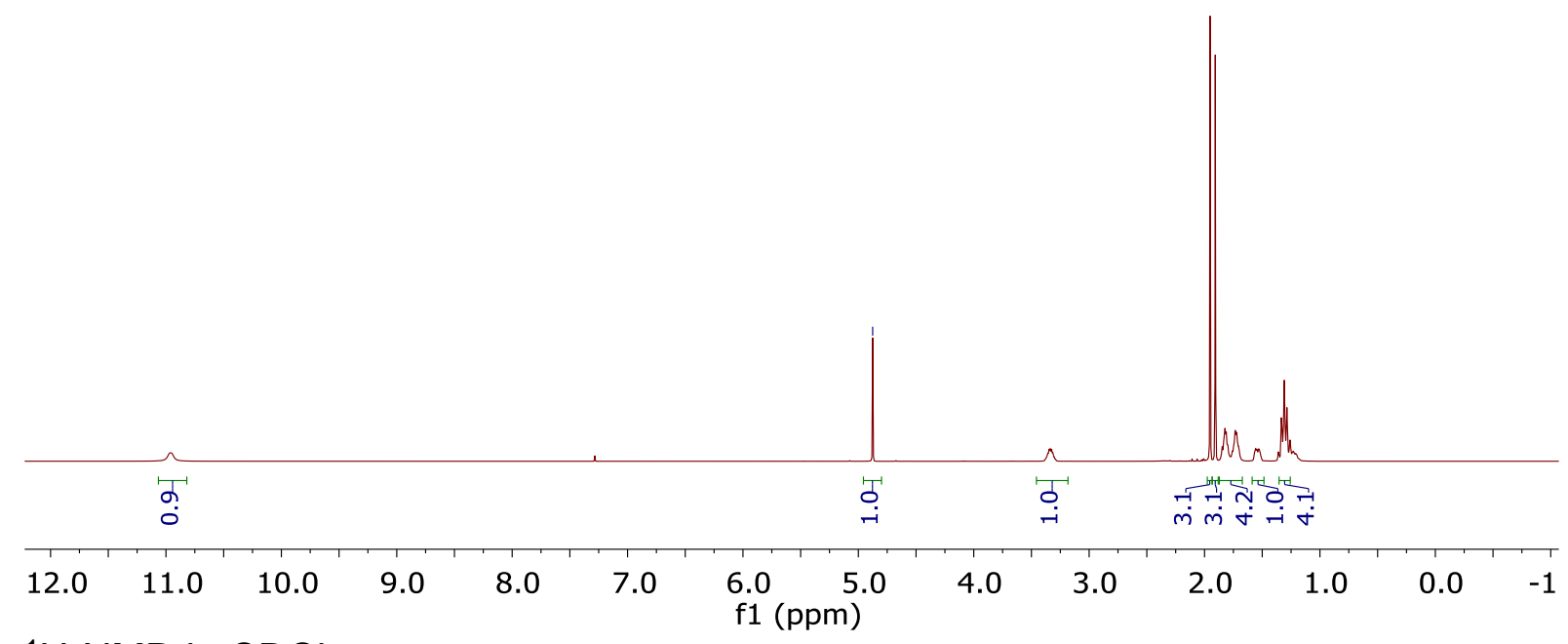

${ }^{1} \mathrm{H}-\mathrm{NMR}$ in $\mathrm{CDCl}_{3}$.

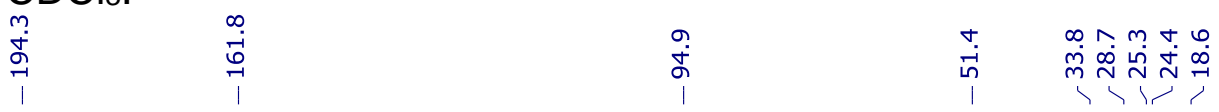<smiles>CC(=O)/C=C(\C)N1CCC(O)CC1</smiles>

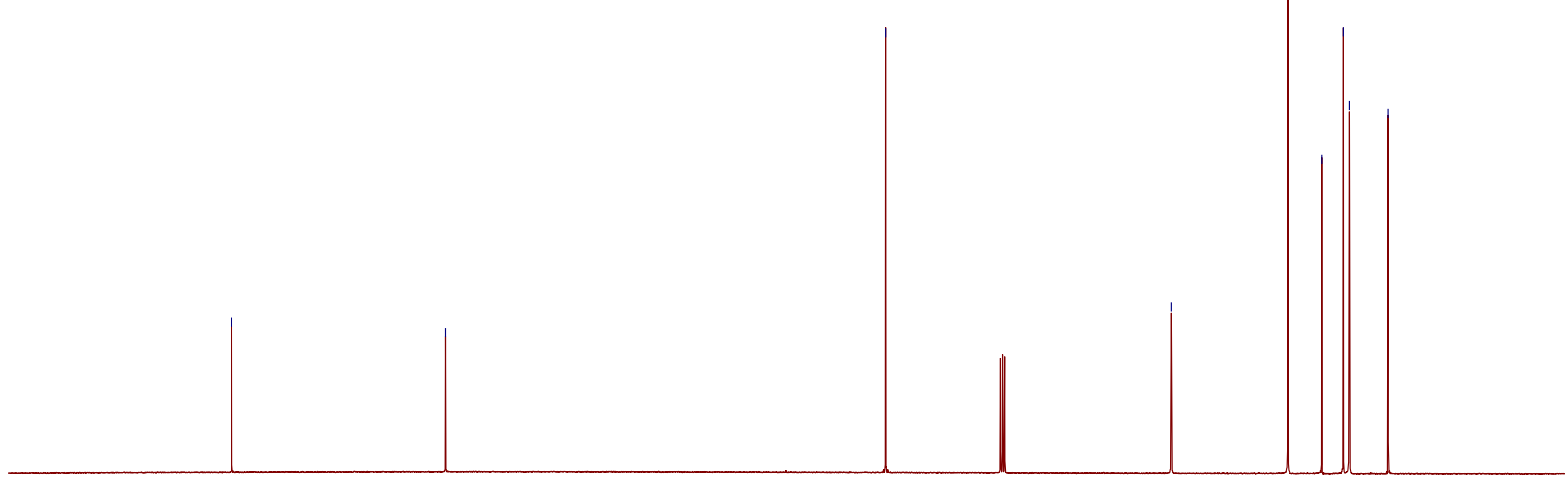

$220210200190180170160150140130120110100 \quad 90 \quad 80 \quad 70 \quad 60 \quad 50 \quad 40 \quad 30 \quad 20 \quad 10 \quad 0$ ${ }^{13} \mathrm{C}-\mathrm{NMR}$ in $\mathrm{CDCl}_{3}$.

f1 (ppm) 

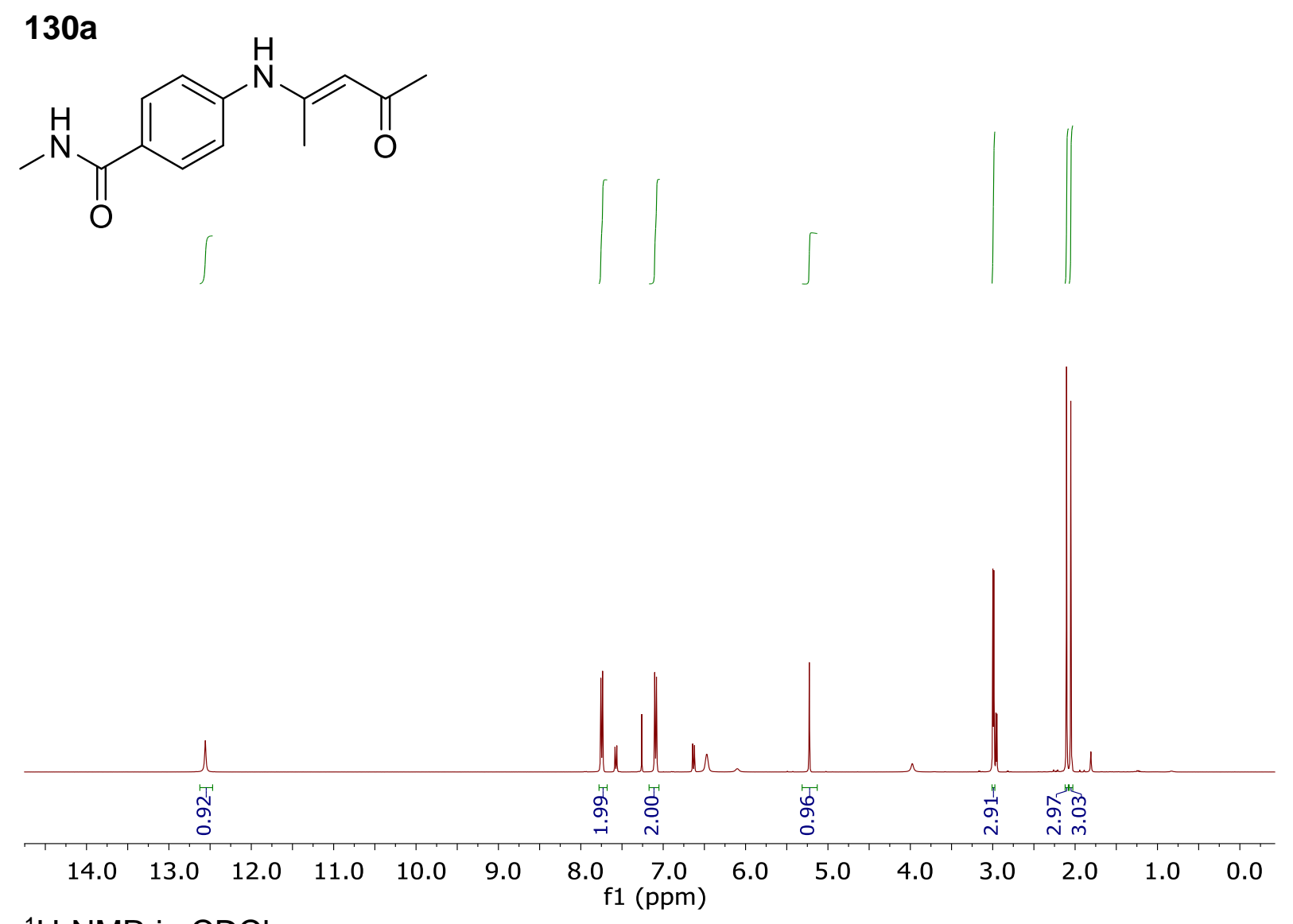
${ }^{1} \mathrm{H}-\mathrm{NMR}$ in $\mathrm{CDCl}_{3}$.

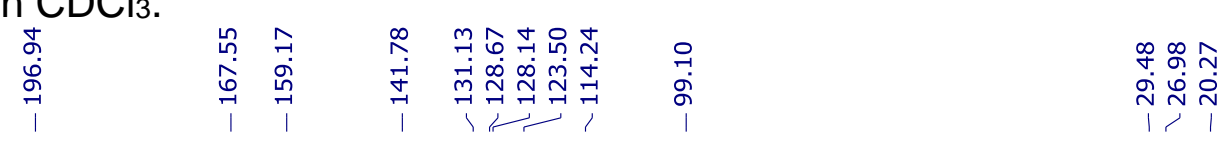<smiles>CNC(=O)c1ccc(N/C(C)=C/C(C)=O)cc1</smiles>

$220210200190180170160150140130120110100 \quad 90 \quad 80 \quad 70 \quad 60 \quad 50 \quad 40 \quad 30 \quad 20 \quad 10 \quad 0$ ${ }^{13} \mathrm{C}-\mathrm{NMR}$ in $\mathrm{CDCl}_{3}$. 


\section{$131 a$}<smiles>CCOC(=O)/C=C/c1ccc(N/C(C)=C/C(C)=O)cc1</smiles>

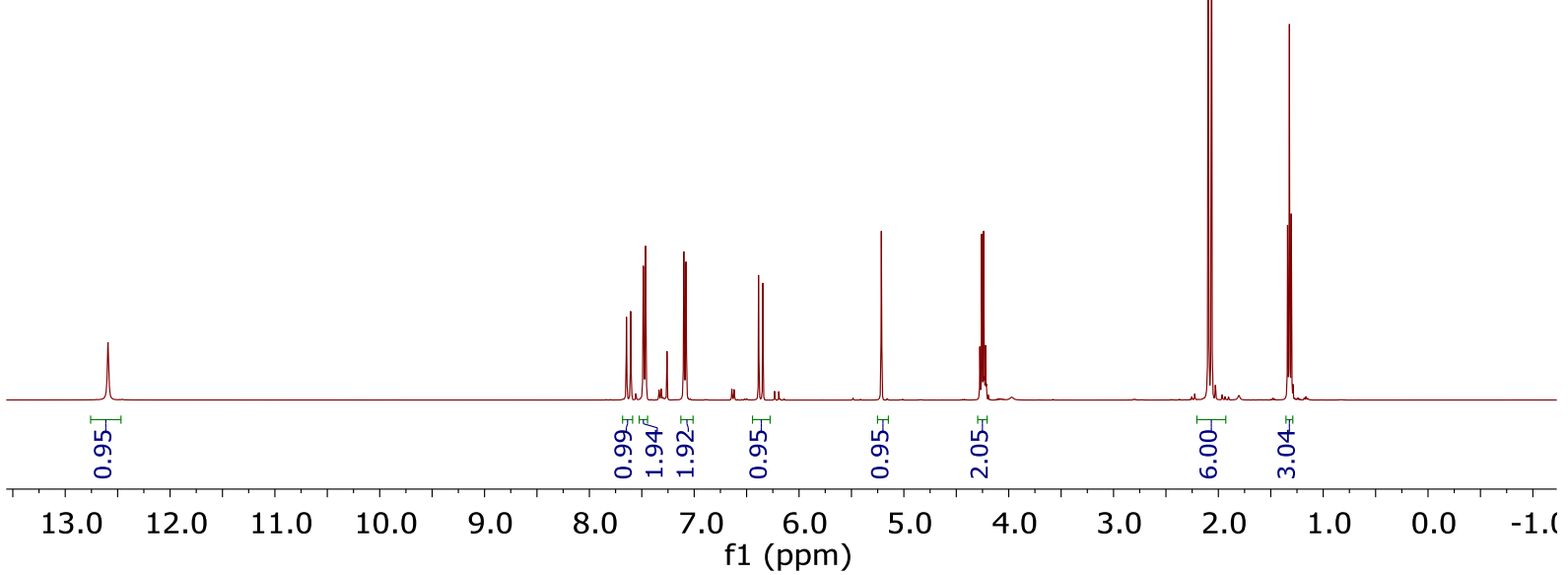

${ }^{1} \mathrm{H}-\mathrm{NMR}$ in $\mathrm{CDCl}_{3}$.

\begin{tabular}{|c|c|c|c|c|c|}
\hline 0 & $\begin{array}{ll}0 & 0 \\
0 & \circ \\
\hat{0} & \infty \\
0 & \stackrel{0}{0} \\
1 & 1\end{array}$ & 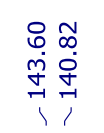 & 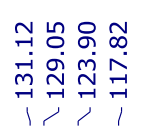 & $\begin{array}{l}0 \\
\stackrel{0}{0} \\
\text { Oे }\end{array}$ & $\begin{array}{l}\vec{b} \\
\dot{0} \\
1\end{array}$ \\
\hline
\end{tabular}<smiles>CCOC(=O)/C=C/c1ccc(N/C(C)=C/C(C)=O)cc1</smiles>

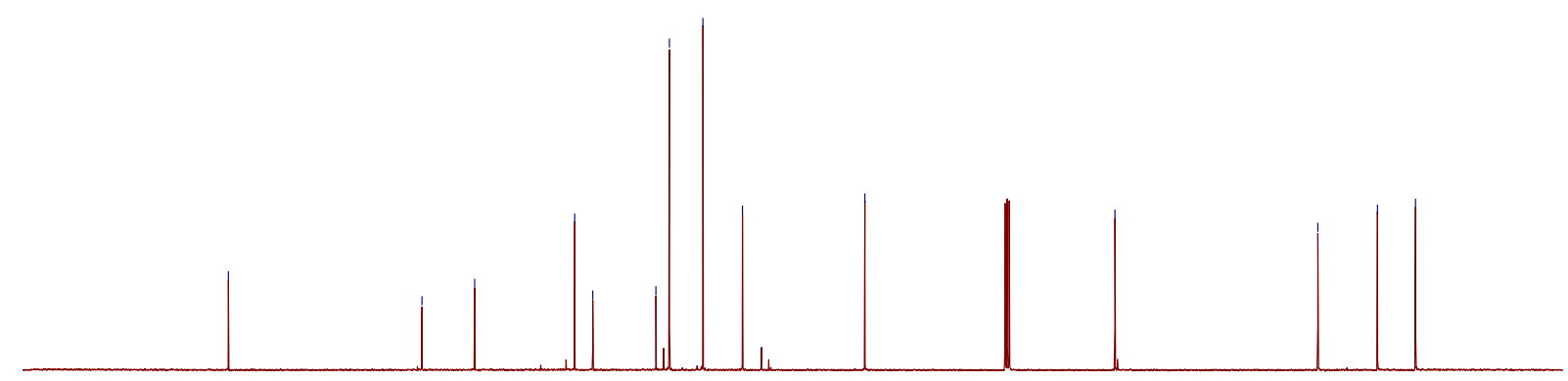

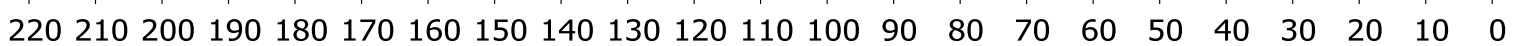
${ }^{13} \mathrm{C}-\mathrm{NMR}$ in $\mathrm{CDCl}_{3}$. 
$132 a$<smiles>CCCCC(=O)/C=C(\C)Nc1ccccc1</smiles>
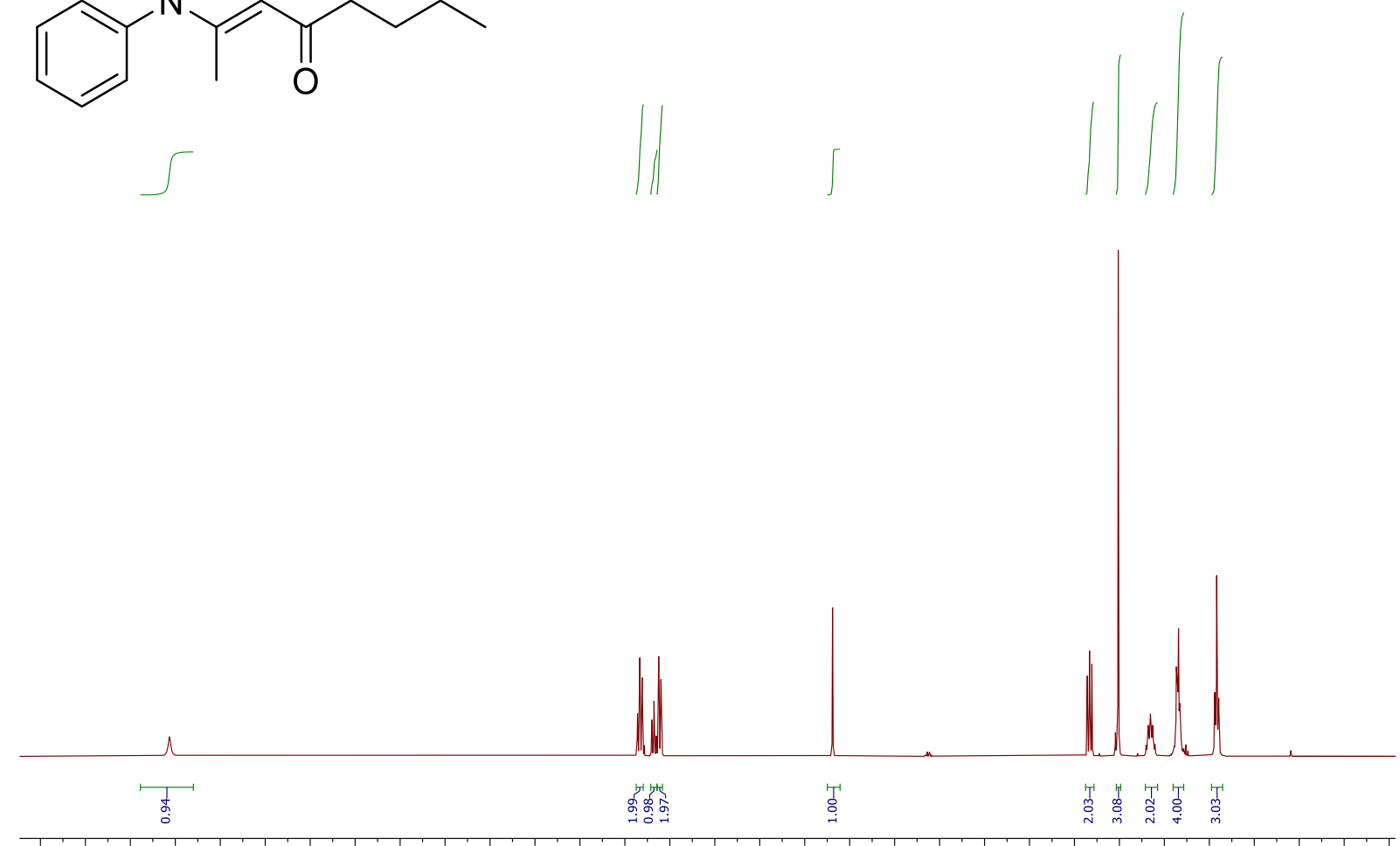

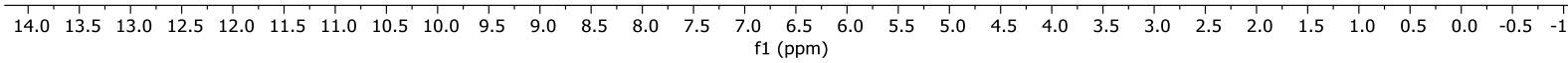
${ }^{1} \mathrm{H}-\mathrm{NMR}$ in $\mathrm{CDCl}_{3}$.<smiles>CCCCC(=O)/C=C(\C)Nc1ccccc1</smiles>

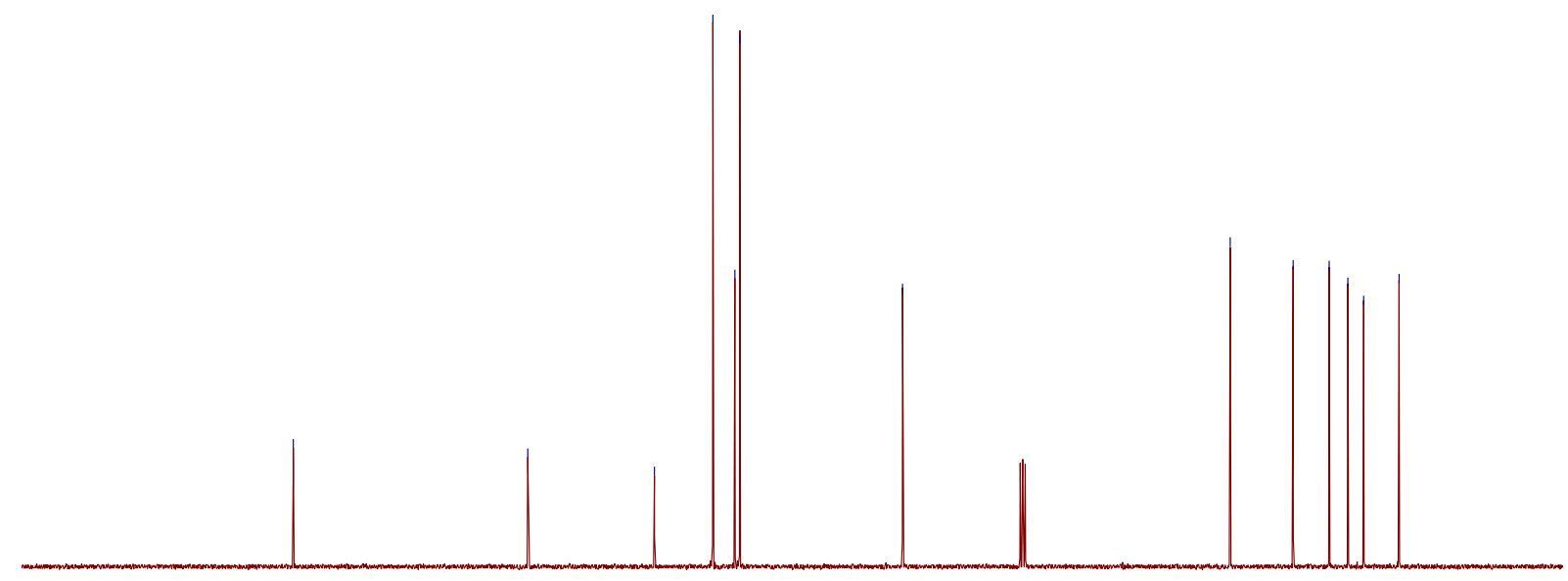

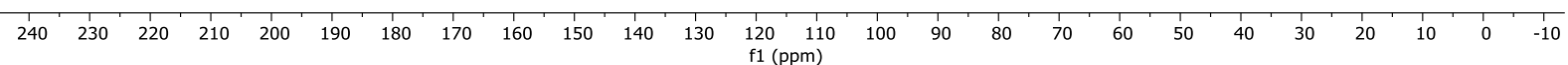
${ }^{13} \mathrm{C}-\mathrm{NMR}$ in $\mathrm{CDCl}_{3}$. 
$132 b$<smiles>CCCCCCC(=O)C(C)(Nc1ccccc1)C(=O)OC</smiles>

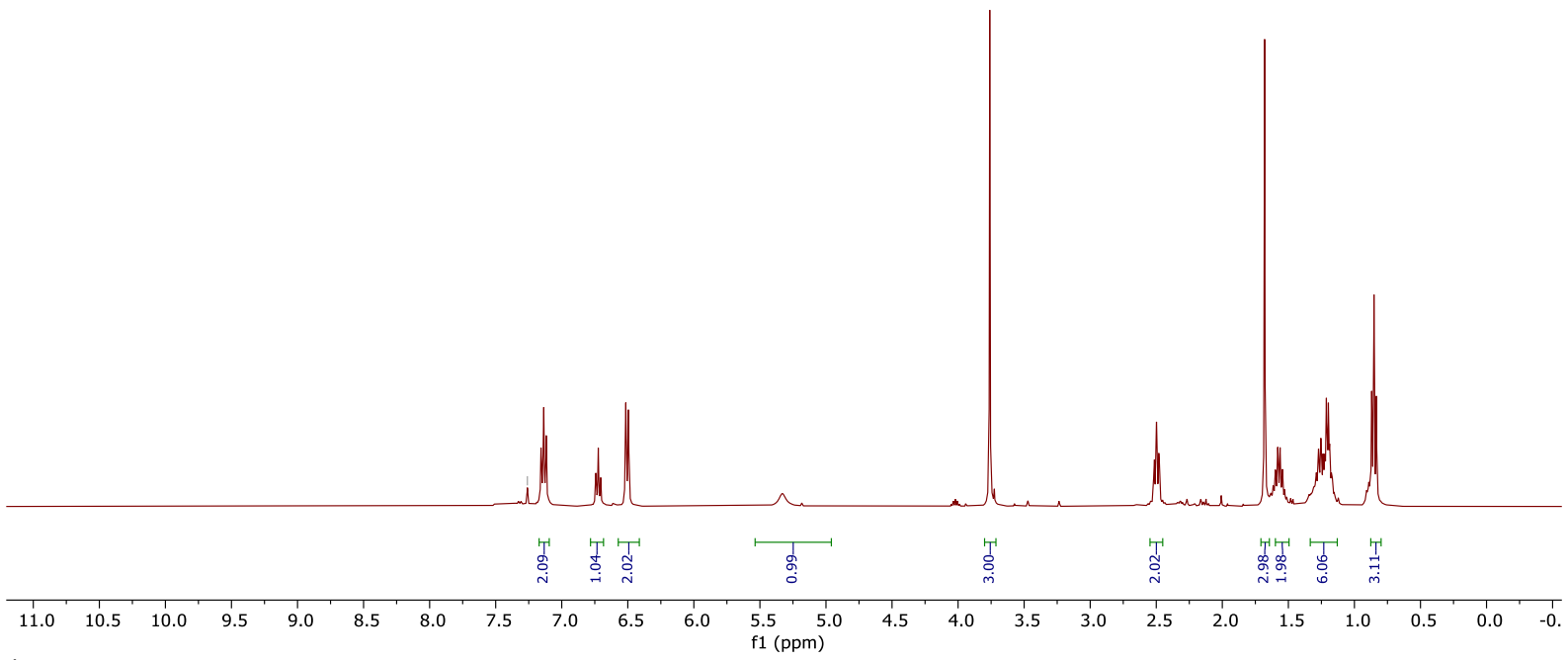

${ }^{1} \mathrm{H}-\mathrm{NMR}$ in $\mathrm{CDCl}_{3}$.

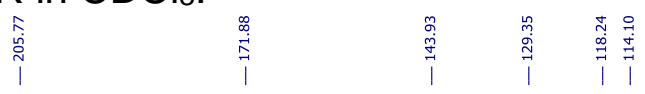<smiles>CCCCCCC(=O)C(C)(Nc1ccccc1)C(=O)OC</smiles>
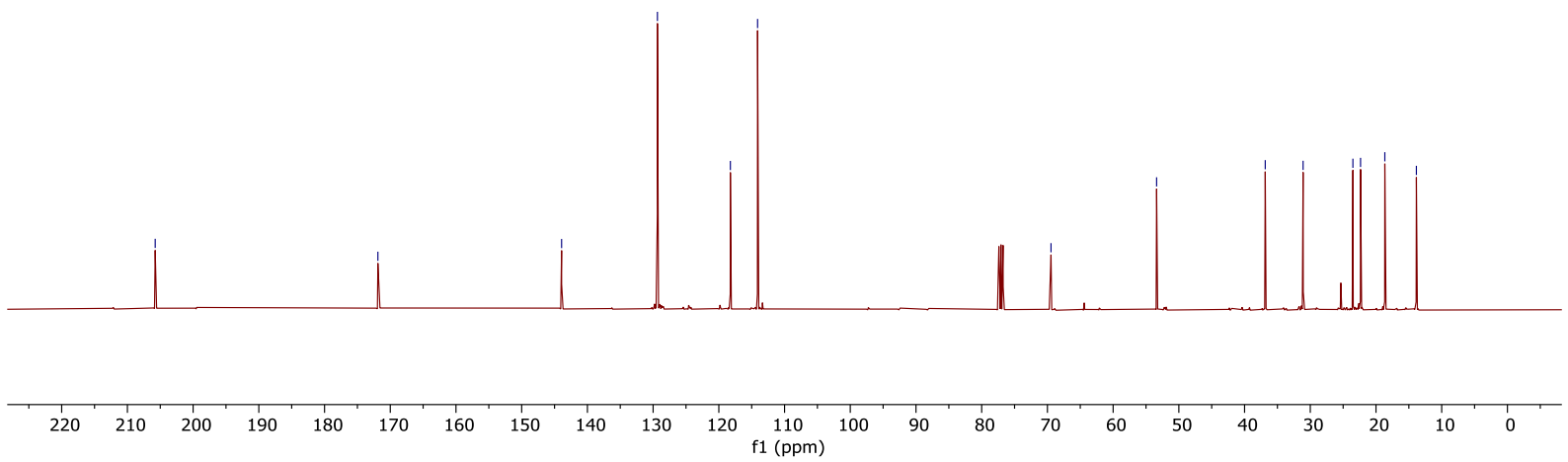

${ }^{13} \mathrm{C}-\mathrm{NMR}$ in $\mathrm{CDCl}_{3}$. 
$133 a$<smiles>CCC(=O)/C=C(\CC)Nc1ccccc1</smiles>

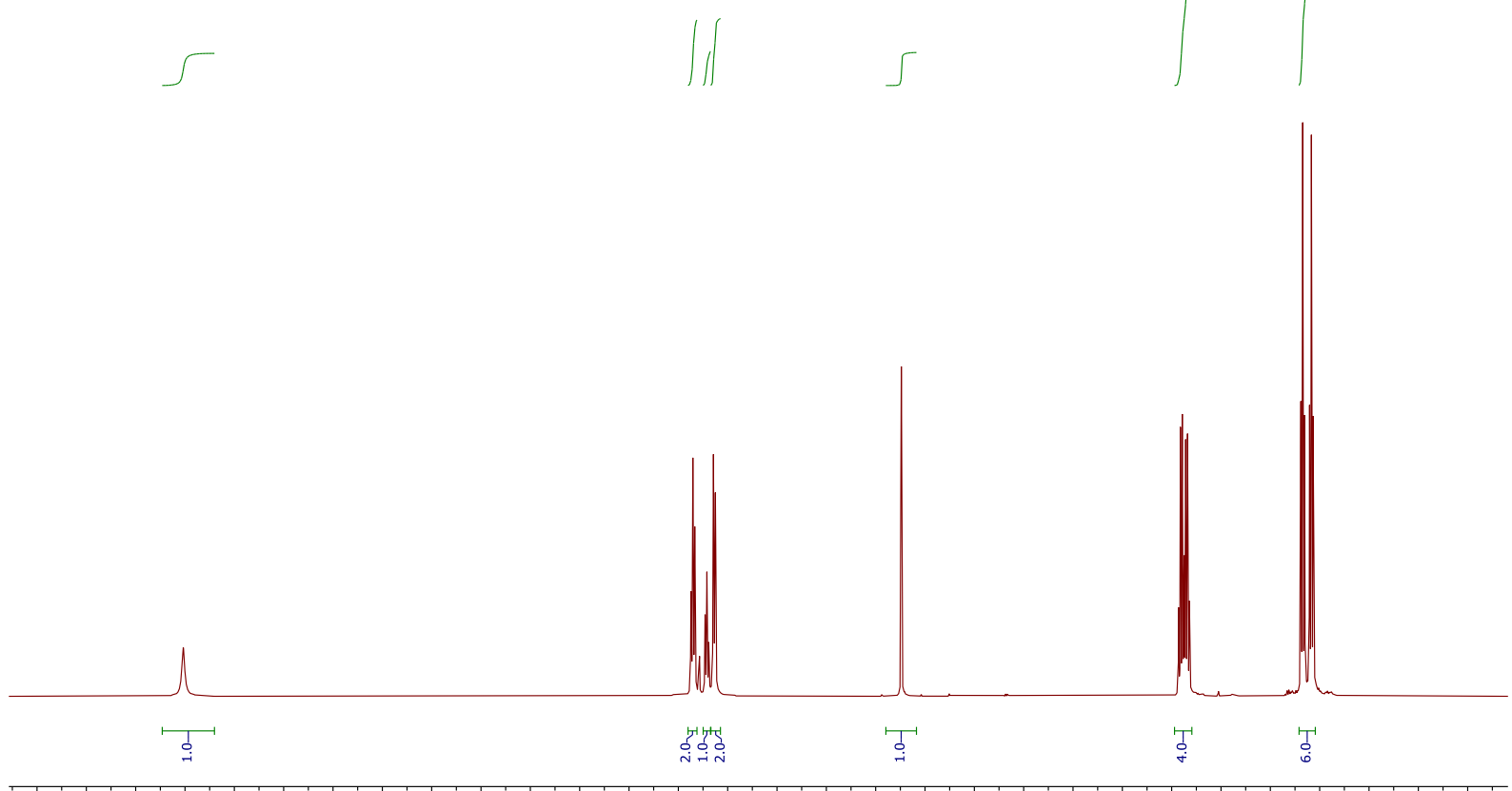

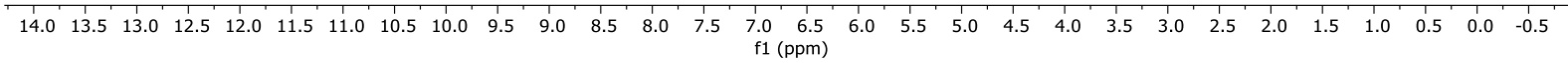
${ }^{1} \mathrm{H}-\mathrm{NMR}$ in $\mathrm{CDCl}_{3}$.

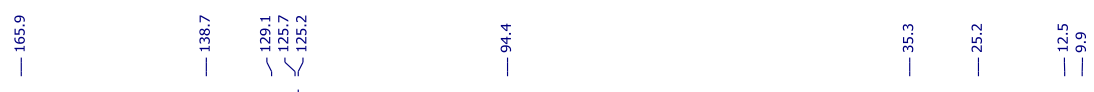<smiles>CCC(=O)/C=C(\CC)Nc1ccccc1</smiles>

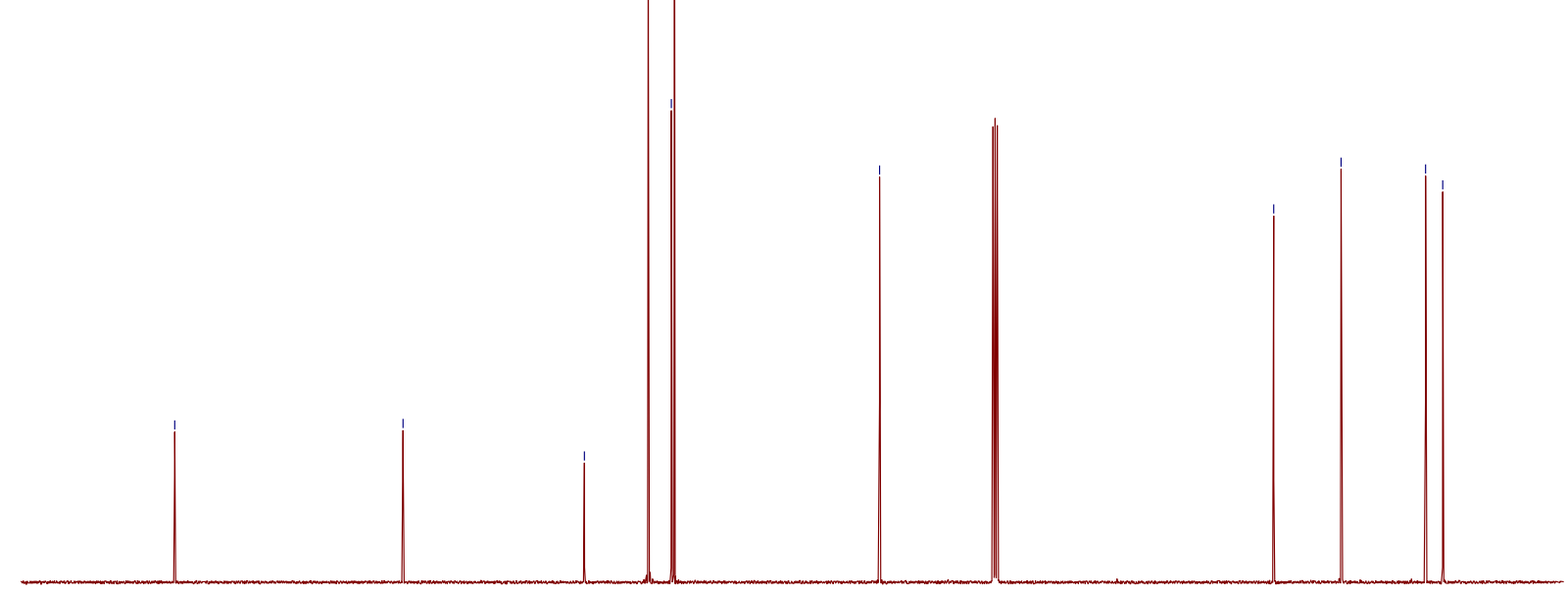

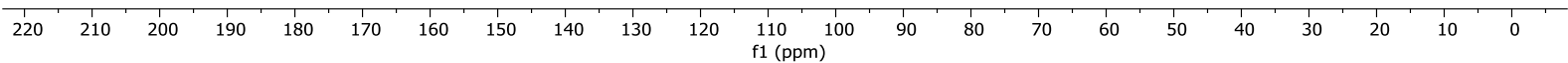
${ }^{13} \mathrm{C}-\mathrm{NMR}$ in $\mathrm{CDCl}_{3}$. 
$133 b$<smiles>CCC(=O)C(Nc1ccccc1)(C(=O)CC)C(=O)OC</smiles>
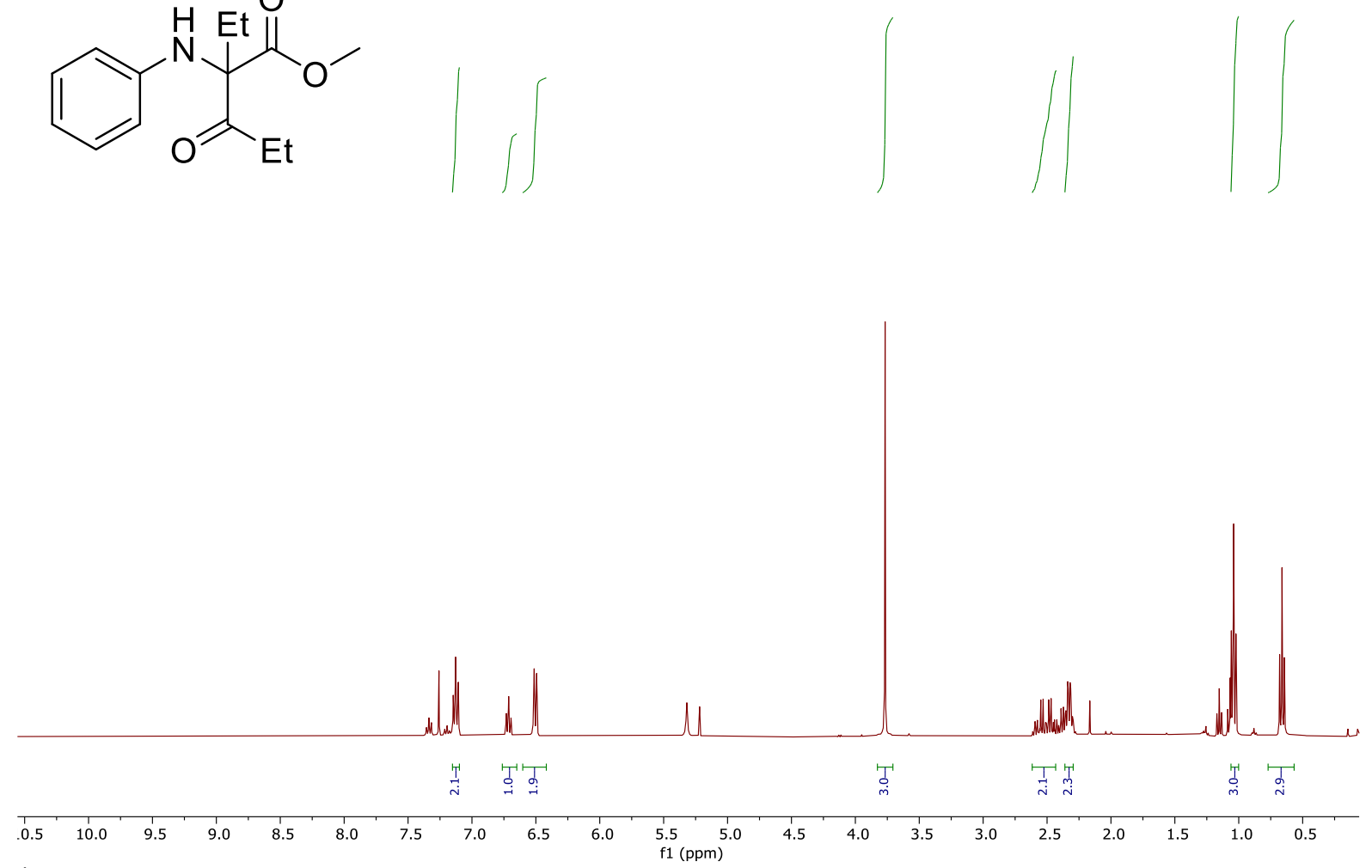

${ }^{1} \mathrm{H}-\mathrm{NMR}$ in $\mathrm{CDCl}_{3}$.

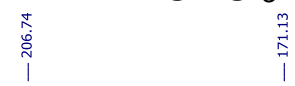<smiles>CCC(=O)C(Nc1ccccc1)(C(=O)CC)C(=O)OC</smiles>

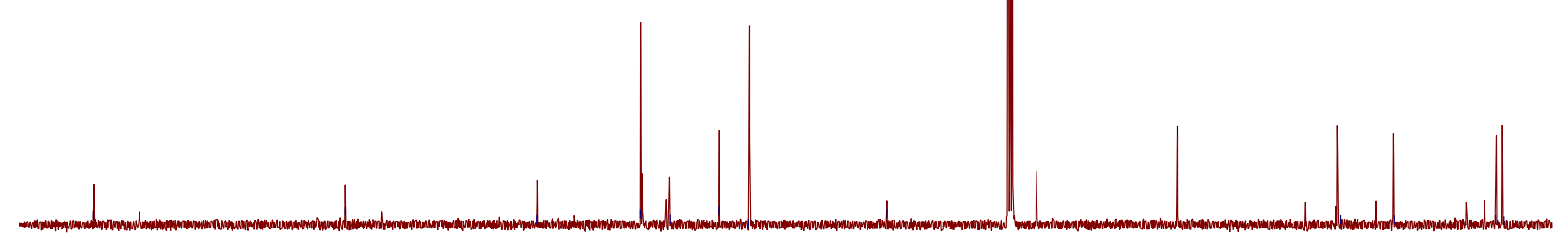

$\begin{array}{llll}210 & 200 & 190 & 180\end{array}$

${ }^{13} \mathrm{C}-\mathrm{NMR}$ in $\mathrm{CDCl}_{3}$. 
$134 a$<smiles>CC(=O)/C(Cl)=C(\C)Nc1ccccc1</smiles>
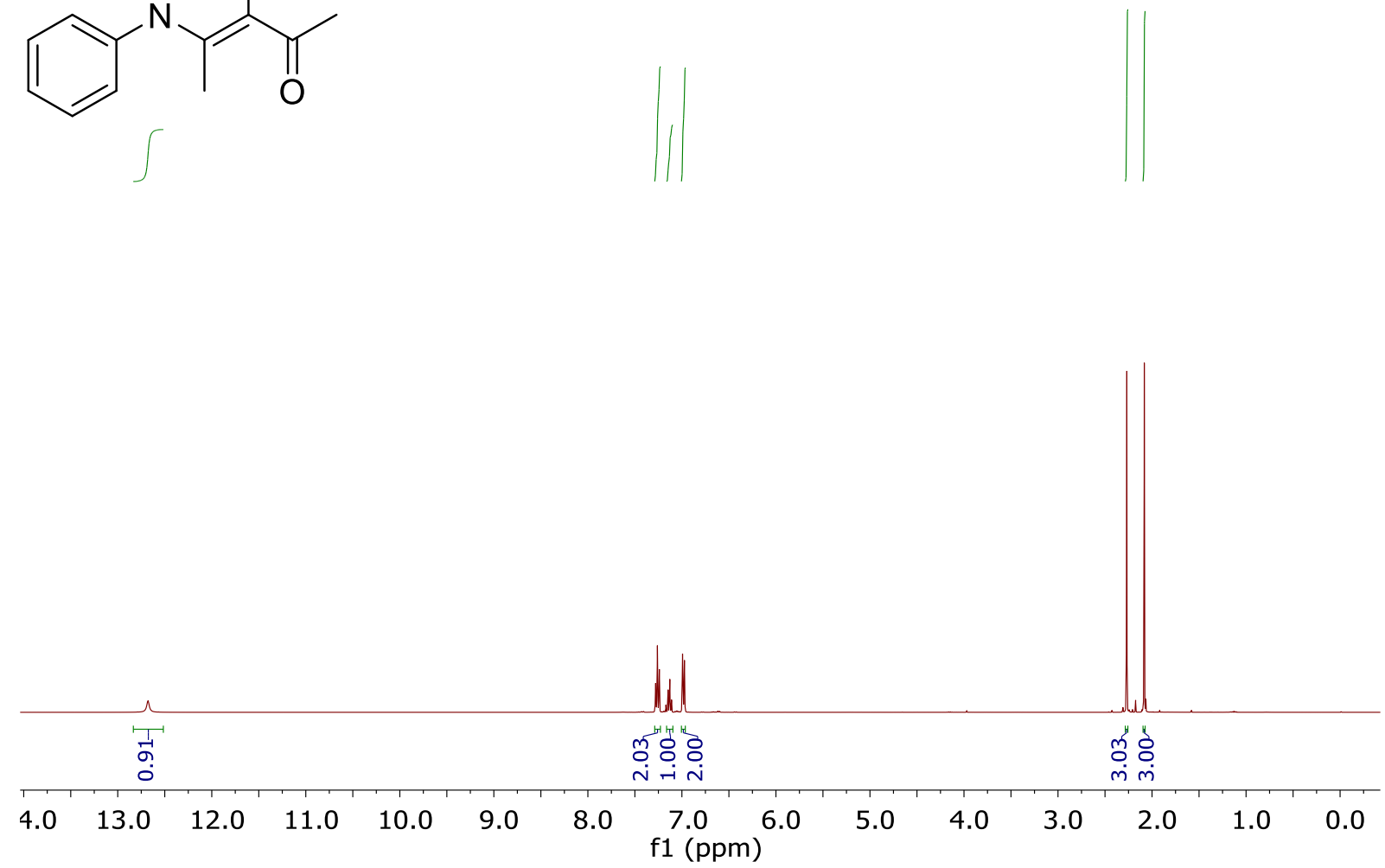

${ }^{1} \mathrm{H}-\mathrm{NMR}$ in $\mathrm{CDCl}_{3}$.

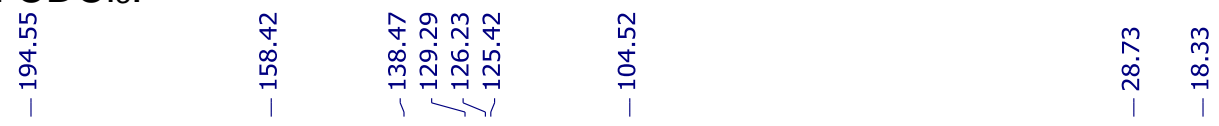<smiles>CC(=O)/C(Cl)=C(\C)Nc1ccccc1</smiles>

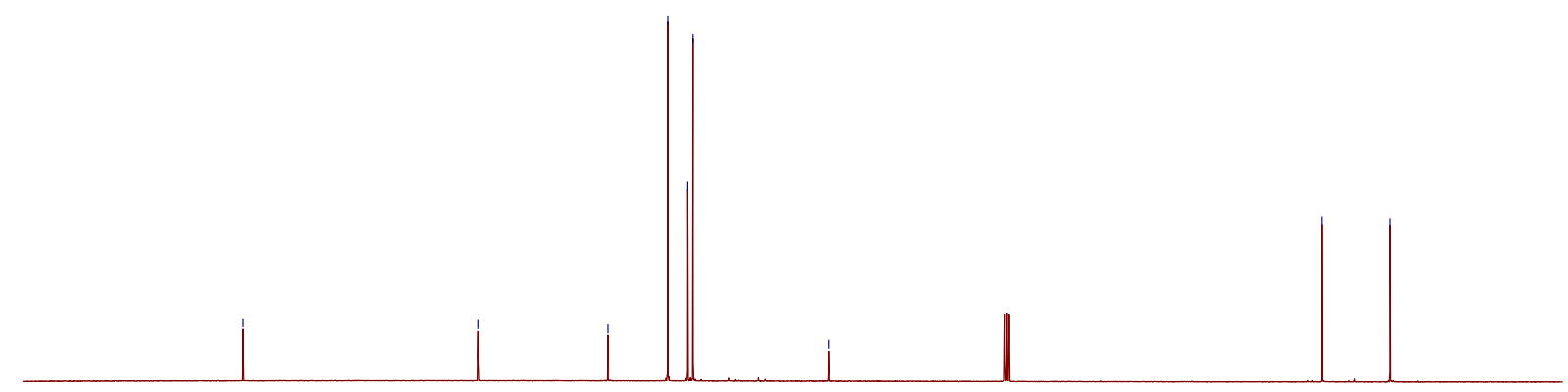

$220210200190180170160150140130120110100 \quad 90 \quad 80 \quad 70 \quad 60 \quad 50 \quad 40 \quad 30 \quad 20 \quad 10 \quad 0$ ${ }^{13} \mathrm{C}-\mathrm{NMR}$ in $\mathrm{CDCl}_{3}$. 


\section{Promovierenden-Erklärung}

\section{der Georg-August-Universität Göttingen Name}

Name: Schilling, Waldemar

Anschrift: Albrecht-Thaer-Weg 8A, 37075 Göttingen

Ich gebe folgende Erklärung ab:

1. Die Gelegenheit zum vorliegenden Promotionsvorhaben ist mir nicht kommerziell vermittelt worden. Insbesondere habe ich keine Organisation eingeschaltet, die gegen Entgelt Betreuerinnen und Betreuer für die Anfertigung von Dissertationen sucht oder die mir obliegenden Pflichten hinsichtlich der Prüfungsleistungen für mich ganz oder teilweise erledigt.

2. Hilfe Dritter wurde bis jetzt und wird auch künftig nur in wissenschaftlich vertretbarem und prüfungsrechtlich zulässigem Ausmaß in Anspruch genommen. Insbesondere werden alle Teile der Dissertation selbst angefertigt; unzulässige fremde Hilfe habe ich dazu weder unentgeltlich noch entgeltlich entgegengenommen und werde dies auch zukünftig so halten.

3. Die Ordnung zur Sicherung der guten wissenschaftlichen Praxis an der Universität Göttingen wird von mir beachtet.

4. Eine entsprechende Promotion wurde an keiner anderen Hochschule im In-oder Ausland beantragt; die eingereichte Dissertation oder Teile von ihr wurden/werden nicht für ein anderes Promotionsvorhaben verwendet. Mir ist bekannt, dass unrichtige Angaben die Zulassung zur Promotion ausschließen bzw. später zum Verfahrensabbruch oder zur Rücknahme des erlangten Grades führen können.

Göttingen, 01.02 .0221 